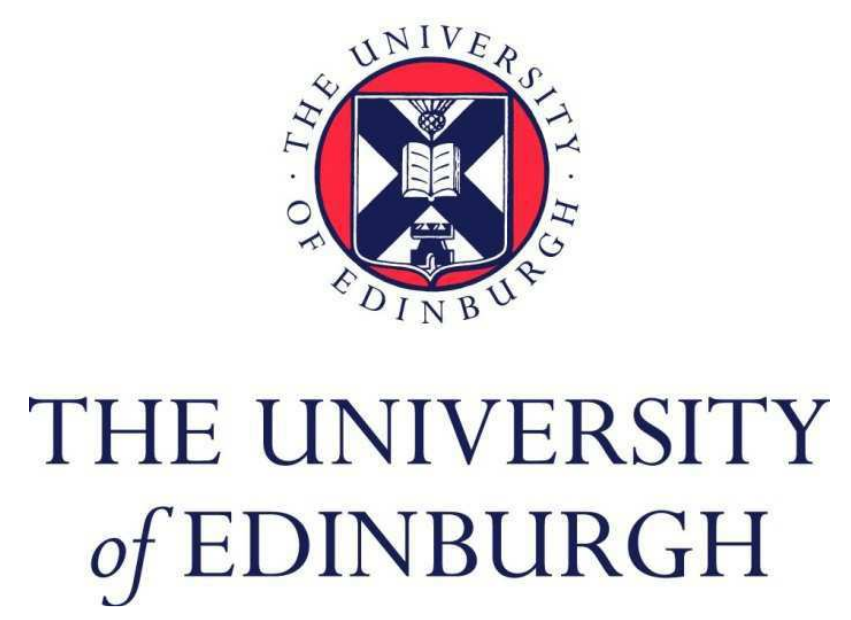

This thesis has been submitted in fulfilment of the requirements for a postgraduate degree (e.g. PhD, MPhil, DClinPsychol) at the University of Edinburgh. Please note the following terms and conditions of use:

This work is protected by copyright and other intellectual property rights, which are retained by the thesis author, unless otherwise stated.

A copy can be downloaded for personal non-commercial research or study, without prior permission or charge.

This thesis cannot be reproduced or quoted extensively from without first obtaining permission in writing from the author.

The content must not be changed in any way or sold commercially in any format or medium without the formal permission of the author.

When referring to this work, full bibliographic details including the author, title, awarding institution and date of the thesis must be given. 


\section{The Value of Electrical Energy Storage: A Comparison between Commercial and System Level Benefits}

Anna Dunbar

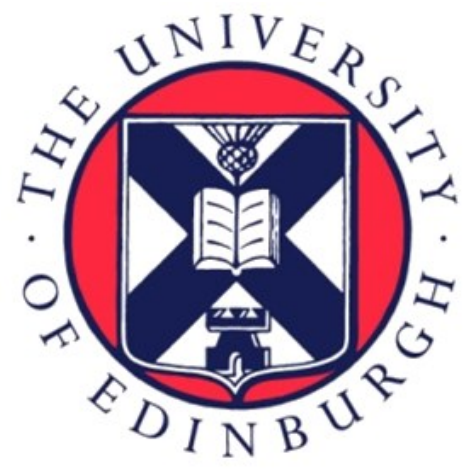

A thesis submitted for the degree of Doctor of Philosophy

The University of Edinburgh

2016 


\section{Lay Summary}

There is a drive to transform the electricity industry from one based largely on fossil fuels to one based on low or zero carbon sources. As a result, the amount of wind power connected to the electricity grid is expected to increase. Wind power is sustainable and affordable, but it is also variable; its output depends on the weather and it cannot be easily controlled. Electricity storage, which works like a rechargeable battery, is a potential solution to this challenge. Storage systems can be charged up during periods when it is windy and too much electricity is produced. Energy can then be released when there is demand for electricity but no wind.

Storage at this scale is expensive and there are a number of regulatory issues with liberalised electricity markets which prevent it from accessing revenue streams. One way storage can earn money is by trading electricity; buying when demand is low and the price cheap, and selling during peak hours when the price is high; however, electricity prices are volatile and future prices are highly uncertain. Furthermore, increasing wind power has the effect of suppressing peak electricity prices and, as a result, reduces opportunities for energy trading. Despite being more valuable, storage could make less money when more wind power is installed. The policy implications of this research indicate a failure in the electricity market in rewarding the true value of electrical energy storage. 


\section{Abstract}

There is a drive to transform the electricity industry in the UK from one based largely on fossil fuels to one based on low or zero carbon sources. The challenge of this transition, enabling a secure and sustainable electricity industry at an acceptable cost to consumers, has been dubbed the Energy Trilemma. Grid-connected electrical energy storage presents a potential solution to this challenge. However, the benefits of storage are split across different sectors of the electricity industry and there are a number of regulatory barriers preventing access to revenue streams. One accessible revenue stream is energy trading or price arbitrage. In current market conditions, arbitrage cannot provide sufficient revenue for electricity storage to cover its capital costs; however, some studies have suggested that with increased penetration of intermittent renewable power, electricity price volatility will increase enabling storage to become commercially viable through price arbitrage alone.

This thesis examines the hypothesis that: Increased wind penetration leads to increased commercial opportunities for energy storage through price arbitrage. A linear programme is used to define the optimum operating strategy for a storage device, subject to the constraints of maximum storage capacity, charging and discharging rates, conversion efficiency and selfdischarge. Initially, historic electricity prices from the British electricity market are used to investigate the value of storage with a low penetration of intermittent wind power. The results show that revenue is dependent on storage characteristics, with the performance of different technologies varying substantially. Furthermore, revenue is highly dependent on changes in market structure and fuel price variations from one year to the next.

The thesis describes the development of a fundamental electricity price model based on the stacked merit order dispatch of thermal generation bidding to produce electricity in a competitive market centred around marginal generation costs. For peaking plant, an exponential uplift in price is applied to represent scarcity of supply. The implications of increasing wind power output are examined using projections of the location and capacity of future wind farms and spatially distributed hind cast wind speed data generated from a mesoscale atmospheric model.

The analysis highlights that despite increased value being placed on storage in an energy system with a high penetration of wind power, opportunities for arbitrage are, in fact, reduced. This is a result of an oversupply of electricity on windy days suppressing peak electricity prices and reducing the daily price spread, which arbitrage exploits. 


\section{Acknowledgements}

Firstly, I would like to thank my supervisor, Professor Gareth Harrison, for his guidance and support throughout the duration of this project. It has been a privilege to have access to his expertise in energy systems and general advice on life. I am also grateful to my second supervisor, Professor Robin Wallace, who provided critical insights at key milestones which have been invaluable in guiding the direction of this thesis.

There are countless other members of IES who have helped and contributed along the way. In particular, I must mention Lucy Cradden, who wrote the code for the aggregate wind power analysis, and Francesca Tagliaferri, who wrote the dynamic programming algorithm. This work could not have been carried out without the previous efforts of Dan Eager and Sam Hawkins, who I have yet to have the pleasure of meeting, but whose work I have regularly referred to. I would also like to thank those who I have shared an office with and who have provided such a great working environment: Steven; James; Mauricio; Andreas; Sotirios and Damilola. I must also mention Susan who has been a great support over the last three years.

I have had many useful discussions with others in the electricity storage industry and I am grateful to all those who have given up their time. In particular, Matthew Barnett and Fernando Morales from Highview Power storage who provided data, commercial insights and advice.

Last, but not least, I would like to thank my parents, Ruth and John, and my husband, Andy, for discussing, distracting, proof checking and generally humouring me throughout this process.

Financial support for this work was provided by an EPSRC Doctoral Training Award, grant number EP/K503034/1. 


\section{Declaration of Originality}

I declare that this work was composed by myself and that the work herein is my own except where explicitly stated otherwise in the text. This work has not been submitted for any other degree or professional qualification except as specified.

Anna Dunbar 


\section{Abbreviations}

AC Alternating Current

BEA British Electricity Authority

BETTA British Electricity Trading and Transmission Arrangements

BSC Balancing and Settlement Code

BSCCo Balancing and Settlement Code Company

CAES Compressed Air Energy Storage

CAPEX Capital Expenditure

CCGT Combined Cycle Gas Turbine

CCL Climate Change Levy

CCS Carbon Capture and Storage

CEA Central Electricity Authority

CEGB Central Electricity Generating Board

CES Cryogenic Energy Storage

CfD Contracts for Difference

CHP Combined Heat and Power

$\mathrm{CO}_{2} \quad$ Carbon Dioxide

CUSC Connection and Use of System Code

DC Direct Current

DCUSA Distribution Connection and Use of System Agreement

DECC The Department of Energy and Climate Change

DLC Double Layer Capacitor

DNO Distribution Network Operator

DP Dynamic Programming

DSIM Dynamic System Investment Model

DSR Demand Side Response

DUKES Digest of United Kingdom Energy Statistics

EES Electrical Energy Storage

EMR Electricity Market Reform

ENA Electricity Network Association

EPRI Electrical Power Research Institute

EPS Emissions Performance Standard

EPSRC Engineering and Physical Science Research Council 


\begin{tabular}{|c|c|}
\hline EROI & Energy Returned on Invested \\
\hline ESN & Electricity Storage Network \\
\hline ESOI & Energy Stored on Invested \\
\hline EU & European Union \\
\hline EUETS & European Union Emissions Trading Scheme \\
\hline FES & Flywheel Energy Storage \\
\hline FOAK & First-of-a-Kind \\
\hline GB & Great Britain \\
\hline GEMA & Gas and Electricity Markets Authority \\
\hline GW & Gigawatt \\
\hline GWh & Gigawatt Hour \\
\hline $\mathrm{h}$ & Hour \\
\hline HMRC & Her Majesty's Revenue and Customs \\
\hline HSE & Health and Safety Executive \\
\hline IMAGES & Integrated Market-fit and Affordable Grid-scale Energy Storage \\
\hline IPP & Independent Power Producer \\
\hline $\mathrm{kV}$ & Kilovolt \\
\hline $\mathrm{kW}$ & Kilowatt \\
\hline $\mathrm{kWh}$ & Kilowatt Hour \\
\hline LCOE & Levelised Cost of Energy \\
\hline LCPD & Large Combustion Plant Directive \\
\hline Li-ion & Lithium Ion \\
\hline LOLP & Loss of Load Probability \\
\hline MRA & Master Registration Agreement \\
\hline MW & Megawatt \\
\hline $\mathrm{MWh}$ & Megawatt Hour \\
\hline $\mathrm{NaNiCl}$ & Sodium Nickel Chloride \\
\hline $\mathrm{NaS}$ & Sodium Sulphur \\
\hline NBP & National Balancing Point (Gas Price) \\
\hline NETA & New Electricity Trading Arrangements \\
\hline NGC & National Grid Company \\
\hline NGET & National Grid Electricity Transmission PLC \\
\hline $\mathrm{NiCd}$ & Nickel Cadmium \\
\hline $\mathrm{NiMH}$ & Nickel Metal Hybrid \\
\hline NOAK & Next-of-a-Kind \\
\hline
\end{tabular}




$\begin{array}{ll}\text { NPV } & \text { Net Present Value } \\ \text { O\&M } & \text { Operation and Maintenance } \\ \text { OCGT } & \text { Open Cycle Gas Turbine } \\ \text { OFFER } & \text { Office of Electricity Regulation } \\ \text { Ofgem } & \text { Office of Gas and Electricity Markets } \\ \text { OFTO } & \text { Offshore Transmission Owner } \\ \text { ONR } & \text { Office of Nuclear Regulation } \\ \text { OPEX } & \text { Operational Expenditure } \\ \text { OTC } & \text { Over the Counter } \\ \text { PCI } & \text { Project of Common Interest } \\ \text { PHS } & \text { Pumped-Hydroelectric Storage } \\ \text { PSB } & \text { Polysulphide Bromide } \\ \text { REC } & \text { Regional Electric Companies } \\ \text { ROC } & \text { Renewable Obligation Certificate } \\ \text { rpm } & \text { Rotations per Minute } \\ \text { SHELT } & \text { Scottish Hydro-Electric Transmission Ltd } \\ \text { SMES } & \text { Superconducting Magnetic Energy Storage } \\ \text { SNG } & \text { Synthetic Natural Gas } \\ \text { SP } & \text { Scottish Power } \\ \text { SPTL } & \text { Scottish Power Transmission Ltd } \\ \text { SSE } & \text { Scottish and Southern Energy } \\ \text { SSEB } & \text { South of Scotland Electricity Board } \\ \text { STC } & \text { System Operator-Transmission Owner Code } \\ \text { TWh } & \text { Terawatt Hour } \\ \text { UAE } & \text { United Arab Emirates } \\ \text { UK } & \text { United Kingdom } \\ \text { UKERC } & \text { UK Energy Research Centre } \\ \text { USA } & \text { United States of America } \\ \text { VOLL } & \text { Value of Lost Load } \\ \text { VRB } & \text { Vanadium Redox Battery } \\ \text { WRF } & \text { Weather Research and Forecasting (Model) } \\ \text { Zero Emission Battery Research Activity } \\ \text { OEA }\end{array}$




\section{Symbols}

$\boldsymbol{A} \quad$ Matrix of constraints computed from conversion and storage efficiencies

$a_{x} \quad$ Fuel conversion coefficient for generator class, $x$

$\boldsymbol{b} \quad$ Vector of maximum storage capacities

$c \quad$ Cost of generation

$C_{T} \quad$ Cash flow in year, $T$

$C_{x} \quad$ Installed generation capacity of marginal generator class, $x$

$\bar{D} \quad$ Average annual demand for future energy scenario

$D \quad$ Demand for future energy scenario

$D$ CAPEX Distribution capital expenditure

$\bar{d} \quad$ Average annual demand for base year

$D_{\max } \quad$ Peak demand for future energy scenario

$d_{\max } \quad$ Peak demand for base year

$d_{t} \quad$ Historic electricity demand at time, $t$

EP Total lifetime electricity production

$e_{x} \quad$ Cost of enriching uranium

$\bar{F} \quad$ Average annual fuel price for future energy scenario

$f \quad$ Vector of prices

$\bar{f} \quad$ Average annual fuel price for base year

$F_{\text {car }} \quad$ Carbon price

$F_{t} \quad$ Fuel price for future energy scenario at time, $t$

$f_{t} \quad$ Fuel price for base year at time, $t$

$F_{x} \quad$ Fuel price for generation class, $\mathrm{x}$

$G C A P E X \quad$ Generation capital expenditure

IC CAPEX Interconnection capital expenditure

lb Lower bound

$N \quad$ Life of investment project

$p \quad$ Power

$P E \quad$ Price of Electricity

$P_{t} \quad$ Price of electricity in period, $t$

$P_{x} \quad$ Power output of the marginal generator class, $x$

$q \quad$ Quantity

$q_{\max }^{C} \quad$ Maximum quantity of electricity which can be charged in a single period 


\begin{tabular}{|c|c|}
\hline$q_{\max }^{D}$ & Maximum quantity of electricity which can be discharged in a single period \\
\hline$q_{t}^{C}$ & Electricity bought in period, $t$ \\
\hline$q_{t}^{D}$ & Electricity sold in period, $t$ \\
\hline$Q^{*}$ & Market clearing quantity \\
\hline$Q^{C}$ & Maximum charging rate \\
\hline$Q^{D}$ & Maximum discharging rate \\
\hline$r$ & Discount rate \\
\hline$R$ & Annual revenue \\
\hline$R^{*}$ & Annual revenue multiplied by minus one \\
\hline$s$ & Forecast error \\
\hline$S C A P E X$ & Storage capital expenditure \\
\hline$S_{\max }$ & Maximum storage capacity \\
\hline$S_{t}$ & State of charge in period, $t$ \\
\hline$T$ & Year \\
\hline$t$ & Settlement period \\
\hline TCAPEX & Transmission capital expenditure \\
\hline$U$ & Wind speed \\
\hline$u b$ & Upper bound \\
\hline$V$ & Variable operation and maintenance cost \\
\hline$V_{x}$ & $\begin{array}{l}\text { Variable operation and maintenance cost per unit output for generator class, } \\
x\end{array}$ \\
\hline$x$ & Vector of decision variables \\
\hline$\alpha$ & Empirically determined uplift coefficient \\
\hline$\beta$ & Empirically determined uplift coefficient \\
\hline$\epsilon_{d}$ & Price elasticity of demand \\
\hline$\epsilon_{s}$ & Price elasticity of supply \\
\hline$\eta$ & Thermal efficiency \\
\hline$\eta_{c}$ & Conversion efficiency \\
\hline$\eta_{s}$ & Storage efficiency \\
\hline$\eta_{x}$ & Thermal efficiency of marginal generator class, $x$ \\
\hline$\pi$ & Price \\
\hline$\pi^{*}$ & Market clearing price \\
\hline$\Pi_{x}$ & Marginal generation cost of the marginal generator class, $x$ \\
\hline$v$ & Carbon produced from burning fuel at $100 \%$ efficiency \\
\hline
\end{tabular}



generator class, $x$

Expected value 


\section{Table of Contents}

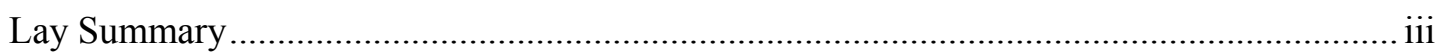

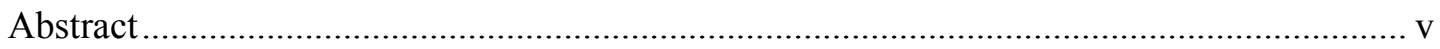

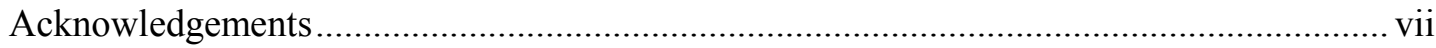

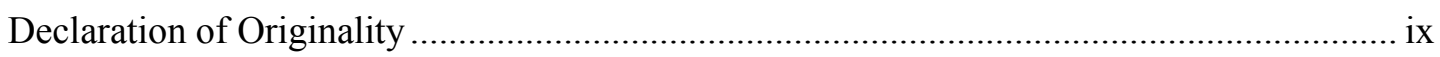

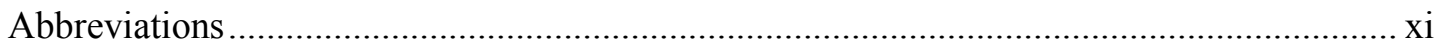

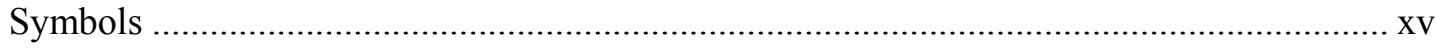

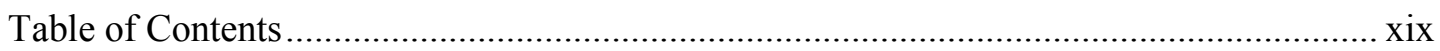

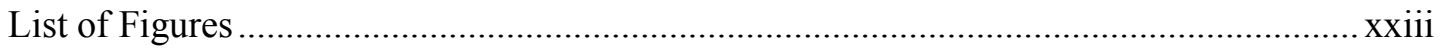

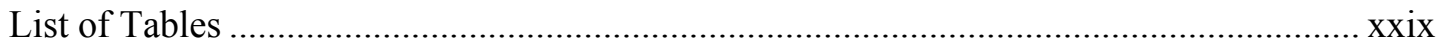

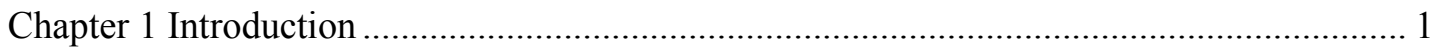

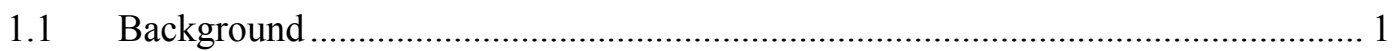

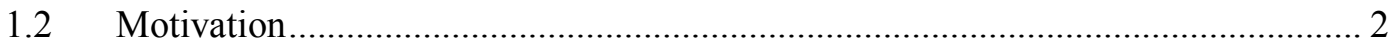

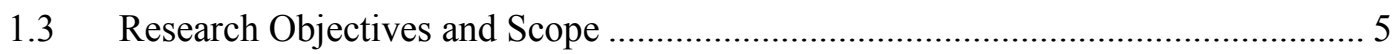

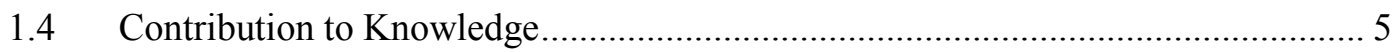

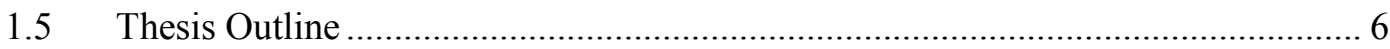

Chapter 2 Electricity Storage Technologies....................................................................... 9

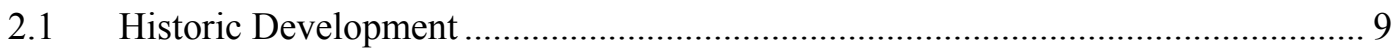

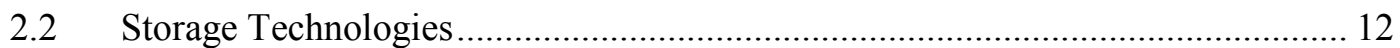

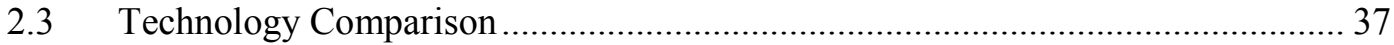

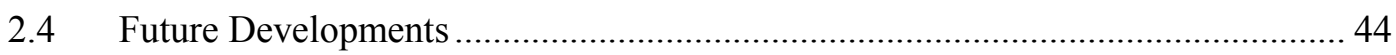

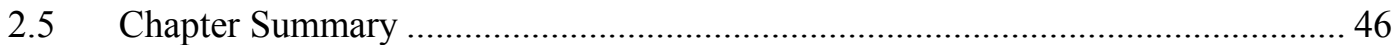




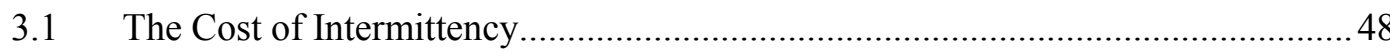

3.2 Applications for Electrical Energy Storage …...................................................5

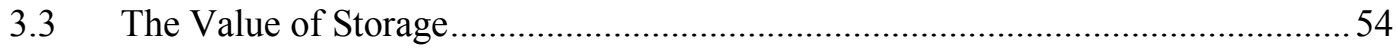

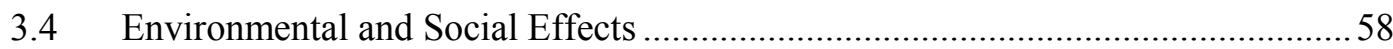

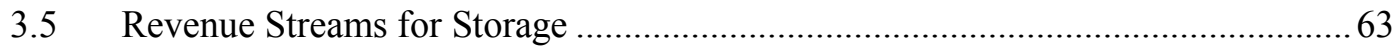

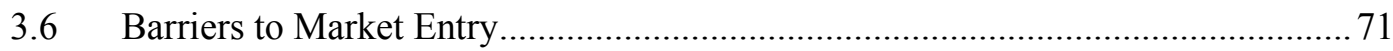

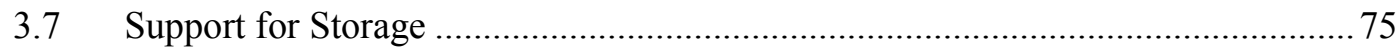

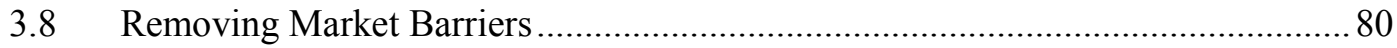

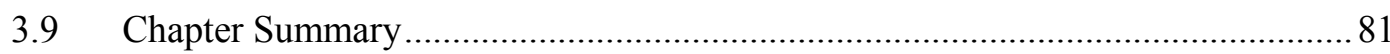

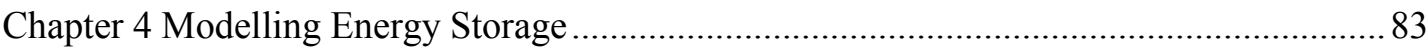

4.1 Justification for Investigating Price Arbitrage Alone ............................................. 83

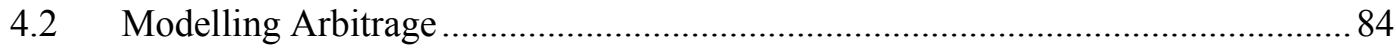

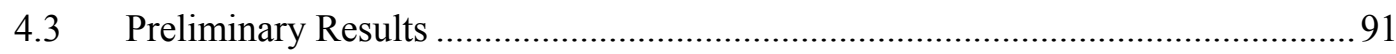

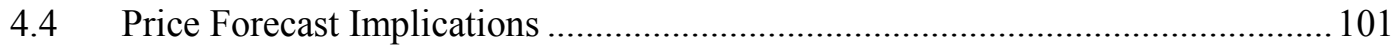

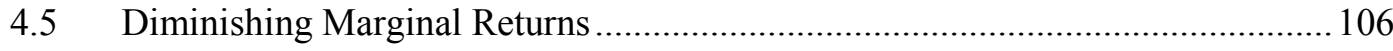

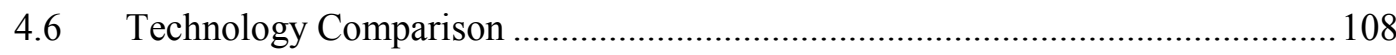

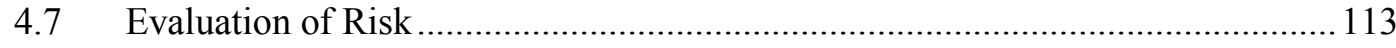

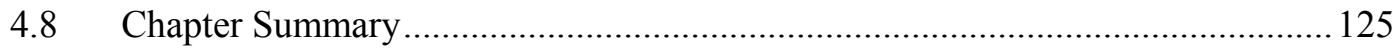

Chapter 5 Electricity Price Model Development............................................................... 127

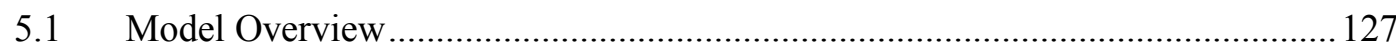

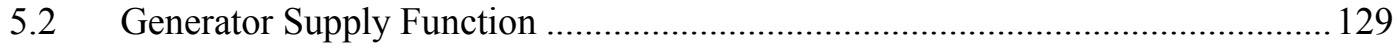

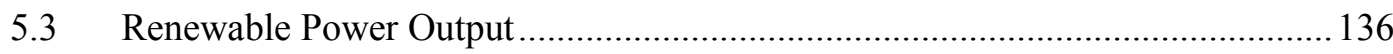

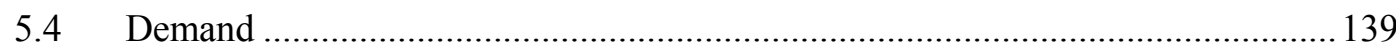

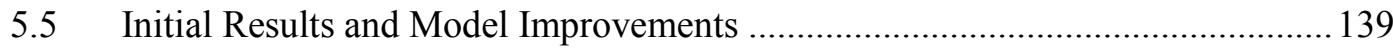

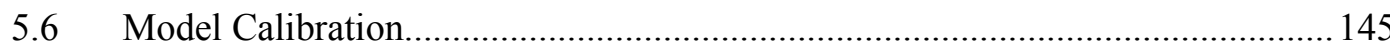




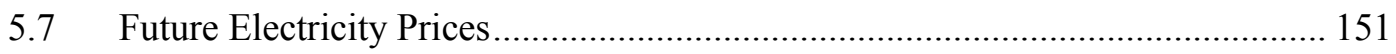

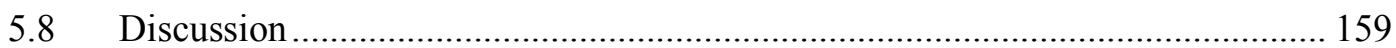

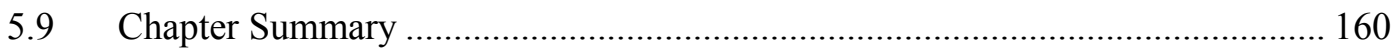

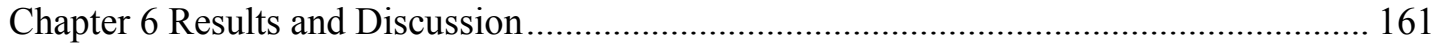

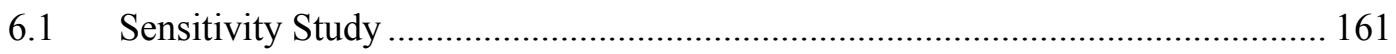

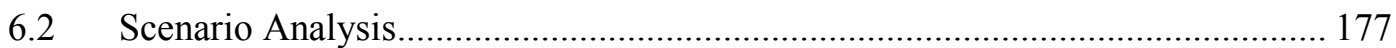

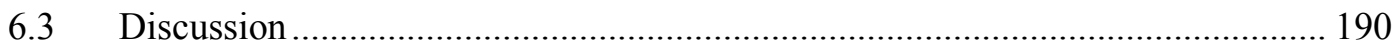

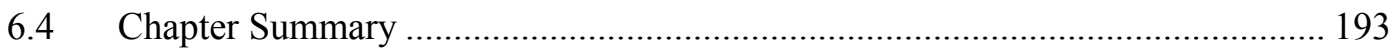

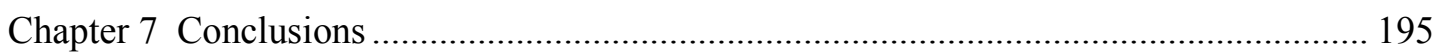

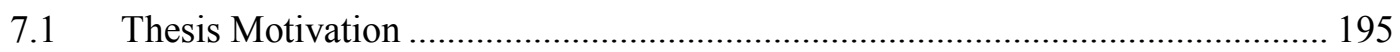

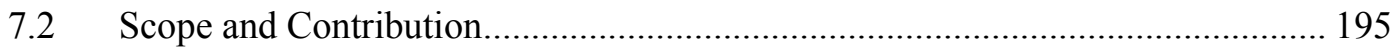

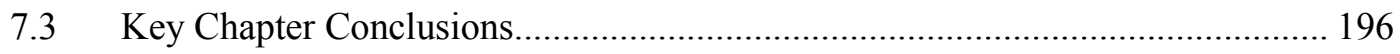

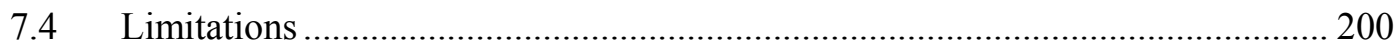

7.5 Recommendations for Further Work ……….................................................. 201

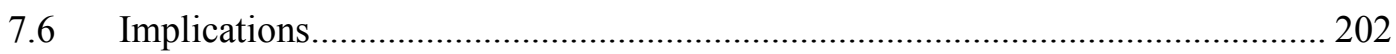

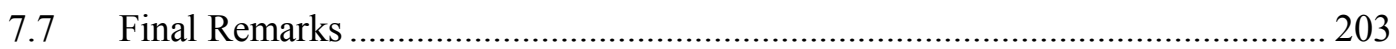

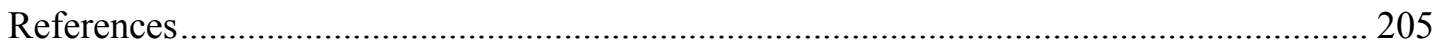

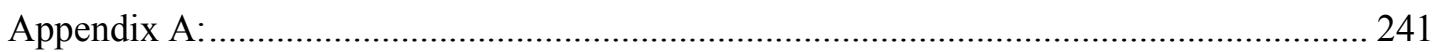

Background information on Electricity Market Design...................................................... 241

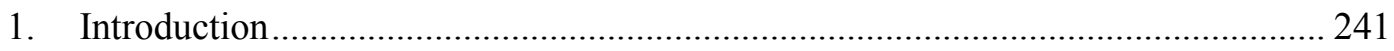

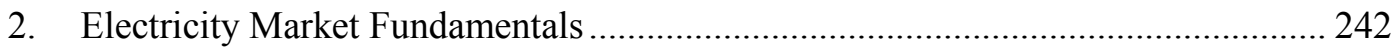

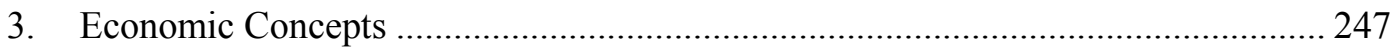

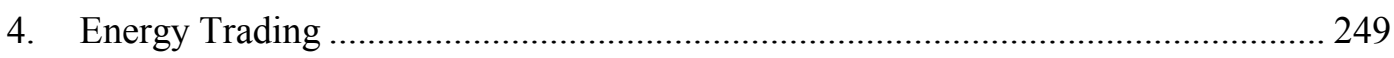




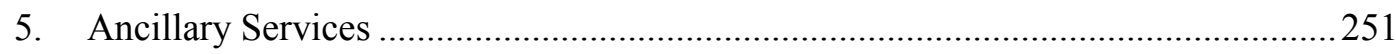

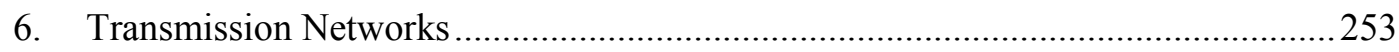

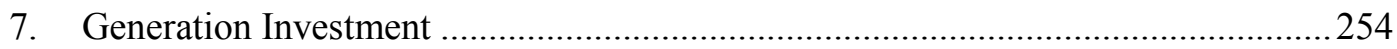

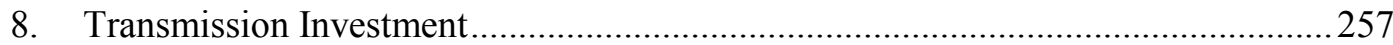

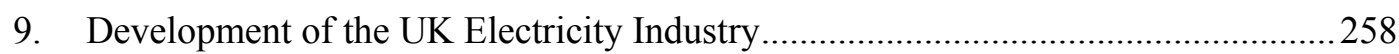

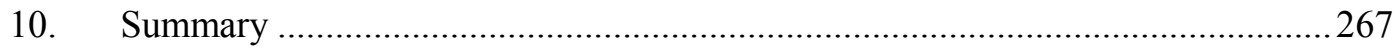

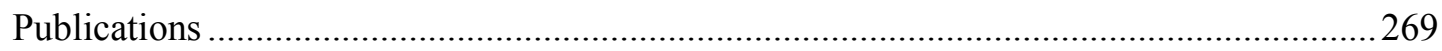




\section{List of Figures}

Figure 1.1 Daily peak electricity price [27] and National Balancing Point gas price [28], 2005

Figure 1.2 Half hourly electricity price [27], demand [29] and wind power output [30], first week of November 2005 4

Figure 2.1 London daily load curves circa 1897 (source [34]) ............................................ 10

Figure 2.2 Electricity storage technology timeline (source [42])

Figure 2.3 Summary of electrical energy storage technologies grouped by type of energy storage.

Figure 2.4 Operation of pumped hydro-electric plant (source [43])

Figure 2.5 World's largest pumped-hydroelectric storage station, Virginia (source [46]).... 13

Figure 2.6 Sankey diagram of pumped-hydroelectric storage (source [48])........................ 14

Figure 2.7 Compressed air energy storage system (based on [55]) .................................... 16

Figure 3.8 Sankey diagram of compressed air energy storage (source [82]) ........................ 17

Figure 2.9 Flywheel electricity storage technology (source [48]) ....................................... 18

Figure 2.10 Sankey diagram of flywheel energy storage (source [48]) ............................... 18

Figure 2.11 Sankey diagram of advanced lead acid battery (source [48]) ........................... 20

Figure 2.12 Sankey diagram of lithium ion battery (source [48]) ….................................. 22

Figure 2.13 Lithium ion battery cost reduction since 2010 and cost projection to 2020 (source [34]) 23

Figure 2.14 Sankey diagram of sodium sulphur battery system (source [48]) ..................... 25

Figure 2.15 Sankey diagram of sodium nickel chloride battery (source [48]) ...................... 26

Figure 2.16 Flow battery system (source [77]) .............................................................. 27

Figure 2.17 Sankey diagram of flow battery system (source [48]) …….............................. 28

Figure 2.18 Flow battery cost reduction since 2010 and cost projection to 2020 (source [34]) .28

Figure 2.19 Sankey diagram of hydrogen storage system (source [48]) ............................. 30

Figure 2.20 Sankey diagram of a double layer capacitor system (source [48]) ..................... 32

Figure 2.21 Superconducting magnetic energy storage system (based on [82]).................... 33

Figure 2.22 Sankey diagram of superconducting magnetic energy storage system (source [48])

Figure 2.23 Cryogenic energy storage process (source [85]) ……………….................... 35

Figure 2.24 Sankey diagram of cryogenic energy storage system (source [48]) ................... 35 
Figure 2.25 Highview's 350kW liquid air energy storage demonstration project (source [85]) 36

Figure 2.26 Sankey diagram of pumped heat energy storage system (source [48]) .................37

Figure 2.27 Storage technologies arranged by power rating and discharge time ...................40

Figure 2.28 Relative development stage of electrical energy storage technology .................. 41

Figure 2.29 Graphical representation of power and energy related storage costs ...................43

Figure 3.1 Benefits of electrical energy storage (source [96]) …….....................................50

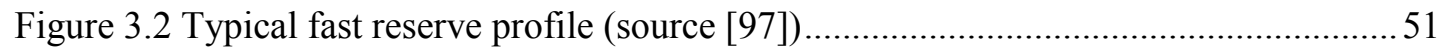

Figure 3.3 Applications of storage arranged by discharge time and power rating (source [109])

Figure 3.4 Annual benefits of (a) bulk and (b) distributed storage in the UK in 2050 (source [2]) .56

Figure 3.5 Estimates of annual global market value for grid connected storage [111], [112], [113] 57

Figure 3.6 Historic global market growth of grid connected electricity storage market (source [111]) .57

Figure 3.7 Energy stored on invested for a range of electrical energy storage technologies (source [115]) 59

Figure 3.8 (top) Average annual global production of elements used in energy storage technologies (2006-2011), (middle) Price of elements, (bottom) Embodied energy used to

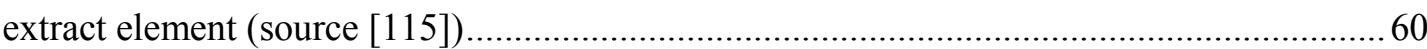

Figure 3.9 Land use of energy storage technologies (source [117]) .....................................61

Figure 3.10 Lifecycle emissions from energy storage devices (source [119]) .......................6 62

Figure 3.11 National Grid expenditure on frequency response services (source [124]) ........65

Figure 3.12 Leighton Buzzard distribution network reinforcement options (source [133])...67

Figure 3.13 Traffic light time bands for distribution use of system charges (source [128]) ..69

Figure 3.14 Energy storage market forecast by application for 2030 (source [45] )..............71

Figure 3.15 Policy tools being implemented in Britain which will impact storage (source [139])

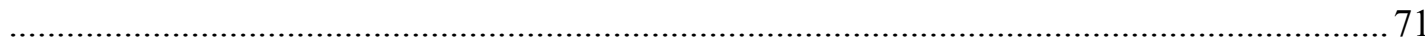

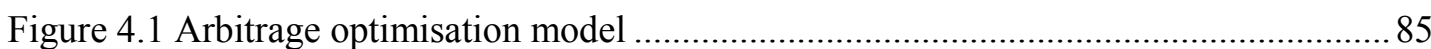

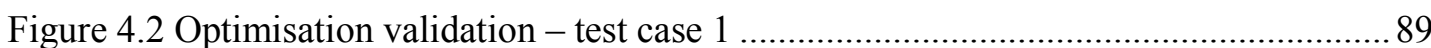

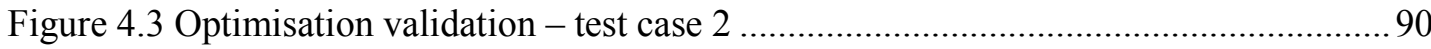

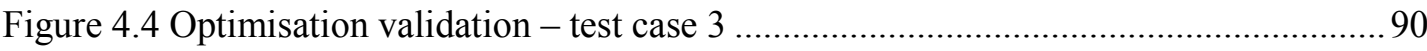

Figure 4.5 Electricity demand for first week in January $2010 \ldots \ldots \ldots \ldots \ldots \ldots \ldots \ldots \ldots \ldots \ldots \ldots \ldots \ldots \ldots . . . .91$

Figure 4.6 Spot market electricity prices for first week in January $2010 \ldots \ldots \ldots \ldots \ldots \ldots \ldots \ldots \ldots \ldots \ldots . . . .91$ 
Figure 4.7 Optimum charging rate for first week in January 2010 …................................. 92

Figure 4.8 State of charge for first week in January 2010 ................................................... 92

Figure 4.9 Monthly revenue and maximum electricity price from 2010 UK market ............ 93

Figure 4.10 Half hour time series of electricity prices for 2010 ........................................ 93

Figure 4.11 Optimum charging rate for first week in August 2010 ..................................... 94

Figure 4.12 State of charge for first week in August 2010 .................................................. 94

Figure 4.13 Monthly revenue and maximum electricity price from 2010 California market 95

Figure 4.14 Variation in annual revenue from 2008-2013 ................................................. 96

Figure 4.152010 annual revenue with varying storage capacity ........................................ 97

Figure 4.16 Normalised state for charge for one week with high and low storage capacity . 97

Figure 4.17 Optimum charging rate for one week with high and low storage capacity ........ 98

Figure 4.182010 annual revenue with varying charging and discharging rate .................... 98

Figure 4.19 Contour map of 2010 annual revenue with varying capacity and charging rate, optimum ratio of charging rate to storage capacity shown by line ........................................ 99

Figure 4.202010 annual revenue with varying conversion efficiency .............................. 100

Figure 4.21 Optimum operating strategy for high and low conversion efficiencies............ 100

Figure 4.222010 annual revenue with varying storage efficiency ..................................... 101

Figure 4.232010 annual revenue as a function of daily self-discharge .............................. 101

Figure 4.24 Performance of practical operating strategies.................................................. 103

Figure 4.25 Depiction of dynamic programming decision process (source [174]).............. 104

Figure 4.26 Loss of revenue with increasing price forecast error...................................... 106

Figure 4.27 Price variation over 24 hours with 2GW, 8GWh of storage (source [181]) ..... 107

Figure 4.28 Price variation over 24 hours with 10GW, 40GWh (source [181]).................. 107

Figure 4.29 Annual revenue for a range of storage technologies from 2008 ...................... 110

Figure 4.30 Annual revenue per unit capacity and per unit charging rate for a range of storage

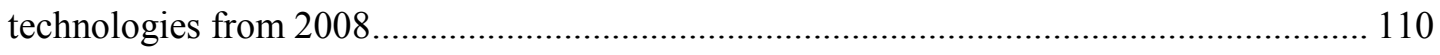

Figure 4.31 NPV for a range of storage technologies based on annual revenue from 2008112 Figure 4.32 NPV for a range of storage technologies with 15 year income based on annual

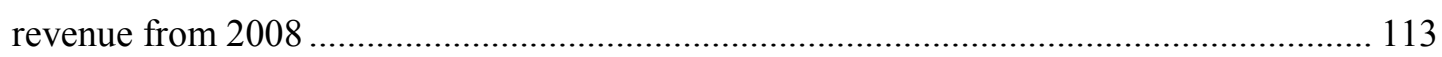

Figure 4.33 Left skewed lognormal probability distribution for device availability ........... 119

Figure 4.34 Normal probability distribution for process equipment cost for first-of-a-kind system 122

Figure 4.35 Normal probability distribution for net present value of cryogenic energy storage

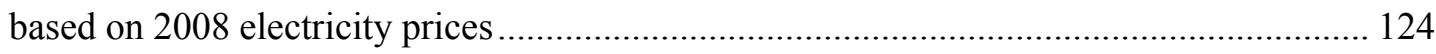

Figure 5.1 Overview of electricity price forecast model...................................................... 128 


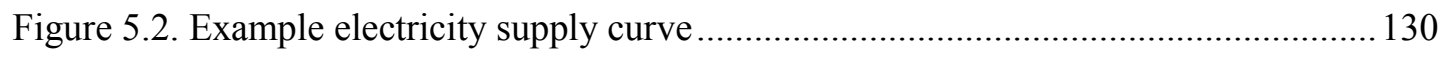

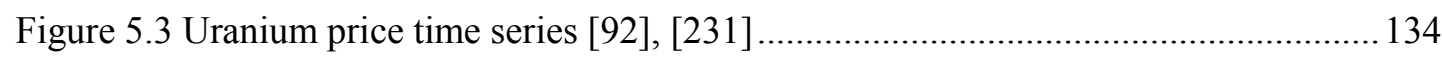

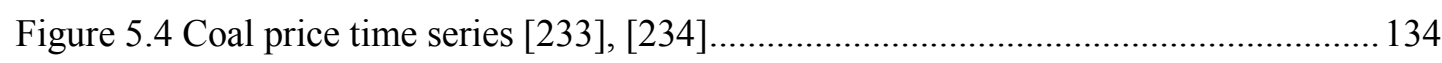

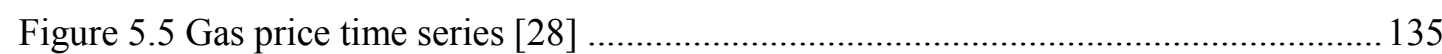

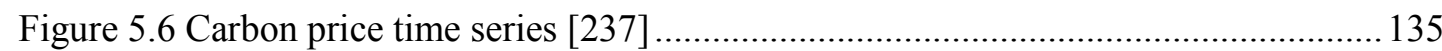

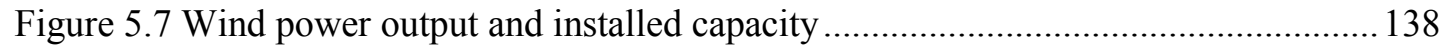

Figure 5.8 Wind power output and electricity demand ....................................................... 138

Figure 5.9 Price duration curve for simulated and historic electricity prices....................... 140

Figure 5.10 Initial model output and historic electricity price time series, 2005-2007 ........ 140

Figure 5.11 Initial model output and historic electricity prices for first two weeks of August 2007

Figure 5.12 Demand net wind power output and generator class capacity limits for first two weeks of august 2007 . 141

Figure 5.13 Model output and historic electricity price time series with high generator efficiencies, 2005-2007 142

Figure 5.14 Model output and historic electricity prices with high generator efficiencies for first two weeks of August 2007. 143

Figure 5.15 Model output and historic electricity prices with high generator efficiencies for first two weeks of August 2007, $\beta=2$ 144

Figure 5.16 Supply curve with linear and hyperbolic price functions for marginal generators 145

Figure 5.17 Historic and simulated electricity prices for second week in August 2007 ...... 147

Figure 5.18 Historic and simulated average monthly electricity prices, 2005-2007 147

Figure 5.19 Location of onshore wind farms used for Scenario 1 (left) weighted by installed capacity (right) 153

Figure 5.20 Location of onshore wind farms used for Scenario 2 (left) weighted by installed capacity (right) .153

Figure 5.21 Comparison of aggregate onshore wind power output per unit from Scenario 1 and Scenario 2 with 2005 wind year (top) 2006 wind year (centre) and 2007 wind year (bottom)

Figure 5.22 Location of offshore wind farms used for Scenario 1 (left) weighted by installed capacity (right) 156

Figure 5.23 Location of offshore wind farms used for Scenario 2 (left) weighted by installed capacity (right) 156 
Figure 5.24 Location of offshore wind farms used for Scenario 3 (left) weighted by installed capacity (right) 156

Figure 5.25 Comparison of aggregate offshore wind power output per unit for different scenarios with 2005 wind year. 157

Figure 6.1 Installed generation capacity 2006 [25] 162

Figure 6.2 Annual revenue from simulated electricity prices with increasing gas price 163

Figure 6.3 Storage state of charge for final two weeks in January with gas price of 40p/therm and $£ 1 /$ therm 164

Figure 6.4 Marginal generation costs with average gas price 10p/therm 165

Figure 6.5 Marginal generation costs with average gas price $£ 1 /$ therm. 165

Figure 6.6 Annual revenue from simulated electricity prices with increasing carbon price 166 Figure 6.7 Annual revenue from simulated electricity prices with varying average annual demand. 168

Figure 6.8 Example electricity supply curve 168

Figure 6.9 Annual revenue from simulated electricity prices with increasing penetration of installed wind capacity 169

Figure 6.10 Time series of wind power output with $40 \mathrm{GW}$ installed capacity for first two weeks in February..... 169

Figure 6.11 Time series of electricity price with no wind capacity and 40GW installed wind capacity for first two weeks in February

Figure 6.12 Storage state of charge for first two weeks in February with no wind and 40GW installed wind capacity.

Figure 6.13 Annual revenue from 2006 using alternative base year time series 172

Figure 6.14 Annual revenue for zero and 40GW installed wind capacity cases varying storage capacity, normalised relative to $600 \mathrm{MWh}$ capacity 173

Figure 6.15 Annual revenue for zero and 40GW installed wind capacity cases varying charging rate, normalised relative to $200 \mathrm{MW}$ charging rate.

Figure 6.16 Contour map of annual revenue with varying capacity and charging rate with $40 \mathrm{GW}$ installed wind capacity, optimum ratio of charging rate to storage capacity shown by line 174

Figure 6.17 Annual revenue for zero and 40GW installed wind capacity cases varying conversion efficiency 175

Figure 6.18 Annual revenue for zero and 40GW installed wind capacity cases varying daily self-discharge 176

Figure 6.19 National Grid Future Energy Scenarios. 178 
Figure 6.20 Thermal generation capacity for National Grid Future Energy Scenarios 20152035

Figure 6.21 Onshore wind capacity for National Grid Future Energy Scenarios 2015-2035

Figure 6.22 Offshore wind capacity for National Grid Future Energy Scenarios 2015-2035

Figure 6.23 Gas price for National Grid Future Energy Scenarios 2015-2035 182

Figure 6.24 Coal price for National Grid Future Energy Scenarios 2015-2035. 183

Figure 6.25 Uranium prices derived from National Grid Future Energy Scenarios 2015-2035

Figure 6.26 Carbon prices for National Grid Future Energy Scenarios 2015-2035 184

Figure 6.27 Average annual demand for National Grid Future Energy Scenarios 2015-2035

Figure 6.28 Peak demand for National Grid Future Energy Scenarios 2015-2035. 185

Figure 6.29 Annual revenue for National Grid Future Energy Scenarios, 2015-2035. 186

Figure 6.30 Thermal capacity / average demand for National Grid Future Energy Scenarios, 2015-2035 187

Figure 6.31 Annual revenue from National Grid Future Energy Scenarios in 2035 with changing base year .189

Figure 6.32 State of charge over 1 week for 2035 Gone Green and No Progression Scenarions .190

Figure 0.1 Monopoly model of the electricity industry (based on [265]) .244

Figure 0.2 Initial purchasing agent model of the electricity industry (based on [265]) .......245

Figure 0.3 Further development of purchasing agent model of the electricity industry (based on [265]) .245

Figure 0.4 Wholesale competition model of the electricity industry (based on [265]) ........ 246

Figure 0.5 Retail competition model of the electricity industry (based on [265]) ................246

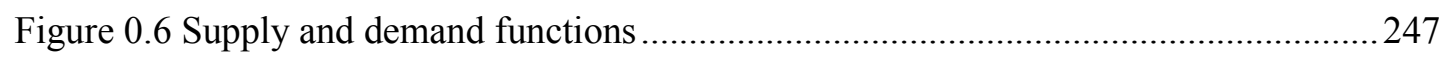

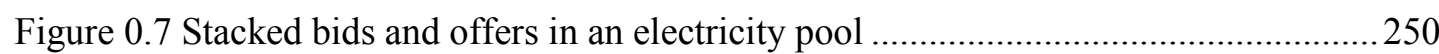

Figure 0.8 Example of electricity price duration curve based on 2003 GB spot market electricity prices [169].. .256

Figure 0.9 BETTA market trading arrangements (source [287]) …....................................261

Figure 0.10 Market share of electricity generation in 2011 (source [288]) ……..................262

Figure 0.11 Distribution network operator companies (source [289]) .263 


\section{List of Tables}

Table 2.1 Summary of storage technology characteristics [37], [42], [45], [75] ................... 38

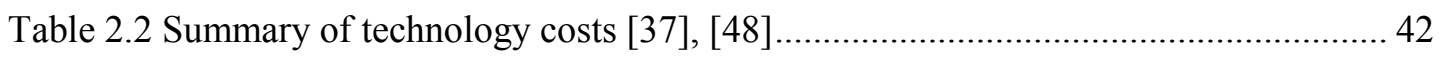

Table 3.1 Triad charges for network zones for 2014-2015 triad season (source [135]) ........ 68

Table 3.2 Estimated cost reductions through innovation for energy storage technologies

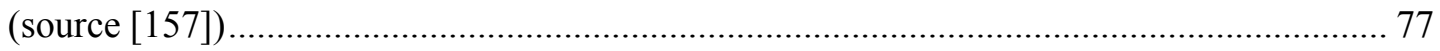

Table 3.3 Policies affecting storage from other countries [96] ............................................ 79

Table 4.1 Storage characteristics used for arbitrage model verification ............................... 88

Table 4.2 Characteristics of UK Power Network's lithium ion battery and Dinorwig PHS 105

Table 4.3 Sample storage technology characteristics .......................................................... 109

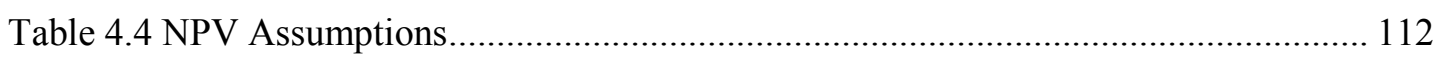

Table 4.5 Risk categories faced by storage technology ................................................... 114

Table 4.6 Cryogenic energy storage characteristics used for risk analysis .......................... 118

Table 4.7 Cryogenic energy storage subassembly costs .................................................. 120

Table 4.8 Cryogenic energy storage subassembly component cost breakdown and uncertainty 121

Table 4.9 Expected net present value for cryogenic energy storage based on 2008 electricity

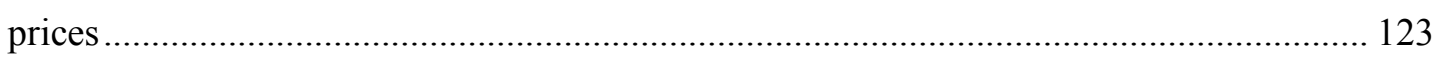

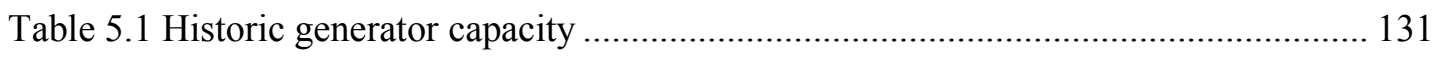

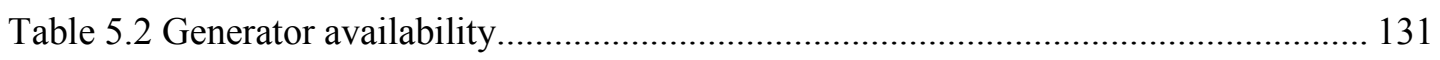

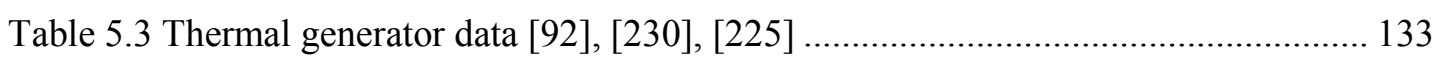

Table 5.4 Storage device characteristics used for price model validation ........................... 146

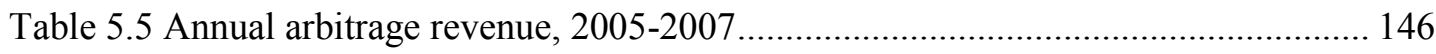

Table 5.6 Annual peak and trough electricity price statistics from historic and simulated data

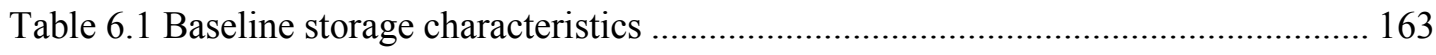

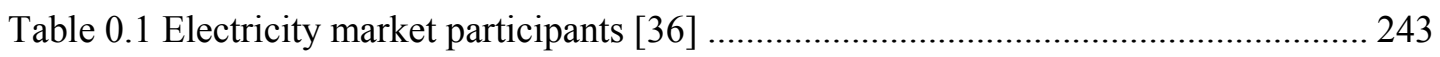





\section{Chapter 1 Introduction}

\section{$1.1 \quad$ Background}

The Climate Change Act 2008 placed a legally binding commitment on the UK Government to achieve an $80 \%$ reduction in UK greenhouse gas emissions relative to 1990 levels by 2050 [1]. This strengthened a drive to transform the electricity industry from one based largely on fossil fuels to one based on low or zero carbon sources. As a result, the number of intermittent renewable and inflexible nuclear generators connected to the grid is expected to increase in coming decades. The integration of these technologies, whilst maintaining an acceptable level of security of supply, is expected to impact costs. The challenge of ensuring a secure and sustainable electricity industry, at an acceptable cost to consumers, has been dubbed the Energy Trilemma.

Grid connected, electrical energy storage (EES) presents a potential solution to this challenge. It is championed as a facilitating technology, enabling intermittent renewable electricity to be integrated with existing electricity systems. It could reduce the requirement for investment in peaking plant and avoid energy curtailment from non-dispatchable generators. It also has the potential to reduce system operating costs and capital expenditure in transmission and distribution infrastructure. EES provides benefits to a number of stakeholders throughout the electricity industry. A study carried out by Imperial College London, for the Carbon Trust reported that by 2050 , the implementation of EES could bring savings of up to $£ 10 \mathrm{bn} / \mathrm{year}$ to the UK [2]. However, the benefits are accrued between regulated and deregulated markets leading to complex contractual arrangements and providing little incentive for any individual actor to invest. Without appropriate financial rewards for EES, its potential to deliver value to the electricity system will not be realised. 


\subsection{Motivation}

Storage of electricity is technically feasible, but it can be inefficient and historically has not been economically viable as a standalone investment, requiring justification at a system level. As the market share of non-dispatchable generators - such as wind and nuclear - increases, the challenge of ensuring that variations in demand can be met is becoming more apparent. As a result, the potential for EES to accommodate this variance is being recognised.

In January 2013 the UK Government announced energy and, specifically, its storage as one of Eight Great Technologies of focus for applied scientific research [3]. The Department of Energy and Climate Change (DECC) has acknowledged a role for EES in the UK in conjunction with demand side response and interconnectors [4]. However, the value of EES is not well understood with estimates for optimum capacity in 2050 ranging from $7 \mathrm{GW}$ to $59 \mathrm{GW}$ [5]. This range has been derived from various system models which have not adequately represented EES. Many have a coarse temporal resolution which underestimates the value of EES in managing short term fluctuations in wind [6] [7]. Others do not model transmission and distribution constraints and neglect storage value in reducing congestion and investment in network infrastructure [8] [9] [10]. Further work is required to quantify these benefits. Grünewald reports that officials have remarked that they are "hunting for objective evidence" on the value of storage [11].

Interest in the commercial value of EES has equally increased in recent years and technical developments are improving efficiencies and reducing costs. Commercial models generally evaluate the techno-economic performance of a specific EES device in a defined market. There are broadly three categories of commercial EES models.

1. Models evaluating the economics of EES when operated with standalone renewable energy systems to provide a firm local energy supply; examples of studies include [12], [13] and [14].

2. Models assessing the added value from dedicated EES backing-up an intermittent renewable plant to allow increased output from embedded generation to be taken up by the grid, for example in [15], [16], [17] and [18].

3. Models estimating the revenue from EES devices operated to maximise profits through price arbitrage in specific electricity markets, including [19], [20], [21] and [22].

The first category of models is of less relevance to this thesis which is concerned with grid connected EES. It is also largely accepted that providing dedicated EES for specific renewable 
generators is not an efficient approach to balancing variations in output. Barnhart commented that coupling EES directly with wind is like "spending $\$ 100$ on a safe to store a $\$ 10$ watch" [23]. Taking a wider system approach allows small variations to be managed by aggregating the output from multiple variable generators [24]. For this reason, the second category of studies is not considered. The third category, estimating revenue from price arbitrage, is investigated further. These revenues could be coupled with other ancillary services; however, these are generally traded through private bilateral exchanges where contract values are not disclosed. These models are less common and are challenging to validate.

Previous arbitrage studies have suggested a dependence of EES revenue on market structure through comparisons of arbitrage in different global markets [22] and variations within a single market from one year to the next [20] as generation capacity and fuel prices change. These have largely used historic electricity prices. It has been suggested that commercial opportunities for EES may emerge in the future with a higher penetration of intermittent renewable energy; however, the level of reward and its dependencies have not been fully defined.

Projecting the future scope for arbitrage is more challenging and this is particularly so for markets such as Great Britain (GB), where substantial structural changes will occur. Historically, peak electricity prices tend to have been driven by gas prices as Figure 1.1 shows. This occurs as mid-merit and peaking generators include a large proportion of gas turbines [25]. Furthermore, as wind power has made only a small contribution and has had little impact on wholesale electricity prices, daily fluctuations in price have been driven principally by variations in demand as shown in Figure 1.2. Over the next decade the contribution from wind generation is expected to increase dramatically. It is often speculated that commercial opportunities for storage will emerge as the penetration of intermittent renewables grows as it has the potential to increase price volatility [26]. More frequent and more acute price differentials would provide additional opportunities for arbitrage which could become a more profitable revenue stream in the future. 


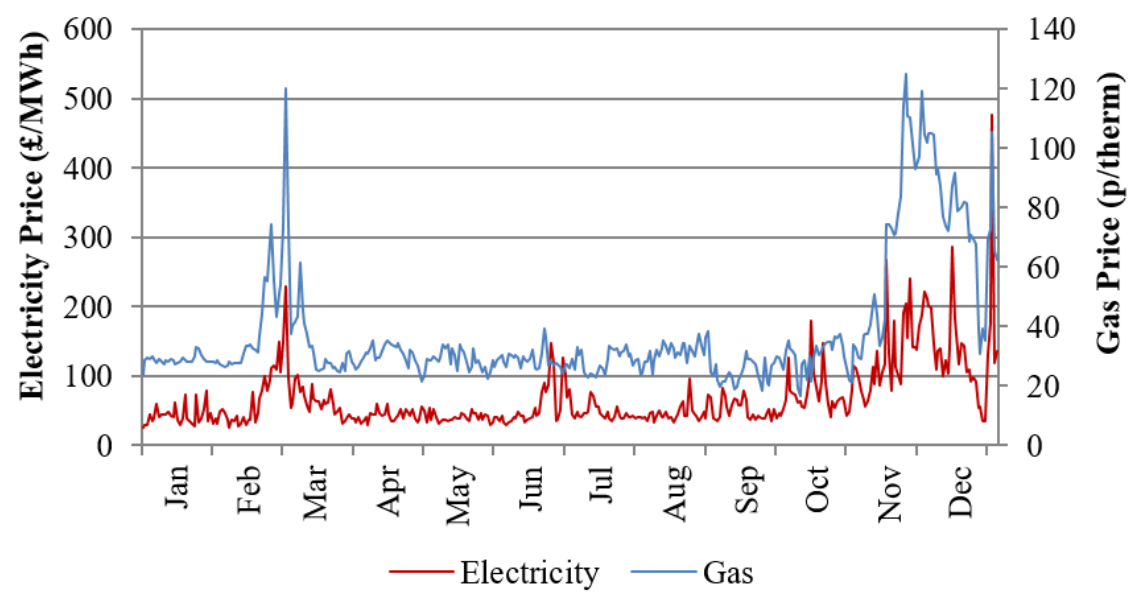

Figure 1.1 Daily peak electricity price [27] and National Balancing Point gas price [28], 2005

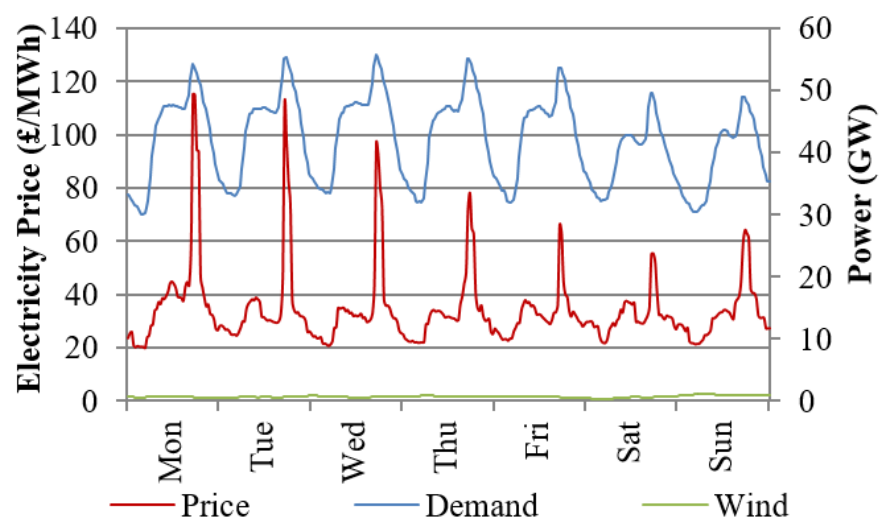

Figure 1.2 Half hourly electricity price [27], demand [29] and wind power output [30], first week of November 2005

A notable analysis of this phenomenon for future GB systems is presented by Grünewald et al [19], finding that $32 \mathrm{GW}$ of wind capacity would enable the gross value of storage to cover its capital costs and investment could be commercially viable through price arbitrage alone. Wind generation was attributed a marginal value equivalent to the opportunity cost of a Renewable Obligation Certificate (ROC) which had the effect of driving costs negative during high wind - low demand periods. An exponential mark-down was applied to electricity prices when demand was lowest to represent generators' preferences not to curtail output, which may be unrealistic. The magnitude of electricity prices will depend on the specific subsidy regime in place. The minimum price will ultimately be limited by the value which could be recovered by a subsidy payment for a renewable generator, and would not be infinitely negative, as an exponential mark-down implies. The study examined four EES technologies in markets with increasing renewable energy capacity. The sensitivity of net present value (NPV), based on 
arbitrage revenue, was tested against a range of variables. The trading margin, or daily price spread, was highlighted as one of the most sensitive parameters. The model was not, however, capable of reflecting variations in gas and carbon prices in the price spread and consequently the effect of these on EES revenue.

There is a rapidly changing portfolio of generation technologies in GB and the potential for large scale development of wind, nuclear or gas capacity to be deployed in the future depending on the prevailing market and political favour. To understand the implications of this on EES, and the business case for storage in the future, the effect of changing market structures and variations in gas and carbon prices, in addition to wind power output, must be established. While investigating future energy scenarios, understanding initial deployments of EES before the technology is deployed at scale is essential. This period of initial market penetration is critical to new technologies as, without overcoming barriers facing the first generation deployments, market penetration at a larger scale is unfeasible.

\subsection{Research Objectives and Scope}

This project aims to investigate the system level and commercial value of EES in GB. It highlights the benefits of EES and some of the regulatory and commercial challenges encumbering its uptake in liberalised electricity markets. The impact of changing market structures, such as generation portfolios, gas and carbon prices, on arbitrage revenue is investigated to provide insights into the commercial value of EES in future energy scenarios.

The objectives are to:

1. review the state of the art of EES technology and scope for future improvements in the absence of non-technical barriers to development;

2. qualitatively assess the benefits of EES to electricity networks;

3. identify routes to market for EES in GB and highlight any commercial or regulatory barriers;

4. develop a techno-economic model to quantify the commercial value of EES through price arbitrage;

5. investigate the value of EES in future energy scenarios with a high penetration of intermittent renewable energy.

\subsection{Contribution to Knowledge}

This thesis will test the hypothesis that: 
Increased wind penetration leads to increased commercial opportunities for energy storage through price arbitrage.

The project provides evidence of the value of EES to electricity networks and highlights deficiencies of liberalised markets in rewarding those benefits commercially. Technoeconomic models have been developed previously; however, these largely assess the commercial value of EES under historic market conditions. There is a distinct gap in the literature of arbitrage models which use electricity prices for potential future energy scenarios, which this thesis addresses. As the electricity sector transitions to a low carbon industry in the future, it is essential that the implications of these changes on enabling technologies, such as EES, are understood. Critically, in this work, an arbitrage model is coupled with a fundamental price model to investigate the value of EES in future energy scenarios. Both of these techniques are commonly used independently in literature and a coupled model has been demonstrated once previously by Grünewald et al [19]. While the framework applied in [19] is credible, there are a number of areas within the model that have limitations. Specifically, the approach to modelling negative electricity prices during periods of low demand and high wind power output. In this work, a different approach to formulating the electricity supply function has led to distinct results which are contrary to previous findings. Furthermore, the level of detail in the price model enables the impact of changing gas and carbon prices on arbitrage revenue to also be investigated, which have not been published previously. A better understanding of how EES is valued in current and future energy scenarios should deliver insights into how policy could best incentivise appropriate investment.

It is expected that the results, analysis and discussion herein will be relevant to anyone with an interest in the development of the EES industry including technology developers, policy makers, regulators, network operators and others involved in the design and operation of the power sector.

\subsection{Thesis Outline}

This thesis is divided into seven chapters.

Chapter 1 provides a brief introduction to the project, states the aims and objectives and outlines the structure of the report.

Chapter 2 reviews EES technologies. A history of the EES industry is presented to provide background information on technology progress to date. EES technologies are then presented by type and compared on the basis of technical ability and cost to highlight the range of storage 
devices available. Potential developments for EES are discussed to identify the scope for future improvements in the absence of non-technical barriers to development.

Chapter 3 discusses the benefits of EES to the energy system as a whole and the commercial opportunities. Issues with integrating large amounts of intermittent renewable energy into the electricity system are reviewed and the associated costs are discussed. A summary of research on the value of EES to the electricity system and the potential size of the EES market are then presented. The environmental and social impacts of EES are also considered. The system benefits are contrasted by the revenue streams available and the regulatory barriers to market entry. Finally, support mechanisms in GB, and internationally, are discussed.

Chapter 4 presents a techno-economic model used to evaluate the revenue available to a storage operator through price arbitrage. Preliminary results, based on historic electricity prices, highlight a dependence on market structures and revenue risk from one year to the next. A comparison of EES technologies demonstrates the range of commercial viability of different devices and which characteristics are most favourable for arbitrage. An assessment of the net present value and investment risk is included to highlight the high costs and risks facing firstof-a-kind deployments of EES.

Chapter 5 describes the model used to simulate electricity prices for future energy scenarios. Development of a competitive model of the electricity market is presented and validated using historic electricity prices. The method used to apply the model to future energy scenarios, with changing fuel and carbon prices, demand and wind power capacity is then explained.

In Chapter 6, the arbitrage and price models are coupled to investigate the impact of changing market conditions on arbitrage revenue. The sensitivity of revenue to individual market variables is explored, confirming the dependence of EES on uncertain market conditions. The characteristics of EES most favourable in markets with a high penetration of wind power are investigated, showing the changes from those favoured historically, a consequence of the transitioning electricity system. Finally, the National Grid Future Energy Scenarios are applied to the model to compare the performance of EES in alternative plausible future electricity systems.

Lastly, Chapter 7 summarises the thesis conclusions. The validity and implications of the results are discussed and recommendations for further work are made. 
Appendix A provides an overview of electricity market design and fundamentals of energy system economics which will be referred to throughout the rest of the thesis. A brief overview of the electricity industry in GB is presented to provide context for the study. 


\section{Chapter 2 Electricity}

\section{Storage Technologies}

On the grid, electricity is produced and consumed almost instantaneously. It is used within a tenth of a second of being generated and less than one tenth of a second of power produced can be stored as electrical energy in the system [31]. EES is a means of converting electrical energy into a form that can be stored so that it can be converted back into electrical energy to be used at a later date. EES has many uses including for portable devices, electric vehicles and grid-scale applications. In this chapter a brief history of EES development is presented followed by a summary of potential grid-scale EES technologies. Technologies are presented by type and then compared on basis of technical and commercial characteristics. Finally, potential future developments which may lead to breakthrough improvements in cost or performance are presented.

In the long term, electric vehicles are expected to be integrated with the grid, however, the principal application of these would be for transportation and so electric vehicle storage technologies are not explicitly examined. If electric vehicles are integrated with the grid, average and peak electricity loads are likely to increase in addition to providing further network storage capacity. The implications of this are discussed in later chapters.

\subsection{Historic Development}

Grid-scale EES has been implemented in GB since the turn of the twentieth century. Leadacid accumulators supplied loads on DC networks when generators were shut down overnight [32]. As consumer demand for electric lighting increased, the shape of the load profile became harder for generators to supply with a distinct evening peak as shown in Figure 2.1. Batteries were considered an ideal solution for load levelling, enabling generators to meet higher peak loads without being oversized for average demand. They also allowed generators to operate at 
a more constant output with higher efficiency. By 1910, all DC generators and some AC generators had their own batteries [33].

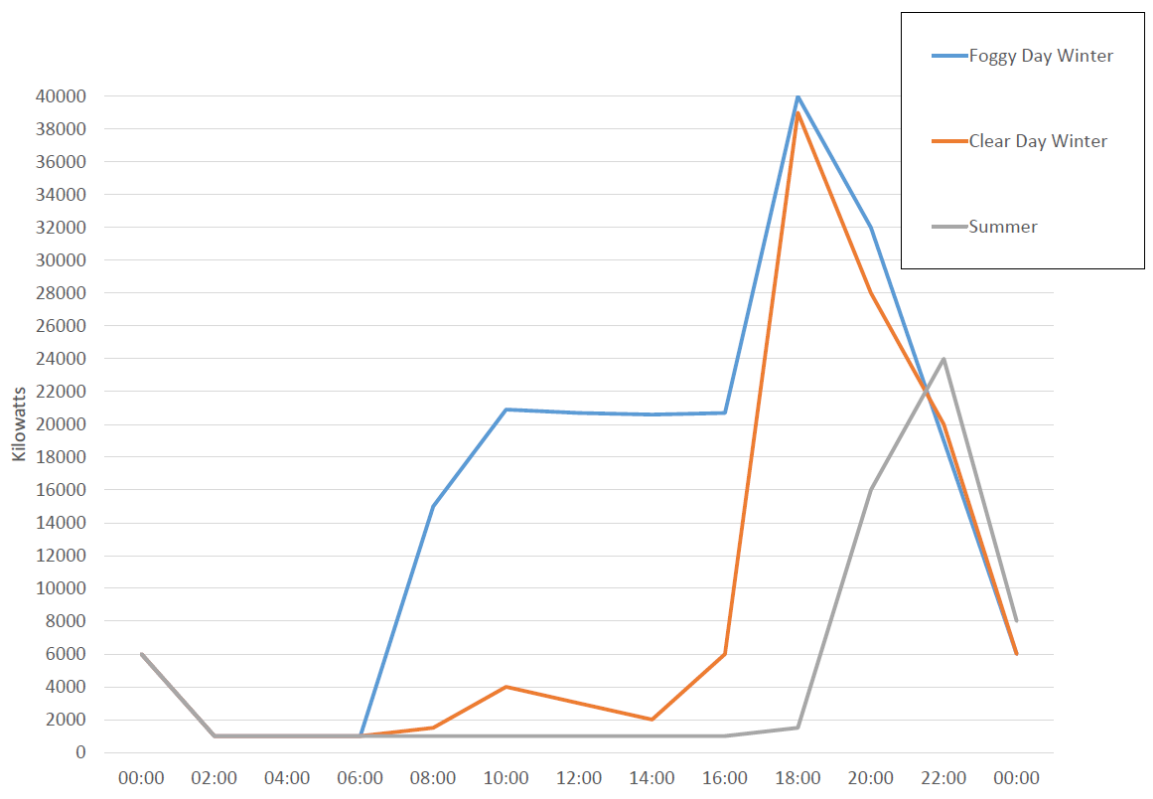

Figure 2.1 London daily load curves circa 1897 (source [34])

In America, utility companies recognised the importance of EES in power networks and Connecticut Light and Power Company built the first centralised pumped-hydroelectric storage (PHS) plant in 1927 which began operating in 1929 [35]. Despite the initial interest in EES, further development of the electricity industry was driven by achieving economies of scale through large central generating stations coupled with extensive transmission and distribution networks [36]. Demand was aggregated and profiles smoothed leading to more efficient operation of power stations reducing the requirement for storage. Interest in gridscale EES stalled and there was little development of technology [37].

In the 1960s, research into nuclear power instigated a second wave of interest in EES. Utilities anticipated future electricity systems with inflexible nuclear plant playing a dominant role. PHS was expected to provide peak power to compliment nuclear base load [38] and a number of projects were developed. There were few alternative EES technologies which could provide the capacity that PHS could and, consequently, there was little implementation of other technologies during this period.

By the 1990s, development of PHS declined for various economic and environmental reasons. Cheap gas prices, high interest rates and technical developments of the combined cycle gas turbine (CCGT) led to the dash for gas [39]. Gas turbines became more competitive for providing peak power than capital intensive pumped-hydroelectric plants. Additionally, 
environmental concerns drew out the permitting process and led to some cancelled projects [11]. It is likely that the re-structuring of the power sector during the 1990s also contributed to the slowdown. As discussed in the previous chapter, many countries liberalised generation markets while retaining a highly regulated transmission network. Adequate policies to support EES technology, which falls between the two, were not considered.

Since the start of the twenty first century, there has been a resurgence of interest in EES as a result of the increasing penetration of intermittent renewable energy. Today, there are 884 grid connected EES projects worldwide [40] with this number increasing as more demonstration projects come online. The US Department of Energy maintains a database of all global energy storage projects where the most up to date information can be found [40]. America and Japan are emerging as marker leaders with the largest number and capacity of EES projects connected to the grid. Despite the increasing number of projects being deployed, the total installed capacity remains relatively small with less than $150 \mathrm{GW}$ known to be in operation globally and only $3.2 \mathrm{GW}$ in the UK as of July 2015 [40]. High capital costs and poorly understood performance characteristics are inhibiting widespread uptake of storage. Furthermore, the market remains fragmented with over 136 companies offering different technology solutions [41]. There remains significant scope for development of EES and for rationalisation of the range of technologies in the future.

An overview of major grid-scale EES developments from 1859 to 2012 is shown in Figure 2.2 .

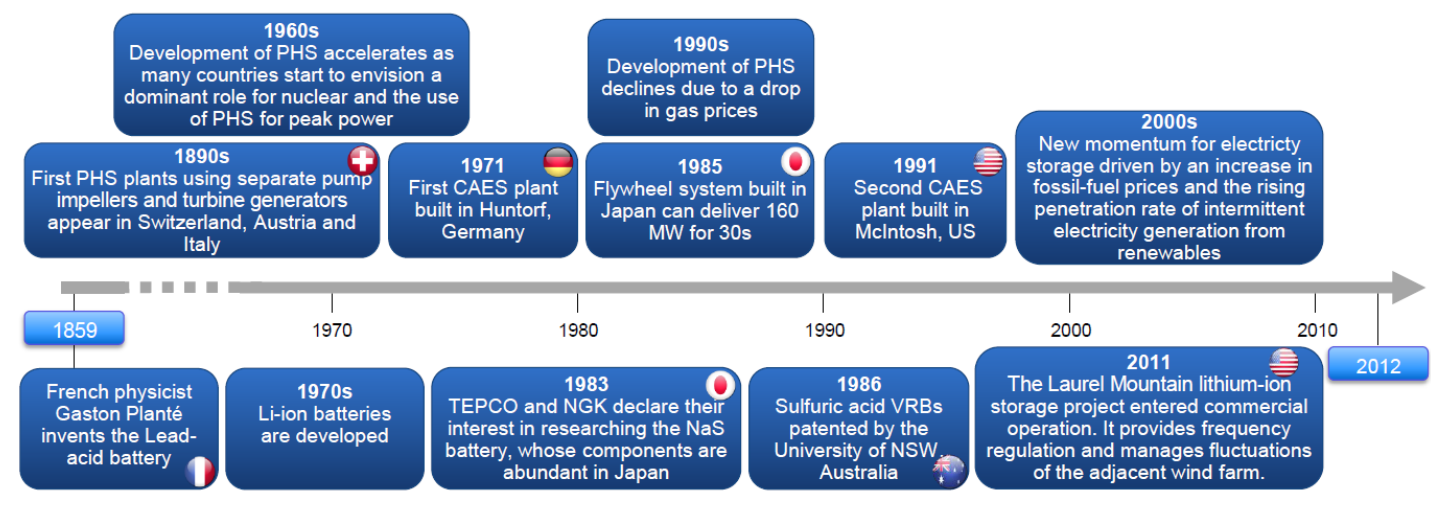

Figure 2.2 Electricity storage technology timeline (source [42])

In parallel to the development of grid-scale EES, industries for portable devices and, more recently, electric vehicles have emerged. Requirements for storage are very different to those for grid-scale storage with a focus on reduction in size and weight and less onerous demands 
on cycle life and storage capacity. However, technology breakthroughs from these industries have the potential to be applied to grid-scale EES in the future.

\subsection{Storage Technologies}

There is a wide range of EES technologies which are grouped in literature by a number of classifications including by technology characteristics or by application. Figure 2.3 lists EES technologies by the form of energy they use. Each technology is described below.

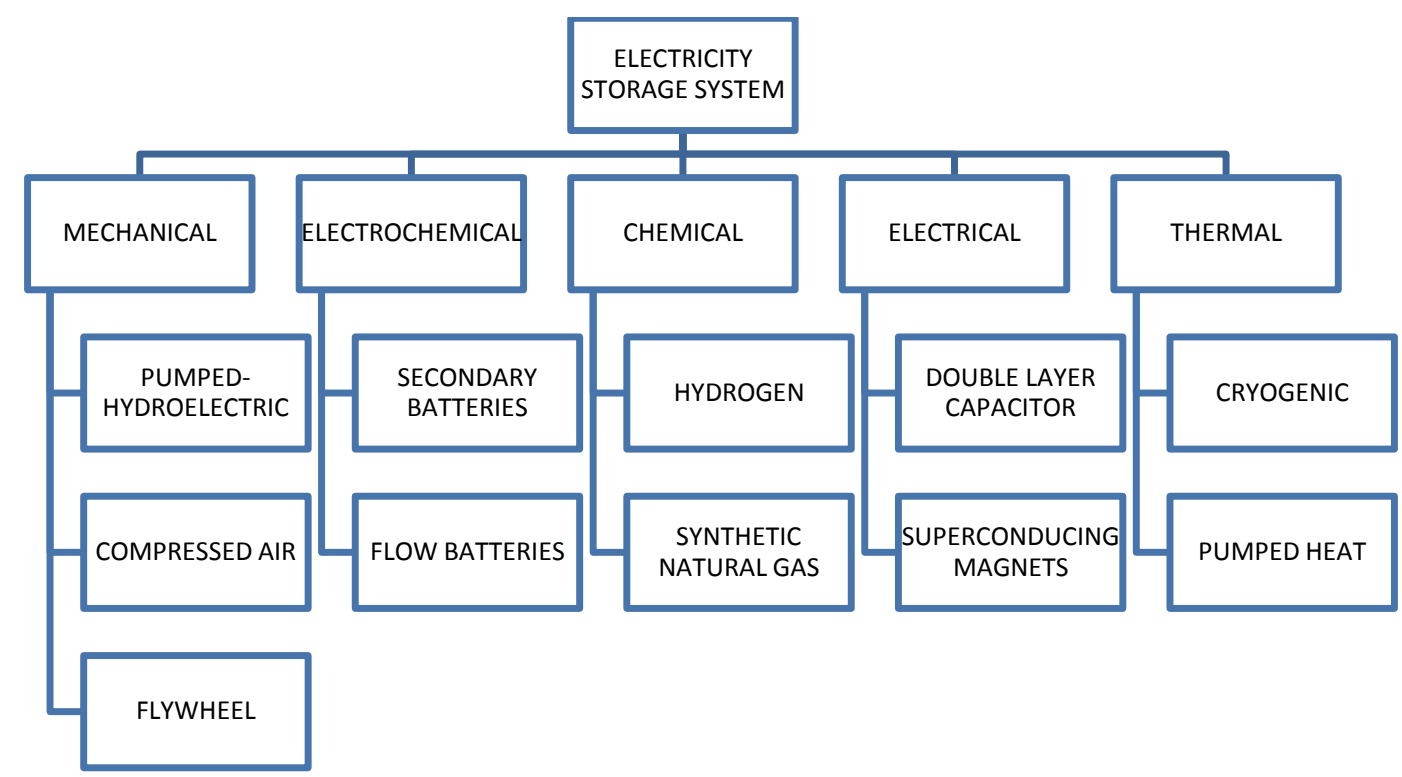

Figure 2.3 Summary of electrical energy storage technologies grouped by type of energy storage

\subsubsection{Mechanical Storage Systems}

\section{Pumped-hydroelectric storage}

Conventional PHS systems have two water reservoirs at different heights. Using electricity, water is pumped from the lower reservoir into the upper reservoir where it is stored as potential energy. When electricity is required, the water is discharged from the upper reservoir, passing through a turbine connected to a generator. An illustration of its operation is shown in Figure 2.4 . 


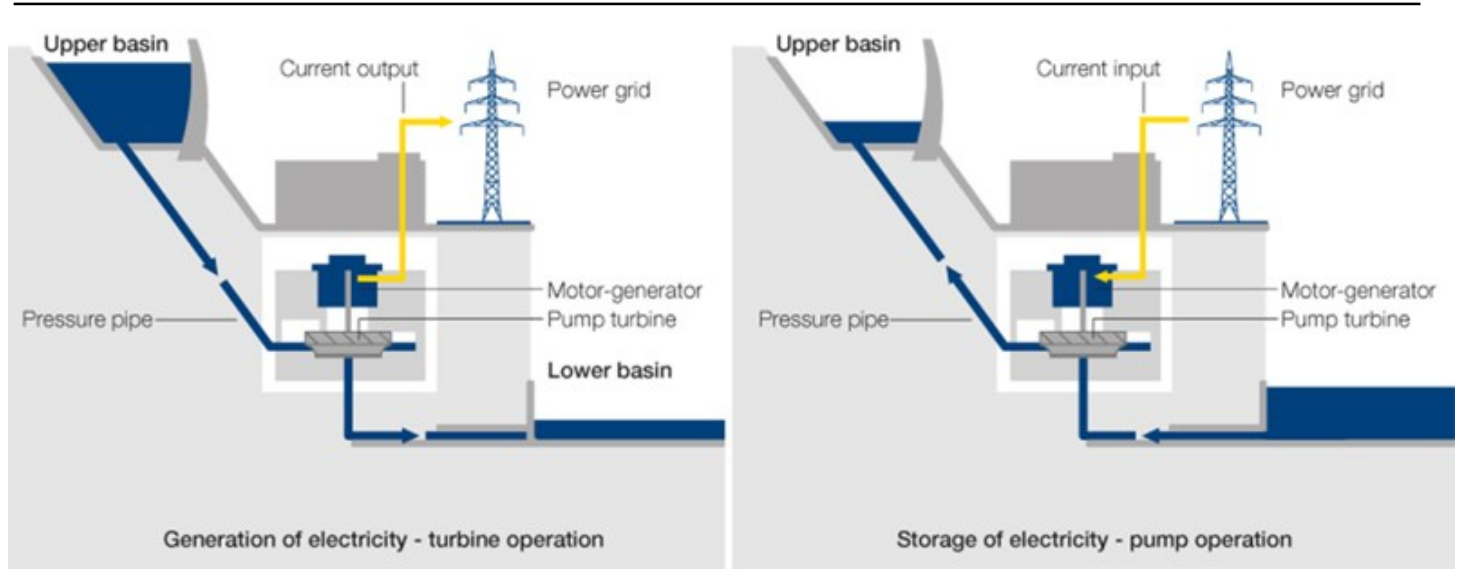

Figure 2.4 Operation of pumped hydro-electric plant (source [43])

PHS is the most commonly used and widely established storage technology. Over 120GW of PHS is installed worldwide making up almost $99 \%$ of global grid-scale EES [44]. The largest PHS plant in the world is in Virginia, USA and has a peak power output of 2100MW [45]. A picture of the upper reservoir is shown in Figure 2.5.

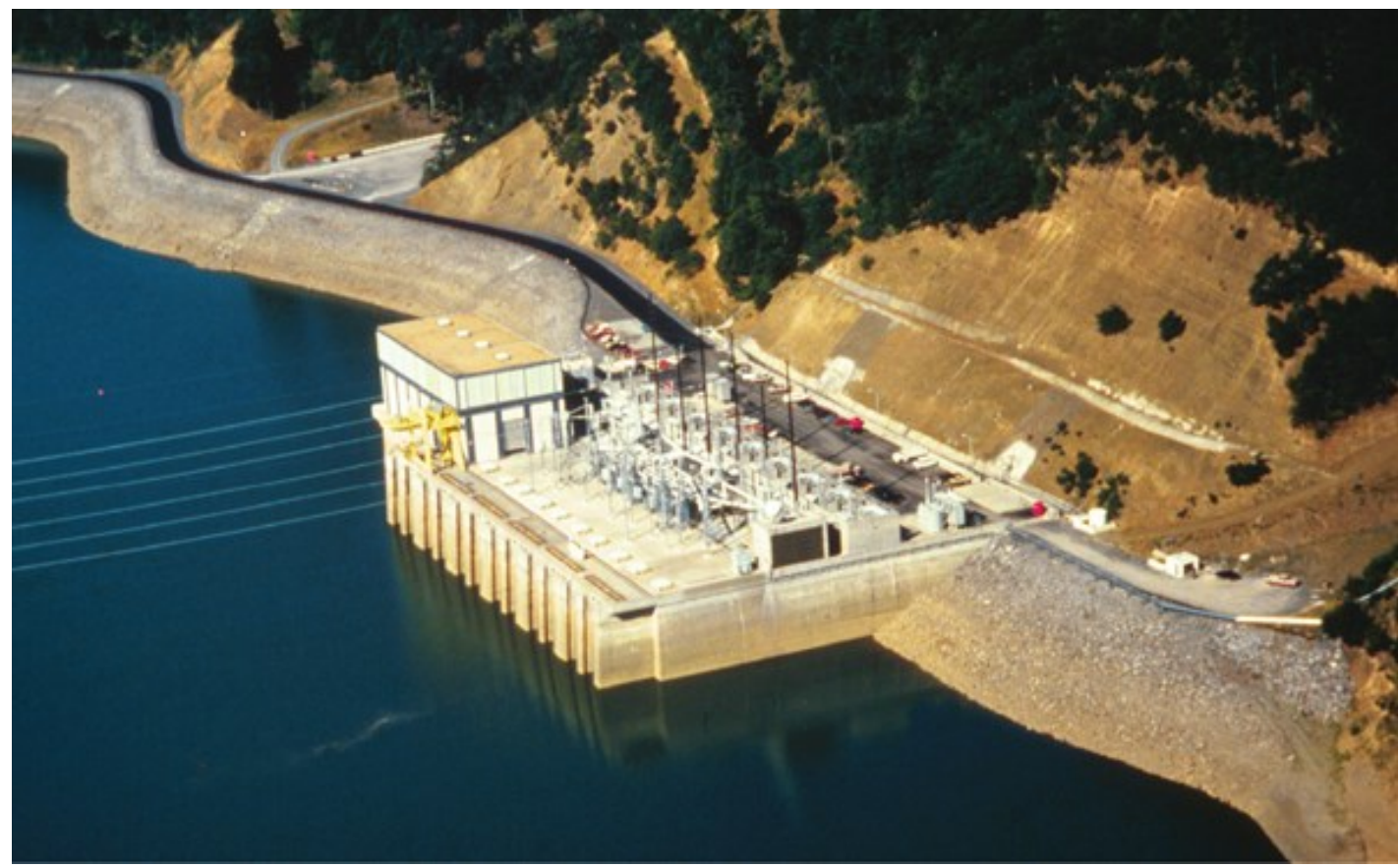

Figure 2.5 World's largest pumped-hydroelectric storage station, Virginia (source [46])

PHS plants can be designed to deliver 100-5000MW power. Discharge times can range from a few hours to a several days and average efficiency is $75-80 \%$ [47]. A Sankey diagram of the losses in the conversion process is shown in Figure 2.6. 


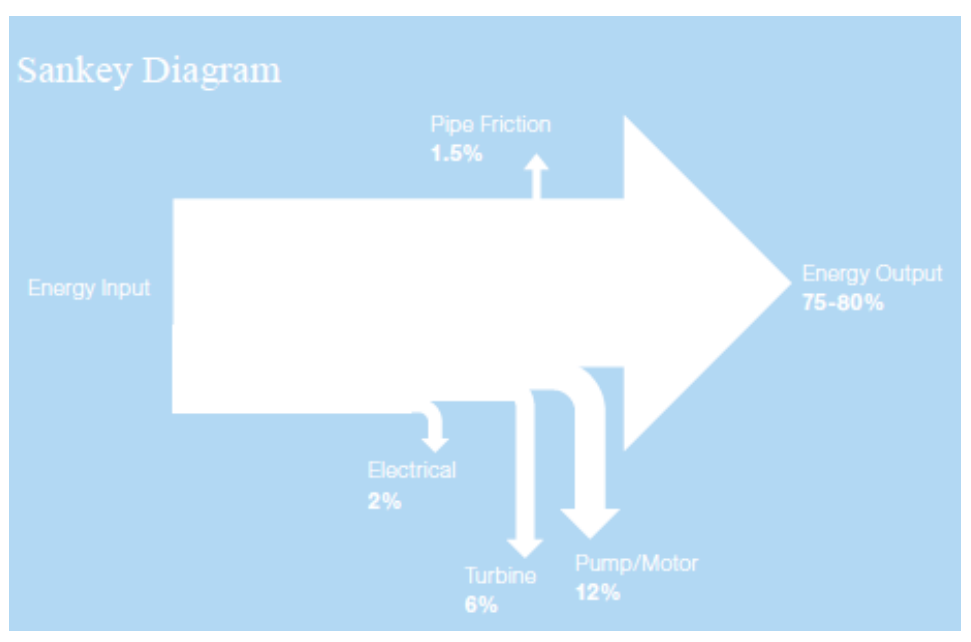

Figure 2.6 Sankey diagram of pumped-hydroelectric storage (source [48])

The advantages of the technology are its long lifetime and practically unlimited cycle stability. Plants can come online and ramp up to full power very rapidly. PHS is a mature technology, which is reliable and has the capability of delivering large power and energy capacities.

The disadvantages of PHS include its dependence on geographical conditions and extensive land use. There are many issues with planning permission because of environmental concerns. Additionally, sites are often located at significant distances from demand centres. PHS plants are capital intensive, requiring considerable civil structures to be built with typical lead times in excess of 10 years. Financial concerns are cited as the primary cause for projects being abandoned [49].

Their flexibility means that there is a wide range of applications which PHS could contribute to, but typically they are used for ancillary services including reserve supplies and provision of black start facilities. The largest plant in the UK is Dinorwig in North Wales which has a storage capacity of $9.1 \mathrm{GWh}$ and, within 12 seconds, can output $1.32 \mathrm{GW}$ of power [50]. It predominantly provides fast response and short term operating reserve services.

As a mature technology, there are few technical developments which are expected to lead to breakthrough improvements in performance or reductions in cost for PHS. Variable speed pumps can provide slightly higher efficiencies and better frequency regulation control. There have also been some improvements in turbine blade performance [49]. Recently, there have been proposals to increase the energy density of PHS through pressurising the water flow. This approach, hydraulic PHS, would use water pressure to lift a heavy weight such as an excavated rock cylinder. However, work in this area remains theoretical and issues with tectonic movements, maintaining water seals and friction must be overcome [51]. 
Developments for existing designs of PHS are limited due to environmental considerations. There are counties with sites still available, but it is estimated that $75 \%$ of PHS sites in Europe have already been exploited [52]. Small scale (mini-hydro) plants are expected to be developed, but these would principally be for isolated communities which operate on local grids. Less conventional solutions are also being sought to increase large PHS capacity. By 2030, over 50\% of Europe's conventional reservoir hydroelectric generators will need to be refurbished and these could be retrofitted with pump facilities during this time. Furthermore, existing PHS sites may be expanded. Scottish Power, which operates Cruachan, one of four large PHS plants in the UK, is currently undertaking a cost benefit feasibility study into doubling its capacity [53]. Underground PHS is also being investigated as an option for new capacity. These systems would make use of existing underground infrastructure such as abandoned mine shafts, quarries or caverns to facilitate reservoirs and pumping and generating machinery. These projects would mitigate many environmental and local planning concerns and also reduce the noise associated with PHS operation [49]. However, high capital costs and identification of suitable underground caverns may be obstacles to development. Offshore PHS has also been proposed; however, capital expenditure and issues with corrosion have prevented any projects being developed to date [49].

\section{Compressed air energy storage}

Compressed air energy storage (CAES) is similar to standard gas turbine technology; however, the compression and expansion cycles are decoupled into two discrete processes. Electricity is used, instead of gas, to compress air and the energy is stored as elastic potential energy [54]. The compressed air is stored in either underground caverns or in storage vessels. When electricity is required, the compressed air is combined with gas, combusted and expanded through a turbine connected to a generator. A sketch of the system is shown in Figure 2.7. 


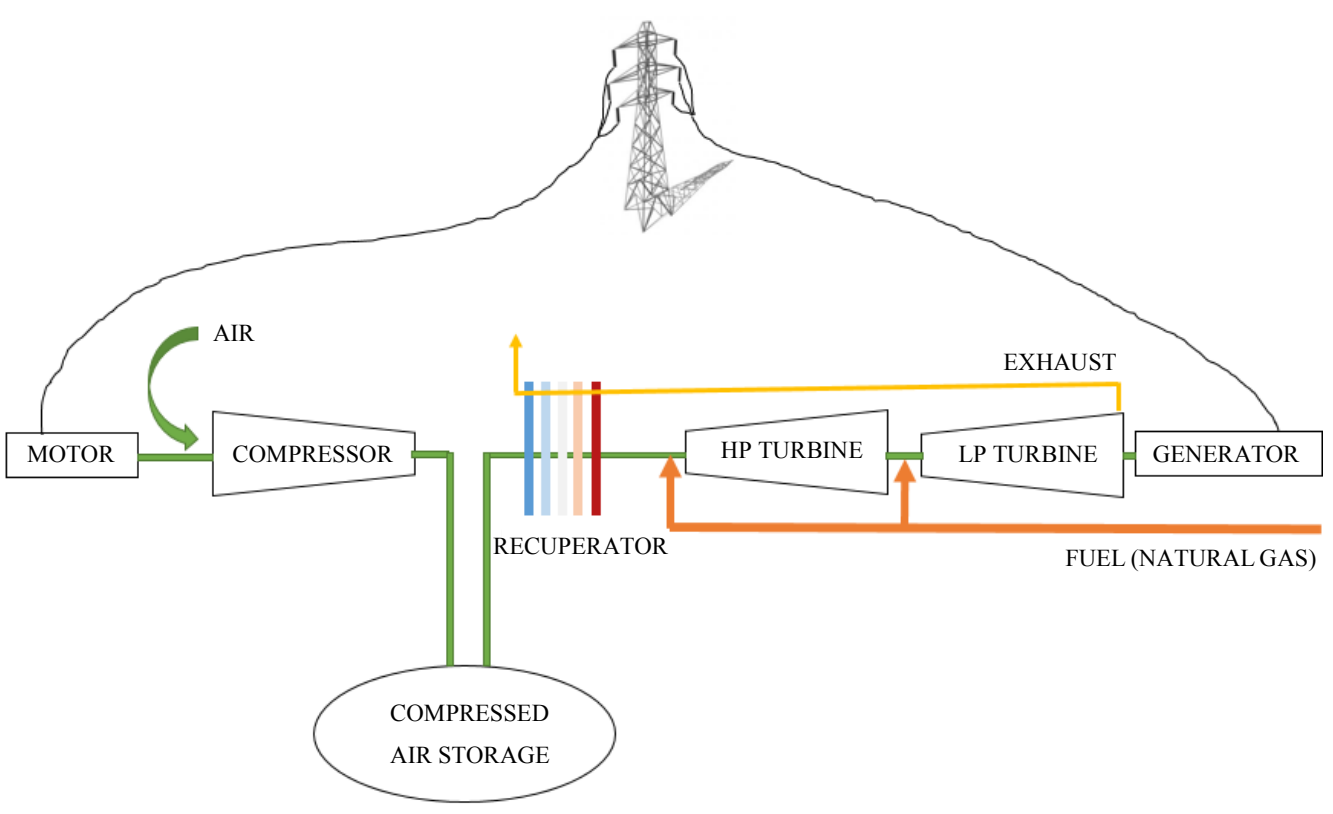

Figure 2.7 Compressed air energy storage system (based on [55])

Typical storage capacities are 50-300MW and CAES is the only commercial technology other than PHS which is capable of providing large capacity with a single unit [37]. Underground storage makes use of existing caverns or abandoned mines and is the most cost effective method of storage. Over ground units tend to be smaller and more expensive [49].

Advantages of CAES include its large capacity and rapid start up time. It is a simple, reliable system with a long lifetime, although slightly shorter than PHS. The systems can tolerate frequent cycling and can operate efficiently at part load. The system can store energy for relatively long periods of time (over a year) and has a fairly low capital cost [37]. CAES can serve many applications, but is suited to load levelling and balancing because of its rapid response time.

Disadvantages include its low roundtrip efficiency. Currently CAES systems dissipate the heat generated during air compression into the atmosphere. Before the air is expanded, it must be reheated which results in efficiencies not much higher than 50\% [48]. A Sankey diagram of the storage process is shown in Figure 2.8. Like PHS, underground storage is geographically constrained and economic projects are limited to locations near rock mines, salt caverns or depleted gas fields. The process burns natural gas relying on a finite resource and emitting fossil fuels. This attribute also means that operation costs are variable, being dependent on gas prices which can be volatile. 


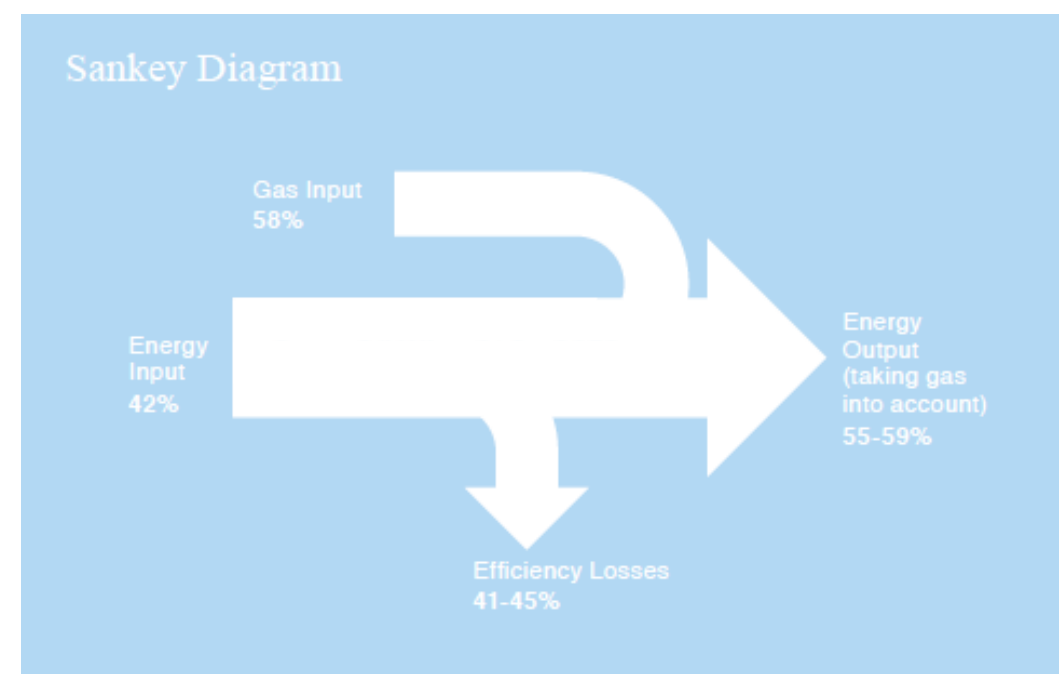

Figure 2.8 Sankey diagram of compressed air energy storage (source [82])

CAES has been used for industrial processes for over 200 years [45]. The first utility scale project was a 290MW plant in Germany using a salt dome for storage. This began operation in 1978 [56]. In 1991 a $110 \mathrm{MW}$ plant with a 26 hour capacity was built in Alabama, USA. The facility cost $\$ 65 \mathrm{~m}$ and uses salt caverns to store air at up to 76Bar [57]. In December 2012, General Compressions completed construction of a 2MW facility in Texas which became the world's third CAES project [58]. Further projects in the USA, Japan and South Africa are planned or under construction.

Research and development is focussed on adiabatic CAES where the heat generated during compression is recycled for use in the expansion process. This is expected to increase efficiency significantly [59]. Operational experience is also expected to bring cost reductions and performance improvements as more projects are implemented. Different configurations of inter-cooling and humidification and simplified high pressure turbo expander train designs are anticipated to bring about further improvements [52].

\section{Flywheel energy storage}

Flywheel energy storage (FES) systems store energy in the angular momentum of a spinning mass. The principal components of the technology are the rotating body or cylinder in a compartment, bearings and a transmission device. Energy is maintained by keeping the flywheel at constant speed. A sketch of a FES system is shown in Figure 2.9 


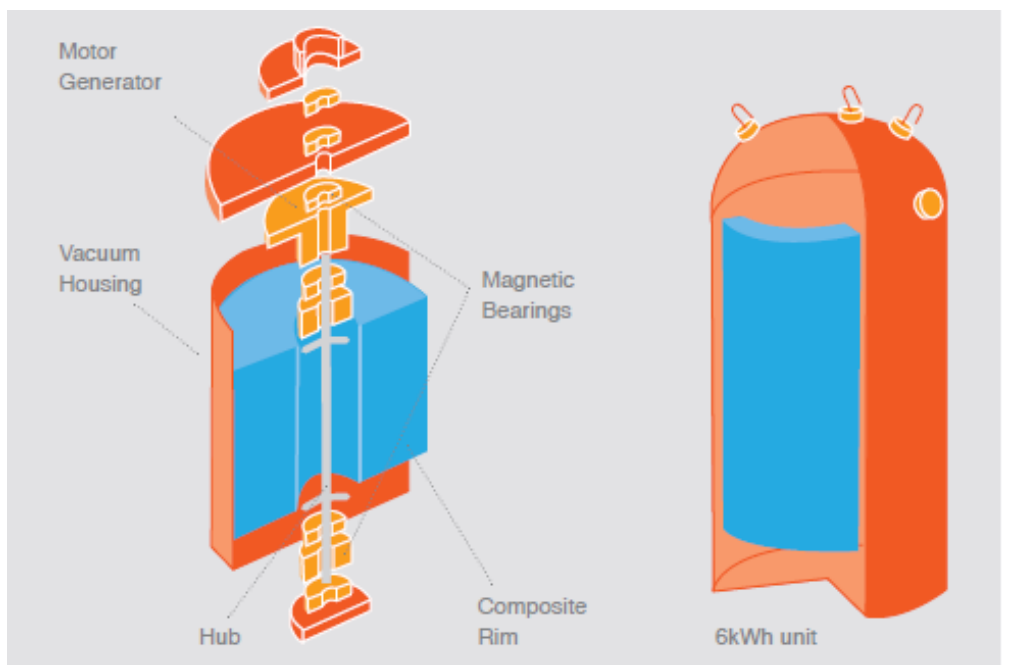

Figure 2.9 Flywheel electricity storage technology (source [48])

The principal advantages of the technology are its cycle stability and long life with little maintenance which can last over several hundreds of thousands of cycles. The device is capable of fully discharging and its performance is almost completely independent of temperature. FES has a high power density and can provide rapid response for high power applications [60].

The principal disadvantage is that FES systems experience very high rates of self-discharge so are not suitable for storing energy over long periods. This is predominantly due to friction losses at the bearings. Furthermore, the components must be housed in a robust container due to the large amount of energy stored in the spinning mass. This, and the high precision engineering required to minimise the losses, leads to high costs.

A Sankey diagram of the FES system is shown in Figure 2.10.

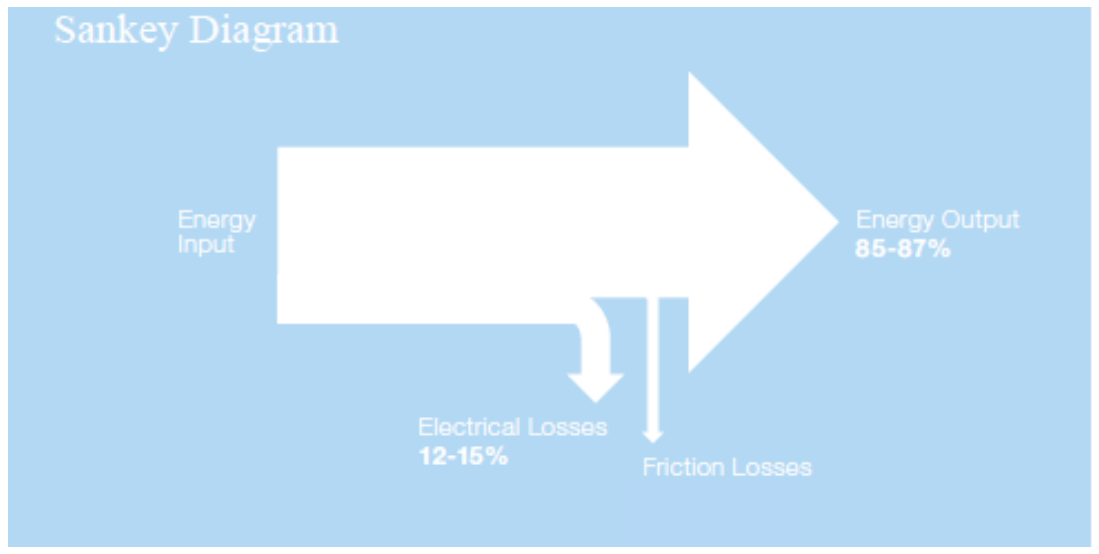

Figure 2.10 Sankey diagram of flywheel energy storage (source [48]) 
First generation FES systems for grid applications have been available since 1970 utilising a steel cylinder spinning on mechanical bearings [45]. Improved devices now use a carbon filament mass, which has high strength, inside a vacuum on magnetic bearings to reduce friction losses. These lead to self-discharge rates of around 1\% per hour compared to mechanical bearings which have typical losses of $5 \%$ over the same time period [61]. FES is used commercially in industrial applications for power quality because of its ability to rapidly charge and discharge [62]; however, there are limited examples of grid-scale projects due to their high cost.

The principal barrier to development is the cost of precision engineering. There are two directions in which the industry is progressing to address this. Firstly, cost reduction is anticipated through mass production. Units are expected to be built up in modules where space is not restricted [63]. Secondly, advanced performance devices are being developed to operate at higher angular velocities, improving the capacity and reducing the cost per megawatt (MW). FES performance is ultimately limited by material properties. To increase the power capacity, flywheels must be made larger and operated at higher speeds. Eventually, the linear speed of the material at the outer radius of the flywheel will be so high that the material will become unstable. Advances in material developments have led to a transition from low speed FES systems operating at approximately 6,000 rotations per minute (rmp) to high speed systems operating between 10,000 and 110,000rpm [64].

\subsubsection{Electrochemical Storage Systems}

\section{Secondary batteries}

Secondary batteries comprise one or more electrochemical cells containing an electrolyte, a positive electrode (anode) and a negative electrode (cathode). When an electrical load is connected to the battery, an electrochemical reaction occurs at the electrodes which generates electricity. When an external voltage is applied across the electrodes, the reaction is reversed and the battery is recharged [37].

There are various types of secondary battery. Characteristics differ between types, but they tend to have low power density with low self-discharge losses and high efficiencies. Large scale deployments are rare with batteries making up less than $1 \%$ of grid-scale EES [65]. This is due to their short cycle life, sensitivity to depth of discharge and high maintenance costs. Additionally, many batteries contain toxic materials with an environmental impact associated with their disposal. 


\section{Lead Acid Batteries}

Lead acid batteries are the most commonly used batteries worldwide. They were invented in 1859 and have been commercially available since 1890. Typically, they are used for emergency power supplies or standalone systems. They are also used as starter batteries in cars. During early electrification, lead acid batteries were employed as grid connected storage as discussed in Section 2.1 [45].

Lead acid batteries have a high roundtrip efficiency of $85-90 \%$ and low self-discharge rates. A Sankey diagram of the conversion process is shown in Figure 2.11. They are a proven technology with an existing infrastructure from the automotive industry for end of life recycling [48].

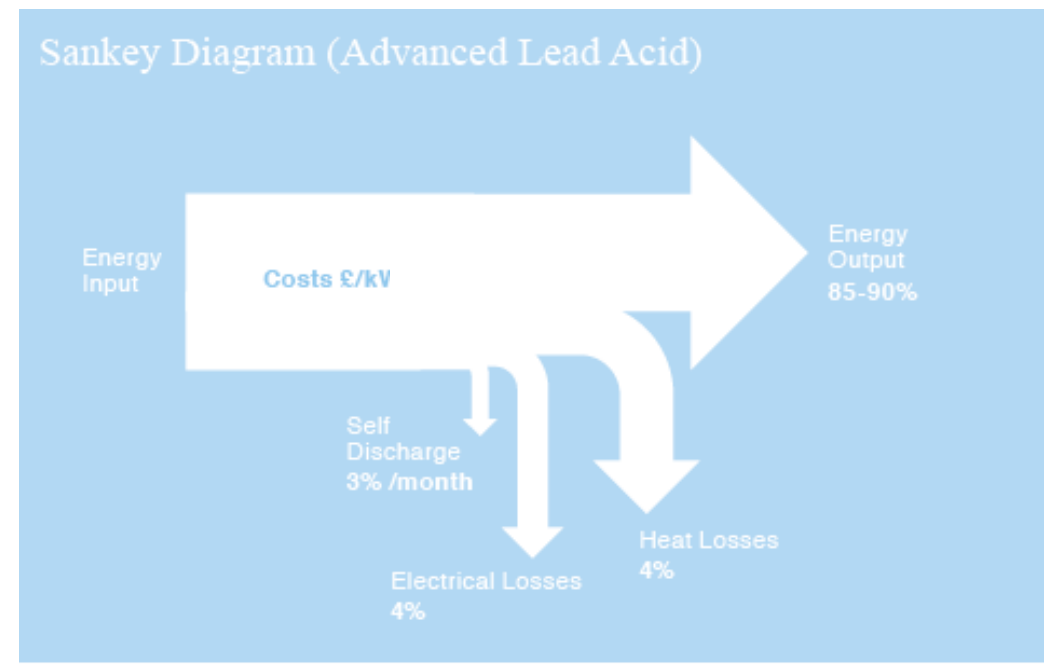

Figure 2.11 Sankey diagram of advanced lead acid battery (source [48])

The cycle life of lead acid batteries is only around 1,000 cycles and they have a low power density. They do not perform well at elevated temperatures and need an integrated thermal management system. The energy capacity is also reduced when there is a high power demand and they use lead which is a hazardous material.

Commercial applications of grid-scale lead acid batteries have included:

1. An $8.5 \mathrm{MW}$ battery with $14 \mathrm{MWh}$ storage capacity operated in Germany to supply frequency and spinning reserve services to West Berlin. In 1993, after 7 years of operation, BEWAG was connected to the West European grid resolving the frequency issues and the storage was decommissioned [66]. 
2. A $10 \mathrm{MW}$ battery with $40 \mathrm{MWh}$ storage capacity in California, USA built as a demonstration project. At the time, this was the world's largest lead acid battery storage system. It was decommissioned in 1997 after nine years of operation as an experimental facility [67].

3. In 1994 a 20MW 14MWh system began operation in Puerto Rico to provide spinning reserve, frequency control and voltage regulation services. It was closed in 1999 after numerous cell failures [68].

The poor cycle life and relatively slow charging rate of lead acid batteries have prevented significant commercial success of the technology for grid applications. However, recent developments, driven predominantly by requirements for hybrid electric vehicles, have led to innovations in advanced lead acid batteries which address some of these deficiencies. Advanced lead acid batteries incorporate carbon into the negative electrode, significantly improving the system cycle life [69].

\section{Nickel Cadmium and Nickel Metal Hydride Batteries}

Nickel cadmium (NiCd) batteries have been available since 1915; however, they have not experienced the same level of commercial success as lead acid batteries largely due to their considerably higher cost and safety concerns [45].

$\mathrm{NiCd}$ batteries have a high tolerance to a range of temperatures and can operate at $-40^{\circ} \mathrm{C}$. They also have a relatively high power density and long cycle life. However, due to the use of highly toxic cadmium, in Europe they are only permitted in static applications and in 2006, they were banned for use by consumers due to environmental concerns about disposing of spent units [45].

Nickel metal hydride (NiMH) batteries were developed in response to concerns over $\mathrm{NiCd}$ batteries however, they have slightly different characteristics such as high energy density but significantly lower maximum power capacity. As a result, they were commonly used in mobile applications including power tools; however, they have since been replaced by lithium ion batteries which are cheaper. The electric vehicle industry does favour NiMH batteries over lithium ion batteries as they are considered more robust and the materials are relatively more abundant [70]. NiMH batteries are not expected to make a significant contribution to gridscale EES in the future. 


\section{Lithium Ion Batteries}

The electrolyte in a lithium ion (Li-ion) battery is formed of lithium salts dissolved in organic compounds. During charging and discharging lithium ions move between the metal oxide cathode and the graphite carbon anode [37].

The concept of lithium ion batteries was suggested in the 1960s and they were first produced when a practical graphite anode was developed by Bell Laboratories. The first commercial batteries were produced by Sony in 1990. Since then, improvements in material science have allowed higher energy density and longer cycle life, of up to 10,000 cycles to be attained [37].

Li-ion batteries have a high efficiency and a flexible discharge time which can range from seconds to weeks. A Sankey diagram of the system is shown in Figure 2.12.

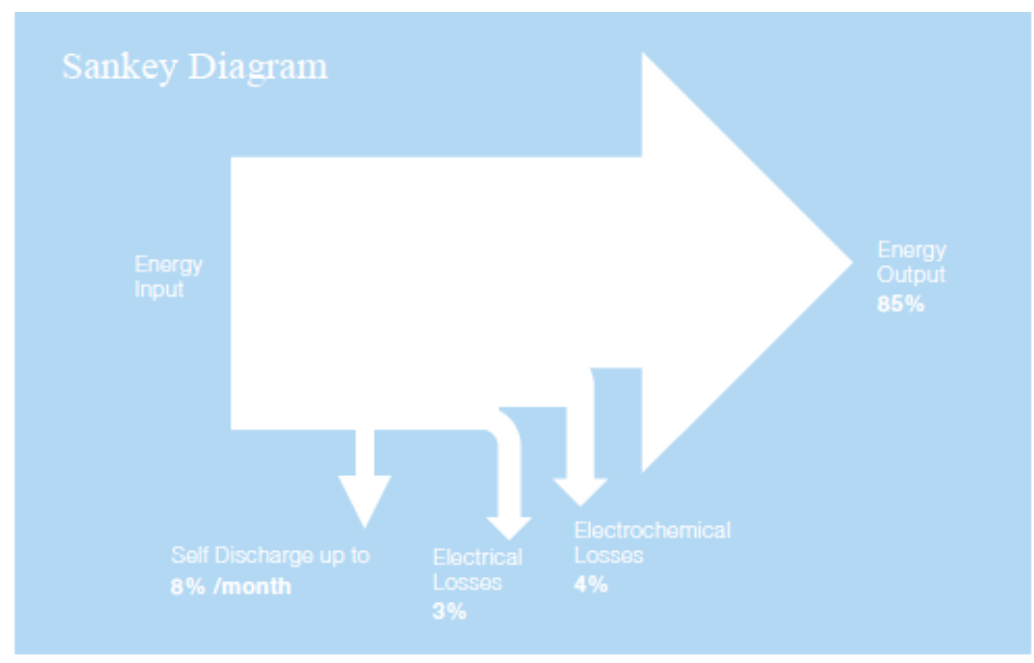

Figure 2.12 Sankey diagram of lithium ion battery (source [48])

They are currently expensive and experience negative effects if overcharged/discharged. There are also issues associated with overheating. As with most metal oxides, they are unstable at high temperatures and can release oxygen which leads to thermal runaway, fires and even explosions. To minimise these risks, the batteries are fitted with monitoring units to avoid overcharging [37]. Further disadvantages include poor performance when cycled over a range of discharge levels and concerns over the finite resource availability of lithium [71].

Li-ion batteries have been the most commonly used storage technology for portable and mobile applications since 2000. Currently, they take over $50 \%$ of the market share [37]. The high cell voltage levels mean a reduced number of cells, and associated electronics, can be used; one Li-ion cell can replace three NiCd cells. These cells can be scaled up for distribution scale EES and research and development over the coming years is expected to drive down 
costs. The main challenge to using $\mathrm{Li}$-ion batteries at a large scale is the high cost of internal protection circuits which prevent overcharging.

Lithium ion batteries are best suited to power quality applications; however, to date there have been a limited number of grid-scale installations. In 2015, there were 230 Li-ion battery installations world-wide totalling $310 \mathrm{MW}$. However, only 7 of these were rated at greater than 10MW [34]. In 2013 UK Power Networks commissioned Europe's largest Li-ion storage facility in Bedfordshire, UK. The $6 \mathrm{MW} / 10 \mathrm{MWh}$ device was installed as a demonstration project to improve the understanding of the economic case for grid connected EES in distribution networks [72]. Despite the limited number of units in operation a recent report by Lux Research indicated that Li-ion batteries would dominate the grid-scale EES market in the future. $90 \%$ of all grid-scale EES projects proposed in 2014 used this technology [73].

In 2015 Tesla announced the launch of its Li-ion battery, Powerwall, intended for consumer use coupled with solar panels at a domestic level. Its intention is to create the infrastructure to enable the emerging electric vehicle industry in which Tesla is a market leader. However, the Powerwall product is also marketed at utility scale for grid applications [74]. Several other companies are working on Li-ion battery development including Kyushi Electric Power and Mitsibushi Heavy Industries. There is significant potential for cost reduction through mass production and refinement of the manufacturing process. Other research is focussed on enhancing materials used for cathodes. The expected cost reduction per kilowatt hour (kWh) of the battery, excluding protective equipment and power electronics, is presented in Figure 2.13 .

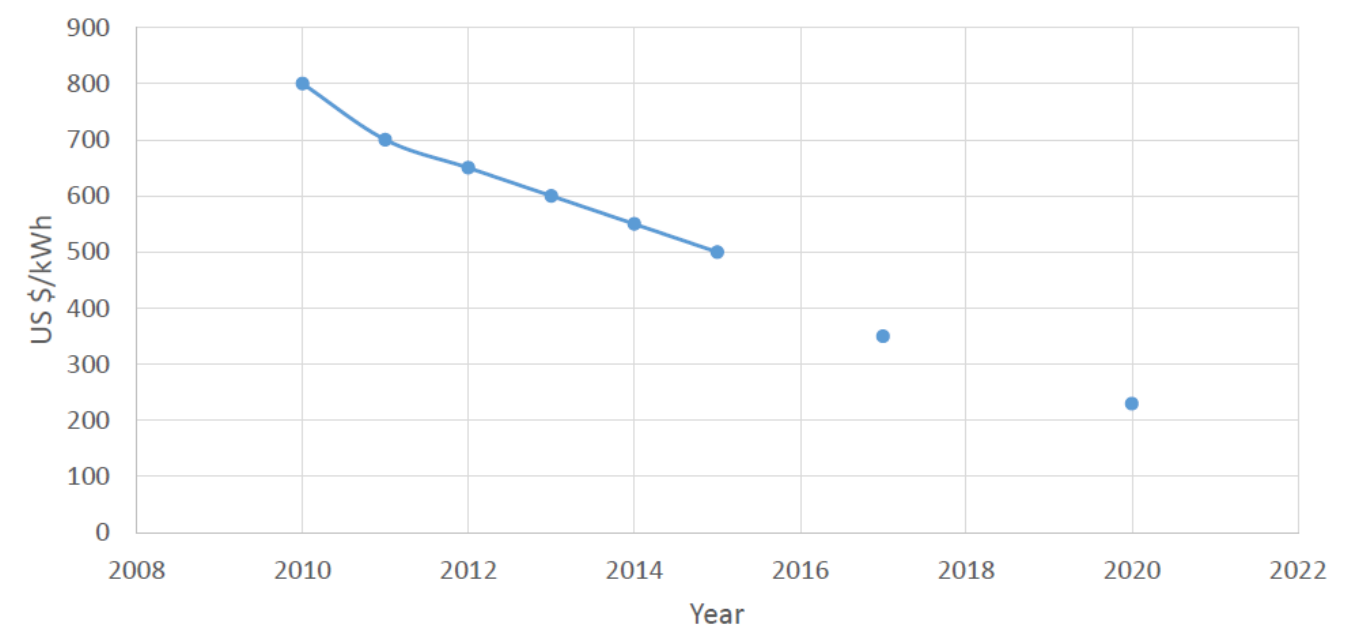

Figure 2.13 Lithium ion battery cost reduction since 2010 and cost projection to 2020 (source [34]) 


\section{Metal Air Batteries}

Metal air batteries consist of an anode made from a pure metal and a cathode connected to a continuous supply of air. Various metals could be used, but currently only zinc air batteries are technically feasible. Lithium would be a more attractive metal to use as it has a high specific energy but there is a high risk of fire from a reaction between the lithium and moisture in the air. The reaction rate of the battery is controlled by varying the flow of air.

To date there has been no grid-scale development of this technology. Electrical recharging of the battery is difficult and inefficient. Generally, the battery unit is replaced and the recharging process undertaken in a separate industrial location. If a more effective means of recharging could be developed, this type of battery could offer low material costs and high specific energy [45].

\section{Sodium Sulphur Batteries}

Sodium Sulphur $(\mathrm{NaS})$ batteries are molten state batteries which use a sulphur coated anode and a molten sodium cathode. The temperature of the battery must be maintained within the range of $300-350^{\circ} \mathrm{C}$ to maintain the molten state of the cathode. When the battery is charged, sodium ions pass into a reservoir through an ion selective conductor. There is no self-discharge because the electrons cannot move across the conductor. When the battery is not in use, it is typically left in a molten state of charge. If it is cooled it must be reheated before it can be used which is time consuming.

These types of battery have a reasonable reputation for cycle life of up to 4,500 cycles. Provided they are kept in a charged state, they have fast response rate in the range of milliseconds. They can provide short bursts of power at six times their standard power rating and so can be used economically for energy shifting combined with power quality applications [45]. Further advantages include the relative abundance of the raw materials [49].

The main disadvantage is that a heat source is required, using energy and reducing the performance of the battery. However, if the batteries are cycled on a daily basis, with appropriate insulation, they can be kept in a molten state from the heat produced by their own reactions. There are also potential safety issues with molten sodium being in close proximity to molten sulphur. These are separated by only a brittle ceramic tube which, if damaged, would allow a runaway thermal reaction. This would lead to fires which could extend beyond the single cell and propagate through the entire battery unit.

A Sankey diagram of the NaS system is shown in Figure 2.14 


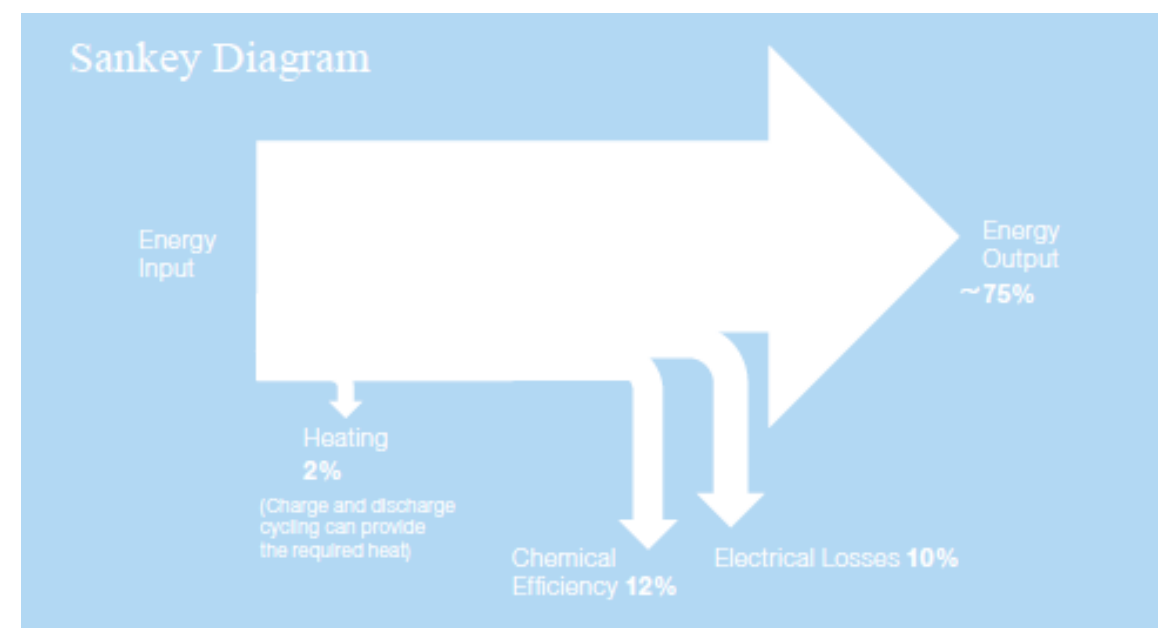

Figure 2.14 Sankey diagram of sodium sulphur battery system (source [48])

There were major development programmes in Canada, Germany, Japan and the USA from 1960-1990, which (excluding Japan) focussed on applications for electric vehicles. For commercial and safety reasons all these development programmes were abandoned apart from the Japanese.

In the 1990s, NaS batteries were manufactured by a single Japanese company, NKG Insulators, which produced units of $50 \mathrm{~kW}$ and typically $300-360 \mathrm{kWh}$ of storage capacity. These are not practical to use as a single unit and so 20 or more batteries are combined to make up a commercial plant with power ratings of approximately $1 \mathrm{MW}$ and energy capacities of 67MWh. Over 200 batteries have been deployed throughout Japan and Germany, France, the USA and UAE also have grid connected units [75].

Highly toxic hydrogen sulphide was released following a fire at a battery facility in September 2011. Following this incident, all production and operation was stopped pending investigation [44]. The investigation concluded that the fire was caused by a manufacturing defect leading to a short circuit in one of the cells. Manufacturing was resumed in 2012 with the addition of fuses and short circuit prevention plates. All units produced prior to the incident were recalled and retrofitted with the safety measures.

\section{Sodium Nickel Chloride Batteries}

Sodium nickel chloride $(\mathrm{NaNiCl})$ batteries consist of a nickel chloride cathode, a beta alumina separator and a liquid sodium anode. Similarly to the NaS battery, electrons cannot move through the beta aluminia and so the battery experiences no self-discharge, although heating can consume up to $14 \%$ of the energy capacity per day [76]). 
These batteries are also known as ZEBRA (Zero Emission Battery Research Activity) Batteries and have been commercially available since 1995. They are high temperature batteries and an operating temperature of $260-360^{\circ} \mathrm{C}$ is required to maintain a molten anode. Similarly to NaS batteries, they are generally left under charge and molten so that they are ready to use when required [45]. They are considered safer than $\mathrm{NaS}$ batteries and are fabricated from low cost materials.

Disadvantages include that preheating of the battery is required, there are safety concerns associated with molten sodium and they are not suitable for applications with short, frequent cycles.

A Sankey diagram of the process is shown in Figure 2.15.

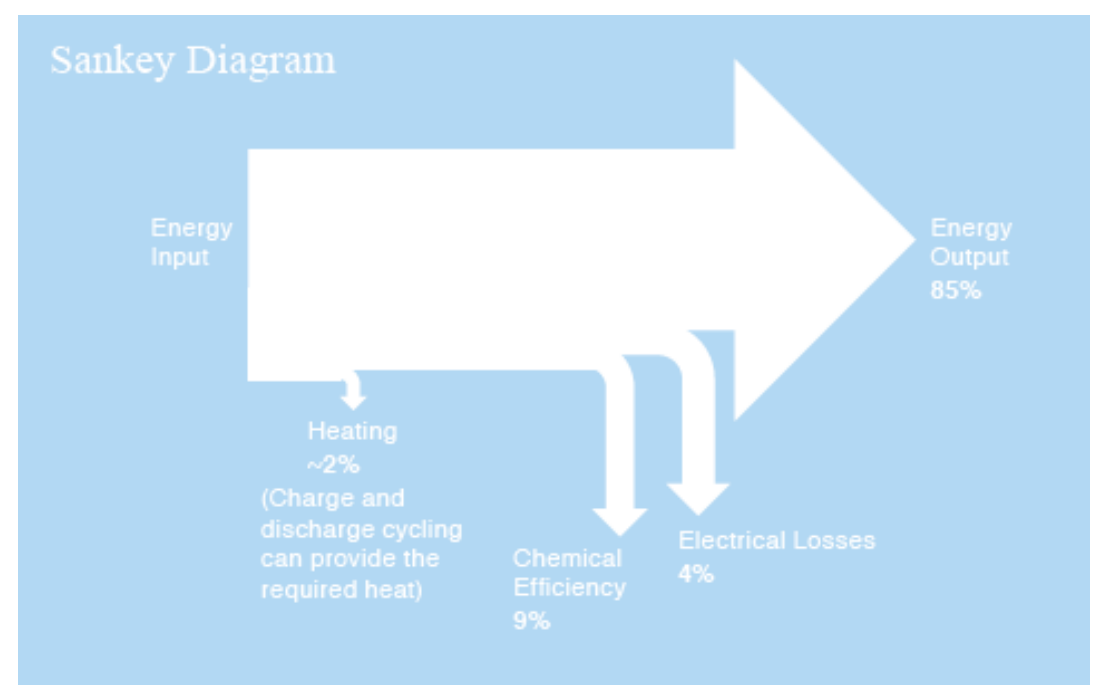

Figure 2.15 Sankey diagram of sodium nickel chloride battery (source [48])

$\mathrm{NaNiCl}$ batteries have been implemented in electric vehicles and future development is focussed on this application with objectives to improve power density. There are higher energy versions available which would suit grid-scale applications including load balancing and asset upgrade deferral. However, there are few examples of these applications in practice. One example is a $400 \mathrm{~kW}$ unit in development in Charlotte NC, USA which is used for smoothing output from solar power [2]. There is expected to be slow development in the future, as the batteries are currently only produced by one company, Beta R\&D. 


\section{Flow batteries}

Flow batteries contain two liquid electrolytes which are separated by an ion selective membrane. When discharging, selected ions are allowed to pass through the membrane and complete chemical reactions to produce electricity. Tanks are used to store the electrolytes which are pumped into the battery when needed. The power depends on the rate of electrode reactions occurring whereas the energy storage capacity is dependent on the tank size. A diagram of the system is shown in Figure 2.16.

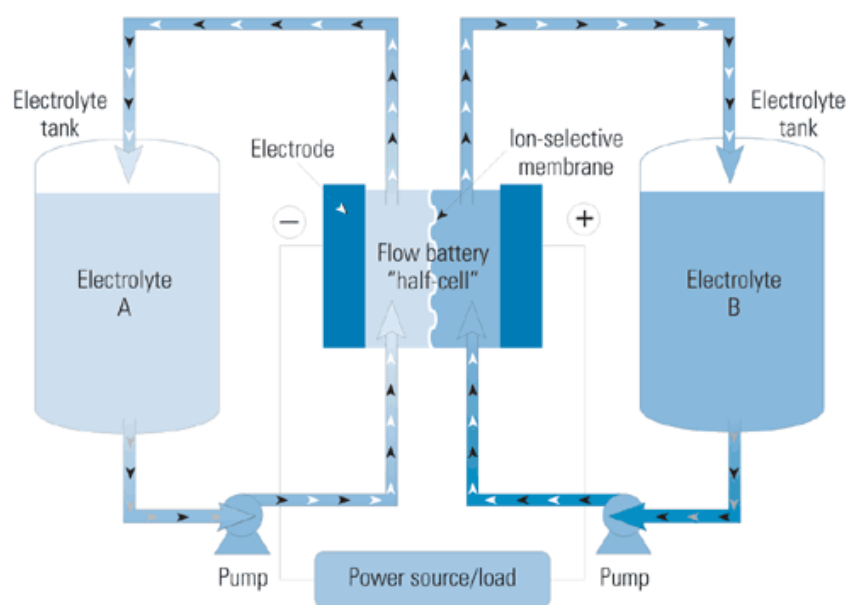

Figure 2.16 Flow battery system (source [77])

Flow batteries have a very long cycle life and are not sensitive to the depth of the discharge cycle. They can be recharged in the time it takes to exchange the tanks of spent electrolyte for ones containing charged electrolyte. The recharging of the electrolyte can then take place out with the system.

Disadvantages include that they have a low energy density and high capital and operation costs associated with the chemical plant pumping system and flow control. Their conversion efficiency is lower than most secondary batteries at $65-75 \%$ [48]. A Sankey diagram of the process is shown in Figure 2.17. 


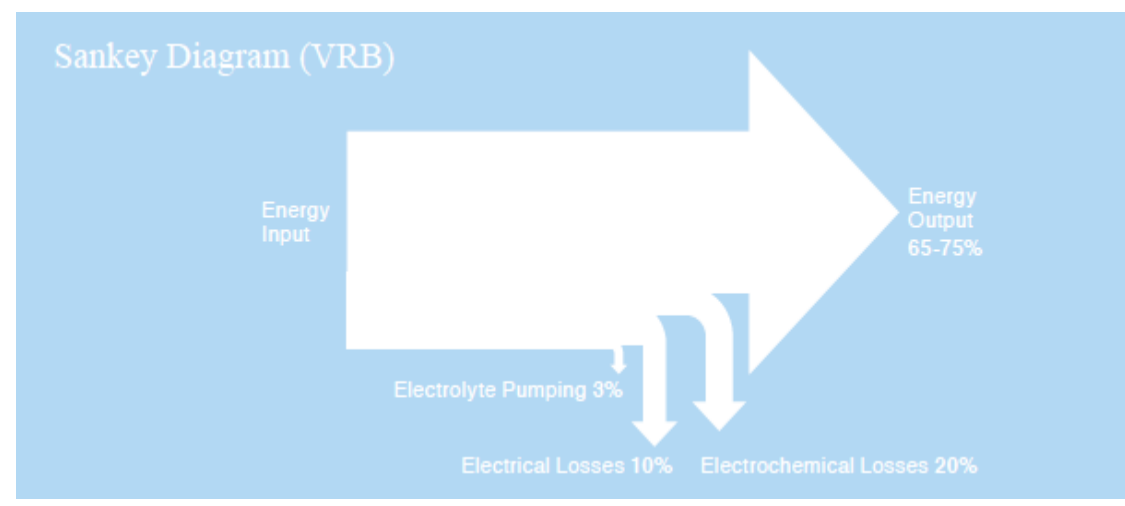

Figure 2.17 Sankey diagram of flow battery system (source [48])

Flow batteries were first used for EES on long term space flights by NASA in the early 1970s. Now, they are considered to have potential for grid applications for storing electricity over longer periods of hours or even days for load balancing and standby power [45]. The technology has only been deployed for a limited number of grid applications. The Huxley Hill Wind Farm in Tasmania utilised a 200kW flow battery which was operational between 2003 and 2008. [78]

Future developments are focussed on safety and operability, but there are also opportunities to improve electrode technology enabling higher power densities. System costs have reduced significantly over the last five years as shown in Figure 2.18; however, there is not expected to be significant scope to reduce this much further in the short to medium term [34].

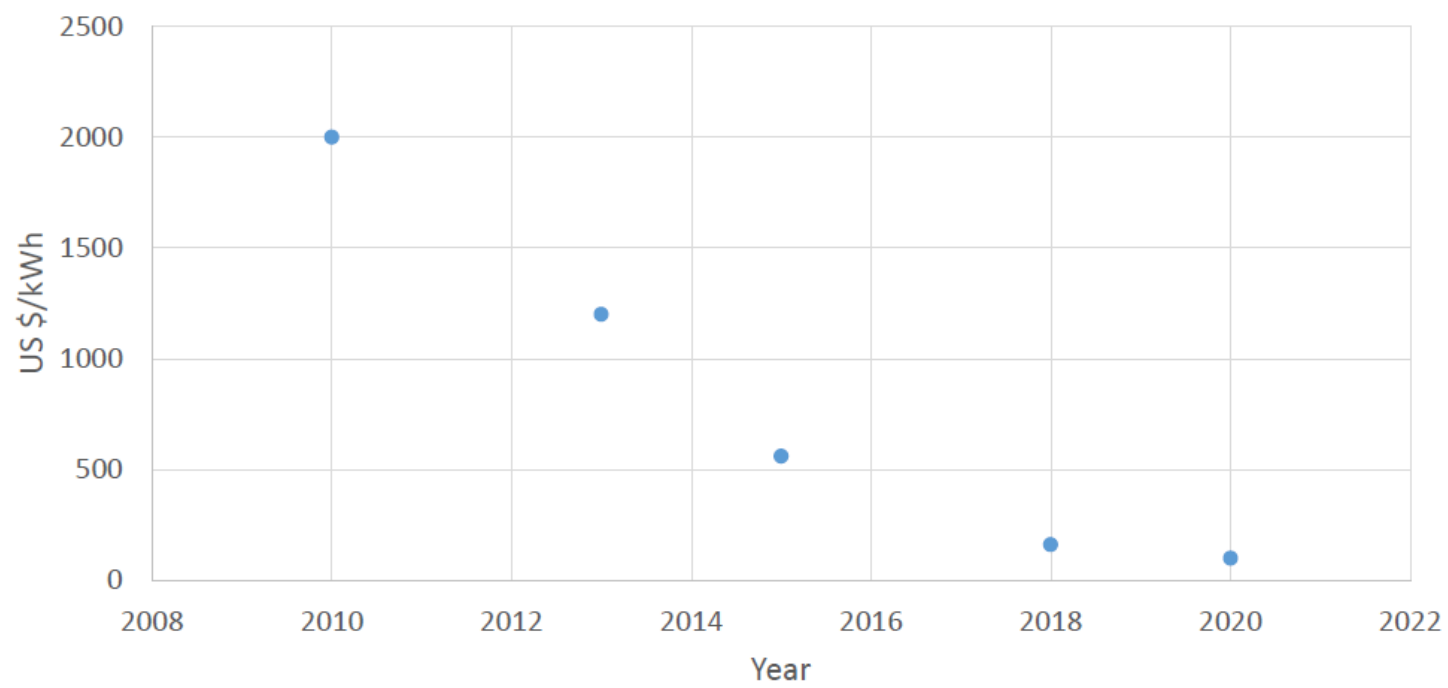

Figure 2.18 Flow battery cost reduction since 2010 and cost projection to 2020 (source [34]) 


\section{Redox Flow Batteries}

Redox flow batteries use liquid or gas electrolytes and charge and discharge through reversible reduction and oxidation reactions. Many different combinations of electrolyte have been investigated, but only zinc/bromine and all vanadium units have been progressed. Vanadium units have proven most successful as there is inevitably some passage of metal ions across the membrane and with the same metal on both sides, the only result is a loss of energy. If different metals are used, there is also a reduction in the battery capacity as the electrolytes degrade [45].

The University of New South Wales, Australia, first developed redox flow batteries in the 1980s. Units up to 500kW and 10h have now been installed by SEI in Japan. Other companies including ZZB Energy and Cellstrom manufacture smaller units which can be combined to make up larger capacity plants [45].

\section{Hybrid Flow Batteries}

Hybrid flow batteries comprise an active mass stored in an electrochemical cell and a liquid electrolyte in a storage tank. They exhibit some features of secondary batteries and others of flow batteries.

Exxon developed a zinc-bromine hybrid flow battery in the early 1970s and several companies are now working on commercialisation of the technology. In the USA, ZBB Energy and Premium Power have developed 1MW 3MWh systems for utility applications. Smaller units for community storage are in development [45].

\subsubsection{Chemical Storage Systems}

There is potential to use electricity to produce hydrogen or synthetic natural gas for storage. This could then be converted back into electricity when required. These gases act as secondary energy carriers as they can be stored or transported to a location where they can be used. The over-all efficiency of the process is low, but it is the only concept which could enable storage of electricity in the terawatt hour (TWh) range. Additionally, the gas could be used for alternative applications including transport, heat and chemical industries as well as for electricity.

\section{Hydrogen}

A hydrogen storage system contains an electrolyser, a tank and a fuel cell. Using electricity, the electrolyser splits water into hydrogen and oxygen. This is an endothermic process which needs a heat source for the reaction to occur. Electricity is generated in the fuel cell using 
hydrogen and oxygen. For economic reasons, the oxygen is not stored during the splitting process but vented to atmosphere and then oxygen is taken from the air for power generation [45].

Various different approaches have been used to store hydrogen including in underground containers at high pressure, as a low temperature liquid and adsorbed in metal hydrides. Small volumes can be stored above ground at pressures of up to 900bar. Larger volumes of thousands of cubic meters can be stored in salt caverns and underground piping systems at pressures of up to 200bar [45].

The advantages of hydrogen storage are that the only emissions at the point of generation are water vapour and there is practically no self-discharge. The gas can be moved from the generation location if the demand is in a different place, but this would incur additional costs and reduce the roundtrip efficiency.

The disadvantages are the extremely low efficiency of around $30-40 \%$, and potential safety issues surrounding hydrogen storage. Additionally, fuel cell technologies are currently very expensive [48]. A Sankey diagram of a hydrogen storage system is shown in Figure 2.19.

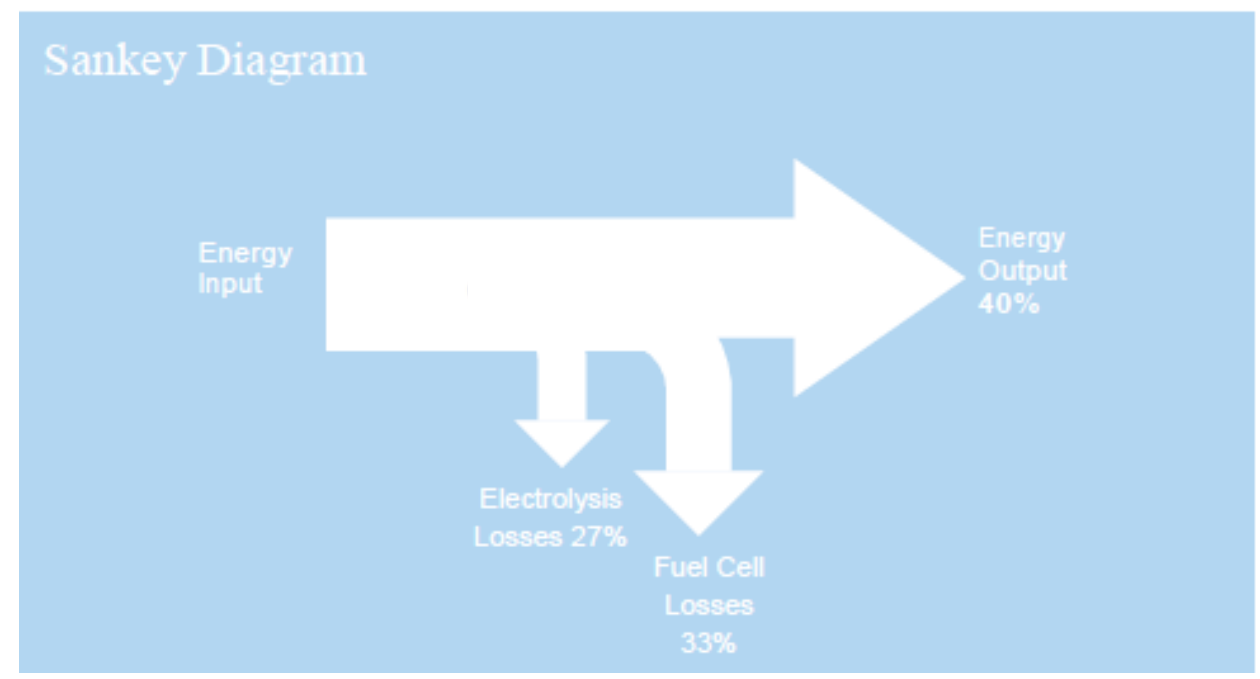

Figure 2.19 Sankey diagram of hydrogen storage system (source [48])

The processes involved use mature technologies which have been implemented extensively in the chemical industry. Transport and containment structures are also based on well-established technologies [48]. Various research and development projects have successfully demonstrated the feasibility of using hydrogen storage, but its low efficiency and high cost have prevented further commercialisation. 


\section{Synthetic natural gas}

An alternative option for chemical storage is to form methane, or synthetic natural gas (SNG). This requires an additional process following the splitting of water to react hydrogen and carbon dioxide to form methane, a process known as methanation. SNG can be kept in pressurised tanks or fed directly into the gas grid. This process could make use of carbon dioxide from fossil fuel emissions.

Methanation is a continuous process so intermediate storage of hydrogen and carbon dioxide are required to ensure a continuous supply is available. Recently the power to methane concept has been piloted in several research and development projects [79].

The main advantages are that methane has a higher energy density than hydrogen and can make use of existing infrastructure; however, it has a very low roundtrip efficiency, even less than that of hydrogen storage and also it creates emissions at the point of electricity generation.

\subsubsection{Electrical Storage Systems}

\section{Double layer capacitors}

Capacitors store an electric charge between two conductors separated by a dielectric insulator. Conventional capacitors have a low energy density. Large, expensive and cumbersome dielectrics are needed if a high capacity is required. Super-capacitors operate based on the same principle as traditional capacitors, but their capacity and discharge current are significantly larger. Double layer capacitors (DLCs), a type of super-capacitor, do not have a conventional solid insulator. Instead, energy is stored at the interface between a porous conductive electrode and a liquid electrolyte ion conductor. The porosity of the electrode significantly increases the surface area [80]. The capacity of DLCs is one to two orders of magnitude larger than conventional capacitors.

The advantages of DLCs are a practically unlimited number of charge and discharge cycles, with a cycle stability of up to $1,000,000$ cycles without degradation. However, regardless of the number of cycles, the solvents used have a time dependent lifespan of 5-6 years. DLCs are a durable technology with a high reliability and minimum maintenance required over their lifetime. Additionally, they can operate in a range of temperatures and environments. They have low inner resistance and so charge and discharge quickly making them well suited to high current loads. DLCs are not susceptible to overcharging and are relatively environmentally friendly and easy to recycle [48]. 
Disadvantages include that the technology is currently very expensive and has a high rate of self-discharge so it is not suitable for long term storage. A Sankey diagram of the system is shown in Figure 2.20. The technology is well suited to applications of voltage and frequency stabilisation.

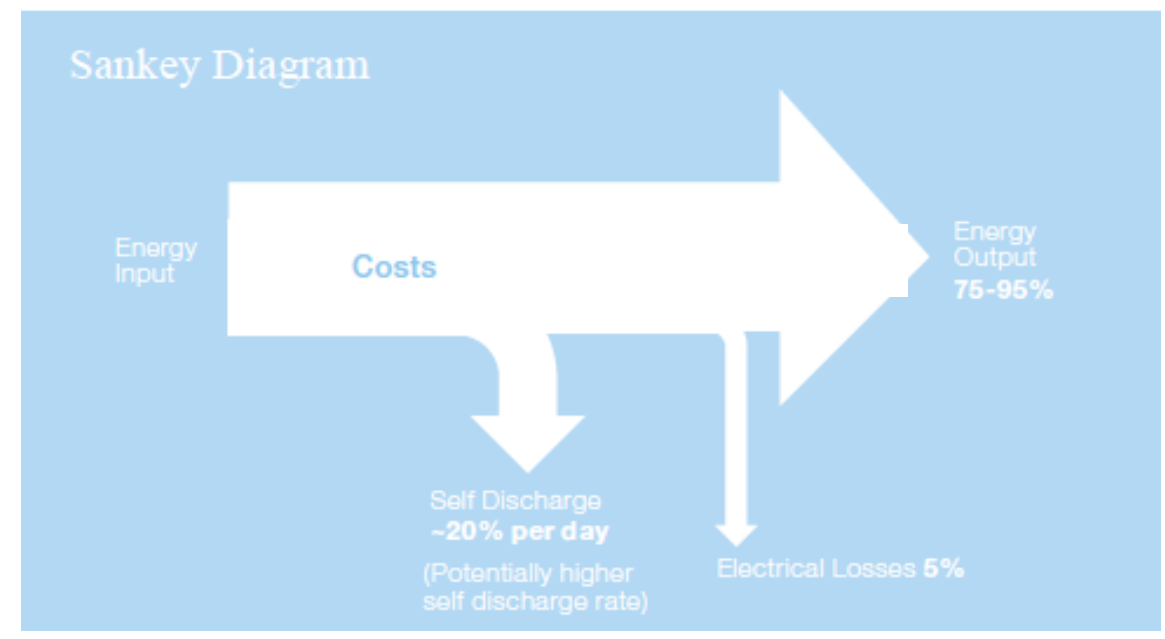

Figure 2.20 Sankey diagram of a double layer capacitor system (source [48])

DLCs have been applied since the 1980s in consumer electronics to bridge short voltage failures and the technology is developing for grid-scale applications [45]. There have been limited examples of installations to date due to the prohibitive cost. This is expected to change with future developments of the technology to improve performance and reduce the cost in parallel with anticipated increased requirements for ancillary services.

Potential developments of the technology focus on three areas [63].

1. Electrode developments to increase the surface area further through use of composite materials and eliminate inter-particle resistances.

2. Electrolyte development to minimise fluid viscosity potentially by raising the fluid temperature.

3. Package development to minimise weight and series resistance.

Significant advances are expected to be made in the coming decades allowing DLCs to make a substantial contribution to grid-scale EES for high power applications in the future.

\section{Superconducting magnetic energy storage}

Superconducting magnetic energy storage (SMES) devices use an electric current to create a magnetic field in a superconductor. The superconductor is cryogenically cooled to below its critical temperature. At steady state, the superconductor offers no resistance to electron flow 
so energy losses are effectively zero. Losses in SMES devices are associated with the cooling system [81]. A schematic of an SMES system is shown in Figure 2.21.

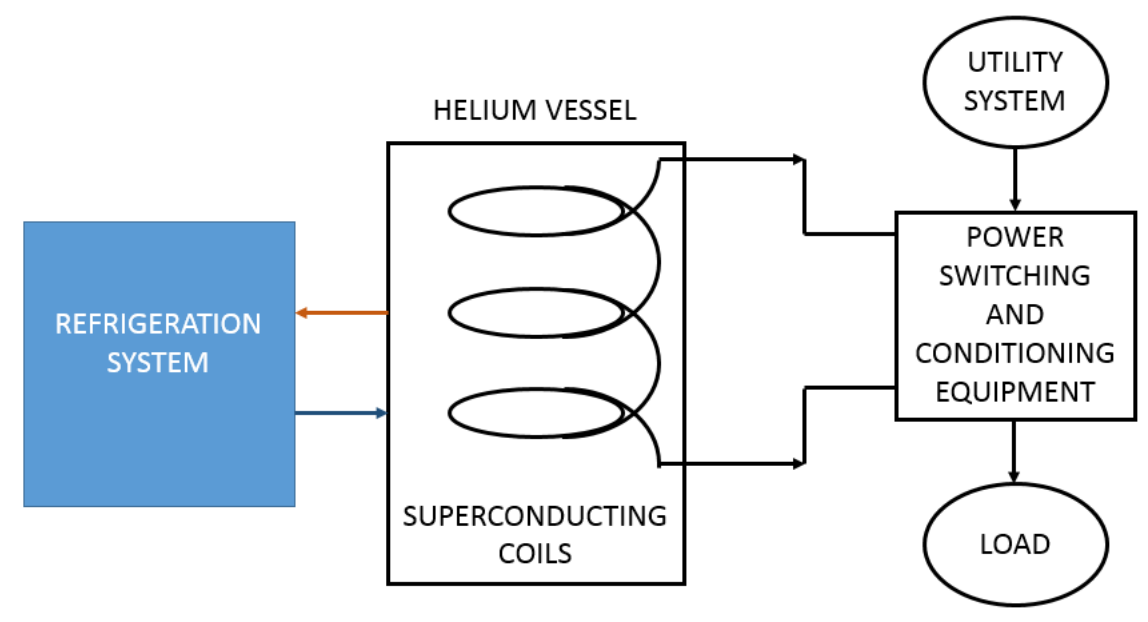

Figure 2.21 Superconducting magnetic energy storage system (based on [82])

SMES devices have an almost infinite lifecycle, are quick to charge and can provide rapid response to power requirements. They have no moving parts, but reliability is dependent on the refrigeration system. For short time periods, they can provide a very large power output and have a relatively high efficiency of around 90\% [48]. A Sankey diagram of the storage process is shown in Figure 2.22. The energy density of the technology is low and the superconducting materials are only available at very high cost. The device also requires continuous cooling [45].

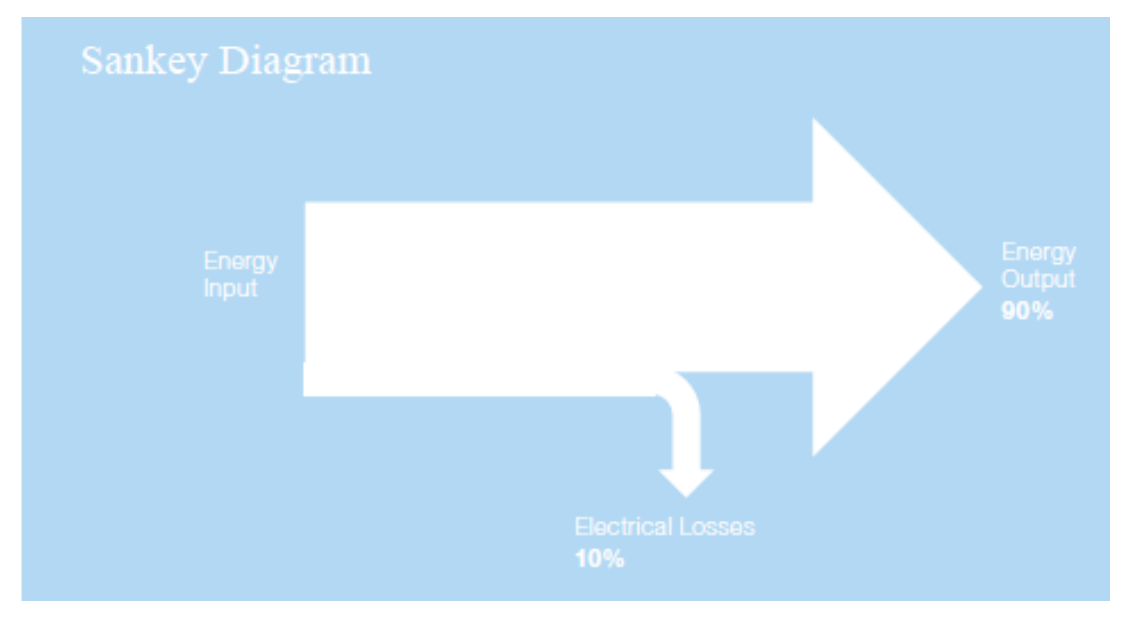

Figure 2.22 Sankey diagram of superconducting magnetic energy storage system (source [48])

SMES was first proposed in 1969 in France; However, it was research at the University of Wisconsin, in 1971, which led to manufacture of the first unit [83]. Following this, there was 
worldwide development of the technology from companies including Hitachi, ISTEC, Wisconsin Public Service Corporation and ACCELL Instruments GmbH [37]. Large SMES systems are commonly used in physics experiments with high power requirements, in particle detectors and for nuclear fusion. There is limited commercial development for grid-scale applications of the technology as it is generally cost prohibitive. There are also environmental concerns with managing the large magnetic field. Where it is available, it is used for power quality and frequency regulation.

Research within the European Union has focussed on developing micro-SMES systems, with a capacity of less than 10MW. Countries such as Germany, Italy, Finland and Spain have made developments in this area. There has been less attention, until recently, paid to high temperature superconductors. However, there have been demonstration projects with superconductors functioning at around $20 \mathrm{~K}$. This is a significant improvement on the critical temperatures of around $4 \mathrm{~K}$ which were needed when superconductivity was first discovered over 100 years ago. Research and development over the years has led to materials with critical temperatures of up to $100 \mathrm{~K}$ being developed; however, these have not yet been demonstrated with SMES technology [63].

Increased temperature superconductors have the potential to allow this technology to become commercially viable; however, there are still several technical issues which must be overcome. For example high temperature superconductors are granular and boundaries between grains limit the transport of current. The technology has the potential to produce devices with $100 \mathrm{MW}$ capacity at $99 \%$ efficiency over a 40 year lifetime. This is anticipated to be a reasonable goal to have reached by 2050 [63].

\subsubsection{Thermal Storage Systems}

\section{Cryogenic energy storage}

Cryogenic Energy Storage (CES) stores liquid air or nitrogen in large volumes at atmospheric pressure in insulated tanks at cryogenic temperatures. The liquid can be liquefied onsite using electricity or imported through existing supply chains from the chemical industry. When electricity is needed, the liquid in the tank is expanded and used to drive a turbine. There are three discrete modules in the system for charging, storing and discharging [84]. A schematic of the process is shown in Figure 2.23 


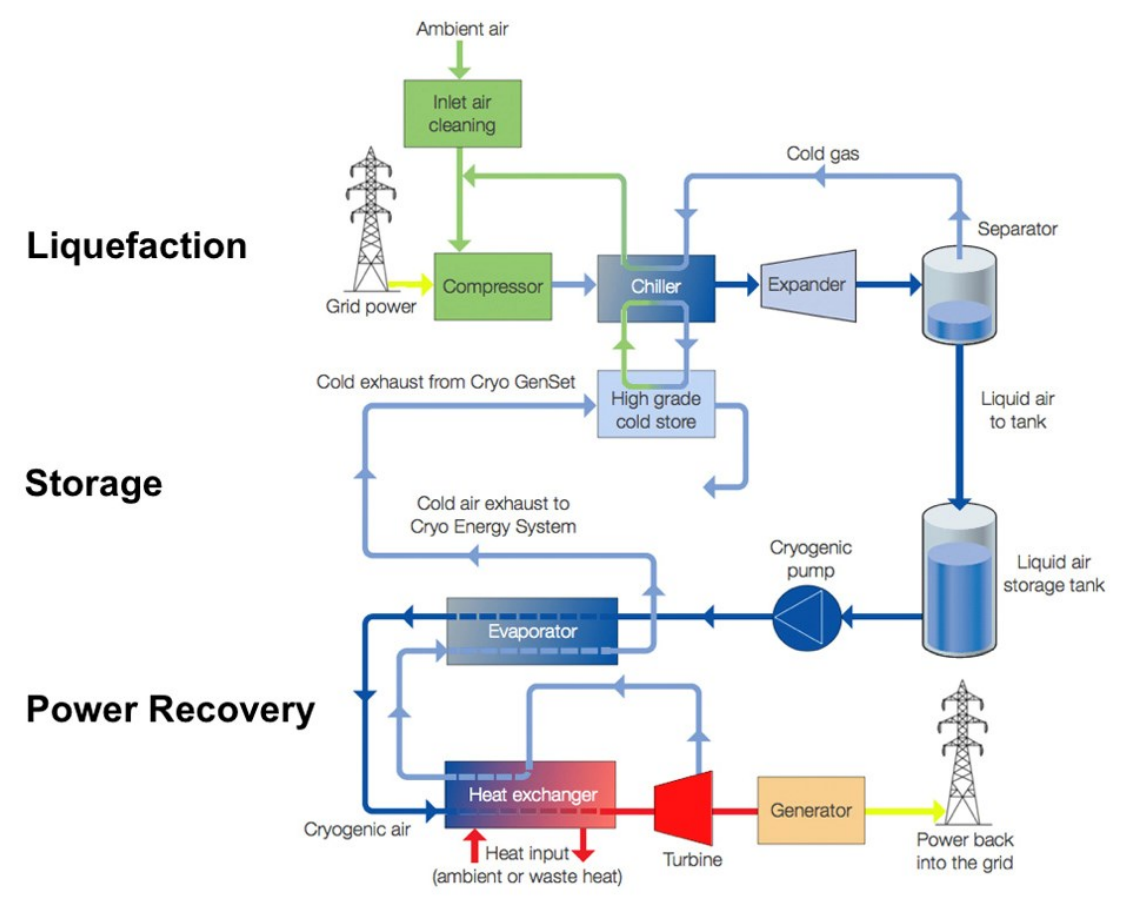

Figure 2.23 Cryogenic energy storage process (source [85])

The advantages of CES are that there is an existing global supply chain of the components, the storage technology is not geographically constrained and there are no emissions associated with the electricity production. However, the technology has poor efficiency if the waste heat is not recovered. A Sankey diagram of the storage process is shown in Figure 2.24. There are also safety concerns regarding warming of the stored liquid which could cause a build-up of high pressure gas. Advocates of the technology argue that the chemical industry, which has extensive experience of liquid nitrogen, have robust procedures in place which can mitigate these risks [85].

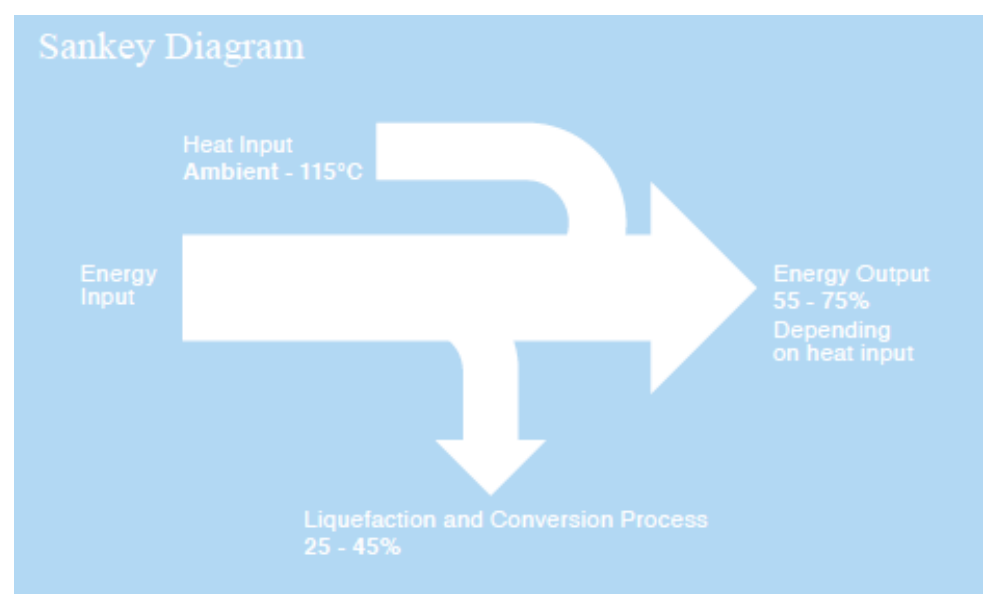

Figure 2.24 Sankey diagram of cryogenic energy storage system (source [48]) 
The technology is in the early stages of development. Highview Power Storage installed the first $350 \mathrm{~kW}$ demonstration plant in the UK in 2012 [85]. A picture of the plant is shown in Figure 2.25. The technology has not yet been demonstrated at the MW scale. CES could make a significant contribution to EES applications which require large volumes of energy to be stored. The storage capacity can be easily increased at low cost by increasing the size or number of storage tanks. Similarly to flow batteries, the storage equipment is decoupled from the charging and discharging equipment, so the capacity and power ratings can be increased independently. There is further information on Highview's liquid air energy storage system in Chapter 5 as the technology is used for an investment case study.

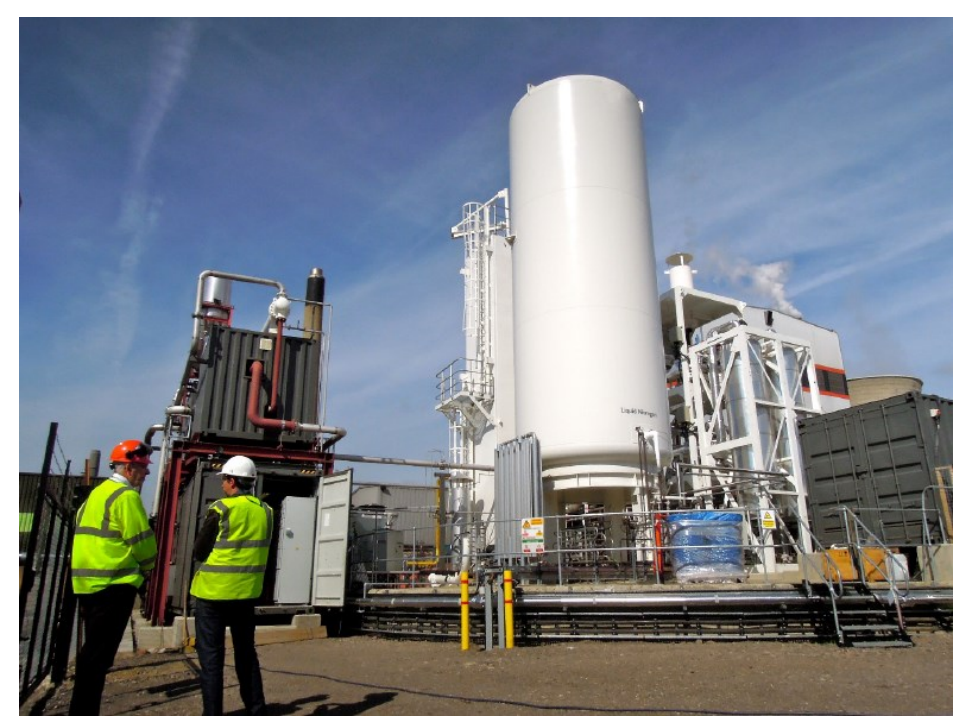

Figure 2.25 Highview's 350kW liquid air energy storage demonstration project (source [85])

\section{Pumped heat energy storage}

Pumped heat energy storage systems store energy as a temperature difference between two containers of mineral particulate such as gravel. Using electricity, heat is pumped out of the first container until it is cooled to approximately $-160^{\circ} \mathrm{C}$. The second container is heated to approximately $500^{\circ} \mathrm{C}$. The containers are stored at a fixed temperature inside insulating covers. When required, the heat pump is reversed and used as an engine to generate electricity from the temperature difference [86].

The system is environmentally friendly with zero operating emissions and it does not use any hazardous materials. It has a long lifespan and can tolerate a large number of charging cycles. The cost of the system is relatively low and it makes use of local materials. The conversion efficiency is $70-80 \%$, similar to that of PHS. A Sankey diagram of the system is shown in Figure 2.26. 


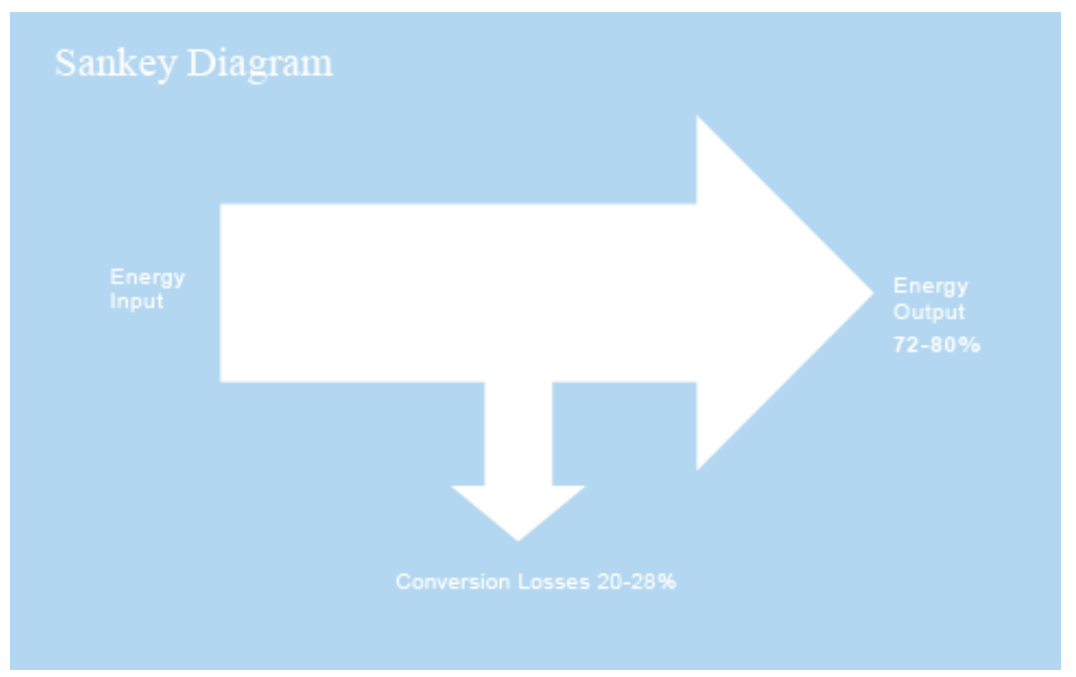

Figure 2.26 Sankey diagram of pumped heat energy storage system (source [48])

The disadvantages are the potentially large footprint of the system and that the technology has not been demonstrated outside of a laboratory to show proof of concept. Isentropic, based in the UK, is currently developing the technology.

There is a wide range of potential applications for pumped heat storage which only exclude markets which require a response time of less than a few minutes. The units are expected to be in the range of $2-5 \mathrm{MW}$ capacity which will be built up to provide plants of larger capacity.

\subsection{Technology Comparison}

\subsubsection{Technical Comparison}

A summary of the characteristics of the EES technologies is shown in Table 2.1 with their typical applications. The applications for EES are explained further in the Chapter 4.

Plotting EES technologies graphically enables a visual comparison of technologies to be made and shows more clearly which devices would be suited to which applications as shown in Figure 2.27. This shows that only PHS and CAES technologies offer high power ratings which can be delivered over very long time-frames for applications of large scale energy management. These technologies have poor power densities and are geographically constrained depending on the availability of appropriate sites for reservoirs or underground storage caverns. 
Table 2.1 Summary of storage technology characteristics [37], [42], [45], [75]

\begin{tabular}{|c|c|c|c|c|c|c|c|c|c|}
\hline Technology & Power Rating & $\begin{array}{l}\text { Discharge } \\
\text { Time }\end{array}$ & $\begin{array}{l}\text { Response } \\
\text { Time }\end{array}$ & $\begin{array}{l}\text { Lifetime } \\
\text { (years) }\end{array}$ & Cycle Life & $\begin{array}{c}\text { Self- } \\
\text { Discharge } \\
\text { per Day }\end{array}$ & $\begin{array}{c}\text { Appropriate } \\
\text { Storage } \\
\text { Duration }\end{array}$ & $\begin{array}{l}\text { Efficiency } \\
\text { (\%) }\end{array}$ & Typical Applications \\
\hline Pumped Hydro & $100-5,000 \mathrm{MW}$ & $1-24 \mathrm{~h}+$ & $\begin{array}{l}\text { Seconds- } \\
\text { Minutes }\end{array}$ & $>50$ & $>15,000$ & Very Small & Hours-Months & $75-85$ & $\begin{array}{l}\text { Time shifting, power } \\
\text { quality, Emergency supply }\end{array}$ \\
\hline Compressed Air & $5-1,000 \mathrm{MW}$ & $1-24 \mathrm{~h}+$ & Minutes & $>25$ & $>10,000$ & Small & Hours-Months & $50-60$ & Time shifting \\
\hline Flywheel & $0-250 \mathrm{~kW}$ & $\begin{array}{l}\text { Miliseconds- } \\
15 \mathrm{mins}\end{array}$ & $<$ Second & $15-20$ & $\begin{array}{c}20,000- \\
1,000,000\end{array}$ & $100 \%$ & $\begin{array}{l}\text { Seconds- } \\
\text { Minutes }\end{array}$ & $85-95$ & Power quality \\
\hline $\begin{array}{l}\text { Lead Acid } \\
\text { Batteries }\end{array}$ & 0-20MW & $\begin{array}{l}\text { Second- } \\
\text { Hours }\end{array}$ & $<$ Second & $3-15$ & $250-1,500$ & $0.1-0.3 \%$ & Minutes-Days & $85-90$ & $\begin{array}{l}\text { Off-grid, emergency supply, } \\
\text { time shifting, power quality }\end{array}$ \\
\hline $\begin{array}{l}\text { Nickel Cadmium / } \\
\text { Nickel Metal } \\
\text { Hydride Batteries }\end{array}$ & 0-40MW & $\begin{array}{l}\text { Seconds- } \\
\text { Hours }\end{array}$ & $<$ Second & $5-20$ & $1,500-3,000$ & $0.2-0.6 \%$ & Minutes-Days & $60-80$ & $\begin{array}{l}\text { Off-grid, emergency supply, } \\
\text { time shifting, power quality }\end{array}$ \\
\hline $\begin{array}{l}\text { Lithium Ion } \\
\text { Batteries }\end{array}$ & $0-10 \mathrm{MW}$ & $\begin{array}{l}\text { Seconds- } \\
24 \mathrm{~h}+\end{array}$ & $<$ Second & $5-15$ & $500-10,000$ & $0.1-0.3 \%$ & Minutes-Days & $85-90$ & $\begin{array}{l}\text { Power quality, network } \\
\text { efficiency, off-grid, time } \\
\text { shifting }\end{array}$ \\
\hline $\begin{array}{l}\text { Metal Air } \\
\text { Batteries }\end{array}$ & $0-10 \mathrm{~kW}$ & $\begin{array}{l}\text { Seconds- } \\
24 \mathrm{~h}+\end{array}$ & $<$ Second & $>1$ & $>1,000$ & Very Small & Hours-Months & $50-70$ & Off-grid \\
\hline
\end{tabular}


Table 3.1 (continued) Summary of storage technology characteristics [37], [42], [45], [75]

\begin{tabular}{|c|c|c|c|c|c|c|c|c|c|}
\hline Technology & Power Rating & $\begin{array}{l}\text { Discharge } \\
\text { Time }\end{array}$ & $\begin{array}{l}\text { Response } \\
\text { Time }\end{array}$ & $\begin{array}{l}\text { Lifetime } \\
\text { (years) }\end{array}$ & Cycle Life & $\begin{array}{c}\text { Self- } \\
\text { Discharge } \\
\text { per Day }\end{array}$ & $\begin{array}{c}\text { Appropriate } \\
\text { Storage } \\
\text { Duration }\end{array}$ & $\begin{array}{l}\text { Efficiency } \\
\quad(\%)\end{array}$ & Typical Applications \\
\hline $\begin{array}{l}\text { Sodium Sulphur } \\
\text { Batteries }\end{array}$ & $50 \mathrm{~kW}-8 \mathrm{MW}$ & $\begin{array}{l}\text { Seconds- } \\
\text { Hours }\end{array}$ & $<$ Second & $10-15$ & $2,500-4,500$ & $\sim 20 \%$ & $\begin{array}{l}\text { Seconds- } \\
\text { Hours }\end{array}$ & $75-85$ & $\begin{array}{l}\text { Power quality, time shifting, } \\
\text { network efficiency, off-grid }\end{array}$ \\
\hline $\begin{array}{c}\text { Sodium Nickel } \\
\text { Chloride Batteries }\end{array}$ & $0-400 \mathrm{~kW}$ & $\begin{array}{l}\text { Seconds- } \\
\text { Hours }\end{array}$ & $<$ Second & $10-15$ & $\sim 1,000$ & $\sim 15 \%$ & $\begin{array}{l}\text { Seconds- } \\
\text { Hours }\end{array}$ & 85 & Time shifting \\
\hline Flow Batteries & $30 \mathrm{~kW}-50 \mathrm{MW}$ & Seconds- $10 \mathrm{~h}$ & Seconds & $5-20$ & $\begin{array}{l}1,000- \\
10,000\end{array}$ & Small & Hours-Months & $65-75$ & $\begin{array}{l}\text { Time shifting, network } \\
\text { efficiency, off-grid }\end{array}$ \\
\hline Hydrogen & $0-500 \mathrm{MW}$ & $\begin{array}{l}\text { Seconds- } \\
24 \mathrm{~h}+\end{array}$ & $\begin{array}{l}\text { Seconds- } \\
\text { Minutes }\end{array}$ & $10-30$ & $\begin{array}{l}10,000- \\
100,000\end{array}$ & $\begin{array}{l}\text { Almost } \\
\text { Zero }\end{array}$ & Hours-Months & 40 & Time shifting \\
\hline $\begin{array}{l}\text { Double Layer } \\
\text { Capacitors }\end{array}$ & $0-300 \mathrm{~kW}$ & $\begin{array}{l}\text { Miliseconds- } \\
60 \mathrm{mins}\end{array}$ & $<$ Second & $4-12$ & $\begin{array}{l}100,000- \\
1,000,000\end{array}$ & $20-40 \%$ & $\begin{array}{l}\text { Seconds- } \\
\text { Minutes }\end{array}$ & $75-95$ & Power quality \\
\hline $\begin{array}{l}\text { Superconducting } \\
\text { Magnets }\end{array}$ & $100 \mathrm{~kW}-10 \mathrm{MW}$ & $\begin{array}{l}\text { Miliseconds- } \\
8 \mathrm{~s}\end{array}$ & $<$ Second & $>20$ & $>100,000$ & $10-15 \%$ & $\begin{array}{l}\text { Seconds- } \\
\text { Minutes }\end{array}$ & 90 & Power quality \\
\hline Cryogenic & $100 \mathrm{~kW}-30 \mathrm{MW}$ & 1-8hours & Minutes & $20-40$ & $\begin{array}{l}\text { Insufficient } \\
\text { Experience }\end{array}$ & $0.5-1 \%$ & Minutes-Days & $55-75$ & $\begin{array}{l}\text { Time shifting, network } \\
\text { efficiency, off-grid }\end{array}$ \\
\hline Pumped Heat & $2-5 \mathrm{MW}$ & $1-24 \mathrm{~h}+$ & Minutes & $5-15$ & $\begin{array}{l}\text { Almost } \\
\text { infinite }\end{array}$ & $0.05-1 \%$ & $\begin{array}{l}\text { Minutes- } \\
\text { Months }\end{array}$ & $72-80$ & Time shifting \\
\hline
\end{tabular}




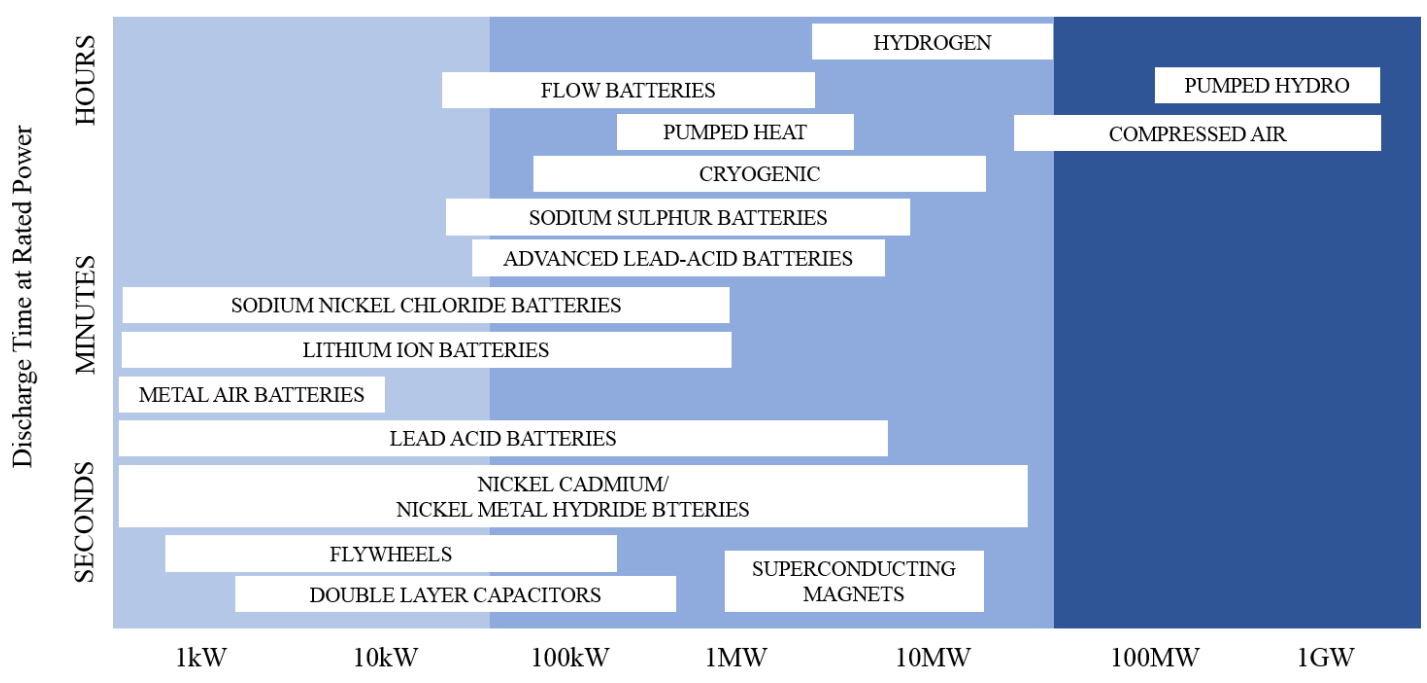

Figure 2.27 Storage technologies arranged by power rating and discharge time

There is, however, a series of technologies serving applications which require a range of $100 \mathrm{~kW}$ to $10 \mathrm{MW}$ of power. These technologies are best suited to distribution scale applications which have varying needs for power and delivery duration. These technologies could replace standing or spinning reserve or assist with peak shaving or load levelling at a local level.

Equally, there is a significant number of smaller power rated technologies which can discharge power rapidly. These technologies are suited to power quality applications such as frequency response and voltage control and will assist in managing smaller, but more frequent fluctuations in generation and consumption. These devices require rapid response rates and a relatively long cycle life in addition to fast discharge times to assist with these services.

Each technology has specific technical features and individual advantages and disadvantages. It is evident that there is no single EES device that will develop as a generic technology solution. Currently a range of technologies with different characteristics is being developed and it is likely that each of these will serve different applications forming multiple niche markets.

\subsubsection{Commercial Comparison}

In addition to exhibiting a range of technical characteristics, storage technologies vary significantly in cost and technology maturity. Figure 2.28 shows the relative development stages of different technologies. While systems may be technically developed, there may not be widespread use of them for grid-scale applications. There are further requirements to 
improve reliability of some technologies before they can be implemented in the electricity industry. Additionally, costs must be reduced to make them commercially viable.

\begin{tabular}{|c|c|c|c|c|c|c|c|c|c|}
\hline \multirow[t]{2}{*}{$\begin{array}{l}\text { METAL AIR } \\
\text { BATTERIES }\end{array}$} & $\begin{array}{l}\text { HYDROGEN (FOR } \\
\text { STATIONARY } \\
\text { APPLICATON) }\end{array}$ & CRYO & GENIC & $\begin{array}{l}\text { DIUM NICKEL } \\
\text { CHLORIDE } \\
\text { BATTERIES }\end{array}$ & $\begin{array}{l}\text { LITHIUMION } \\
\text { BATTERIES }\end{array}$ & \multicolumn{2}{|c|}{$\begin{array}{c}\text { NICKEL CADMIUM/ } \\
\text { NICKEL METAL HYDRIDE BTTERIES }\end{array}$} & $\begin{array}{l}\text { LEAD ACID } \\
\text { BATTERIES }\end{array}$ & FLYWHEELS \\
\hline & \multicolumn{8}{|c|}{ DEVELOPMENT STAGE } & \\
\hline $\begin{array}{c}\text { COMPRESSED } \\
\text { AIR } \\
\text { (ADIABATIC) }\end{array}$ & $\begin{array}{r}\text { SUPERCONDUC } \\
\text { MAGNETS }\end{array}$ & CTING & $\begin{array}{l}\text { PUMPED } \\
\text { HEAT }\end{array}$ & $\begin{array}{l}\text { FLOW } \\
\text { BATTERIES }\end{array}$ & $\begin{array}{l}\text { ADVANCED } \\
\text { LEAD ACID } \\
\text { BATTERIES }\end{array}$ & $\begin{array}{l}\text { DOUBLE LAYER } \\
\text { CAPACITORS }\end{array}$ & $\begin{array}{l}\text { SODIUMSULPHUR } \\
\text { BATTERIES }\end{array}$ & $\begin{array}{l}\text { COMPRESSED AIR } \\
\text { (DIABATIC) }\end{array}$ & $\begin{array}{l}\text { PUMPED } \\
\text { HYDRO }\end{array}$ \\
\hline
\end{tabular}

Figure 2.28 Relative development stage of electrical energy storage technology

A summary of technology costs is outlined in Table 2.2. These costs are based on values reported by Chen at al [37] which are similar to those given by the Electrical Power Research Intitute (EPRI) [48], but somewhat smaller than the costs reported by Arup [75]. Arup takes into account increases in cost estimates for near term scope of supply, or first-of-a-kind (FOAK) costs. In the longer term, once technologies have been proven at demonstration scale, the costs from Chen et al and EPRI are expected to be more appropriate. These should be representative of the costs of next-of-a-kind (NOAK) devices. It is difficult to access reliable cost data due to the commercially sensitive nature of the information and the lack of practical experience of EES projects. Developers may provide different costs based on different analysis assumptions in addition to differences in actual technology cost. Furthermore, project costs vary depending on size and location. A cost range is given for each technology to attempt to address these challenges. It should be noted that cost estimates vary by orders of magnitude between different sources. Values are left blank where there is insufficient information available.

Storage costs are formed from power related costs $(\$ / \mathrm{kW})$ and energy related costs $(\$ / \mathrm{kWh})$. For technologies which have discrete charging/discharging and storage modules such as flow batteries and CES, these costs are associated with the respective equipment for each function and can be scaled independently. For other devices, such as secondary batteries, these are approximations. In these cases, the power rating and storage capacity cannot be sized independently and the costings are more complex. 
Table 2.2 Summary of technology costs [37], [48]

\begin{tabular}{|c|c|c|c|}
\hline \multirow{2}{*}{ Technology } & \multicolumn{3}{|c|}{ Cost } \\
\hline & $\mathbf{\$} / \mathbf{k W}$ & $\$ / \mathbf{k W h}$ & \$/kwh/Cycle \\
\hline Pumped Hydro & $600-2,000$ & $5-100$ & $0.1-1.4$ \\
\hline Compressed Air & $400-800$ & $2-50$ & $2-4$ \\
\hline Flywheel & $250-350$ & $1,000-5,000$ & $3-25$ \\
\hline Lead Acid Batteries & $300-600$ & $200-400$ & $20-100$ \\
\hline \multicolumn{4}{|l|}{ Nickel Cadmium / Nickel Metal } \\
\hline Hydride Batteries & $500-1,500$ & $800-1,500$ & $20-100$ \\
\hline Lithium Ion Batteries & $1,200-4,000$ & $600-2,500$ & $15-100$ \\
\hline Metal Air Batteries & $100-250$ & $10-60$ & - \\
\hline Sodium Sulphur Batteries & $1,000-3,000$ & $300-500$ & $8-20$ \\
\hline Sodium Nickel Chloride Batteries & $1,500-3000$ & $100-200$ & $5-10$ \\
\hline Flow Batteries & $600-2,500$ & $150-1,000$ & $5-80$ \\
\hline Hydrogen & $10,000+$ & - & - \\
\hline Double Layer Capacitors & $100-300$ & $300-2,000$ & $2-20$ \\
\hline Superconducting Magnets & $200-300$ & $1,000-10,000$ & - \\
\hline Cryogenic & $200-300$ & $3-30$ & $2-4$ \\
\hline Pumped Heat & - & $30-60$ & - \\
\hline
\end{tabular}

The storage and power related costs from Table 2.2 are presented graphically in Figure 2.29 which shows the range of cost estimates for each technology. This supports the conclusion that different EES technologies will be suited to different applications. Devices with the lowest costs per kWh but relatively higher costs per $\mathrm{kW}$, such as CAES and PHS, would be applied to applications where large volumes of energy, but low power delivery requirements are 
needed. Alternatively, those with high costs per $\mathrm{kWh}$, but low costs per $\mathrm{kW}$, such as FES and DLCs, would be better applied where high power but low energy requirements are needed.

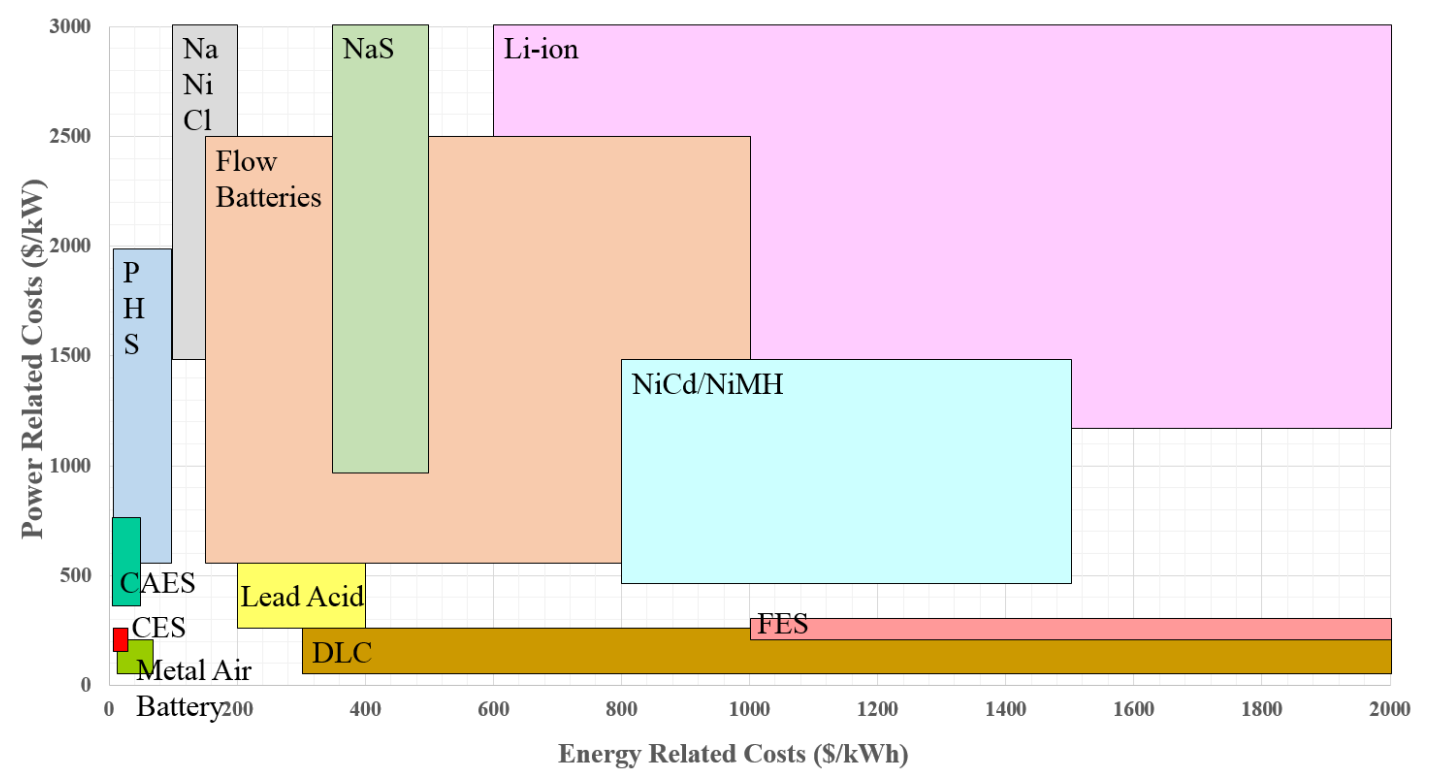

Figure 2.29 Graphical representation of power and energy related storage costs

Different applications can provide value in different ways and so it is not appropriate to directly compare the costs of all technologies. The cost per cycle is included to provide a metric for evaluating cost of energy storage for applications which require a frequent charge/discharge cycle. Some developers calculate a levelised cost of energy (LCOE) to provide a suitable metric on which storage costs can be compared to generation technologies. This is calculated, excluding discounting, by:

$$
L C O E=\frac{(C A P E X+O \& M+E+D)}{E P}
$$

where $C A P E X$ is the capital expenditure, $O \& M$ is the operation and maintenance cost including replacement costs for any components, $E$ is the cost of electricity used for charging over the lifetime of the device, $D$ is the decomissioning cost and EP is the total lifetime electricity production. This calculation provides a single metric for cost comparisons to be made; however, there are a number of underlying assumptions which are depend on the specific technology type and electricity market under analysis. This includes the number of cycles carried out over the device lifetime and the cost of wholesale electricity. Furthermore, valuing storage with a metric which is directly comparible to generation technologies does not reflect the value of storage in providing flexibility. On an LCOE basis, storage cannot compete with generation technology as it is a net consumer of electricity. 
Whatever metric is used to compare costs of EES, in most cases, a step change in technology cost is required to enable grid-scale applications.

\section{$2.4 \quad$ Future Developments}

There is no single EES technology which is yet commercially viable, capable of making a return for investors on a large scale, for grid applications. Technologies which are mature have high capital costs or significant environmental impacts and those which have potential to provide large power ratings have not yet been demonstrated. With a renewed interest in the industry, developments are expected to reduce costs leading to increased levels of deployment.

PHS is expected to remain the dominant technology being developed in countries where sites are still available. In 2013 the SBC Energy Institute reported 8.2GW of PHS under construction and a further $8.3 \mathrm{GW}$ planned which increases total global capacity of PHS by approximately $13 \%$ [42]. Additionally, existing plant is expected to be upgraded with variable speed turbines and underground and underwater PHS may develop in the longer term.

It is anticipated that CAES will develop rapidly in the USA where there are numerous suitable natural sites for storage caverns. There is further interest in Germany, South Korea and Canada [42]. With substantial funding being channelled into this technology, demonstration of adiabatic CAES is expected to improve the roundtrip efficiency leading to further deployments. However, the Boston Consulting Group anticipates that CAES will only form an interim solution with the market potential declining after 2020 due to the technology's reliance on fossil fuels and limited flexibility [87].

Battery technology has not changed substantially since its invention over 200 years ago and limited deployment of large scale batteries is expected without significant reduction in price and improvement in cycle life. Improvements in safety standards and recycling techniques are expected as the industry develops. To date, developments have been made through empirical methods and there has been a lack of understanding of the fundamental processes which occur at the interfaces between electrodes and electrolytes. Advances in analytical and computational tools to understand these processes at an atomic level and time-scales down to femtosecond range will vastly improve knowledge in this area [88]. This may allow for improved designs with enhanced characteristics. Lead acid batteries are not expected to make a significant contribution to grid applications; however, Li-ion batteries are proving to be popular and Japan announced a three year subsidy scheme for this technology in 2012. The uptake of NaS batteries is expected to increase in the future; however, longer term, flow batteries may take 
the majority share of the grid battery market [42]. Battery research must also take into account the resource scarcity of rare earth metals.

Flywheels will benefit from mass production and high performance engineering.; however, several failed projects in 2011 provided set-backs to for the technology. In USA, a project for frequency regulation was abandoned and in a lab in Germany, an engineer was killed which highlighted safety concerns [42]. Efforts are being made to optimise storage for longer durations for use in electric vehicles. Breakthroughs in technology from this industry may prove to be advantageous for grid-scale EES. However, FES devices are expected to contribute only to niche applications of voltage and frequency stabilisation due to their high levels of self-discharge.

Radical developments are anticipated for SMES and DLCs as EES. Developments in material science are expected to provide significant improvements in performance. Research on synthetic control of material architectures to refine grain size, structure and compositions could lead to breakthroughs in capacity, power and lifetime [88]. However, these devices are only expected to be used for high power, short duration applications.

Cryogenic energy storage and metal air batteries are expected to attract significant research interest due to their advantages of being environmentally benign. However, development of these technologies is anticipated to occur over a longer timescale due to a lack of experience to draw on from their use as electricity storage in other industries.

The focus for hydrogen development will be for use in hybrid vehicles. Cost and efficiency are expected to improve with research and more robust and reliable supply chains may be established. If there is an opportunity for this technology to transition into the grid-scale EES industry in the future, these developments will be advantageous.

The principal barrier to development of EES technology is high capital cost. Renewed interest in the industry has led to resources becoming available for research and development. This is expected to provide breakthroughs in performance and reductions in cost of many of the technologies discussed. To establish the commercial potential of each technology, further evidence is needed on the expected lifetime of the devices and the impact of duty cycles. Demonstration projects planned over the next decade will assist with obtaining this information. 


\subsection{Chapter Summary}

A brief history of grid-scale EES was presented which highlighted the phases of development of EES in GB. Firstly, batteries were coupled with early generators to efficiently supply variable demand. Secondly, PHS was built in response to the expectation of large scale deployment of inflexible nuclear generators. Today, a third phase of EES development is underway in anticipation of a large penetration of intermittent renewable generation. Many different technologies are being developed. In this chapter, these were presented individually and then compared on a basis of technical and commercial characteristics. The range of advantages and disadvantages of each technology demonstrated the range of applications to which EES could be applied. These applications are discussed in more detail in the following chapter. Finally, potential future developments of EES technologies were discussed. Despite a number of existing grid-scale projects, EES requires significant research and development, to reduce costs and improve performance, and further demonstration of novel technologies, to provide operational experience and evidence of reliability and cycle life. 


\section{Chapter 3 System}

Benefits and Commercial Opportunities

This chapter discusses the system benefits and commercial opportunities of EES. The 'cost' of intermittency is discussed before introducing the applications of EES and its estimated value to the electricity system. The environmental and social implications of EES are then examined to highlight the additional benefits which are not readily monetised. Detrimental environmental impacts and the long term sustainability of EES devices are also considered to ensure that the overall objective of resolving the Energy Trilemma is not compromised by the short term goal of balancing variable renewables. These discussions establish a role for EES, highlighting the net positive contribution it could play in enabling a secure, sustainable and affordable electricity system. Understanding the value of storage at a system level highlights the benefits it could provide.

The revenue streams available to EES are then presented. Due to complex regulations in the electricity sector, these are distinct from its applications. Regulatory barriers which lessen and, in some cases, prevent access to income are also presented. Support for EES is discussed, firstly in GB followed by other countries which are taking action to reduce the market barriers EES faces. Finally, action which could be taken to remove the barriers to deployment of storage are suggested.

This chapter draws together literature on the potential system value of EES demonstrating the benefits that storage could provide to the electricity system. By contrast, it highlights the 
barriers to market entry for EES which currently exist establishing a disparity which exists between the commercial and system level benefits of EES.

\subsection{The Cost of Intermittency}

The penetration of variable renewable generation has increased in recent years as efforts have been made to decarbonise the electricity industry. This is expected to increase further in the coming decades as the cost of onshore and offshore wind and solar generators reduces and as wave and tidal technologies are proven commercially [89]. In addition, under the LCPD, many coal generators in Europe, which previously provided dispatchable generation ${ }^{1}$, are being mothballed [90]. These changes are expected to bring increased costs to the electricity system. The potential to minimise these costs has prompted a resurgence of interest in EES. To understand the role and value of EES, the cost of intermittency must first be understood.

When discussing the impact of intermittency, it is useful to consider the definitions of the terms below:

1. System adequacy: the ability of the electricity system to meet the demand of all consumers at all times. It is a measure used for long term planning and capacity investment decisions [91];

2. System reliability: the ability of the system to manage unexpected events such as generator outages. It is related to the short-term management of the system and is a measure of system security [91];

3. Loss of load probability (LOLP); the probability, in a fixed time period, of an occurrence where there is insufficient capacity to meet demand [92]. It does not indicate the frequency, duration or severity of the capacity shortage;

4. Capacity credit: the amount of conventional generation in an electricity system which can be displaced by an intermittent generator. There are several variations on how it is calculated. The UK Energy Research Centre (UKERC) defines it as the amount of thermal capacity which can be replaced by an intermittent generator without increasing the LOLP [91].

There is a significant body of research on the cost of intermittency spanning over 25 years. A brief summary is presented below which draws substantially from [91] where a more thorough review of the literature can found. The majority of the research to date focusses on costs

\footnotetext{
${ }^{1}$ Dispatchable generation refers to electricity output which can be turned on, off or adjusted on demand.
} 
associated with integrating wind power; reflecting the relative maturity of this technology compared to other renewable generators.

Studies conclude that with wind power penetration of up to $20 \%$ in the UK, an additional 10 $20 \%$ reserve capacity is needed to maintain sufficient system adequacy. This leads to additional costs of approximately $£ 5 / \mathrm{MWh}$ [91]. A wide range of cost values is reported, suggesting that costs are sensitive to specific assumptions in each model. In all cases, as more wind power is deployed, the costs rise because the capacity credit of wind diminishes as its penetration increases [93].

Costs associated with maintaining system reliability are also estimated at approximately $£ 5 / \mathrm{MWh}$. Similarly, this would increase with higher penetrations of wind as issues become more difficult to manage when the remainder of the system is less capable of flexibility [91].

In addition to costs associated with maintaining system adequacy and reliability, there is expected to be reduced efficiency of thermal plant running at part load in response to increased wind power output. Estimates of the effect of this range from negligible impact up to a reduction in potential fuel savings of 7\% [91]. As with other costs, this would be expected to increase as the penetration of wind power increased.

As more wind power is deployed, more is curtailed, primarily due to network constraints limiting the amount of electricity which can be transmitted from wind farms to loads. Estimates of curtailment of wind power output range from zero to up to $7 \%$ [91] with $20 \%$ installed wind capacity. As wind capacity increases, without significant enhancement of transmission and distribution infrastructure, larger quantities of electricity will be curtailed on more frequent occasions due to grid constraints. In addition, there is the potential for wind power to be curtailed for periods where output exceeds demand.

Increased intermittent power will also have an effect on the wholesale price of electricity. Research shows that significant penetrations of wind power will be likely to reduce the average electricity price - as a result of the minimal marginal price of wind power - but increase the range and variability - due to its variable output [94], [95]. While lower average prices may be beneficial, price spikes, allowing peaking generators to recover their fixed costs, are expected to become less frequent and more volatile. As a result, the market for generation capacity will be higher risk and investment capital more expensive [26].

Solutions which provide flexibility would be beneficial to reduce the costs associated with integrating large amounts of intermittent renewable energy into the existing electricity system. 
They will become essential if complete decarbonisation of the sector is ever achieved. EES is one technology which could provide this flexibility. Alternative solutions, such as enhanced interconnections, DSR and smart grid technologies, are also expected to contribute. However, storage is unique in that it can provide flexibility independently of demand as well as offering services which can improve system control and power quality.

\subsection{Applications for Electrical Energy Storage}

The benefits that EES can bring to the electricity system are summarised in Figure 3.1. These span a range of applications across the entire value chain of the industry including generation, transmission, distribution and operational savings. The principal applications for EES are outlined below.

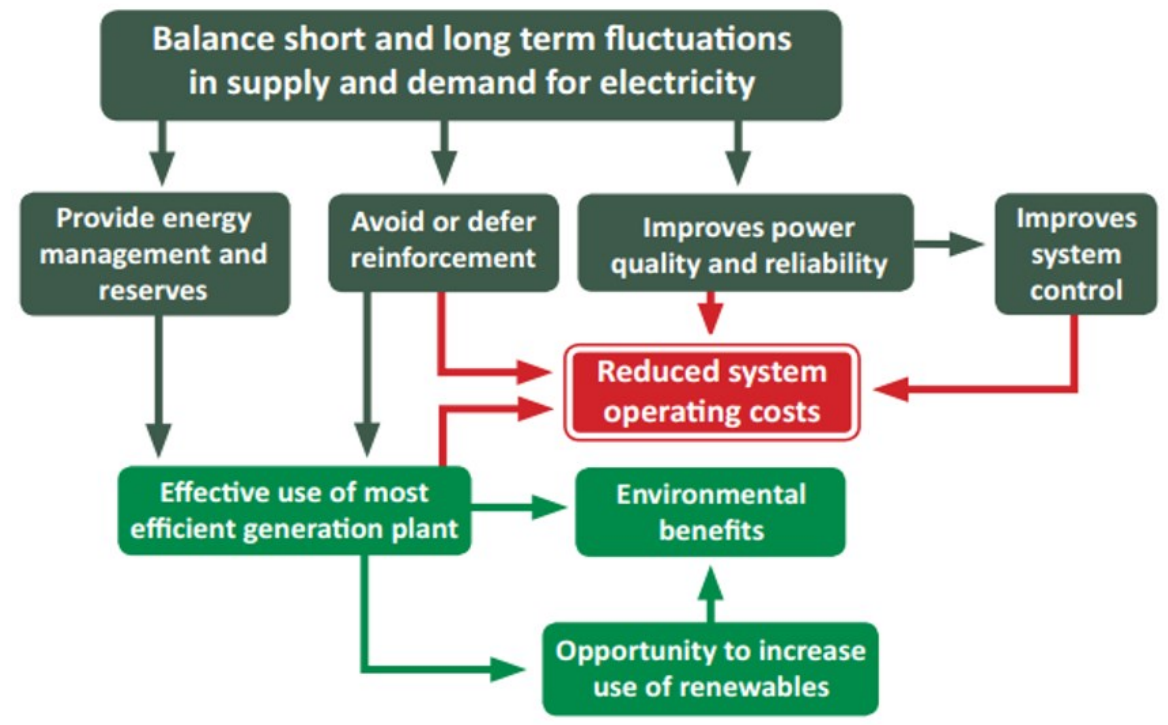

Figure 3.1 Benefits of electrical energy storage (source [96])

\subsubsection{Provision of Ancillary Services}

Ancillary services are procured through bilateral contracts and spot markets. Any EES device which is capable of meeting the required criteria would be able to bid in these markets to offer provision of ancillary services.

\section{Fast reserve}

Fast reserve services must respond automatically to an electronic request from the system operator when the service is required. In GB, devices must be capable of ramping up to full 
power at a rate of at least $25 \mathrm{MW}$ per minute and must provide a minimum of 50MW for up to 15 minutes [97]. Example response profiles are shown in Figure 3.2.

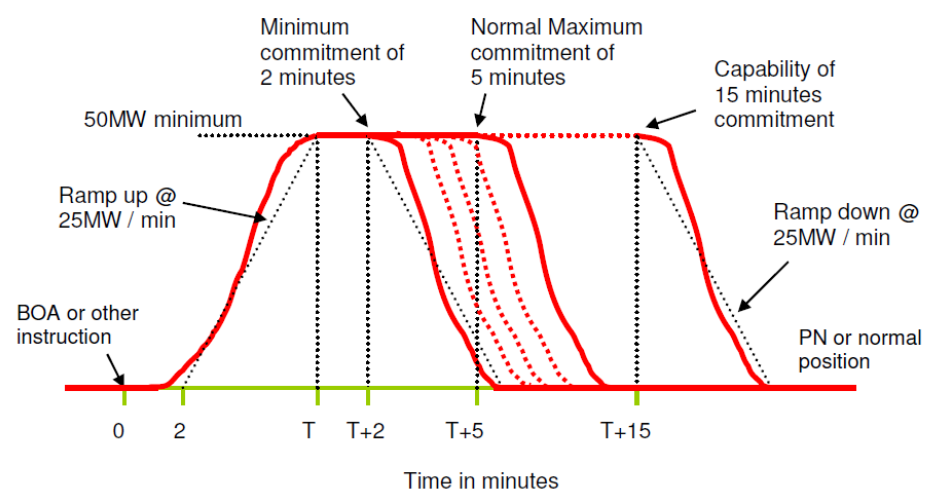

Figure 3.2 Typical fast reserve profile (source [97])

\section{Frequency response}

To ensure security of supply, the system frequency must be maintained at a nominal level within specified limits. If the consumption exceeds generation, the system frequency will reduce; if generation is greater than consumption, the system frequency will increase.

Regulation services handle rapid fluctuations in frequency. These are rapid response generators which are connected to the grid and operate with a governor to allow automatic response to changes in frequency [98]. Load following services also make use of online equipment to manage fluctuations, but over slightly longer time periods than regulation services. These tend to be over intervals of around ten minutes rather than minute to minute. Regulation and load following services are preventative security measures which are provided continuously with fairly small actions that are largely predictable.

Primary frequency response is a regulation service which is activated within seconds of a change in frequency to recover it back to its nominal level. After a fixed time period, secondary frequency response mechanisms come online to replace primary response services. The time at which secondary response mechanisms are activated varies between different countries, but is usually in the range of 30 seconds to 15 minutes. If required for longer periods of time, usually greater than an hour, tertiary response (or reserve) can be called upon by the system operator.

In GB, frequency response services are provided through mandatory frequency response, which all large generators connected to the grid are obliged to supply, and firm frequency response, procured through a separate tender process [99]. Generators are considered to be 
large if their installed capacity is greater than 100MW in England and Wales. In SPTL and SHELT transmission territories, the limits are 30MW and 10MW respectively [100]. To provide firm frequency response, devices must have a minimum power output of 10MW [101].

\section{Reactive Power Services}

Voltage must also be maintained within prescribed limits to make the transmission system secure and reduce the likelihood of insulation failures. Provision of voltage control is also called reactive power support service. Voltage control is provided by both generators which can control their reactive power output and by separate reactive power resources such as mechanically switched capacitors, reactors and tap changing transformers [102].

Similarly to frequency response, in GB, these services are provided through both obligatory services, mandatory for large generators, and enhanced reactive power services, which are procured through a separate tender process [103].

\section{Black start capability}

Following a system failure, black start services may be required. Restarting large thermal generators requires electric power to drive auxiliary equipment such as pumps and fans. This will not be available if the system has collapsed [104]. Often small diesel generators or hydro plants are used for these services. Sufficient black start services must be available to ensure prompt restart of the system at any time.

Partial or total shut down of the GB system is not common and black start services are rarely called upon, but high reliability is essential when they are. Requirements for providing black start services include [105]:

- capability to start at least one generation unit without external supply of electricity;

- ability to maintain voltage and frequency when loaded in discrete blocks of between 30 and 50MW; and

- ability to provide at least three sequential black start supplies in case tripping occurs during the re-instatement period.

\subsubsection{Peak Shaving}

EES can store electricity during off peak hours and release it to serve peak demand. This would reduce the requirement for investment in peaking generators and distribution network infrastructure, which is also sized for local peak loads [75]. 


\subsubsection{Load Levelling}

By charging and discharging in response to variations in demand, EES could be used to average out fluctuations in load throughout the day allowing thermal generators to run at a constant, optimal level improving their efficiency [44].

\subsubsection{Integration of Renewable Power}

Smoothing the output from renewable generators would allow them to be integrated more easily into the electricity system. EES could prevent curtailment of renewable output by storing electricity during periods of grid congestion and releasing it when there is spare transmission capacity. This would offset investment requirements in transmission network infrastructure by making more effective use of existing assets [106].

\subsubsection{Long Term Storage}

There are concerns that a system with a high penetration of wind power may experience a high pressure weather system during winter leading to several days with significantly reduced output [44]. EES with long term storage capability and large capacity could be used to overcome these challenges. Historically, daily electricity consumption in January in the UK has been just over 1TWh [107]. An electricity storage device must be capable of storing 3TWh of electricity and discharging power at approximately $60 \mathrm{GW}$ to meet peak demand if it were to deliver electricity over a period of 3 days with no other power output. There is not currently an EES device capable of storing such large volumes of electricity. An extension of this application is seasonal storage of electricity to even out variations in demand over the year and allow a reduction in generation capacity. Historically, electricity demand has not varied significantly from summer to winter, with average demand increasing from approximately 30 $35 \mathrm{MW}$ in July to 40MW in January [107]. However, if heating is electrified, this will change significantly. In 2014, domestic gas demand reduced from approximately $120 \mathrm{TWh}$ in the first quarter of the year to approximately $30 \mathrm{TWh}$ in the third quarter [108]. This change is attributed to increased requirements for gas heating over the winter months. To manage such fluctuations, after accounting for conversion efficiencies, storage capacities of several hundred terawatt hours would be required. This is not expected to be an economically viable application of EES [44].

\subsubsection{Consumer Applications}

Industrial consumers who require an uninterrupted power supply could use EES for backup power, replacing diesel generators which are traditionally used for this purpose. EES could 
also be used in consumer homes to shift demand to off peak times. However, this would require time of use tariffs as an incentive, which are not common in GB.

\subsubsection{Comparison of Applications}

The applications presented require different characteristics such as speed of response, discharge time, storage capacity, power rating and frequency of cycles. Figure 3.3 shows a selection of applications arranged by power rating and discharge time.

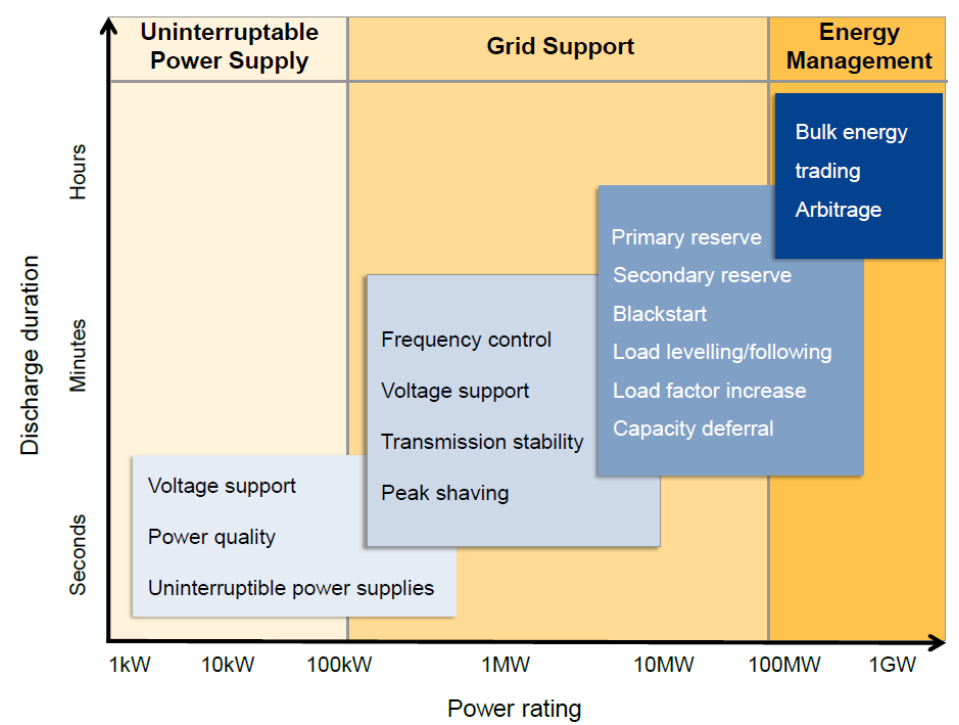

Figure 3.3 Applications of storage arranged by discharge time and power rating (source [109])

This can be compared to Figure 2.27 in the previous chapter which shows storage technologies arranged by the same characteristics. This highlights which technologies are best suited to which applications, for example, PHS and CAES are well suited to provision of energy management, whereas FES and DLCs would be more useful for uninterruptible power supply services. It is evident that no single EES technology could fulfil all the applications. In order to deliver the range of applications which EES can serve, a range of diverse technologies must be developed.

\subsection{The Value of Storage}

\subsubsection{System Value}

Many studies attempt to quantify the system savings which could be made through implementing EES. These studies model the entire electricity system and optimise the inputs for the lowest total system cost. A wide range of values is reported with optimum storage capacity in the UK in 2050 ranging from $7 \mathrm{GW}$ to $59 \mathrm{GW}$ [5]. There are two principal reasons 
for this inconsistency: inadequacies in modelling EES, which requires high spatial and temporal resolution to capture the full range of benefits, and uncertainty surrounding the landscape of the future electricity system.

Many system optimisation models have coarse temporal resolutions which implement investment decisions over a timescale of several years [6]. By comparison, system balancing, to which EES could contribute significant savings, occurs over a timescale of seconds, minutes or hours. Other studies, which have a shorter time step and consider the value of EES in the balancing market, do not capture the longer term benefits of storage in reducing the need for generation capacity [8] [9] [10]. Furthermore, both system and balancing models commonly over simplify transmission and distribution networks, neglecting the benefits of EES in reducing grid congestion.

A report by Imperial College, London for the Carbon Trust [2] attempts to address some of these inadequacies by modelling the electricity system in the UK over a range of time scales and aggregating savings across different sectors. The system model, DSIM (Dynamic System Investment Model), was developed to carry out the analysis, which optimised the total electricity system costs: a combination of short term operational costs and long term investment costs. The transmission network was represented by 5 different zones: Scotland, North England and Wales, the Midlands, London and South England and Wales. Distribution areas were represented by statistically configured networks. EES was included using generic storage characteristics, selected to be technology agnostic. While this enabled a technology neutral approach to be taken, it risked that the characteristics, and corresponding costs, were not representative of any feasible EES technology. Headline results estimated that, at the lowest cost, EES could bring savings of over £10bn per year to the UK by 2050. Figure 3.4 shows the allocation of annual savings of transmission connected (bulk) and distribution connected (distributed) storage where: OPEX is operational expenditure, CAPEX is capital expenditure, $G$ is generation, $T$ is transmission, $I C$ is interconnection, $D$ is distribution and $S$ is storage. The savings in system CAPEX and OPEX are shown as positive, while the EES cost is represented as a negative saving. A range of EES costs were investigated and the optimum capacities and resulting system savings calculated for each. The marker on each bar shows the net savings; the total system savings subtract the EES costs. The results show that the largest savings were attributed to system OPEX followed by generation CAPEX. 


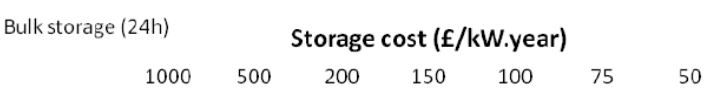

(a)

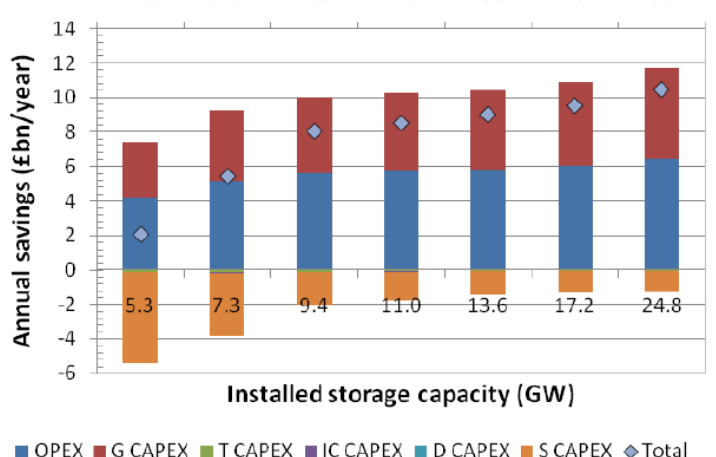

Distributed storage (24h) Storage cost ( $\mathrm{f} / \mathrm{kW}$.year)

(b)

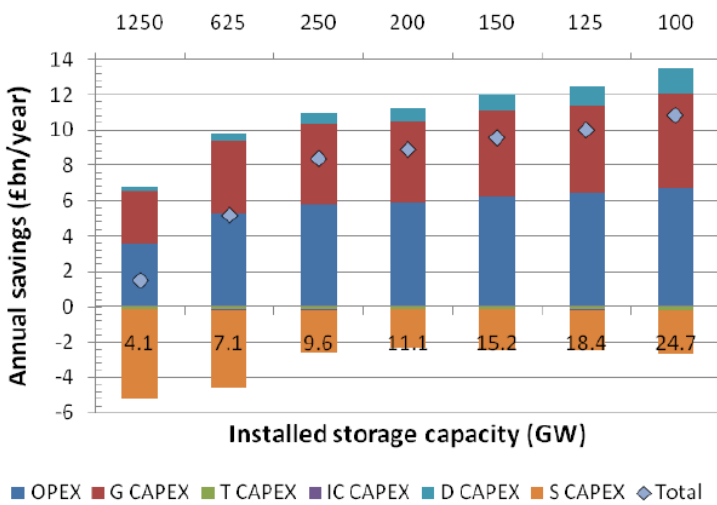

Figure 3.4 Annual benefits of (a) bulk and (b) distributed storage in the UK in 2050 (source [2])

Alternative scenarios and competing sources of flexibility were also investigated. Results showed that the annual savings of EES reduced by $£ 0.9 \mathrm{bn} /$ year after introducing DSR, but there was less impact from both increased interconnection and flexible generation with reductions of $£ 0.6 \mathrm{bn} / \mathrm{year}$ and $£ 0.4 \mathrm{bn} /$ year respectively [110].

Most recently, Strbac has concluded that the true value of storage is only evident when considering stochastic planning of the electricity system [110]. For any deterministic model, systems will be optimised without EES, favouring conventional generation; however, when the uncertainty of future energy scenarios is considered, the flexibility that storage provides significantly reduces the over-all costs.

\subsubsection{Market Value}

In addition to academic research, commercial studies have attempted to quantify the future market value of EES. These estimates also vary significantly between analysts; however, 
details of modelling are not largely in the public domain so it is difficult to determine the reasons for discrepancies. A range of valuations of the annual global market size from Lux Research [111], Pike Research [112] and SBI Energy [113] is shown in Figure 3.5. Despite the considerable variation, all reports show that the size of the EES market will increase significantly within the next decade.

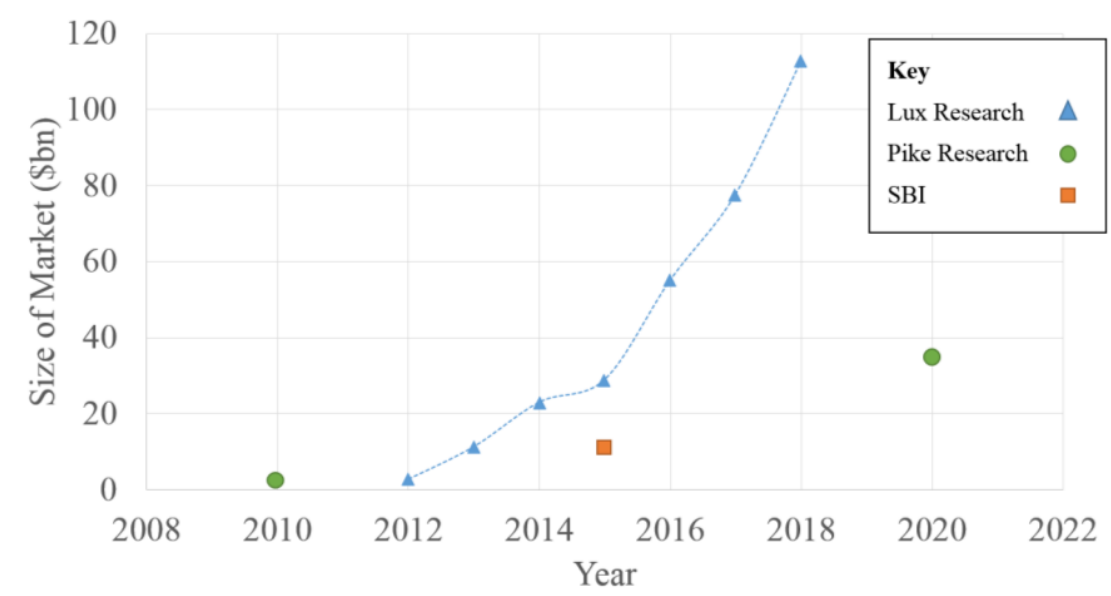

Figure 3.5 Estimates of annual global market value for grid connected storage [111], [112], [113]

Figure 3.6 shows the historic growth of the market from 2006-2012 based on the sum of the cost of projects which were deployed globally each year. Many demonstration projects are completed at a high cost and often at a loss to developers in an attempt to de-risk the technology by proving its technical viability [111]. Additionally, government funding has skewed the historic market size where start up grant capital has been available to support early stage technologies. Many forecasts are based this on historic growth which creates an overly optimistic perception of the market potential based on unsustainable sources of funding [111].

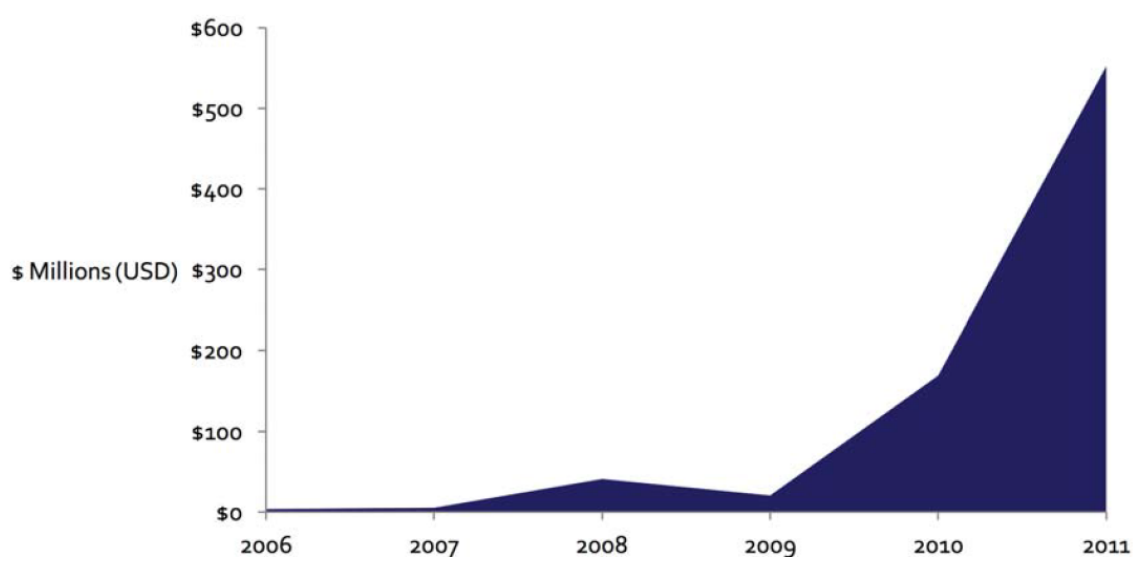

Figure 3.6 Historic global market growth of grid connected electricity storage market (source [111]) 


\subsection{Environmental and Social Effects}

There are additional environmental and social benefits of EES which are not captured in system models.

1. Savings from reduced curtailment of renewable energy and more efficient use of conventional generators are commonly reported in terms of fuel savings which do not capture the true environmental value of reducing emissions.

2. Using EES to reduce peak demand would contribute to more stable wholesale electricity prices reducing the risk associated with investing in generation capacity and lowering the cost of capital [20].

3. Creation of a new industry would contribute to the economy bringing additional jobs associated with manufacturing, installation and maintenance of EES. Estimates for the net contribution of the EES to the UK economy range from $£ 6$ bn- $£ 34$ bn by 2050 [5].

Despite the benefits, there are also detrimental impacts which must be considered. When assessing the contribution of EES, the interim objective of integrating more renewable energy must not take precedence over the ultimate goal of achieving a secure, sustainable and affordable electricity system. The energy used to produce, operate and maintain the EES unit must be considered in conjunction with its direct environmental impacts. As with the costs of EES technologies, these effects are challenging to quantify as they are dependent on the specific technology, project location and its application. The electricity source used during charging and displaced during discharging will also affect the environmental impact of EES.

\subsubsection{Energy Stored on Energy Invested}

Various measures are used to compare the energy required to generate electricity including the energy returned on invested (EROI) ${ }^{2}$ and the energy payback time ${ }^{3}$ [114]. These parameters cannot objectively be compared between generators and EES technologies, which are net consumers of electricity. Instead, Barnhart and Benson [115] propose a new parameter of energy stored on invested (ESOI) to quantify the lifecycle energetic costs of EES. ESOI is the ratio of the total electrical energy discharged from a device in its lifetime to its embodied primary energy. This accounts for the expected depth of discharge and number of cycles a

\footnotetext{
${ }^{2}$ EROI is the ratio of usable energy the plant produces over its lifetime to the total energy invested to make the energy usable.

${ }^{3}$ Energy payback time, also called the energy amortization time, is the time after which the energy produced by a generation plant equals the energy invested.
} 
device would complete in its life and so should only be used to compare EES devices intended for similar applications. Figure 3.7 shows the ESOI calculated for CAES, PHS and a number of battery storage devices.

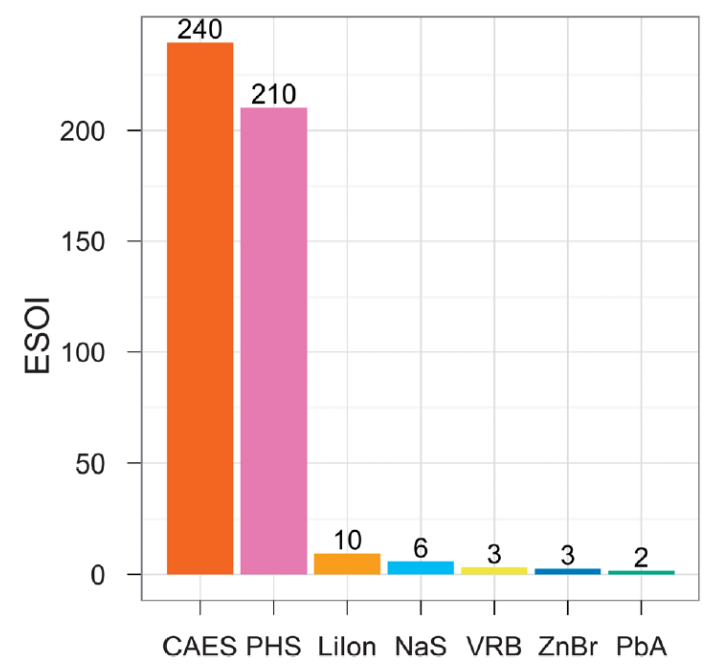

Figure 3.7 Energy stored on invested for a range of electrical energy storage technologies (source [115])

The low ESOI of batteries is mainly due to the energy intense processes used to mine the raw materials and the short cycle life of the devices. A conventional lead acid battery has an ESOI of two implying that it is only capable of storing twice as much energy as was used to produce it. PHS and CAES have significantly higher ESOI values as they use less energy intensive materials and have significantly longer cycle lives.

\subsubsection{Material Resource Requirements}

In addition to energy costs for fabrication, EES devices require raw materials which may make the technologies themselves unsustainable. Research in this area relates predominantly to batteries which generally depend more on scarce materials than other EES devices [116]. Figure 3.8 shows the global production, cost and embodied energy of various elements used in EES technologies plotted against the element abundance in the Earth's crust. The element abundance is reported by lithospheric mass fraction which is the ratio of the mass of the element present in the lithosphere ${ }^{4}$ to the total mass of the lithosphere. The colour of the

\footnotetext{
${ }^{4}$ The Earth's lithosphere is its outermost shell which includes the crust, from which elements are mined, and a portion of the upper mantle [301]
} 
element corresponds to the EES technology it is used in. The blue lines represent a linear regression of the data with the grey areas showing the $95 \%$ confidence interval for the data.
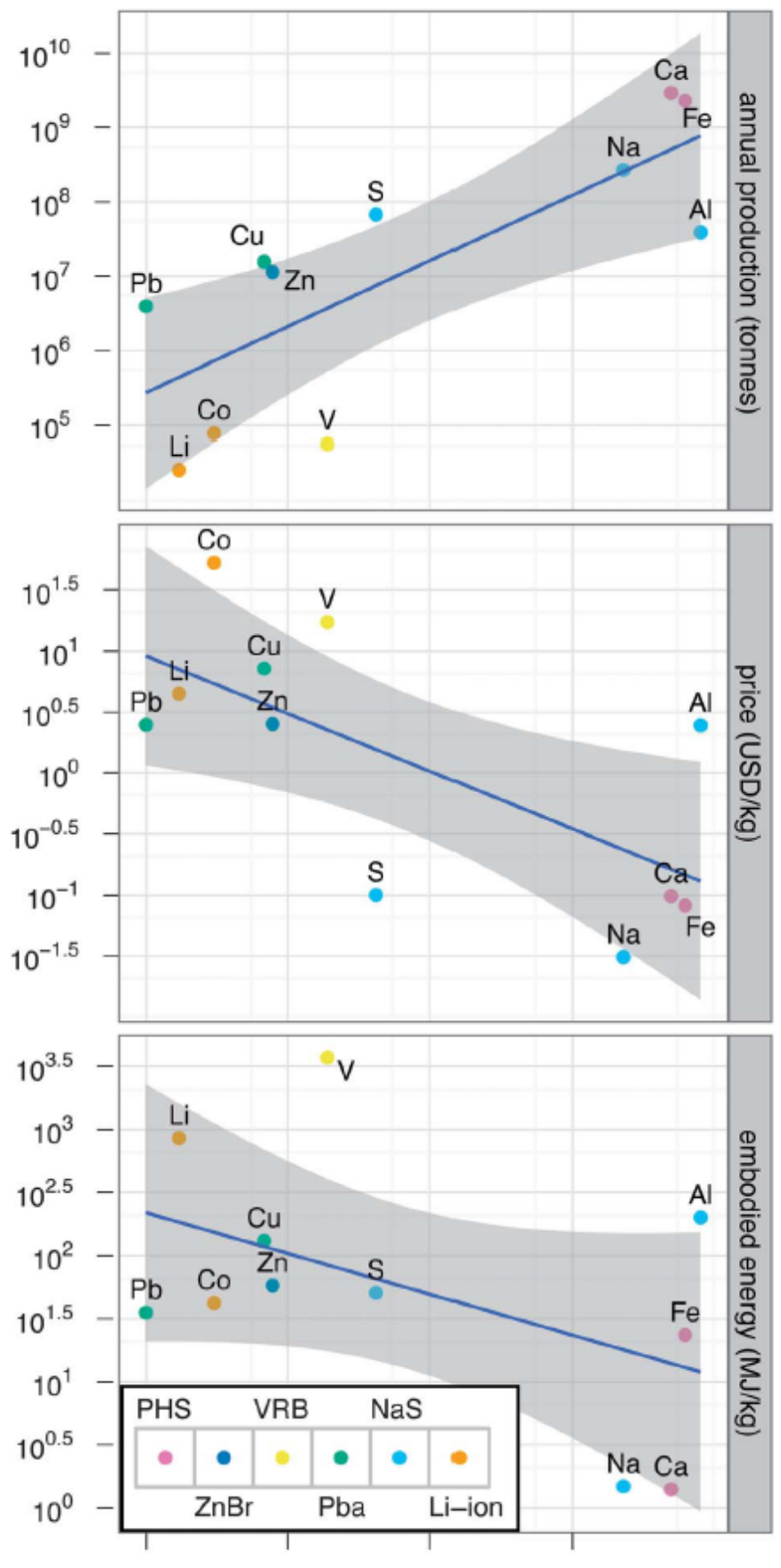

$10^{-5} \quad 10^{-4} \quad 10^{-3} \quad 10^{-2}$

abundance (lithospheric mass fraction)

Figure 3.8 (top) Average annual global production of elements used in energy storage technologies (2006-2011), (middle) Price of elements, (bottom) Embodied energy used to extract element (source [115]) 
The graphs demonstrate the generally lower production, higher cost and higher embodied energy of the rarer materials [115]. Notable is the relatively low abundance of elements essential for Li-ion and $\mathrm{NaS}$ batteries. Further information on the lifecycle analysis of battery technologies can be found in [116].

\subsubsection{Environmental Performance}

Conversely to research on material resource requirements, studies on the environmental performance of EES focus on PHS. Figure 3.9 shows the land use per MW for a range of storage technologies. PHS has the largest footprint as a result of its low energy density [117]. Other studies report considerably higher figures for PHS of up to $4,000 \mathrm{~m}^{2} / \mathrm{MW}$ [106]. Batteries, which tend to have high power densities benefit from smaller physical footprints.

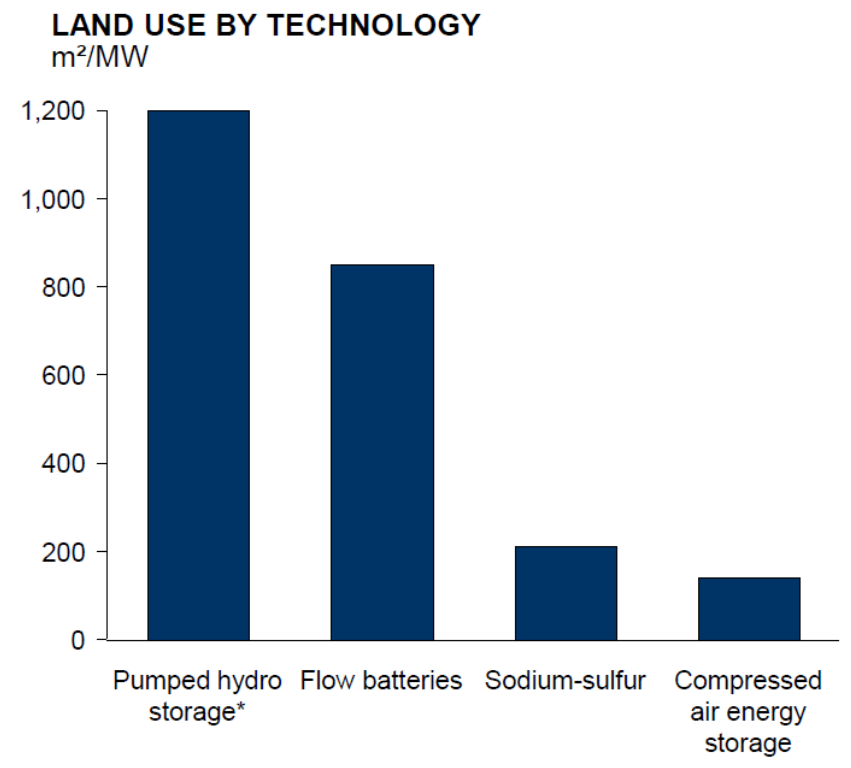

Figure 3.9 Land use of energy storage technologies (source [117])

The principal impacts of PHS are a result of water and land use which have consequential effects on local biodiversity and visual impacts. Specific case studies on the environmental impact of operational PHS projects can be found in [118].

\subsubsection{Greenhouse Gas Emissions}

CAES emits carbon dioxide $\left(\mathrm{CO}_{2}\right)$ directly when discharging electricity as it combusts natural gas. The quantities are estimated to be around one third of a conventional gas turbine at 100150 grams $\mathrm{CO}_{2}$ equivalent per $\mathrm{kWh}$ [117]. Adiabatic CAES, which recycles the heat generated during compression for use during the expansion process, could eliminate these emissions; however, the technology has not yet been proven. 
PHS reservoirs may also cause greenhouse gas emissions. If biomass is left in place during flooding of reservoirs, it may decay both aerobically and anaerobically, producing $\mathrm{CO}_{2}$ and methane. If biomass is cleared before flooding, there would be an increase in atmospheric $\mathrm{CO}_{2}$. Furthermore, reservoirs transport carbon sediment from the surrounding ecosystem into the atmosphere. There is a tendency for shallow reservoirs in hot climates to be more problematic; however, there is no consensus in the research community on the impact of gas emissions from reservoirs, with valuations ranging from 0.2-152 grams $\mathrm{CO}_{2}$ equivalent per $\mathrm{kWh}$ [118].

Further to direct operational emissions, the net emissions of EES depend on the electricity used to charge the device. Figure 3.10 shows the lifecycle $\mathrm{CO}_{2}$ emissions from CAES, PHS, a Vanadium-redox battery (VRB) and a polysulfide-bromide-redox flow battery (PSB) as a function of the emissions from the primary electricity source used during charging.

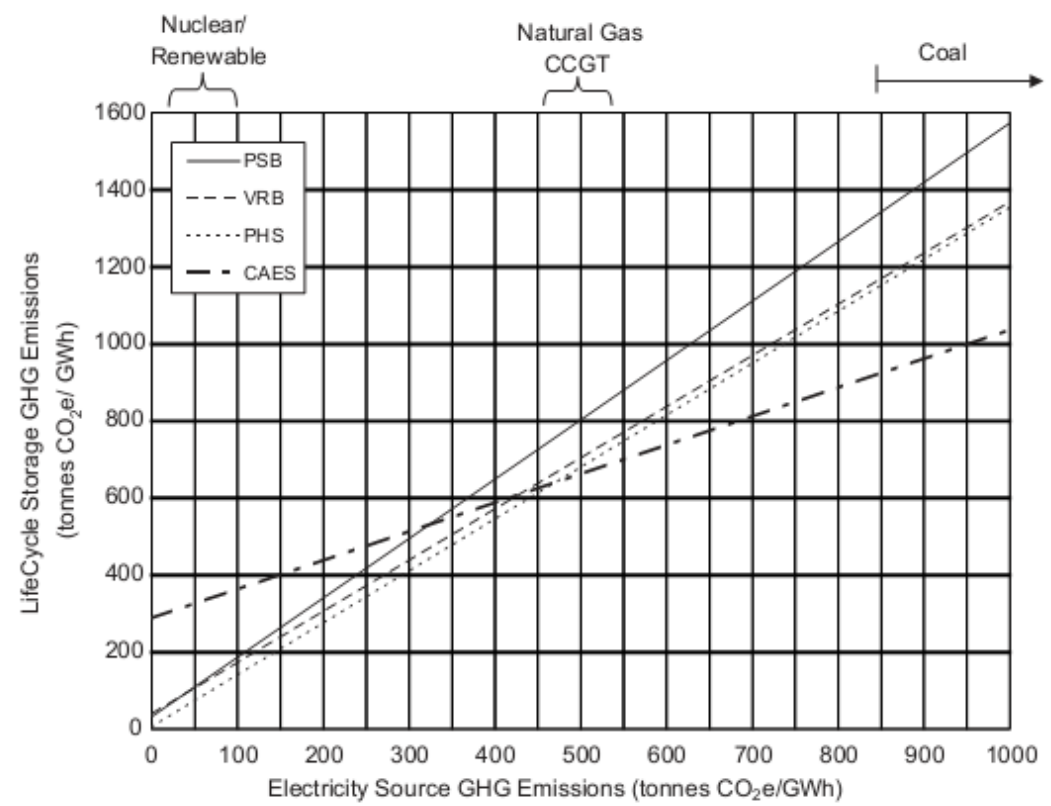

Figure 3.10 Lifecycle emissions from energy storage devices (source [119])

As CAES combines natural gas with compressed air during the discharging process, a relatively small portion of primary electricity is used resulting in the shallow slope seen in Figure 3.10. Emissions from CAES and PHS are equal when the primary electricity generator produces approximately 425 tonnes $\mathrm{CO}_{2}$ equivalent per $\mathrm{GWh}$ which is roughly equal to current emissions from the most efficient CCGTs [119]. If the primary electricity generator is coal, CAES is the least polluting storage solution; however, if the primary generator is renewable or nuclear, PHS has lower emissions. In general, it is fossil fuel generation which is displaced when energy storage is discharged, which would further reduce the net system $\mathrm{CO}_{2}$ emissions. Studies of lifecycle emissions of EES conclude that any combination of renewables or nuclear 
generation with energy storage will produce significantly less $\mathrm{CO}_{2}$ emissions than a fossil fuel based generation system [119], reaffirming the value of EES.

\subsection{Revenue Streams for Storage}

It is evident that there are benefits of EES; however, without accessible revenue streams, sufficient to cover the costs and risks associated with EES, it will not be implemented. While some applications, such as ancillary services, can provide direct sources of income, some sources of revenue are not directly related to a single application. Furthermore, some benefits of EES are not rewarded financially in the current market.

\subsubsection{Energy Trading}

The most apparent source of income for EES is through energy trading, or arbitrage, purchasing electricity off-peak when the price is low and selling it during peak hours when the price is high. Many economic case studies are based on arbitrage [20], [120], [121], [22], [122]. The economic evaluation is similar to that of a generator; however, the primary energy is traded on the same market as the final product. Retail customers are generally charged a fixed tariff for electricity and so energy trading requires interaction with wholesale electricity markets.

The majority of electricity in GB is traded through private bilateral exchanges in forward markets [123]. These provide long term contracts with comparatively stable prices. The price spread between peak and overnight prices is approximately $£ 4 / \mathrm{MWh}$ for summer contracts and $£ 8 / \mathrm{MWh}$ for winter contracts which does not provide sufficient revenue for EES [124].

Around 3\% of electricity is traded on the power exchange, a spot market operating up to an hour ahead of real time [123]. As a market of last resort, the spot market exhibits high price volatility which is reflected by more significant price spreads between peak and off-peak hours. As a result, the spot market presents more favourable prices to enable EES to trade; however, the revenue is also higher risk [22].

\subsubsection{Participation in the Capacity Market}

In GB, There were concerns that with increasingly high levels of intermittent generation and market flaws, such as short term demand side inelasticity, there were insufficient market signals to trigger the required investment in generation capacity. To address this, a capacity mechanism was introduced as outlined below as part of the recent Electricity Market Reform (EMR) [125]: 
- A forecast of future peak demand is made on behalf of Government by Ofgem, the System Operator or other technical experts;

- Ministers agree the total amount of capacity required to ensure security of supply based on this forecast;

- The agreed volume of capacity is contracted through a competitive central auction run by the System Operator 4-5 years ahead of the delivery year;

- The participants successful in the auction enter into capacity agreements and receive an agreed revenue stream to cover the cost of the plant capacity. In return, the plant is committed to provide electricity when called upon for a stress event. If they cannot deliver when required, they face high penalties;

- The cost of the capacity payment is split between the electricity suppliers in the delivery year.

The capacity market provides an additional revenue stream for EES. The first auction results were released in December 2014 for delivery of capacity in 2018. The clearing price of $£ 19.30 / \mathrm{kW} /$ year was lower than expected [126]. As a result no new EES projects were enabled through the capacity mechanism. The only EES which was able to compete was existing PHS which was only eligible for contracts of a single year. Thermal generation accounted for over $80 \%$ of the contracted capacity [126]. EES provides additional benefits when compared to conventional generation; however, when competing directly, thermal generators provide a lower risk and lower cost investment than FOAK deployments of EES.

In addition to competing with existing generators, capacity providers face considerable penalties if they fail to deliver during a stress event. These penalties present significant barriers to entry for new technologies which do not have sufficient operational experience to provide confidence in their reliability. Furthermore, specific to EES, is the challenge of providing energy for the open ended duration of any stress event [109]. EES has a finite storage capacity and cannot provide power indefinitely. Penalties are linked to the value of lost load which has been estimated at up to $£ 17,000 / \mathrm{MWh}$ [127]. The scale of the penalties faced is currently prohibitive for many EES devices.

\subsubsection{Ancillary Service Markets}

Some specific ancillary services are outlined in Section 3.2.1 which could provide direct revenue streams for EES. In general, ancillary services have minimum power requirements which providers must meet and many small to medium sized EES projects would not qualify. To enable these devices to participate, aggregator services can be employed. Aggregators 
provide access to ancillary service markets for smaller energy resources which are combined to provide a single larger megawatt response which would fulfil qualification criteria [128]. While this does carry additional contractual costs, a single agreement can be arranged enabling access to multiple ancillary service markets [128].

Ancillary service markets are continuously evolving and products and services vary from one year to the next [128]. National Grid does not usually contract services for periods longer than two years and revenues can be unpredictable which makes them relatively high risk. Figure 3.11 shows National Grid's spending on frequency response services from 2007-2015. This shows a general increase in spending on frequency response, largely expected due to the increased volume of intermittent wind power connected to the system. However, there is an unexpected reduction in spending in 2013-2014. When queried on this, National Grid commented that it did not reflect the contracted volumes of frequency response services, which did not change considerably from the previous year, but was due to changes in the company's tender strategy which was continuously under review [124]. This highlights one aspect of risk that the ancillary service markets are exposed to.

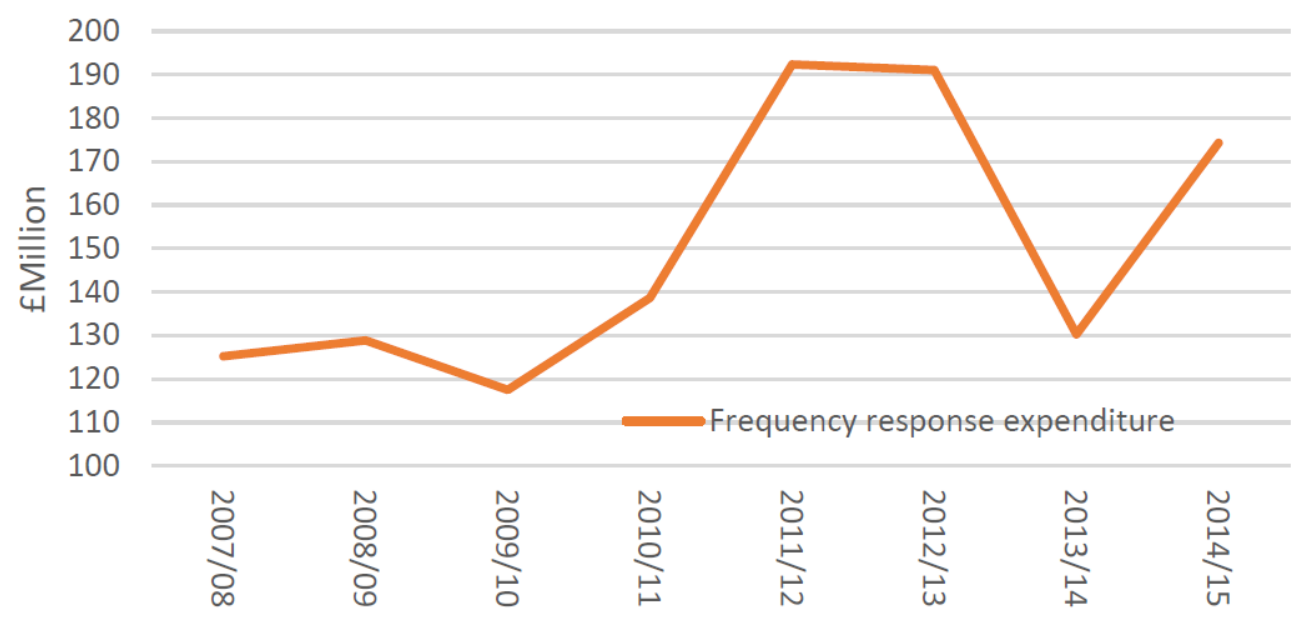

Figure 3.11 National Grid expenditure on frequency response services (source [124])

Spending on fast reserve and black start services has increased over the last decade [129] and Ofgem anticipates that these trends will continue in the future [130]. Despite the uncertainty, the size of the ancillary service markets is expected to grow providing more potential opportunities for EES. In addition to growing requirements for existing services, new service markets are expected to emerge including inertia services and additional balancing reserve services [128]. 
System inertia is currently provided by synchronous generators from thermal plant connected to the grid. This is slowly being removed as conventional generators are mothballed and more non synchronous generation, including wind and solar, is connected. A reduction in system inertia will cause the rate of change of system frequency to increase in response to an incident such as the loss of a generator [128]. As an alternative to procuring additional frequency response, National Grid could use synthetic inertia from a new 'system inertia service' to provide rapid frequency response for periods of $0.5-4$ seconds. This would present an ideal market for some EES technologies and would reward the speed within which some systems can respond. A similar service has been introduced in North America to provide additional compensation for provision of rapid response [128] and Ireland have also proposed a new synchronous inertia response service [131].

\subsubsection{Distribution Network Services}

An important application of EES is its potential to defer spending on traditional distribution network reinforcement. In economic models this is accounted for as a system saving [110] and is not currently a direct source of revenue. UK Power Networks is investigating the business case for EES in distribution networks through a demonstration project called Smarter Network Storage. The project is supported by $£ 13.2 \mathrm{~m}$ of funding from the Low Carbon Network Fund and an additional $£ 5.5 \mathrm{~m}$ from UK Power Networks and other partners [132].

The Smarter Network Storage project is testing a 6MW, 10MWh lithium ion battery facility at Leighton Buzzard primary substation. The substation comprises two $33 / 11 \mathrm{kV} 38 \mathrm{MVA}$ transformers fed by two $33 \mathrm{kV}$ overhead lines with a winter rating of 35.6MVA [133]. Peak demand at Leighton Buzzard has exceeded the firm capacity limit for between 9 and 37 days in each of the last 5 years. During these periods, additional capacity has been transferred from neighbouring parts of the network; however the transfer capacity limit is 2MVA and the demand is forecast to grow with expectations that the limits will be breached in the future. The conventional response would be to reinforce the network with a third $33 \mathrm{kV}$ circuit and $38 \mathrm{MVA}$ transformer providing an additional 36MVA of firm capacity which is expected to be over rated for a number of years [133]. Reducing peak demand at Leighton Buzzard could delay the need for reinforcement for a number of years or potentially avoid it completely. The installation of a distribution-connected EES device is being trialled to investigate the operation strategy and commercial arrangements which could enable this. The traditional and storage reinforcement options for the Leighton Buzzard site are shown in Figure 3.12. 


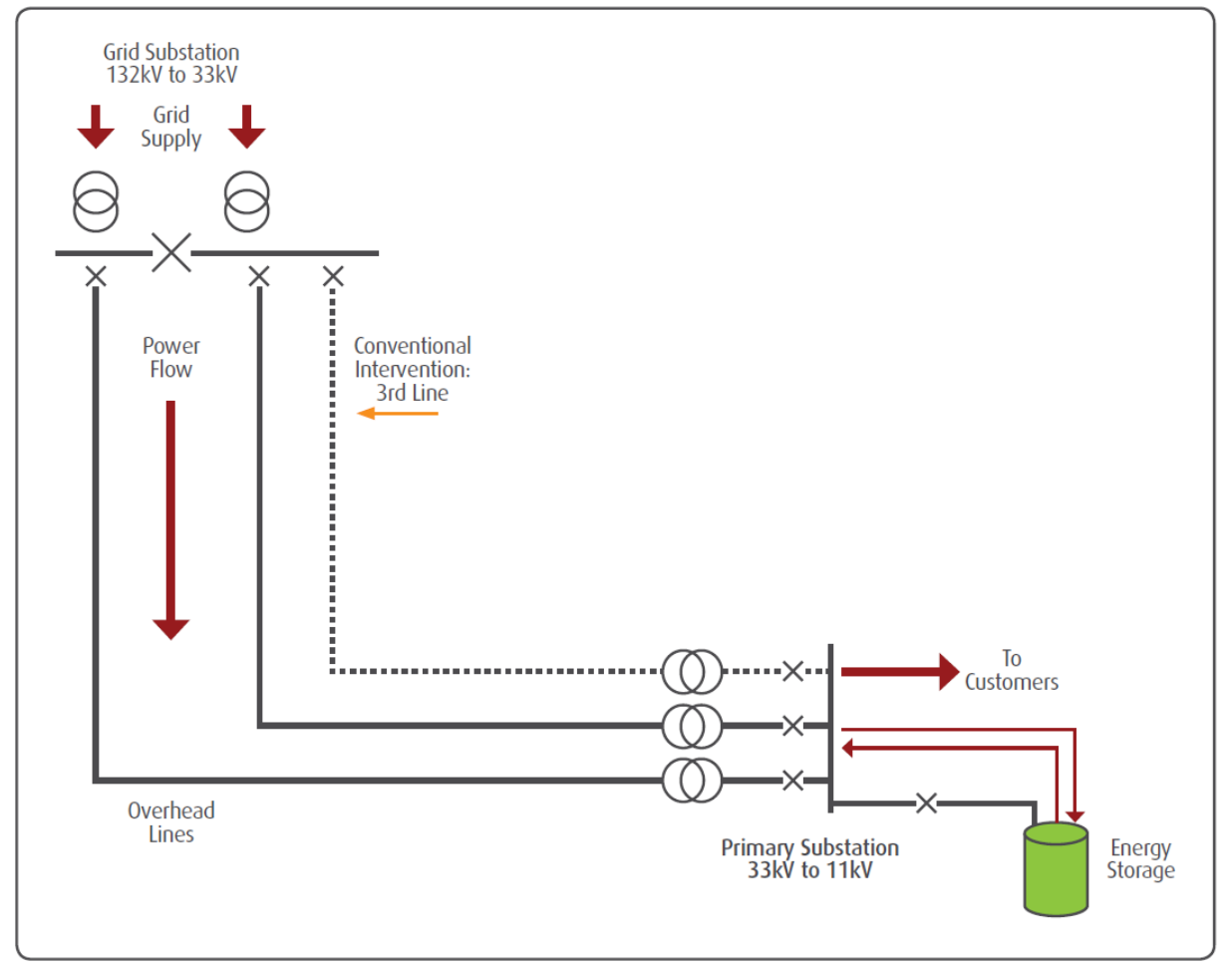

Figure 3.12 Leighton Buzzard distribution network reinforcement options (source [133])

UK Power Networks has proposed a number of business models which could provide revenue to an EES device from a DNO ranging from direct ownership by the DNO to arrangements for contracted services. Details of these can be found in [133]. There are also a number of challenges associated with the operation of EES in a distribution network. These are discussed in Section 3.6.

\subsubsection{Consumer Markets}

\section{Large Domestic Consumers}

Opportunities for domestic consumers to benefit from EES may emerge in the future. Domestic solar power producers benefit from a feed-in-tariff payment for the electricity they generate and additionally an export tariff paid for $50 \%$ of the electricity they produce [134]. This is calculated, in the absence of time of use meters, on the assumption that $50 \%$ of the electricity produced is consumed locally and the remainder is exported to the National Grid. This ratio is fixed regardless of how much electricity the domestic producer consumes. As a result, owners can benefit from reduced electricity bills, without affecting their payments, during periods where they can use the electricity generated from their solar panels. With the implementation of domestic EES, they could store the electricity they produce when it is sunny to use it when they needed it. Retail electricity prices are not currently high enough to justify 
an investment in domestic EES, but if prices increase significantly there may be a viable business case for this application in the future.

\section{Commercial Consumers}

There is scope for commercial consumers to gain revenue from EES by providing relief for electricity suppliers from transmission and distribution use of network charges.

The cost of operating the transmission network is driven by peak demand and is recovered via a system called triad charging. Triad season runs from November to February each year and, at the end of the season, the three half hourly periods with maximum system demand are identified. Suppliers are charged their customer's average consumption in each network zone, during each triad period, multiplied by the charge for that zone [135]. The charges for each network zone for 2014-2015 are shown in Table 3.1 which demonstrates the higher prices in the relatively more populous areas.

Table 3.1 Triad charges for network zones for 2014-2015 triad season (source [135])

\begin{tabular}{lc}
\hline \multicolumn{1}{c}{ Network Zone } & $\begin{array}{c}\text { Triad Charge } \\
(\mathfrak{f} / \mathbf{k W})\end{array}$ \\
\hline Northern Scotland & 11.04 \\
Southern Scotland & 16.78 \\
Northern & 22.34 \\
North West & 25.18 \\
Yorkshire & 25.48 \\
Merseyside and North Wales & 26.63 \\
East Midlands & 28.21 \\
Midlands & 29.20 \\
Eastern & 29.89 \\
South Wales & 27.54 \\
South East & 32.82 \\
London & 34.08 \\
Southern & 33.75 \\
South West & 33.55 \\
\hline
\end{tabular}

If customers can avoid consuming electricity, or generate it locally from EES, during the triad periods they can save money for electricity suppliers. Generators can typically negotiate 90 $95 \%$ of the share of triad savings from a supplier [135]. However, triad periods are not known 
in advance and most generators run for 25-30 hours during a triad season in response to warnings from market forecasters. Payment is only received for a maximum of the three actual half hour triad periods where savings are made [135]. Triad savings are unlikely to be a sufficient revenue stream to justify the cost of EES on their own; however, they could provide additional income to a storage operator at a high value for a small number of hours per year enabling alternative revenue streams to be accessed for the remainder.

The cost of delivering electricity through the distribution system is recovered by local DNOs through distribution use of system charges which vary regionally and depend on the voltage level at which the customer is connected. Large consumers, connected at high voltage levels, are charged through traffic light systems which define the peak periods throughout the year [128]. An example of the periods defined by the traffic light system is shown in Figure 3.13.

\begin{tabular}{|l|r|r|c|}
\hline \multicolumn{3}{|c|}{ Time Bands for Half Hourly Metered Properties } \\
\hline Time periods & Red Time Band & Amber Time Band & Green Time Band \\
\hline $\begin{array}{l}\text { Monday to Friday } \\
\text { (Including Bank Holidays) } \\
\text { All Year }\end{array}$ & $16.00-19.00$ & & \\
\hline $\begin{array}{l}\text { Monday to Friday } \\
\text { (Including Bank Holidays) } \\
\text { All Year }\end{array}$ & & $07.00-16.00$ & \\
\hline $\begin{array}{l}\text { Monday to Friday } \\
\text { (Including Bank Holidays) } \\
\text { All Year }\end{array}$ & & $19.00-23.00$ & \\
\hline & & & $00.00-07.00$ \\
\hline $\begin{array}{l}\text { Saturday and Sunday } \\
\text { All Year }\end{array}$ & & & $23.00-24.00$ \\
\hline Notes & All times are in UK Clock time & $00.00-24.00$ \\
\hline
\end{tabular}

Figure 3.13 Traffic light time bands for distribution use of system charges (source [128])

Prices during the red time band are typically only slightly lower than domestic consumer rates and can be orders of magnitude larger than those in other time bands [136]. A consumer could make use of EES to charge during the green time band and discharge during the red time band, receiving significant revenue.

\subsubsection{Aggregating Revenue Streams}

It is generally concluded from economic models that currently deployment of EES for a single application is not economically viable [137]. Commercial trials from the Smarter Network Storage project support this conclusion [128]. Ancillary service markets do not provide sufficient revenue to justify investment in EES, when conventional generators can provide similar services at a lower cost, and applications which alleviate peak demand lead to 
underutilisation. It is accepted that multiple revenue streams will need to be aggregated to make a business case for EES.

Some sources of income are not compatible with others, such as black start services, which must maintain $100 \%$ availability for their application. Other ancillary services, require automatic response to electronic instructions from the system operator. This priority would need to be accounted for when providing multiple services. Additionally, each application will have associated costs for example for trading, administration and licences. Each of these must also be taken into account.

Xian et al proposed a method to allow multiple participants to share the use of an EES device [137]. Value is achieved through a series of auctions selling the power and energy capacity of the device to different users. Each auction takes place in a sequential time horizon with the user bounded by the EES constraints and, additionally, by the constraints imposed by the previous users' actions. This may lead to some users not gaining as much value as they would have if using a dedicated storage device independently; however, the sum of the aggregated values would be significantly greater than any single application. Market arrangements to enable this form of trading do not currently exist and would be complex and expensive to establish. It would rely on individual users cooperating with others, despite their own benefits being suboptimal as a result, and on the free exchange of information [137].

Figure 3.14 shows the applications of EES divided into centralized and decentralized markets and their estimated value in 2030 . This demonstrates that the potentially profitable markets span both parts of the sector. There are regulatory obstacles preventing interaction between the centralized and decentralized parts of the electricity industry which would prevent the methods proposed by Xian et al [137] being implemented. The division of benefits across regulated and deregulated sectors presents one of the major challenges facing EES. This is discussed, with other barriers to market entry, in the following section. 


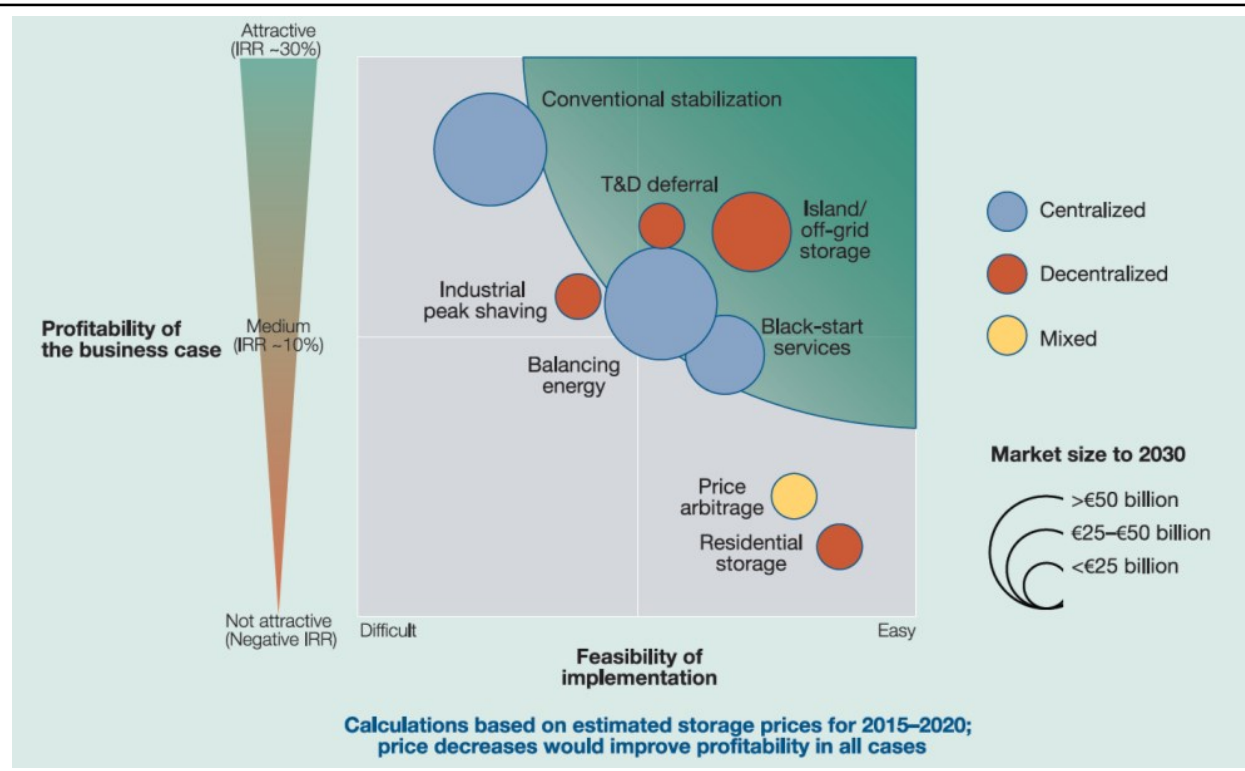

Figure 3.14 Energy storage market forecast by application for 2030 (source [45])

\subsection{Barriers to Market Entry}

Some specific issues, such as the risks associated with participation in the capacity market, are discussed above but there are broader barriers to market entry for EES. Figure 3.15 shows the policy tools being implemented in GB which could affect EES. Each of these policies is being consulted on separately which highlights the level of complexity surrounding the policy environment. EES technologies which can contribute to energy management, such as PHS and CAES, have high capital costs and long service lives. For projects to be funded, investors need confidence in future revenue streams for decades ahead. However, as an enabling technology, revenues depend on the future development of the electricity system which is largely uncertain [138]. Specific regulatory barriers are discussed below.

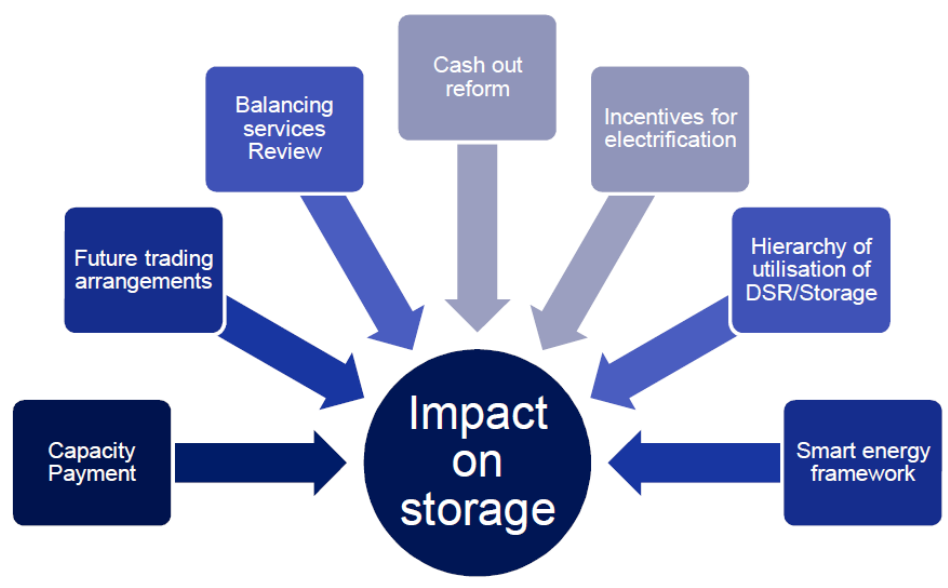

Figure 3.15 Policy tools being implemented in Britain which will impact storage (source [139]) 


\subsubsection{Classification of Storage}

In the absence of a dedicated asset class to define EES, it is classified as generation plant [109]. This presents several issues which are discussed below. The allocation of a distinct asset class for storage in the UK, to remove these complications, is one of the recommendations made by the Electricity Storage Network, the UK industry body [96].

\section{Grid access fees}

The classification of EES presents uncertainty surrounding grid access fees. Although a generation licence is granted, EES is a net consumer of electricity. In most EU countries EES pays fees as both a consumer and a producer [140]. It has been argued that EES should be exempt from paying any access fees as its operation could contribute to alleviating grid congestion [65].

\section{Climate Change Levy}

The Climate Change Levy (CCL) is a tax paid on generation of electricity in GB. Originally, renewable generation was exempt from payment; however, from August 2015, this exemption was removed and all generators are now eligible to pay [141]. EES consumes electricity which has been produced by a primary generator and, as such, has already been subject to CCL payments. However, as EES holds a generation licence, it may be subject to payment of the CCL a second time for the electricity it discharges. As part of the Smarter Network Storage Project, UK Power Networks received exemption from CCL payments as the lithium ion battery being used was not considered a generator when assessed as an individual case study. It is unclear if other forms of EES which comprise rotating generators, such as PHS, CAES and CES, would receive the same exemption [109].

\section{Unbundling Requirements}

The separation of centralized and decentralized activities is referred to as unbundling and is enforced through legislation at both EU and GB levels. At EU level, the Third Energy Package specifies that "without effective separation of networks from activities of generation and supply (effective unbundling), there is an inherent risk of discrimination not only in the operation of the network, but also in the incentives for vertically integrated undertakings to invest adequately in their networks" [142]. In GB, the Electricity Act 1989 [143] and later amendments prohibit any company from holding both generation and transmission, distribution or supply licences. 
Small generators, with an output of less than 50MW, are exempt from licencing requirements. The exemption is applied per site and the capacity is not aggregated, so DNOs could legally own multiple small scale distribution connected EES units. However, DNOs are prohibited from undertaking activities which may distort the generation or supply markets. Trading of electricity by a DNO would not be permitted, so a third party would be required to manage the flow of electricity to and from the EES device and DNOs would not be able to receive any revenue associated with energy trading.

Furthermore, under distribution licence agreements, DNOs are subject to de-minis business restrictions which limit their activities which are not directly related to the distribution of electricity. These specify that annual turnover from all non-distribution related activity cannot exceed $2.5 \%$ of total turnover [144]. Analysis from the Smarter Network Storage Project estimates that DNOs would be able to deploy between 10 and 20 EES devices of equivalent size to the lithium ion battery demonstration project to remain under this threshold [109]. However, this could restrict other business activities. The regulations also specify that no more than $2.5 \%$ of total investment is allowed to be in non-distribution related activities [144]. The definition of EES as a distribution or non-distribution related investment is harder to determine; however, it is expected that this will be less restrictive than limitations on turnover [109].

\section{Risk Appetite}

The regulatory framework within which DNOs operate is complex and legislation relating to EES is ambiguous. While exemptions appear to exist which would enable the ownership of storage by DNOs, in practice, there is little implementation. As regulated organisations DNO profits are restricted and investments in the business are characterised as low risk, low return [31]. Furthermore, the reliability of infrastructure is valued highly with significant penalties imposed if there are service failures. As a result, DNOs are risk averse with a preference for using proven traditional technologies over higher risk innovative solutions [138]. This does not present a favourable environment for the development of novel EES technology despite the longer term and broader benefits it could offer.

\subsubsection{Undervaluation of flexibility}

Further to direct regulatory barriers for EES, there are indirect mechanisms which serve to reduce the value of flexibility in the electricity market. In GB, if a market participant generates or consumes more electricity that they have contracted prior to gate closure, they must pay an imbalance settlement at the 'cash-out' price. The current method used to determine the 
imbalance settlement dampens the cash-out price through the mechanisms outlined below. As a result, the incentive for participants to balance their contracts prior to gate closure, which could be achieved using EES, is reduced [109].

1. The principal imbalance price is a weighted average of the most expensive trades required to balance the system which means that participants are not necessarily incentivised to reduce the cost of their individual imbalances.

2. When short term operating reserve is exercised through the balancing mechanism the utilisation fees contribute to the cash-out price. However, the fee prices are fixed during the tender process which is in advance of the delivery period so they do not reflect the system scarcity in real time.

3. During periods of extreme system stress, the system operator can instruct DNOs to reduce their local demand through voltage control enforcing brownouts or even blackouts. A brownout is a drop in voltage on the electricity system. The terms comes from the dimming experienced by incandescent lighting as a result of the voltage sag. A blackout refers to a complete power outage of part (or all) of the electricity system. These actions are not included in calculation of the cash-out price and so system imbalance prices are further dampened during these periods.

The dampening of cash-out prices results in flexibility being undervalued by market participants. Although Ofgem has launched an Electricity Balancing Significant Code Review to attempt to address these issues, at the time of writing the proposed changes were not approved [145].

\subsubsection{Environmental Legislation}

Environmental legislation presents a further barrier to EES project development. While it is essential that projects are adequately assessed, to ensure there are not significant environmental impacts outweighing the benefits, the complexity adds significant costs and risks during project development [118]. Legislation spans national and EU planning frameworks which are approved at different stages by different organisations.

Despite local impacts, EES projects contribute to the wider objective of decarbonising the electricity sector. Often planning decisions are made at a local level where authorities do not have the expertise or resources to fully assess projects. In Ireland, the Strategic Infrastructure Act has been introduced to accelerate projects of strategic importance through the approval process. It is expected that PHS and CAES projects would fall within this category [140]. It has been suggested that including EES in national plans, such as these, would enable decisions 
to be made by higher authorities at an earlier stage which would help to reduce costly planning delays [118].

\subsection{Support for Storage}

Despite the challenges there is support for EES, both in GB and internationally. Changes are being implemented to promote EES, reduce costs and lessen the regulatory obstacles it faces.

\subsubsection{Great Britain}

Over the last decade in GB support for EES has increased and funding has been made available for fundamental research and development, innovation and demonstration projects.

In 2006, the Supergen Energy Storage Consortium was founded to accelerate the development of energy storage systems [146]. In 2012, the Engineering and Physical Science Research Council (EPSRC) funded two Grand Challenge projects for energy storage with $£ 5.5 \mathrm{~m}$ awarded to the Energy Storage for Low Carbon Grids project led by Imperial College London [147] and $£ 3 \mathrm{~m}$ awarded to the Integrated Market-fit and Affordable Grid-scale Energy Storage (IMAGES) project led by Warwick University [148]. In the same year, the Energy Storage Research Network was established to develop a more integrated energy storage research community in the UK [149].

These developments have led to a more focussed research community and 2012 saw a number of high profile reports published which highlighted the value of storage and the regulatory challenges it faces.

1. Imperial College London produced an important study for the Carbon Trust, Strategic Assessment of the Role and Value of Energy Storage Systems in the UK Low Carbon Energy Future [2]. This highlighted the significant benefits from development of gridscale storage, but also concluded that the current UK market framework did not allow these benefits to be realised commercially. This report is commonly quoted by industry and lobby groups [96].

2. DECC published its report, Electricity System: Assessment of Future Challenges [150]. This was welcomed by the energy storage industry as formal recognition by the Government of the role that storage could play in the electricity sector in the future. It committed to continued support for storage technology innovation and also highlighted a need to develop commercial arrangements to allow owners and operators to capture an appropriate return for the benefits that storage can provide. 
3. The Low Carbon Innovation Coordination Group published its report, Technology Innovation Needs Assessment for Electricity Networks and Storage [5], estimating the potential for up to $59 \mathrm{GW}$ of grid connected storage capacity by 2050. It highlighted that some technologies are likely to be deployed more extensively than others depending on technology improvements and also on regulation and commercial factors.

Following these developments, in 2013, the Minister for Universities and Science, announced an additional $£ 30 \mathrm{~m}$ of capital funding from EPSRC for research into grid-scale EES technology [151]. Five university proposals were awarded funds to create dedicated research and development facilities. Additionally, in 2014, the Supergen Energy Storage Hub was established to facilitate the development of research projects to larger scale prototypes. The consortium of seven academic and fourteen industrial and governmental partners was awarded $£ 4 \mathrm{~m}[152]$.

At the same time, funding for technology developers was made available. The Energy Technologies Institute announced project funding and an equity investment of $£ 14 \mathrm{~m}$ in Isentropic Ltd to build a full scale demonstrator of its pumped heat EES device [153] and DECC announced a series of funding including [154]:

1. The Energy Entrepreneurs Fund Scheme;

2. The Energy Storage Component Research Feasibility Study Competition; and

3. The Energy Storage Technology Demonstration Competition.

Similarly, Ofgem established the Low Carbon Network Fund of up to $£ 500 \mathrm{~m}$ to support projects sponsored by DNOs to trial new technology, operating and commercial arrangements [155]. Several EES demonstration projects have been funded by this including UK Power Networks' Smarter Network Storage project.

There is also support for EES emerging at European level. The Horizon2020 work programme specified 3 calls for EES projects with collective budgets of $€ 44 \mathrm{~m}$ and $€ 26 \mathrm{~m}$ in 2014 and 2015 respectively [156].

The level of funding for innovation of EES is significant and the Carbon Trust estimates that this could lead to average cost reductions for EES technologies of 39\% by 2050 [157]. The estimated savings for each technology are shown in Table 3.2. 
Table 3.2 Estimated cost reductions through innovation for energy storage technologies (source [157])

\begin{tabular}{lcc}
\hline \multicolumn{1}{c}{ Technology } & 2020 Cost Reduction (\%) & 2050 Cost Reduction (\%) \\
\hline PHS & - & 20 \\
CAES & 10 & 30 \\
NaS Batteries & 20 & 40 \\
Flow Batteries & 20 & 40 \\
Lithium Ion Batteries & 20 & 50 \\
FES & 15 & 30 \\
Supercapacitors & 5 & 25 \\
Thermal to Electric & 20 & 50 \\
\hline Weighted Average & 15 & 39 \\
\hline
\end{tabular}

$23 \%$ of the total cost reduction by 2050 is expected to be achieved through additional fundamental research and development and the remaining 16\% through learning by doing [157]. Both the academic and developer led innovation is essential for this to be achieved.

Despite the innovation support, initiating cost reduction and a technology push for EES, without fundamental changes to the regulatory system, there will be no market pull. Many proponents of EES argue that it should receive a direct subsidy from targeted support through either CfD or the capacity market arrangements [158]. This would recognise that EES is a developing technology and has not yet benefited from the cost reductions that mature technologies experience [158]. When questioned on this, DECC maintains the need to remain technology neutral to ensure that flexibility is provided at the lowest cost to consumers [159]. Conversely, DECC does recognise that not all low carbon generators are at the same level of development and offers technology specific CfDs for different generation technologies [160]. Furthermore, in addition to direct subsidies, paid for by consumers, nuclear generators also receive unquantified support from tax payers in the form of underwritten insurance, underwritten political risk, guaranteed finance and funding for the Nuclear Decommissioning Authority [161]. These additional measures significantly reduce the risk of large infrastructure nuclear projects which would not be feasible in their absence. Large scale EES such as PHS and CAES would benefit considerably from similar support which is not available for them.

During the Committee Stage of the EMR Energy Bill, Baroness Worthington commented in relation to EES that "... if you spend that kind of money on the demonstration but you do not 
have a policy to secure the route to market, that is effectively wasted money. Markets are useful but policy is needed to implement change." [158]

Some changes are being made to improve the planning process at regional and European level. In Scotland PHS has been included in the Third National Planning Framework which sets out a national plan for large infrastructure projects [162]. This is expected to streamline the planning process for PHS in Scotland. The European Commission has also identified strategic energy infrastructure priorities including EES. These have been termed Projects of Common Interest (PCIs) which must benefit at least two member states, contribute to market integration and further competition, enhance security of supply and reduce $\mathrm{CO}_{2}$ emissions. PCIs can benefit from a rationalized permitting progress and access to EU funding, but must be connected to the transmission system, be rated at a minimum of $225 \mathrm{MW}$ and contribute a minimum of $250 \mathrm{GWh}$ of electricity generation per year [109].

\subsubsection{International Experience}

\section{USA}

Regulation of EES in the USA is further complicated by inter/intra state regulation. The Federal Regulatory Commission regulates interstate transactions while state bodies, such as the Public Utility Commissions, regulate utility management, operations, electricity rate structure and capacity acquisition within their respective states [163]. Various innovative funding is available for EES in the USA. Full details are available in [163]. Three key legislative changes which are helping to remove regulatory barriers faced by EES are discussed below.

\section{The California Assembly Bill 2514}

In 2013, the California Public Utilities Commission adopted a $1.325 \mathrm{GW}$ procurement target for energy storage with biannual targets increasing every two years from 2016-2020. These are split into specific capacity targets for transmission connected, distribution connected and behind-the-meter EES and explicit requirements for each utility company [163].

\section{ERCOT Charging Regime Changes}

The Electricity Reliability Council of Texas (ERCOT) is the Public Utility Commission of Texas. ERCOT legislation specifies that generators are compensated for electricity on a nodal pricing basis which depends on grid congestion between transmission network nodes. Demand loads, however, pay for electricity based on the average price of the nodes in the regional zone that they are in. EES was previously charged zone prices as a consumer when charging; however, changes in classification have enabled nodal prices to now apply to EES during both 
charging and discharging to provide an incentive for EES to be sited and operated more efficiently.

\section{Rewards for Fast Frequency Response}

The Federal Regulatory Commission has introduced compensation for performance in the wholesale regulation market. In the regulation market, resources provide rapid response to manage fluctuations in system-wide supply and demand. Order 755 has modified the compensation regime to reward faster acting resources with higher payments [163].

Each of these changes is contributing to creating a market pull for EES which does not currently exist in the UK.

\section{Ireland}

The island of Ireland is an interesting example as, with limited interconnection to the rest of Europe and increasing penetrations of wind power, it is experiencing the problems associated with intermittency ahead of GB. In addition to some existing revenue streams that EES can access, new ancillary services are being proposed in Ireland which will create new markets. This includes services for: synchronous inertial response, fast frequency response, ramping, fast post fault active power recovery and dynamic reactive response [140]. These proposals are currently under review and their impact on deployment of EES is unknown.

\section{Other}

Several other countries have specific policies which support EES directly. These are listed in Table 3.3. These all contribute to enabling EES deployment, although some distort the market for electricity and do not necessarily promote the most efficient use of EES.

Table 3.3 Policies affecting storage from other countries [96]

\begin{tabular}{ll}
\hline \multicolumn{1}{c}{ Country } & \multicolumn{1}{c}{ Policy } \\
\hline Germany & $\begin{array}{l}\text { A subsidy is available to cover } 30 \% \text { of the cost of storage associated } \\
\text { with domestic solar power. New storage has also been made exempt } \\
\text { from network usage fees. }\end{array}$ \\
Italy & $\begin{array}{l}\text { A transmission and distribution target of } 75 \mathrm{MW} \text { of battery storage by } \\
\end{array}$ \\
& \\
Japan & Government subsidies are available for home owners and companies to \\
& install batteries with domestic solar generators. Utilities are also allowed
\end{tabular}


to curtail wind and solar power for up to 30 days (provided it is not more than $8 \%$ of their annual power production) without providing any compensation. In addition to this, some utilities have specified that wind developers must install secondary batteries onsite to mitigate wind variability.

Puerto Rico All renewable generation projects must include $30 \%$ of their capacity as storage.

\subsection{Removing Market Barriers}

While some berries to market entry for EES could be readily overcome with changes to legislation, there are many areas which would require further consideration of the wider implications on the energy market before specific recommendations could be made. Some examples of ways in which barriers to market entry for EES could be removed, and the subsequent broader impacts, are discussed below:

1. Removal of the open ended definition of stress events on the capacity market would reduce the risk of penalties for EES which can only deliver capacity for a finite period. This may not, however, be sufficient to serve the requirements of the electricity system where the duration of a stress event is not known with certainty. One approach would be to provide categories for different technologies to complete in with specific requirements applying to different technologies. This would result in an approach which was not technology neutral and may favour technologies which would not necessarily best serve the system requirements at the lowest cost.

2. Reclassification of EES from a generating asset so it had its own licence may remove multiple market barriers. For example, depending on the specific requirements of the new licence, it could clarify the uncertainty surrounding grid access frees, the payment of the CCL and the complexities surrounding ownership and operation of EES by DNOs.

3. Providing longer term fixed contracts for ancillary services would provide EES with guaranteed income for a larger proportion of its lifetime and provide certainty for investors to fund projects. This is unlikely to happen as the requirement for ancillary services changes from one year to the next and new technologies could provide services at lower cost if these are contracted annually. 
4. Some proponents of EES argue that it should be eligible for a subsidy payment, such as a percentage of a Feed-in-Tariff when co-located with renewables. However, this could skew the market and unduly favour EES being co-located with renewables which would not necessarily be the most efficient solution to providing flexibility.

Enabling EES, as a new technology, to penetrate the market is complex and requires consideration of the policy impact of all areas of the electricity industry.

\subsection{Chapter Summary}

This chapter has reviewed the issues associated with integrating variable renewable power with the existing electricity system. Research has shown the cost of intermittency to be less than $£ 10 / \mathrm{MWh}$ for wind penetrations of less than $20 \%$, but that this will increase as more wind power is deployed. To resolve these issues, technologies which offer flexibility will be required. EES is one solution to this problem. It has multiple applications across a range of different timescales including: provision of ancillary services, peak shaving, load levelling, smoothing renewable power and provision of backup power. The applications can be categorised by their required characteristics such as discharge time, speed of response and capacity and it is evident that no single EES technology can deliver all the applications.

Attempts have been made to quantify the value EES can bring to the electricity system. This is challenging due to the span of temporal and spatial resolutions which must be modelled to capture the range of benefits it provides. Additionally, as a cost saving technology, its value is ultimately dependent on the cost of the future electricity system which is inherently uncertain. Despite this, there is general consensus that EES is valuable to the electricity industry. Similarly, analysts have attempted to quantify the market value of EES and equally, there is a wide range of estimates with consensus that the market value will increase in the future.

EES brings additional benefits, which are not fully accounted for financially, of reduced system emissions, stable wholesale electricity prices and new jobs. However, there are also detrimental impacts. Batteries make use of mined elements which are energy intensive and finite and PHS has a significant environmental footprint. Despite this, and the $\mathrm{CO}_{2}$ emissions that EES devices cause, research concludes that any renewable electricity system with EES will produce significantly less $\mathrm{CO}_{2}$ than an equivalent fossil fuel based system.

Regardless of system benefits, it is the commercial valuation of storage which will determine its success. The revenue available to EES is challenging to access. It is evident that income streams from multiple sources will need to be aggregated if EES is to become commercially 
viable. However, revenues are split between regulated and deregulated parts of the electricity industry. Market barriers prevent access to all of these simultaneously and the current costs and risks of EES are prohibitively high. There is some support for EES innovation to reduce costs and enable proof of concept through demonstration projects. However, without any direct subsidy, in the current regulatory system there is no market pull for EES and it is unlikely to be developed without changes to legislation.

This chapter highlights the commercial value of EES and the barriers to market entry which currently exist establishing a disparity between the commercial and system level benefits which EES can provide. The following chapters of this thesis examine the commercial value of EES quantitatively through arbitrage alone. It examines the change in the commercial value of EES through arbitrage in future energy scenarios, leading to a reduction, or otherwise, in the gap between the system and commercial value of EES. 


\section{Chapter 4 Modelling Energy Storage}

This chapter describes a model developed and used to assess the commercial value of EES through price arbitrage. Preliminary results demonstrate the variation in performance of EES in different global markets and changes in revenue from one year to the next. Changes in revenue for different storage characteristics are also investigated. The implications of the assumptions of perfect foresight of electricity prices and the representation of storage as a price taker in the model are then examined. It is expected that these will have minimal impact on the results in the context of this thesis.

The performance of different technologies is compared to demonstrate the range of revenue available to different EES devices and the calculation of net present value (NPV) is introduced to compare the lifetime revenue to the system costs. Finally, the importance of evaluating risk, in addition to profit, is considered and a risk model is presented using characteristics of a CES device as a case study to demonstrate the challenges facing novel EES technologies.

The work reported in sections 4.2 and 4.4 has been presented at the Institution of Engineering and Technology $3^{\text {rd }}$ Renewable Power Generation Conference [164]. A copy of the manuscript is available in the end of this thesis.

\subsection{Justification for Investigating Price Arbitrage Alone}

Commercial models generally evaluate the techno-economic performance of a specific EES device in a defined market. While there are many revenue streams which EES can access, arbitrage is one which can be readily quantified over the duration of an EES asset. Electricity prices are publically available, whereas other ancillary markets are traded through private bilateral contracts which make it challenging to validate quantitative results. Furthermore, ancillary service contracts are awarded for only one to two years at a time and so it can be challenging to justify their inclusion in an EES project business case. 
Previous arbitrage studies have suggested a dependence of EES revenue on market structure through comparisons of arbitrage in different global markets [22] and variations within a single market from one year to the next [20] as generation capacity and fuel prices change. These have largely used historic electricity prices. It has been suggested that commercial opportunities for EES may emerge in the future with a higher penetration of intermittent renewable energy; however, the level of reward and its dependencies have not been fully defined.

One notable exception, which investigates future energy scenarios, is presented by Grünewald et al [19], finding that $32 \mathrm{GW}$ of wind capacity would enable the gross value of storage to cover its capital costs and investment could be commercially viable through price arbitrage alone. This suggests that, in future energy scenarios, the disparity between the commercial and system level value of storage may reduce. This finding, and the challenges in assessing the rewards from other ancillary services, justify the investigation of arbitrage alone to quantify the commercial value of EES in the remainder of this thesis. The later chapters investigate future energy scenarios, while this chapter uses historic electricity prices to verify and validate the arbitrage model.

While there is a wealth of research on the broad topic of EES, as highlighted above, only one study has been identified which investigates the commercial value of EES from arbitrage in future energy scenarios. The work presented in the following chapters is compared to existing literature where possible but is, in some cases, limited due to the limited previous work in this specific field.

\subsection{Modelling Arbitrage}

Many studies attempt to quantify the revenue available through price arbitrage. They generally make the assumption that the EES is a small device compared to the total generation capacity in the market [120], [122], [165], [166]. A small device is a price taker i.e. its own participation in the electricity market will have no impact on the price. This may be valid for initial deployments of EES; however, for the full system value of EES to be realised, large volumes must be deployed, and the participation of EES in the energy market will affect prices. This is discussed in more detail in Section 4.5.The majority of these studies also assume perfect visibility of electricity prices and use historical price data to estimate the maximum revenue which could have been achieved by EES in previous years. This assumes that prices are known with certainty, which is unrealistic and discussed further in Section 4.4; however, the approach 
provides an optimum solution of the maximum revenue available which provides a benchmark for comparison.

Both deterministic and stochastic approaches to optimisation of EES have been applied, for example, in [22] and [120] respectively. The stochastic method presented by Barbour et al [120] seemed to present few benefits over the simpler deterministic methods, as the constraints described could equally be applied in a linear optimisation programme. Furthermore, it did not guarantee that the final result converged on the optimum solution. When using this approach compared to modelling the same constraints in a linear programme, the model described in [120] was found to consistently underestimate the optimum revenue by around $5 \%$. Simpler deterministic methods, such as those discussed by Connolly et al [22] and Lund et al [121] oversimplified the problem, for example, by assuming the same operation schedule for a storage device each day, rather that changing the schedule to suite the changing price profiles.

Here, a deterministic, linear optimisation strategy was employed as presented by Byrne and Silvia-Monroy [167] and outlined in Figure 4.1. This did not over simplify and over constrain the problem as in [22] or [121] or unnecessarily introduce stochastic methods which were not required as in [120].

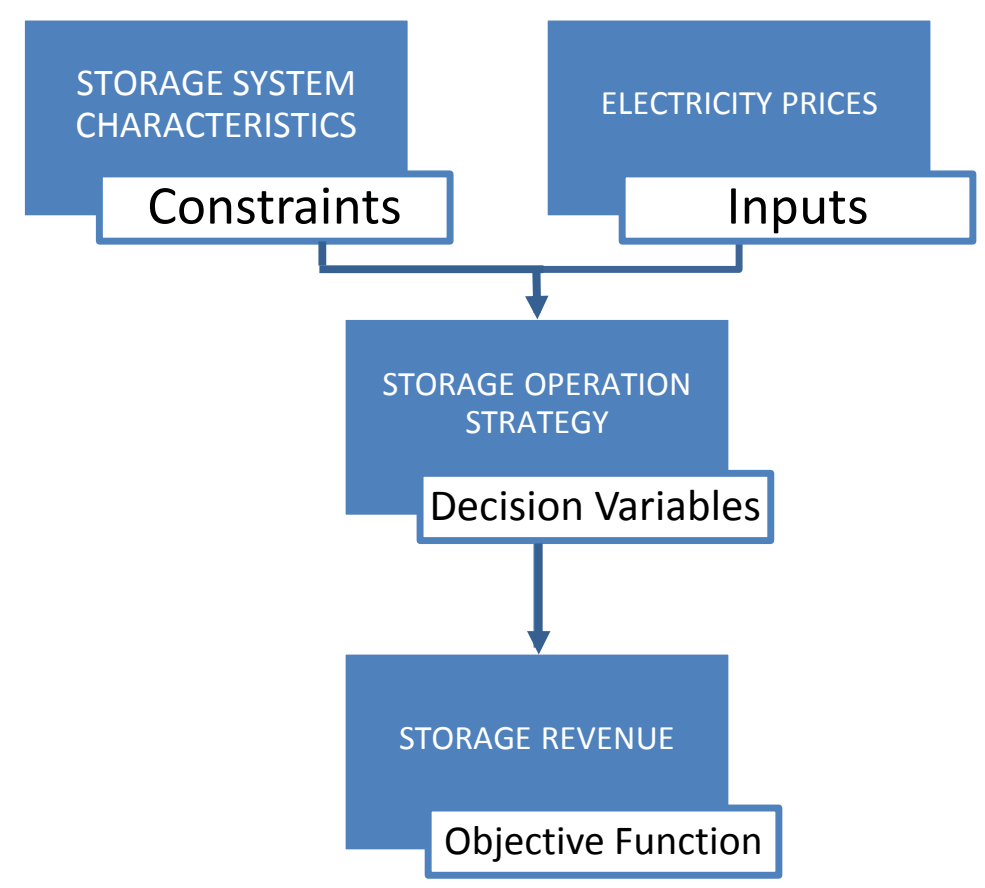

Figure 4.1 Arbitrage optimisation model 
- Inputs: a time series of half hourly electricity prices.

- Constraints: maximum charging and discharging rates, maximum storage capacity, storage efficiency and rate of self discharge.

- Decision variables: amount of electricity to charge or discharge (buy or sell) at each half hour time period.

- Objective function: to maximise revenue; the sum of the product of the electricity price and the net electricity sold at each half hour period for the duration of the optimisation.

Historic market index data defines the price, $P_{t}(£ / \mathrm{MWh})$, of electricity for each half hour settlement period, $t$, in the UK. It reflects the value of wholesale electricity in the short-term market and was used as an input for the linear optimisation.

The EES device characteristics, defined below, formed the problem system constraints.

$S_{\max } \quad$ Storage capacity (MWh) - the total amount of electricity that could be stored by the device.

$Q^{C} \quad$ Charging rate (MW) - the maximum rate at which the EES device consumed electricity when recharging.

$Q^{D} \quad$ Discharging rate (MW) - the maximum rate at which the EES device could deliver electricity.

$\eta_{c} \quad$ Conversion efficiency (\%) - the ratio of energy delivered to energy consumed excluding any losses due to self-discharge.

$\eta_{s} \quad$ Storage efficiency (\%) - the percentage of electricity retained in storage over each time period excluding any changes as a result of charging or discharging.

From the charging and discharging rates, the maximum quantity of electricity (MWh) which can be charged, $q_{\max }^{C}$, or discharged, $q_{\max }^{D}$, in a single half hour time period was defined.

The decision variables for the EES operator were how much electricity to buy, $q_{t}^{C}$, and sell, $q_{t}^{D}$, during each time period. The state of charge of the EES device, $S_{t}$, was defined by Equation (4.1) and subject to the constraints given in Equations (4.2), (4.3) and (4.4).

$$
\begin{aligned}
& S_{t}=\eta_{s} S_{t-1}+\eta_{c} q_{t}^{C}-q_{t}^{D} \\
& 0 \leq S_{t} \leq S_{\max }
\end{aligned}
$$




$$
\begin{aligned}
& 0 \leq q_{t}^{C} \leq q_{\max }^{C} \\
& 0 \leq q_{t}^{D} \leq q_{\max }^{D}
\end{aligned}
$$

The objective was to maximise the annual revenue, $R$, which was the sum of the price multiplied by the net quantity sold during each settlement period. This is defined in Equation (4.5).

$$
R=\Sigma P_{t}\left(q_{t}^{D}-q_{t}^{C}\right)
$$

Linear programming was used to calculate the revenue that could be achieved with perfect foresight of electricity prices as demonstrated by Byrne and Silva-Monroy [167]. $R^{*}$ was defined as $-R$ to formulate the problem as a standard minimisation problem with the objective defined by Equation (4.6), subject to the constraints in Equations (4.7) and (4.8)

Minimise:

$$
R^{*}=-\boldsymbol{f}^{T} \boldsymbol{x}
$$

Subject to:

$$
\begin{aligned}
& \boldsymbol{A x} \leq \boldsymbol{b} \\
& l b \leq x_{t} \leq u b
\end{aligned}
$$

where $\boldsymbol{x}$ is a vector of decision variables, units of electricity to buy or sell, and $\boldsymbol{f}$ a vector of prices for each half hour period throughout the year. $\boldsymbol{A}$ is a matrix of constraints computed from the conversion and storage efficiencies and $\boldsymbol{b}$ a vector based on the maximum storage capacity. $l b$ and $u b$ are lower bounds and upper bounds on the charging and discharging rates; zero and the maximum charging/discharging rate, respectively. A standard linear programming function implemented in MatLab, "linprog $(f, A, b,[],[], l b, u b)$ ", was used to solve the objective function and define the optimum operation strategy to maximise annual revenue.

Revenue was optimised on a weekly basis with the additional constraint that the state of charge must be zero at the start and end of each week. This reduced the computational time, but had minimal impact on total revenue with variations of less than $2 \%$ compared to optimisation on a daily, monthly or annual basis.

The following assumptions were also applied:

- The EES device had $100 \%$ availability throughout the year; there was no planned or unplanned maintenance time allowed for. The concerns surrounding this assumption are discussed during a specific case study at the end of this chapter. 
- The network was represented as a single bus system and the EES device was not subjected to network capacity constraints, which is reasonable in GB where there is currently no locational pricing.

- The device characteristics were constant over the period of investigation. This was acceptable for short periods; however, did not represent appropriate degradation in performance of some batteries, for example, as the cycle life progressed.

- The conversion efficiency was modelled during charging only i.e. the discharge cycle was $100 \%$ efficient. This may have impacted the absolute value of revenue, but would not affect the comparison between values for a single technology which the latter part of the thesis focusses on.

- The ramp rate was negligible compared to the time period. This is valid as a time period thirty minutes was applied and the majority of EES technologies have a response time of a few second as presented in Chapter 2.

- The cost of charging and discharging (in addition to the cost of electricity) was negligible. This was reasonable for most EES devices, but the economics of CAES, which also burns natural gas during its discharge cycle, would not be represented appropriately.

- The interest rate was negligible over the time period considered. This is expected to be valid for the thirty minute time period.

The model was verified using locational marginal price data from the California Independent System Operator for node TAP78_6_B1 in the day ahead market for 2010-2011 [168] and the EES characteristics listed in Table 4.1. These were used by Byrne and Silva-Monroy and reported in [167], producing identical results. The following chapters of this thesis use this method to investigate arbitrage in future energy scenarios which was not carried out in [167].

Table 4.1 Storage characteristics used for arbitrage model verification

\begin{tabular}{lcc}
\hline Characteristic & Value & Units \\
\hline Storage capacity, $S_{\max }$ & 32 & MWh \\
Charging rate, $Q^{C}$ & 8 & MW \\
Discharging rate, $Q^{D}$ & 8 & MW \\
Conversion efficiency, $\eta_{c}$ & 80 & $\%$ \\
Storage efficiency, $\eta_{s}$ & 100 & $\%$ per half hour \\
\hline
\end{tabular}


The model was validated against three test scenarios with intuitive solutions for the optimum storage schedule. An artificial input price was used for each scenario with specific storage characteristics for each case and the scenario run for a duration of one week.

1. The test price was a square wave varying between $£ 100 / \mathrm{MWh}$ and $£ 200 / \mathrm{MWh}$ with a period of 24 hours. The storage capacity was $100 \mathrm{MWh}$ with $8 \mathrm{MW}$ charging and discharging rates and $100 \%$ storage and conversion efficiencies. The optimisation performed as expected; charging at its maximum rate for the first 12hours, when the price was low, followed by discharging at its maximum rate for the subsequent 12 hours, when the price was high. This pattern was repeated over the full week as shown in Figure 4.2.

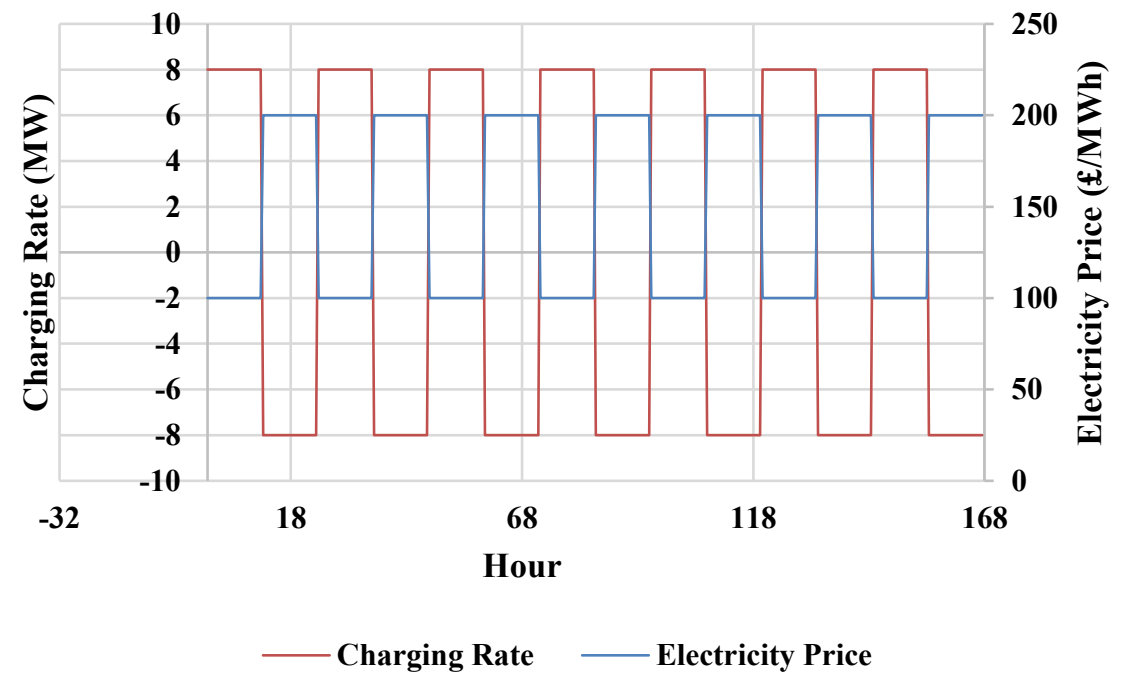

Figure 4.2 Optimisation validation - test case 1

2. The test price was the same as (1), but the storage efficiency was reduced from $100 \%$ to $90 \%$. This is equivalent to a self-discharge of $36 \%$ in 24 hours. As expected, the charging rate followed a similar square wave, but minimised the time over which energy was stored, as shown in Figure 4.3. 


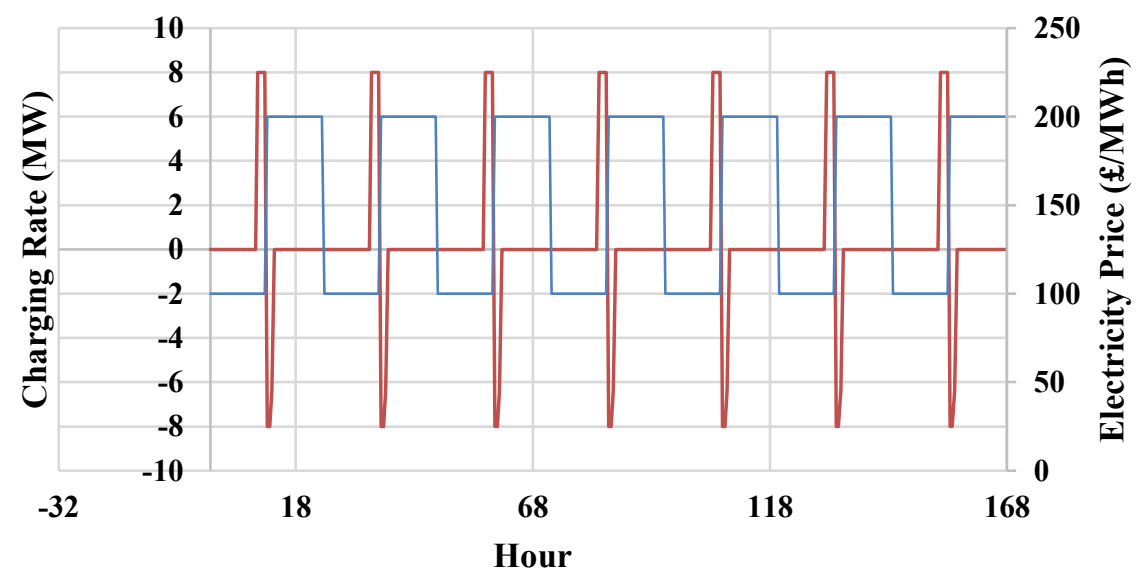

_Charging Rate _ Electricity Price

Figure 4.3 Optimisation validation - test case 2

3. The test price was a sine wave with a period of 24 hours varying from zero to $£ 100 / \mathrm{MWh}$. For this scenario, the conversion efficiency was set to $10 \%$ and the storage efficiency $100 \%$ with the power and capacity fixed as before. The results, shown in Figure 4.4, demonstrate that, as expected, the EES device only charges and discharges electricity when it can be bought for less than $10 \%$ of the selling price. The device charges for a longer duration than it discharges for as the storage efficiency is modelled during the charging cycle only i.e. it can only discharge at its rated power for $10 \%$ of the time compared to charging at its rated power.

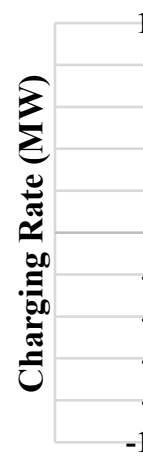

$-32$
10

8

8
6

6

4
$\mathbf{0}$
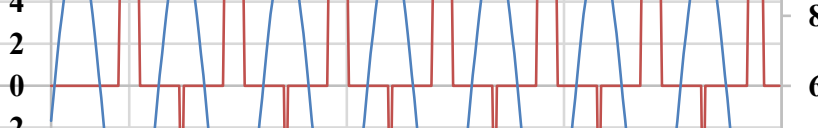

.

$-6$

$-10$

18

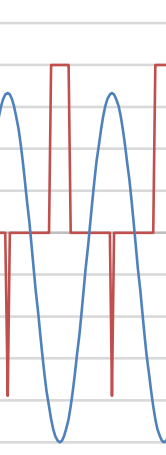

68

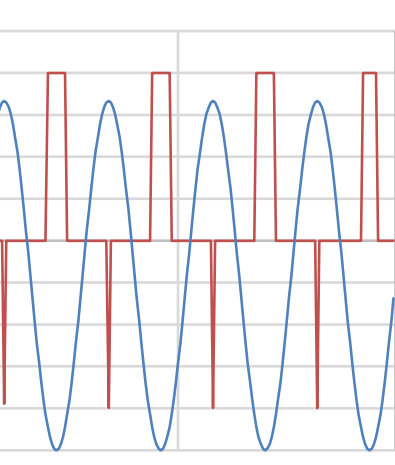

118
168

120

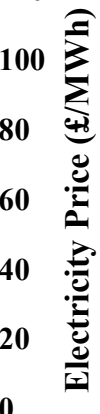

Hour

Electricity Price Charging Rate

Figure 4.4 Optimisation validation - test case 3

The test cases demonstrate the optimisation results in EES schedules which would be expected. 


\subsection{Preliminary Results}

\subsubsection{Performance in the UK Market}

The 2010 Market Index Data from the ELEXON Power Exchange [169] was used with the storage characteristics listed in Table 4.1 to investigate the performance of EES in the UK market. The annual revenue available through price arbitrage was $£ 181,415$. The optimum operating strategy for the device was to charge and discharge on a daily basis in line with the variations in electricity price, which were driven by daily demand behaviour. Figures 4.2 to 4.5 show the demand, electricity price, optimum charging schedule and state of charge of the device for the first week in January, which demonstrate these similar diurnal patterns. From Figure 4.7 and Figure 4.8 it can be seen that the EES device reached its maximum charging and discharging rate and utilised its full range of storage capacity almost every day.

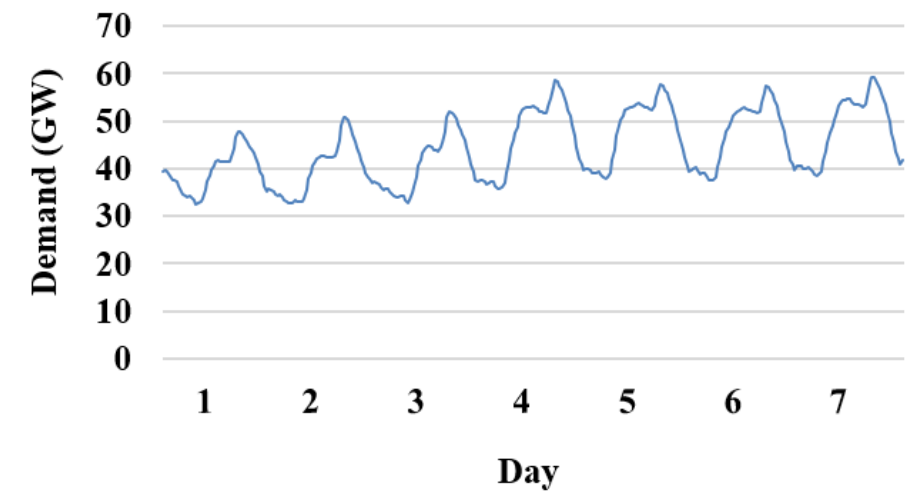

Figure 4.5 Electricity demand for first week in January 2010

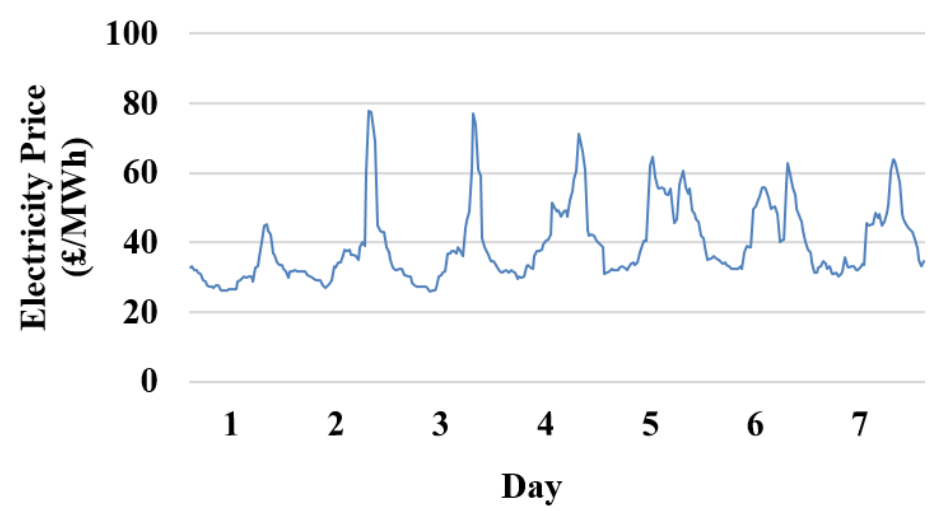

Figure 4.6 Spot market electricity prices for first week in January 2010 


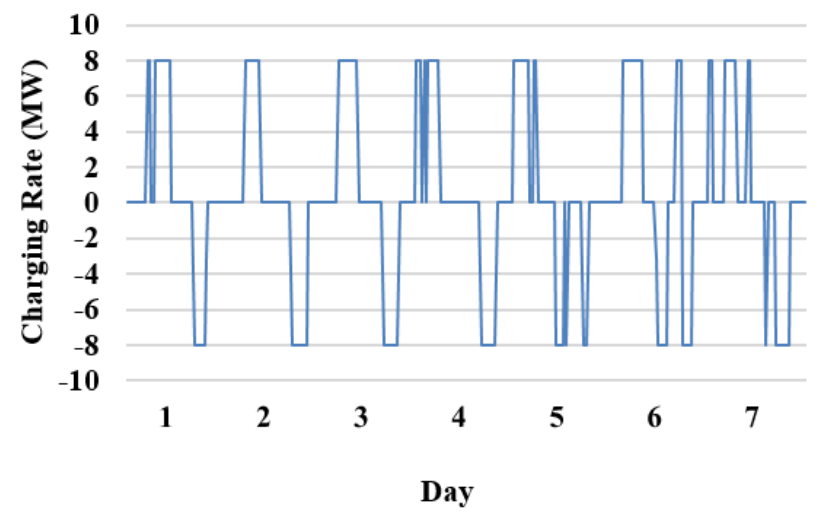

Figure 4.7 Optimum charging rate for first week in January 2010

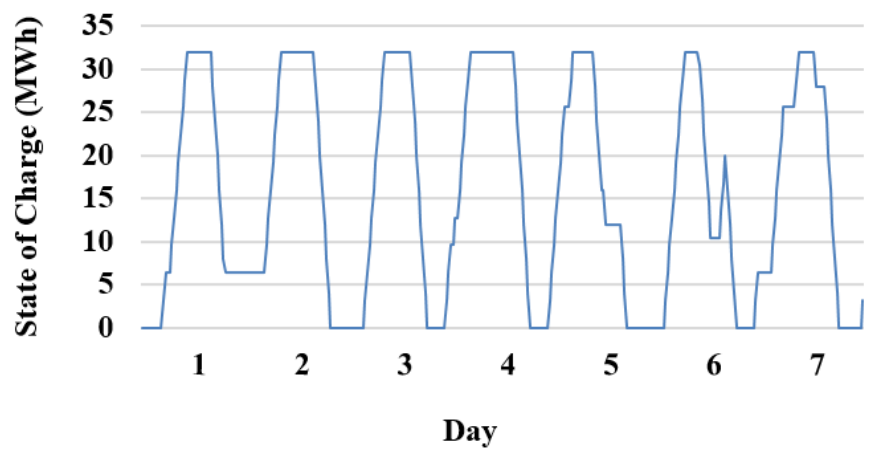

Figure 4.8 State of charge for first week in January 2010

A higher daily price spread would provide a larger difference between the price the EES operator paid to charge the device and the price at which electricity was sold during discharging, increasing the total revenue available. Figure 4.9 shows the total annual revenue gained in each month of the year and the maximum electricity price in that month. This shows that significantly higher revenue was achieved in January, November and December: the months with the highest maximum prices. Although the monthly maximum price is not a direct measure of daily price volatility, it gives an indication of individual price spikes which may have led to significantly higher revenue being achieved in a single cycle. Figure 4.10 shows the time series of electricity prices for the entire year with half hour resolution, which clearly displays the higher price spikes and volatility in the months where the largest revenue was achieved. 


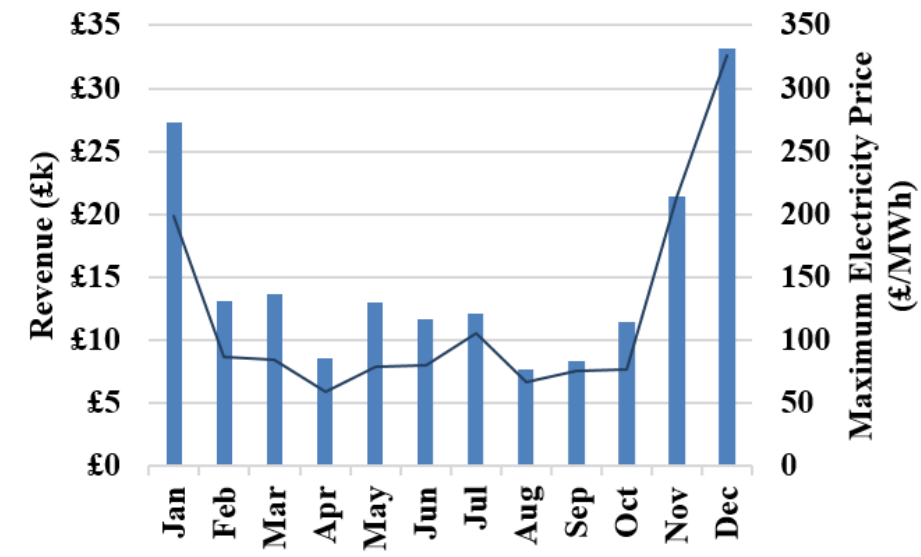

Revenue Maximum Electricity Price

Figure 4.9 Monthly revenue and maximum electricity price from 2010 UK market

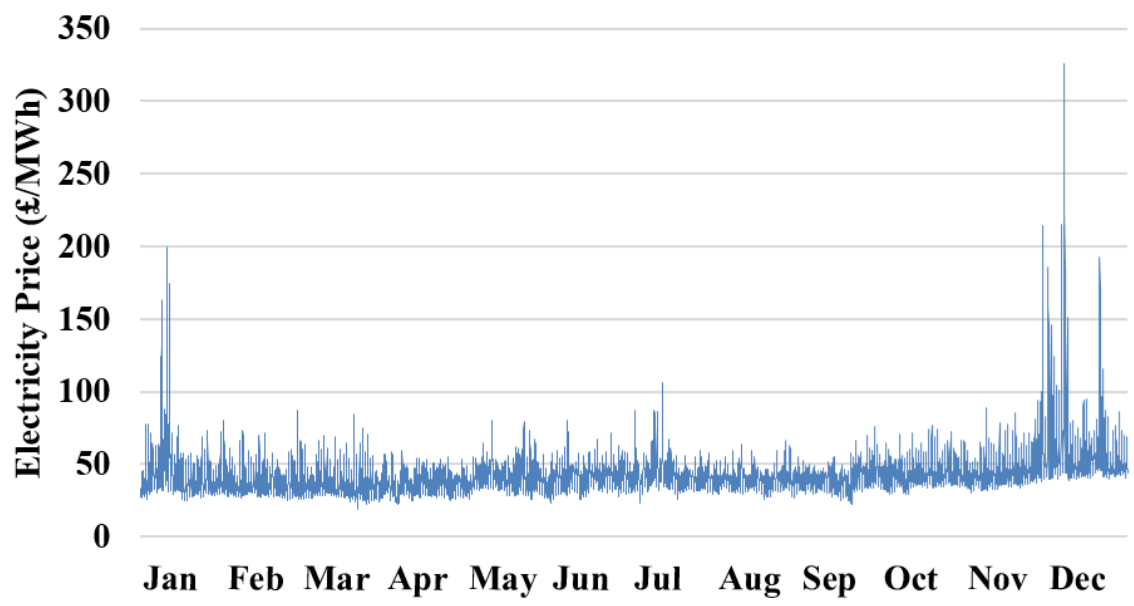

Figure 4.10 Half hour time series of electricity prices for 2010

Conversely, August, which returned the lowest revenue of all the months in the year, did not exhibit any substantial price spikes. Figure 4.11 and Figure 4.12 show the optimum charging strategy and state of charge for the device for the first week in August. Similarly to the first week in January, the device reached its maximum charging and discharging rates in each of its daily cycles; however, the full range of storage capacity was not utilised every day. This demonstrates that, in addition to making less profit from each unit of electricity cycled through the device, fewer units of electricity were bought and sold in response to the reduced price spread. 


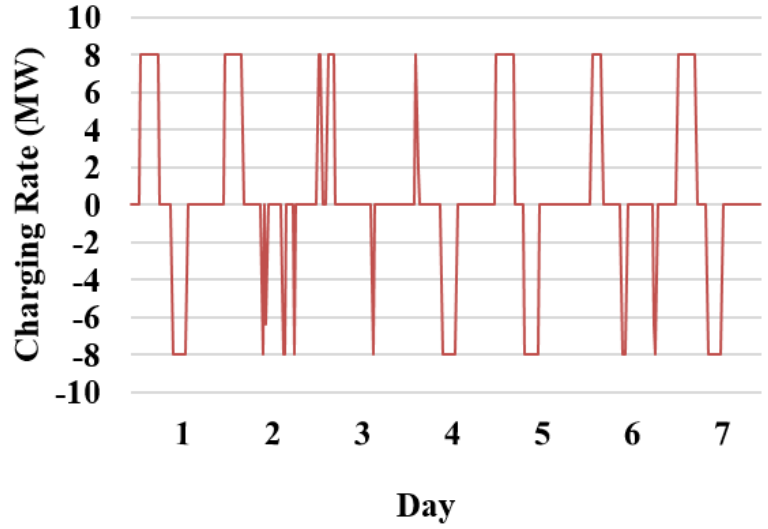

Figure 4.11 Optimum charging rate for first week in August 2010

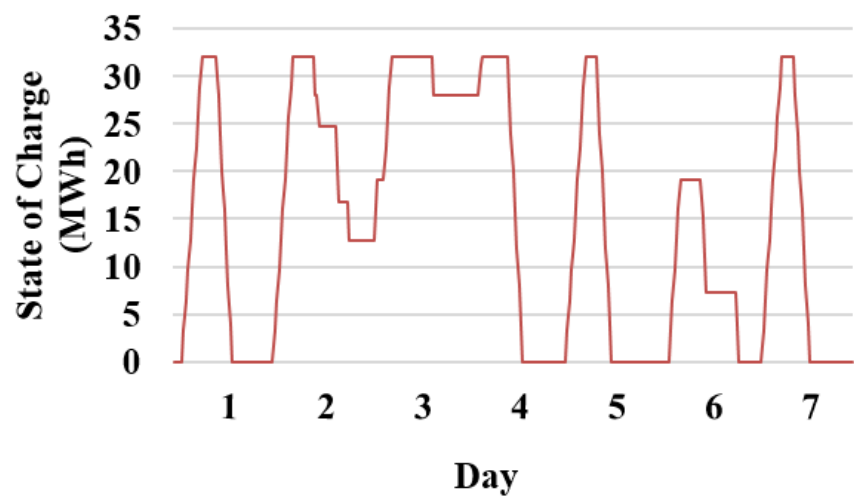

Figure 4.12 State of charge for first week in August 2010

Higher average electricity prices would also lead to reduced revenue as conversion losses would be more costly. For example, if 5MWh of electricity were purchased at a cost of $£ 10 / \mathrm{MWh}(5 M W h \times £ 10 / M W h=£ 50)$ and sold, after conversion losses of $20 \%$, at a price of $£ 40 / \mathrm{MWh}(4 M W h \times £ 40 / M W h=£ 160)$, the revenue would be $£ 110$. If the prices were all increased by $£ 10 / \mathrm{MWh}$, i.e. the electricity was bought at $£ 20 / \mathrm{MWh}(5 M W h \times £ 20 / M W h=$ $£ 100)$ and sold for $£ 50 / \mathrm{MWh}(4 M W h \times £ 50 / M W h=£ 200)$, the revenue would be $£ 100$, a reduction of $£ 10$. Both the mean price and the price range affect EES revenue in addition to the frequency of price peaks.

\subsubsection{Market Variation}

Comparing the revenue available in the UK to that in California highlights the dependence on the market structure and the local environment. The revenue available and the maximum 
electricity price for each month in the Californian market in 2010 is shown in Figure 4.13. Results are shown in US\$.

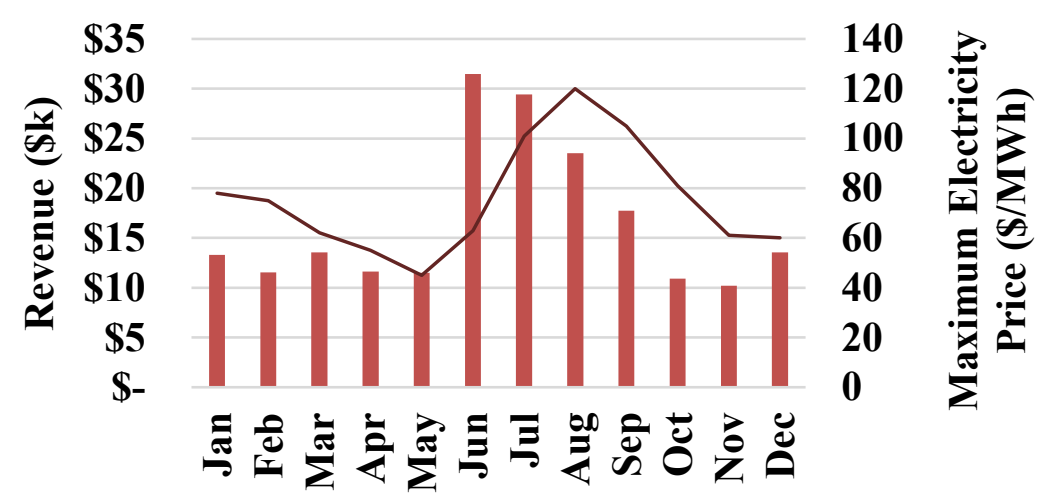

Revenue Maximum Electricity Price

Figure 4.13 Monthly revenue and maximum electricity price from 2010 California market

The total annual revenue was $\$ 198,331$ (approximately $£ 130,000$ at average 2010 exchange rates) which is $28 \%$ less than the revenue which could have been achieved in the UK market. Furthermore, the highest revenue was achieved in the summer months compared to the winter months in the UK. This reflects differences between the markets and the periods of highest demand which, in California, tend to be due to air conditioning loads in the summer [170]. Connolly et al [22] compare arbitrage value of a PHS plant in 13 different global markets and similarly highlighted the range of values which could be achieved. California was not investigated, but GB was shown to return an average profit compared to the range of markets investigated with Alberta, Canada performing best with over two times the return of GB and the Nordic region, the worst, performer returning less than $15 \%$ of GB. This demonstrates the dependence of value on regulatory frameworks and consumer behaviour. Connolly et al [22] state that a range of issues will affect the electricity price such as market structure, regulation, demand and plant portfolio, but do not attempt to analyse the implications of each of these on arbitrage revenue, which this thesis addresses in subsequent chapters.

Equally, revenue can vary within a market from one year to the next. Figure 4.14 shows the annual revenue available to the EES device from 2008-2013 based on historic Market Index Prices in the UK. There was a reduction in revenue of 52\% between 2008 and 2009 and an increase of 65\% between 2011 and 2012. 


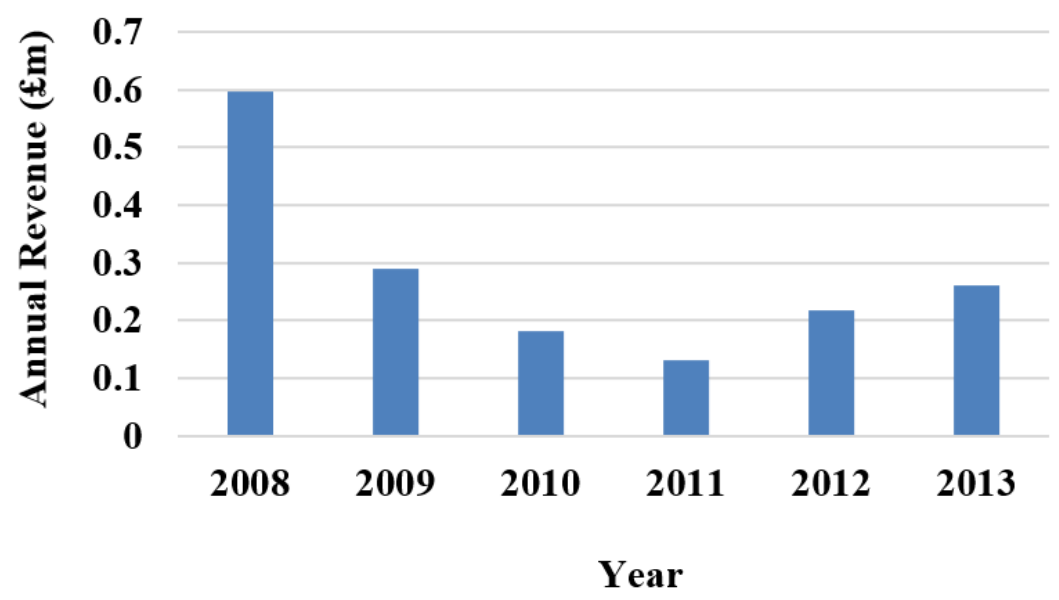

Figure 4.14 Variation in annual revenue from 2008-2013

Similar results were found by Connolly et al [22] and Barbour et al [120] with variations from year to year of 50\% and 75\% identified, respectively. Sioshansi et al [20] attributed variations in the PJM market to the changing generation mix and cost of fuel. These results further highlight the dependency of revenue on market conditions and level of risk associated with an investment in EES. None of the previous works attempt to quantify the impact of varying market factors on electricity prices from one year to the next and subsequently their impact on arbitrage revenue.

\subsubsection{Varying Storage Characteristics}

In addition to varying market conditions, the revenue available will vary depending on the storage system characteristics, which, to some extent, an investor may control. The cost associated with an increase in either storage capacity, charging or discharging rate is dependent on the specific technology, as discussed in Chapter 2. Figure 4.15 shows the change in revenue with increasing storage capacity using 2010 UK Market Index Prices. The remaining characteristics were kept constant at the values given in Table 4.1. 


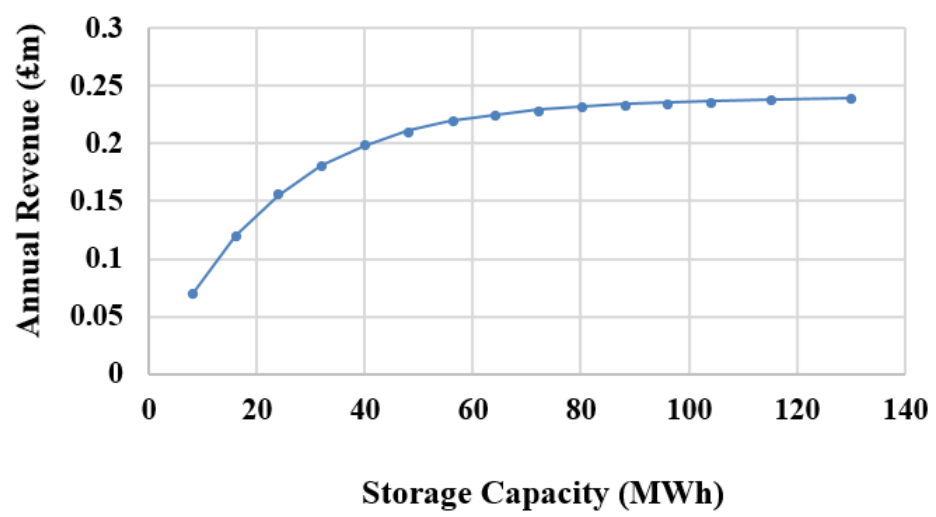

Figure 4.152010 annual revenue with varying storage capacity

The results show an increase in revenue at a diminishing rate as the storage capacity increased. Initially, as the storage capacity increased, the device stored more electricity during each cycle enabling it to displace more units and make more revenue. However, as the storage capacity continued to increase, the charging rate became the limiting constraint. The device was unable to charge and discharge quickly enough to make full use of the maximum storage capacity. Figure 4.16 shows the normalised state of charge over the same week with a high (80MWh) and low $(8 \mathrm{MWh})$ maximum storage capacity. For the low storage capacity, the device utilised the full range every day. For the high storage capacity, the device was only fully charged during two cycles throughout the week, with only around $50 \%$ of the capacity used on four of the days. Figure 4.17 shows the optimum charging schedule for both devices for the same week. It can be seen that the maximum charging and discharging rates were applied for longer durations for the device with the high storage capacity than with the low capacity where they were less restrictive.

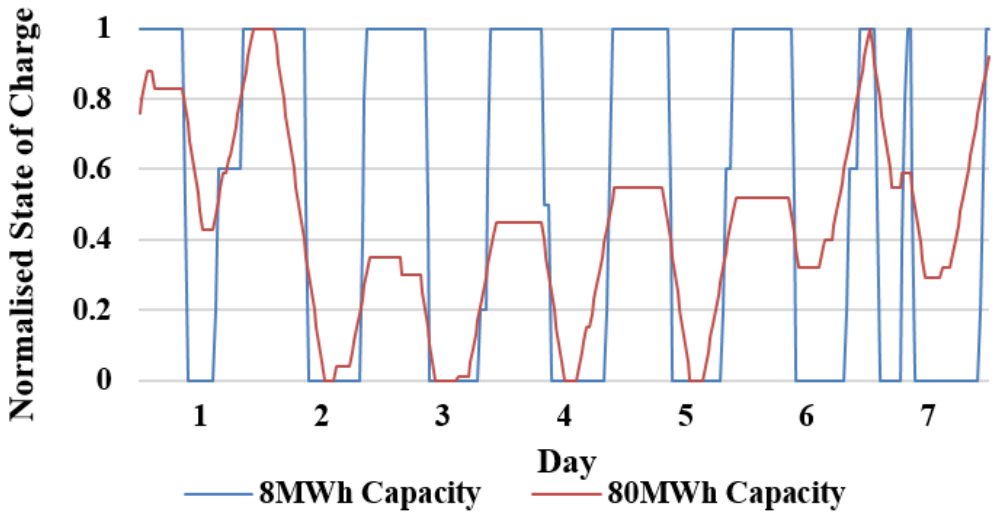

Figure 4.16 Normalised state for charge for one week with high and low storage capacity 


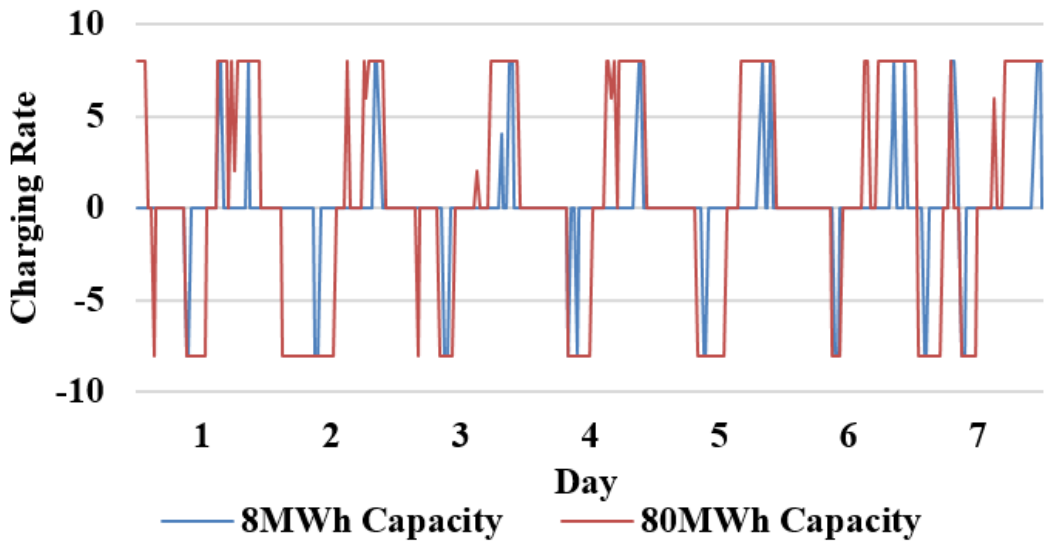

Figure 4.17 Optimum charging rate for one week with high and low storage capacity

Figure 4.18 shows the annual revenue for the same EES device with varying charging and discharging rates. The storage capacity was fixed at $32 \mathrm{MWh}$. Although the charging and discharging rates may be discrete processes in some EES devices, in many technologies they are coupled and so it was assumed that the maximum rates for both charging and discharging were the same. The results show a similar increase in revenue at a diminishing rate as the charging rate increased. At low charging rates, the storage capacity was less restrictive, so increasing the charging rate led to an increase in revenue; however, at higher charging rates, the storage capacity became more restrictive and increasing the charging rate further provided little additional benefit.

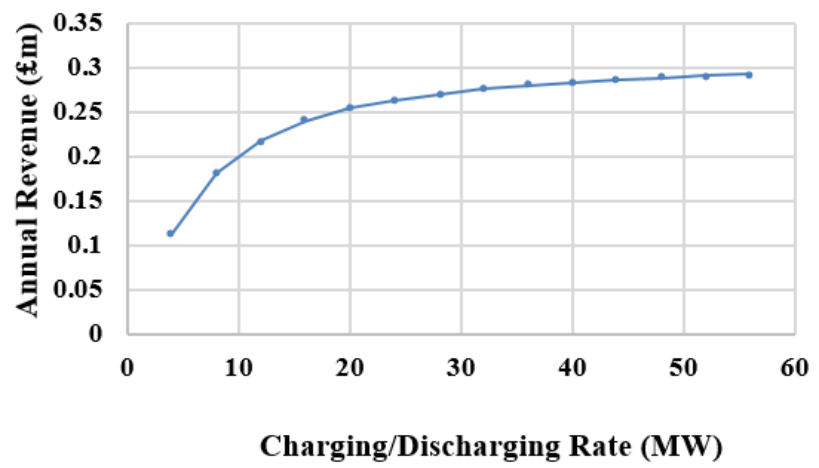

Figure 4.182010 annual revenue with varying charging and discharging rate

The results show that the revenue was dependent on both the storage capacity and the charging rate; however, these characteristics must be optimised together. Increasing one without the other would not continue to yield additional gains. Figure 4.19 shows a contour map of constant revenue for a wider range of both storage capacity and charging rate. If the per unit 
cost of an EES device were constant for energy storage and power delivery related costs, the optimum ratio of charging rate to storage capacity would be approximately $17 \mathrm{MW}: 100 \mathrm{MWh}$ as represented approximately by the dashed line on the graph. However, different technologies have different energy and power related costs, as discussed in Chapter 2, and so would have different optimal ratios of these characteristics.

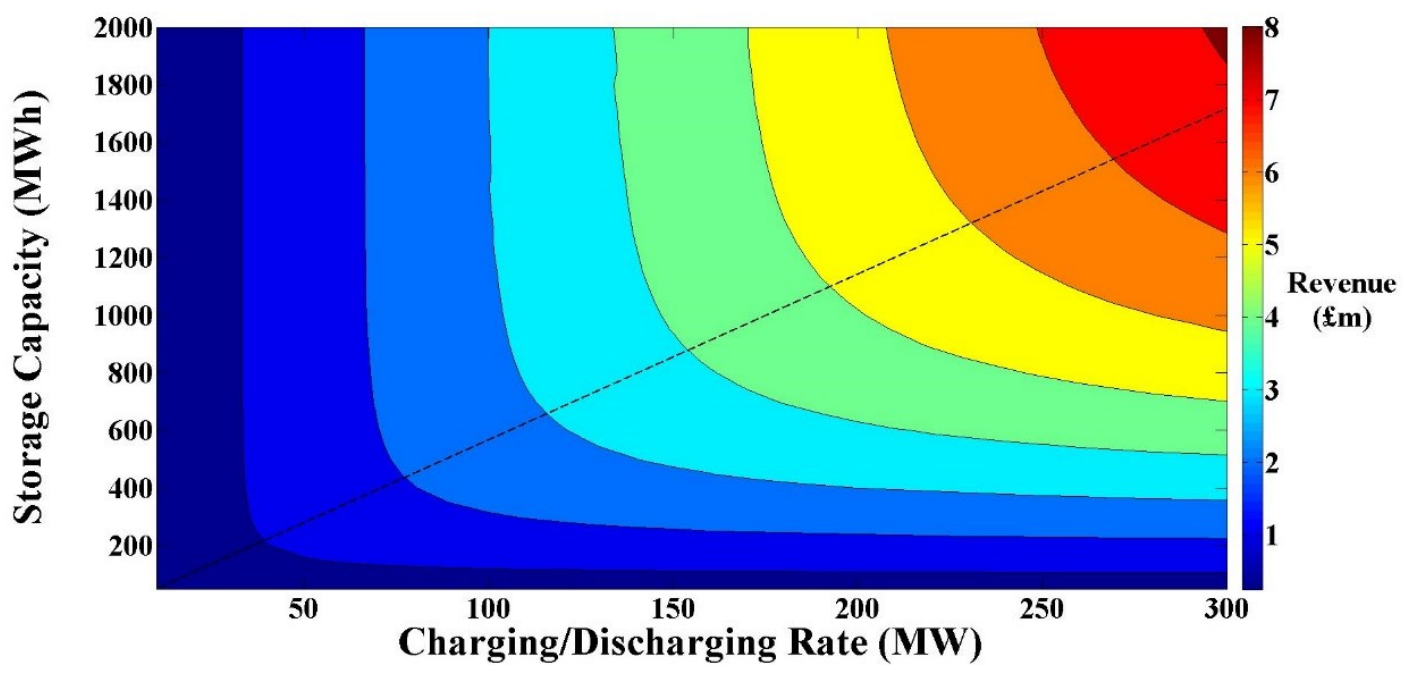

Figure 4.19 Contour map of 2010 annual revenue with varying capacity and charging rate, optimum ratio of charging rate to storage capacity shown by line

Figure 4.20 shows the change in revenue with varying conversion efficiency. As expected, higher conversion efficiencies led to higher annual revenue. With lower conversion efficiencies, more electricity was lost during each cycle and so lower net revenue was returned. Furthermore, the lower daily price spreads, which would have been profitable at higher efficiencies, became unprofitable and so the lower efficiency devices completed fewer charging and discharging cycles. This reduction in the number of cycles can be seen in Figure 4.21 which shows the optimum charging rate over the same period of 200 hours for a device with a conversion efficiency of 50\% compared to one with $100 \%$ conversion efficiency. The effect of reducing both the revenue achieved per cycle and the number of cycles leads to the exponential shape of the curve in Figure 4.20. As a result, marginal increases in conversion efficiency could lead to significant increases in revenue, even for the most efficient EES devices. 


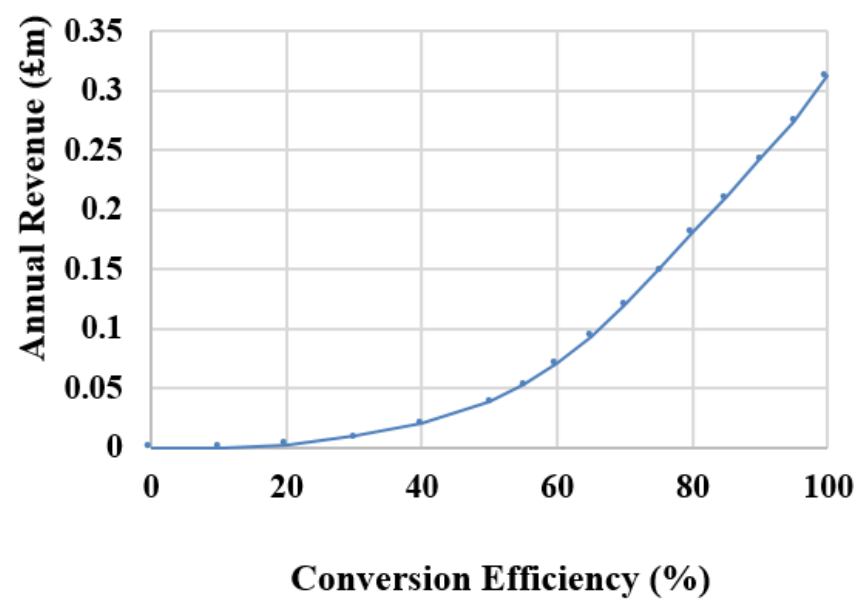

Figure 4.20 2010 annual revenue with varying conversion efficiency

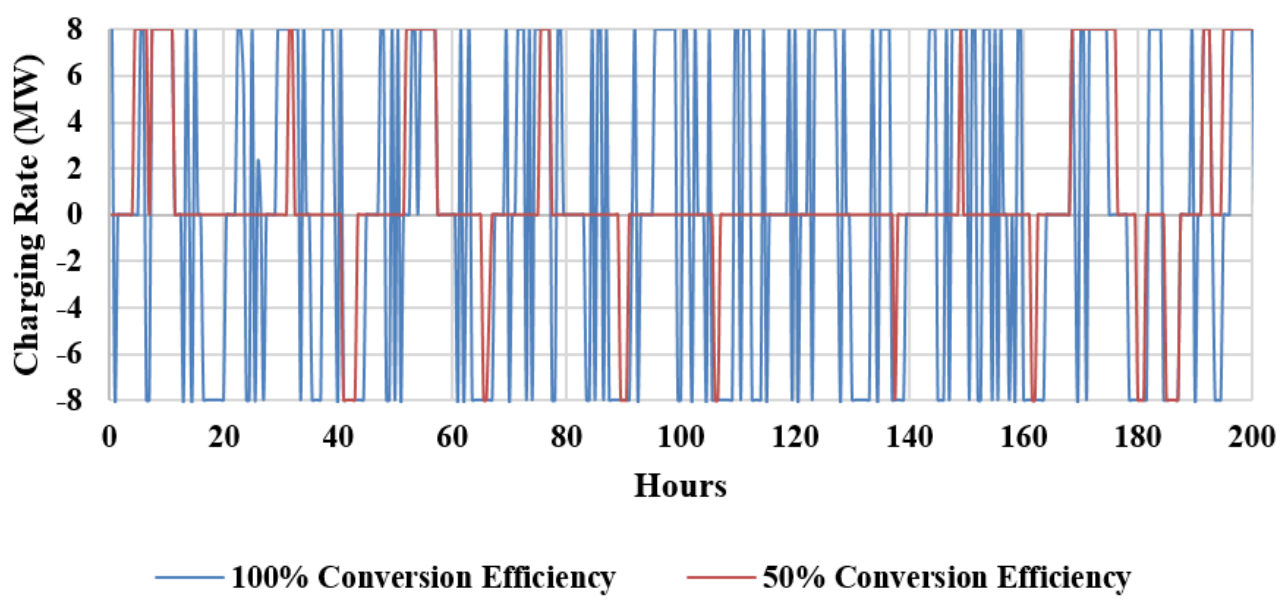

Figure 4.21 Optimum operating strategy for high and low conversion efficiencies

Figure 4.22 shows the change in annual revenue with increasing storage efficiency, defined as the energy sustained by the storage during a half hour time period. The results show, similarly to conversion efficiency, that marginal increases in storage efficiency provided significant gains, even at the highest efficiencies. The same results are shown as a function of selfdischarge per day in Figure 4.23. These results show that EES technologies with high conversion and storage efficiencies are important for arbitrage applications. 


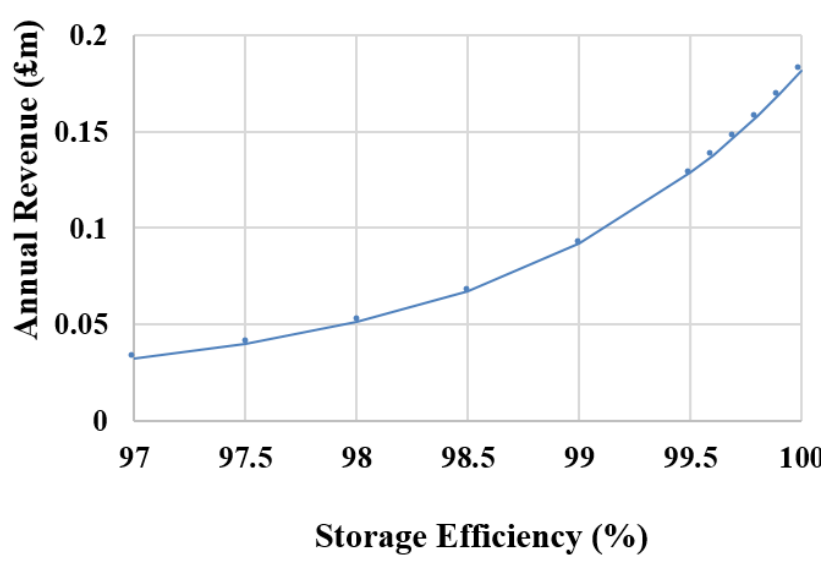

Figure 4.222010 annual revenue with varying storage efficiency

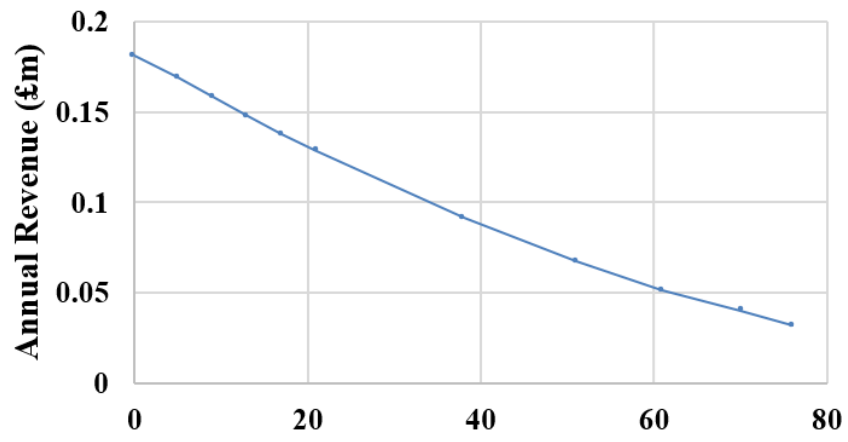

Self Discharge per Day (\%)

Figure 4.232010 annual revenue as a function of daily self-discharge

\subsection{Price Forecast Implications}

Spot market prices, used for this analysis, exhibit significant volatility making them difficult, if not impossible, to predict accurately; a prerequisite of the assumption of perfect foresight. In practice, operators will not have perfect foresight and so alternative approximate optimisation methods must be used with price forecasts. Various approaches have been taken to address this ranging from simple, deterministic methods using historic prices in place of actual forecasts, to more complex techniques implementing stochastic price forecasting methods. Grünewald [11] investigated the gain in revenue an operator could receive with foresight over an increasing time horizon. He showed that by increasing foresight from 1 hour to 4 hours, revenue improvements of up to $22 \%$ could be achieved; however, he maintained some degree of foresight for all his work. Historically, foresight beyond 12 hours was of no 
additional value, as storage devices typically followed a daily cycle in line with electricity prices.

\subsubsection{Deterministic Methods}

Deterministic methods optimise the revenue using projected electricity prices or apply a fixed operation strategy which has been optimised for preceding prices. Walawalkar et al [122] assumed a fixed daily storage cycle: devices were charged overnight and discharged during the same pre-defined peak hours each day. Sioshansi et al [20] determined an optimal charging strategy using prices from the preceding two week period and applied this to the current two week period; using this approach, approximately $85 \%$ of the optimal revenue was achieved.

Lund et al [121] and Connolly et al [22] compared different practical strategies - without foresight of electricity prices - to an optimal strategy - with perfect foresight - for CAES and PHS, respectively. With the practical strategies implemented, CAES could achieve $80-90 \%$ of its optimum revenue [121]. For PHS, the operator required "very accurate price predictions" to avoid a significant loss in profit. The accuracy required was not, however, quantified [22].

Using a similar approach, the four strategies outlined below were applied to investigate the impact on EES revenue.

1. For each day of the year, the optimum operating schedule from the previous day was applied.

2. For each week, the optimum operating schedule from the previous week was applied.

3. The same daily schedule was applied based on the optimum strategy for the average daily prices from the previous year.

4. The same weekly schedule was applied based on the optimum strategy for the average weekly prices from the previous year.

The optimal revenue was calculated assuming perfect foresight and then applying each of the strategies above for 2006-2010. The average percentage of the optimum annual revenue achieved for each strategy is shown in Figure 4.24. 


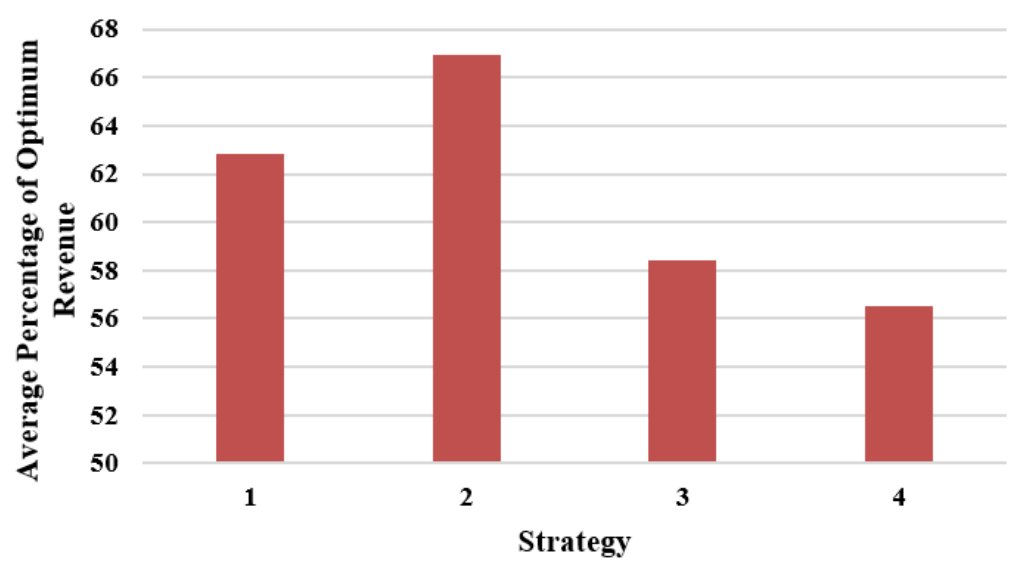

Figure 4.24 Performance of practical operating strategies

The results show that optimising using recent historic data (strategy 1 and 2) achieved better results than optimising using average annual data (strategy 3 and 4). This indicates that there was likely to be seasonal variation in the optimum operating schedule. Optimising using the previous week's prices also provided better results that optimising using the previous day. This suggests that there was also variation within the week. Strategy 2 provided the best results, but still only achieved $67 \%$ of the revenue available with perfect foresight. This is less than the $80-90 \%$ identified by other authors [20], [122], [121]; however, these studies investigated various US markets which exhibit different price profiles.

A deterministic approach to forecasting is simplistic and computationally fast to implement. Mokrian and Stephen [171] commented that it is crude and performs poorly compared to alternative forecasting techniques. They demonstrated stochastic models implementing price forecasts with correlated random variables with Gaussian noise or geometric Brownian motion as alternatives. However, deterministic methods can be useful to provide an indication of the lower bound on the revenue which could be achieved through arbitrage. More sophisticated strategies, which any storage operator would implement in reality, would enable higher revenue to be gained. The assumption of perfect foresight and application of a deterministic approach provide an envelope within which the actual storage revenue would lie.

\subsubsection{Dynamic Programming}

An alternative method to applying a deterministic forecast to a linear optimisation programme is to use dynamic programming (DP) which can be used with stochastic price forecasts such as those mentioned above. The work carried out relating to DP was completed in collaboration with Francesca Tagliaferri, another PhD student, and presented in [164]. Francesca Tagliaferri 
wrote the DP algorithm; however, formulation of the problem and analysis of the results were carried out independently.

DP was formalised by Bertsekas [172] and has been used for a variety of applications [173]. In DP, a problem is defined by its state at each time period. The set of states for the entire problem is called the state space. At each period a number of decisions, or actions, could be taken leading to a number of subsequent states. Each action incurs a cost and/or a reward as shown in Figure 4.25. The sequence of actions taken at each time step forms the policy [174].

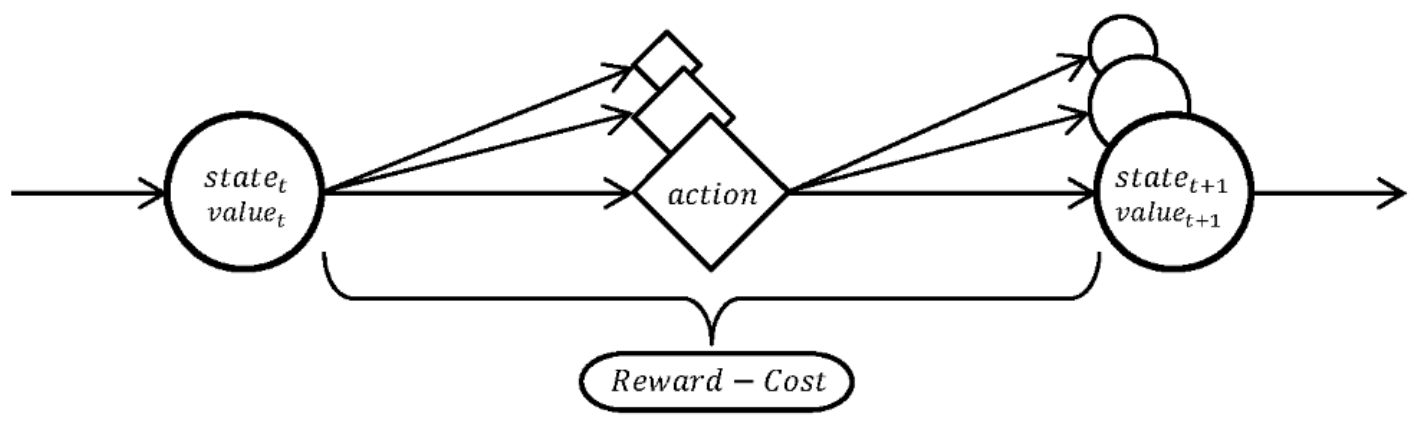

Figure 4.25 Depiction of dynamic programming decision process (source [174])

DP discretizes a problem's state space and uses backwards recession to derive the policy which results in the maximum global reward [175]. For EES, the state space is described by two dimensions; the electricity price and the state of charge of the device. The state of charge is discretized, for example, into $1 \mathrm{kWh}$ steps. The action space would be the decision to charge or discharge, ranging from the maximum amount of electricity which could be discharged per time step to the maximum amount of electricity which could be charged, represented by a discrete series of steps [171]. DP models expand rapidly with an increasing number of states and so algorithms are typically limited to problems with less than three or four state variables. This rapid expansion is known as the 'curse of dimensionality' [171]. The computational intensity also limits the solvers to Markovian processes by which the probability of the next state can be described entirely by the current state i.e. no history of state information is needed [175]. This is acceptable for the state of charge; however, it restricts the price models which could be applied, the most accurate of which model prices as a continuous process [171]

Price forecasting is a broad and complex field of research in its own right and it is out with the scope of this thesis to compare forecasting techniques. Electricity price processes are characterised by high volatility, large spikes, reversion to a daily pattern and seasonality [176]. Forecast accuracies depend on the electricity market being examined as well as the forecasting method being used. Instead, the impact of price forecast accuracy on revenue was considered. 
DP was applied to the optimisation problem shown in Figure 4.1. Instead of perfect foresight of future electricity prices, a forecast was assumed with an associated error. The objective of the DP algorithm was to generate a policy $\left\{q_{t}^{D}-q_{t}^{C}\right\}$, of optimal decisions to charge or discharge. The objective function is defined by Equation (4.9) and has the same constraints as the linear programme defined in Equations (4.7) and (4.8).

Minimise

$$
\epsilon\left(R^{*}\right)=\epsilon\left[\sum P_{t}\left(q_{t}^{D}-q_{t}^{C}\right)\right]
$$

$\epsilon$ represents the expected revenue with respect to the probability distribution of the electricity prices. The price forecasts were artificially generated by adding a random variable with uniform distribution over an interval $[-s, s]$ to the actual prices, where $s$ was the maximum error of the forecast. The average error of the forecast was zero and the absolute average error half the maximum error, $s$.

The annual revenue was calculated using 2013 electricity prices. Real storage scenarios were investigated to determine the impact of price forecast accuracy on existing projects. Characteristics for a lithium ion battery were used based on the battery system demonstrated as part of the UK Power Networks Smarter Network Storage Project [177]. This is a small scale storage device connected to the distribution network. Results were compared to a large scale PHS device based on the characteristics of Dinorwig [178]. The EES system characteristics are listed in Table 4.2.

The DP was run using randomly generated forecasts with maximum errors of $1 \%, 2 \%, 5 \%$, $10 \%, 20 \%, 30 \%, 40 \%$ and $50 \%$. Ten simulations were run for each level of forecast error. The maximum percentage difference in revenue between simulations for the same error was $3 \%$.

Table 4.2 Characteristics of UK Power Network's lithium ion battery and Dinorwig PHS

\begin{tabular}{lccc}
\hline Characteristic & $\begin{array}{c}\text { Lithium Ion } \\
\text { Battery }\end{array}$ & PHS & Unit \\
\hline Storage capacity, $S_{\max }$ & 10 & 10100 & MWh \\
Charging rate, $Q^{C}$ & 6 & 1728 & MW \\
Discharging rate, $Q^{D}$ & 6 & 1728 & MW \\
Conversion efficiency, $\eta_{c}$ & 65 & 75 & $\%$ \\
Storage efficiency, $\eta_{s}$ & 99.5 & 100 & $\%$ per half hour \\
\hline
\end{tabular}

The results, shown in Figure 4.26, show that the revenue reduced at an increasing rate with increasing forecast error for both the lithium ion battery and the PHS. For the lithium ion 
battery, with perfect foresight, the optimum revenue which could be achieved was $£ 47,248$. With DP, as the maximum error was increased from zero to $50 \%$, the revenue reduced to $63.6 \%$ of the optimum. For the large PHS the optimum revenue which could be achieved was $£ 50 \mathrm{~m}$. The optimality was slightly more sensitive to forecast accuracy than the smaller scale battery. As the maximum error was increased from zero to $50 \%$, the revenue reduced to $56 \%$ of the optimum.

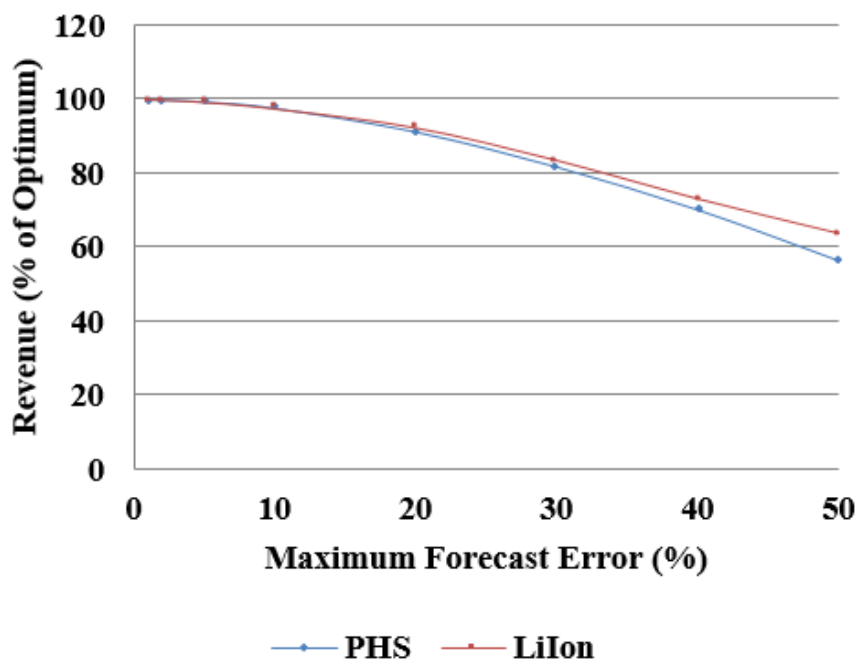

Figure 4.26 Loss of revenue with increasing price forecast error

For both technologies, over $95 \%$ of the optimum revenue was attained with a maximum forecast error of 10\%. Hu and Taylor [179] implied that this level of accuracy of forecast could be readily realised in the short-term British electricity market. As the reduction is relatively small the assumption of perfect foresight appears to be a not unreasonable simplification, and is used henceforth.

\subsection{Diminishing Marginal Returns}

The assumption that EES devices are price takers is reasonable for single devices and valid for small capacities which could be deployed in the short to medium term. However, EES creates extra demand when electricity selling prices are low, forcing the price up. It provides additional supply when prices are high depressing the selling price. If network capacities of EES increase significantly, a smoothing effect of electricity price differentials, upon which arbitrage depends, would result, and operators' income would fall. This diminishing marginal value of storage, termed 'self-cannibalisation' [180], depresses the commercial value of EES. The effect is more pronounced than standard supply and demand markets which exhibit diminishing marginal returns. 
Figure 4.27 and Figure 4.28 demonstrate the effect of self-cannibalisation, showing the range of daily prices over the year with a small amount of EES $(2 \mathrm{GW}, 8 \mathrm{GWh})$ and a large amount $(10 \mathrm{GW}, 40 \mathrm{GWh})$, respectively. With a large amount of storage the price spikes, which previously occurred on $25 \%$ of the days, are eliminated and the median price range is almost a flat line demonstrating the reduced opportunities for arbitrage.

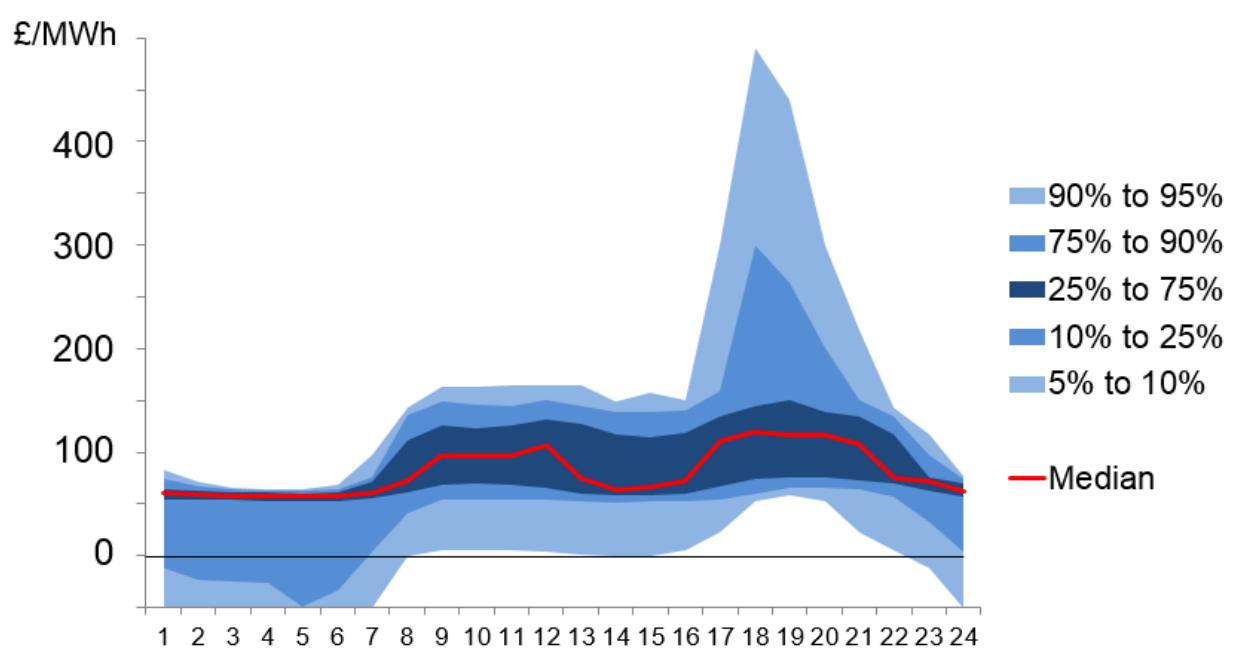

Figure 4.27 Price variation over 24 hours with 2GW, 8GWh of storage (source [181])

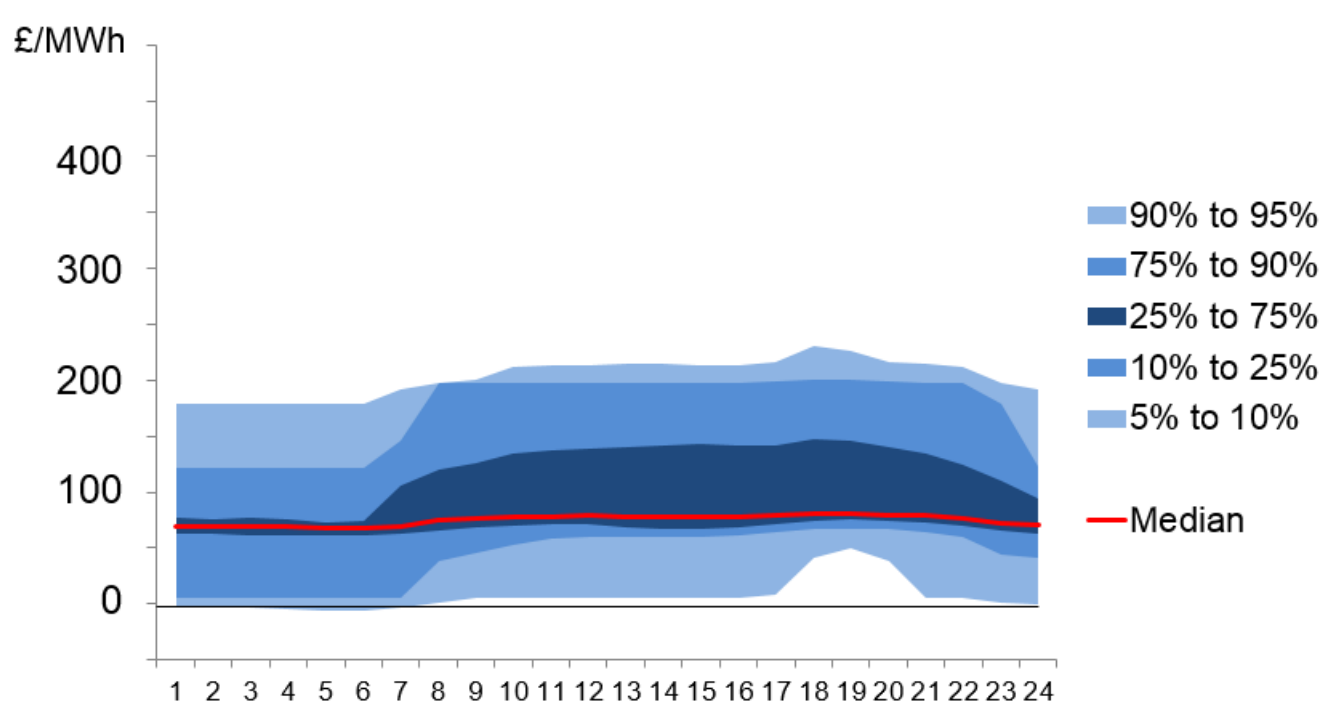

Figure 4.28 Price variation over 24 hours with 10GW, 40GWh (source [181])

In addition to recent work by Green [181], discussed above, Sioshansi [182] is one of the few authors to address the assumption of storage as a price taker. He showed that while selfcannibalisation reduced the commercial value of EES, significant external welfare gains were achieved through price smoothing. A simple economic model demonstrated that producer 
profit was reduced while consumer surplus was more significantly increased resulting in a transfer of wealth from producers to consumers and a net global welfare gain. The impact of different ownership models on achieving the welfare gain was also investigated. A joint ownership model of EES, with merchant traders, consumers and generators owning a share of the total storage capacity was shown to enable the optimum operational strategy with maximum global benefits. With a single type of owner, a merchant operator would come closest to the optimum, despite sensitivity to a reduction in the electricity price spread.

A price elastic model could be used to adjust electricity prices to modest levels of EES; however, this thesis focusses on early stage EES technology development, where MW rather than GW of capacity are likely to be installed. For these amounts of storage, the assumption that it is a price taker is expected to be valid.

\subsection{Technology Comparison}

Section 4.3.3 demonstrated that arbitrage revenue would vary depending on the EES characteristics. Different technologies exhibit different combinations of characteristics and as such, revenue will vary between them. A selection of representative EES technologies is shown in Table 4.3. These show feasible characteristics for each technology with a full range listed in Table 2.1 in Chapter 2.

The revenue available to each EES technology was investigated using 2008 electricity prices. Figure 4.14 shows that 2008 was the most profitable year for EES with over double the revenue achieved compared to any other year investigated. This year was selected to give the best case scenario for annual revenue. The results are shown in Figure 4.29. 
Table 4.3 Sample storage technology characteristics

\begin{tabular}{|c|c|c|c|c|c|c|}
\hline Technology & $\begin{array}{c}\text { Life } \\
\text { (Years) }\end{array}$ & $\begin{array}{c}\text { Storage } \\
\text { Capacity } \\
\text { (MWh) }\end{array}$ & $\begin{array}{c}\text { Maximum } \\
\text { Charging } \\
\text { Rate (MW) }\end{array}$ & $\begin{array}{c}\text { Maximum } \\
\text { Discharging } \\
\text { Rate (MW) }\end{array}$ & $\begin{array}{c}\text { Conversion } \\
\text { Efficiency } \\
(\%)\end{array}$ & $\begin{array}{c}\text { Storage } \\
\text { Efficiency } \\
\text { (\%/half hour) }\end{array}$ \\
\hline Pumped-hydroelectric Energy Storage (PHS) & 60 & 2000 & 200 & 200 & 80 & 100 \\
\hline Compressed Air Energy Storage (CAES) & 30 & 1500 & 150 & 150 & 59 & 100 \\
\hline Flywheel Energy Storage (FES) & 15 & 0.05 & 0.25 & 0.25 & 87 & 82.54 \\
\hline Batteries & 15 & 5 & 5 & 5 & 80 & 99.54 \\
\hline Superconducting Magnetic Energy Storage (SMES) & 20 & 0.01 & 5 & 5 & 90 & 99.66 \\
\hline Double Layer Capacitor (DLC) & 10 & 0.1 & 0.3 & 0.3 & 85 & 99.26 \\
\hline Cryogenic Energy Storage (CES) & 30 & 10 & 10 & 10 & 75 & 99.98 \\
\hline Pumped Heat & 15 & 50 & 5 & 5 & 80 & 99.98 \\
\hline
\end{tabular}




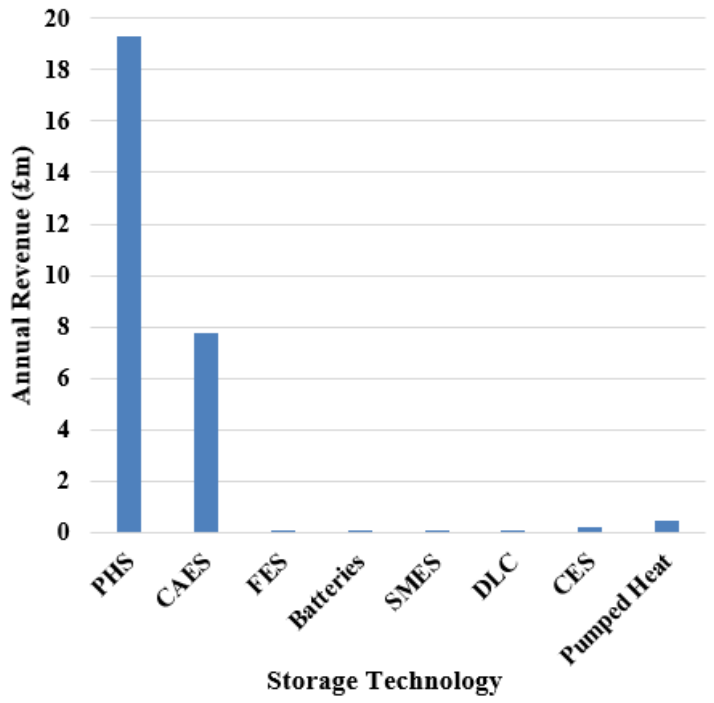

Figure 4.29 Annual revenue for a range of storage technologies from 2008

The results show that PHS and CAES returned significantly higher revenue than any other technology. However, these are represented by significantly larger EES devices, in both storage capacity and power rating, which would cost significantly more. Multiple units of the smaller EES technologies could be deployed to reach the size of the larger PHS or CAES devices which may lead to an equivalent revenue. Figure 4.30 shows the annual revenue per unit capacity and per unit charging rate for each of the technologies.

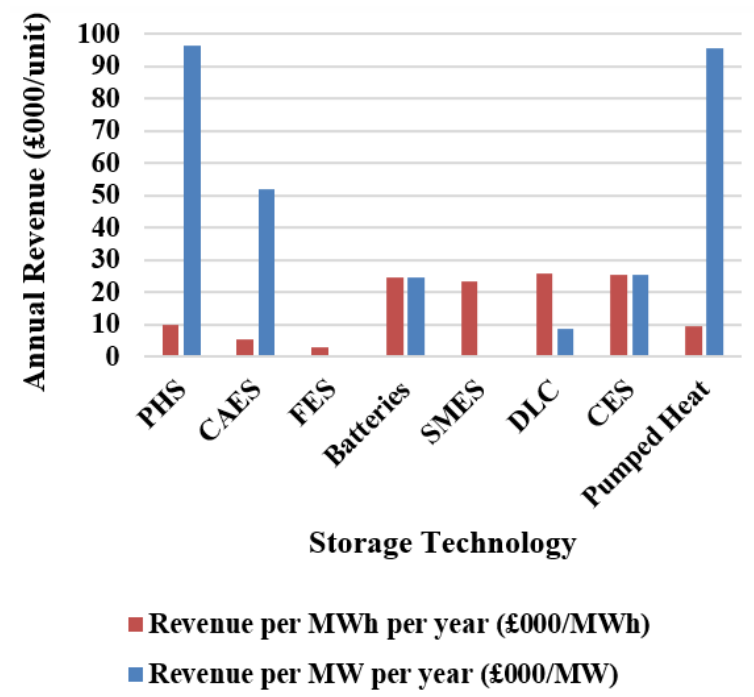

Figure 4.30 Annual revenue per unit capacity and per unit charging rate for a range of storage technologies from 2008 
This shows that pumped heat is competitive with PHS on a revenue per MW basis and that there is much less variation in performance per MWh with PHS and CAES among the technologies with the worst return. Capacity and power rating also effect costs of EES technologies and so revenue per MW or per MWh cannot be used to compare commercial value; the total system costs must also be considered to provide a commercial evaluation of a project. Generally, an internal rate of return is used as a measure of the economic case for a project with a threshold percentage considered a 'good investment'. This is not practical here as many of the technologies will not make a positive return from arbitrage and so an internal rate of return cannot be calculated. Instead, the Net Present Value (NPV) is used.

NPV is a measure of the value of an investment based on the capital spent compared to the discounted revenue ${ }^{5}$ over the lifetime of the project [183]. It provides a measure of the rate of return on a project. The NPV is calculated using Equation (4.10)

$$
N P V=\sum_{T=0}^{N} \frac{C_{T}}{(1+r)^{T}}
$$

where $T$ is the year, $N$ is the lifetime of the project in years, $C_{T}$ is the cash flow in year $T$ and $r$ is the discount rate. To calculate the NPV of the EES technologies listed in Table 4.3 it was assumed that the project was built and capital released instantaneously in year zero followed by the first year revenue being achieved at the end of year one. Following this, revenue was accrued annually at the end of each year. This is unlikely to be accurate for capital intensive civil projects, such as PHS, which can take over a decade to build [184]. The cost of capital should also be taken into account to calculate the true NPV of specific projects; however, for a high level comparison these simplifications were considered acceptable. Although the revenue would vary each year, for this preliminary assessment, it was assumed that the revenue was fixed based on the annual revenue from 2008. The capital costs were taken as the mean values for each technology from the range listed in Table 2.2 in Chapter 2. A discount rate of $10 \%$ was applied. The assumptions are summarised in Table 4.4 and the results shown in Figure 4.31 .

${ }^{5}$ Discounted revenue applies a reduction to future income to take into account the preference of investors to have cash immediately rather than having to wait for it, known as the time value of money. 
Table 4.4 NPV Assumptions

\begin{tabular}{lc}
\hline \multicolumn{1}{c}{ Assumption } & Value \\
\hline Capital Spend & Lump sum at commissioning \\
Life & Technology specific \\
Discount Rate & $10 \%$ \\
Period revenue accrued & Annual \\
Annual revenue & Based on 2008 arbitrage revenue \\
\hline
\end{tabular}

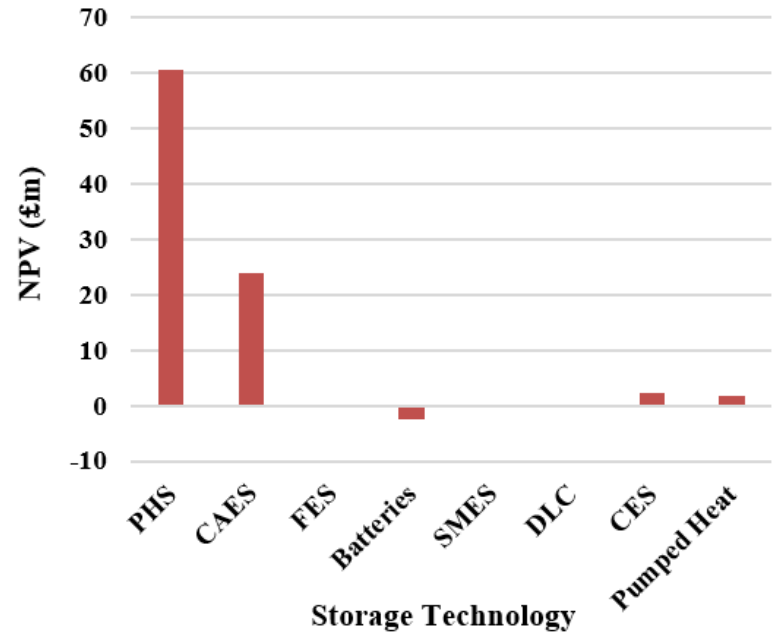

Figure 4.31 NPV for a range of storage technologies based on annual revenue from 2008

The results show that the only technologies which returned a positive NPV were PHS, CAES, CES and pumped heat. PHS and CAES are the more mature technologies which have, to some extent, already come down their cost curves, returning the highest NPV. The capital cost of CES and pumped heat are expected to reduce over the coming decades as more of these projects are deployed, which would lead to a higher NPV for these technologies. Although the result for CAES is slightly misleading, as the cost of fuel used during the discharging process is not accounted for in the model, these are the four technologies which are expected to contribute to energy management in the electricity system which would be rewarded through arbitrage. The other devices would be more useful for power quality applications, as discussed in Chapter 3, which would gain revenue through dedicated ancillary service contracts.

Carrying out the same analysis using electricity prices from any other year, which was not as profitable for EES, did not return a positive NPV for any technology. This further highlights the risk associated with revenue which is dependent on an unpredictable electricity market. 
Investors tend to be risk averse and favour early returns, which is partially accounted for in the discount rate applied in the calculation of the NPV. However, for large infrastructure projects in the electricity sector, some analysts disregard revenue from later years entirely to represent the high risk associated with changes in the market [185]. This penalises devices with a longer lifetime but better reflects an investor's preference for quick returns. Eager et al [185] used income available only for the first 15 years of lifetime to calculate the NPV. Applying that approach to EES technologies reduced the value of PHS and CAES most significantly, but they still remained the most economically favourable, followed by CES and pumped heat as shown in Figure 4.32.

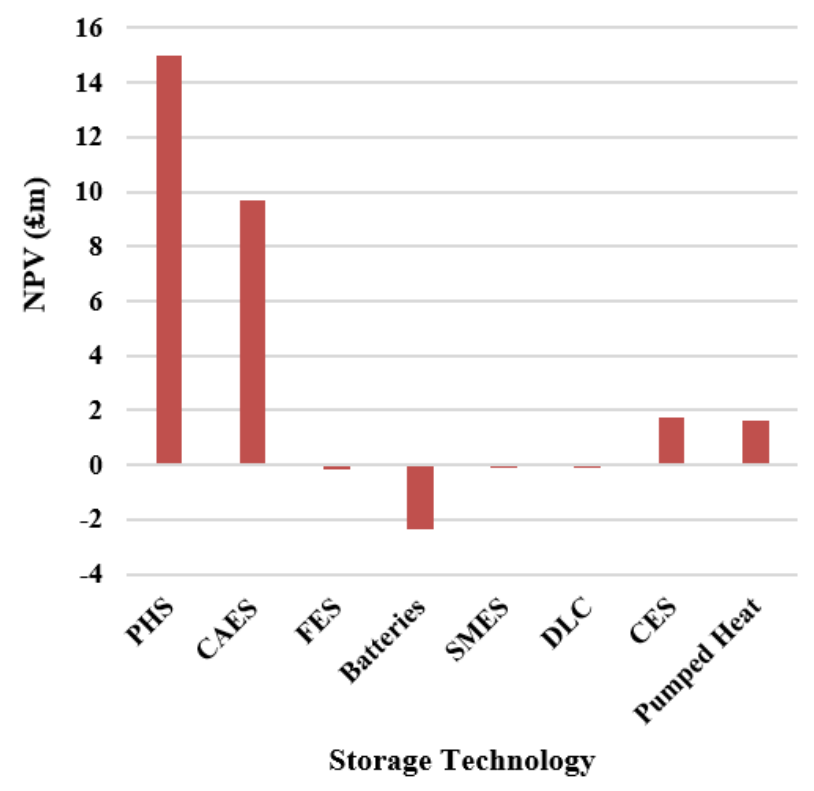

Figure 4.32 NPV for a range of storage technologies with 15 year income based on annual revenue from 2008

\subsection{Evaluation of Risk}

To fully reflect the level of risk in a project, it is useful to carry out a risk analysis which takes into account the range of risks associated with an investment. Some of the commercial risks facing EES are mentioned above, such as the change in revenue from one year to the next and the dependence on market structures. Novel technologies also carry higher technical risks including uncertainty surrounding cost and performance. Evidence shows that uncertainty and risk delay investment decisions [186] and that a low return on a low risk investment may be more favourable than a higher expected return at a higher risk [187]. Risks can be categorised as technical or commercial, which largely relate to the specific project and market conditions, respectively. The potential risks facing EES technologies are listed in Table 4.5. 
Table 4.5 Risk categories faced by storage technology

\begin{tabular}{cc}
\hline Technical Risks & Commercial Risks \\
\hline Construction costs & Fuel costs \\
Operational costs & Demand growth \\
Lead time & Emissions costs \\
Availability & Degree of competition \\
Performance & Cost of capital \\
Decommissioning costs & Credit risk \\
& Regulatory changes \\
& Planning process \\
\hline
\end{tabular}

Commercial risks are largely associated with changing market structures and future energy costs, which an EES developer can have little control over. These are discussed in the following chapter. This section examines the impact of technical risks on a project's commercial viability using realised historic electricity prices. Various approaches can be used to assess the risk. These are discussed below.

\subsubsection{Methods of Risk Analysis}

\section{Qualitative Assessment}

In qualitative risk assessments risks are identified and rated for their impact and probability of occurrence. Although described as qualitative, these are allocated a number on a sliding scale (e.g. 1-5). The two scores are multiplied to give a resulting risk rating [188]. This enables risks to be ranked in order of importance to identify where project resources should be focussed. Although this method is common practice in project management [188] it is crude and relies largely on engineering insight to identify and quantify the impact and probability of risks.

\section{Sensitivity Analysis}

Sensitivity analysis is applied to models and calculates an output, such as revenue, based on the expected value of variable inputs, such as capital costs. The responsiveness of the result to a change in value of a specific variable is then measured [189]. Changes are generally applied uniformly across all variables without consideration of how likely the changes are to occur. The approach is also limited in its ability to assess the impact of changing multiple variables together where input values are not mutually exclusive. Stress testing can be applied to 
sensitivity analysis where an extreme value of a chosen variable is applied to investigate its impact [189].

\section{Scenario Analysis}

Scenario analysis provides an assessment of the impact of changing multiple dependent variables where various possible combinations of inputs are investigated [189]. Stress tests can also be applied to scenario analysis where extreme scenarios are created. Similarly to sensitivity analysis, the probability of each scenario is not normally considered.

\section{Monte Carlo Method}

The Monte Carlo method is a technique which uses repeated random sampling to obtain an expected outcome and its probability distribution, which is a measure of risk [190]. Inputs such as capital costs, availability and performance are modelled by probability distributions rather than single expected values. Models are subject to repeated simulations where each run uses input values selected from the distributions. Completing sufficient numbers of simulations leads to the outputs converging to a single expected value and enables the probability distribution of the output to be computed. It has been proven that the expected output using the Monte Carlo method, which is statistically the most likely result, is not necessarily the same as the output derived deterministically using each input's mean value [191].

Probability distributions of outputs enable investors to make decisions which are specific to their individual risk appetite. For example, Eager et al [185] require the NPV of new generation plant to be twice the project fixed costs at a $95 \%$ confidence level. This information can only be achieved by statistical methods of risk analysis such as the Monte Carlo technique.

A single EES technology was selected to carry out a risk analysis case study using the Monte Carlo technique. Work in this and previous chapters established a precedent that EES technologies have different cost and performance characteristics and must be modelled individually. Equally, the risks associated with inputs vary between technologies: mature technologies are represented by narrower input distributions demonstrating the higher certainty with which variables are known. To carry out a thorough risk analysis accurate and detailed information is required which can be challenging to obtain. In particular, cost information can be commercially sensitive and is not readily available. It is not within the scope of this thesis to complete a risk analysis for multiple technologies, rather a single technology is used to demonstrate the issues associated with novel EES projects. 


\subsubsection{Case Study}

A CES device developed by Highview Power Storage was selected for the case study. It is a novel technology, which highlights the risks associated with early stage development. Detailed cost and performance data was made available by the company [192]. With limited operational experience, the reliability of the data from a source promoting the technology must be considered. However, it was largely in agreement with, or greater than, the published information on CES presented in Chapter 3 and so was considered to be reasonable. An overview of the company and technology development is given below to provide context for the analysis.

\section{The Company}

Highview Power Storage is a privately funded UK company developing utility scale CES. It has raised over $£ 26 \mathrm{~m}$ of equity investment with NTR plc, an international developer and operator of renewable energy, as a major shareholder [193]. It is a small company with 16 staff based in London.

Highview Power Storage has one patent granted for its CES technology and five further patents pending. It has positioned itself as an engineering service and intellectual property licencing company, not a manufacturer or operator of energy storage. Following proof of concept, it intends to design units for customers' specific applications. This way, it hopes to access a broad range of market applications for its technology [194]. The company is listed in the 2012 Global Cleantech 100 [195] and has won several awards for its technology.

\section{Development}

Highview Power Storage was founded in 2002 to develop a piston engine running on liquid nitrogen called the Dearman Engine. In 2005 the company began working with the University of Leeds and the Institute of Engineering Thermophysics at the Chinese Academy of Sciences to develop utility scale CES. In 2008, a lab scale proof of concept system was built. Following successful testing, the company made the decision to position itself with industrial and utility scale storage as its first market. It was awarded a $£ 1.1 \mathrm{~m}$ grant from DECC through the Low Carbon Investment Fund for development of its pilot plant [196]. The 350kW, 2.5MWh prototype device was hosted by SSE in Slough with a generator connected to the grid in 2010 using an external supply of liquid air to generate electricity. In 2011 a liquefier was added to form a fully integrated EES device. A range of operating conditions were tested at the pilot plant until the end of 2014 when it was disconnected from the grid and moved to The 
Birmingham Centre for Cryogenic Storage where it is undergoing re-commissioning for use as a research facility [197].

In 2014 Highview Power Storage was awarded a further £8m from DECC’s Energy Storage Technology Demonstration Competition in conjunction with energy and waste management company, Viridor [198]. The funding was awarded to build a 5MW CES demonstrator to operate at Viridor's landfill gas generation plant near Manchester. Construction is underway and the plant is expected to be operational in 2016. The company is also working on the design of multi MW projects that can deliver 15-200MWh of electricity at power ratings of 5-50MW.

\section{Technology}

An introduction to CES technology is outlined in Chapter 2, Section 2.2.5. When charging, electricity is used to refrigerate air from the surrounding environment. The air is cooled and turns to liquid at $-196^{\circ} \mathrm{C}$. It is then separated and liquid nitrogen stored in insulated, but unpressurised vessels. When discharging, nitrogen is vaporised and the high pressure gas used to turn a turbine and generate electricity. Harnessing and recycling the cold air from the process roughly halves the energy required for the liquefaction increasing the total round trip efficiency to around $50 \%$ which is commonly quoted in literature [37]. Additionally, harnessing waste heat from other industrial processes could increase the roundtrip efficiency further to approximately $70 \%$. Low grade heat at around $115^{\circ} \mathrm{C}$ is required to vaporise nitrogen [199]. This is commonly available from industrial plants as waste as it is not high enough temperature to be useful for many other applications.

The technology uses modular components which have been proven in the industrial gases industry. This reduces the risk associated with project costs and the component life is well understood. The only novel aspect is the detail of the design of the high pressure turbine [199]. Further details on the novel aspects of this technology can be found in [200].

Liquid nitrogen has a high volumetric energy density of $0.12-0.2 \mathrm{kWh} /$ litre, several ten times that of compressed air and about a hundred times that of elevated water [37]. Highview Power Storage estimates that a complete CES device with $10 \mathrm{MW}$ rated power and $90 \mathrm{MWh}$ capacity would have a footprint of approximately $4,000 \mathrm{~m}^{2}$ (one acre) [201]. Its high energy density provides an added benefit that electricity available at remote off grid locations can be used to produce liquid nitrogen which could be transported to a central location to produce electricity locally. The high volumetric density also means that liquid air can be used to store large quantities of energy. A cryogenic tank with a $150,000 \mathrm{~m}^{3}$ capacity is currently used for storage 
of liquid natural gas [202]. If such a container were used to store liquid nitrogen, the storage capacity would be around 18-30GWh.

CES does carry associated safety risks including cold hazards, pressure build up and oxygen deficiency [203]. However, these should not prevent the technology being widely deployed. Cold hazards can be managed with suitable materials for low temperature service and appropriate insulation systems [203]. Pressure build up in fuel tanks is commonly solved with pressure relief valves and burst discs [203]. Oxygen deficiency would require stricter regulating. Liquid nitrogen stored for substantial periods will boil off in time as ambient heat penetrates the insulation. This will cause the pressure in the vessel to rise, and gas to be released through a relief valve. If the tank is housed in an enclosed space with inadequate ventilation, this could lead to the concentration of nitrogen causing the air to become unbreathable. Anyone entering the space would be at risk of asphyxiation. This risk could be eliminated by mandating appropriate passive ventilation and monitoring equipment [203].

To date, liquid nitrogen production facilities have required continuous operation [203]. This conflicts with its use for grid-scale energy storage which requires the production of liquid air during times where electricity demand is low and not during peak hours [204]. However, developments have been made to design processes which are suitable for quick start ups, shut downs and variable operations.

Despite some challenges, CES devices are expected to be deployed in increasing numbers in the future.

\section{Technical Inputs}

It is anticipated that future CES systems will have higher capacity and charging rates than the prototype projects piloted by Highview Power Storage. Costs were provided for two larger EES systems, the characteristics of which are specified in Table 4.6. These were both used for the risk analysis.

Table 4.6 Cryogenic energy storage characteristics used for risk analysis

\begin{tabular}{lccc}
\hline Characteristic & System A & System B & Units \\
\hline Storage capacity, $S_{\max }$ & 100 & 250 & MWh \\
Charging rate, $Q^{C}$ & 20 & 50 & MWh \\
Discharging rate, $Q^{D}$ & 20 & 50 & MWh \\
Conversion efficiency, $\eta_{c}$ & 60 & 60 & $\%$ \\
Storage efficiency, $\eta_{s}$ & 99.98 & 99.98 & $\%$ per half hour \\
\hline
\end{tabular}


The storage efficiency of $99.979 \%$ per half hour represents the boil off of nitrogen from the storage tank, as ambient heat penetrates the insulation, equivalent to a rate of approximately $1 \%$ per day. Although some sources report lower boil off rates for larger tanks of between 5\% every 100 days [205] and $0.2 \%$ per day [206], it is accepted that smaller tanks, such as those which would be used for the CES systems specified in Table 4.6, would experience higher rates due to their larger ratio of surface area to volume.

It was assumed that the system was adequately designed for these characteristics and their values were known with certainty. The lifetime of the device was 30 years and the uncertainty associated with this was accounted for in the model by completely discounting revenue gained after 15 years. An annual planned maintenance time of 7 days was also assumed to occur over a week during the summer months, when revenue was expected to be lowest [201]. The days with the actual lowest revenue, on which it would be optimum to carry out maintenance, would not be known in advance. To represent this down time, the average revenue from the lowest quartile of performing weeks over the year was subtracted from the annual revenue.

An availability of $94.6 \%$ was achieved at the CES pilot plant in Slough [207]. In the model availability was applied, in addition to the planned maintenance outages, as a fixed percentage reduction to the total annual revenue in the absence of a more thorough investigation into failure mode probabilities and repair times. The availability was not known with certainty and so was represented by the left skewed lognormal distribution shown in Figure 4.33. The distribution had an upper bound of $100 \%$, mean of $94.6 \%$ and standard deviation of $5 \%$ (absolute).

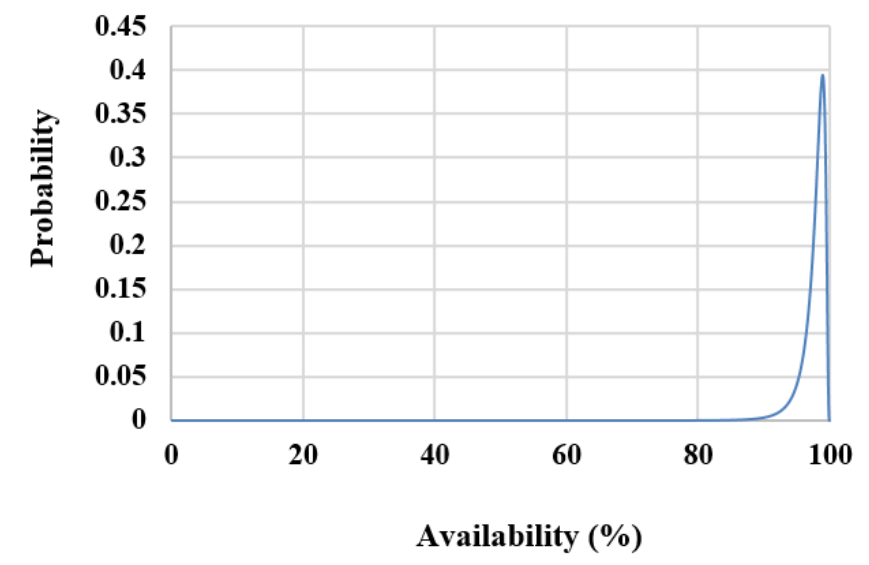

Figure 4.33 Left skewed lognormal probability distribution for device availability 


\section{Cost Inputs}

CES costs were provided for the three subassemblies in the system: the liquefier (charging equipment), the power recovery unit (discharging equipment) and the storage tank. The data is listed in Table 4.7. Costs were provided for a FOAK device and a NOAK device for both system sizes. The NOAK costs were calculated by cost analysts at Highview Power Storage estimating cost reductions through learning by doing and manufacturing at scale [192]. A learning rate of $17.5 \%$ was applied each time there was a doubling in the number of CES systems deployed, up to a predefined lower bound. This was based on similar rates which have been observed in CCGT cost evolution since the 1990s [192]. Although much of the equipment is expected to be off-the-shelf from the industrial gases industries, the scale and assembly of the components is novel and the company anticipates opportunities for cost reduction which justify this learning rate. The number of projects deployed and the lower bounds on cost were not disclosed. A six tenths factor was applied to estimate the economies of scale; assuming that as the quantities increased, the cost increased by the same ratio to an exponent of sixtenths as shown in Equation (5.10) [208].

$$
\frac{\text { Cost }_{1}}{\text { Cost }_{n}}=\left(\frac{\text { Quantity }_{1}}{\text { Quantity }}\right)^{6 / 10}
$$

Table 4.7 Cryogenic energy storage subassembly costs ${ }^{6}$

\begin{tabular}{lccc}
\hline System & $\begin{array}{c}\text { Liquefier } \\
(\mathfrak{f m})\end{array}$ & $\begin{array}{c}\text { Power Recovery } \\
\text { Unit (fm) }\end{array}$ & $\begin{array}{c}\text { Tank } \\
(\mathfrak{f m})\end{array}$ \\
\hline FOAK System A & & & \\
NOAK System A & & & \\
FOAK System B & & & \\
NOAK System B & & & \\
\hline
\end{tabular}

In addition, a breakdown of component costs from each subassembly was provided, as a percentage of the subassembly cost. An estimate of the certainty with which each component cost was known was also assessed through discussions with Engineers at Highview Power Storage [209]. The data is listed in Table 4.8. This was largely in agreement with the accuracy range estimates in the EPRI Electricity Storage Handbook [106] based on simplified mature technology estimates and preliminary commercial cost estimates. Components which have a

\footnotetext{
${ }^{6}$ Commercially sensitive information has been removed from this thesis
} 
more established supply chain, such as the storage tank, have significantly lower uncertainty estimates than the more novel aspects of the technology, such as the turbines.

Table 4.8 Cryogenic energy storage subassembly component cost breakdown and uncertainty ${ }^{7}$

\begin{tabular}{|c|c|c|c|c|}
\hline \multirow[b]{2}{*}{ Subassembly } & \multirow[b]{2}{*}{ Component } & \multirow{2}{*}{$\begin{array}{c}\text { Cost }(\% \text { of } \\
\text { Subassembly } \\
\text { Cost })\end{array}$} & \multicolumn{2}{|c|}{ Uncertainty (\%) } \\
\hline & & & FOAK & NOAK \\
\hline \multirow[t]{10}{*}{ Liquefier } & Process equipment & & 20 & 10 \\
\hline & Cold box assembly & & 5 & 2 \\
\hline & Recycle compressor & & 20 & 10 \\
\hline & Air compressor and drier & & 10 & 5 \\
\hline & Control and instrumentation & & 20 & 10 \\
\hline & Electrical equipment & & 20 & 10 \\
\hline & Cooling equipment & & 1 & 1 \\
\hline & Engineering and site supervision & & 20 & 5 \\
\hline & Construction & & 20 & 10 \\
\hline & Miscellaneous & & 20 & 10 \\
\hline Tank & Tank & & 2 & 1 \\
\hline Power & Turbines & & 20 & 15 \\
\hline recovery & Cryogenic Pumps & & 20 & 15 \\
\hline \multirow[t]{7}{*}{ unit } & Control and Instrumentation & & 20 & 10 \\
\hline & Heat Exchangers & & 10 & 5 \\
\hline & Pipework & & 20 & 10 \\
\hline & Electrical & & 20 & 10 \\
\hline & Engineering and Supervision & & 20 & 5 \\
\hline & Construction & & 20 & 10 \\
\hline & Miscellaneous & & 20 & 10 \\
\hline
\end{tabular}

Each component cost was represented by a normal distribution in the risk model with the cost estimate used as the mean value and the uncertainty range as the expanded uncertainty. The expanded uncertainty is defined as the standard deviation of a distribution multiplied by a coverage factor, which is a positive number greater than one. A coverage factor of two was

\footnotetext{
${ }^{7}$ Commercially sensitive information has been removed from this thesis
} 
used, which resulted in a distribution with $95.5 \%$ probability of being within the expanded uncertainty limits [210]. An example of the probability distribution for the process equipment for a FOAK device with the characteristics of system A is shown in Figure 4.34. The expected (mean) value was $£ \mathrm{~m}, \square$ of the liquefier subassembly cost, and the expanded uncertainty was $£ \square \mathrm{m}, \square \%$ of the mean component cost. With a coverage factor of two, the standard deviation was $£ 0.315 \mathrm{~m}$.

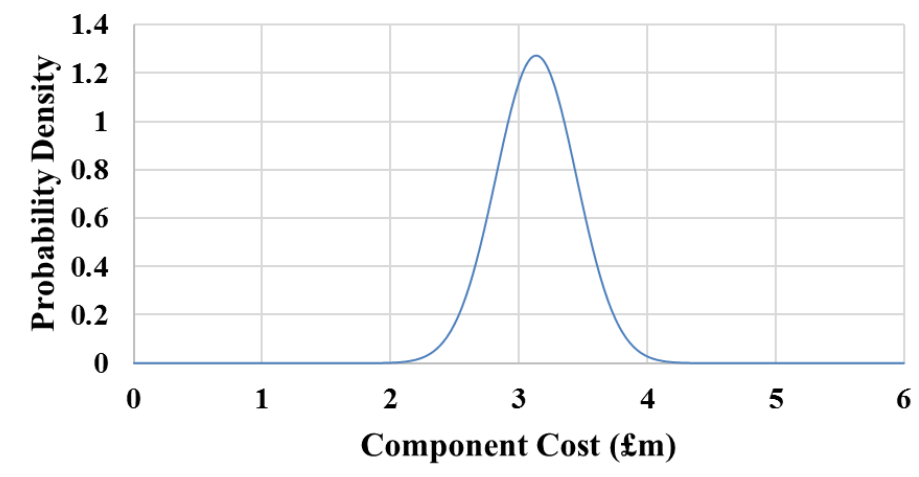

\section{Figure 4.34 Normal probability distribution for process equipment cost for first-of-a-kind} system

Between FOAK projects and NOAK projects the costs and uncertainty ranges reduced as there would be more established supply chains and more confidence in cost estimates. System A and B were examined using both FOAK and NOAK data for comparison. For the NOAK devices, the standard deviation of the lognormal probability distribution characterising the availability was reduced from $5 \%$ to $2 \%$ to represent the increased certainty with which this would be known.

In additional to the capital expenditure (CAPEX), the following operational expenditure (OPEX), supplied by Highview Power Storage [201] was included in the model ${ }^{8}$ :

- fixed OPEX of $\llbracket \%$ CAPEX per year;

- variable OPEX of $£ / M W h$ consumed for liquefaction; and

- overheads and insurance costs of $£$ per year.

The model was run for 100,000 iterations to allow the output of NPV to converge towards a single expected value for each of the systems.

\footnotetext{
${ }^{8}$ Commercially sensitive information has been removed from this thesis
} 


\section{Results}

The expected NPV for system A and B for FOAK and NOAK devices based on annual revenue from 2008 electricity prices is shown in Table 4.9. It is notable that for all cases investigated the NPV was negative. This is contrary to the preliminary results, shown in Figure 4.32, which suggested CES could return a positive NPV of $£ 1.72 \mathrm{~m}$. There were a number of details in the analysis which led to these differences, including the size of the EES system analysed. Secondly, more accurate cost estimates provided by Highview Power Storage were used for the risk analysis which were generally higher than the estimates found in literature used for the preliminary results. Cost estimates for operation and maintenance were also included, which were initially assumed to be negligible. This was the largest cause of the discrepancy. OPEX added additional annual costs of $2-4 \%$ of CAPEX, which had a significant impact on the NPV. Finally, the risk analysis included more accurate modelling of the device performance including downtime for planned and unplanned maintenance which were excluded in the preliminary work. The results shown in Table 4.9 are expected to provide a more accurate assessment of the NPV and are in agreement with previous studies which suggest that arbitrage alone is not a viable source of revenue for EES [137].

Table 4.9 Expected net present value for cryogenic energy storage based on 2008 electricity prices

\begin{tabular}{lc}
\hline System & $\begin{array}{c}\text { Expected NPV } \\
(\mathfrak{f m})\end{array}$ \\
\hline FOAK System A & -40.14 \\
NOAK System A & -19.10 \\
FOAK System B & -63.63 \\
NOAK System B & -29.21 \\
\hline
\end{tabular}

The results show that the expected NPV was higher i.e. a lower negative result, for the NOAK device than for the FOAK device for system A and B due to the reduction in expected costs. The results also show that the expected NPV was higher for system A, the smaller device, than for system B, when comparing FOAK with FOAK and NOAK with NOAK. Increasing the size of the system from $A$ to $B$, increased the annual revenue from $£ 0.9 \mathrm{~m}$ to $£ 2.3 \mathrm{~m}$; however, the CAPEX and OPEX increased more significantly resulting in the lower NPV. The normal probability distributions representing the NPV for each system are shown in Figure 4.35. The larger spread of the FOAK systems show the higher risk associated with novel technologies compared to the narrower distribution for the NOAK devices. The uncertainty was measured 
as a percentage of the capital cost for each component and so system A, the smaller device with the lower cost, had the narrower distribution, representing the lower risk.

The Monte-Carlo simulation was used to demonstrate the risk profile for an EES project and the reduction in risk as it moved from FOAK to NOAK providing more additional confidence to investors. A deterministic simulation would have produced a similar result; however, it would not have provided an equivalent profile of project risk to be established.

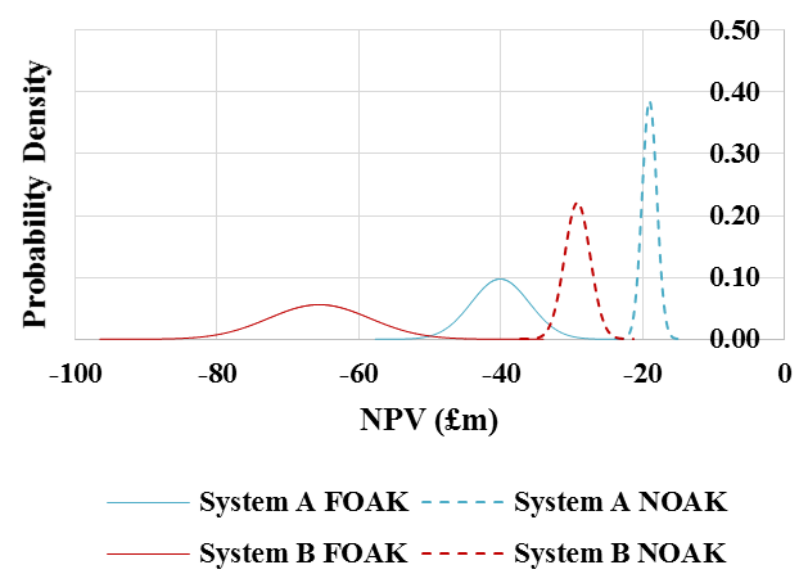

Figure 4.35 Normal probability distribution for net present value of cryogenic energy storage based on 2008 electricity prices

The results show that the expected costs and risks associated with a novel EES technology should reduce as more devices are deployed. Initially, financial support may be needed to enable developers to deploy high cost, high risk FOAK projects which would, in turn, enable NOAK costs to be realised. The innovation support funding discussed in Chapter 4 will assist, to some extent, with prototype project funding; however, without longer term, guaranteed revenue support for EES, there is little scope for further early stage projects to be funded. In the short term, even with access to multiple revenue streams, EES costs and risks are prohibitively high. Proponents of EES argue that in addition to the potential for reduced costs, opportunities for revenue will increase in the future as more variable output wind power is integrated into the electricity system [11]. This is discussed in subsequent chapters. Grünewald et al [19] considered that without targeted support for EES existing mature generation technologies may be favoured, despite not providing the range of benefits of EES in the long term. Due to the nature of large generators with long service lives, once this capacity has been built, the benefits of additional EES would be diminished and future opportunities for financially independent EES projects eliminated. 


\subsection{Chapter Summary}

This chapter has presented a model to assess the commercial value of EES by optimising revenue through price arbitrage. Investigating the optimum operating strategy showed that devices charged and discharged on a daily basis following diurnal variations in electricity prices which were driven by daily demand cycles. Revenue was affected by mean electricity prices, daily price spreads and the frequency of cyclic price fluctuations. Variations in consumer behaviour, market structures and fuel prices between different global markets and within the same market from one year to the next, led to different opportunities for EES and demonstrated the risk associated with investments in the technology.

Varying technical characteristics showed that revenue increased as storage capacity and power rating increase; however, the constraints were not mutually exclusive and required joint optimisation. Furthermore, arbitrage revenue was highly sensitive to both conversion and storage efficiency.

An investigation into the implications of perfect foresight of electricity prices showed that forecast errors of up to $10 \%$ could be tolerated with less than $5 \%$ reduction in revenue suggesting that the assumption would have minimal impact on the results. The assumption of EES as a price taker was also discussed. If the impact of EES operation on electricity prices was considered, increasing storage capacity would lead to reduced marginal returns for an investor, but increased social welfare. This would inflate the difference between the commercial opportunity available to a storage operator and value of EES to society. Without dedicated support to reward EES for the external welfare benefits it provides, and to assist in reducing short term investment risk, the opportunity for the long term system benefits to be realised may be diminished.

Comparing individual technologies highlighted the range of device performance and cost characteristics and demonstrated that modelling multiple EES technologies using generic characteristics is not an acceptable representation. The results showed PHS, CAES, CES and pumped heat were the most feasible options available as EES for energy management applications.

A more detailed case study of CES showed that the preliminary modelling results were optimistic in their values of NPV based on arbitrage revenue. Once more accurate cost data and operation and maintenance were accounted for, the results supported previous conclusions that justification of investment in EES is not viable through arbitrage alone. Additionally, the importance of including an analysis of risk in addition to the value of an investment was 
discussed. A Monte Carlo risk analysis was used to assess the impact of uncertainty in cost estimates and performance on the NPV. This showed that the uncertainty reduced in addition to the value increasing between FOAK and NOAK devices.

In addition to cost and technical risk reducing in the future, there is the potential for revenue to increase. The value of storage has been demonstrated to be highly dependent on market frameworks. In future energy scenarios, with potentially large penetrations of variable renewable generation and changing fuel prices, electricity price patterns will change, leading to changing revenues for EES. The potential impact of these changes is discussed in the following chapters. 


\section{Chapter 5 Electricity Price Model}

Development

This chapter presents the model developed to simulate time series of electricity prices for future energy scenarios. In the following chapter, this is coupled with the storage arbitrage model, described previously, to investigate the impact of changing market structures and fuel and carbon prices on EES revenue. The work reported in this chapter has been published in a journal paper by Dunbar et al [211], a copy of this is available at the end of this thesis.

An overview of the competitive model of the GB electricity market is presented. The techniques used to create supply and demand curves are explained referencing historic data, which is later used to validate the model. Initial comparisons between historic and simulated electricity prices are presented and improvements made to the electricity price functions. The treatment of future fuel and carbon prices, wind power output and demand are then presented. Finally, the implications of representing wind power output as negative demand and the treatment of errors in the model are discussed.

\subsection{Model Overview}

The complexity of electricity price processes, in addition to their significance to the power industry has motivated numerous researchers in the field of electricity price forecasting [212]. Many different techniques have been applied [213], which can generally be categorized as either stochastic or fundamental models [214]. Stochastic models focus on addressing short term characteristics of spot electricity markets, but provide little insight into price formation. As such, they are not good at modelling future scenarios where electricity systems may be different to current structures. Fundamental price models rely on accurate forecasts of demand, fuel prices and, in a system with a large penetration of wind power, wind speeds, among other 
variables. These are challenging to predict, particularly over long time horizons; however, such models have been shown to be representative of the power exchange and are commonly used for modelling scenarios of future energy prices [215], [94].

The purpose of the electricity price model was not to attempt to predict future electricity prices, but to give an indication of wholesale price volatility for different scenarios to enable the potential value of EES to be explored under different generating capacity and fuel price assumptions. The model represented prices in the GB power exchange and was validated using historic electricity price data from the ELEXON price portal [27], which provides market index prices as a half-hourly time series of the average trades through the power exchange.

The model was based on the assumption of perfect competition in the electricity market. Supply and demand curves were constructed for each half hour time period and the price of electricity determined by the market clearing price: where the curves intersected. The generator supply function was calculated by stacking thermal plant in merit order of increasing marginal cost. An industry supply curve was used, where all generators of the same technology were assumed to have the same bidding behaviour, rather than individual generator supply functions being calculated. This reduced the model complexity and has been shown to be a reasonable approximation of the market in GB [216]. Renewable power output was assumed to have zero marginal generation cost and was deducted from electricity demand to calculate the dispatachable power which was required from thermal generators in the system; this formed the net demand curve. Assuming zero marginal generation costs for renewable power output assumes that no subsidy is paid. This is reasonable as subsidies are expected to be removed as technology costs reduce; the UK Government recently announced an end to subsidies for all new onshore wind farms as early as 2016 [217]. As a result, in the model, the price of electricity never falls below zero; the implications of this are discussed in Section 5.8. An overview of the model is shown in Figure 5.1.

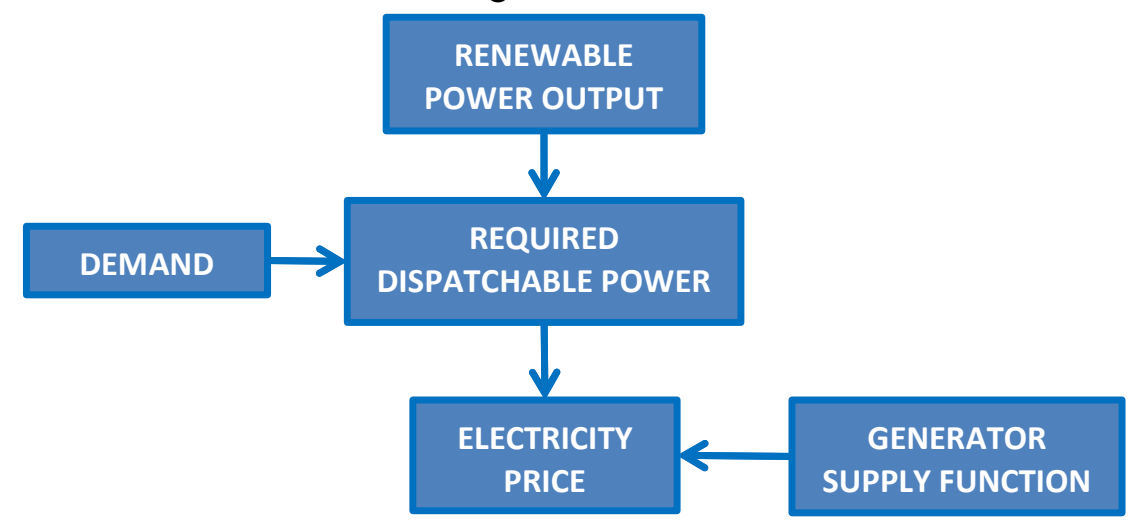

Figure 5.1 Overview of electricity price forecast model 
The model was developed and validated using historic data from 2005-2007. These years were selected as robust data sets for coal, gas and nuclear fuel prices in addition to wind resource data were available. For other years, this information could not be sourced at as high a temporal resolution.

\subsection{Generator Supply Function}

Thermal generators were grouped into four classes of plant; nuclear, coal, CCGT and OCGT and stacked in merit order of increasing generation cost. It was assumed that each class of generator would bid to produce electricity at a price around its marginal generation cost. In the $\mathrm{UK}$, there are no regulations requiring them to do this; however, it is likely to be the case in a competitive market and studies have shown it to be a reasonable assumption [218], [219]. To represent a competitive market, the price bid by each class was bound by the lower limit of its own marginal cost and the upper limit of the marginal cost of the next class in the merit order stack [180]. A linear relationship between the price of electricity, $P E$, and power output was assumed between these upper and lower limits, defined by:

$$
P E=\Pi_{x}\left[1+\frac{\Pi_{x+1}-\Pi_{x}}{\Pi_{x}} \frac{P_{x}}{C_{x}}\right]
$$

where $\Pi_{x}$ is the marginal generation cost, $x$ denotes the merit order of the marginal generator (i.e. for baseload $x=1$ and for peaking plant $x=4$ ) and $P_{x}$ and $C_{x}$ are the power output and installed capacity of the marginal generator, respectively. All generators were paid the market clearing price which was set by the highest merit order generator that was scheduled to run. The difference between the market clearing price and the generator's short run marginal cost is known as a scarcity rent and provides income to cover the plant's long run fixed costs [36].

Peaking plant was the last class of generator to be dispatched. Its maximum price was not limited by a more expensive generator and so it bid to produce electricity at a premium price to reflect scarcity of supply. This price uplift, significantly higher than the marginal generation cost, enables peaking generators, which generate for a limited number of hours, to recover their fixed costs and is a feature of electricity markets in GB [26] and worldwide [220], [221]. Grünewald [180] and Eager [222] used forms of exponential function to represent the price of electricity bid by peaking generators. Both these uplift functions were investigated; but neither was found to adequately represent the shape of price curve. An alternative exponential was found to be more representative and was implemented in the model:

$$
P E=\Pi_{x}\left[\beta e^{\alpha\left(\frac{P x}{C_{x}}\right)}\right]
$$


where $\alpha$ and $\beta$ are constants which were determined empirically and used to define the extent of the uplift applied when capacity was scarce. The calibration of these scalars is presented in Section 5.6. Figure 5.2 shows an example aggregate supply curve.

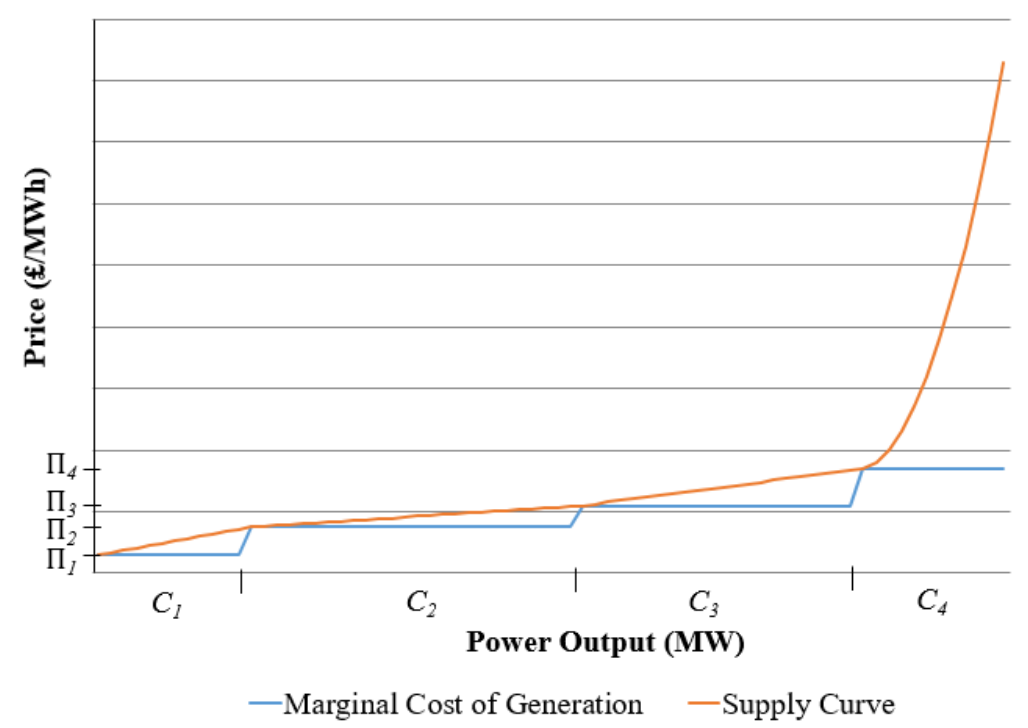

Figure 5.2. Example electricity supply curve

\subsubsection{Generation Capacity}

There is a wide range of generation capacity contributing to electricity production in GB. To simplify the model, only 4 classes of generation were considered. Smaller peaking generators including oil, as well as pumped-hydroelectric, were grouped with OCGTs and other base load generators, such as run-of-river hydro, were grouped with coal. This approach, used by Eager [92], gives a reasonable representation of the British system without significantly increasing the complexity of the model. Grünewald [11] simplifies his model further, considering only three classes of thermal generation defined generically as base load, mid-merit and peaking plant. While this approach allows easier implementation of the model, it does not enable adequate representation of the different marginal generation costs of different types of generator. Instead of calculating the marginal cost for each classification of generation, a single approximate value was assigned to each class by Grünewald, which meant that sensitivity of electricity price to variations in fuel and carbon price could not be represented.

Historic generator capacity data was obtained from the National Grid Interim Seven Year Statement [223]. Although this data was published ahead of time and based on projected capacities, it provides a detailed breakdown of individual generators allowing the plant to be assigned to each of the categories outlined above. Actual plant capacities were subsequently published in the Digest of United Kingdom Energy Statistics (DUKES) [25]. The information 
was verified against these figures; however, DUKES alone was not sufficiently detailed to distinguish CCGT from OCGT generation. The historic capacities of each class of generator are listed in Table 5.1.

Table 5.1 Historic generator capacity

\begin{tabular}{lcccc}
\hline \multirow{2}{*}{ Year } & \multicolumn{4}{c}{ Capacity (MW) } \\
\cline { 2 - 5 } & Nuclear & Coal & CCGT & OCGT \\
\hline 2005 & 12,134 & 26,060 & 21,737 & 11,898 \\
2006 & 11,984 & 26,060 & 22,567 & 12,268 \\
2007 & 11,086 & 26,060 & 23,397 & 12,836 \\
\hline
\end{tabular}

The plant capacity was assumed to be fixed throughout the year and GB was considered an islanded network with no interconnector capacity.

\subsubsection{Availability}

To reflect plant downtime for maintenance, the capacities listed in Table 5.1 were multiplied by the generator availabilities given Table 5.2 [224]. This applied a constant reduction in capacity to generator output throughout the year, assuming a significantly large number of generators in each class and that the likelihood of shut down was constant throughout the year. Although it is likely that there would be increased planned maintenance during the summer months, this was a reasonable simplification given the level of generator classification.

Table 5.2 Generator availability

\begin{tabular}{lc}
\hline Generator Class & Availability (\%) \\
\hline Nuclear & 78 \\
Coal & 86 \\
CCGT & 87 \\
OCGT & 95 \\
\hline
\end{tabular}

\subsubsection{Marginal Generation Cost}

Marginal generation costs can be obtained from a number of sources including reports by Mott MacDonald [225] and the former Department of Trade and Industry [226]. Green [227] and Eager et al [185] compute marginal generation costs from fuel price, plant thermal efficiency 
and carbon price. This allows flexibility to investigate the impact of changes in these variables on electricity price.

Generation costs of thermal generators, $c$, were calculated based on the cubic power output function presented by Wood et al [228]. The cubic function was simplified, assuming that all variable costs were linear and, additionally, the costs associated with emissions were incorporated. Start up and zero load costs were neglected. It is only during peak hours that start-up costs make a significant contribution to marginal costs [95]. As discussed above, during these hours generators will bid to produce electricity at substantially inflated prices and the accuracy of the marginal cost at these times is less critical. The cost function is given as:

$$
c(p)=d p+V+\frac{v F_{c a r}}{\eta}
$$

where $p$ is the power output, $d$ is a coefficient derived from thermal efficiency and fuel price, $V$ is the operation and maintenance cost, $v$ is the amount of carbon produced from burning fuel at $100 \%$ efficiency, $\eta$ is the thermal efficiency and $F_{c a r}$ is the price of carbon. From this the short run marginal cost was calculated using:

$$
\Pi_{x}=\frac{1}{\eta_{x}}\left(a_{x} F_{x}+v_{x} F_{c a r}\right)+V_{x}+e_{x}
$$

where $\eta_{x}$ is the thermal efficiency of the marginal generator, $x, a_{x} F_{x}$ is cost of fuel, with $F_{x}$ the fuel price relating to each class of generator. Specifically, nuclear and coal fuel prices were in $£ / \mathrm{kg}$ while gas prices were in $£ /$ therm. $a$ was required to convert the units of price into $£ / \mathrm{MWh}$. $e_{x}$ is the cost of enriching the fuel, which only applies to nuclear generators, and $v_{x}$ and $V_{x}$ are the carbon emissions and operation and maintenance costs per unit output, respectively. The conversion coefficients for each fuel type were calculated using equations (5.5)-(5.7):

$$
a_{n u c}=\frac{8.9}{24 \times 45}=8.24 \times 10^{-3} \mathrm{~kg} / \mathrm{MWh}
$$

where $8.9 \mathrm{~kg}$ of uranium $\left(\mathrm{U}_{3} \mathrm{O}_{8}\right)$ is needed to produce $1 \mathrm{~kg}$ of converted, enriched and fuel fabricated (CEFF) uranium and a burn up rate of $45 \mathrm{MWday} / \mathrm{kg}$ is used [229].

$$
a_{\text {coal }}=\frac{3.6}{0.024}=150 \mathrm{~kg} / \mathrm{MWh}
$$

where $0.024 \mathrm{GJ}$ of energy is available per $\mathrm{kg}$ of coal [230] and $1 \mathrm{MWh}=3.6 \mathrm{GJ}$.

$$
a_{\text {gas }}=3.6 \times 9.48=34.12 \text { therms } / \mathrm{MWh}
$$

where 9.48 therms is equivalent to $1 \mathrm{GJ}$ of energy. 
The data used for each generator class is listed in Table 5.3. Thermal generators are established technologies. While performance may improve over time due to technology developments, it was assumed that there would not be significant changes and these inputs remained fixed for all future energy scenarios.

Table 5.3 Thermal generator data [92], [230], [225]

\begin{tabular}{lcccc}
\hline $\begin{array}{l}\text { Generator } \\
\text { Type }\end{array}$ & $\begin{array}{c}\text { Thermal } \\
\text { Efficiency } \\
\mathbf{( \% )}\end{array}$ & $\begin{array}{c}\text { Carbon } \\
\text { Emissions } \\
(\mathbf{k g} / \mathbf{M W h})\end{array}$ & $\begin{array}{c}\text { Variable } \\
\text { Operating Cost } \\
(\mathbf{f} / \mathbf{M W h})\end{array}$ & $\begin{array}{c}\text { Enrichment } \\
\text { Cost } \\
(\mathfrak{f} / \mathbf{M W h})\end{array}$ \\
\hline Nuclear & 36 & 0 & 1.8 & 2.5 \\
Coal & 35 & 285 & 2.0 & 0 \\
CCGT & 53 & 185 & 2.2 & 0 \\
OCGT & 39 & 185 & 2.7 & 0 \\
\hline
\end{tabular}

\section{Fuel prices}

Fuel markets are volatile and prices depend on a number of complex factors ranging from natural disasters and adverse weather conditions to regulatory regimes and political conflicts. Time series of historic fuel price data were used to ensure that intra-annual volatility was captured.

\section{Nuclear}

The NUEXCO exchange value of monthly uranium fuel prices are published by TradeTech [231]. This is the longest running uranium price indicator series in the nuclear fuel industry [232]. The monthly time series data for 2005-2008 is shown in Figure 5.3 (Data Source 1). Alongside the average annual price quoted by Eager [92]. The values are similar, but the monthly average prices provide better resolution and so were used in the model. 


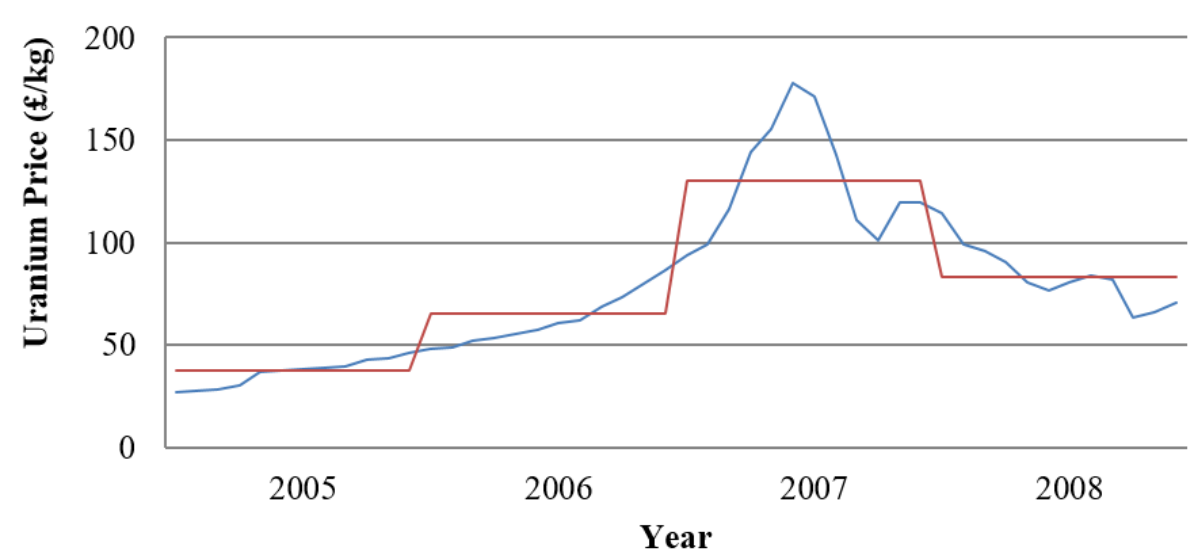

—Data Source 1: Monthly Average Data ——Data Source 2: Annual Average Data

Figure 5.3 Uranium price time series [92], [231]

\section{Coal}

Coal prices are published in the DECC reports on Quarterly Energy Prices [233] and are shown in Figure 5.4 (Data Source 1). BP also publishes the Northwest Europe marker price of coal in its Statistical Review of World Energy [234]; however, only a single annual price is published, as shown in Figure 5.4 (Data Source 2). There is a small discrepancy, but the DECC prices are specific to GB and provided a better resolution and so were used for the model.

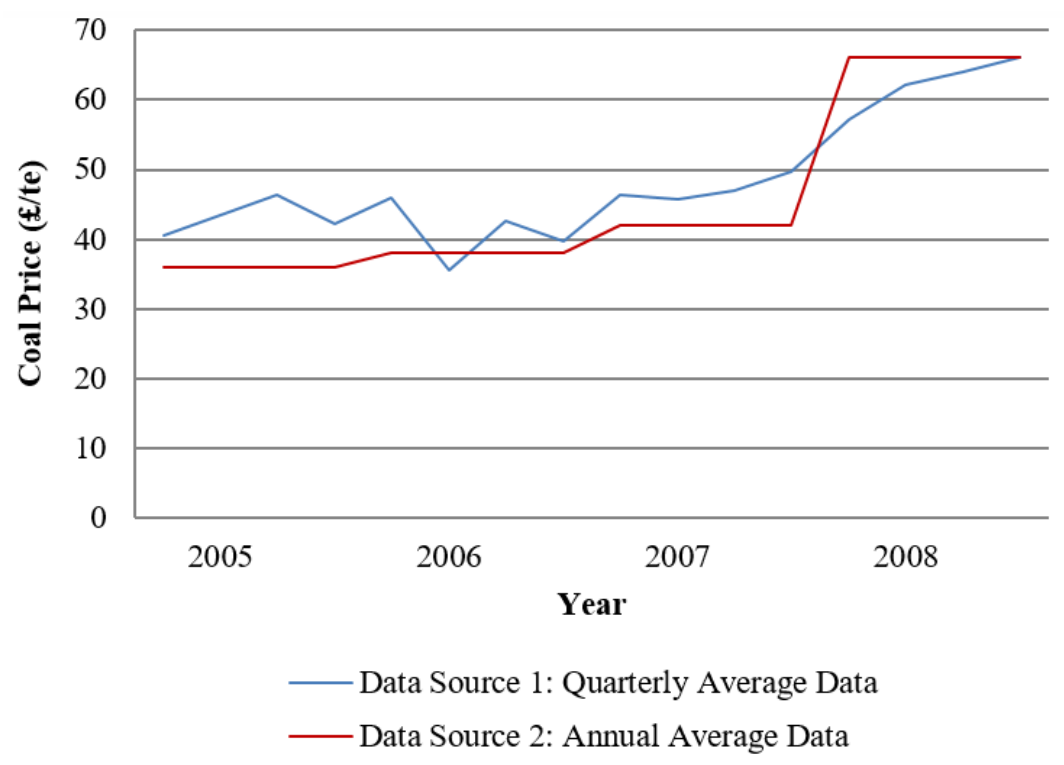

Figure 5.4 Coal price time series [233], [234]

Gas

The UK National Balancing Point (NBP) is a virtual exchange for trading natural gas [235]. It has been in existence since the 1990s and is the most liquid gas market in Europe [236]. Trades at the NBP are made via the On-the-day Commodity Market which is operated by ICE- 
ENDEX (previously APX-ENDEX). Historic daily gas price data from 2005-2007 was provided by ICE-ENDEX [28]. The average monthly prices from this data were compared to values reported to be sourced from Argus [235] and Platts [236], although the detailed monthly average prices could not be accessed from these primary sources. The maximum difference between different data sets for the same month was $20 \%$.

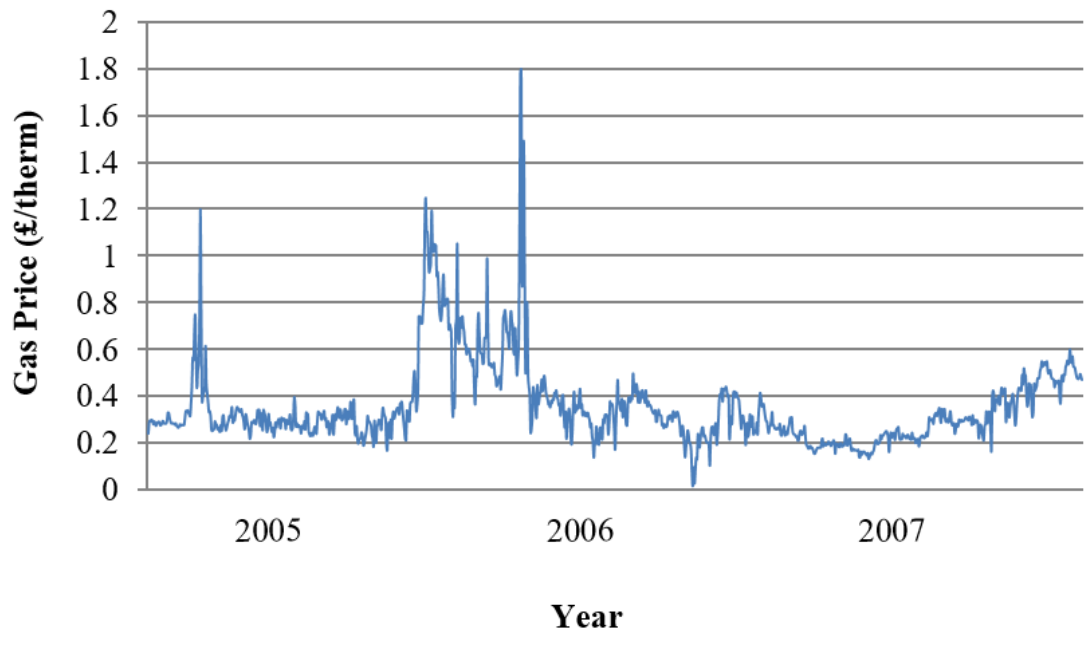

Figure 5.5 Gas price time series [28]

\section{Carbon prices}

Carbon emissions are a by-product of thermal electricity production. Similarly to fuel prices, carbon prices have a direct impact on marginal generation costs, they can be volatile and are highly dependent on political regulation. Historic daily carbon prices were sourced from the European Environment Agency [237]. These are shown in Figure 5.6.

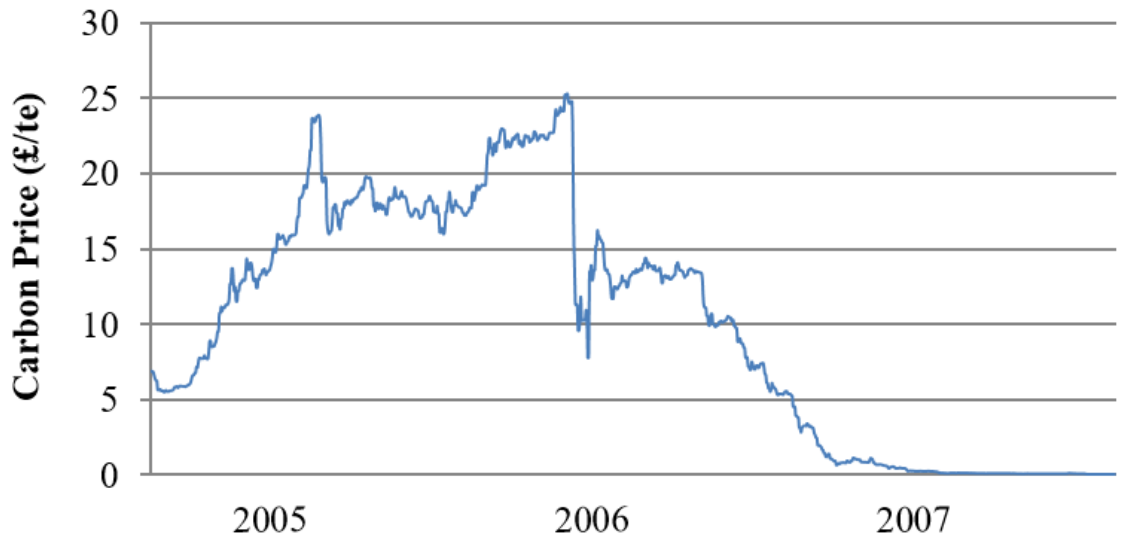

Year

Figure 5.6 Carbon price time series [237] 


\subsection{Renewable Power Output}

Wind power was the only form of renewable energy considered in the model. Other forms of renewable generation were grouped with classes of thermal capacity as discussed above. The implications of larger penetrations of other variable renewables including solar, wave and tidal generation are discussed in Chapter 6. It was assumed that wind power had zero marginal cost and was always dispatched when available. The UK was conceptually modelled as a single bus transmission system and network constraints were neglected. It was assumed that wind power output was not curtailed unless electricity demand was fully satisfied.

\subsubsection{Wind Speed Data}

Wind speed measurements are available from the UK Meteorological Office [238], which have been used in other similar analyses [95], [11]. This data is only available at specific met mast locations which may be some distance from wind turbine sites. Additionally, topography local to the met masts, which are often located in urban areas, could affect the boundary conditions which would influence the wind speed measurements [239]. There are also limited offshore locations where data is available.

Hind-cast data from atmospheric models can provide more accurate wind speed data at a higher and more regular spatial resolution. Hawkins [30] used the Weather Research and Forecasting (WRF) model [240] to produce hourly wind speeds for the UK and surrounding waters. Wind speeds were produced at a spatial resolution of $3 \mathrm{~km}$ by $3 \mathrm{~km}$ and extracted at various heights above ground or sea level. This allowed the wind speed to be obtained at a height closer to the actual turbine hub height, rather than the met mast height which can be considerably lower. When using met mast data, the wind speed is commonly transformed to the required hub height using the power law [241]. This method can cause issues when assuming values for the shear exponent. For example, when it is sunny, the reduction in shear exponent due to increased turbulence in the boundary layer can be difficult to represent [92]. Further information on the wind speed model can be found in [30].

\subsubsection{Power Output}

The MatLab code used to calculate the aggregate wind power output described in this section was written by Lucy Cradden, a Research Fellow at the University of Edinburgh.

The DECC RESTATS planning database [242] details renewable energy projects which are operational, under-construction or consented in GB. For the years under investigation, the planning report data was used to identify onshore wind farms, their location, capacity and the 
date that they commenced electricity production. Each wind farm was considered to be producing power from the start of the year in which the database stated it was commissioned and operational. The time series of wind speed data corresponding to each farm location was extracted from the wind model at $80 \mathrm{~m}$. This was the height at which wind speeds were available, which was expected to be closest to the average turbine hub height.

Manufacturers' power curves can be used to estimate power output from wind farms [243], [244]. However, these are obtained in controlled environments and often don't include the effects of short-term variations in wind speed and direction, the presence of turbulence or other dynamic effects due to interactions between turbines [245], [246]. As a result, manufacturers' power curves often overestimate power output [247]. Hayes et al [248] demonstrated a 10\% error in power output calculated from the manufacturer's power curve compared to measured data for a UK wind farm. To overcome these issues a number of approaches have been used to develop aggregate wind farm power curves [245], [249], [250]. These conclude that aggregate power curves should exhibit the following characteristic changes from manufacturers' power curves [30]:

1. Power is generated below the cut in speed;

2. rated power is reached at a higher wind speed;

3. output drops below the rated power earlier than the cut-out speed; and

4. some power is generated after the turbine cut-out speed.

Further information on these differences and their causes is available in [30]. A model for obtaining an equivalent aggregate power curve for a wind farm in the UK was presented in [248]. This is approximated as a function of the wind speed, $U$, in equation (5.8).

$$
\text { Power Output }=\frac{1}{1+e^{-\frac{U-9.7}{1.8}}}
$$

This was used to calculate the power output for each wind farm in the UK. The output per unit was multiplied by the wind farm capacity for each site. Although the power curve was specific to the wind farm investigated in [248] it was applied as a generic power curve in this model. It was not feasible to empirically obtain aggregate power curves for each individual wind farm.

The power output from all the wind farms was summed to give an aggregate power output for onshore wind. The data was interpolated linearly to obtain a time series of power output at half-hourly intervals. The power output at each time period was then reduced by $10 \%$ to 


\section{Electricity Price Model Development}

account for availability, assuming $90 \%$ of the wind fleet was operating continuously. This is a somewhat conservative estimate for onshore wind [251].

The process was repeated for offshore wind farms under the same assumptions. Offshore wind farms are likely to have a hub height greater than $80 \mathrm{~m}$ and, for the years used to validate the model, the availability was expected to have been less than $90 \%$. These inaccuracies are likely to have little impact as the total power output from offshore wind turbines in the UK made little contribution to meeting electricity demand in the years 2005-2007.

The time series of total wind power output for 2005-2007 is shown in Figure 5.7. This is the sum of onshore and offshore power output. The increasing annual installed capacity over these years is also shown. Figure 5.8 shows wind power output and electricity demand on the same scale which demonstrates the minor contribution that wind power made to serving electricity demand during this period.

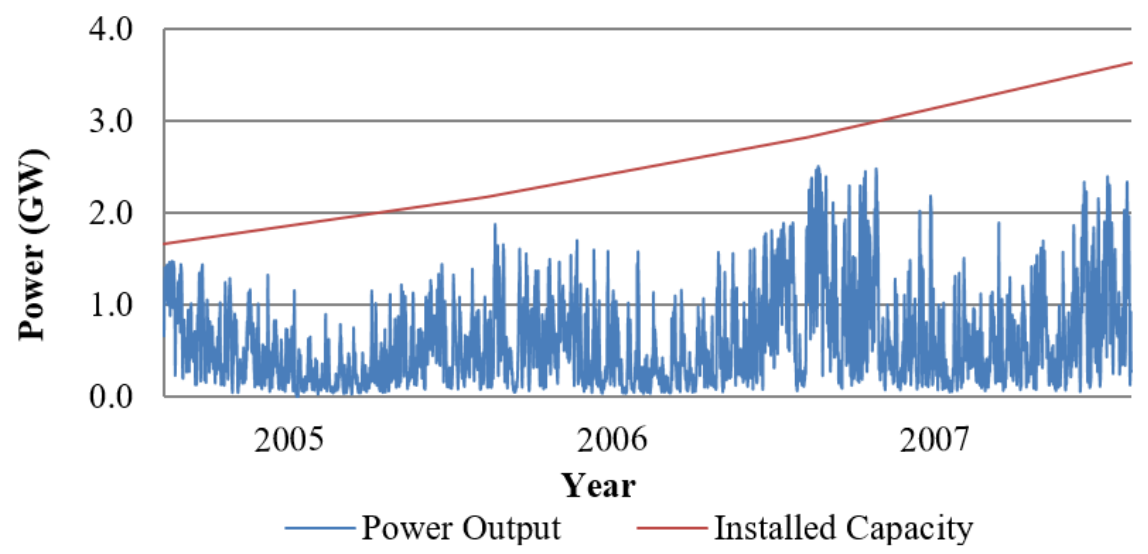

Figure 5.7 Wind power output and installed capacity

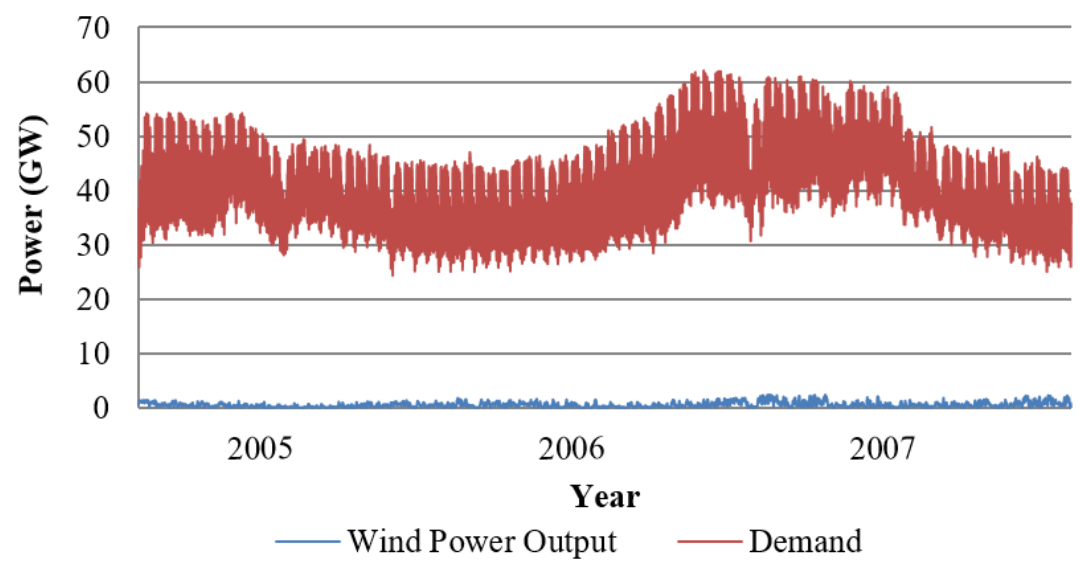

Figure 5.8 Wind power output and electricity demand 


\subsection{Demand}

Historic demand data is available from National Grid [251]. This provided a time series of half hourly demand for the UK. Electricity was assumed to have zero price elasticity of demand (i.e. consumers would not respond to changes in electricity price), which is a reasonable assumption for historic electricity prices [21] but may change in the future with the introduction of smart grids and demand side response. The implications of this are discussed in Chapter 7.

The electricity supply curve and the demand were calculated at each half hourly time step and, using the basic principles of supply and demand, the intersection of the inelastic demand and the supply function define the electricity price at that time.

\subsection{Initial Results and Model Improvements}

This section compares preliminary outputs from the price model to historic electricity prices over a three year period. Initially, prices are compared by examining the price duration curve. This gives an indication of if the correct magnitude of prices are generated by the model. Following this, the full three year time series are investigated to provide evidence of the uplift function operating correctly. Examining a two week period of the time series shows that diurnal price fluctuations are appropriately captured by the model; however, the magnitude of the daily price spread is not adequately represented; a feature which is critical to arbitrage revenue. Subsequently, modifications to the model are described, which compensate for previously overestimated daily price troughs and underestimated daily price peaks. For this section the uplift coefficients, $\alpha$ and $\beta$, were arbitrarily fixed at values of 1.8 and 1 , respectively. Calibration of these coefficients, discussed in the following section, should correct any errors in electricity price during periods where peaking generators are used.

\subsubsection{Price Magnitude}

Figure 5.9 shows the price duration curve over three years, from 2005-2007, for simulated and historic electricity prices from the ELEXON Power Exchange [27]. This indicates that the simulated prices were of the correct order of magnitude, although slightly higher than historic prices. The difference was most significant for the highest prices with the maximum simulated price reaching $£ 619 / \mathrm{MWh}$, while historically, the maximum price was $£ 550 / \mathrm{MWh}$. Calibration of $\alpha$ and $\beta$ should correct the overestimation of the extreme prices. 


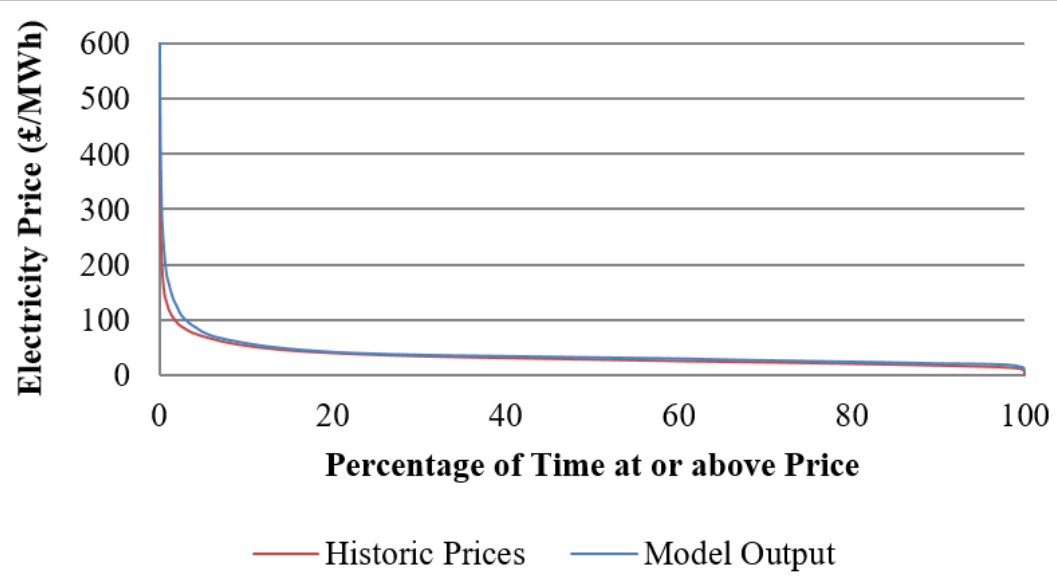

Figure 5.9 Price duration curve for simulated and historic electricity prices

\subsubsection{Price Uplift Function}

Figure 5.10 shows the time series of the results shown in the price duration curve. The general shape of the two time series is similar, with extreme prices most prevalent during the winter months. This suggests that the model correctly identified periods of scarce capacity and applied the price uplift during these periods. Occasional price spikes, such as those in the summer of 2006, were not captured by the model. These were likely to be caused by isolated events, such as an unexpected generator shut downs, which are not adequately characterised by the simplified approach used to model generator availability. However, these occasions were rare and would have little impact on EES revenue over its lifetime.

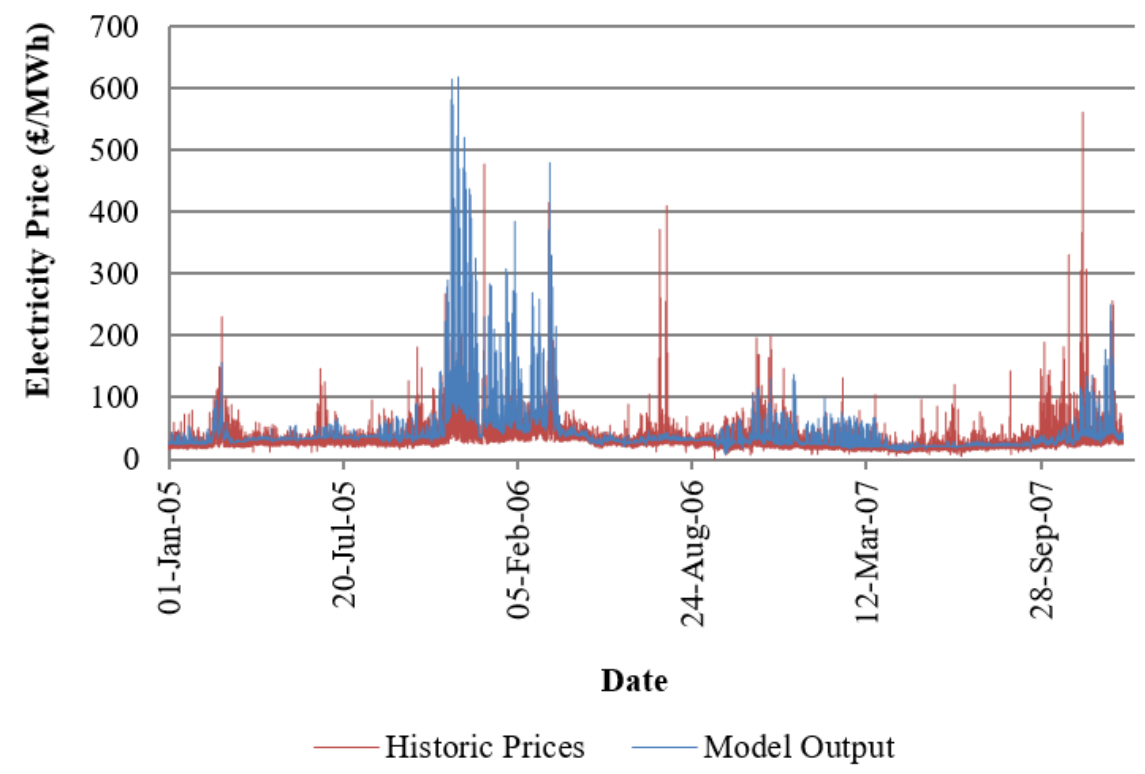

Figure 5.10 Initial model output and historic electricity price time series, 2005-2007 


\subsubsection{Daily Price Spread}

Also evident from Figure 5.10 is the frequently larger price spread in historic prices than in the model. The model consistently overestimated the lowest prices and underestimated the peaks when the uplift function was not applied. This is evident on inspection of prices over a shorter duration. Figure 5.11 shows electricity prices for the first two weeks of August 2007 as an extreme example of this. Figure 5.12 shows the demand net wind power output for the same period. $1^{\text {st }}$ August 2007 was a Wednesday so the reduced net demand on days 4 and 5 and 11 and 12 are over the weekends. The capacity limits for each class of generator are also shown.

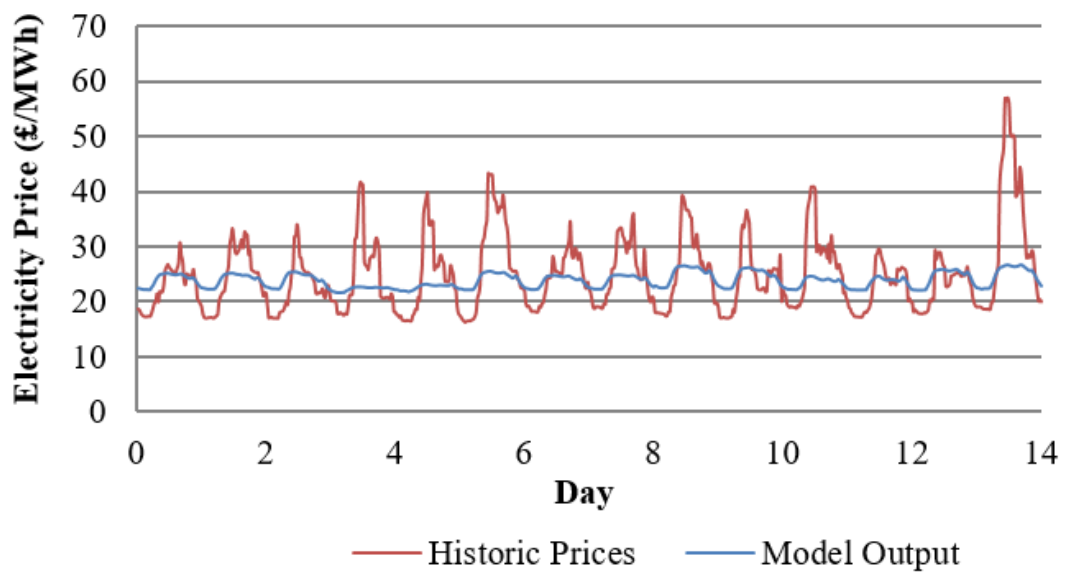

Figure 5.11 Initial model output and historic electricity prices for first two weeks of August 2007

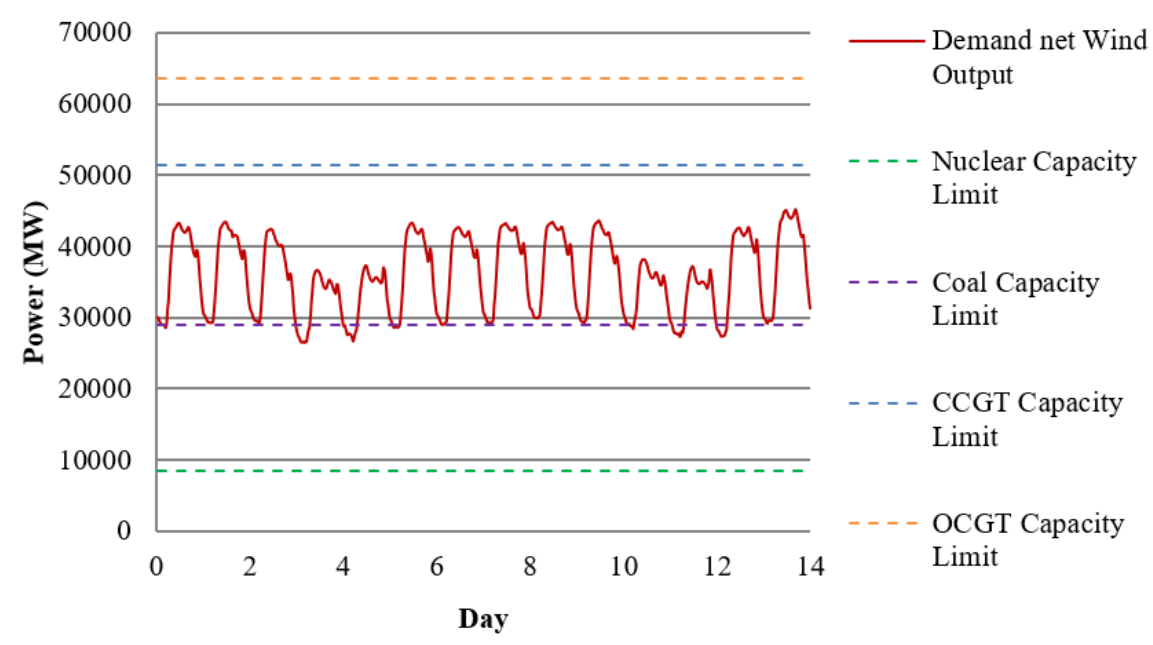

Figure 5.12 Demand net wind power output and generator class capacity limits for first two weeks of august 2007 


\subsubsection{Correcting Minimum Prices}

The overestimation of the minimum prices may be attributed to the calculation of marginal costs used to form the supply curve. Average generator efficiencies were used to calculate the marginal costs for each class of generator. In reality, the supply curve will be made up of many individual supply curves with the most efficient generators setting the lowest marginal cost for each class. The performance of less efficient generators was not important in the model, as it was accounted for in the linear increase in price bid by each class. It was assumed that the competitive price bid by the marginal generator was always higher than its marginal cost. Mott MacDonald [225] reports high, medium and low generator efficiencies for different classes of generator. The efficiencies in the model were changed from the average values listed in Table 5.3, to the high efficiency figures from this report; $46 \%, 60 \%$ and $46 \%$ for coal, CCGT and OCGT respectively. Figure 5.13 shows the simulated and historic prices for 2005-2007 using the high efficiencies. Figure 5.14 shows the same data for the first two weeks in August 2007. These graphs show that the minimum simulated electricity prices were significantly closer to the historic prices. However, the simulated price peaks were also reduced, increasing the difference between the peaks in the model and the historic data.

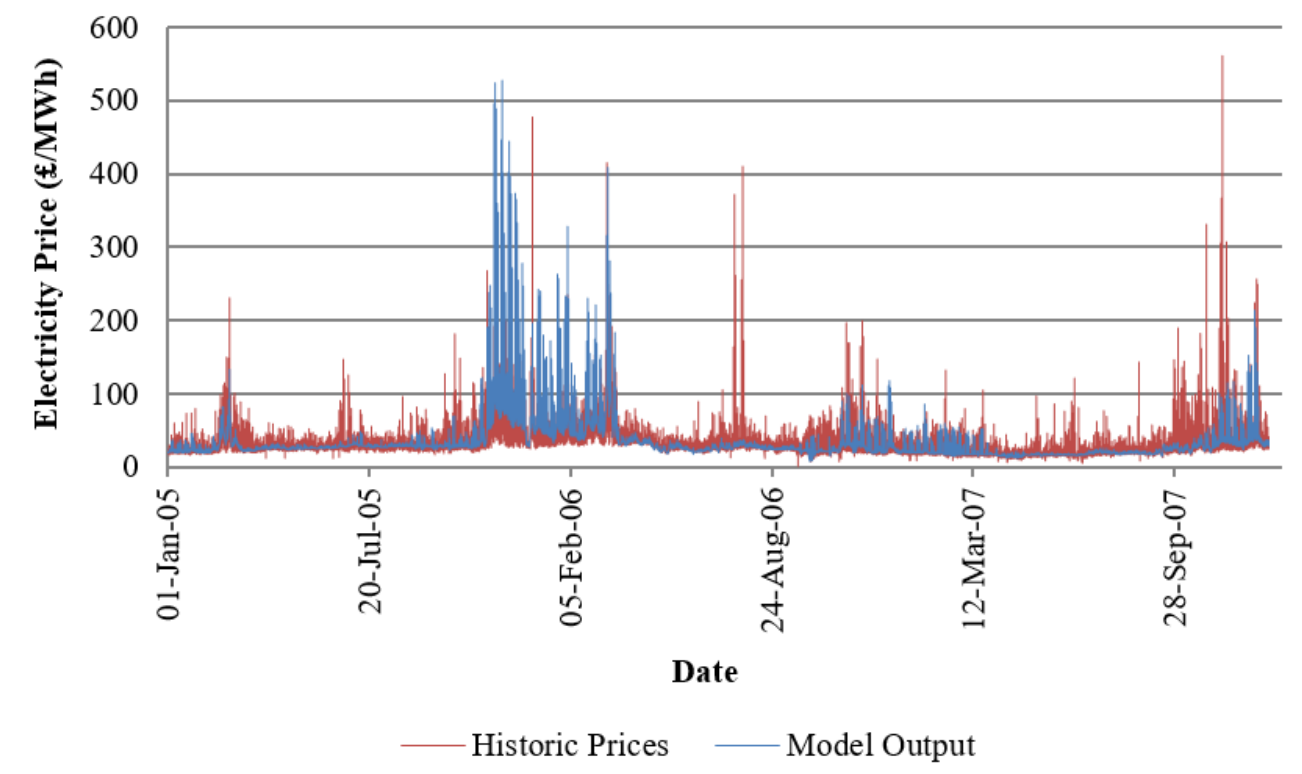

Figure 5.13 Model output and historic electricity price time series with high generator efficiencies, 2005-2007 


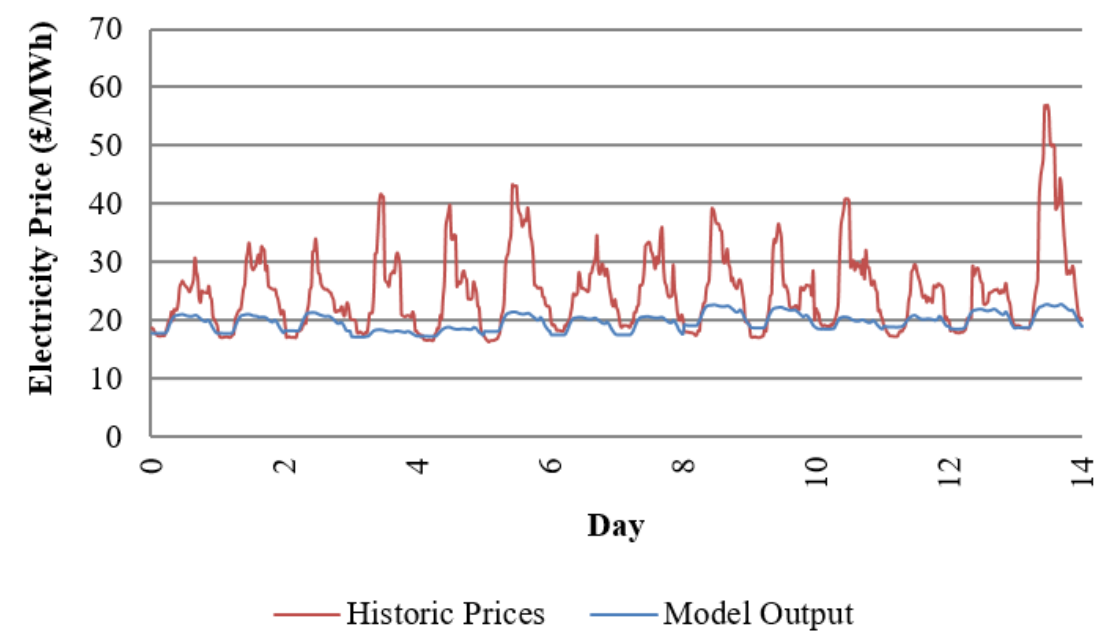

Figure 5.14 Model output and historic electricity prices with high generator efficiencies for first two weeks of August 2007

\subsubsection{Correcting Price Peaks}

Figure 5.12 shows that, in the model, CCGT was the marginal generator during every price peak for the first two weeks in August 2007. OCGT (peaking plant) was not required. Calibrating $\alpha$ and $\beta$, would have had no effect on these prices. This suggests that some form of uplift function should be applied to the prices bid by CCGT generators, in addition to that applied to OCGT generators, during this period to better reflect the price peaks. It is reasonable to assume in a competitive market that the maximum price bid by a CCGT generator would not be restricted by the marginal generation cost of the cheapest OCGT generator, but by the minimum price that OCGT generator would bid to produce electricity. To reflect this, the same linear relationship between price and output was assumed for CCGT, but the minimum price bid by OCGT, including the uplift function, was used as the upper limit. This is described by equation (5.9) where the variables are previously defined.

$$
P E_{C C G T}=\Pi_{C C G T}\left[1+\frac{\left(\Pi_{O C G T}\left[\beta e^{\alpha\left(\frac{P_{O C G T}}{C_{O C G T}}\right)}-\Pi_{C C G T}\right)\right.}{\Pi_{C C G T}} \frac{P_{C C G T}}{C_{C C G T}}\right]
$$

When the marginal generator is CCGT, there is no power output from OCGT (i.e. PoCGT $=0$ ) so this can be simplified to:

$$
P E_{C C G T}=\Pi_{C C G T}\left[1+\frac{\left(\beta \Pi_{O C G T}-\Pi_{C C G T}\right)}{\Pi_{C C G T}} \frac{P_{C C G T}}{C_{C C G T}}\right]
$$


With $\beta=1$, as assumed for initial investigations, equation (5.10) simply represents a linear increase in price for CCGT with the upper and lower limits set at the marginal generation costs for CCGT and OCGT, respectively, as previously assumed. Increasing $\beta$ increases the maximum price bid by CCGTs, in addition to the price bid by OCGTs. To demonstrate this, Figure 5.15 shows the simulated and historic electricity prices for the first two weeks of August 2007 with $\alpha=1.8$ and $\beta=2$. While not identical, the price peaks from the model are of a much closer magnitude to the historic data. The actual values of $\beta$ and $\alpha$ must be determined such that the complete time series of model electricity prices reflects the historic market data.

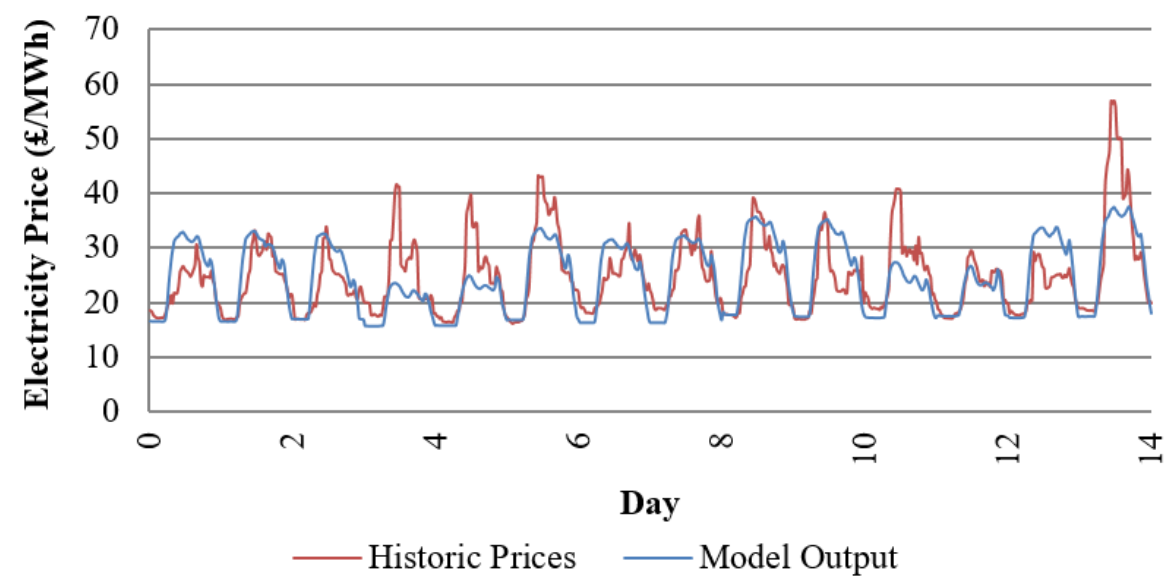

Figure 5.15 Model output and historic electricity prices with high generator efficiencies for first two weeks of August 2007, $\beta=2$

A further feature evident from Figure 5.15 is the extended duration of the price peaks in the model compared to the historic data. This was improved by replacing the linear supply curves of the marginal generators with the hyperbolic function described by equation (5.11). This was expected to better represent the complexities of supply curve, such as differing ages and efficiencies of plant within each class of generation [92], than the linear approximation.

$$
P E=\Pi_{\chi}\left[1+\frac{\Pi_{x+1}-\Pi_{\chi}}{\Pi_{\chi}} \frac{\cosh \left(\frac{P x}{C_{\chi}}\right)-1}{\cosh (1)-1}\right]
$$

The change this made to the generator supply function is shown in Figure 5.16. For both price functions, $\beta=1$. Equation (5.12) was used to calculate the price of electricity for the third marginal class of generator in the hyperbolic supply curve. This incorporates the hyperbolic function in equation (5.11) and the uplift described by equation (5.10).

$$
P E=\Pi_{x}\left[1+\frac{\beta \Pi_{\chi+1}-\Pi_{\chi}}{\Pi_{\chi}} \frac{\cosh \left(\frac{P_{x}}{C_{\chi}}\right)-1}{\cosh (1)-1}\right]
$$


The calibration of the uplift function and the final values of the coefficients, $\alpha$ and $\beta$, used in the model are defined in the next section.

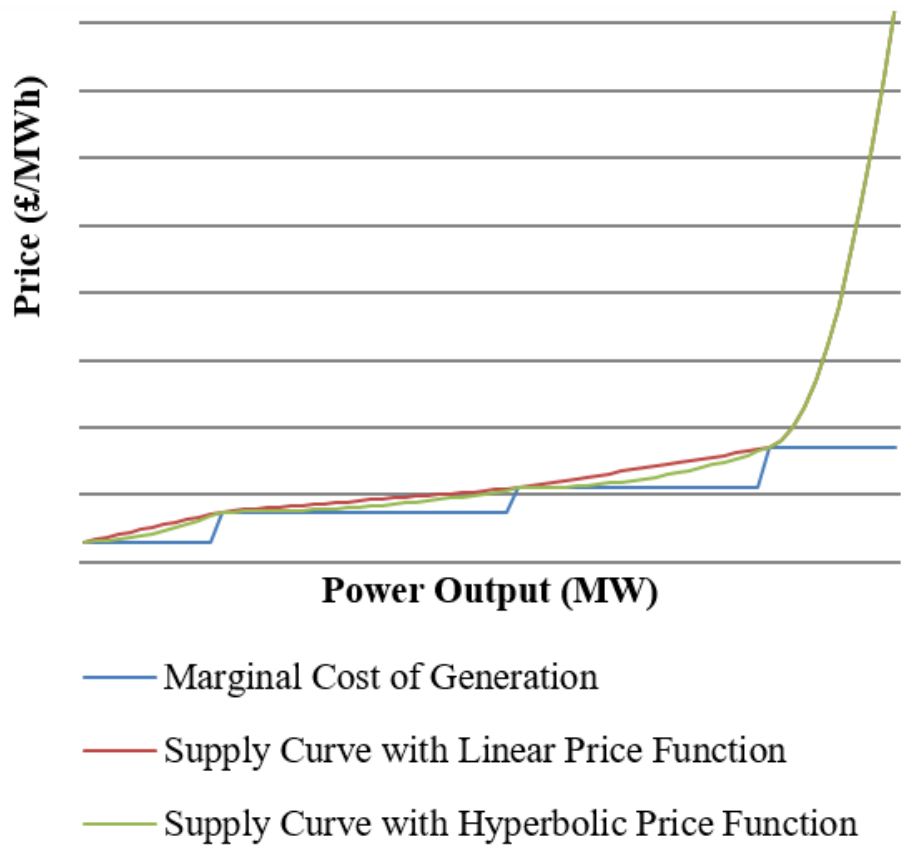

Figure 5.16 Supply curve with linear and hyperbolic price functions for marginal generators

\subsection{Model Calibration}

As discussed in the previous chapter, a number of characteristics of the electricity price time series affected the EES revenue including the frequency and magnitude of peaks and troughs as well as the mean price. The uplift coefficients, $\alpha$ and $\beta$, were calibrated to minimise the difference between the revenue calculated from historic and simulated electricity prices using the storage arbitrage model and through inspection of the electricity price time series from 2005-2007. Using revenue and inspection, rather than a single statistical measure from the time series, allowed multiple characteristics, which may have impacted the operation of EES, to be accounted for.

Alternative approaches used to calibrate uplift functions include assigning a Value of Lost Load (VOLL) to the price when the capacity margin is zero [92] or pricing to ensure that the investment case for peaking plant remains viable [19]. These methods only use the most extreme prices and are most relevant when investigating system scarcity and investment cases for capacity. They introduce challenges of accurately defining VOLL and peaking plant costs, respectively. Furthermore, in this model, installed generation capacities were defined exogenously and were not dependent on simulated electricity prices. They were expected to 
be reflective of future scenarios, but would not necessarily represent the system margin accurately enough to employ these methods.

The arbitrage algorithm described in the previous chapter was used to calculate the annual revenue using simulated and historic electricity prices. The EES device characteristics listed in Table 5.4 were used. The optimum values of $\alpha$ and $\beta$ were found to be 1.62 and 1.41, respectively. The annual revenue calculated using historic and simulated electricity prices is shown in Table 5.5.

Table 5.4 Storage device characteristics used for price model validation

\begin{tabular}{lcc}
\hline Characteristic & Value & Units \\
\hline Storage capacity, $S_{\max }$ & 200 & MWh \\
Charging rate, $Q^{C}$ & 20 & MWh \\
Discharging rate, $Q^{D}$ & 20 & MWh \\
Conversion efficiency, $\eta_{c}$ & 75 & $\%$ \\
Storage efficiency, $\eta_{s}$ & 100 & $\%$ per half hour \\
\hline
\end{tabular}

Table 5.5 Annual arbitrage revenue, 2005-2007

\begin{tabular}{ccc}
\hline Year & $\begin{array}{c}\text { Revenue from historic } \\
\text { market index prices } \\
(\mathfrak{f m})\end{array}$ & $\begin{array}{c}\text { Revenue from simulated } \\
\text { electricity prices } \\
(\mathfrak{f m})\end{array}$ \\
\hline 2005 & 0.892 & 0.839 \\
2006 & 0.831 & 0.914 \\
2007 & 0.875 & 0.845 \\
\hline
\end{tabular}

The results show that the model did not consistently over or under estimate the revenue which was within $10 \%$ of the revenue based on historic electricity prices in every year. An average annual revenue, across the three years, of $£ 0.87 \mathrm{~m}$ was achieved using both historic and simulated prices. Figure 5.17 and Figure 5.18 show a comparison of the historic and simulated electricity prices for the second week in August, 2007 and the monthly average values over the three year period. 


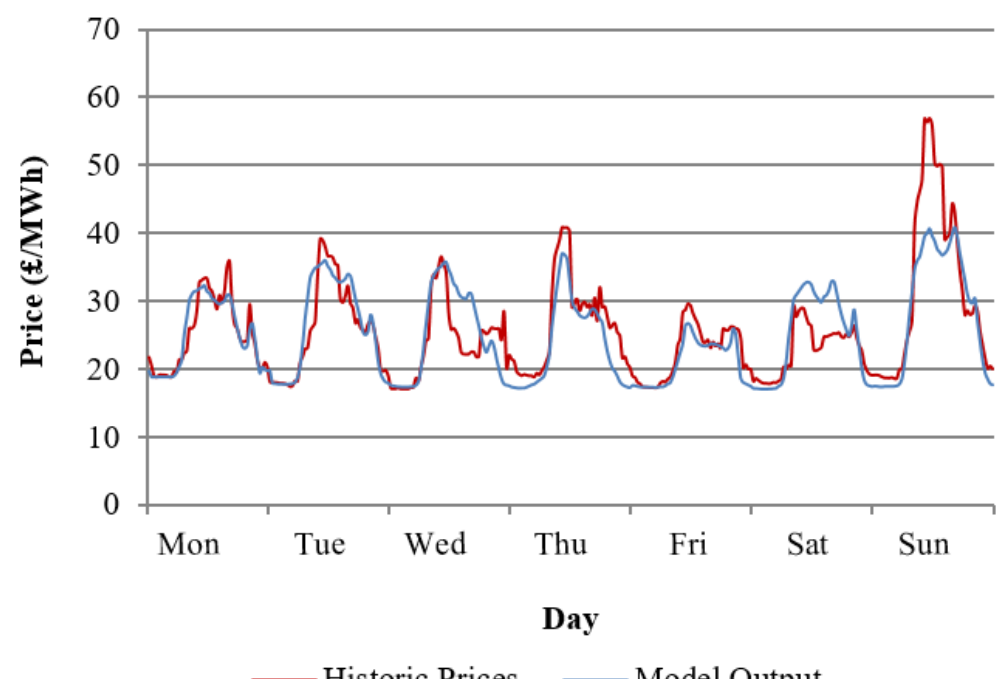

Figure 5.17 Historic and simulated electricity prices for second week in August 2007

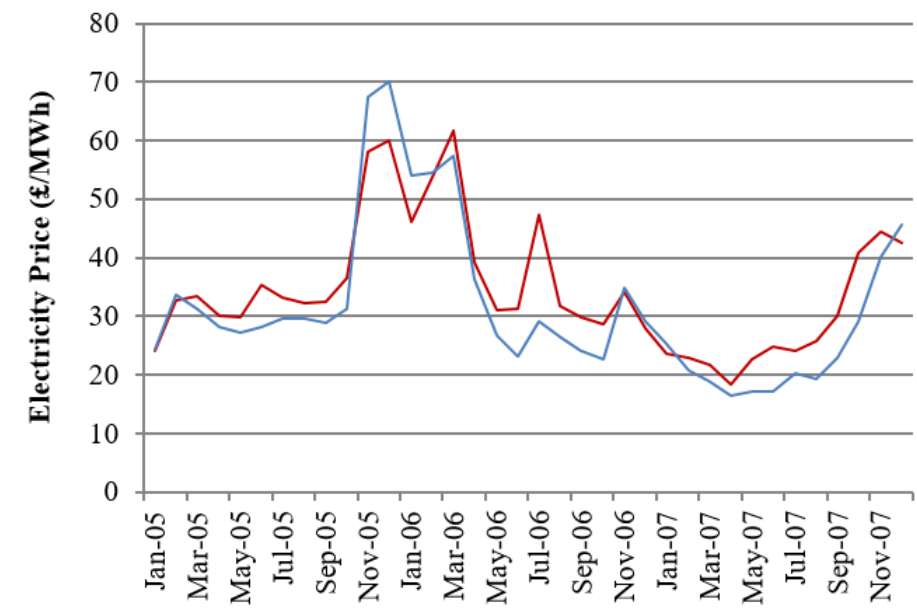

Month

Figure 5.18 Historic and simulated average monthly electricity prices, 2005-2007

These show that the model adequately captured the basic features of the market. Critically, the good fit of the half hourly prices shows that the diurnal variations, to which arbitrage is particularly sensitive [19], were well represented. The model was not expected to reproduce an identical time series of historic electricity prices as there are a number of simplifications compared to the real market including; the assumption of perfect competition, transmission constraints, availability and generator classifications. 
Table 5.6 presents the annual minimum and maximum electricity prices from historic and simulated data. The mean and standard deviation of the daily troughs and daily peaks for each year are also presented. Historically, there was significant variation in maximum daily price but little variation in the minimum daily price.

A comparison of the price statistics shows that the simulations adequately represented the key characteristics of the historic electricity price time series that were significant to arbitrage. As 'one-off' extreme measurements, the absolute maximum and minimum values were less critical than the mean and standard deviation data which was in better agreement. The peak statistics showed better agreement than the trough statistics. The maximum difference between historic and simulated mean peak values in any year was $7 \%$. The maximum difference between the mean trough values for 2006 was 326\%; however, this was an absolute error of only $£ 14.29 / \mathrm{MWh}$. The better representation of peak values was possible through calibration of the uplift coefficients $\alpha$ and $\beta$ whose values did not affect the trough characteristics. The simulated results showed slightly higher standard deviation of peak prices compared to historic data which may have provided additional price volatility to compensate for the minor overestimation in minimum prices. The error in the peak standard deviation was negligible in 2007 where the difference between the mean trough values was also smallest.

The model should be validated using historic data for years which were not used to calibrate the uplift coefficients; however, data at a sufficiently high temporal resolution could not be sourced for all variables out with the years 2005-2007. The wind speed model data was available from 2001-2011. Prior to 2005, there were significant gaps in the demand data available from National Grid and, from 2008-2011, daily gas prices could not be sourced. Utilities have long term contracts with fixed gas prices to hedge against the risks of price variation and it could be argued that publically available quarterly gas prices would be sufficient. However, when using fixed quarterly gas prices in the model, the intra-weekly price variation (and intra-day variation) to which arbitrage is particularly sensitive was not adequately represented. It is likely that while utilities make use of long term contacts for forward and future electricity production, they inevitably trade in the spot gas market to make up shortfalls and sell surplus; these actions feed through into the power exchange. This effectively means that the generators' marginal costs are reflected by the short term variations.

Ideally, more recent years with a higher penetration of wind power would be used for validation. In recent years, National Grid has published wind power output from wind farms connected to the transmission system. However, this is not appropriate to use as it does not 
adequately capture the approximately $1 \mathrm{GW}$ of embedded wind generation. These challenges in sourcing accurate data make it difficult to truly validate the price model. As such, the uplift coefficients may produce electricity prices reasonably for the years for which they were calibrated (2005-2007), but it would be misleading to argue that they would provide an accurate representation of the magnitude of the price spikes in future years. The shape of the price profile is, however, well represented by the model, as shown by Figure 5.17. As a result, while the absolute values of electricity prices may not be accurate for future scenarios, the resulting trends in arbitrage revenue investigated in the following chapter should remain valid. The results have been used to identify patterns of increasing or decreasing revenue and have deliberately not been compared to other costs or other revenues in light of this. 
Table 5.6 Annual peak and trough electricity price statistics from historic and simulated data

\begin{tabular}{lcccccc}
\hline Year & \multicolumn{2}{c}{$\mathbf{2 0 0 5}$} & \multicolumn{2}{c}{$\mathbf{2 0 0 6}$} & \multicolumn{2}{c}{$\mathbf{2 0 0 7}$} \\
\hline \multirow{2}{*}{ Price Statistic (f/MWh) } & Historic & Simulated & Historic & Simulated & Historic & Simulated \\
Minimum Price & Data & Data & Data & Data & Data & Data \\
Daily Trough Mean & 11.86 & 17.38 & 1.09 & 8.62 & 5.92 & 9.02 \\
Daily Trough Standard Deviation & 23.18 & 28.41 & 4.38 & 18.67 & 17.06 & 13.23 \\
Maximum Price & 4.41 & 6.44 & 6.70 & 5.95 & 4.47 & 4.11 \\
Daily Peak Mean & 476.91 & 617.18 & 414.71 & 513.60 & 553.30 & 451.06 \\
Daily Peak Standard Deviation & 67.49 & 72.26 & 69.93 & 70.27 & 60.50 & 63.32 \\
\hline
\end{tabular}




\subsection{Future Electricity Prices}

In future energy scenarios a number of variables will change including generation capacity, fuel prices and demand. The specific scenarios investigated are presented in the following chapter. This section describes the method used to generate time series of fuel and carbon prices, wind power output and demand.

\subsubsection{Fuel and Carbon Prices}

Average annual prices for gas, coal, uranium and carbon were specified for each future energy scenario. Time series data from a historic base year was scaled to match the average future fuel prices to ensure that intra-annual volatility was maintained. The fuel price for each half hour period, $F_{t}$, was calculated according to:

$$
F_{t}=\frac{\bar{F}}{\bar{f}} f_{t}
$$

where $\bar{F}$ is the average price of the fuel, $\bar{f}$ is the average price of fuel from the base year and $f_{t}$ is the base year fuel price at time $t$. The carbon prices were calculated using the same method.

\subsubsection{Wind Power Output}

Several approaches have been taken in the literature to produce aggregate time series for future wind power output. Broadly, a spatial distribution of wind farms is assumed and historic wind speed profiles applied to determine the aggregate power output using a method such as that described in Section Error! Reference source not found.. Techniques vary in their ssumptions of future wind farm locations. Grünewald [11] used an optimisation algorithm to position wind capacity in geographically diverse areas. This did not consider planning restrictions or network constraints, both of which are critical factors in the development of wind farms. This is unlikely to be a realistic representation of future distributions. Green and Vasilakos [95] assumed that offshore installations would be focussed in areas where National Grid was intending to upgrade transmission lines and placed additional capacity in regions which aligned with these locations. This would not necessarily be the optimum future position and may, equally, not be the final positions where wind farms are deployed. Onshore, installed wind capacity was scaled at existing locations for future years. This assumed that the spatial distribution in the future would be the same as it is currently. Here, different approaches were taken to onshore and offshore wind farm locations described below. 


\section{Onshore}

Two scenarios of onshore wind farm locations were investigated. Scenario 1 was based on the historic locations of wind farms in GB. The DECC RESTATS planning database [242] was used to identify the location and capacity of all wind farms which were operational by 2013 . The locations are show on the left hand map in Figure 5.19 with the right hand heat map weighted by installed capacity. The total installed capacity was $6.7 \mathrm{GW}$. Scenario 2 was based on all onshore wind farms listed in the DECC RESTATS planning database in 2014 [242]. This lists all renewable electricity projects in the UK with an intended installed capacity greater than $1 \mathrm{MW}$ from inception through to operation. Although many projects listed may not be built, these represent practical locations which developers are considering and it is reasonable to assume that some form of feasibility study would have been carried out prior to listing a project in the database. As such, the projects listed represent realistic options for future onshore wind farm locations. The locations are shown in Figure 5.20 (left) and weighted by installed capacity (right). The total installed capacity for Scenario 2 was $19.0 \mathrm{GW}$, which includes the $6.7 \mathrm{GW}$ already operating.

The spatial distribution of wind farms in Scenario 1 was relatively diverse with the highest installed capacity focussed slightly in southern Scotland. In Scenario 2, the capacity was increased by over $200 \%$. Although the locations appear to be spread throughout GB, the relative increase in southern Scotland is largest with some additional weighting of capacity in west Wales.

The aggregate wind power output per unit was calculated using the method described in Section Error! Reference source not found. for both scenarios using wind speed data from 005, 2006 and 2007. This base year, from which the wind speeds were used, was termed the wind year. The time series output for Scenario 1 was plotted against Scenario 2 for each year and the results are shown in Figure 5.21. 


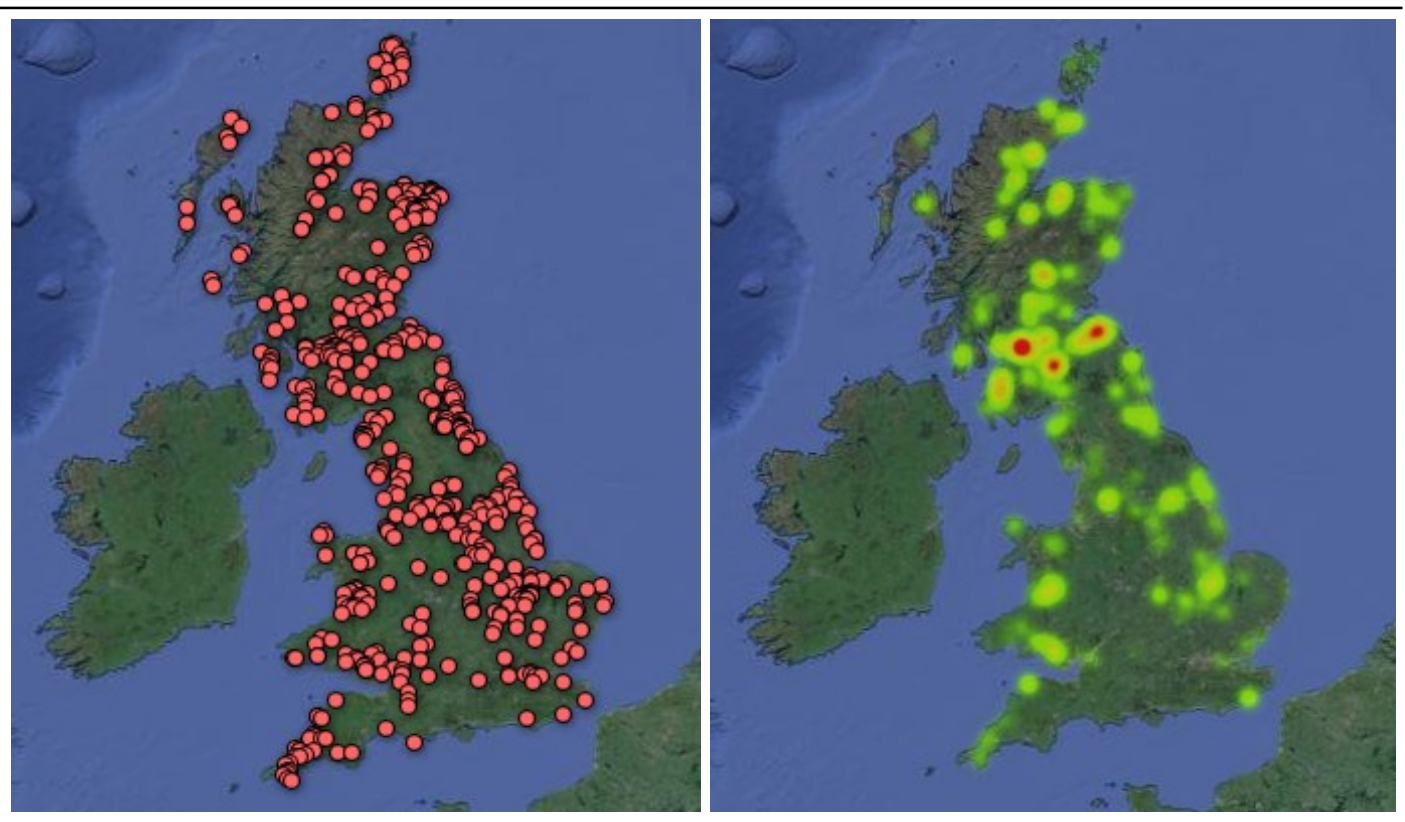

Figure 5.19 Location of onshore wind farms used for Scenario 1 (left) weighted by installed capacity (right)
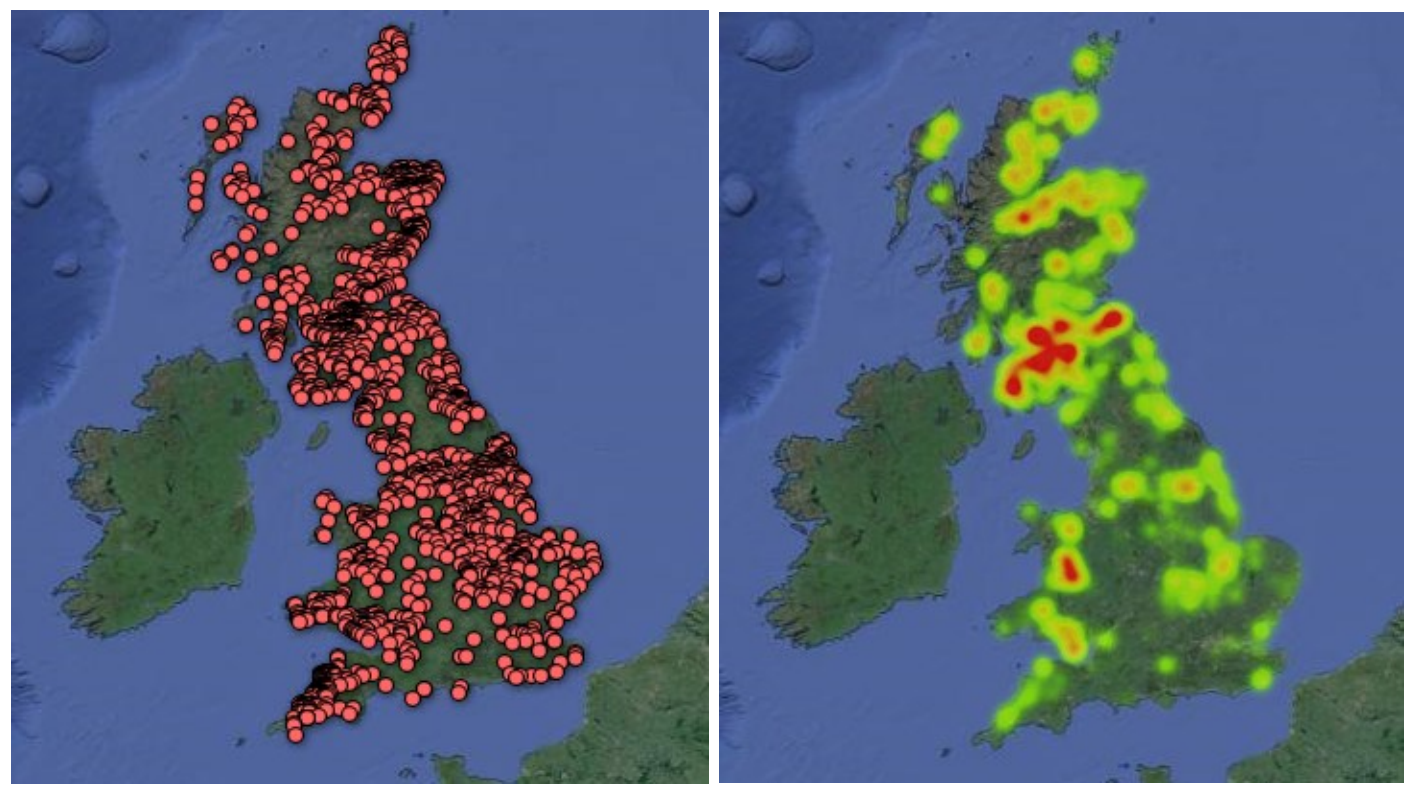

Figure 5.20 Location of onshore wind farms used for Scenario 2 (left) weighted by installed capacity (right) 


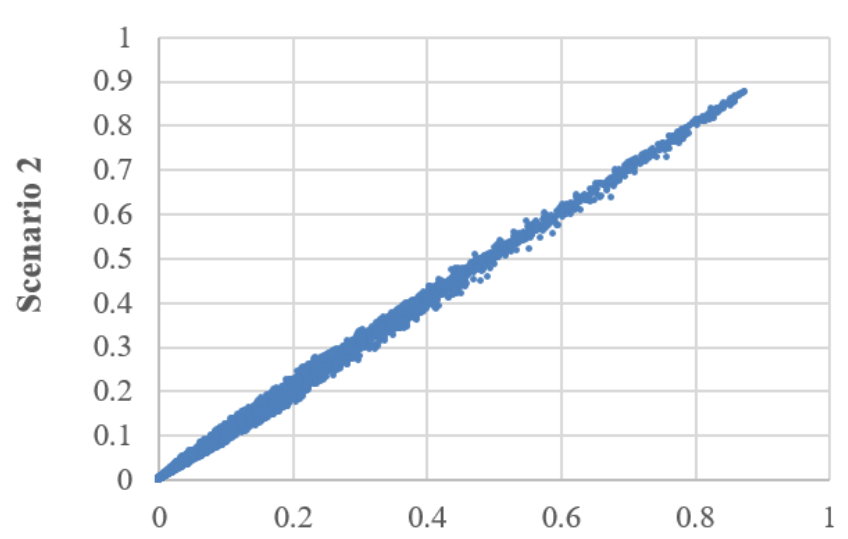

Scenario 1
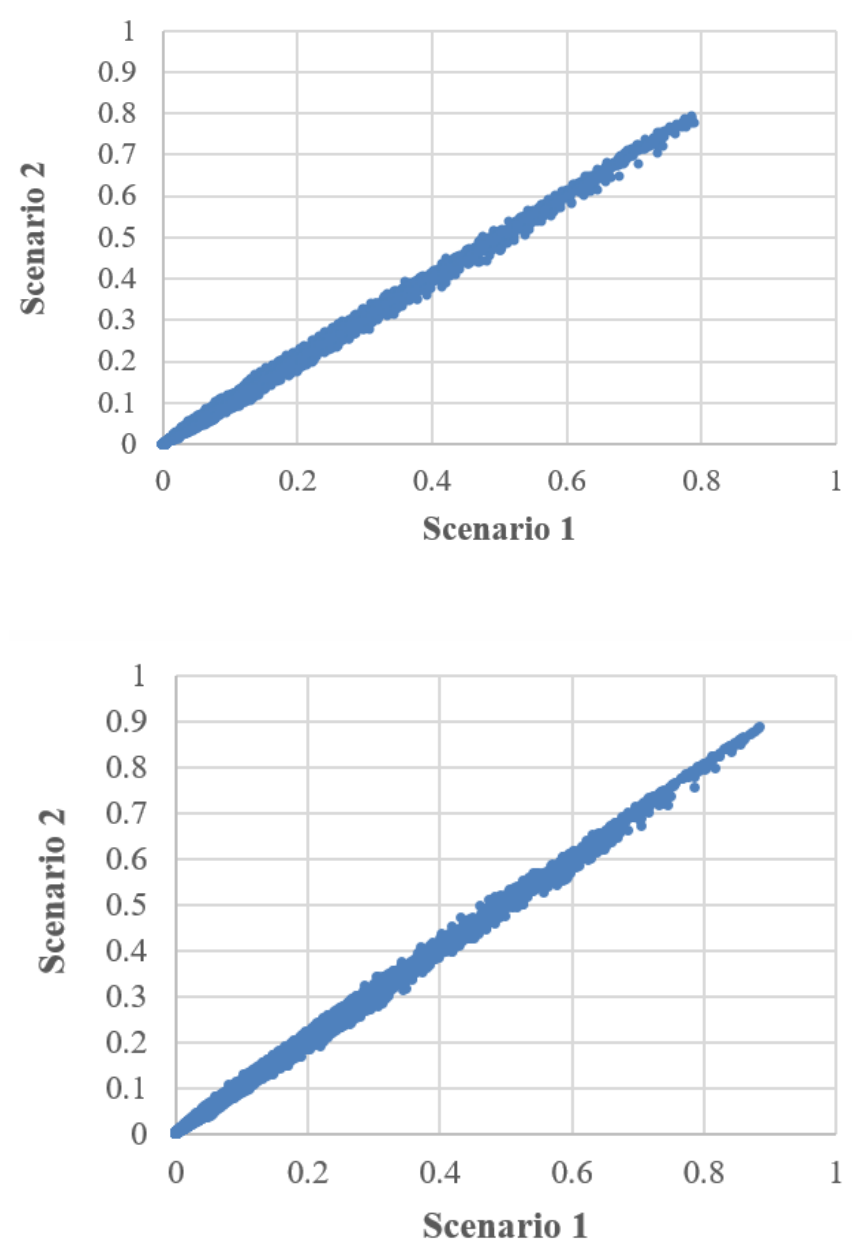

Figure 5.21 Comparison of aggregate onshore wind power output per unit from Scenario 1 and Scenario 2 with 2005 wind year (top) 2006 wind year (centre) and 2007 wind year (bottom)

The graphs of each wind year exhibited the same slope of 1.01, indicating very similar output for both scenarios despite the slight change in spatial distribution of capacity between Scenario 1 and Scenario 2. The coefficient of determination (R-squared) from a linear regression was 
0.998 or greater for every wind year investigated. As there was little change in output between the scenarios, for future energy scenarios, the installed capacity of onshore wind was scaled linearly from the base year.

\section{Offshore}

Historically, installed offshore wind capacity has been significantly less than onshore; however, that is anticipated to change in the future. Three scenarios of spatial distributions of offshore wind were investigated. Scenario 1, shown in Figure 5.22, was based on the actual operational capacity in 2013 totalling 3.65GW installed. Scenario 2, shown in Figure 5.23, was based on all the projects listed in the DECC RESTATS planning database [242] including those which were not yet permitted, constructed or operational; this represented a total installed capacity of $21.0 \mathrm{GW}$. Scenario 3, shown in Figure 5.24, used the same installed capacities and locations in Scenario 2, plus additional capacity to represent wind farms which may be developed as part of the Crown Estate Round 3 seabed leasing round [252]. The exact locations of wind farms within each zone specified in the leasing round were approximated to match the anticipated capacity which may be developed in that area [253]. The total installed capacity in Scenario 3 was $51.8 \mathrm{GW}$.

Figure 5.22 shows that the historic installed capacity of offshore wind farms is not geographically diverse with capacity concentrated in near shore locations in the north west and south east of England. In the future, it is expected that there will be increasing installed capacity in Scotland and sites further offshore as shown in Figure 5.23 and Figure 5.24. The aggregate per unit output for each scenario was plotted against each other scenario and is shown in Figure 5.25 with a wind year of 2005. The results showed similar patterns of correlation when alternative wind years were used. 


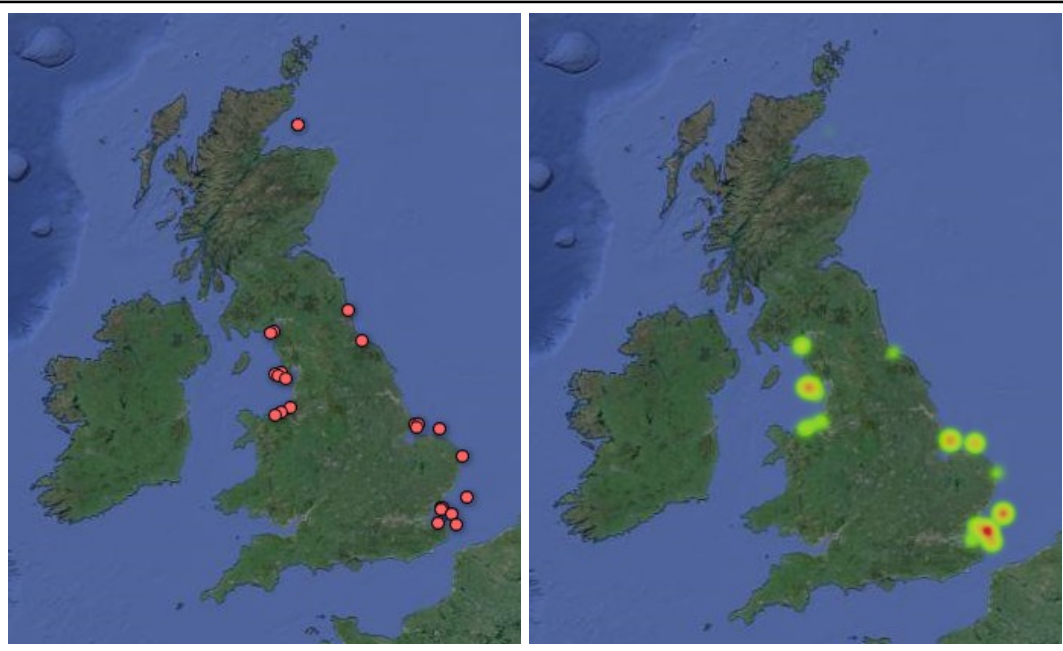

Figure 5.22 Location of offshore wind farms used for Scenario 1 (left) weighted by installed capacity (right)
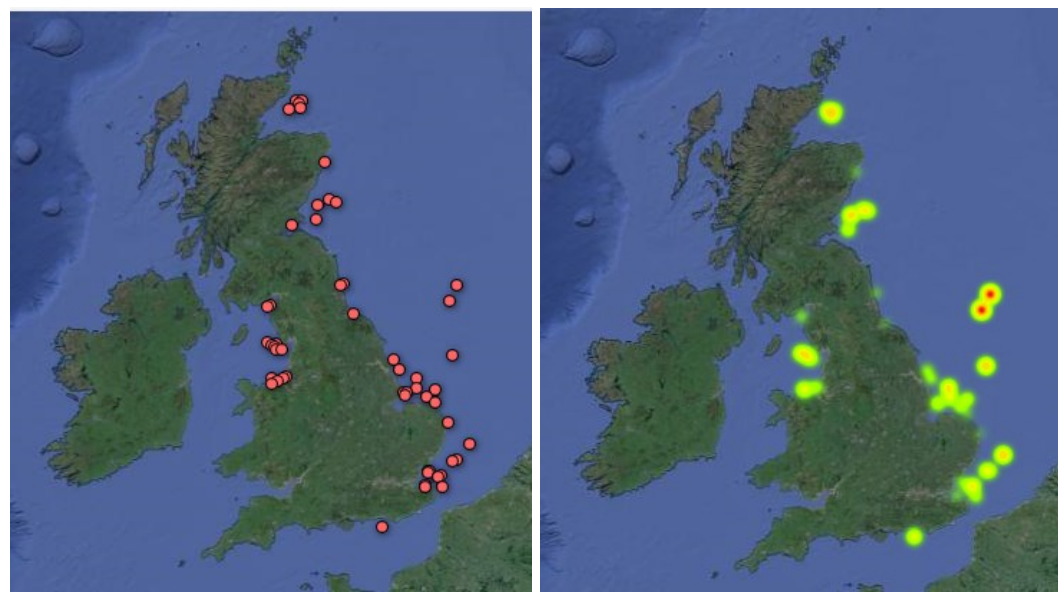

Figure 5.23 Location of offshore wind farms used for Scenario 2 (left) weighted by installed capacity (right)
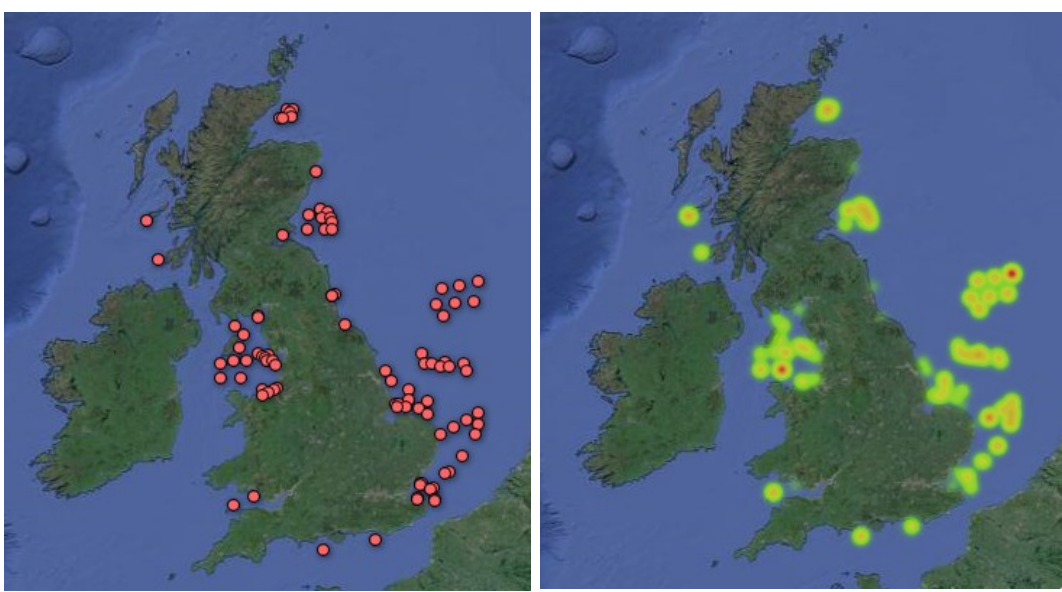

Figure 5.24 Location of offshore wind farms used for Scenario 3 (left) weighted by installed capacity (right) 


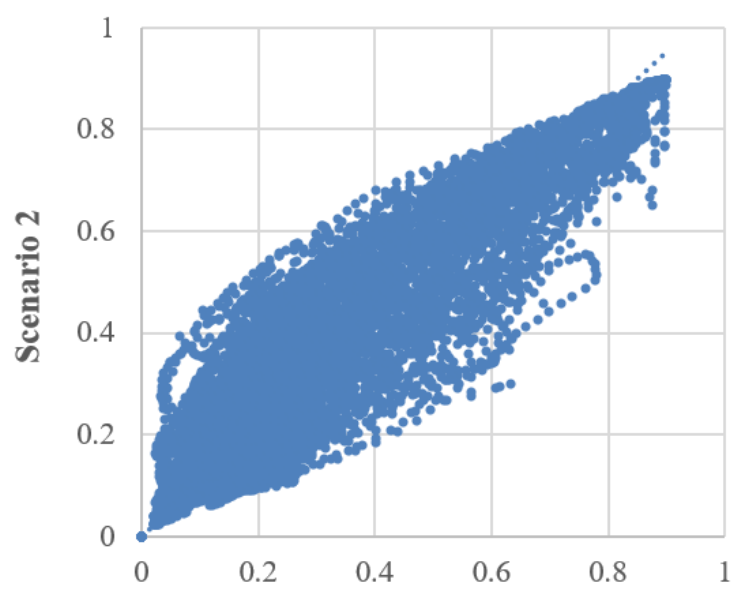

Scenario 1

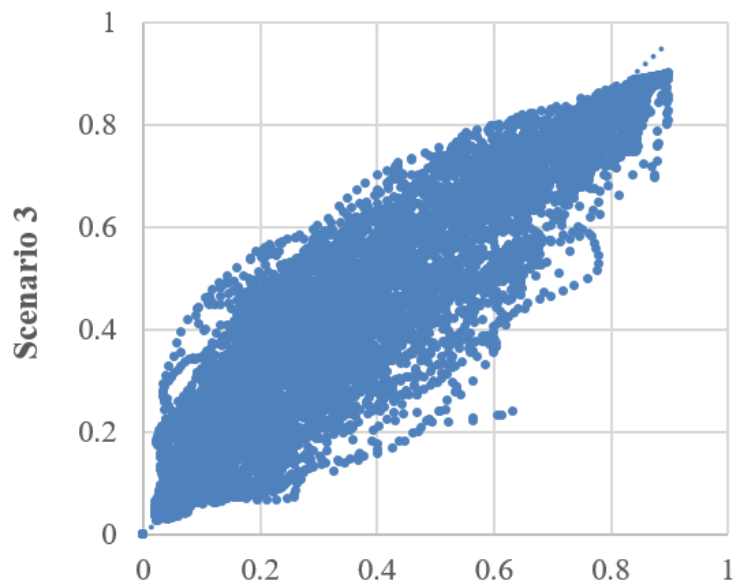

Scenario 1

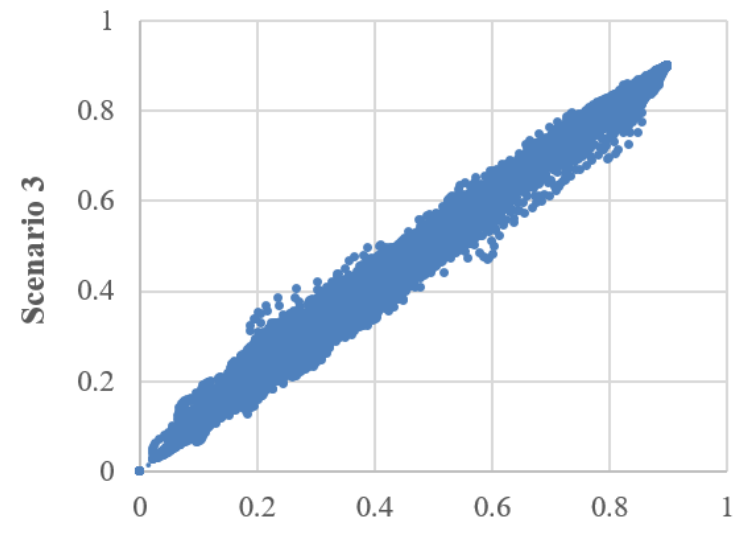

Scenario 2

Figure 5.25 Comparison of aggregate offshore wind power output per unit for different scenarios with 2005 wind year 
The output from Scenario 1 showed little correlation with the output from Scenario 2 or 3 as Scenario 1 had significantly less diverse locations of offshore capacity. Scenarios 2 and 3 had somewhat similar spatial distributions and the per unit output from these scenarios showed some linear correlation; however the relationship was signficantly weaker than that shown in Figure 5.21 of the onshore scenarios. This demonstrates the importance of the assumed wind farm locations for offshore capacity. Fewer, larger capacity wind farms make up the offshore fleet and so the location of each has more impact on the aggreagte power output compared to numerous, smaller capacity farms forming the onshore fleet which have less individual influence.

Offshore wind power output would have most impact in the future scenarios with a large capacity of installed offshore wind. These are likely to be best represented by Scenario 3 . Hence, the offshore farm locations in Scenario 3 were scaled to match the capacity required for future energy scenarios.

\subsubsection{Demand}

The average and peak electricity demand were specified for each future energy scenario. Time series demand data from each historic base year was transformed to match the future average and peak demand values. Equations (5.14) and (5.15) were used to calculate the future electricity demand at any time period, $D$

$$
d_{1}=d_{t}-\bar{d}+\bar{D}
$$

where $d_{l}$ is an interim time series of demand, $d_{t}$ is the base year demand at time $t, \bar{d}$ is the base year average demand and $\bar{D}$ is the average demand in the future scenario

$$
D=\left(d_{1}-\bar{D}\right) \frac{D_{\max }-d_{1}}{d_{\max }-d_{1}}+\bar{D}
$$

$D_{\max }$ is the future peak demand and $d_{\max }$ is the peak of the interim time series. This approach is similar to that used by Eager [92] where a historic time series was scaled by a growth rate each year to reflect changes in demand but maintain patterns of use. Eager's approach assumed a regular growth rate which was not necessarily representative of real changes in demand due to changing global and economic factors. It is expected that scaling demand to match peak and average values from future energy scenarios would provide a better representation than applying a generic growth rate. Green and Vasilakos also used a similar method in [94] applying a fixed growth rate of $1.1 \%$ per year. It is unlikely that this fixed rate of change would be representative of real demand growth as step changes in the use of heat pumps, electric 
vehicles and energy efficiency improvement (for example) would lead less uniform changes. The limitation of the method used here, and by previous authors, is that it does not represent changes in demand behaviour which may develop in the future. These will be different for different scenarios, for example, depending on demand side response and the uptake of electric heat pumps and electric vehicles, which have distinct demand profiles [254]. Creating a bottom up aggregate demand curve would give a more realistic output than simply scaling historic demand; however, this detail would introduce additional complexities and is out with the scope of this thesis.

Unless otherwise stated, the same base year was used for fuel and carbon prices, wind speed and demand in an attempt to maintain any relationship between fuel costs, weather and consumer behaviour.

\subsection{Discussion}

\subsubsection{Wind as a Price Setter}

The analysis models wind as negative load; however, in the UK, it is only embedded generation below 50MW capacity which truly behaves in this way. Larger wind farms forecast their output in advance and trade in forward markets. Instead of contributing to negative demand, wind farm output would adjust the supply function for each half hour period. Thermal generation would be required to respond not only to changes in demand, but also to wind forecast errors. Moreover, the assumption that thermal plant is dispatched in merit order is a simplification. In reality, the network responds in different ways to variation in wind and demand and, consequently, there are different cost implications.

If there was a significant market share of wind and wind generators were paid a subsidy for electricity, generators may not bid to produce electricity at zero marginal cost. These effects would change the electricity price at the lower end of the supply curve and arguably may drive it negative. For example, this could be up to the equivalent value of any subsidy which could be claimed. While this would have some impact on the results, trends in arbitrage revenue would be unlikely to be affected as the magnitude and frequency of price spikes would remain largely unchanged. Furthermore, recent analysis has shown that, in the UK, negative prices are not a significant feature of the market in any year through to 2040 [255].

\subsubsection{The Impact of Errors}

The price time series produced by the model is the out turn price and represents a projection of future prices rather than a forecast. The errors in the model would be expected to be 
consistent across a range of scenarios and small compared to uncertainties associated with other inputs such as future gas prices and carbon prices and the variation in wind power output from one year to the next. Due to the high level of uncertainty associated with future energy scenario inputs, the results are used to identify trends in revenue over several years, rather than to compare the absolute value with storage costs and assess the return on investment for a storage device in the future. It is believed that a lack of explicit treatment of forecast error within the modelling will therefore not have a substantial impact on the results and conclusions.

\subsection{Chapter Summary}

This chapter has presented the development of a model used to simulate future electricity prices. Historically, prices have been driven by gas prices and demand cycles. Wind power has made little contribution to demand and variations in output have had little impact on price. In the future this is expected to change as large penetrations of intermittent renewable power are connected to the grid. The impact of these changes on electricity prices will have implications for EES revenue.

A fundamental electricity price model was developed assuming perfect competition in the electricity market. Thermal generators were grouped into four categories and stacked in merit order of increasing marginal cost to form the supply curve. An exponential uplift in price was applied to peaking generators. Wind power output was calculated and deducted from consumer demand to form a net demand curve, assumed to be completely inelastic. The model was calibrated using the revenue achieved through price arbitrage from 2005-2007. This was considered more appropriate than using any individual statistical measurement from the price time series as a number of characteristics, including the frequency and magnitude of price spikes as well as average prices, would affect EES revenue.

In the following chapter, this price model is coupled with the arbitrage model described previously and used to investigate the impacts of changing variables in future energy scenarios on EES. 


\section{Chapter 6 Results and}

Discussion

Chapter 4 demonstrated the high costs and project risks facing novel EES technologies. It also implied there were additional risks facing EES as a result of changing market structures and environmental and political changes between markets and between years. This chapter couples the arbitrage model outlined in Chapter 4 with the electricity price model described in Chapter 5 to investigate the impact of specific market variables on EES. The output of a time series of electricity prices was used as an input for the arbitrage optimisation. This soft coupling of models is adequate given the assumptions described in previous chapters and has been used in this way previously [11]. Initially a sensitivity study is carried out to evaluate the impact of independent factors on arbitrage revenue and EES operation. Following this, specific scenarios are investigated to examine plausible future combinations of these market variables.

Selected results from Section 6.2 have been published in a journal paper by Dunbar et al [211]. Portions of Section 6.1 were presented at the Offshore Energy and Energy Storage Symposium, 2015 [256] and have been accepted for publication in the Institution of Engineering Technology journal, Renewable Power Generation: Offshore Energy Storage Special Issue. Copies of these manuscripts are available at the end of this thesis.

\subsection{Sensitivity Study}

The electricity price model, described in Chapter 6, produces a time series of electricity prices given the inputs of generation capacity, fuel prices, carbon prices, demand and wind speed profiles. This time series was used as the input for the arbitrage model to carry out a revenue sensitivity study. The potential future value of many of the variables is highly uncertain and investors will need to understand how EES performs across a range of outcomes. Sensitivity studies are a useful method for appraising uncertainty, as discussed in Chapter 5, and allow the influence of individual factors to be investigated. It is critical that the relative importance 
of key EES characteristics is understood in the context of uncertain market variables. This will enable technology choices to be developed which are robust to changing market conditions rather than solutions which are 'optimal' in today's market, but which may become redundant as the electricity sector evolves.

The sensitivity study was conducted by individually adjusting key external parameters from initial baseline values to investigate the impact of each factor on arbitrage revenue. These parameters included gas and carbon prices, average demand, and installed wind capacity. In a future energy system these would not vary independently of each other and additional variables, such as thermal generation capacity and underlying patterns of demand, would also change; however, these were kept constant to investigate each effect in isolation and gauge its significance. The impact of changing thermal generation capacity is discussed qualitatively. A scenario analysis, with combinations of multiple changing variables, was subsequently carried out and results are presented in Section 6.2.

The baseline case used historic data from 2006, including time series of fuel [28] and carbon [237] prices, generation capacity [25], demand times series [29] and wind speed time series [30]. This ensured a degree of coherence in the underlying data. The installed capacity of each class of generator is shown in Figure 6.1. Typically coal generation was dispatched before CCGT in the merit order. The installed wind capacity was less than $3 \%$ of the total generation capacity.

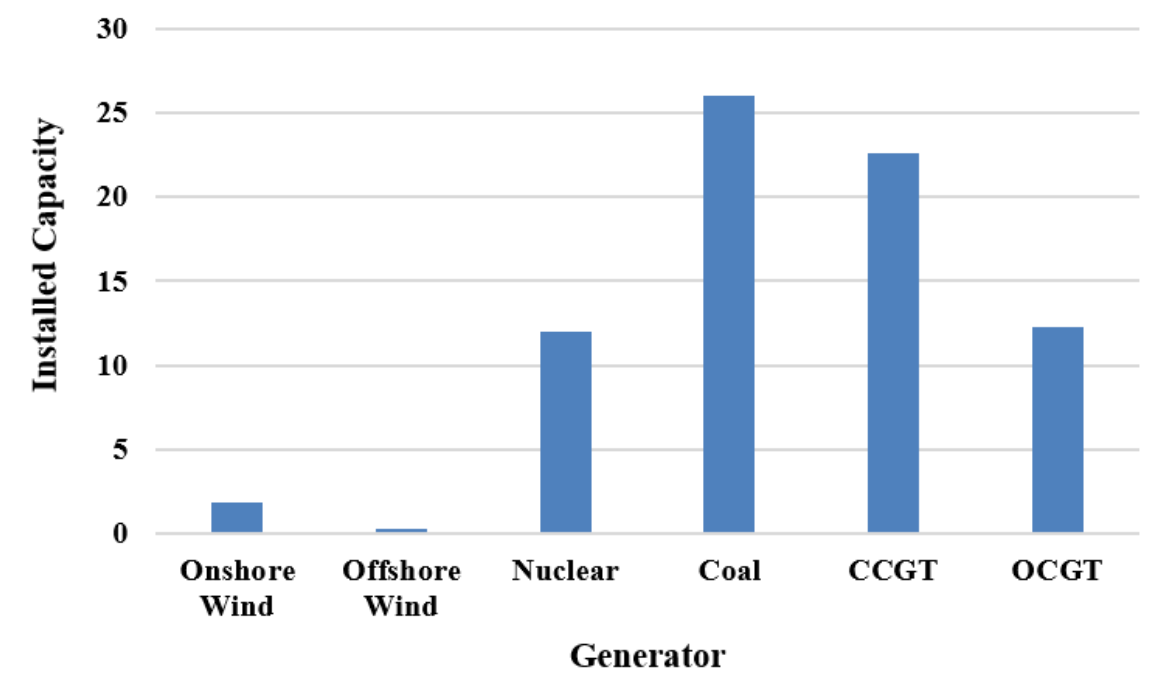

Figure 6.1 Installed generation capacity 2006 [25] 
Initially, the EES characteristics were fixed at the baseline values listed in Table 6.1. These depict a moderate scale device that acts as a price-taker with a power-to-storage ratio of 1:10, reasonable round trip efficiency and no other losses.

Table 6.1 Baseline storage characteristics

\begin{tabular}{lcc}
\hline Storage Constraint & Unit & Value \\
\hline Maximum storage capacity & MWh & 200 \\
Maximum charging/discharging rate & MW & 20 \\
Conversion efficiency (round trip) & $\%$ & 75 \\
Storage efficiency & $\% /$ day & 100 \\
\hline
\end{tabular}

\subsubsection{Market Variables}

\section{Gas price}

The National Grid Future Energy Scenarios [257] provide information on plausible pathways for future, uncertain energy landscapes out to 2035. The 2014 Future Energy Scenarios estimate that, in a high price scenario, gas prices would be slightly less than $£ 1 /$ therm by 2035 . The arbitrage algorithm was therefore run for simulated electricity prices with average gas prices increasing from $10 \mathrm{p} /$ therm to $£ 1 /$ therm. For each average gas price, the remaining inputs from the baseline year were used and the electricity price simulated at each half hour for 365 days to enable the annual revenue to be determined. The time series of historic gas prices from the 2006 baseline year was scaled in each case to maintain a constant intra-annual volatility of gas prices for each run.

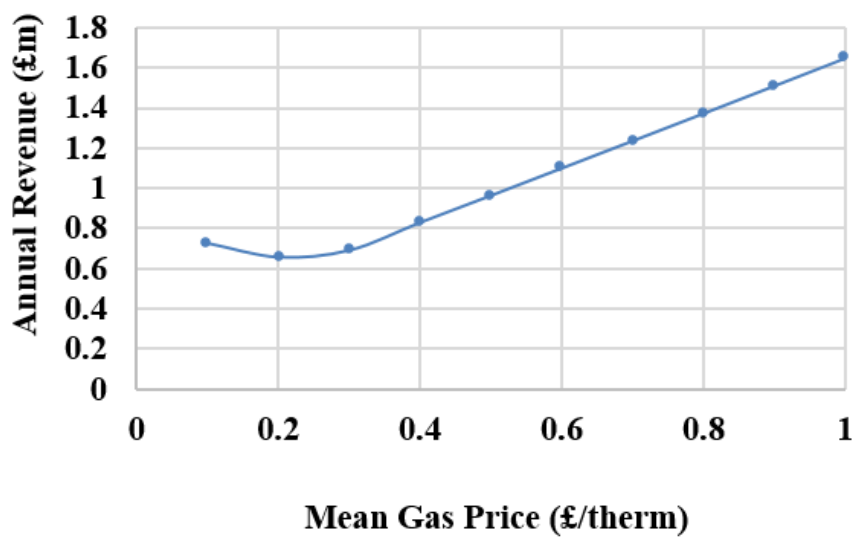

Figure 6.2 Annual revenue from simulated electricity prices with increasing gas price 
The results, shown in Figure 6.2, indicate that for gas prices greater than 30p/therm the arbitrage revenue increased approximately linearly with gas price. Gas turbines were the most expensive thermal generators dispatched and their marginal prices set the daily peak electricity prices. Figure 6.3 shows the EES device state of charge over a two week period with an average gas price of $40 \mathrm{p} /$ therm and $£ 1 /$ therm. This shows that the optimum operating schedule in both cases was almost identical. The device charged and discharged on a daily basis in line with daily demand cycles. Wind power output had little influence on electricity prices compared to variations in demand as the baseline installed capacity was small. Despite the similar operational pattern, the higher gas price led to a larger daily price spread enabling more revenue to be made during each cycle.

For the lowest gas prices, revenue increased. This was because the lowest gas prices reduced the marginal generation cost of gas sufficiently that it became cheaper than coal for some periods. During these periods, coal was the peaking generator and gas generation contributed to base load during off peak hours. Lower gas prices reduced the price of off peak generation which increased the daily price spread, enabling more revenue to be achieved during a storage cycle.

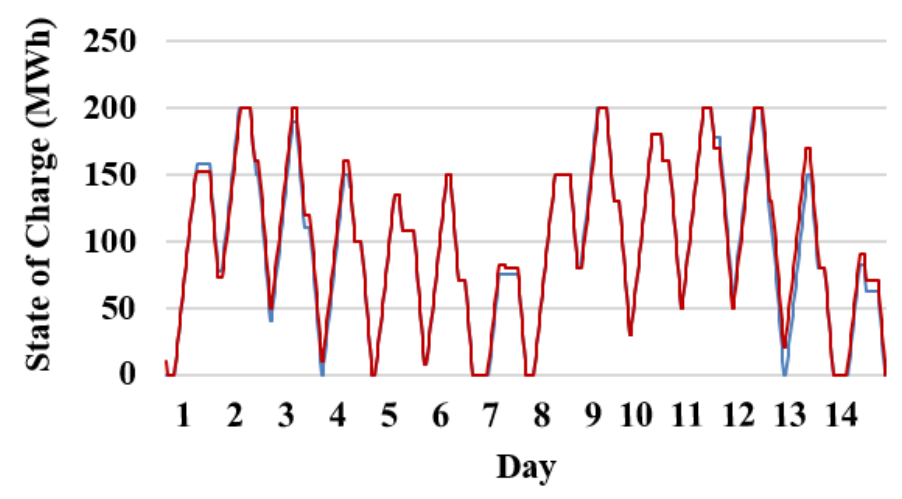

_ Gas Price 40p/therm — Gas Price £1/therm

Figure 6.3 Storage state of charge for final two weeks in January with gas price of 40p/therm and $£ 1 /$ therm

Figure 6.4 shows the marginal generation costs across the year in $£ / M W h$ with an average gas price of $10 \mathrm{p} /$ therm. This shows periods where coal had the highest marginal generation cost and was dispatched as peaking plant. Figure 6.5 shows the marginal generation costs in $£ / \mathrm{MWh}$ with an average gas price of $£ 1 /$ therm, which shows that OCGTs were the most expensive generator for all periods of the year. The variation in marginal costs for each generator type are a result of the time series of fuel prices used. Gas prices were available at a 
daily resolution, while coal prices were only available as monthly averages and nuclear prices as quarterly average prices.

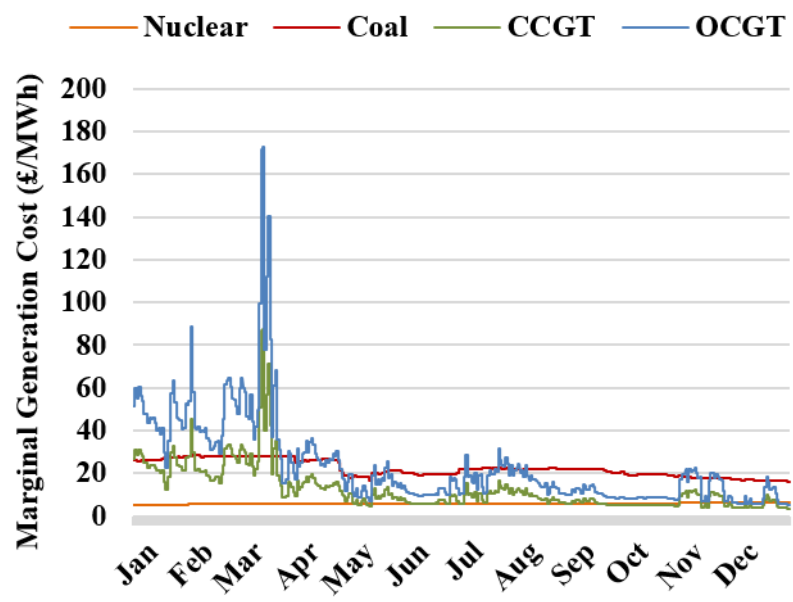

Figure 6.4 Marginal generation costs with average gas price 10p/therm

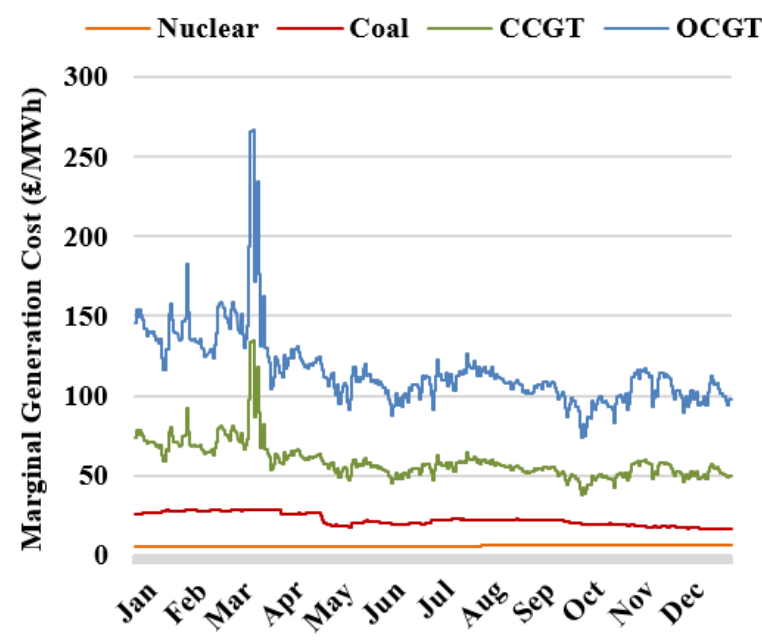

Figure 6.5 Marginal generation costs with average gas price £1/therm

\section{Carbon price}

Historically, the carbon price has always been below £20/tonne [237] and the 2014 National Grid Future Energy Scenarios estimate that by 2035 it could increase to between $£ 30 /$ tonne and $£ 75 /$ tonne [257]. The analysis was repeated for simulated annual time series of electricity prices with increasing average carbon price from $£ 10 /$ tonne $(£ 0.01 / \mathrm{kg})$ to $£ 100 /$ tonne $(£ 0.1 / \mathrm{kg}$ ). The time series of carbon prices within the year was scaled from 2006 to the average value. 


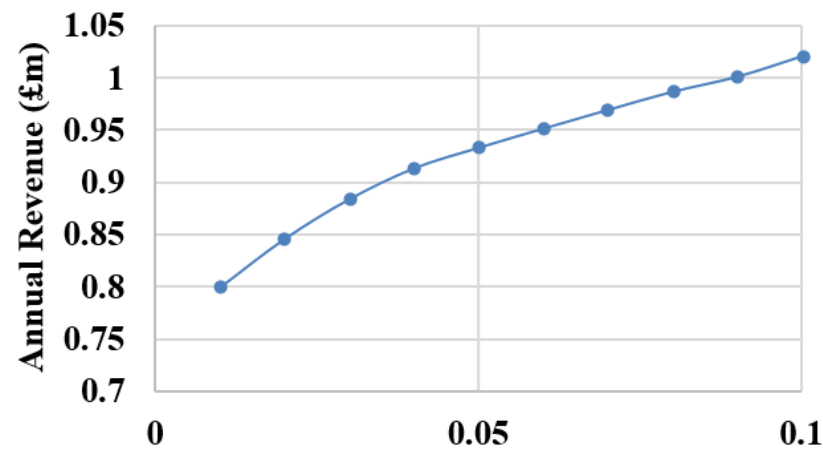

Mean Carbon Price ( $(\mathfrak{k g g})$

Figure 6.6 Annual revenue from simulated electricity prices with increasing carbon price

The results, shown in Figure 6.6, demonstrate that revenue increased with carbon price, albeit at a diminishing rate. Increasing the carbon price increased both gas and coal marginal generation costs, but did not affect nuclear generation costs. For many periods, increasing the carbon price raised the daily peak electricity prices, increasing the price spread and enabling the EES device to gain additional revenue. For some off-peak periods the second marginal generator - commonly coal - was required, which set the off peak electricity prices. Increasing the carbon price increased coal generation costs more significantly than gas generation costs, reducing the price spread during periods where coal was required for off peak generation. As the carbon price increased further the price spread, and opportunity for arbitrage, was reduced during these periods, leading to diminishing gains in revenue.

If the carbon price were to increase further, the marginal generation cost for coal would increase such that it was more expensive than gas generation and the merit order of the generators would switch. This was the case, for example, with a mean carbon price of $£ 0.25 / \mathrm{kg}$. Coal was the third marginal generator for the majority of the year, with CCGT being the second and OCGT, as previously, providing peaking capacity. In this case, the annual arbitrage revenue increased to $£ 1.36 \mathrm{~m}$ as the price spread between peak and off peak prices was magnified. This phenomenon shows that there is not a simple linear relationship between carbon price and arbitrage revenue.

The EES device followed a similar strategy to that shown in Figure 6.3 over the range of carbon prices investigated. The EES revenue was significantly less sensitive to carbon price than to gas price. There was an increase in revenue of less than $30 \%$ with an order of magnitude increase in carbon price (from $£ 10$ to $£ 100 /$ tonne). This compared to an increase in revenue of over $125 \%$ for an order of magnitude increase in gas price (from $10 \mathrm{p}$ to $£ 1 /$ therm). This 
demonstrates the relatively modest influence of the current range of expected carbon prices on arbitrage revenue compared to the impact of changing gas prices.

\section{Demand}

In 2006, average electricity demand was approximately 40GW. It is anticipated that this will remain constant, or may even reduce, in the future as energy efficiency measures are implemented [257]. Alternatively, if large numbers of heat pumps or electric vehicles are deployed, both average and peak demand could significantly increase, changing the pattern of electricity demand. A detailed examination of scenarios of potential changes in the shape of demand patterns is beyond the scope of the sensitivity study but is discussed further in Section 6.3. Retaining the underlying pattern of demand from 2006, and with peak demand kept constant, average demand was increased in $2 \mathrm{GW}$ increments from $30 \mathrm{GW}$ to $50 \mathrm{GW}$ to investigate the impact on EES revenue. The electricity generation capacity was fixed at 2006 levels, so increasing demand represented a reduction in the average capacity margin. In a competitive market, this would lead to increased electricity prices incentivising investors to build more generators. This would, in turn, restore a greater average capacity margin and reduce prices restoring market equilibrium. The static market model used, with exogenously determined generation capacity, does not fully reflect these changes, but allows variations in demand to also be a proxy for the capacity margin.

The results, shown in Figure 6.7 indicate an increase in annual revenue with rising average demand at an increasing rate. For low average demand, representing a high average capacity margin, commonly the low merit order generators were able to serve demand throughout the day. This was delivered by the left hand side of the supply curve discussed in Chapter 6, an example of which is shown in Figure 6.8. In this region prices are low and price elasticity of supply is also low, demonstrated by the shallow curve, resulting in a small price spread. At higher levels of demand, the higher merit order generators including peaking plant were required. This was delivered through generation represented by the right hand side of the supply curve. Here, prices are higher, and price elasticity of supply is also higher, demonstrated by the steep shape of the curve. As a result, for the same daily variation in demand, the price spread was increasingly larger enabling higher revenue to be achieved. 


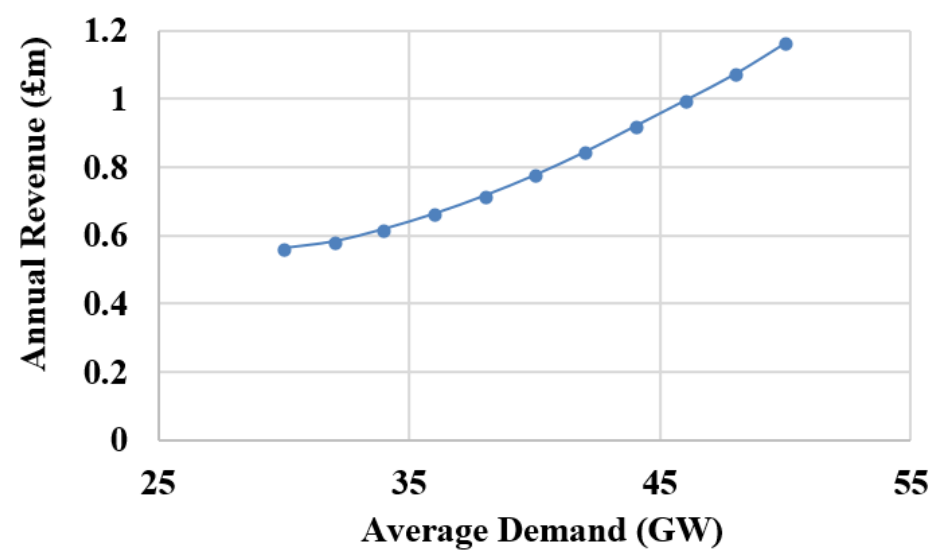

Figure 6.7 Annual revenue from simulated electricity prices with varying average annual demand

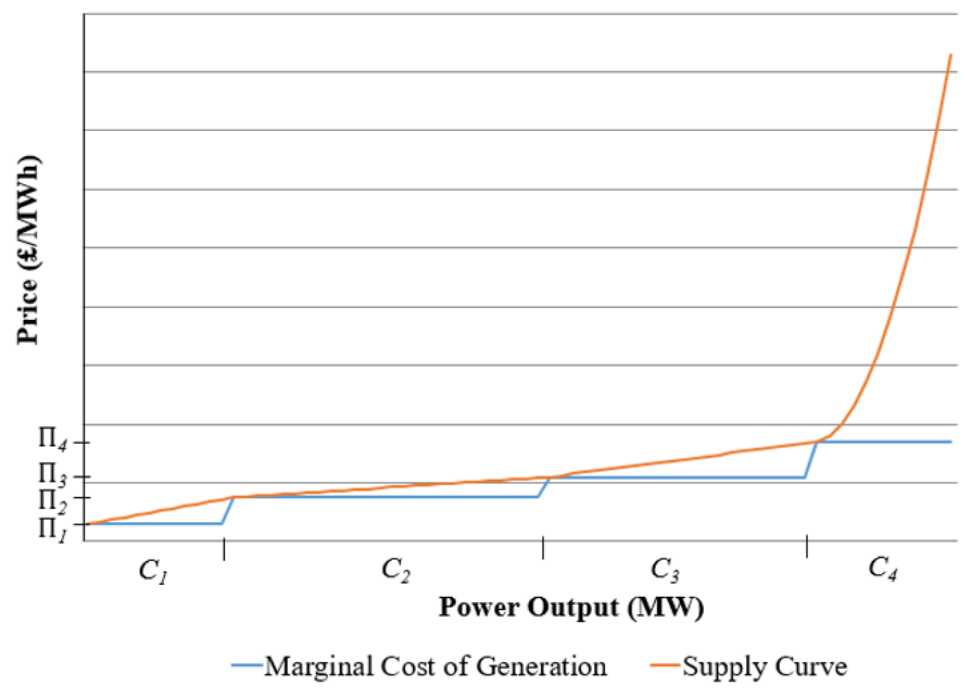

Figure 6.8 Example electricity supply curve

\section{Wind}

The arbitrage model was run for simulated annual time series of electricity prices with increasing installed wind capacity from zero to $40 \mathrm{GW}$. The ratio of offshore to onshore capacity was fixed at 3:2. Again, the remaining inputs, including the wind speed distributions, were taken from the baseline year, 2006. Retaining all other generation capacity as per 2006 (Figure 6.1 ), $40 \mathrm{GW}$ of installed wind represents $35 \%$ of the total generation capacity in GB.

The results, shown in Figure 6.9, indicate that the revenue reduced as the wind capacity increased, but tending to an asymptote. This was due to lower variation in electricity price with increased wind power output. Figure 6.10 shows the wind power output for two weeks in 
February with $40 \mathrm{GW}$ of installed wind capacity. Figure 6.11 shows the electricity prices for the same period for scenarios with zero and $40 \mathrm{GW}$ of installed wind capacity.

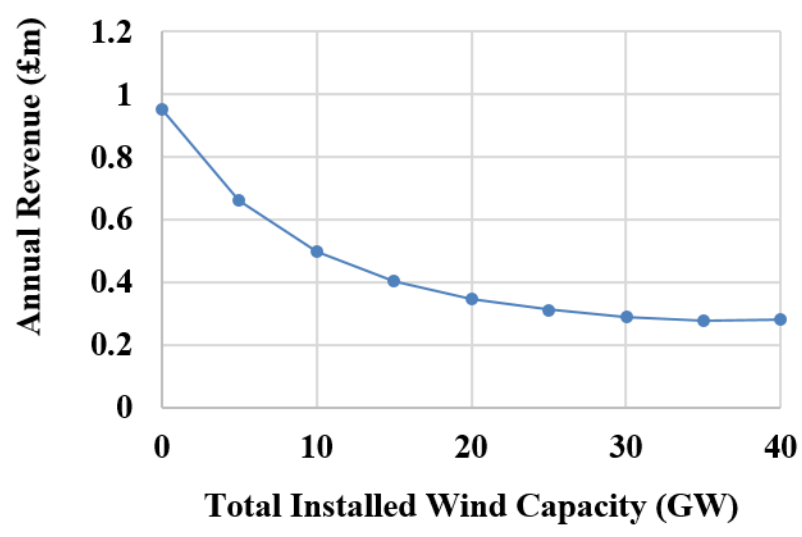

Figure 6.9 Annual revenue from simulated electricity prices with increasing penetration of installed wind capacity

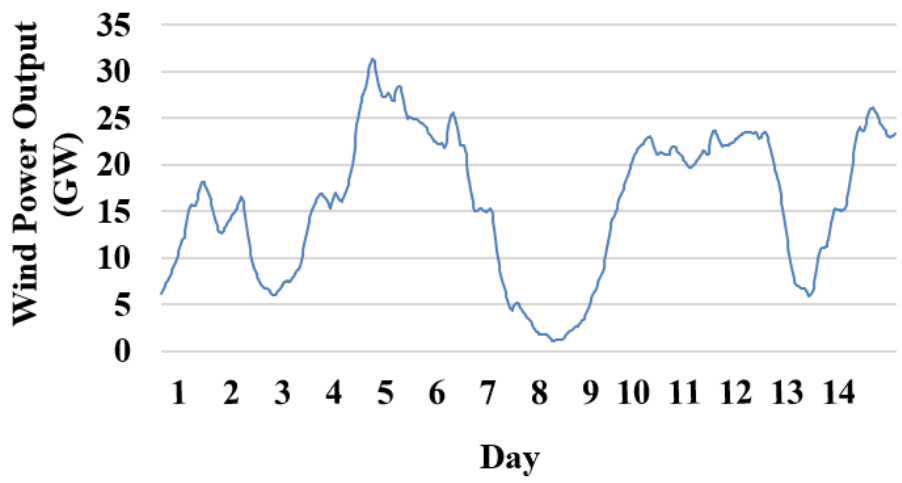

Figure 6.10 Time series of wind power output with 40GW installed capacity for first two weeks in February

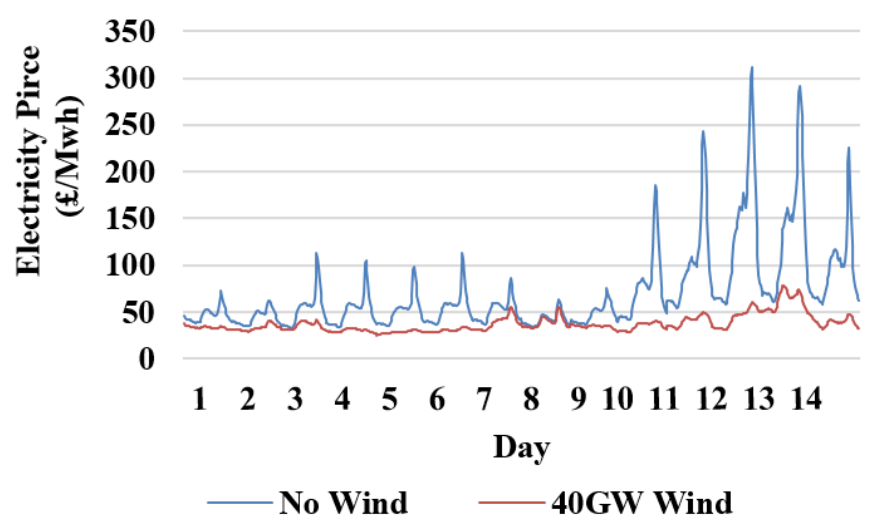

Figure 6.11 Time series of electricity price with no wind capacity and 40GW installed wind capacity for first two weeks in February 
Prices were similar for both scenarios between days 8 and 9 when the wind power output was low. With 40GW of installed wind capacity, during periods of high wind power output, peak prices were significantly reduced, which led generally to lower price variation. This is a result of the shape of the supply curve (Figure 6.8), which was steep during periods of high net demand (low wind production), but shallow during periods of low net demand (high wind production). The increased wind output with $40 \mathrm{GW}$ of capacity reduced scarcity of supply, leading not only to reduced average prices, but also reduced variation in price, despite increased variation in thermal output; this contributed to a decrease in arbitrage revenue.

The pattern of electricity price variation with a high penetration of intermittent wind power was largely in agreement with other studies [26], which additionally concluded that these less frequent price spikes were likely to be of a larger magnitude to enable peaking plant to cover its fixed cost over fewer operational hours. Modelling this phenomenon would have required a dynamic model, where the uplift coeffients $\alpha$ and $\beta$ were recalibrated each year depending on the capacity margin and peaking plant investment case. This was not carried out here, but is an area for further development and is discussed in Chapter 7. The general result of reduced frequency of prices spikes and magnitude of peak prices during periods of high wind power output were also seen in [26] and [92].

Figure 6.12 shows the state of charge of the EES device for the same two weeks. With no installed wind capacity the device charged and discharged once a day in line with the variation in electricity price driven by demand patterns. The EES device did not reach its maximum storage capacity or fully discharge on every cycle. This has different implications for different EES technologies, for example, some battery devices can experience significantly reduced lifetimes when they are regularly cycled without reaching their full storage capacity or fully discharging. With 40GW of installed wind the device charged and discharged less frequently with only four distinct cycles over the two week period, but fully discharged between each cycle. This pattern is similar to the four distinct cycles of wind power output shown in Figure 6.10 and is in line with the typical frequency of synoptic weather patterns that dominate UK climate. Additionally with 40GW of installed wind capacity, the EES device was limited for longer periods of time by its maximum capacity of 200MWh. 


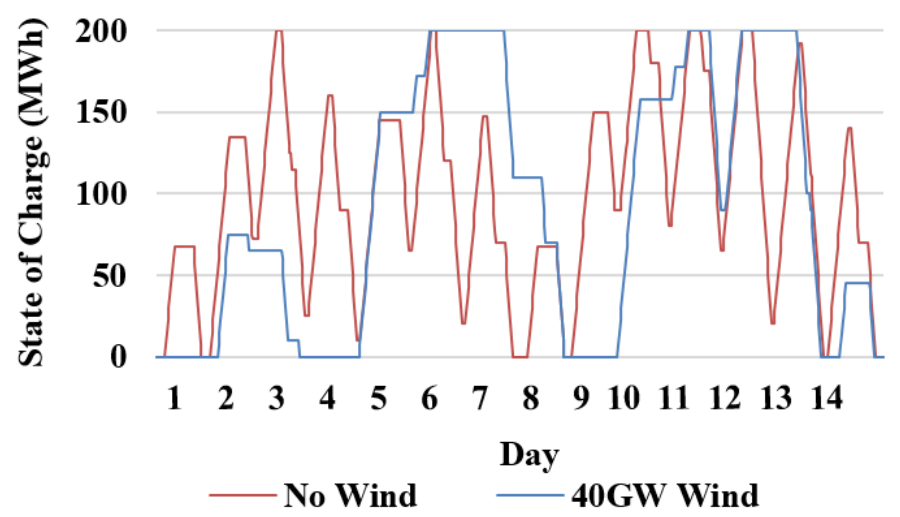

Figure 6.12 Storage state of charge for first two weeks in February with no wind and 40GW installed wind capacity

\section{Thermal generation capacity}

Revenue sensitivity to changes in thermal generation capacity are more challenging to assess explicitly. As discussed above, variations in average demand provide an indication of sensitivity to average capacity margin. However, changes in the capacity of individual classes of generation will have different effects depending on whether the net thermal capacity changes (changing the capacity margin) or if another class of generation is adjusted to compensate. Increasing the relative proportion of the lower merit order generators will flatten a larger portion of the supply curve leading to frequently lower daily price spreads, reducing revenue. Increasing the relative proportion of peaking plant would lead to the steeper region of the supply curve occupying a larger range of power output. This would result in more frequent larger price spreads and increased revenue. In a competitive market, it is likely that generators would, in fact, respond to these changes and modify their bidding behaviour, leaving the supply curve largely unchanged; however, the static nature of the price model cannot reflect this.

\section{Base year}

For all the variables discussed above, time series of fuel prices, demand and wind speeds were taken from the same base year, 2006. These inter-annual patterns change from one year to the next and will affect EES revenue. To examine the effect of this, the underlying time series of demand, wind and seasonal fuel price patterns from different base years were employed with the average fuel and carbon prices remaining at 2006 levels. Only 2005, 2006 and 2007 were investigated as these were the years where similar daily resolution of fuel prices were available and the underlying weather patterns were reasonably diverse. 
Figure 6.13 shows the resulting annual revenue for each of the years. It can be seen that there are substantial differences between them with 2005 and particularly 2007 revenue substantially below 2006. The swing in revenue of 54\% between 2006 and 2007 demonstrates the inherent risk facing EES investors from variations in patterns of demand, fuel prices and wind speeds; factors influenced by circumstances outside even the electricity sector.

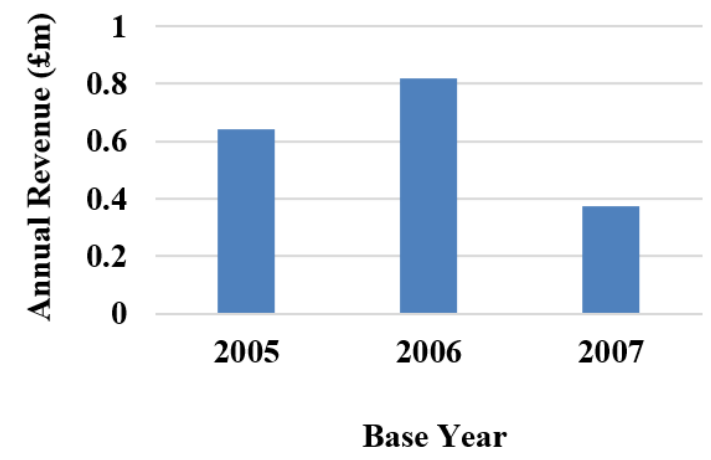

Figure 6.13 Annual revenue from 2006 using alternative base year time series

\subsubsection{Storage System Characteristics}

The external factors examined in Section 6.1.1 are outside the direct control of EES investors. However, it may be possible to engineer device characteristics to minimise potential negative impacts or enhance the positive impacts of these external factors. Understanding the value of different technology characteristics in the context of changing markets will enable development of EES systems which are robust to uncertain future circumstances. Equally, if the characteristics of EES which are promoted through the market are not those which provide the most value to the energy system then alternative support mechanisms may be required.

\section{Storage capacity and charging rate}

The majority of the variables investigated in Section 6.1.1 did not affect the optimum operating strategy of the EES device. In these cases, the sensitivity of revenue to storage capacity and charging rate did not change significantly as the variables changed. However, Figure 6.12 showed that there was a substantial change in the operating schedule with $40 \mathrm{GW}$ of installed wind capacity compared to no installed wind. This suggests that the storage capacity and charging rate may be valued differently in energy systems with different penetrations of wind. This was investigated by comparing the change in annual revenue for devices with increasing storage capacity and charging rate for cases with no installed wind capacity and with 40GW of wind capacity. 
Figure 6.14 shows the change in revenue with increasing storage capacity and constant charging and discharging rate for cases with zero and $40 \mathrm{GW}$ of installed wind. The remaining characteristics were fixed at the values listed in Table 6.1. The results are normalised relative to the revenue from devices with a $600 \mathrm{MWh}$ capacity, the maximum investigated for each case: these were $£ 0.96 \mathrm{~m}$ and $£ 0.38 \mathrm{~m}$ for the zero and $40 \mathrm{GW}$ wind cases, respectively. The normalised revenue shows the distinct difference in sensitivity to storage capacity more visibly than the absolute values. Figure 6.15 shows the change in revenue with increasing charging rate and constant storage capacity for the same cases, again normalised relative to the revenue from devices with the maximum charging rate tested $(200 \mathrm{MW})$. The absolute revenue for a 200MW device was $£ 2.72 \mathrm{~m}$ and $£ 0.69 \mathrm{~m}$ for zero and $40 \mathrm{GW}$ of wind capacity, respectively.

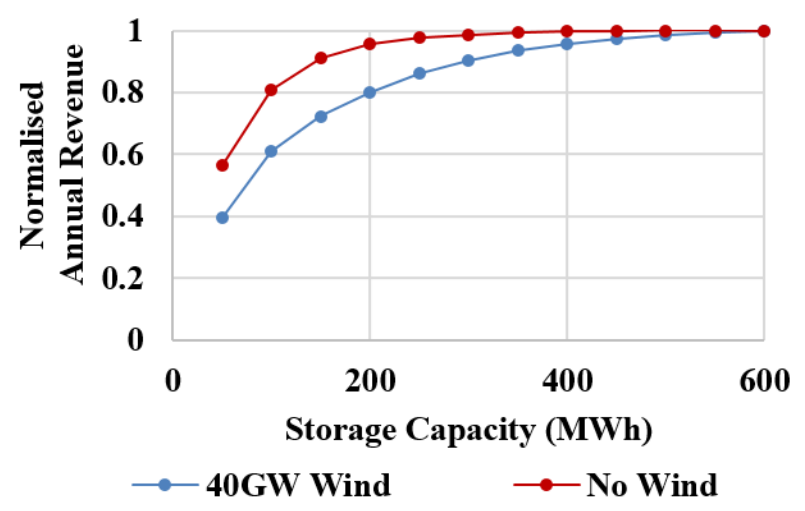

Figure 6.14 Annual revenue for zero and 40GW installed wind capacity cases varying storage capacity, normalised relative to 600MWh capacity

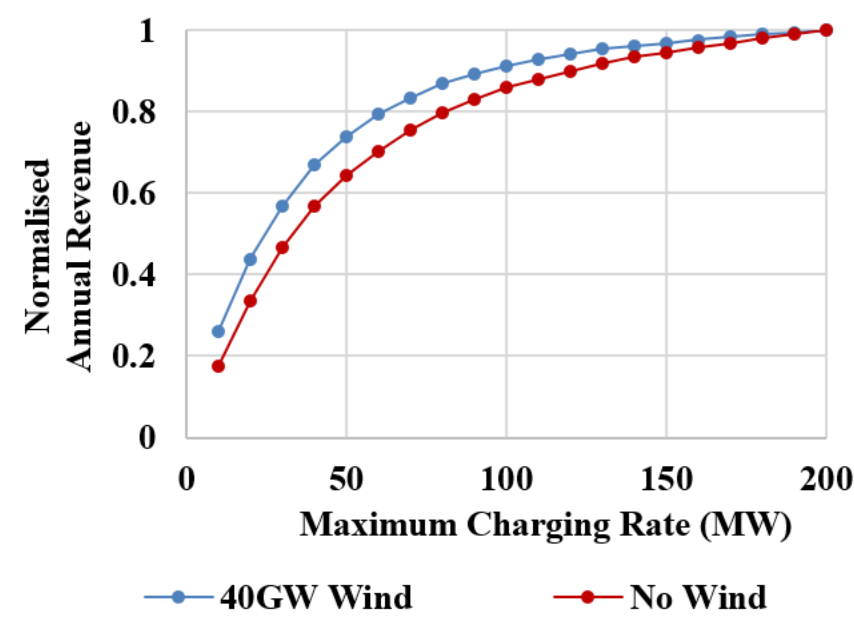

Figure 6.15 Annual revenue for zero and 40GW installed wind capacity cases varying charging rate, normalised relative to $200 \mathrm{MW}$ charging rate

Figure 6.14 and Figure 6.15 show that the increase in revenue from independently increasing storage capacity and charging rate decreased as the capacity and charging rate increased. For 
larger storage capacities the full range was utilised less frequently and increasing the capacity further yielded fewer opportunities to store more electricity and generate additional revenue. Similarly, the highest charging rates were the least restrictive on revenue so increasing them further, with the storage capacity fixed, yielded fewer benefits. These results are similar to those shown in Section 4.3.3. With no installed wind capacity, the arbitrage revenue was less sensitive to the storage capacity than with $40 \mathrm{GW}$ of installed wind; however, it was more sensitive to the charging rate. With no wind, the EES performed best by charging and discharging on a daily basis limited by the maximum charging rate on each cycle, as seen in Figure 6.12. With $40 \mathrm{GW}$ of wind capacity, however, the EES performed best by charging, discharging and storing energy over longer periods of time with fewer cycles, leading to the storage capacity becoming the more restrictive constraint. These results suggest that devices with higher storage capacity to power ratios may perform better in markets with a large penetration of wind power compared those with little installed wind capacity.

Figure 6.16 shows a contour map of constant revenue for a wider range of both storage capacity and charging rate for the case with $40 \mathrm{GW}$ installed wind. The line on the graph shows the ratio of charging rate to storage capacity which would maximise the arbitrage revenue, approximately $1 \mathrm{MW}: 10 \mathrm{MWh}$. This compares to a ratio of $1.7 \mathrm{MW}: 10 \mathrm{MWh}$ presented in Chapter 5 using 2010 electricity prices, when there was only approximately $5 \mathrm{GW}$ installed wind.

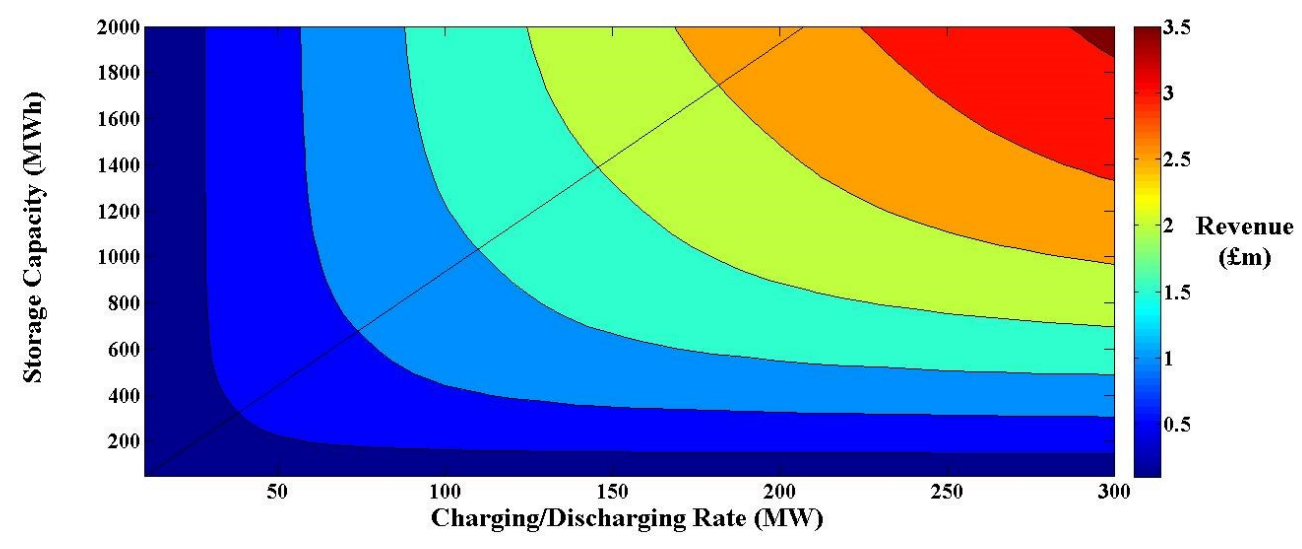

Figure 6.16 Contour map of annual revenue with varying capacity and charging rate with $40 \mathrm{GW}$ installed wind capacity, optimum ratio of charging rate to storage capacity shown by line

\section{Efficiency}

With higher electricity prices, conversion losses are relatively more costly and arbitrage revenue is more sensitive to efficiency. Increasing fuel and carbon prices led to higher 
electricity prices and increased sensitivity to round-trip efficiency and self-discharge. Increased wind capacity, on the other hand, led to frequently reduced electricity prices. However, similarly, this led to increased sensitivity to round-trip efficiency and self-discharge, as shown in Figure 6.17 and Figure 6.18. The higher levels of wind power not only reduced average electricity prices but also reduced the variation between wholesale price peaks and troughs. As a result, conversion processes needed to be more efficient to return the same revenue. This is contrary to previous studies, which have reported that high penetrations of wind power may lead to less value being placed on energy, and conversion efficiency becoming less important. Grünewald et al [19], for example, state that with very high wind penetration "any renewable energy that has to be curtailed, undergoes an effective conversion efficiency at $0 \%$. At this point even a conversion chain with low efficiency may improve the overall system efficiency". Strbac et al [2] also assert that conversion efficiency is of "secondary importance". For whole system optimisations, a low efficiency EES device may yield benefits, but from a commercial perspective, when revenue is accrues through price arbitrage, high conversion efficiency is critical.

Furthermore, with high wind penetrations, electricity prices cycled over longer durations and energy was stored over longer periods making revenue more sensitive to self-discharge.

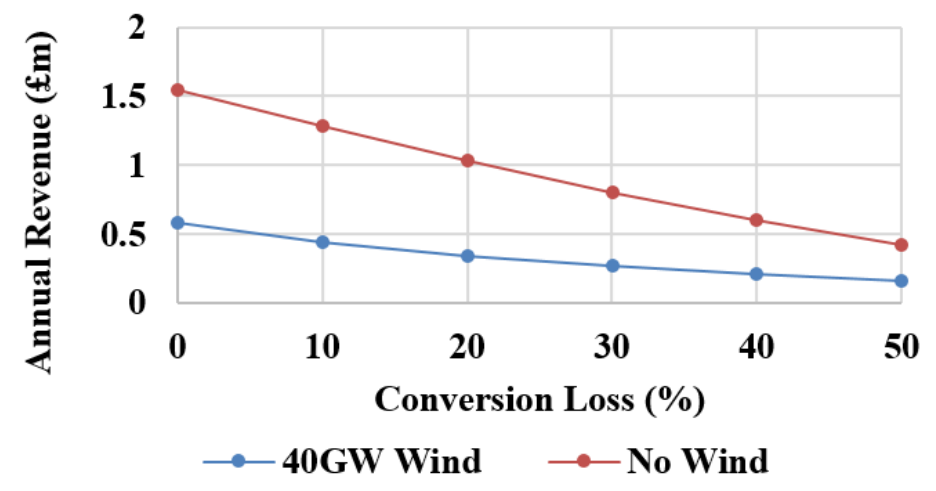

Figure 6.17 Annual revenue for zero and 40GW installed wind capacity cases varying conversion efficiency 


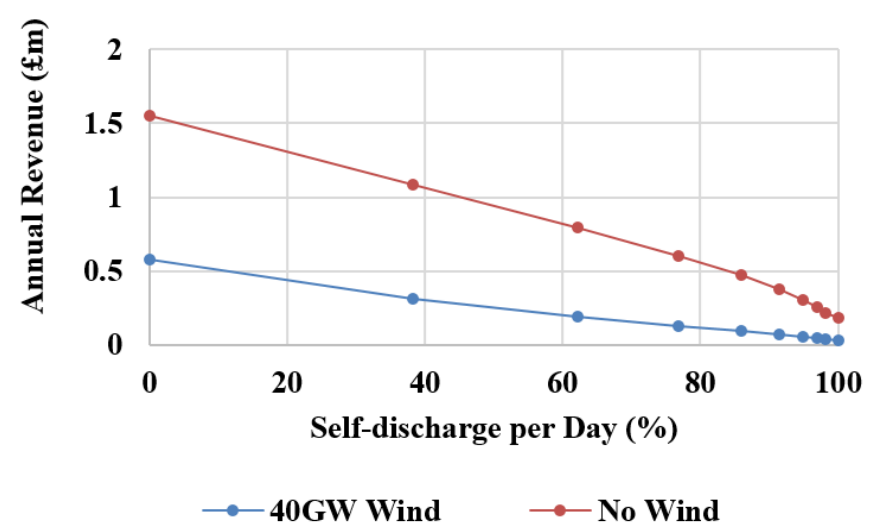

Figure 6.18 Annual revenue for zero and 40GW installed wind capacity cases varying daily self-discharge

\subsubsection{Implications}

Systematic examination of the sensitivity of revenue available to an EES operator to key external factors showed that revenue increased with rising average gas prices, carbon prices and demand, but reduced as wind capacity increased. Year-to-year variation in patterns of fuel prices, wind speeds and demand indicated revenue could change by over $50 \%$, demonstrating that the revenue risks facing EES arise not only from uncertain mid- to long-term market conditions, but also from variations in underlying economic, climate and behavioural patterns from one year to the next.

As a facilitating technology, EES was sensitive to a number of changing market variables, but increased wind capacity was a game changer. It not only affected the revenue available to EES, but also the way in which it was operated and the characteristics which were rewarded by the market. Evidence, reported in Chapter 4, Section 4.1, has shown that as the penetration of wind power increases, the associated system costs increase and the value of flexible solutions, such as EES, grows. Conversely, as the penetration of wind power increases, arbitrage revenue lessens, amplifying the disparity between the system value of, and the commercial reward for EES.

With higher wind penetration wholesale prices became more strongly influenced by wind power output than by diurnal patterns of demand behaviour. This reduced the number of cycles carried out by the EES device and led to energy being stored for longer periods of time. As a result, revenue was more sensitive to storage capacity and less sensitive to charging rate, leading to lower power to capacity ratios being better compensated financially compared to cases with low wind penetration. This is challenging for investors, suggesting that EES device characteristics designed for current market conditions may not be optimum if the penetration 
of wind power changes significantly in the future. Consequently, smaller, modular EES devices are likely to be preferable to large, monolithic projects and devices with modular storage and power related equipment, which could be expanded independently as the electricity system evolves, may provide safer investment options. Grünewald et al [19] highlighted that wider benefits, such as energy security, increase with longer storage durations (lower power to capacity ratios) implying that these features provide most value to the system. This suggests that the market may tend to promote characteristics which are also optimum from a system-wide perspective.

Conversely, with wide-spread wind deployment, the arbitrage market also promoted highly efficient EES, which is not necessarily critical from a system perspective. With high wind penetration the marginal cost of electricity is reduced and conversion efficiency becomes less critical to the over-all electricity system value [110]. If wind power were frequently curtailed, even EES with low efficiency would provide an improvement to shedding non-dispatchable energy [19]. This suggests that, in the case of efficiency, the market does not provide appropriate incentives for technologies which enable optimum system-wide solutions.

\subsection{Scenario Analysis}

As discussed in Chapter 4, sensitivity studies are useful to measure changes in response to a single variable; however, they are limited in their ability to assess the impact of multiple changing variables which, in reality, will not vary independently of one another. In the case of an uncertain future energy market this could include, for example, increased carbon prices resulting in wider deployment of low carbon generation. Scenario analyses enable the impact of multiple dependent variables to be investigated.

A wide range of scenarios have been developed for future energy systems in GB including scenarios based on system optimisation and capacity investment decisions. The UK Energy Research Centre [258] developed optimum scenarios for the future energy landscape using the MARKAL Model, while Eager [92] developed a model which defined decisions to build or mothball generation capacity based on price signals in the market. Any of these approaches could be used to determine scenarios for the future, and equally, any scenario could be applied to the model. In this thesis, the 2014 National Grid Future Energy Scenarios [257] were investigated. Future Energy Scenarios are published annually by National Grid and are defined through discussion with stakeholders and operational experience. The input data are clearly defined and the majority is publicly available. The scenarios take into account a range of socio- 
and techno-economic factors to describe plausible future energy systems. For each scenario, there is a range of changing inputs such as fuel costs, generation mix and demand.

Four scenarios were considered representing alternative pathways for the energy system in GB with varying emphasis on affordability and sustainability, as shown in Figure 6.19. The scenarios were classified as: Low Carbon Life; Gone Green, No Progression and Slow Progression. Key details of each scenario are highlighted below with more detailed information available in [257].

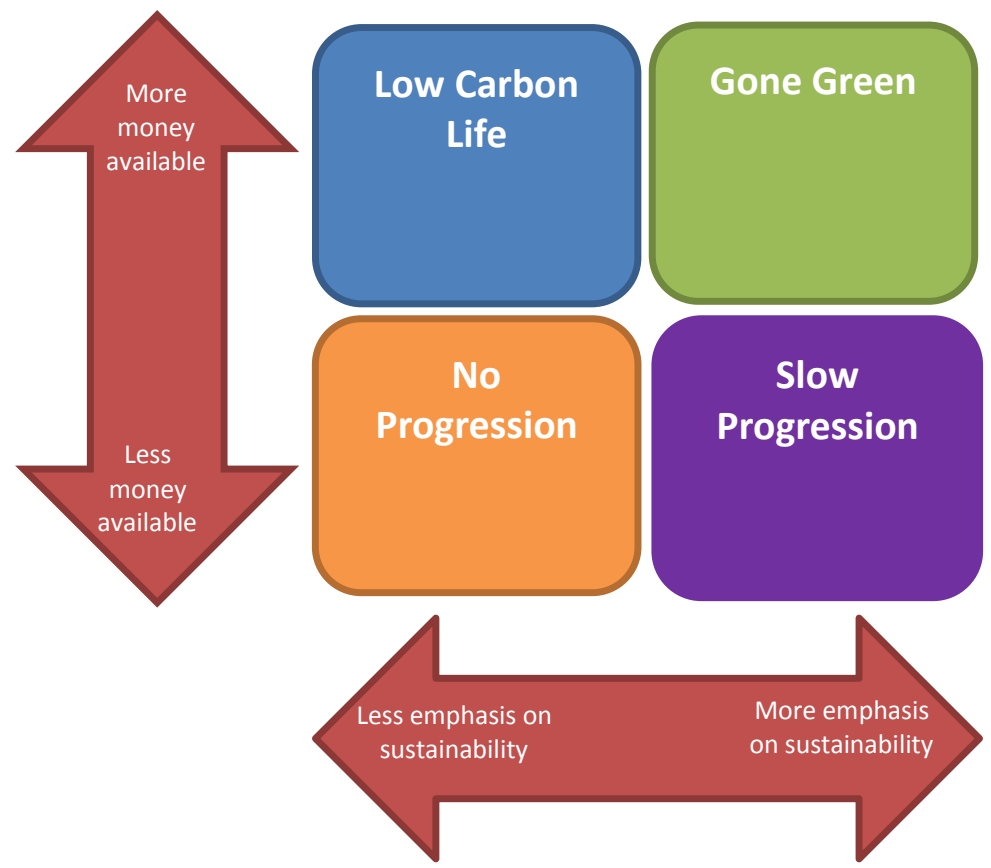

Figure 6.19 National Grid Future Energy Scenarios

\subsubsection{Scenario Overview}

\section{Low Carbon Life}

The Low Carbon Life scenario represents a world of high disposable income and low sustainability. There is short term political volatility unsettling investor confidence in sustainable technology followed by a long term consensus towards decarbonisation. Consumers and government have capital available to spend but little political will to decarbonise. This results in a low uptake of heat pumps and poor developments in energy efficiency. Greater economic prosperity, however, leads to higher demand in both domestic, commercial and industrial sectors. There is increased nuclear generation and carbon capture and storage (CCS). 


\section{Gone Green}

The Gone Green scenario has a high emphasis on sustainability with high economic growth. There is European cross party political support of sustainability. Heat, transport and power sector policies are aligned and renewable energy and carbon targets are met. Strong economic growth enables substantial research and development activities leading to new and potentially disruptive technologies. A significant number of heat pumps and electric vehicles are deployed and energy efficiency is improved. A significant amount of thermal generation is built to manage intermittency of the large numbers of renewable generators which have been deployed.

\section{Slow Progression}

The Slow Progression scenario represents a world with the same intentions to move towards a sustainable energy sector as the Gone Green scenario, but with slower economic growth and less capital available. Renewables are favoured over CCS and nuclear power and 2020 renewable energy targets are met. There is a strong emphasis on reducing consumption; however energy efficiency measures are delayed due to affordability. Similarly, uptake of heat pumps and electric vehicles is minimal. Consumers are driven by price and are responsive to time of use tariffs through smart meters.

\section{No Progression}

The No Progression scenario represents a future with political volatility and focus on short term measures of affordability. There is little drive to change policy and regulation which remain the same as today. Sustainability is viewed as low priority and the 2020 renewable energy targets are missed. Economic recovery is slow and there is little investment in innovation so only incremental changes to existing technology are realised and the focus is on gas generation to maintain security of supply. There is restricted build of new nuclear plant and no CCS. Energy consumption is constrained due to limited disposable income. There is little drive for energy efficiency and so there is higher domestic demand than the Slow Progress scenario; however, the slow economic recovery means there is less industrial and commercial demand. There is limited uptake of heat pumps and electric vehicles.

\subsubsection{Market Evolution}

The evolution of market variables from 2015 to 2035 in each of the scenarios is presented below. 


\section{Thermal generation capacity}

The capacity of each type of thermal generation for each scenario is shown in Figure 6.20. In the scenario data CCGT and OCGT are not differentiated and so the total gas generation capacity was combined with other peaking generators and assumed to comprise $65 \%$ CCGTs and 35\% OCGTs, which is roughly in line with historic years. Interconnector capacity was neglected. A separate grouping is shown for CCS/biomass/CHP (combined heat and power) and coal to demonstrate the differences between the scenarios; however, these were all assumed to operate as base load and were grouped together with coal generation for use in the electricity price model.

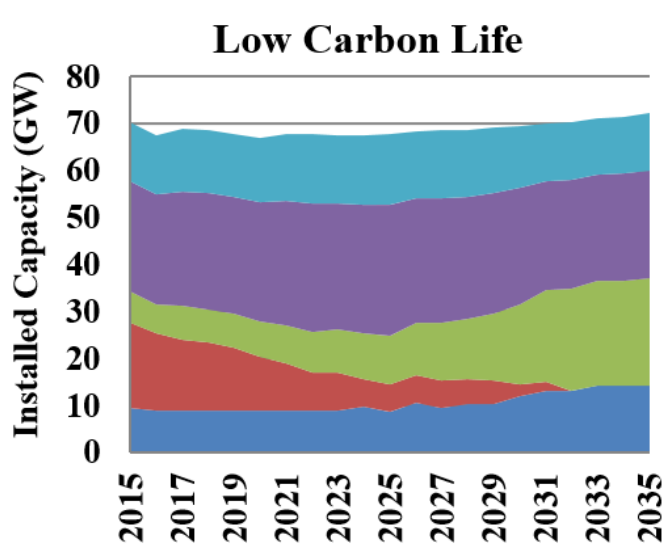

Year

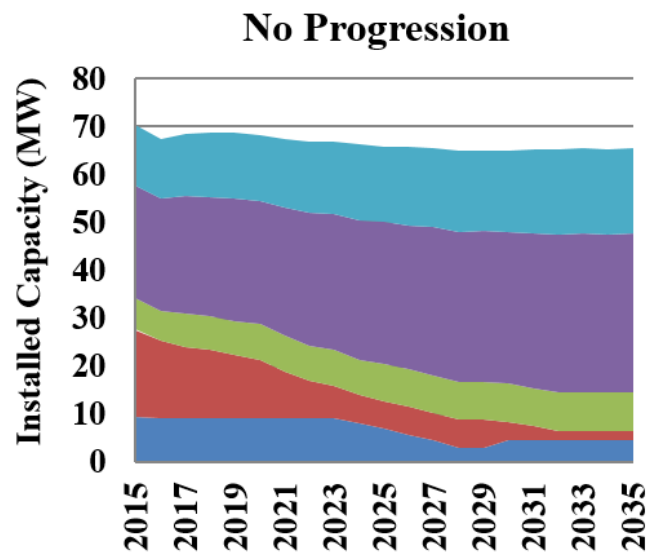

Year

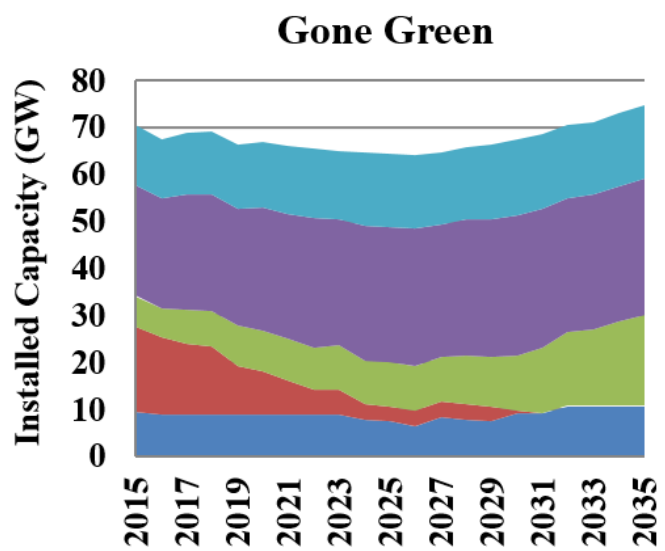

Year

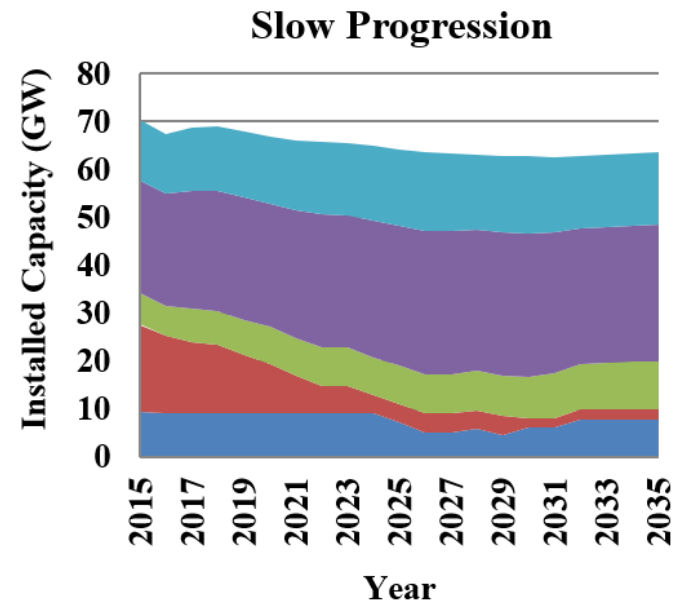

Nuclear Coal $\backsim$ CCS/Biomass/CHP $\backsim$ CCGT $\square$ OCGT

Figure 6.20 Thermal generation capacity for National Grid Future Energy Scenarios 20152035 
Up to 2020 generation sources remain largely constant with minor changes as plant becomes compliant with low carbon legislation such as the Large Combustion Plant Directive. There is little variation between the scenarios over this period. From 2020-2026 the impact of the Industrial Emissions Directive is most visible with the majority of coal stations closing by 2023. During the final ten years, there is clearer differentiation between the scenarios representing the uncertainty associated with the longer-term development of the power industry.

\section{Wind generation capacity}

The increase in onshore and offshore wind power capacity in each of the scenarios is shown in Figure 6.21 and Figure 6.22, respectively.

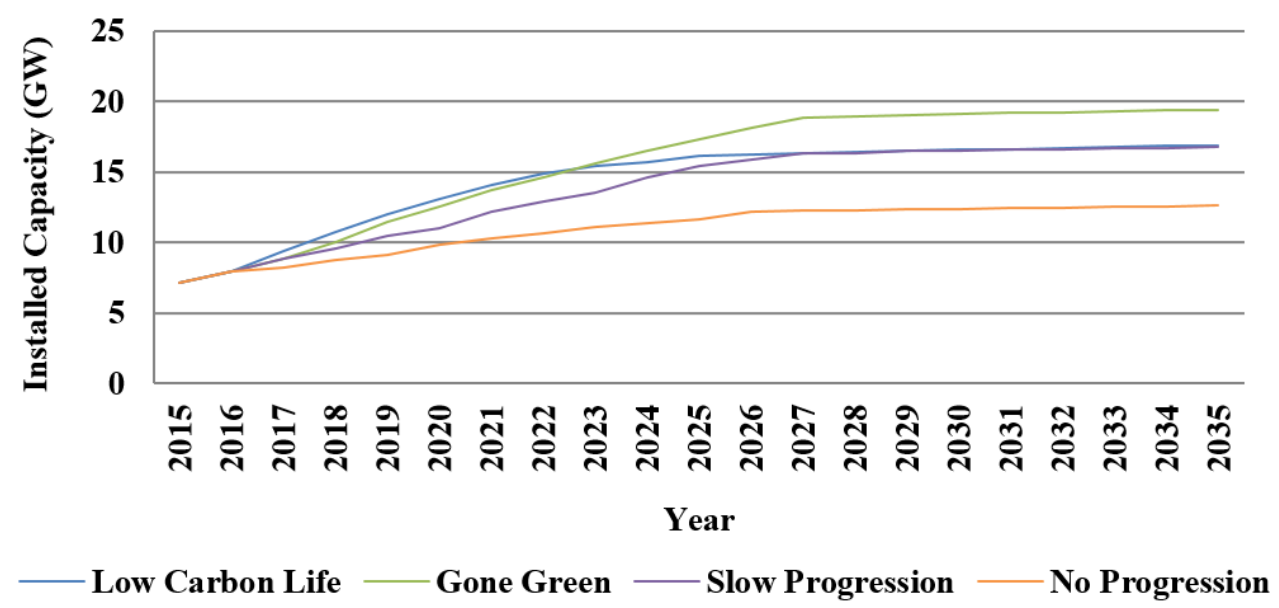

Figure 6.21 Onshore wind capacity for National Grid Future Energy Scenarios 2015-2035

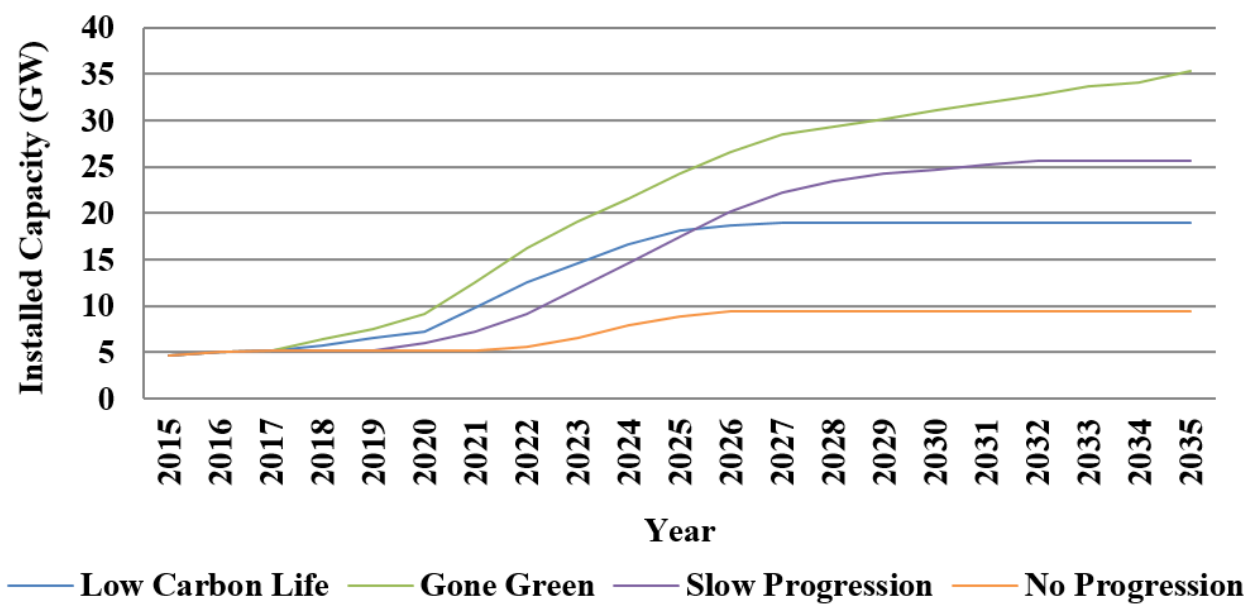

Figure 6.22 Offshore wind capacity for National Grid Future Energy Scenarios 2015-2035 
The Low Carbon Life scenario has an increase in nuclear generation and CCS but limited wind development to 2035, although still more than the No Progression scenario as more capital is available. The Gone Green scenario has the highest emphasis on low carbon generation with the highest levels of both onshore and offshore installed wind capacity. The Slow Progression scenario also places emphasis on clean technologies but with less available capital, there is less significant deployment of wind power. In the No Progression scenario cost of generation is more important than sustainability leading to little development of wind power, particularly in later years.

\section{Fuel prices}

Figure 6.23 shows the average annual gas price from 2015-2035 from the National Grid Future Energy Scenarios. Prices decrease slightly until the end of the decade as global gas supply increases through commissioning of new liquid natural gas liquefaction plants and demand in Europe remains relatively low. After 2020 demand is assumed to increase in China and India driving up global prices. The two scenarios reflect the level of uncertainty associated with proposed liquefaction export projections from North America, global economic recovery, the level of increasing demand in Asia and the potential restart of nuclear power generation in Japan. The high case is applied in the Slow Progression and No Progression scenarios and the base case for the Gone Green and Low Carbon Life scenarios.

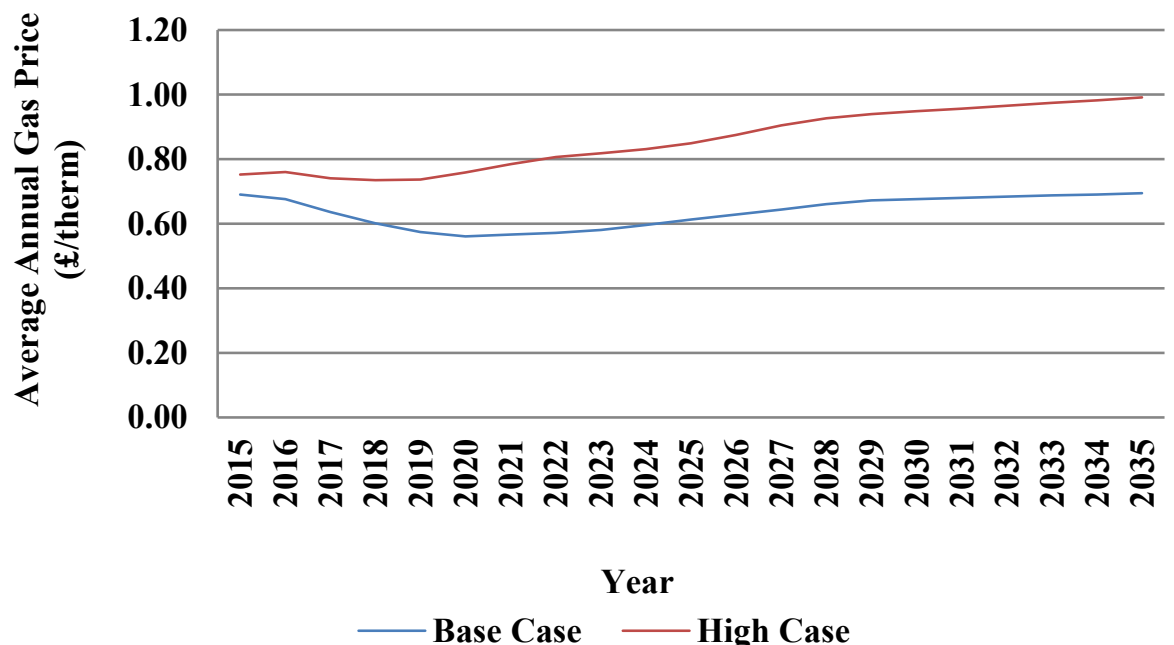

Figure 6.23 Gas price for National Grid Future Energy Scenarios 2015-2035

The change in average coal price to 2035 is shown in Figure 6.24. In recent years, coal prices have decreased as there has been surplus supply in the global market with cheap shale gas in the US replacing coal for electricity generation. Despite this, prices are expected to rise 
steadily to 2035 due to increasing demand from developing countries. This is expected to be most notably from Asia; however, demand in China may slow as it moves towards a cleaner energy market in an attempt to reduce concerns over air pollution. The same coal prices are applied in all the scenarios.

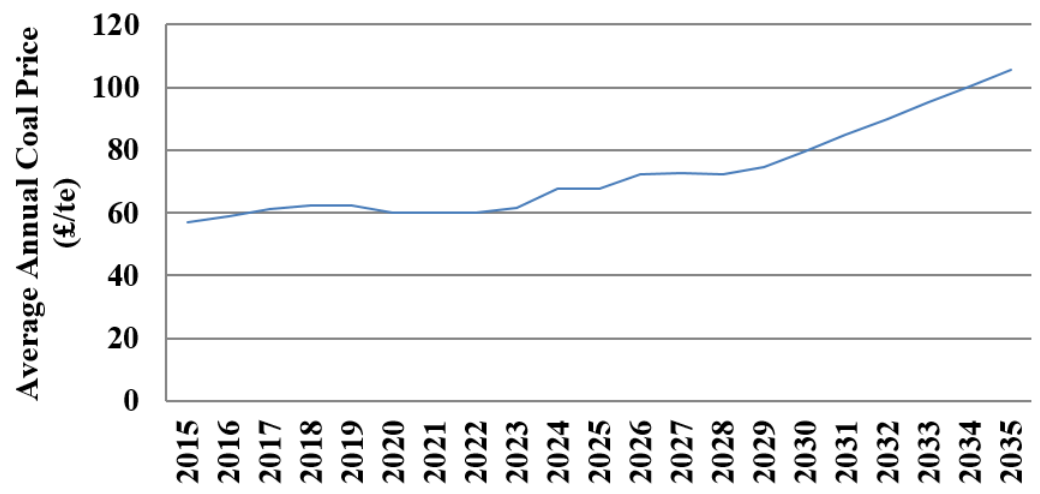

Year

Figure 6.24 Coal price for National Grid Future Energy Scenarios 2015-2035

Uranium prices are not provided directly in the National Grid Future Energy Scenarios. The trend in base load power price is used to infer the uranium price. Although this may not be entirely accurate, it is expected to give a reasonable indication of changes in uranium price. Furthermore, the model is not largely sensitive to the price of raw uranium as additional costs, such as fuel enrichment and operation and maintenance, make up a significant proportion of the relatively low marginal generation cost for nuclear power. The average annual uranium prices used for all scenarios are shown in Figure 6.25.

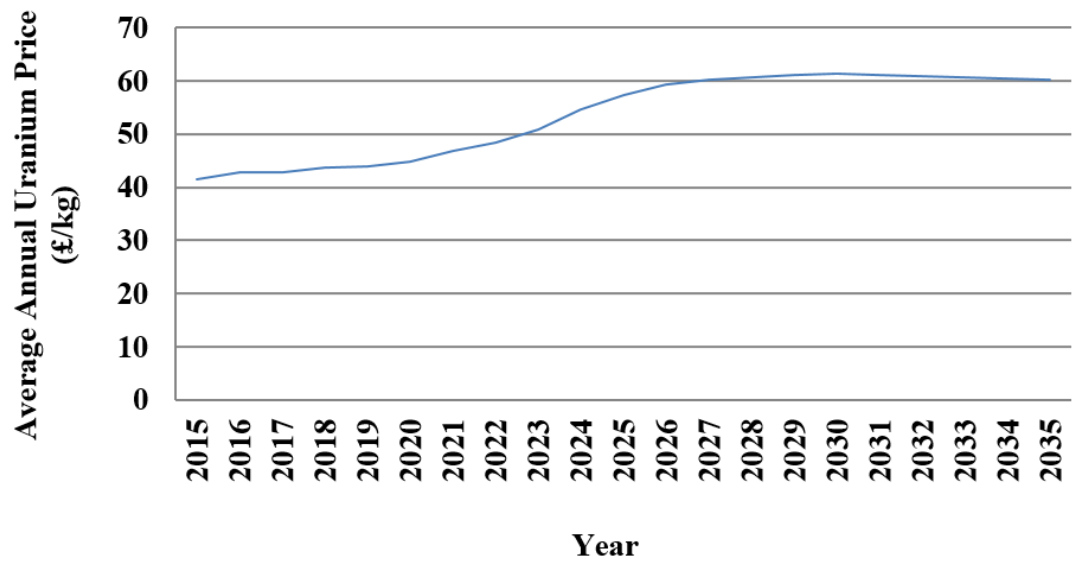

Figure 6.25 Uranium prices derived from National Grid Future Energy Scenarios 2015-2035 


\section{Carbon prices}

The carbon price to 2035 is shown in Figure 6.26. A UK carbon floor price was introduced in 2013 to guarantee a minimum price for carbon emissions from electricity generators in the UK. This was achieved through a Carbon Price Support payment on top of the EU ETS. The support rate will be capped at $£ 18$ until 2020; however during this period, the EU ETS price is expected to increase resulting in a small increase in total carbon price. After 2020, it is expected that there will be little further increase in price due to adverse effects which would be imposed on businesses.

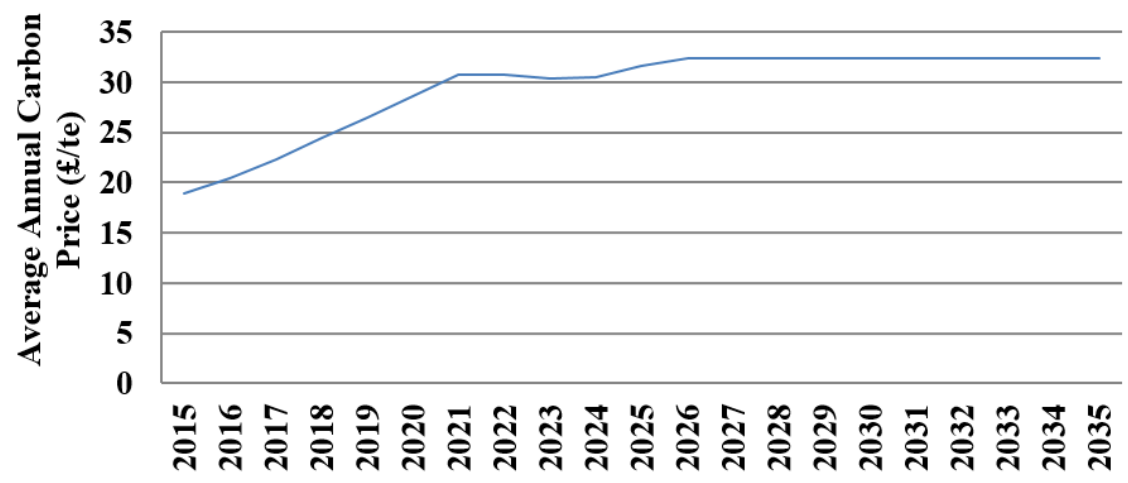

Year

Figure 6.26 Carbon prices for National Grid Future Energy Scenarios 2015-2035

\section{Demand}

Annual and peak demand data up to 2035 is shown in Figure 6.27 and Figure 6.28, respectively.

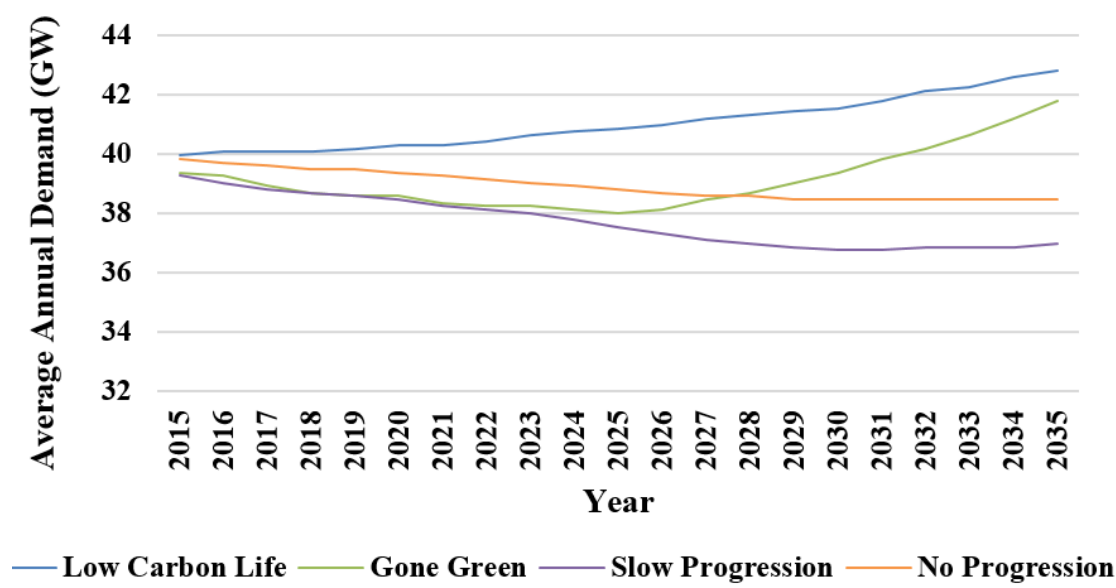

Figure 6.27 Average annual demand for National Grid Future Energy Scenarios 2015-2035 


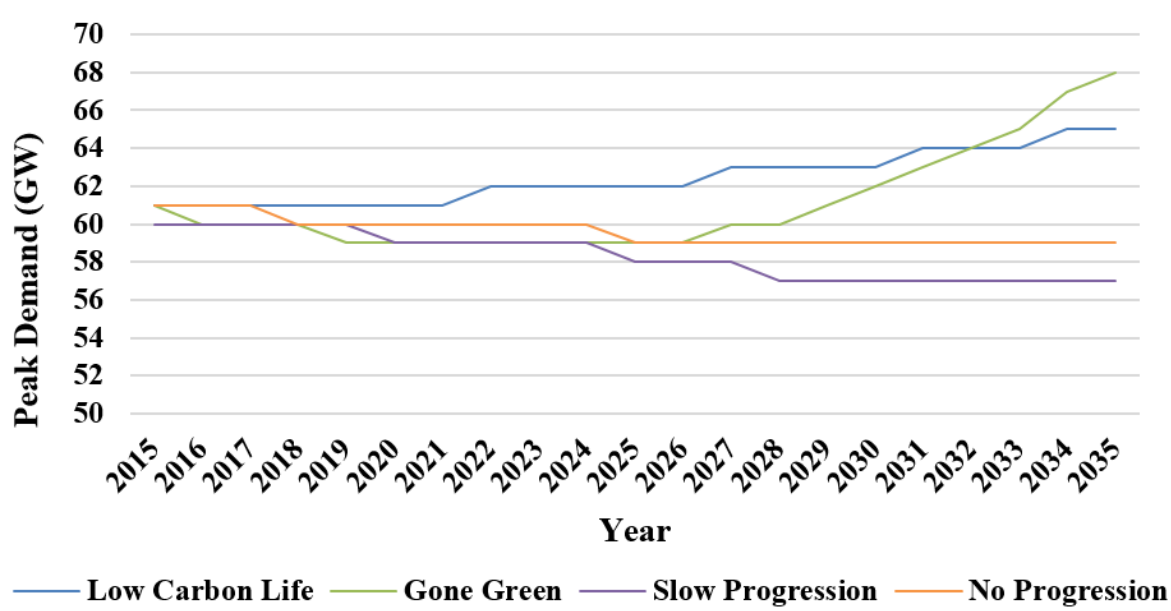

Figure 6.28 Peak demand for National Grid Future Energy Scenarios 2015-2035

The Low Carbon Life scenario shows improvements in energy efficiency overtaken by growth in demand as a result of the rapid economic recovery. In the Gone Green scenario, improvements in energy efficiency lead to a reduction in demand up to 2025. Following this, there is a sharp increase in demand due to a number of changing factors including limitations to further efficiency improvements and increased numbers of households. The electrification of heat and transport lead to further increases and a steep rise in peak demand. For the Slow Progression scenario there are some improvements in efficiency coupled with limited consumption leading to a continuous reduction in demand to 2035. In the No Progression scenario, there is similarly limited consumption, but also limited improvements to efficiency leading to demand reducing at a lower rate to the Slow Progression scenario.

\subsubsection{Results}

For all Future Energy Scenario simulations a base year of 2006 was used. Time series of fuel prices, carbon prices, wind speeds and electricity demand were scaled from this base year to maintain any existing relationships between these variables. The storage system characteristics in Table 6.1 were used. The annual revenue available from the storage device for each scenario from 2015-2035 is shown in Figure 6.29. 


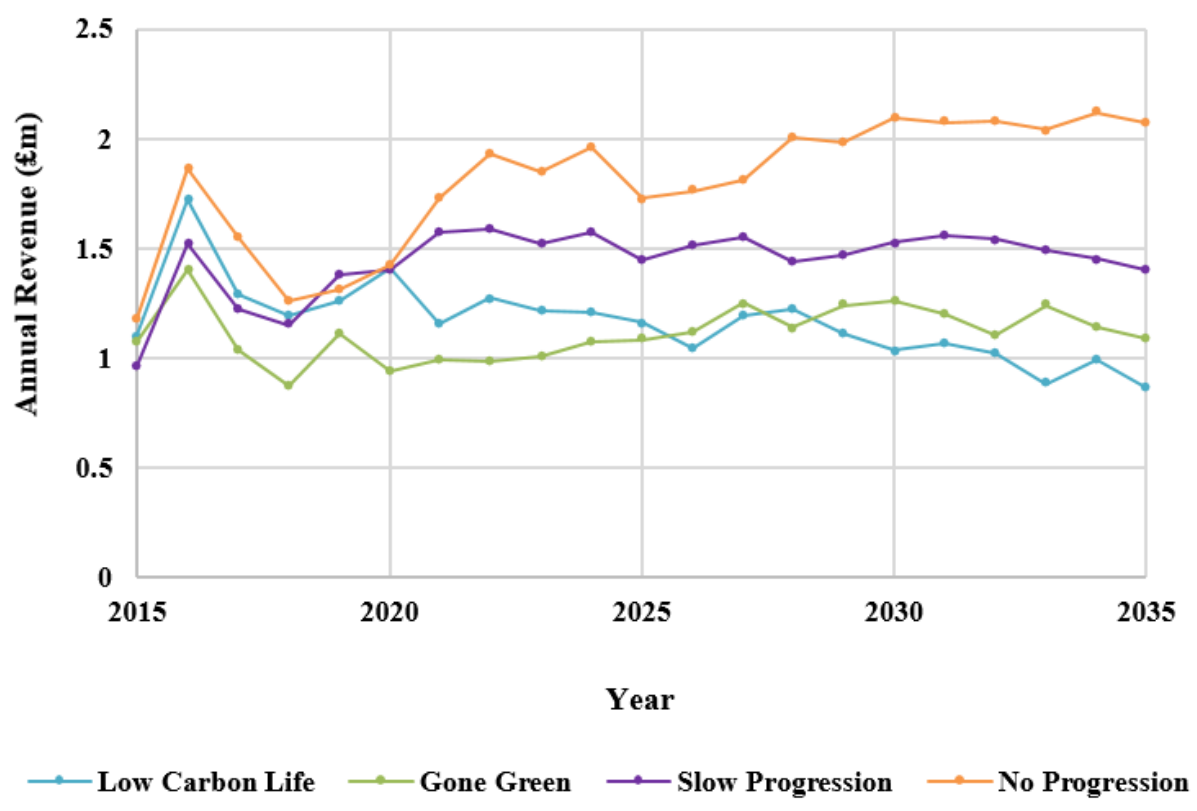

Figure 6.29 Annual revenue for National Grid Future Energy Scenarios, 2015-2035

All the scenarios showed a similar trend in the initial years with a sharp increase in revenue in 2016 followed by a reduction of similar magnitude in 2017. This is likely to be a result of the reduction in coal capacity across all scenarios in 2016, reducing the capacity margin, which was recovered in 2017 when additional CCGT was brought online. Over these years demand did not vary significantly in any of the scenarios and so the changes in capacity were reflective of changes in the system capacity margin. When capacity was scarce, the prices were increasingly inflated creating additional opportunities for arbitrage and higher annual revenue. Figure 6.30 shows the ratio of total thermal capacity to average annual demand for the scenarios from 2015-2035. The arbitrage revenue peak in 2016 corresponds to the acute reduction in this ratio. It is also evident that there are additional drivers of arbitrage revenue, as in later years the changes in revenue do not correspond solely to changes in the ratio of thermal capacity to average demand. 


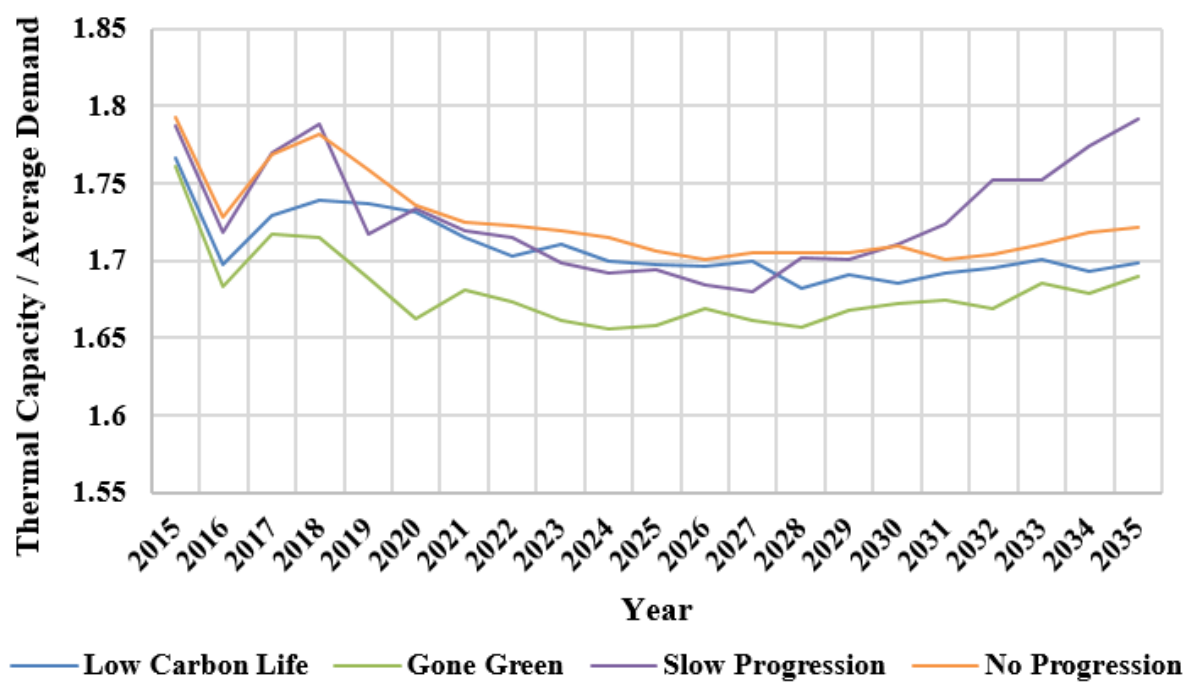

Figure 6.30 Thermal capacity / average demand for National Grid Future Energy Scenarios, 2015-2035

In the No Progression scenario, there was a general increase in revenue from 2018 to 2024. This was driven by the reducing capacity margin in that scenario. The reduction in revenue in 2023 may be a result of the reduction of coal capacity in this year. From 2025 to 2035, there was also a trend of increasing revenue, likely to be driven by increasing gas prices, which rose at a reduced rate from 2030 to 2035 . The reduced revenue in 2025 coincides with a reduction in peak demand in the same year. Changes in peak demand are not captured in Figure 6.30, but reductions reflect relative increases in capacity leading to a reduced frequency of peak electricity prices and reduced opportunity for arbitrage.

The Slow Progression scenario did not show the same increase in revenue from 2020 to 2025 which is likely to be due to the increase in installed wind capacity over these years. There was a marked reduction in revenue in the 2030s, which coincides with an increase in thermal generating capacity coupled with declining demand during this period.

The Gone Green scenario showed a slower increase in revenue from 2020 to 2030. This was also likely to be driven principally by steadily increasing gas prices, which did not rise as steeply as in the No Progression or Slow Progression cases. The increase in installed wind capacity was likely to have driven the reduction in annual revenue from 2030 to 2035 . Additionally, gas prices plateaued during this period.

Revenue in the Low Carbon Life scenario follows a similar trend, inversely related to the ratio of thermal capacity to average demand. The lower gas prices and higher installed wind power led to reduced revenue compared to the Slow Progression and No Progression scenarios. In 
the later years investigated, there was higher thermal capacity and lower peak demand than the Gone Green scenario which may explain the lower annual revenue.

The Gone Green and Slow Progression scenarios, which used the base case gas prices, led to significantly lower average annual revenue than the No Progression and Slow Progression scenarios, which used the high gas prices. The scenarios which used the base case gas prices represented the situations where there was more available capital to invest in new technologies and energy efficiency measures. These are likely to be the same cases where there would be research and development funding and investment capital to develop EES projects and reduce the technology costs. Ironically, in these cases the market would provide the lowest financial return. In the Slow Progression and No Progression scenarios, there is less available capital to spend and so, despite potentially higher revenue available for EES, creating a market pull, the upfront capital costs for technology development would be less likely to be available in these cases. The No Progression scenario, in particular, demonstrates the case where EES would provide least value to the system, with minimal installed wind capacity, but the highest return to the investor. Conversely, the Gone Green scenario, with the highest installed wind power, which would benefit most from EES, returns the lowest financial reward for over $50 \%$ of the years investigated.

The same base year was used for each year simulated removing the stochastic uncertainty in wind speeds, demand and fuel price patterns from one year to the next. Despite this, arbitrage revenue varied significantly and erratically with variations of up to $60 \%$ from year to year and over $100 \%$ between different scenarios in the same year. The unpredictable revenue presents a challenging environment for investment in long term EES projects which, in some cases, have a lifetime of over 50 years. The results support the conclusions of Grünewald et al [19] that commercialisation of storage, as a facilitating technology, is dependent on a number of uncertainties including the future generation portfolio, the regulatory environment in the energy sector and global economic trends. Arbitrage revenue is dependent on the individual behaviour of each of these characteristics, but also on the complex interactions between them. These uncertainties increase the cost of finance for storage technologies and reduce the chance of successful market uptake.

When also including the uncertainty from changing patterns of demand, wind speeds and fuel and carbon prices, arbitrage revenue is even more variable. Figure 6.31 shows the annual revenue from 2035 for each of the Future Energy Scenarios with a base years of 2005, 2006 and 2007. This demonstrates the risk to arbitrage revenue from one year to the next. All scenarios show an increase in revenue from 2005 to 2006 of greater than $30 \%$ and a reduction 
from 2006 to 2007 of over $40 \%$. This demonstrates, that it is variations in demand and fuel price, in addition to variations in wind power output, which drive arbitrage revenue as even the no progression scenario, with the lowest installed wind power, exhibited substantial changes with different base years. It is, in fact, the Gone Green scenario, with the highest penetration of wind power in 2035, which demonstrated the smallest variation between different base years.

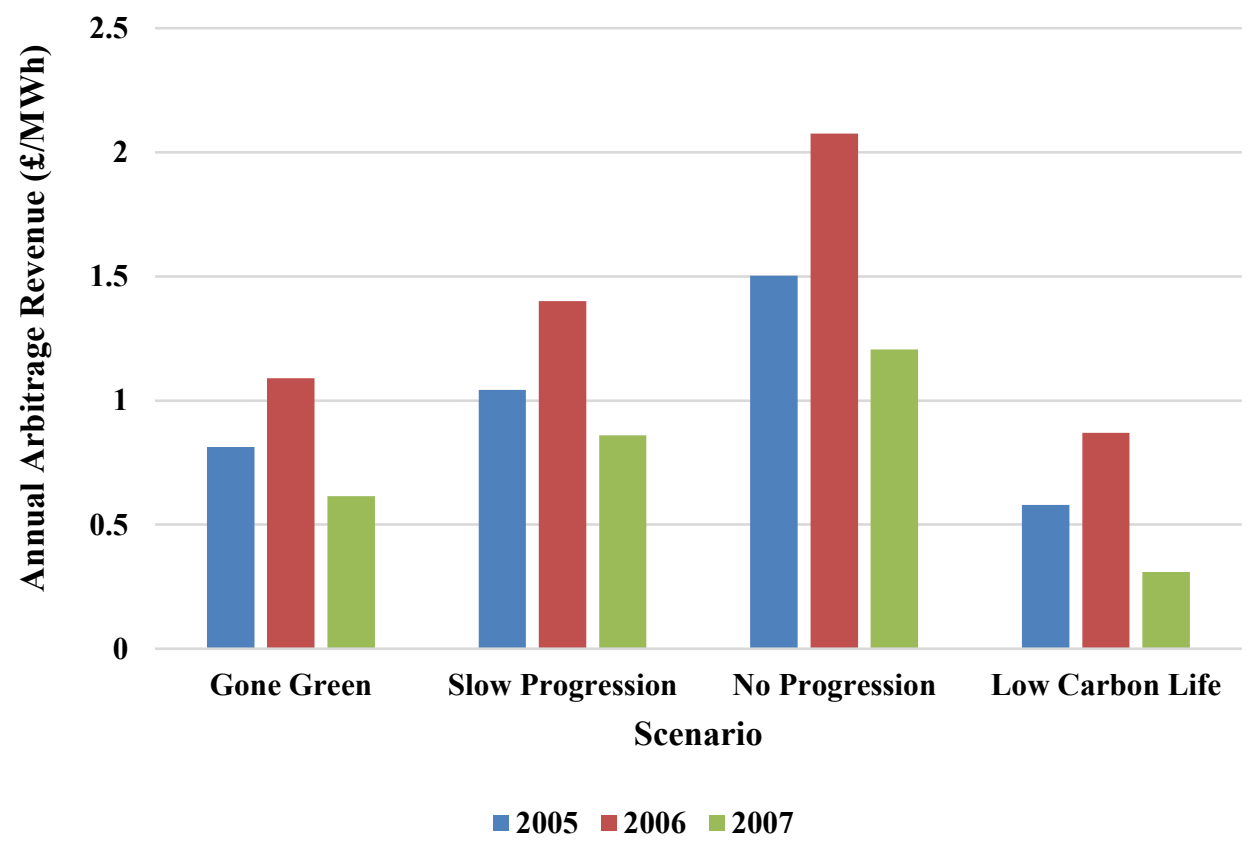

Figure 6.31 Annual revenue from National Grid Future Energy Scenarios in 2035 with
changing base year

Figure 6.32 shows the state of charge of the EES device for a week long period in 2035 for the Gone Green and No Progression scenarios. These were the scenarios with the highest and lowest installed wind capacity, respectively. This shows that, in the No Progression Scenario, the device charges and discharges on a daily basis, similar to the pattern with zero installed wind capacity shown in Figure 6.12, driven by patterns of daily demand. In the Gone Green scenario, however, the optimum operating strategy is to charge and discharge only three times over the week long period, demonstrating that in this scenario the optimum EES operation is driven by the cycles of wind speed. As discussed in Section 6.1.2, this drives the optimum EES characteristics demonstrating that different devices may be more or less favourable in alternative future energy scenarios. 


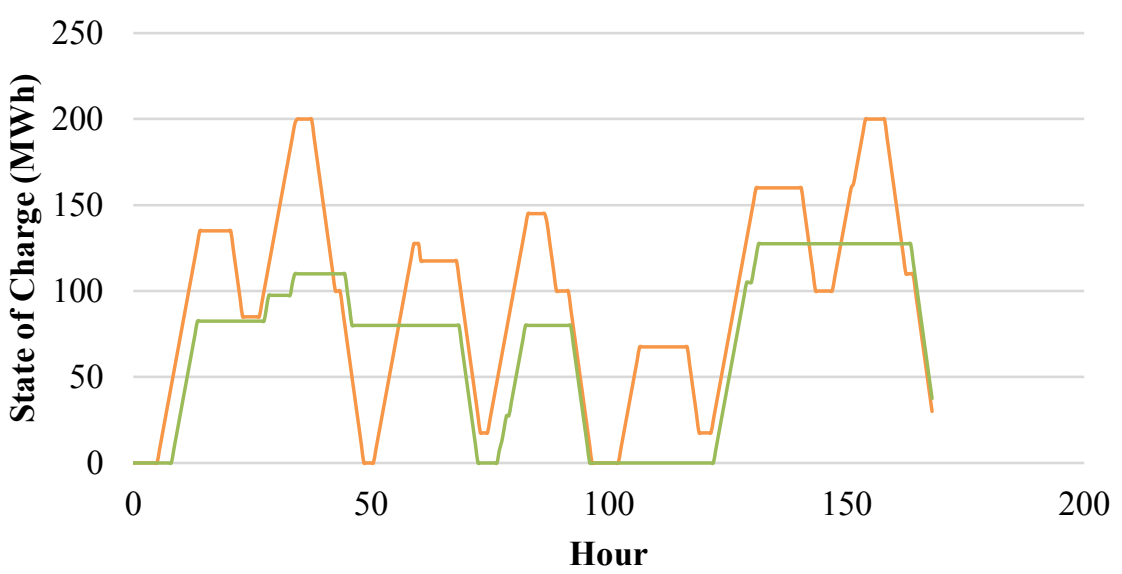

- No Progression $\quad$ Gone Green

\section{Figure 6.32 State of charge over 1 week for 2035 Gone Green and No Progression} Scenarions

\subsection{Discussion}

Historically, technical development of EES has been driven by economic, environmental and political conditions. Until recently, the majority of grid connected EES projects were established prior to the restructuring of the electricity markets in the 1990s when generation projects were planned centrally and the power sector optimised from a whole system perspective. Following the restructuring, with EES classified as a generation asset, challenges arose surrounding how to accrue value from the highly regulated transmission and distribution sectors, to which EES also provided many benefits. Despite these challenges, the potential for a large penetration of intermittent renewable power has led to a recent resurgence of interest in EES and its commercial viability.

There are expected to be substantial system benefits through deployment of EES, due to the anticipated increase in system balancing costs and requirement for increased flexibility. EES has the potential so provide multiple ancillary services; minimising system operating costs, assisting with network management; reducing the requirement for investment in expensive transmission and distribution network infrastructure and reducing curtailment of renewable energy. In addition, development of the EES industry could create jobs, stabilise wholesale electricity prices and reduce carbon emissions from the electricity sector. System studies conclude that there is substantial value and system-wide cost savings to be made from the implementation of EES. 
There is a range of EES technologies which are suited to specific applications; no single device can serve all flexibility requirements. Devices which can respond quickly with high power output are better suited to power quality applications, while devices which can deliver a large amount of energy over longer durations, such as CES, CAES and PHS are better suited to energy management applications. These tend to have higher capital costs, longer lead times and longer lifetimes creating high risk projects which are not well suited to investment from the private sector. There are many other regulatory challenges facing EES such as restrictions on ownership and operation of generation assets by DNOs, high penalties on open ended stress events in the capacity market and undervaluation of flexibility in the balancing market. Classification of EES as a generation asset present further challenging in accruing revenue from multiple different value streams and many ancillary services are offered as short term contracts which cannot provide EES with the guaranteed future income needed to finance projects. These challenges highlight some of the deficiencies of liberalised markets in enabling access to monetary rewards for the benefits that EES provides.

It has been argued despite these regulatory barriers, an investment in EES could be justified in the future through income from arbitrage revenue alone. With increased penetration of intermittent renewables, prices may become more volatile creating additional opportunities for energy trading. A linear optimisation model was developed to determine the optimum charging and discharging strategy for a storage device given the device constraints of maximum charging and discharging rate, storage capacity, conversion efficiency and self-discharge. Using historic electricity prices, this confirmed that revenue was not sufficient to cover the costs of a storage project. It also highlighted the risks associated with novel technologies and the risk to arbitrage revenue from one year to the next dependent on volatile electricity prices. There was, however, a clear and predictable optimum pattern of charging and discharging following the regular daily cycle of electricity prices driven by patterns of demand behaviour. In the future, as more variable renewable capacity is installed, these price patterns will change leading to changes in the optimum operation strategy for and EES device and changes in revenue. An electricity price model was developed and coupled with the arbitrage optimisation to investigate these effects.

The results from the sensitivity and scenario analysis presented in this chapter show that EES revenue is, indeed, dependent on prevailing generation technology and patterns of demand which dictate the variation in wholesale electricity price. Critical aspects which could affect EES revenue which have not been examined include high penetrations of other renewable 
generation, such as solar, wave or tidal, and significant changes in patterns of demand as a result of wide spread uptake of electric vehicles, heat pumps or demand side response (DSR).

In the model, the only renewable generation considered was wind power. This is the most mature technology and accounts for the highest installed capacity in GB. The cost of solar power has reduced significantly in recent years and is continuing to fall [259], making it more competitive with conventional generation and economically viable in countries, such as the UK, which do not have the sunniest climates. Solar power is not included in the model, but may make a significant contribution to generation in GB in the 2020s. Like wind power, solar output would be represented in the model as non-dispatchable with zero marginal cost. Unlike wind power, it follows a more regular daily cycle of peak generation around midday and no generation overnight (out with daylight hours). There is also significant seasonal variation. This would change the pattern of electricity prices, supressing daytime peaks, particularly over the summer months, but having little effect during hours of darkness. If solar power was the prominent form of generation, storage devices which could charge and discharge on a daily basis, would be best rewarded by arbitrage, rather than those better suited to longer durations promoted by wind power.

Wave and tidal generation are unlikely to be deployed in large numbers over the timescales investigated in the scenario analysis; however, longer term these may also form a substantial share of the generation mix. Tidal power output is regular, following a daily cycle which can be accurately predicted years in advance. Wave power, driven predominantly by the wind, is less regular, and can only be accurately forecast over shorter time horizons. The impact of each of these on electricity prices will depend on the natural resource, technical characteristics and spatial distributions of the devices in addition to the installed capacity relative to other renewable and thermal generators. The presiding generation regime, upon which arbitrage revenue is dependent, is continuously changing and there are a number of vastly different potential long term outcomes.

Changes in patterns of demand will also change wholesale electricity prices and impact arbitrage revenue. It is anticipated that electric vehicles will be used during the day and charged overnight, increasing demand during off peak hours. This would contribute towards reducing daily variations in electricity price and reduce opportunities for arbitrage. The effect would be more pronounced if there were incentives to charge vehicles when electricity was cheap. Electric vehicles would act as proxy EES devices and accelerate the effect of selfcannibalisation discussed in Chapter 5. The extent of this depends on the uptake of electric vehicles relative to traditional consumption levels as well as consumer charging behaviour. 
Equally, increased participation in DSR, for example through the role out of smart meters and implementation of time of use tariffs, would tend to flatten the demand curve, reducing opportunities for arbitrage. There are, however, significant socio-political concerns surrounding smart devices in the home and ownership of consumer data which are delaying widespread uptake. Increased use of electric heat pumps on the other hand, could increase opportunities for arbitrage. Heat demand follows a daily cycle with a significant reduction overnight with seasonal variation. Depending on the extent of the electrification of heat, there could be increased opportunities for EES.

It is clear that the electricity market is complex and that wholesale electricity prices, driven by a number of uncertain variables, cannot be accurately predicted over long time-scales. These uncertainties have huge impacts on the return from an EES project over its lifetime. Without certainty in future revenue, it is unlikely that large EES projects will be supported by private funding. It is also evident that increasing penetrations of renewable energy, which increase the value of flexibility in the electricity system, do not necessarily increase EES revenue, and may, in fact, reduce it. In addition, the influence of wind power on electricity prices leads to a less regular optimum daily cycle of charging and discharging for an EES device making it more challenging to schedule a regular operation strategy. This supports the conclusion drawn by the UK Energy Research Centre that providing system reserves "is not ... a purely economic issue" [260]. Alternatively, it may be concluded that there is a market failure and that power system economics do not adequately reflect the true system costs. For EES to be commercially viable, alternative methods of remuneration must be considered where a higher economic reward is issued for flexibility services than for simply delivering energy, as is currently the case. This suggests that the regulatory barriers, currently restricting EES from aggregating multiple revenue streams across regulated and deregulated parts of the electricity industry, need to be resolved. The results imply that liberalised electricity markets do not currently provide sufficient financial rewards for the benefits of EES to be realised.

\subsection{Chapter Summary}

This chapter coupled the arbitrage model, described in Chapter 4, with the electricity price model, presented in Chapter 5 to investigate the impact of changing market variables on EES revenue.

The sensitivity study varied inputs independently to investigate their impact on arbitrage revenue. This showed that rising gas prices, carbon prices and demand led to increased annual revenue, but increased wind capacity reduced revenue. With higher wind penetrations 
wholesale electricity price patterns also changed, changing the way in which EES was operated. As a result, revenue was more sensitive to capacity, but less sensitive to charging rates. Conversion efficiency and self-discharge also became more important. In addition to long-term evolution of the electricity market, annual changes in wind speed, fuel price and demand profiles could lead to changes in revenue of over $50 \%$ from one year to the next.

The National Grid 2014 Future Energy Scenarios were investigated to assess the changing revenue to 2035 for different future energy systems in GB. These scenarios demonstrate plausible combinations of changing inputs in the electricity market with alternative priorities of affordability and sustainability. The results showed the No Progression scenario, with least installed wind power, led to the highest annual revenue for EES. Up until 2025, the Gone Green scenario, with the least installed wind, returned the lowest revenue. There was a significant range in revenue between different scenarios, with changes of up to $100 \%$. There was also substantial variation within each individual scenario from one year to the next, despite using a single base year for wind speed, demand and fuel price patterns. There was no underlying trend of increasing or reducing revenue as the scenarios developed. This demonstrated that arbitrage revenue was dependent on the individual behaviour of electricity market variables, but also on the complex interaction between them. 


\section{Chapter 7}

\section{Conclusions}

This final chapter presents the conclusions of the thesis. Following a review of the motivation, the objectives of the thesis are addressed. The key chapter conclusions are summarised followed by consideration of the limitations in the approach taken and opportunities for further work. Finally, the implications of the results are discussed and some closing remarks are made addressing the hypothesis from Chapter 1.

\subsection{Thesis Motivation}

Legally binding targets to reduce greenhouse gas emissions have led to increased levels of variable renewable electricity generation, a trend which is expected to continue in the coming decades. In recent years, concerns surrounding the cost of integrating these technologies with existing electricity systems have risen on the political agenda. EES is a potential solution which could provide the flexibility needed to enable the integration of large penetrations of variable power without compromising system security.

As the market share of non-dispatchable generators increases, the potential value of EES becomes more evident. Although the system value is important, it is the commercial value which will drive its development. Without appropriate financial incentives for investment in EES, its potential to deliver value to the electricity system will not be realised.

\subsection{Scope and Contribution}

This project investigated the system level and commercial value of EES in GB. Regulatory and commercial challenges have resulted in an inconsistency between the social value and financial reward for EES and are encumbering its uptake in liberalised electricity markets. The objectives, which are addressed in Section 7.3, were to: 
1. review the state of the art of EES technology and scope for future improvements in the absence of non-technical barriers to development;

2. qualitatively assess the benefits of EES to electricity networks;

3. identify routes to market for EES in GB and highlight any commercial or regulatory barriers;

4. develop a techno-economic model to quantify the commercial value of EES through price arbitrage;

5. investigate the value of EES in future energy scenarios with a high penetration of intermittent renewable energy.

Techno-economic models have been developed previously; however, these largely assess the value of EES in historic market conditions. As the electricity sector evolves, from one based on fossil fuels to one based largely on low carbon generation, it is essential that the implications of these changes on enabling technologies, such as EES, are understood. This research combined a state of the art mesoscale atmospheric wind model with an electricity price model, applying a novel price function to represent scarcity of supply. This was coupled with a price arbitrage model to investigate the impact of changing market variables on EES revenue. The level of detail in the price model, which has not been implemented in an investigation on EES previously, enabled an assessment of the impact of changing fuel and carbon prices on storage revenue. A better understanding of how EES is valued commercially in current and future energy scenarios should deliver insights into how policy could best incentivise appropriate investment.

\subsection{Key Chapter Conclusions}

\subsubsection{Technology Development}

Chapter 2 reviewed EES technologies. A history of the industry highlighted key technological advances and the economic, environmental and political conditions which enabled their development. Largely, deployment of grid connected EES occurred prior to the re-structuring of the power sector in the 1990s. Many countries liberalised generation markets while retaining highly regulated transmission networks. This introduced a key regulatory issue for EES which provides benefits to both sectors. There is evidence of a recent resurgence of interest in EES, as a result of the increasing penetration of variable renewable electricity; however, beyond demonstration plants and state funded projects, widespread deployment is yet to be seen.

A review of EES technologies was presented by type then compared on technical ability and cost. This highlighted the range of devices with different combinations of characteristics suited 


\section{Conclusions}

to different applications. PHS and CAES were the only technologies which offered sufficiently high power ratings suited to delivering energy over long time-frames. A range of battery technologies were found to be better suited to distribution scale applications with varying requirements for power and delivery duration. FES, DLCs and SMES can discharge large amounts of electricity over short periods making them suitable for power quality applications including frequency response and voltage control services.

While no EES technology is yet commercially viable on a large scale for grid applications, further improvements in performance and cost are expected across a range of devices. Existing PHS plants may be upgraded with variable speed turbines and underground and underwater PHS could be developed in the longer term. Advances in analytical and computation tools to understand processes at atomic scale are expected to improve understanding of reactions which occur at the interfaces between electrodes and electrolytes in batteries. Improvements in safety standards and recycling techniques are also anticipated as the industry becomes more established. In the medium term, lithium ion and sodium ion batteries are expected to be market leaders, but in the long term, flow batteries may take the largest share of the grid battery market. Mass production and high performance engineering are anticipated to result in reductions in cost and improved storage efficiency of FES and material science may lead to significant performance improvements for SMES and DLCs. In the absence of non-technical barriers to development, there is significant scope for technological improvements in EES devices.

\subsubsection{System Value}

Chapter 3 presented evidence on the cost of integrating variable renewable generators with the existing electricity systems. This highlighted the costs associated with maintaining acceptable levels of system adequacy and reliability in addition to losses in efficiency from running thermal plant at part load and the cost of curtailment. Furthermore, reduced running hours for peaking plant and increased volatility of wholesale electricity prices lead to higher costs of capital for investment in generation capacity, indirectly increasing system costs. EES provides a potential solution to this challenge. It could provide ancillary services minimising system operating costs, contribute to peak shaving and load levelling reducing the requirement for investment in expensive peaking plant and assist with network management reducing the need for investment in transmission and distribution network infrastructure and avoiding curtailment from renewable generators. As the penetration of variable renewables increases, system costs increase, and the potential benefits of EES also increase, expected to be of the 
order of $£ 10$ bn per year by 2050 [2]. Further benefits of job creation, stable wholesale electricity prices and environmental benefits would be created in addition to this value.

\subsubsection{Route to Market and Regulatory Barriers}

Despite the benefits EES can provide, revenue streams are challenging to access. Energy trading, or arbitrage, is the simplest method for an EES device to monetise the benefits it provides; however, this generally requires access to the wholesale electricity market, which carries a significant administrative cost. Delivery of ancillary services and, in recent years, participation in the capacity market could provide revenue for EES; however, contracts are provided for short durations and values change from one year to the next. These are not capable of providing the secure annual income required to invest in capital intensive projects which provide services over a number of decades. Provision of transmission and distribution network services, such as reducing peak demand to defer network capacity upgrades, are unlikely to provide sufficient revenue streams for EES on their own, but they could provide additional income to a storage operator at a high value for a short number of hours, enabling alternative revenue streams to be accessed for the remainder of the time. It is evident that there is a requirement to aggregate a number of revenue streams to make storage commercially viable; however, issues surrounding the exchange of information and capital between regulated and deregulated sectors of the industry currently prevents this. Additional market barriers include arrangements surrounding the Climate Change Levy, the undervaluation of flexibility in the imbalance settlement and complex local environmental legislation. These barriers highlight the deficiencies of liberalised markets in enabling access to monetary rewards for the benefits that EES provides.

\subsubsection{Modelling}

The latter part of the thesis addressed the final objectives of developing a techno-economic model of EES and investigating its value in future energy scenarios. Arbitrage was selected as a specific revenue stream which was representative of the commercial value of EES. Although it was recognised that multiple revenue streams are likely to be required to form a business case, market arrangements which enable aggregation of multiple incomes do not yet exist. Furthermore, the commercial nature of ancillary services and transmission and distribution contracts make it challenging to verify models and provide quantitative comparisons. Investigating a single source of income enabled key trends to be identified which provided insights into the drivers of arbitrage value, a more useful outcome than inaccurately modelling numerous markets. It had also been suggested that in future energy scenarios, with a high 


\section{Conclusions}

penetration of intermittent wind power, arbitrage revenue alone could justify an investment in EES.

Chapter 4 presented the linear optimisation model developed to calculate annual revenue using an input of historic electricity prices and the storage characteristic constraints. This was used, with market index prices from GB, to investigate the commercial value of EES. This highlighted the variation in revenue from one year to the next and the variation between different global markets. There was a clear relationship between the cyclical patterns of electricity demand and the daily charging cycle of the EES device. Comparing the costs and revenues for a case study of a CES device returned a negative NPV supporting previous conclusions that the economic case for EES could not be made through arbitrage alone. The importance of including an analysis of risk in addition to the value of an investment was discussed. A Monte Carlo risk analysis was used to assess the impact of uncertainty in cost estimates and performance on NPV, highlighting the risk facing an investor in any novel EES technology.

Chapter 5 described the model developed to simulate electricity prices for future energy scenarios. Wind power was represented using projections of the location and capacity of future wind farms and spatially distributed hind cast wind speed data generated from a mesoscale atmospheric model. This was coupled with the arbitrage model to assess the value of EES under different market conditions in Chapter 6. A sensitivity study showed that arbitrage revenue increased with rising gas prices, carbon prices and demand, but reduced with increasing penetrations of wind power. This implies that the inconsistency between the financial reward for EES and the system value which it provides is likely to be greater in an energy system with a high capacity of installed wind power. The novel uplift function applied in the price model and the treatment of wind power with zero marginal cost led to this distinct result which has not been demonstrated previously.

With high penetrations of wind power, the optimum EES characteristics also changed. Devices with a low power to capacity ratio performed better than in energy systems with little installed wind. This is likely to deter investment in large, monolithic projects as the commercial value may depreciate as the electricity market evolves. Conversion and storage efficiency became more critical in systems with high wind capacity as devices cycled less frequently and stored electricity over longer time periods. These characteristics have been shown to be of less importance from a whole system perspective suggesting that, in the case of efficiency, the market does not provide appropriate incentives for optimal system-wide solutions. 
Finally, the National Grid Future Energy Scenarios were investigated to compare the performance of EES in alternative plausible future energy systems. This showed the complexity of the electricity market and the range of combination of variables which could emerge as the sector develops, impacting EES significantly. By 2030, the range of annual revenue varied by over $100 \%$ between different scenarios. Equally, there was significant variation in revenue from one year to the next within the same scenario. Without certainty in future revenue, it is unlikely that large EES projects will be funded, even with substantial reductions in cost and improvements in technology performance.

\subsection{Limitations}

There are a number of limitations to the modelling approach which must be considered. The arbitrage model assumed perfect foresight of electricity prices. This was a reasonable approximation for historic electricity prices as it was shown that $95 \%$ of the optimum revenue could be attained with a price forecast error of up to $10 \%$. Historically, electricity prices, and storage operation, followed a regular daily cycle. An error in the electricity price forecast would be unlikely to significantly alter the operation schedule, resulting in the minimal impact on revenue. However, in future energy systems with a large penetration of wind power, electricity prices and storage operation strategies followed less regular patterns, driven by variations in wind power output. Errors in price forecasting may have a more significant impact in these cases. While this may affect the absolute value of revenue, the impact would be likely to be similar from one year to the next and so the trends in revenue in the sensitivity study and scenario analysis would be expected to remain largely unchanged.

A further assumption in the arbitrage model was that EES was a price taker and its operation would not affect electricity prices. This is valid when the capacity of installed EES is small compared to the total generation capacity, which is likely to remain the case in GB for the foreseeable future. However, if large capacities of EES were installed, the effect would be to smooth electricity prices, increasing demand during off peak hours and increasing supply to reduce price peaks. This would inherently reduce the opportunities for arbitrage, reducing the financial reward for EES, but increase the system benefits, providing more stable prices and a lower risk environment for generation capacity investments.

The dispatch and pricing model in itself is a simplification of both the price setting process and the operation of the GB system. The nature of the BETTA system makes it extremely difficult to model but the broad fundaments are likely to be a fair representation of a competitive system. In terms of system operation, the use of economic dispatch underplays 


\section{Conclusions}

the role of considerations such as spinning and standing reserve. These are an additional system cost, but one which EES could reduce. Further work, using models that explicitly handle reserve would be valuable.

A final limitation in the modelling approach was the representation of GB as a single bus bar with no network constraints. As locational marginal pricing is not currently used in GB, the arbitrage value would not be largely affected by this. However, representation of the network would provide insights into the optimal positioning of EES in the transmission and distribution networks which could provide substantial system savings.

The scenarios investigated included a number of projections about the future including the price of fuel, installed generation capacity and technology performance. Economic projections are difficult to make given the level of uncertainty surrounding market structure, technological progress, investment decisions, policy and consumer behaviour. As such, the results must be interpreted with a degree of caution. The absolute values of annual revenue are unlikely to be accurate and an evaluation of NPV, comparing lifetime income to technology costs for future energy scenarios, was actively excluded from the analysis. Similarly, it was decided not to investigate a specific technology and how its performance and costs may evolve over the time horizon, but to focus on a set of generic storage characteristics which were representative of a real technology used for arbitrage applications to investigate trends in revenue in isolation.

\subsection{Recommendations for Further Work}

The model could be extended to include alternative forms of renewable energy, such as wave, tidal and solar power. This would allow the relative impacts of different generating regimes to be investigated. It would also be beneficial to investigate the impact of changing patterns of demand on arbitrage revenue. DSR, heat pumps and electric vehicles are not currently represented in the price model. These emerging industries could develop over similar time scales to EES and, depending on the scale of their uptake, may have a significant impact on its operation.

A substantial extension of the project could combine the price model with a dynamic capacity investment model. This would better reflect the evolution of thermal generation capacity based on market signals and provide corrections to dampen or heighten the price peaks (on which arbitrage depends) in response to these changes. This would provide the foundations to include a feedback mechanism between EES operation and the electricity price, which would enable the effects of self-cannibalisation to be investigated and a comparison between investment in EES capacity and thermal generation to be made. 


\section{Conclusions}

The opportunities for participation in the newly formed capacity market in the UK were discussed. These were limited for EES as contracts were awarded for short durations and penalties were intolerably high for not delivering during an open-ended stress event. However, the impact of the capacity mechanism on wholesale electricity prices was not considered. This would be interesting to model as it is designed to reduce price spikes and flatten wholesale electricity prices. The level of capacity contracted through the capacity market is likely to impact the value of EES revenue. Subsidising technologies, such as existing gas turbines and new build diesel generators, through the capacity mechanism in the short term, may inadvertently reduce the market for novel technologies, such as EES, which could provide these services at a lower cost in the long run.

A further recommendation is to include a representation of transmission and distribution networks in the model. As discussed above, this is unlikely to have a significant impact on arbitrage revenue in the absence of locational marginal pricing; however, additional opportunities to monetise benefits may arise. For example, curtailment of renewable power, due to network capacity constraints, may provide commercial opportunities for EES.

Finally, a more structured review of the electricity market and ways in which barriers to market entry for EES could be removed would be beneficial to the EES industry. While this may be straight forward from the perspective of EES alone, when considering the broader implications of changes to regulation on other generators, loads and providers of flexibility, it quickly becomes more complex.

\subsection{Implications}

While the results of this thesis must be interpreted with caution and understanding of the assumptions and simplifications required to create a tractable model, the general conclusions can provide some useful insights. The research has demonstrated a clear disparity between the commercial and social value of EES and it is hoped that the results will inform the debate around the challenges facing the industry. There are regulatory barriers which restrict access to financial rewards for the full range of benefits which can be provided. Some of these could be easily resolved, for example, through creation of a new category of licence for EES and clarity on exemption from payment of the Climate Change Levy. There are other more substantial issues which are unlikely to be resolved without significant electricity market reform.

The most substantial challenge facing EES is the level of uncertainty in the electricity industry. There are many alternative futures which could evolve from the current system which are 


\section{Conclusions}

drastically different from one another, each with significantly different roles for EES. This is a consequence of the influence of the political system, operating on a five year cycle, compared to the lifetime of EES devices, which can be over 50 years, in addition to the rapid technological changes in generation capacity. Without commitment of guaranteed future income or public funding to pay for projects, it is unlikely that large scale, grid connected EES will be deployed.

\subsection{Final Remarks}

This thesis has taken an interdisciplinary approach to investigate the value of EES in liberalised electricity markets using GB as a case study. It has drawn on literature from engineering, mathematics, climate and policy research. The work has enhanced the body of knowledge surrounding the implications of wind power on electricity prices and specifically, the performance of EES technology in future energy systems.

The risks facing investors in EES can be largely grouped into three categories; market, technology and environmental factors, which each lead to uncertainty. In addition to the risk, currently the high cost and poor performance of EES technologies, combined with regulatory barriers to the market, restrict their commercial viability. Despite a higher value being placed on EES in energy systems with a high penetration of wind power, oversupply of electricity during windy periods leads to frequently suppressed peak electricity prices and reduced price differentials. This thesis provides evidence which negates the hypothesis that:

Increased wind penetration leads to increased commercial opportunities for energy storage through price arbitrage. 


\section{References}

[1] UK Government, "The Climate Change Act," Crown, London, 2008.

[2] G. Strbac, M. Aunedi, D. Pudjianto, P. Djapic, F. Teng, A. Sturt, D. Jackravut, R. Sansom, V. Yufit and N. Brandon, "Strategic Assessment of the Role and Value of Energy Storage Systems in the UK Low Carbon Energy Future," Carbon Trust, London, 2012.

[3] D. Willetts, "Eight Great Technologies," 2013. [Online]. Available: http://www.policyexchange.org.uk/images/publications/eight\%20great\%20techn ologies.pdf. [Accessed 2015 March 26th].

[4] Department of Energy and Climate Change, "Planning our electric future: a white paper for secure, affordable and low-carbon electricity," July 2011. [Online]. Available:

https://www.gov.uk/government/uploads/system/uploads/attachment_data/file/4 8129/2176-emr-white-paper.pdf. [Accessed 20th June 2015].

[5] Low Carbon Innovation Coordination Group, "Technology Innovation Needs Assessment Electricity Networks and Storage,” August 2012. [Online]. Available: www.lowcarboninnovation.co.uk/document.php?o=15. [Accessed 23rd March 2015].

[6] P. Ekins, J. Skea, M. Winskel, D. Howard, N. Eyre and A. Hawkes, "Making the Transition to a Secure and Low-Carbon Energy System," UK Energy Research Centre, 2009.

[7] R. Kannan, "Role of electricity and hydrogen storage in low carbon energy system - modelling in temporal Markal model," in Proceedings of the International Energy Workshop, London, 2008.

[8] G. Bathurst and G. Strbac, "Value of combining energy storage and wind in short term energy and balancing markets," Electric Power Systems Research, vol. 67, pp. 1-8, 2003. 
[9] P. Pelacci and D. Poli, "The influence of wind generation on power system reliability and the possible use of hydrogen storages," Electric Power Systems Research, vol. 80, no. 3, pp. 249-255, 2010.

[10] P. Barton and D. Infield, "Energy storage and its use with intermittent renewabable energy," IEEE Transactions on Energy Conversion, vol. 19, no. 2, pp. 441-448, 2004.

[11] P. Grünewald, The Role of Electricity Storage in Low Carbon Energy Systems: Technoeconomic Drivers and Transitional Barriers, $\mathrm{PhD}$ Thesis: Imperial College London, Centre for Energy Policy and Technology Faculty of Natural Sciences, 2012.

[12] W. X. Shen, "Optimally sizing of solar array and battery in a standalone photovoltaic system in Malaysia," Renewable Energy, vol. 34, no. 1, pp. 348-352, 2009 .

[13] A. Jakhrani, A. Othman, A. Rigit, S. Samo and S. Kambph, "A novel analytical model for optimal sizing of standalone photovoltaic systems," Energy, vol. 46, no. 1, pp. 675-682, 2012.

[14] D. Ipsakis, S. Voutetakis, P. Seferlis, F. Stergiopoulos, S. Papadopoulou, C. Elmasides and C. Keivanidid, "Energy management in stand-alone power system for the production of electrical energy with long term hydrogen storage," Computer Aided Chemical Engineering, vol. 25, pp. 1125-1130, 2008.

[15] B. Nyamdash, E. Denny and M. O'Malley, "The viability of balancing wind generation with large scale energy storage," Energy Policy, vol. 38, pp. 7200$7208,2010$.

[16] A. Cavallo, "Controllable and affordable utility-scale electricity from intermittent wind resources and compressed air energy storage (CAES)," Energy, vol. 32, pp. 120-127, 2007. 
[17] B. Ge, W. Wang, D. Bi, C. B. Rogers, F. Z. Peng, A. T. de Almeida and H. AbuRud, "Energy storage system-based power control for grid-connected wind power farm," International Journal of Electrical Power and Energy Systems, vol. 44, no. 1, pp. 115-122, 2013.

[18] M. Korpass, A. Holen and R. Hildrum, "Operation and sizing of energy storage for wind power plants in a market system," International Journal of Electrical Power and Energy Systems, vol. 25, no. 8, pp. 599-606, 2003.

[19] P. Grünewald, T. Cockerill, M. Contestabile and P. Pearson, "The role of large scale storage in a GB low carbon energy future: Issues and policy challenges," Energy Policy, vol. 39, no. 9, pp. 4807-4815, 2011.

[20] R. Sioshansi, P. Denholm, T. Jenkin and J. Weiss, "Estimating the value of electricity storage in PJM: Arbitrage and some welfare effects," Energy Economics, vol. 31, no. 2, pp. 269-277, 2009.

[21] T. Muche, "A real option-based simulation model to evaluate investments in pumped storage plants," Energy Policy, vol. 37, no. 11, pp. 4851-4862, 2009.

[22] D. Connolly, H. Lund, P. Finn, B. Mathiesen and M. Leahy, "Practical operation strategies for pumped hydroelectric energy storage (PHES) using electricity price arbitrage," Energy Policy, vol. 39, pp. 4189-4196, 2011.

[23] N. Ni, "Energy storage solutions to enhance grid economics," Renewable Energy Focus, 31 October 2013. [Online]. Available: http://www.smartgridnews.com/artman/publish/Technologies_DG_Renewables/ Stanford-says-Don-t-use-batteries-with-wind-but-pumped-hydro-is-OK6159.html/\#.UpXB_p1FAy9.. [Accessed 20th November 2013].

[24] G. Strbac, "Quantifying the System Costs of Additional Renewables in 2020," October 2002. [Online]. Available: http://webarchive.nationalarchives.gov.uk/+/http:/www.berr.gov.uk/files/file213 52.pdf. [Accessed 27th January 2014]. 
I. MacLeay, K. Harris and A. Annut, "Digest of United Kingdom Energy Statistics (DUKES)," $2010 . \quad$ [Online]. Available: $\mathrm{http}: / /$ www.google.co.uk/url?sa=t\&rct=j\&q=\&esrc=s\&source=web\&cd=7\&cad= rja\&uact=8\&ved=0ahUKEwib09La857JAhVDuxQKHf4FCr0QFghAMAY\&url $=$ http $\% 3 \mathrm{~A} \% 2 \mathrm{~F} \% 2$ Frefman.etmodel.com\%2Fpublications\%2F1548\%2Fdownload\&usg=AFQjCNEtrt111GOs6 ZXM1u1rxWJ0kpuA5w\&sig2=-Y3puv7GTaTqwz-R. [Accessed 12 August 2013].

[26] J. Cox, "The impact of intermittency: How wind variability could change the shape of the British and Irish electricity markets," 1st July 2009. [Online]. Available: http://www.poyry.co.uk/sites/www.poyry.uk/files/202_0.pdf. [Accessed 27th March 2014].

[27] ELEXON, "ELEXON Portal Website," 02 July 2012. [Online]. Available: https://www.elexonportal.co.uk/news/latest?cachebust=ib0snesorc. [Accessed 26 June 2013].

[28] ICE ENDEX, "ICE Download Centre," [Online]. Available: http://www.iceendex.com/. [Accessed 1109 2014].

[29] National Grid, "Metered half-hourly electricity demands," Optimum Media Ltd, 2013. [Online]. Available: http://www.nationalgrid.com/uk/electricity/data/demand+data/. [Accessed 28 August 2013].

[30] S. Hawkins, A High Resolution Reanalysis of Wind Speeds over the British Isles for Wind Energy Integration, University of Edinburgh: PhD Thesis, 2012.

[31] S. Stoft, Power System Economics: Designing Markets for Electricity, New York: IEEE Press Wiley-Interscience, 2002.

[32] J. Baker and A. Collinson, "Electrical energy storage at the turn of the millenium," Power Engineering Journal, vol. 13, no. 3, pp. 107-112, 1999. 
[33] R. H. Schallenberg and E. Newson, "The Anomalous Storage Battery: An American Lag in Early Electrical Engineering," Technology and Culture, vol. 22, pp. $725-752,1981$.

[34] A. Price, Storage Technologies and Future Developments, Presentation from the IET Energy Storage: What's Next for the Grid Conference, London: The Electricity Storage Network, 4th June 2015.

[35] D. Hay, Hydroelectric Development in the United States, 1880-1940, Edison Electric Institute, 1991.

[36] D. Kirschen and G. Strbac, Fundamentals of Power System Economics, Wiley, 2010.

[37] H. Chen, T. N. Cong, W. Yang, C. Tan, Y. Li and Y. Ding, "Progress in electrical energy storage system: A critical review," Progress in Natural Science, vol. 19, no. 3, pp. 291-312, 2009.

[38] C. Yang and R. Jackson, "Opportunities and barriers to pumped-hydro energy storage in the United States," Renewable and Sustainable Energy Reviews, vol. 15, no. 1, pp. 839-844, 2011.

[39] M. Winskel, "When systems are overthrown: The 'Dash for Gas' in the British electricity supply Industry," Social Studies of Science, vol. 32, no. 4, pp. 563-598, 2002.

[40] Sandia National Laboratories, "Global Energy Storage Database," [Online]. Available: http://www.energystorageexchange.org/projects. [Accessed 21st July 2015].

[41] Navigant Research, "Nearly 40 New Advanced Energy Storage Projects Kicked Off in the First Half of 2013," 8th August 2013. [Online]. Available: http://www.navigantresearch.com/newsroom/nearly-40-new-advanced-energystorage-projects-kicked-off-in-the-first-half-of-2013. [Accessed 21st July 2015]. 
SBC Energy Institute, "Leading the Energy Transition Factbook Electricity Storage," $\quad 2013 . \quad$ September Anline]. Available: http://files.energystorageforum.com/SBC_Energy_Institute_Electricity_Storage \%20Factbook_vf.pdf. [Accessed 21 st July 2015].

[43] Hydro Equipment Association, "Pump Storage Power Plants," [Online]. Available: http://www.thehea.org/hydropower/special-focus/pump-storagepower-plants/. [Accessed 22nd July 2015].

[44] G. Fuchs, B. Lunz, M. Leuthold and D. U. Sauer, "Technology Overview on Electricity Storage," June 2012. [Online]. Available: http://www.sefep.eu/activities/projectsstudies/120628_Technology_Overview_Electricity_Storage_SEFEP_ISEA.pdf. [Accessed 13th October 2013].

[45] Internation Electrochemical Commission, "Electrical Energy Storage White Paper," 2011. [Online]. Available: http://www.iec.ch/whitepaper/pdf/iecWPenergystorage-LR-en.pdf. [Accessed 21st September 2013].

[46] Dominion, "Bath County Pumped Storage Station," [Online]. Available: https://www.dom.com/corporate/what-we-do/electricity/generation/hydropower-stations/bath-county-pumped-storage-station. [Accessed 22nd July 2015].

[47] J. P. Deane, B. Gallachoir and E. McKeogh, "Techno-economic review of existing and new pumped hydro energy storage plant," Renewable and Sustainable Energy Reviews, vol. 14, no. 4, pp. 1293-1302, 2010.

[48] Arup, "A five minute guide to electricity storage," 2012. [Online]. Available: http://www.arup.com/Publications/5_minute_guide_to_electricity_storage.aspx. [Accessed 23 April 2013].

[49] S. Sabihuddin, A. E. Kiprakis and M. Mueller, "A numerical and graphical review of energy storage technologies," Energies, vol. 8, pp. 172-216, 2015.

[50] E. Williams, Dinorwig: the electric mountain, London: National Grid Company, 1991. 
[51] R. Klar, M. Aufleger, T. Sant, V. Neisch and R. N. Farrugia, "Buoyant Energy Balancing wind power and other renewables in Europe's Oceans," in 2nd Offshore Energy and Energy Storage Symposium, Edinburgh, 2015.

[52] R. Loisel, A. Mercier, C. Gatzen, N. Elms and H. Petric, "Valuation framework for large scale electricity storage in a case with wind curtailment," Energy Policy, vol. 38, no. 11, pp. 7323-7337, 2010.

[53] Hydro World, "Scottish Power mulls expansion to Cruachan pumped-storage hydropower plant," 13th February 2014. [Online]. Available: http://www.hydroworld.com/articles/2014/02/scottish-power-mulls-expansionto-cruachan-pumped-storage-hydropower-plant.html. [Accessed 22nd July 2015].

[54] H. Chen, X. Zhang and J. T. C. Liu, "Compressed Air Energy Storage," in Energy Storage - Technologies and Applications, ISBN: 978-953-51-0951-8, InTech, DOI: 10.5772/52221, 2013, pp. 978-953.

[55] Salt Cavern Information Centre, "Compressed Air Energy Storage (CAES) in Salt Caverns," [Online]. Available: http://web.evs.anl.gov/saltcaverns/uses/compair/index.htm. [Accessed 11 April 2013].

[56] H. Ibrahim, "Energy storage systems - Characteristics and comparisons," Renewable and Sustainable Energy Reviews, vol. 12, no. 5, pp. 1221-1250, 2008.

[57] EA Technology, "Review of Electrical Energy Storage Technologies and Systems and of their Potential for the UK," 2004. [Online]. Available: http://www.wearemichigan.com/JobsAndEnergy/documents/file15185.pdf. [Accessed 27th September 2013].

[58] General Compression, "What We Do - Texas Dispatchable Wind 1, LLC," 2013. [Online]. Available: http://www.generalcompression.com/index.php/tdw1. [Accessed 11 April 2013]. 
[59] P. Moser, "Projects and Technologies: ADELE - Adiabatic compressed-air energy storage (CAES) for electricity supply," RWE, [Online]. Available: http://www.rwe.com/web/cms/en/365478/rwe/innovation/projectstechnologies/energy-storage/project-adele-adele-ing/. [Accessed 22 April 2013].

[60] R. Dell and D. Rand, "Energy storage - a key technology for global energy sustainability," Journal of Power Sources, vol. 100, no. 1-2, pp. 2-17, 2001.

[61] M. Strasik, P. Johnson, A. Dat, J. Mittleider, M. Higgins, J. Edwards, J. Scindler, K. McCray, C. Mclver, D. Carlson, J. Gonder and J. Hull, "Design, Fabrication, and Test of a $5-\mathrm{kWh} / 100-\mathrm{kW}$ Flywheel Energy Storage Utilizing a HighTemperature Superconducting Bearing," IEEE Transactions on Applied Superconductivity, vol. 17, no. 2, pp. 2133-2137, 2007.

[62] Beacon Power, "Frequency Regulation," [Online]. Available: http://beaconpower.com/solutions/frequency-regulation.asp. [Accessed 22 April 2013].

[63] J. Hall and E. Bain, "Energy storage technologies and electricity generation," Energy Policy, vol. 36, pp. 4352-4355, 2008.

[64] H. Lui and J. Jiang, "Flywheel energy storage - an upswing technology for energy sustainability," Energy and Building, vol. 39, pp. 599-604, 2007.

[65] DG ENER Working Paper, "The Role and Future Challenges of Energy Storage," [Online]. Available: https://www.google.co.uk/url?sa=t\&rct=j\&q=\&esrc=s\&source=web\&cd=1\&cad $=$ rja \&uact $=8 \&$ ved $=0$ CCAQFjAAahUKEwimqIKYwPTIAhXFPhQKHdgICjg\& url=https $\% 3 \mathrm{~A} \% 2 \mathrm{~F} \% 2 \mathrm{Fec}$.europa.eu $\% 2 \mathrm{Fenergy} \% 2 \mathrm{Fsites} \% 2 \mathrm{Fener} \% 2 \mathrm{Ffiles} \% 2 \mathrm{Fe}$ nergy_storage.pdf\&usg=AFQjCNGhmfa3eycsq-IZQLzjem9eCKjbOQ\&sig2=I. [Accessed 3rd November 2015].

[66] J. Garche, Encyclopaedia of Electrochemical Power Soures, Elsevier, 2009.

[67] D. Rand, Valve-Regulated Lead Acid Batteries, Elsevier, 2004. 
[68] California Energy Commission, "Puerto Rico's 20MW Battery Energy Storage," in US Department of Energy Workshop of Electricity Storage, 2005.

[69] B. B. McKeon, J. Furukawa and S. Fenstermacher, "Advanced lead-acid batteries and the development of grid-scale energy storage systems," Proceedings of the IEEE, vol. 102, no. 6, pp. 951-963, 2014.

[70] D. Linden and B. R. Thomas, Handbook for Batteries Third Edition, McGrawHill, 2002.

[71] W. Tahil, "The Trouble with Lithium: Implications of Future PHEV Production for Lithium Demand," December 2006. [Online]. Available: http://tyler.blogware.com/lithium_shortage.pdf. [Accessed 7th October 2013].

[72] UK Power Networks, "Smarter Network Storage Brochure," October 2013. [Online].

Available:

http://innovation.ukpowernetworks.co.uk/innovation/en/Projects/tier-2projects/Smarter-Network-Storage-(SNS)/. [Accessed 23rd July 2015].

[73] J. Gifford, "Report: Lithium ion batteries dominate emerging grid storage market," pv-magazine.com, 5th March 2015. [Online]. Available: http://www.pvmagazine.com/news/details/beitrag/report--lithium-ion-batteries-dominateemerging-grid-storage-market_100018455/\#ixzz3fmXxqtr5. [Accessed 21st July 2015].

[74] Tesla, "Powerwall: Tesla Home Battery," [Online]. Available: http://www.teslamotors.com/en_GB/powerwall. [Accessed 23rd July 2015].

[75] D. Rastler, "Electricity Energy Storage Technology Options: A White Paper Primer on Applications, Costs and Benefits," December 2010. [Online]. Available:

https://www.parliament.nsw.gov.au/prod/parlment/committee.nsf/0/7482f370da 08bf4eca257a38000982f6/\$FILE/CSIRO\%20attachment\%201c\%20\%20US\%20storage\%20economics.PDF. [Accessed 1st November 2013].

[76] Woodbank Communications, “Zebra Batteries," [Online]. Available: http://www.mpoweruk.com/zebra.htm. [Accessed 23 April 2013]. 
[77] Tantaline, "Flow Batteries," [Online]. Available: http://www.tantaline.com/FlowBatteries-568.aspx. [Accessed 23rd July 2015].

[78] R. Castellano, Alternative Energy Technologies: Opportunities and Markets, Old City Publishing, 2010.

[79] United States Environmental Protection Agency, "Superfund Landfill Methaneto-Energy Pilot Project," December 2010. [Online]. Available: https://cluin.org/greenremediation/docs/Landfill_Methane_Final_Report_051011.pdf. [Accessed 12th March 2014].

[80] J. San Matin, I. Zamora, J. San Matin, V. Aperribay and P. Eguia, "Energy Storage Technologies for Electric Applications," in International Conference on Renewable Energies and Power Quality, Canary Islands, 2011.

[81] PEW Centre on Global Climate Change, "Electric Energy Storage,” August 2011. [Online]. Available: http://www.c2es.org/technology/factsheet/ElectricEnergyStorage. [Accessed 26 April 2013].

[82] F. Rahman, S. Rehman and M. Abdul-Majeed, "Overview of energy storage systems for storing electricity from renewable energy sources in Saudi Arabia," Renwable and Sustainable Energy Reviews, vol. 16, no. 1, pp. 274-283, 2012.

[83] S. Molokac, L. Grega and P. Rybar, "Using MRI devices for the energy storage purposes," Acta Montanistica Slovaca, vol. 12, no. 2, pp. 278-284, 2007.

[84] P. Taylor, R. Bolton, D. Stone, X. Zhang, C. Matrin and P. Upham, "Factsheet to accompany the report "Pathways for energy storage in the UK"," The Centre for Low Carbon Futures, 2012.

[85] Highview Power Storage, “Technology," 2013. [Online]. Available: http://highview-power.com/wordpress/?page_id=1405. [Accessed 12 April 2013].

[86] Isentropic, “Isentropic's PHES Technology," [Online]. Available: http://www.isentropic.co.uk/our-phes-technology. [Accessed 12 April 2013]. 
C. Pieper and H. Rubel, "Revisiting Energy Storage - There Is a Business Case," February 2011. [Online]. Available: https://www.bcg.com/documents/file72092.pdf. [Accessed 25th May 2014].

[88] Office of Basic Energy Sciences, "Basic Research Needs for Electrical Energy Storage," July 2007. [Online]. Available: http://science.energy.gov/ /media/bes/pdf/reports/files/ees_rpt.pdf. [Accessed 20th June 2013].

[89] Department of Energy and Climate Change, "UK Renewable Energy Roadmap Update," November 2013. [Online]. Available: https://www.gov.uk/government/uploads/system/uploads/attachment_data/file/2 55182/UK_Renewable_Energy_Roadmap_-_5_November__FINAL_DOCUMENT_FOR_PUBLICATIO__.pdf. [Accessed 17th October 2014].

[90] European Commission, "Large Combustion Plant Directive,” 2001.

[91] R. Gross, P. Heptonstall, D. Anderson, T. Green, M. Leach and J. Skea, "The Costs and Impacts of Intermittency: An assessment of the evidence on the costs and impacts of intermittent generation on the British electricity network," 1st March 2006. [Online]. Available: http://www.ukerc.ac.uk/asset/BCD3C9C4FCC1-4DAD-896A95960CD61D51/. [Accessed 9th January 2014].

[92] D. Eager, Dynamic Modelling of Generation Capacity Investment in Electricity Markets with High Wind Penetrations, University of Edinburgh: PhD Thesis, 2012.

[93] K. R. Voorspools and W. D. D'haeseleer, "An analytical formula for the capacity credit of wind power," Renewable Energy, vol. 31, no. 1, pp. 45-54, 2006.

[94] R. Green and N. Vasilakos, "The long-term impact of wind power on electricity prices and generating capacity," in IEEE Power and Energy Society General Meeting, San Diego, 24th-29th Jul 2011.

[95] R. Green and N. Vasilakos, "Market behaviour with large amounts of intermittent generation," Energy Policy, vol. 38, no. 7, pp. 3211-3220, 2010. 
[96] The Electricity Storage Network, "Development of Electricity Storage in the National Interest," May 2014. [Online]. Available: http://www.electricitystorage.co.uk/index.php/download_file/view/59/178/. [Accessed 3rd November 2015].

[97] National Grid, "Fast Response Service Description," April 2013. [Online]. Available:

http://www2.nationalgrid.com/WorkArea/DownloadAsset.aspx?id=11757. [Accessed 3rd November 2015].

[98] M. Peydayesh and R. Baldick, "The effects of very fast response to frequency fluctuation," in 31st USAEE/IAEE North American Conference; Transition to a sustainable energy era; opportunities and challenges, Austin, 2012.

[99] National Grid, “The Grid Code Issue 5 Revision 14,” 26th August 2015. [Online]. Available:

http://www2.nationalgrid.com/WorkArea/DownloadAsset.aspx?id=39212. [Accessed 3rd November 2015].

[100] National Grid, "Manatory Frequency Response," [Online]. Available: http://www2.nationalgrid.com/UK/Services/Balancing-services/Frequencyresponse/Mandatory-Frequency-Response/. [Accessed 19th August 2015].

[101] National Grid, "Firm Frequency Response," [Online]. Available: http://www2.nationalgrid.com/UK/Services/Balancing-services/Frequencyresponse/Firm-Frequency-Response/. [Accessed 19th August 2015].

[102] B. Kirby and E. Hirst, "Ancillary Service Details: Voltage Control," 1998. [Online]. Available: http://www.osti.gov/scitech/servlets/purl/607488. [Accessed 4th May 2015].

[103] National Grid, "Reactive Power Services," [Online]. Available: http://www2.nationalgrid.com/uk/services/balancing-services/reactive-powerservices/. [Accessed 19th August 2015]. 
[104] National Grid, "Balancing Services, Black Start," [Online]. Available: http://www2.nationalgrid.com/uk/services/balancing-services/systemsecurity/black-start/. [Accessed 16th July 2015].

[105] National Grid, "Black Start Service Description," October 2012. [Online]. Available:

http://www2.nationalgrid.com/WorkArea/DownloadAsset.aspx?id=7056.

[Accessed 3rd November 2015].

[106] A. A. Akhil, G. Huff, A. B. Currier, B. C. Kaun, D. M. Raster, S. B. Chen, A. L. Cotter, S. T. Bradshaw and W. D. Gauntlett, "DOE/EPRI 2013 Electricity Storage Handbook in Collaboration with NRECA," July 2013. [Online]. Available: http://www.sandia.gov/ess/publications/SAND2013-5131.pdf. [Accessed 16th February 2014].

[107] National Grid, "Historical Demand Data," [Online]. Available: http://www2.nationalgrid.com/uk/Industry-information/Electricity-transmissionoperational-data/Data-Explorer/. [Accessed 2nd November 2015].

[108] UK Government, "Energy Trends Section 4 - Gas,” 29th October 2015. [Online]. Available:

https://www.gov.uk/government/uploads/system/uploads/attachment_data/file/4 62554/Gas.pdf. [Accessed 2nd November 2015].

[109] Pöyry, "Smarter Network Storage Low Carbon Network Fund - Electricity storage in GB: SNS4.13 - Interim Report on the Regulatory and Legal Framework,”2014. [Online]. Available: http://poyry.co.uk/sites/www.poyry.co.uk/files/smarternetwork-storage-lcnf-interim-report-regulatory-legal-framework.pdf. [Accessed 29th January 2015].

[110] G. Strbac, Role and Value of Energy Storage in the Future GB Electricity System, London: IET seminar Energy Storage: What's Next for the Grid?, 4th June 2015. 
[111] Warshay, "Grid Storage under the Microscope: Using Local Knowledge to Forecast Global Demand,” 2012. [Online]. Available:

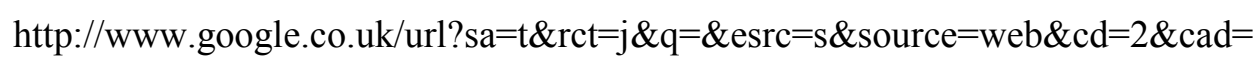
rja\&uact $=8 \& v e d=0 C C s Q F j A B a h U K E w i i s K b R u \_T I A h W J 1 R o K H X Q d A X E \& u r$ l=http $\% 3 \mathrm{~A} \% 2 \mathrm{~F} \% 2 \mathrm{Fcdn} 2$.hubspot.net $\% 2 \mathrm{Fhub} \% 2 \mathrm{~F} 86611 \% 2 \mathrm{Ffile}-15811852$ pdf\%2Fdocs\%2Fresearch\%2520downloads\%2F2012\%2Fgrid_storage_state-of. [Accessed 3rd November 2015].

[112] Navigant Research, "Grid-Scale Energy Storage Market to Reach \$35bn by 2020,” 31st August 2010. [Online]. Available: http://www.navigantresearch.com/newsroom/grid-scale-energy-storage-marketto-reach-35-billion-by-2020. [Accessed 20th August 2015].

[113] SBI Energy, "Energy Storage Technologies in Utility Markets Worldwide," 1st August 2010. [Online]. Available: http://www.marketresearch.com/SBIv775/Energy-Storage-Technologies-Utility-Worldwide-2435740/. [Accessed 3rd November 2015].

[114] D. Weißback, G. Ruprecht, A. Huke, K. Czerski, S. Gottlieb and A. Hussein, "Energy intensities, EROIs and energy payback times of electricity generating power plants," Energy, vol. 52, pp. 210-221, 2013.

[115] C. J. Barnhart and S. M. Benson, "On the importance of reducing the energetic and material demands of electrical energy storage," Energy and Environmental Science, vol. 6, pp. 1083-1092, 2013.

[116] J. L. Sullivan and L. L. Gaines, "A Review of Battery Life-Cycle Analysis: State of Knowledge and Critical Needs," 2010. [Online]. Available: https://anl.box.com/s/mcw0r17a55gok9imea1hexde3wgo9fjk. [Accessed 17th January 2015].

[117] SBC Energy Institute, "Leading the Energy Transition Factbook: Energy Storage," $\quad$ February $2013 . \quad$ [Online]. Available: https:/www.sbc.slb.com/ /media/Files/SBC\%20Energy\%20Institute/SBC\%20E nergy\%20Institute_Electricity_Storage\%20Factbook_vf1.pdf. [Accessed 24th February 2014]. 
[118] A. Wänn, P. Leahy, M. Reidy, S. Doyle, H. Dalton and P. Barry, "Environmental Performance of Existing Energy Storage Installations,”2012. [Online]. Available:

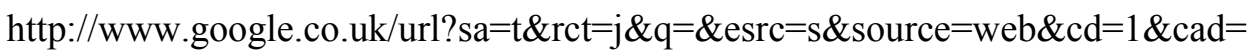
rja\&uact=8\&ved=0CCAQFjAAahUKEwj2kfb1v_TIAhXF8RQKHQK1DvY\&ur $\mathrm{l}=\mathrm{http} \% 3 \mathrm{~A} \% 2 \mathrm{~F} \% 2 \mathrm{Fwww}$.storeproject.eu\%2Fdocuments\%2Fresults\%2Fen_GB\%2Fenvironmentalperformance-of-existing-energy-storage-install. [Accessed 3rd November 2015].

[119] P. Denholm and G. L. Kulcinski, "Life cycle energy requirements and greenhouse gas emissions from a large scale energy storage system," Energy Conversion and Management, vol. 45, no. 13-14, pp. 2153-2172, 2004.

[120] E. Barbour, G. I. A. Wilson, I. G. Bryden, P. G. McGregor, P. A. Mulheran and P. J. Hall, "Towards an objective method to compare energy storage technologies: development and validation of a model to determine the upper boundary of revenue available from electrical price arbitrage," Energy \& Environmental Science, vol. 5, pp. 5425-5436, 2012.

[121] H. Lund, G. Salgi, B. Elmegaard and A. Andersen, "Optimal strategies of compressed air energy storage (CAES) on electricity spot markets with fluctuating prices," Applied Thermal Engineering, vol. 29, no. 5-6, pp. 799-806, 2009.

[122] R. Walawalker, J. Apt and R. Mancini, "Economics of electric energy storage for energy arbitrage and regulation in New York," Energy Policy, vol. 35, pp. 2558$2568,2007$.

[123] G. I. A. Wilson, P. G. McGregor, D. G. Infield and P. J. Hall, "Grid-connected renewables, storage and the UK electricity market," Renewable Energy, vol. 36, no. 8, pp. 2166-2170, 2011.

[124] A. J. Miranda, Techno-Economic Evaluation of Cruachan Power Station Extension, University of Edinburgh MSc Thesis, 2015. 
[125] Department of Energy and Climate Change, "EMR Annex C - Capacity Market: Design and Implementation Update," November 2012. [Online]. Available: https://www.gov.uk/government/uploads/system/uploads/attachment_data/file/6 5637/7104-emr-annex-c-capacity-market-design-and-implementation.pdf.

[Accessed 2nd December 2014].

[126] National Grid, "Final Auction Results T-4 Capacity Market Auction 2014," 16th December 2014.

[Online]. Available: https://www.emrdeliverybody.com/Capacity\%20Markets\%20Document\%. [Accessed August 25th 2015].

[127] Ofgem, "Electricity Balancing Significant Code Review - Draft Policy Decision Impact Assessment," 30th July 2013. [Online]. Available: https:/www.ofgem.gov.uk/sites/default/files/docs/2013/07/ebscr-draft-policydecision-impact-assessment_1.pdf. [Accessed 3rd November 2015].

[128] UK Power Networks, "Smarter Network Storage Low Carbon Network Fund SDRC 9.3 - Commercial Arrangements for Integrated Use of Flexibility (SNS4.6)," $2014 . \quad$ [Online]. Available: http://innovation.ukpowernetworks.co.uk/innovation/en/Projects/tier-2projects/Smarter-Network-Storage-(SNS)/ProjectDocuments/SNS4.6_SDRC+9.3+-+CA+for+IU+of+Flexibility_v1.0.pdf. [Accessed 3rd November 2015].

[129] National Grid, “Monthly Balancing Services Summary 2014/2015,” March 2015. [Online]. Available: http://www2.nationalgrid.com/UK/Industryinformation/Electricity-transmission-operational-data/Report-explorer/ServicesReports/. [Accessed 27th August 2015].

[130] K. Marr, "Electricity security of supply: A commentary on National Grid's Future Energy Scenarios for the next three winters," 17th July 2015. [Online]. Available: https://www.ofgem.gov.uk/publications-and-updates/electricity-security-supplyreport. [Accessed 3rd November 2015].

[131] M. Barlow, S\&C Electric Europe - Operation of Energy Storage, London: IET Seminar Energy Storage: What's Next for the Grid?, 4th June 2015. 
[132] UK Power Networks, "Smarter Network Storage: Project Overview,” November 2013. [Online].

Available:

http://innovation.ukpowernetworks.co.uk/innovation/en/Projects/tier-2projects/Smarter-Network-Storage-(SNS)/Project-Documents/OverviewSmarter-Network-Storage.pdf. [Accessed 3rd November 2015].

[133] UK Power Networks, "Smarter Network Storage - Business Model Consultation," July $2013 . \quad$ [Online]. Available: http://innovation.ukpowernetworks.co.uk/innovation/en/Projects/tier-2projects/Smarter-Network-Storage-(SNS)/Project-Documents/Smarter-NetworkStorage-Business-model-consultation.pdf. [Accessed 3rd November 2015].

[134] Energy Saving Trust, "Feed-in Tariffs," [Online]. Available: http://www.energysavingtrust.org.uk/feed-tariffs. [Accessed 25th August 2015].

[135] Flexitricity, “Triad guidance notes," 2013. [Online]. Available: http://www.flexitricity.com/docLibrary/Triad\%20guidance\%20notes\%202013.p df. [Accessed 25th August 2015].

[136] Western Power Distribution, "Use of System Charging Statement," 1st April 2015. [Online]. Available: https://www.westernpower.co.uk/docs/systemcharges/2015-Charging-Statements/MIDE-LC14-Complete-2015-V1-10publish.aspx. [Accessed 2nd November 2015].

[137] H. Xian, E. Delarue, W. D'haeseleer and J. Glachant, “A novel business model for aggregating the values of electricity storage," Energy Policy, vol. 39, pp. 1575$1585,2011$.

[138] P. Grünewald, T. Cockerill, M. Contestabile and P. Pearson, "The socio-technical transition of distributed electricity storage into future networks - System value and stakeholder views," Energy Policy, vol. 50, pp. 449-457, 2012.

[139] A. Patten, Energy Storage: Challenges in Policy Faced by Utilities, London: IET Conference: Power in Unity - A Whole System Approach, 16th September 2013. 
[140] A. Wänn and P. Leahy, "Energy Storage Action List: Promoting Energy Storage in Ireland," 2012. [Online]. Available:

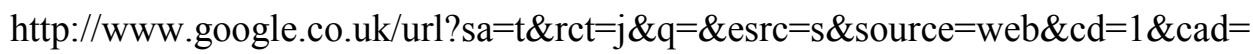
rja\&uact=8\&ved=0CCMQFjAAahUKEwi7nYLKv_TIAhUDXhQKHY_7A90\& url=http $\% 3 \mathrm{~A} \% 2 \mathrm{~F} \% 2 \mathrm{Fwww}$.store-project.eu\%2Fdocuments\%2Ftarget-countryresults\%2Fen_GB\%2Fenergy-storage-action-list-in-ireland\&usg=AFQ.

[Accessed 3rd November 2015].

[141] "Climate Change Levy Version 2," 31 July 2015. [Online]. Available: https://www.ofgem.gov.uk/sites/default/files/docs/2015/07/ccl_exemption_remo val_faq_v2_0.pdf. [Accessed 28th August 2015].

[142] European Commission, "Directive 2009/72/EC," 13th July 2009. [Online]. Available:

http://www.google.co.uk/url?sa=t\&rct=j\&q=\&esrc=s\&source=web\&cd=1\&cad= rja\&uact $=8 \&$ ved $=0 \mathrm{CCMQFjAAahUKEwiU9vPKwPTIAhWFVxQKHcvlCJ8 \& u}$ rl $=$ http $\% 3 \mathrm{~A} \% 2 \mathrm{~F} \% 2$ Feurlex.europa.eu\%2FLexUriServ\%2FLexUriServ.do\%3Furi\%3DOJ\%3AL\%3A200 9\%3A211\%3A0055\%3A0093\%3AEN\%3APDF\&usg=AFQjCNFSo. [Accessed 3rd November 2015].

[143] UK Government, “Electricity Act,” Crown, London, 1989.

[144] Ofgem, "Electricity Distribtuion Price Control Revenue Reporting: Regulatory Instructions and Guidance - Version 4," March 2008. [Online]. Available: https:/www.ofgem.gov.uk/ofgem-publications/47065/revenue-rigs-v4-newtemplate-draft.pdf. [Accessed 28th August 2015].

[145] Ofgem, "Electricitry Balancing Significant Code Review," 2nd April 2015. [Online]. Available: https://www.ofgem.gov.uk/electricity/wholesalemarket/market-efficiency-review-and-reform/electricity-balancing-significantcode-review. [Accessed 28th August 2015].

[146] Supergen, ESPRC, “The UK Energy Storage Consortium,” Supergen, ESPRC, 2010. [Online]. Available: http://www.energy-storage.org.uk/. [Accessed 17 May 2013]. 
[147] EPSRC, "Energy Storage for Low Carbon Grids," 1st October 2012. [Online]. Available:

http://gow.epsrc.ac.uk/NGBOViewGrant.aspx?GrantRef=EP/K002252/1. [Accessed 31st August 2015].

[148] EPSRC, "Integrated, Market-fit and Affordable Grid-scale Energy Storage," 15th March 2012. [Online]. Available: http://gow.epsrc.ac.uk/NGBOViewGrant.aspx?GrantRef=EP/K002228/1. [Accessed 31st August 2015].

[149] Energy Storage Research Network, "About ESRN," [Online]. Available: http://www.esrn.co.uk/. [Accessed 31st August 2015].

[150] Department of Energy and Climate Change, "Electricity System: Assessment of Future Challenges," 9th August 2012. [Online]. Available: https://www.gov.uk/government/uploads/system/uploads/attachment_data/file/4 8549/6098-electricity-system-assessment-future-chall.pdf. [Accessed 21st August 2014].

[151] EPSRC, "Willetts Announces $£ 85$ Million Research Capital Fund," Engineering and Physcial Science Research Council, 4 April 2013. [Online]. Available: http://www.epsrc.ac.uk/newsevents/news/2013/Pages/85millionresearchcapitalfu nd.aspx. [Accessed 20 May 2013].

[152] EPSRC, "New SUPERGEN Hub to set UK's energy storage course," 1st May 2014. [Online].

Available: https://www.epsrc.ac.uk/newsevents/news/newsupergenthub/. [Accessed 31st August 2015].

[153] Energy Technologies Institute, "ETI invest $£ 14 \mathrm{~m}$ in energy storage breakthrough with Isentropic," 12th June 2012. [Online]. Available: http://www.eti.co.uk/etiinvest-14m-in-energy-storage-breakthrough-with-isentropic/. [Accessed 31st August 2015]. 
[154] Department of Energy and Climate Change, "Innovation funding for low-carbon technologies: opportunities for bidders,” DECC, 8 May 2013. [Online]. Available: https://www.gov.uk/innovation-funding-for-low-carbon-technologiesopportunities-for-bidders. [Accessed 20 May 2013].

[155] Ofgem, "Low Carbon Network Funds," [Online]. Available: https://www.ofgem.gov.uk/electricity/distribution-networks/networkinnovation/low-carbon-networks-fund. [Accessed 31st August 2015].

[156] J. Radcliffe, Energy Storage Innovation, Glasgow: Scottish Hydrogen Fuel Cell Association event Powering the Future on Energy Storage and Energy Conversion, 7th March 2014.

[157] E. Lounsbury, The Future of Energy Storage in the UK, London: IET Conference: Power in Unity - A Whole System Approach, 16th September 2013.

[158] The Electricity Storage Network, "About Us," The Electricity Storage Network, 2011. [Online]. Available: http://www.electricitystorage.co.uk/electricitystorage-network_about.html. [Accessed 20 May 2013].

[159] Department of Energy and Climate Change, "Electricity Market Reform: Capacity Market - Detailed Design Proposals," June 2013. [Online]. Available: https://www.gov.uk/government/uploads/system/uploads/attachment_data/file/2 09280/15398_TSO_Cm_8637_DECC_Electricity_Market_Reform_web_optimi sed.pdf. [Accessed 20th February 2014].

[160] Department of Energy and Climate Change, "2010 to 2015 government policy: UK energy security,” 2015. [Online]. Available: https://www.gov.uk/government/policies/maintaining-uk-energy-security-2. [Accessed 16th November 2015].

[161] M. McAdam, "The Nuclear Option - What are the True Costs," 21st October 2013. [Online]. Available: http://www.aquamarinepower.com/blog/the-nuclear-optionwhat-are-the-true-costs/. [Accessed 31st August 2015]. 
[162] The Scottish Government, "National Planning Framework 3," 2014. [Online]. Available: http://www.gov.scot/Resource/0045/00453683.pdf. [Accessed 11th May 2015].

[163] USA Department of Energy, “Grid Energy Storage,” December 2013. [Online]. Available:

http://www.google.co.uk/url?sa=t\&rct=j\&q=\&esrc=s\&source=web\&cd=3\&cad= rja\&uact=8\&ved=0CCwQFjACahUKEwjYpL6QuPTIAhVH1BoKHX_C7s\&u $\mathrm{rl}=\mathrm{http} \% 3 \mathrm{~A} \% 2 \mathrm{~F} \% 2 \mathrm{Fwww}$.sandia.gov\%2Fess\%2Fdocs\%2Fother\%2FGrid_Ener gy_Storage_Dec_2013.pdf\&usg=AFQjCNF6AOSmnpruJBrUGJGb3DBM6bsV Rg\&sig. [Accessed 3rd November 2015].

[164] A. Dunbar, F. Tagliaferri, I. M. Viola and G. P. Harrison, "The implications of price forecast accuracy on the optimality of storage revenue," in IET $3 r d$ Renewable Power Generation Conference, Naples, 2014.

[165] F. Graves, T. Jenkin and D. Murphy, "Opportunities for electricity storage in deregulated markets," The Electricity Journal, vol. 12, pp. 46-56, 1999.

[166] F. Figueiredo, P. Flynn and E. Cabral, "The economics of energy storage in 14 deregulated power markets," Energy Studies Review, vol. 14, pp. 131-152, 2006.

[167] R. Byrne and A. Silvia-Monroy, "Estimating the Maximum Potential Revenue for Grid Connected Electricity Storage: Arbitrage and Regulation,” December 2012. [Online]. Available: http://www.sandia.gov/ess/publications/SAND20123863.pdf. [Accessed 20th February 2014].

[168] California Independent System Operator, "California ISO open access same-time information system (OASIS) site," [Online]. Available: http://oasis.caiso.com. [Accessed 18th June 2013]

[169] ELEXON, "ELEXON Portal: Market Index Price and Volume," 1st September 2010. [Online].

Available:

https://www.elexonportal.co.uk/article/view/188?cachebust=9t4u231wwk.

[Accessed 16th July 2015]. 
[170] R. E. Brown and J. G. Koomey., "Electricity use in California: Past trends and present usage patterns," Energy Policy, vol. 31, no. 9, pp. 849-864, 2003.

[171] P. Mokrian and M. Stephen, "A Stochastic Programming Framework for the Valuation of Electricity Storage," in 26th USAEE IAEE North America Conference, Detroit, 2006.

[172] D. P. Bertsekas, Dynamic Programming and Stochastic Control, New York: Academic Press Inc, 1976.

[173] H. Ben-Ameur, M. Breton, L. L'Ecuyer and L. Karoui, “A dynamic programming approach for pricing options embedded in bonds," Journal of Economic Dynamics and Control, vol. 31, pp. 2212-2233, 2007.

[174] M. Gil-Pugliese and F. Olsina, "Risk-Constrained Forward Trading Opitimization by Stochastic Approximate Dynamic Programming," in Dynamic Programming and Bayesian Inference, Concepts and Applications, INTECH, 2014.

[175] D. J. White, Markov Decision Processes, Manchester: Wiley, 1993.

[176] N. Amjady and F. Keynia, "Day ahead price forecasting of electricity markets by a mixed data model and hybrid forecast method," International Journal of Electrical Power and Energy Systems, vol. 30, no. 9, pp. 533-546, 2008.

[177] UK Power Networks, “Smarter Network Storage Homepage," January 2013. [Online]. Available: http://innovation.ukpowernetworks.co.uk/innovation/en/Projects/tier-2projects/Smarter-Network-Storage-(SNS)/Project-Documents/Smarter-NetworkStrorage-Brochure-Oct+2013.pdf. [Accessed 22nd April 2014].

[178] Electric Mountain, "Dinorwig Power Station," [Online]. Available: http://www.electricmountain.co.uk/en-GB/Dinorwig. [Accessed 22 April 2014].

[179] 1. Hu and G. Taylor, "A novel hybrid technique for short-term electricity price forecasting in UK electricity markets," Journal of International Council of Electrical Engineering, vol. 4, no. 2, pp. 114-120, 2014. 
[180] P. Grünewald, "Electricity storage in future GB networks - a market failure?," in British Institute of Energy Economics 9th Conference, Oxford, 19th-20th Sept 2012.

[181] R. Green, Storage in the Energy Market, Edinburgh: Keynote Presentation in Offshore Energy and Energy Storage Symposium, 3rd July 2015.

[182] R. Sioshansi, "Welfare impacts of electricity storage and the implications of ownership structure,” Energy Journal, vol. 37, pp. 173-198, 2010.

[183] J. Black, N. Hashimzade and G. Myles, A Dictionary of Economics Fourth Edition, Oxford: Oxford University Press, 2012.

[184] Centre for Low Carbon Futures, "Factsheet to accompany the report "Pathways for Energy Storage in the UK"," [Online]. Available: http://www.lowcarbonfutures.org/sites/default/files/PHS_final_0.pdf. [Accessed 19th October 2015].

[185] D. Eager, J. Bialek and T. Johnson, "Validation of a dynamic control model to simulate investment cycles in electricity generating capacity," in IEEE PES General Meeting, Minnesota, 25th-29th Jul 2010.

[186] Bloomberg New Energy Finance, "Weakest Quarter for Clean Energy Investment since 2009," 15 April 2013. [Online]. Available: http://about.bnef.com/pressreleases/weakest-quarter-for-clean-energy-investment-since-2009/. [Accessed 23 May 2014].

[187] W. Blythe, "Factoring Risk into Investment Decisions, UKERC Working Paper," November 2006. [Online]. Available: www.ukerc.ac.uk/asset/563AEB7C-D958409D-9661C423131B5D57/. [Accessed 1st May 2014].

[188] Office of Government Commerce, Managing Successful Projects with PRINCE2, London: The Stationery Office, 2009. 
[189] International Actuarial Association, "Stress Testing and Scenario Analysis," July 2013.

[Online].

Available:

http://www.actuaries.org/CTTEES_SOLV/Documents/StressTestingPaper.pdf.

[Accessed 23rd May 2014].

[190] F. Roques, W. Nuttall and D. Newberry, Using Probabilistic Analysis to Value Power Generation Investments under Uncertainty, Cambridge: Cambridge working papers in economics, Electricity Policy Research Group, 2006.

[191] S. Reutlinger, Techniques for Project Appraisal under Uncertainty (World Bank), Balitmore: The John Hopkins University Press, 1970.

[192] Personal Correspondence, Email from Matthew Brett, Head of Business Development, Highview Power Storage, London, Feb 2014.

[193] Highview Power Storage, "Highview Power Storage Company," [Online]. Available: http://www.highview-power.com/company/. [Accessed 19th October 2015].

[194] IMechE, "Highview Power Storage - Secure, clean power," 31 January 2012. [Online]. Available: http://www.imeche.org/docs/default-source/2011-pressreleases/Highview_2pager.pdf?sfvrsn=0. [Accessed 26 September 2013].

[195] Cleantech Group, "Global Cleantech 100," 2012. [Online]. Available: http://www.cleantech.com/global100/global-cleantech-100/. [Accessed 25 September 2013].

[196] Department of Energy and Climate Change, "Electricity System: Assessment of Future Challenges - Annex," 9th August 2012. [Online]. Available: https://www.gov.uk/government/uploads/system/uploads/attachment_data/file/4 8550/6099-elec-system-assess-future-chall-full.pdf. [Accessed 15th September 2014].

[197] Highview Power Storage, "Pilot Plant 350kW/2.5MWh," [Online]. Available: http://www.highview-power.com/liquid-air-energy-storage-laes-pilot-plant-july2011-november-2014/. [Accessed 19th October 2015]. 
[198] Department of Energy and Climate Change, “ $£ 8$ million boost for energy storage innovation," 13th February 2014. [Online]. Available: https://www.gov.uk/government/news/8-million-boost-for-energy-storageinnovation. [Accessed 19th October 2015].

[199] Y. Li, H. Chen, C. Tan and Y. Ding, "An integrated system for thermal power generation, electrical energy storage and CO2 capture," International Journal of Energy Research, vol. 35, pp. 1158-1167, 2011.

[200] The Dearman Engine Company, "Dearman Technology Summary," 11 April 2013. [Online]. Available: http://liquidair.org.uk/files/DEC2.pdf. [Accessed 26 September 2013].

[201] G. Brett, "Science and Technology Facilities Council - Highview Power Storage Presentation," 2 November 2011. [Online]. Available: http://www.stfc.ac.uk/resources/pdf/garethbrett.pdf. [Accessed 26 September 2013].

[202] S. Cornot-Gandolphe, O. Appert, R. Dickel, M. Chabrelie and A. Rojey, "The Challenges of Further Cost Reductions for New Supply Options (Pipelne, LNG, GTL)," in 22nd World Gas Conference, Tokyo, 2003.

[203] Liquid Air Energy Network, "Liquid air in the energy and transport systems: Opportunities for industry and innovation in the UK," 2013. [Online]. Available: http://www.liquidair.org.uk/files/full-report.pdf. [Accessed 19th October 2015].

[204] P. Agrawal, A. Nourai, L. Markel, R. Fioravanti, P. Grodon, N. Tong and G. Huff, "Characterization and Assessment of Novel Bulk Storage Technologies," Sandia National Laboratories, New Mexico, 2011.

[205] G. Brett and S. Leech, "Revolutionary, low-cost plant technology for energy security and flexibility," in Siemens: Investing in our Industrial Infrastructure, Manchester, 2012.

[206] Liquid Air Energy Network, "Liquid Air," SCS Marketing, [Online]. Available: http://www.liquidair.org.uk/liquid-air. [Accessed 26 September 2013]. 
[207] T. Peters, "International Events and Missions: UK-China Energy Storage Technologies Report," 30 August 2012. [Online]. Available: http://www.raeng.org.uk/international/pdf/Shafiq/Toby_Peters.pdf. [Accessed 26 September 2013].

[208] J. Sweeting, Project Cost Estimating: Principles and Practices, Rugby: Institution of Chemical Engineers, 1997.

[209] Personal Correspondence, Email from Fernando Morales-Rojo, Business Analyst, Highview Power Storage, London, Feb 2014.

[210] I. Leito, "The Normal Distribution," [Online]. Available: https://sisu.ut.ee/measurement/31-normal-distribution. [Accessed 1st December 2015].

[211] A. Dunbar, L. C. Cradden, A. R. Wallace and G. P. Harrison, "Impact of wind power on abritrage revenue for electricity storage," IET Generation, Transmission \& Distribution, doi:10.1049/iet-gtd.2015.0139, 2015.

[212] M. Shahidehpour, H. Yamin and Z. Li, Market Operations in Electric Power Systems: Forecasting, Scheduling and Risk Management, New York: Wiley-IEEE Press, 2002.

[213] L. Wu and M. Shahidehpour, "A hybrid model for day-ahead price forecasting," IEEE Transactions on Power Systems, vol. 25, no. 3, pp. 1519-1530, 2010.

[214] N. V. Karakatsani and D. W. Bunn, "Intra-day and regime-switching dynamics in electricity price formation," Energy Economics, vol. 30, no. 4, pp. 1776-1797, 2008 .

[215] R. J. Green and D. M. Newbery, "Competition in the British electricity spot market," Journal of Political Economy, vol. 100, no. 5, pp. 929-953, 1992.

[216] J. E. Evans and R. J. Green, "Why Did British Electricity Prices Fall after 1998?," in Cambridge Working Papers in Economics CWPE 0326, Massachusetts, 2005. 
[217] Department of Energy and Climate Change, "Changes to onshore wind subsidies protect investment and get the best deal from bill payers," 18th June 2015. [Online]. Available: https://www.gov.uk/government/news/changes-to-onshorewind-subsidies-protect-investment-and-get-the-best-deal-for-bill-payers.

[Accessed 24th June 2015].

[218] J. Boucher and Y. Smeers, "Alternative models of restructured electricity systems, Part 1: No market power," Operational Research, vol. 49, pp. 821-838, 2001.

[219] C. Metzler, B. F. Hobbs and J. S. Pand, "Nash-Cournot equilibria in power markets on a linearized DC network with arbitrage: Formulations and properties," Networks and Spatial Economics, vol. 3, pp. 123-150, 2003.

[220] R. Sioshansi and S. Oren, "How good are supply function equilibrium models: An empirical analysis of the ERCOT balancing market," Journal of Regulatory Economics, vol. 31, pp. 1-35, 2007.

[221] J. B. Bushnell, E. T. Mansur and C. Saravia, "Vertical arrangements, market structure, and competition: An analysis of restructured US electricity markets," American Economic Review, vol. 98, no. 1, pp. 237-266, 2008.

[222] D. Eager, "A dynamic control model of the UK electricity generating capacity investment market: engineering tools applied to an economic problem," in YEEES (Young Energy Engineers and Economists Seminar), Cambridge, 8th-9th Apr 2010.

[223] National Grid, "Interim Great Britain Seven Year Statement," November 2004. [Online]. Available: http://www.nationalgrid.com/NR/rdonlyres/1907A417F615-45B7-8345-0615725122BF/7347/Interim_GB_SYS.pdf. [Accessed 27 February 2014].

[224] UKERC, "UK MARKAL 2007 Electricity Generating Technologies," 2007. [Online]. Available: http://data.ukedc.rl.ac.uk/cgibin/dataset_catalogue//view.cgi.py?id=4. 
[225] Mott MacDonald, "UK Electricity Generation Costs Update," June 2010. [Online].

Available:

https://www.gov.uk/government/uploads/system/uploads/attachment_data/file/6 5716/71-uk-electricity-generation-costs-update-.pdf. [Accessed 12th August 2013].

[226] Department of Trade and Industry, "The Energy Challenge: Energy Review Report 2006,” July 2006. [Online]. Available: https://www.gov.uk/government/uploads/system/uploads/attachment_data/file/2 72376/6887.pdf. [Accessed 12th August 2013].

[227] R. Green, "Carbon tax or carbon permits: the impact on generators' risk," The Energy Journal, vol. 29, pp. 67-89, 2008.

[228] A. J. Wood, B. F. Wollenberg and G. B. Sheblé, Power Generation, Operation and Control, 3rd Edition, Wiley, 2013.

[229] World Nuclear Association, "The Economics of Nuclear Power," November 2008. [Online]. Available: http://www.worldnuclear.org/uploadedfiles/org/info/pdf/economicsnp.pdf. [Accessed 12th August 2013].

[230] Department of Energy and Climate Change, "Digest of United Kingdom Energy Statistics Annex A: Energy and commodity balances, conversion factors and calorific 2012 values,” [Online]. Available: https://www.gov.uk/government/uploads/system/uploads/attachment_data/file/6 5884/5959-dukes-2012-annex-a.pdf. [Accessed 12th August 2013].

[231] TradeTech, “Uranium Prices - NUEXCO Exchange Value,” [Online]. Available: http://www.uranium.info/nuexco_exchange_value.php. [Accessed 2 September 2014].

[232] TradeTech, "Uranium Prices - Overview," 2011. [Online]. Available: www.uranium.info. [Accessed 23rd March 2014]. 
Department of Energy and Climate Change, "Quarterly Energy Prices," $\begin{array}{lll}\text { September } 2009 . & \text { [Online]. Available: }\end{array}$ http://webarchive.nationalarchives.gov.uk/20100406130654/http://decc.gov.uk/ media/viewfile.ashx?filepath=statistics/publications/prices/1_20091008133755_ e_@@_qepsep09.pdf\&filetype=4. [Accessed 3rd March 2014].

BP, "Statistical Review of World Energy," http://www.bp.com/en/global/corporate/about-bp/energy-economics/statisticalreview-of-world-energy.html, 2014.

[235] S. Alterman, "Natural Gas Price Volatility in the UK and North America," February 2012. [Online]. Available: http://www.oxfordenergy.org/wpcms/wpcontent/uploads/2012/02/NG_60.pdf. [Accessed 20th November 2015].

[236] European Commission, "Quarterly Report on European Gas Markets: Market Observation for Energy Volume 7, Issue 3," 2014. [Online]. Available: https://ec.europa.eu/energy/sites/ener/files/documents/quarterlygas_q3_2014_final_0.pdf. [Accessed 2nd November 2015].

[237] European Environment Agency, "EUA Future Prices 2005-2011," 18th October 2011. [Online]. Available: http://www.eea.europa.eu/data-and-maps/figures/euafuture-prices-200520132011\#tab-documents. [Accessed 20th November 2015].

[238] UK Meteorological Office, "Met Office Integrated Data Archive System (MIDAS) Land and Marine Surface Stations Data (1853-current)," Centre for Environmental Data Archive, [Online]. Available: http://badc.nerc.ac.uk/view/badc.nerc.ac.uk_ATOM_dataent_ukmo-midas. [Accessed 12 February 2014].

[239] B. P. Hayes and S. Z. Djokic, "Modelling of wind generation at all scales for transmission system analysis," IET Generation, Transmission and Distribution, vol. 7, no. 10, pp. 1144-1154, 2013. 
[240] W. C. Skamarock, J. B. Klemp, J. Dudhia, D. O. Gill, D. M. Barket, X.-Y. Huang, W. Wang and J. G. Powers, "A Description of the Advanced Research WRF Version 3," Mesoscale and Microscale Meteorology Division, National Centre for Atmospheric Research, Colorado, 2008.

[241] Met Office, "Small-scale Wind Energy - Technical Report," August 2008. [Online]. Available: https://www.carbontrust.com/media/85174/small-scalewind-energy-technical-report.pdf. [Accessed 23rd January 2015].

[242] Department of Energy and Climate Change, "DECC Planning Database - Monthly Extract," January 2014. [Online]. Available: https://restats.decc.gov.uk/app/reporting/decc/monthlyextract. [Accessed 17th February 2014].

[243] T. Boehme, A. R. Wallace and G. P. Harrison, "Applying time series to power flow analysis in networks with high wind penetration," IEEE Transactions on Power Systems, vol. 22, pp. 951-957, 2007.

[244] R. Karki, P. Hu and R. Billington, "A Simplified wind power generation model for reliability evaluation," IEEE Transactions on Energy Conversion, vol. 21, pp. 533-540, 2007.

[245] K. Kaiser, W. Langreder, H. Hohlen and J. Hojstrup, Wind Energy: Turbulence Correction for Power Curves, Springer Link, 2007, pp. 159-162.

[246] European Standard, Wind turbine power performance testing, EN61400-12-1, 2006.

[247] H. Holttinen, Hourly wind power variations and their impact on the Nordic power system operation, Helsinki University of Technology: Licentiate Thesis, 2003.

[248] B. P. Hayes, I. Ilie, A. Porpodas and S. Z. Djokic, "Equivalent Power Curve Model of a Wind Farm Based on Field Measurement Data," in IEEE Power and Energy Society PowerTech, Trondheim, 19th-23rd Jun 2011. 
A. Tindal, C. Johnson, M. Leblanc, K. Harman and E. Rareshide, "Site specific adjustments to wind turbine power curves," in AWEA Windpower Conference, Houston, 1st-4th Jun 2008.

[250] J. E. McLean, "TradeWind Project - Equivalent Wind Power Curves," Garrad Hassan, 2008.

[251] K. Harman, R. Walker and M. Wilkinson, "Availability trends observed at operational wind farms," in European Wind Energy Conference, Brussels, 31st March-3rd April 2008.

[252] The Crown Estate, "R3 Site Selection at National and Project Levels FINAL v2 060613," [Online]. Available: http://www.thecrownestate.co.uk/energy-andinfrastructure/downloads/round-3-offshore-wind/. [Accessed 3rd March 2015].

[253] RenewableUK, "Offshore Wind Energy Development Rounds," [Online]. Available: $\quad$ http://www.renewableuk.com/en/renewable-energy/windenergy/offshore-wind/development-rounds.cfm. [Accessed 2nd March 2015].

[254] National Grid, "UK Future Energy Scenarios," 2014. [Online]. Available: http://www2.nationalgrid.com/WorkArea/DownloadAsset.aspx?id=34300. [Accessed 27th August 2014].

[255] Baringa, "Negative pricing in the GB wholesale electricity market," 7th July 2015. [Online]. Available: https://www.gov.uk/government/uploads/system/uploads/attachment_data/file/4 41809/Baringa_DECC_CfD_Negative_Pricing_Report.pdf. [Accessed 17th December 2015].

[256] A. Dunbar, A. R. Wallace and G. P. Harrison, "Storage characteristics rewarded by arbitrage and the implications of offshore wind power," in Offshore Energy and Energy Storage Symposium, Edinburgh, 1st-3rd July 2015.

[257] National Grid, "Future Energy Scenarios," 2014. [Online]. Available: http://fes.nationalgrid.com/. [Accessed 11th January 2016]. 
[258] J. Skea, P. Ekins and M. Winskel, "Energy 2050: The Transition to a Secure, Low Carbon Energy System for the UK,” Earthscan, London, 2011.

[259] National Geographic, "Solar Energy Sees Eye-Popping Price Drops," 2nd October 2015.

[Online].

Available:

http://news.nationalgeographic.com/energy/2015/10/151002-solar-energy-seeseye-popping-price-drops/. [Accessed 22nd January 2016].

[260] D. Anderson, "Electricity Generation Costs and Investment Decisions: A Review. Working Paper," February 2007. [Online]. Available: www.ukerc.ac.uk/asset/032EAA3B-0686-43F5-8DA6D868861FD94E/.

[Accessed 5th April 2013].

[261] D. Parker, The official History of Privatisation Vol. 1: The formative years 19701987, London: Routledge, 2009.

[262] F. P. Sioshansi and W. Pfaffenberger, Electricity Market Reform: An International Perspective, Elsevier Global Energy Policy and Economics Series, 2006.

[263] W. Boyes and M. Melvin, Fundamentals of Economics, Sixth Edition, SouthWestern, 2008.

[264] M. Hessenhausen, "Privatisations in Europe's liberalised electricity markets - the cases of the United Kingdon, Sweden, Germany and France," Stiftung Wissenschaft und Politik, Berlin, 2007.

[265] S. Hunt and G. Shuttleworth, Competition and Choice in Electricity, Chichester: Wiley, 1996, pp. 21-30.

[266] G. Gutiérrez-Akcaraz and G. B. Sheblé, "Electricity market dynamics: Oligopolistic competition," Electric Power Systems Research, vol. 76, pp. 695$700,2006$.

[267] K. Case and R. Fair, Principles of Economics, Fifth Edition, Prentice-Hall, 1999. 
[268] Ofgem, "Liquidity in the GB wholesale energy markets," 3rd July 2009. [Online]. Available: https://www.ofgem.gov.uk/publications-and-updates/liquidity-greatbritain-gb-wholesale-energy-markets. [Accessed 16th July 2015].

[269] National Grid, "What are Reserve Services?," [Online]. Available: http://www2.nationalgrid.com/uk/services/balancing-services/reserve-services/. [Accessed 16th July 2015].

[270] International Electrotechnical Commission, "Efficient Electrical Energy Transmission and Distribution," 2007. [Online]. Available: http://www.iec.ch/about/brochures/pdf/technology/transmission.pdf. [Accessed 2nd March 2015].

[271] L. Liu and A. Zobian, "The Importance of Marginal Loss Pricing in an RTO Environment," The Electricity Journal, vol. 15, no. 8, pp. 40-45, 2002.

[272] F. Elkarmi and N. AbuShikhah, Power System Planning Technologies and Applications, Engineering Science Reference, 2012.

[273] European Wind Energy Association, "Market Design: A position paper from the Eurpoean Wind Energy Industry," June 2015. [Online]. Available: http://www.ewea.org/fileadmin/files/library/publications/position-papers/eweaposition-paper-market-design.pdf. [Accessed 12th November 2015].

[274] H. L. Willis, Power Distribution Planning Reference Book Second Edition, Revised and Expanded, New York: Routledge, 2004.

[275] Ofgem, "Updated household energy bills explained factsheet 98," July 2013. [Online].

Available: http://www.ofgem.gov.uk/sites/default/files/docs/2013/01/household_energy_bil 1s_explained_udjuly2013_web.pdf. [Accessed 7th Oct 2014].

[276] UK Government, "Electricity Lighting Act,” Crown, London, 1982. 
[277] Centre for the Study of Regulated Industries, "Regulation of the UK Electricity Industry," $2002 . \quad$ [Online]. Available: http://www.bath.ac.uk/management/cri/pubpdf/industry_briefs/electricity_gillian _simmonds. [Accessed 20th March 2013].

[278] UK Government, "Electricity Act,” Crown, London, 1947.

[279] R. J. Gilbert, “International Comparisons of Electricity Regulation,” Cambridge University Press, Cambridge, 1996.

[280] F. McGovern, "Electricity - The Experience of Offer," in Regulatory Review, Bath, Centre of the Study of Regulated Industries, 1993.

[281] M. G. Pollitt, "Lessons from the history of independent system operators in the energy sector," Energy Policy, vol. 47, pp. 32-48, 2012.

[282] The National Archives, "Records of the Office of Electricity Regulation," 1999. [Online]. Available: http://discovery.nationalarchives.gov.uk/details/r/C141. [Accessed 16th July 2015].

[283] S. Hesmondhalgh, "Is NETA the Blueprint for Wholesale Electricity Trading Arrangements of the Future?," IEEE Trans. Power Systems, vol. 18, pp. 548-554, 2003.

[284] D. Newbery, "Power Markets and Market Power," Energy Journal, vol. 16, no. 3, pp. 41-66, 1995.

[285] X. Cui, "The UK Electricity Markets: Its Evolution, Wholesale Prices and Challenge of Wind Energy," University of Stirling, PhD Thesis, 2010.

[286] J. M. Griffin and S. L. Puller, Electricity Deregulation: Choices and Challenges, Chicago: University of Chicago Press, 2009.

[287] National Grid, "NETS Seven Year Statement: Chapter 10 - Market Overview," 2011. [Online]. Available: http://amazonaws.com/zanran_storage/www.nationalgrid.com/contentpages/126 556511.pdf. [Accessed 20th July 2013]. 
[288] D. Wadham and J. Bremen, "Electricity Regulation in the UK: Overview," Practical Law Company, 1 April 2013. [Online]. Available: http://uk.practicallaw.com/1-523-9996?q=*\&qp=\&qo=\&qe=\#. [Accessed 16 May 2013].

[289] Energy Network Association, "Electricity Distribution Map,” Energy Networks Association Ltd, 2013. [Online]. Available: http://www.energynetworks.org/info/faqs/electricity-distribution-map.html. [Accessed 16 May 2013].

[290] Department of Energy and Climate Change, "Domestic Electricity Payments in Great Britain: Assessment of payment timeliness," November 2012. [Online]. Available:

http://www.gov.uk/government/iploads/systems/uploads/attachment_data/file/49 758/domestic_electricity_payments_in_great_britain_-_timelines.pdf. [Accessed 2nd December 2014].

[291] Ofgem, "Ofgem - About us," Ofgem, 2007. [Online]. Available: http://www.ofgem.gov.uk/About\%20us/Pages/AboutUsPage.aspx. [Accessed 16 May 2013].

[292] National Grid, "The Connection and Use of System Code," [Online]. Available: http://www2.nationalgrid.com/UK/Industry-information/Electricitycodes/CUSC/The-CUSC/. [Accessed 16th July 2015].

[293] European Commission, "Renewable Energy Directive," 2009.

[294] UK Government, "The Electrical Safety, Quality and Continuity Regulations," 2002.

[295] UK Government, "Energy Act,” Crown, London, 2013.

[296] Department of Energy and Climate Change, "Policy: Maintaining UK Energy Security," DECC, 14 March 2013. [Online]. Available: https://www.gov.uk/government/policies/maintaining-uk-energy-security-2/supporting-pages/electricity-market-reform. [Accessed 16 May 2013]. 
[297] Department of Energy and Climate Change, "EMR Annex A - Feed-in Tariff with Contracts for Difference: Operational Framework," November 2012. [Online]. Available:

https://www.gov.uk/government/uploads/system/uploads/attachment_data/file/6 5635/7077-electricity-market-reform-annex-a.pdf. [Accessed 2nd December 2014].

[298] The Carbon Trust, "EU ETS Phase II allocation: implications and lessons," May 2007. [Online]. Available: https://www.carbontrust.com/media/84888/ctc715euets-allocation-implications-and-lessons.pdf. [Accessed 16th April 2013].

[299] HM Revenue and Customs, "Carbon price floor: reform and other technial amendments," $2014 . \quad$ [Online]. Available: https://www.gov.uk/government/uploads/system/uploads/attachment_data/file/2 93849/TIIN_6002_7047_carbon_price_floor_and_other_technical_amendments. pdf. [Accessed 3rd September 2015].

[300] Department of Energy and Climate Change, "Electricity Market Reform: Update on the Emissions Performance Standard," 2012. [Online]. Available: https://www.gov.uk/government/uploads/system/uploads/attachment_data/file/4 8375/5350-emr-annex-d--update-on-the-emissions-performance-s.pdf. [Accessed 23rd November 2014].

[301] H. Holland and K. Turekian, Treatise on Geochemistry, Elsevier, 2014. 


\section{Appendix A:}

\section{Background information on}

\section{Electricity Market Design}

This appendix provides an introduction to electricity market design and the fundamentals of energy system economics. It describes the role and relationship of different market participants and explains key economic concepts relevant to the electricity sector. This draws substantially from [36] where further information and examples can be found. The finals sections review a brief history of the development of the electricity industry in GB to provide context for the analysis carried out in this thesis.

\section{Introduction}

Traditionally, electricity was supplied to consumers by local distribution companies. The companies were either vertically integrated, responsible for generation and transmission of electricity, or they purchased electricity and were only responsible for distribution and supply. Consumers had no choice of service provider as companies held monopolies in specific geographical areas [36].

These models were largely successful and delivered electricity to increasing numbers of people with improved reliability. However, by the 1980s, economists argued that liberalised electricity markets were needed to provide further incentives for innovation and that monopolies could not offer maximum cost efficiency for consumers [261].

In a monopoly market, long term planning and optimisation tools are used to determine investment across the entire system. Some economists argue that this is beneficial to stimulate technological progression [262]. Large enterprises can better bear the costs associated with innovation and central planning reduces some elements of risk associated with future uncertainty. However, it is generally accepted that monopolies also lead to inefficiencies and higher costs for consumers. If ineffective decisions are made, the costs are passed onto customers. Competitive markets are driven by profits and are more cost effective [263]. 
In 1986, Chile was the first country to liberalise its electricity market followed by GB in 1990 and Norway in 1991 [262]. Today many countries have established wholesale energy markets including; all European Union member states, Australia, Brazil, New Zealand and many parts of the USA [264]. Market operation varies between countries; however, the goal of all market designers is to ensure that the industry operates in the best interest of society to ensure secure and affordable electricity. Recently, a third objective has come to the fore, to minimise emissions; however, the importance given to this depends on national politics and government priorities. Together, these three objectives form the Energy Trilemma.

\section{Electricity Market Fundamentals}

\subsection{Market Participants}

The organisations listed in Table 0.1 play a role in the electricity industry and will be referred to throughout this thesis. 
Table 0.1 Electricity market participants [36]

\begin{tabular}{|c|c|}
\hline Participant & Role \\
\hline $\begin{array}{l}\text { Vertically integrated } \\
\text { utilities }\end{array}$ & $\begin{array}{l}\text { Own generation plant as well as transmission and distribution } \\
\text { networks. }\end{array}$ \\
\hline Generating companies & Produce and sell electricity to the wholesale market. \\
\hline Distribution companies & Own and operate distribution networks. \\
\hline Retailers & $\begin{array}{l}\text { Purchase electricity on the wholesale market and sell it to } \\
\text { consumers. They cannot directly own distribution } \\
\text { infrastructure, but are often subsidiaries of distribution } \\
\text { companies. }\end{array}$ \\
\hline Market operators & $\begin{array}{l}\text { Run a system to match bids and offers from sellers and buyers } \\
\text { of electricity. }\end{array}$ \\
\hline $\begin{array}{l}\text { Independent system } \\
\text { operators }\end{array}$ & $\begin{array}{l}\text { Responsible for maintaining security of the power system. } \\
\text { Often the independent system operator is the market operator } \\
\text { for the market of last resort. }\end{array}$ \\
\hline Transmission companies & $\begin{array}{l}\text { Own and maintain transmission equipment which is operated } \\
\text { under the instructions of the independent system operator. }\end{array}$ \\
\hline Regulators & $\begin{array}{l}\text { Usually a governmental body which is responsible for } \\
\text { overseeing the operation of the industry. They approve the } \\
\text { rules and regulations for the electricity market. }\end{array}$ \\
\hline Consumers & $\begin{array}{l}\text { Purchase electricity from retailers and rent a connection to the } \\
\text { power system from distribution companies. Large consumers } \\
\text { may take an active role in the wholesale electricity market or } \\
\text { provide flexible demand services to the independent system } \\
\text { operator. }\end{array}$ \\
\hline
\end{tabular}

\subsection{Models of Competition}

Hunt and Shuttleworth [265] identify four phases of development of electricity markets as they progress from regulated monopolies through to fully competitive markets. These are presented below and help to explain the rationale of the structure of liberalised electricity markets today. 


\section{Monopoly}

The traditional monopoly model of the electricity industry is shown in Figure 0.1. This could include a vertically integrated monopoly or a monopoly for generation and transmission with further monopolies for distribution and supply in each geographical area. Bilateral trading could occur between the utilities in different areas which would take place at wholesale level. Alternatively, prices may be set through an agreed rate system.

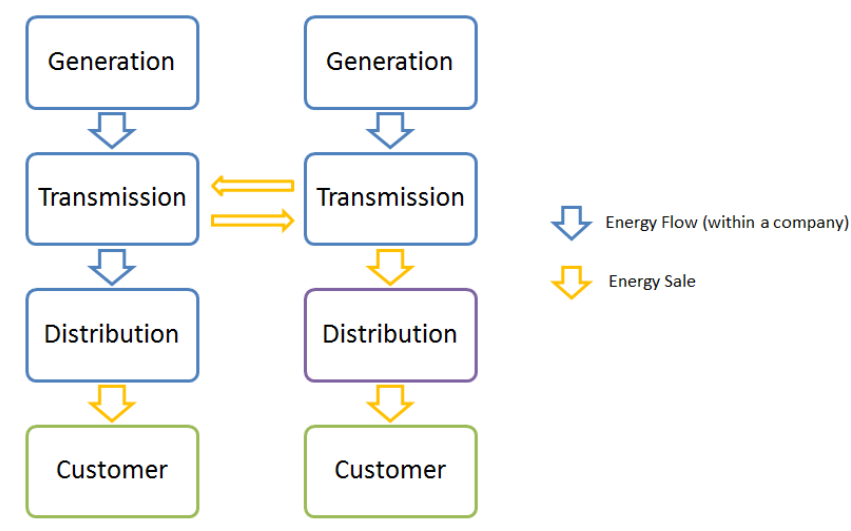

Figure 0.1 Monopoly model of the electricity industry (based on [265])

\section{Purchasing agency}

The purchasing agency model, shown in Figure 0.2, shows the introduction of competition within the electricity industry. Several independent power producers (IPPs) generate electricity and are connected to the network. They sell the power they generate to the principal utility which acts as a purchasing agency but does not own all the generation capacity. A further development of this model introduces multiple distribution companies as shown in Figure 0.3 . In this model, there is no vertically integrated utility. The IPPs sell electricity to the purchasing agency and the distribution companies purchase electricity from it to sell to consumers. In this scenario, the purchasing agency must be regulated as it would have monopoly power over the distribution companies and monopsony power ${ }^{9}$ towards the IPPs [36]. This model has the advantage of introducing some competitive elements into the electricity industry without the complexity and cost of establishing a fully competitive market. However, with the regulation of the purchasing agency, prices would not be as cost reflective as a free market.

\footnotetext{
${ }^{9}$ Monopsony power describes a market condition where there are several sellers, but only one buyer who has the ability to drive prices down. This is sometimes referred to as a buyer's monopoly.
} 


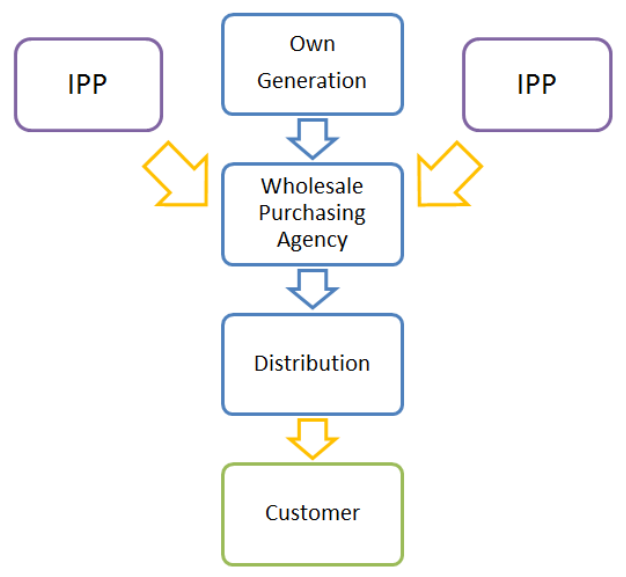

Figure 0.2 Initial purchasing agent model of the electricity industry (based on [265])

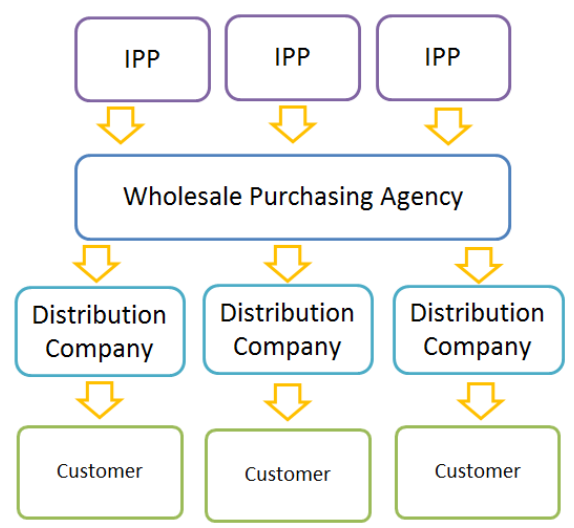

Figure 0.3 Further development of purchasing agent model of the electricity industry (based on [265])

\section{Wholesale competition}

The wholesale competition model shown in Figure 0.4 shows a further level of liberalisation. Distribution companies purchase energy directly from generating companies through a wholesale market. At the wholesale level, the spot market and the transmission network remain centralised. Wholesale prices are then determined by supply and demand rather than being set by the regulator, which is a more competitive process. Distribution companies maintain monopolies over customers in their local areas and, as such, retail prices must remain regulated. This leaves distribution companies exposed to losses if there are any unexpected increases in the wholesale market price. 


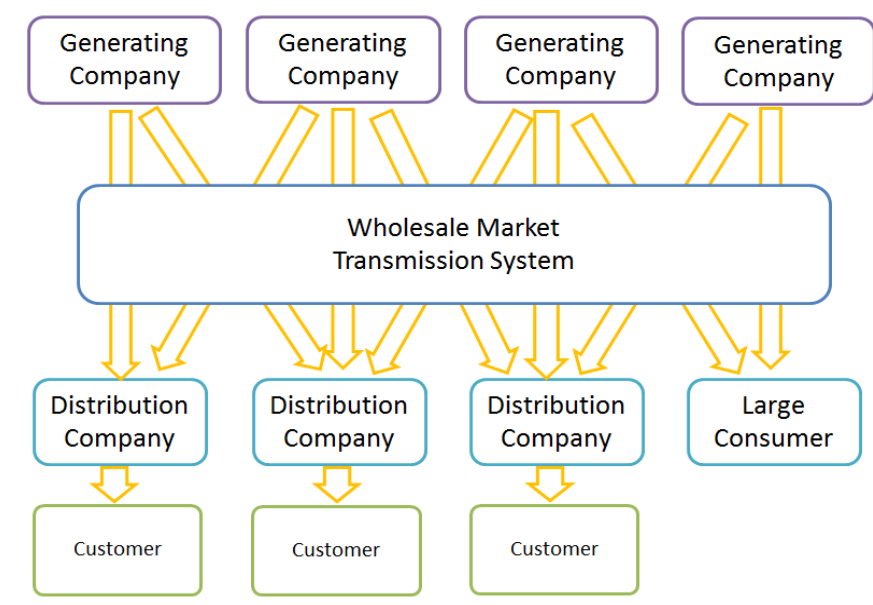

Figure 0.4 Wholesale competition model of the electricity industry (based on [265])

\section{Retail competition}

Small consumers cannot afford to participate directly in the wholesale market due to high transaction costs. To enable price competition for retail customers, a retail market must be established as shown in Figure 0.5. Retail competition removes monopoly power over customers so prices no longer need to be regulated which allows the most efficient cost of energy to be passed on to consumers. For retail competition to work effectively, customers must be able to switch readily between competing utilities. In practice this is not always the case. Transmission and distribution networks are natural monopolies and remain regulated. This model is implemented in most modern liberalised electricity markets.

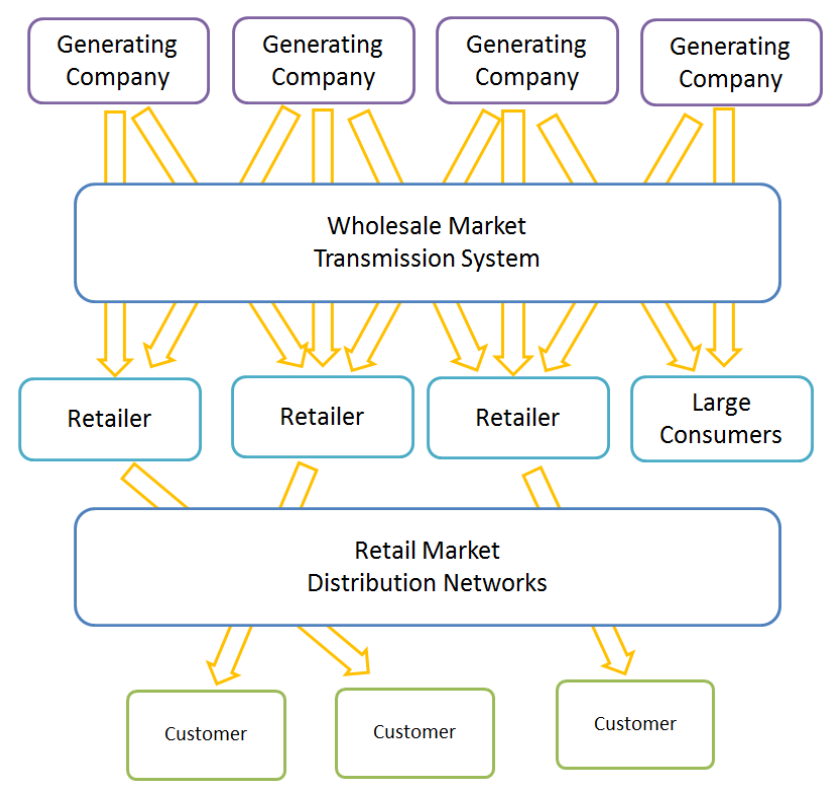

Figure 0.5 Retail competition model of the electricity industry (based on [265]) 


\section{Economic Concepts}

This section introduces some fundamental economic concepts which provide a foundation for the work in this thesis. A more comprehensive summary can be found in Chapter 2 of [31].

\subsection{Supply and Demand}

The supply and demand functions, shown in Figure 0.6, represent the relationship between the price of a product and the quantity sold in competitive markets. As prices increase fewer consumers will buy units. Similarly, as prices reduce, fewer suppliers will sell the product. The market clearing price, $\pi^{*}$, and the quantity, $Q^{*}$, represent market equilibrium where the two lines intersect.

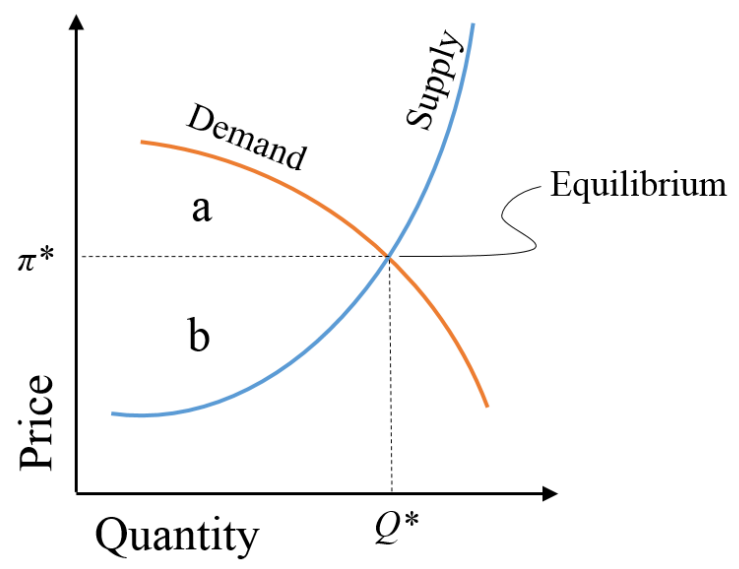

Figure 0.6 Supply and demand functions

\subsection{Global Welfare}

Consumer surplus is the difference between the price paid for products purchased and the value of those products to consumers. This is represented by the area above the market clearing price, but below the demand curve marked ' $a$ ' in Figure 0.6. The supplier profit is the difference between the cost of producing the products and the price they are sold to consumers for. This is represented by the area above the supply curve but below the market clearing price marked ' $b$ ' in Figure 0.6.

The sum of the consumer surplus and the supplier profit equates to the global welfare of the system. This is maximised at market equilibrium where the supply and demand curves intersect. Higher profits could be achieved if products were sold at a higher price; however, fewer units would be sold and the consumer surplus would be significantly smaller. This would lead to a net reduction in global welfare. Equally, if the price were lower than the equilibrium 
price, consumer surplus may increase, but supply would be restricted and profits reduced, again, leading to a net reduction in global welfare.

\subsection{Perfect Competition and Monopoly Power}

The model shown in Figure 0.6 assumes that there is perfect competition between all suppliers. This depends on two key assumptions:

1. Every supplier is a price taker i.e. no single supplier has a market share large enough to control the price of a product.

2. There is transparency in the market and consumers have visibility of all prices available to them [31].

When any individual company has a large enough share of the market that they are able to influence the price they are said to have monopoly power. Suppliers with monopoly power can withhold capacity which artificially shifts the supply curve to the left and increases the price of the product. This is known as exercising market power. The result is to increase profit for suppliers but, as discussed above, the consumer surplus will be reduced and the overall result is a reduction in global welfare. The loss of welfare is also known as a deadweight loss [36].

An oligopoly is a market structure where a small number of competing firms dominate, rather than a single firm, as in a monopoly. Oligopolies are commonly found in electricity industries including the GB market where there are six major generation companies. These are discussed in more detail in Section 9. Firms in oligopolies are interdependent and must take into account their competitors actions when making their own decisions, for example, on price setting. Barriers to market entry make it difficult and expensive for new firms to enter oligopoly markets. The dominant firms exploit natural barriers and manipulate the market to create new ones to maintain their position [266].

\subsection{Price Elasticity}

The price elasticity of demand is a measure of how sensitive the level of demand is to a change in price [267]. It is defined as:

$$
\epsilon_{d}=\frac{\frac{d q}{q}}{\frac{d \pi}{\pi}}=\frac{\pi}{q} \frac{d q}{d \pi}
$$

where $q$ is quantity, $\pi$ is price and $\epsilon_{d}$ is the price elasticity of demand. If $\epsilon_{d}$ is negative then demand is considered price responsive: a fall in price corresponds to a rise in demand and vice 
versa. If the magnitude is greater than one, demand is considered elastic, or more responsive. If it is less than one, the demand is inelastic, or less responsive.

Similarly, levels of supply respond to changes in price and the price elasticity of supply is used to measure its sensitivity. This is defined in equation $(0.2)$ where $\epsilon_{s}$ is the price elasticity of supply and other parameters are defined above [267]. Price elasticity of supply is similar to price elasticity of demand, but it is derived from the supply curve rather than the demand curve shown in Figure 0.6.

$$
\epsilon_{S}=\frac{\frac{d q}{q}}{\frac{d \pi}{\pi}}=\frac{\pi}{q} \frac{d q}{d \pi}
$$

\section{Energy Trading}

It is commonly accepted that there are two principal failures of electricity markets [31]:

1. Consumers do not respond to short term changes in price leading to almost zero price elasticity of demand.

2. Consumers are able to take power from the grid without a contract.

These failures prevent the market from functioning successfully without regulation and lead to additional complexities. As a result, energy is traded over different timescales through a combination of open and managed market arrangements which are described below.

\subsection{Open Energy Market}

Electricity is traded ahead of real time on the open energy market. These trades can be bilateral or through electricity pools.

\section{Bilateral trading}

Bilateral trading occurs directly between market participants. A key characteristic is that deals are made directly between buyers and sellers and prices are set by the participants, not by any third party.

Customised long term contracts are negotiated privately for large amounts of energy over long time periods. These are referred to as forwards and futures contracts. They have high transaction costs and so are too expensive to use to trade smaller quantities. These contracts are attractive in highly vertically integrated markets [36]. 
Forward trading also occurs over the counter (OTC). These contracts are pre-defined with standard terms and so have lower transaction costs, but prices are not made public. Price disclosure for OTC trades is reliant on price reporters and informal market intelligence [268]. These trades take place closer to real time than customised long term contracts and allow participants to refine their position as forecasts become more accurate.

Electronic trading allows participants to buy and sell electricity in a more transparent marketplace. Bids and offers are submitted by generators and suppliers and matched automatically. When a match is made, the price is made public. These trades are fast with low transaction cost and occur up until the market closes, allowing generators and retailers to adjust their positions just before delivery [36].

\section{Electricity pools}

'Pools' are a less common form of commodity trading, but do feature in electricity markets as they are seen as a natural evolution from centralized systems. Bids and offers are submitted to a pool which is managed by a third party. The bids from generators are ranked in increasing price order to determine the supply curve. Similarly, offers from suppliers are stacked to produce a demand curve. The point at which the two curves intersect gives the market clearing price (also known as the system marginal price) and this price is paid to (and by) all generators (and consumers) [36].

An electricity pool provides a centralised form of system management where the price paid is publically available.

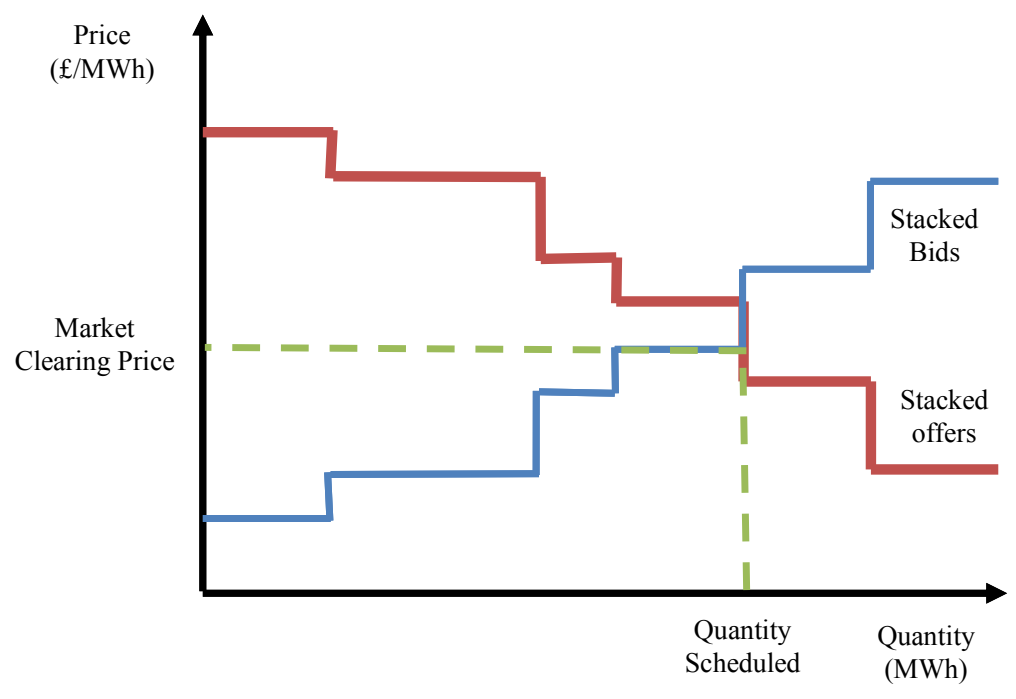

Figure 0.7 Stacked bids and offers in an electricity pool 


\subsection{Managed Spot Market}

The system operator is ultimately responsible for ensuring security of supply and so trading must stop ahead of real time to allow the system to be finally balanced. The point at which trading ceases is known as gate closure. The interval between gate closure and real time varies in different countries. System operators prefer long intervals to give them sufficient time to plan and execute balancing. Market participants, however, prefer short gate closures to enable them to make more accurate forecasts and reduce the risk they are exposed to.

At gate closure, producers and suppliers inform the system operator of their contractual positions. The system operator uses this information to run a managed spot market. Bids and offers are made by balancing resources to increase or reduce generation or demand. The system operator decides which bids and offers to accept, but the parties responsible for the imbalances pay the cost of the balancing activities. These markets are also called balancing or reserve markets.

Balancing services are offered by generating units which are not operating at full output to either increase or reduce their production. Equally, the demand side can provide balancing services by offering to reduce consumption. Offers of balancing resources that are made close to real time present a risk to the system operator that the prices will be high. To reduce the risk exposure balancing resources can be purchased in advance under longer term contracts.

\section{Ancillary Services}

In addition to the balancing services discussed above, there are other ancillary services which are required to ensure the secure and reliable operation of the electricity system [31]. These, including types of balancing mechanisms, are outlined below.

\subsection{Frequency Response}

To ensure security of supply, the system frequency must be maintained at a nominal level within specified limits. If the consumption exceeds generation, the system frequency will reduce; if generation is greater than consumption, the system frequency will increase.

Regulation services handle rapid fluctuations in frequency. These are rapid response generators which are connected to the grid and operate with a governor to allow automatic response to changes in frequency [98]. Load following services also make use of online equipment to manage fluctuations, but over slightly longer time periods than regulation services. These tend to be over intervals of around ten minutes rather than minute to minute. 
Regulation and load following services are preventative security measures which are provided continuously with fairly small actions that are largely predictable.

\subsection{Reserve Services}

Reserve services are intended to handle larger, less predictable power deficits such as outages of generators and are brought online as corrective actions [269]. They are generally classified as spinning and non-spinning (standing) reserve. Spinning reserve can provide a faster response while standing reserve has a start-up time associated with it. These services are used for relatively long periods until replacement generators can be brought online.

\subsection{Voltage Control}

Voltage must also be maintained within prescribed limits to make the transmission system secure and reduce the likelihood of insulation failures. Provision of voltage control is also called reactive power support service. Voltage control is provided by both generators which can control their reactive power output and by separate reactive power resources such as mechanically switched capacitors, reactors and tap changing transformers [102].

\subsection{Stability Services}

Some system operators require additional network security services such as inter-trip schemes to mitigate transient stability problems. In the event of a fault, these schemes automatically disconnect generation and/or load to maintain system stability [36].

\subsection{System Restoration}

Following a system failure, black start services may be required. Restarting large thermal generators requires electric power to drive auxiliary equipment such as pumps and fans. This will not be available if the system has collapsed [104]. Often small diesel generators or hydro plants are used for these services. Sufficient black start services must be available to ensure prompt restart of the system at any time.

\subsection{Provision of Ancillary Services}

Ancillary services are either compulsory for system users to provide or a separate market is created for their provision, depending on the service.

One example of compulsory provision of ancillary services is the requirement for generators connected to the grid to be capable of operating within a specific voltage range and be equipped with a voltage regulator. These compulsory requirements are not always 
economically efficient. It may be more expensive for some units to provide services than others and may encourage inefficient investment. Furthermore, it provides little incentive for innovation [36].

Some services are provided through ancillary service markets such as long term contracts for black start capabilities and inter-trip schemes. In some cases, spot markets are preferred because of interactions with the energy market. While these should provide more efficient markets, the number of participants for certain services is small and so they are susceptible to abuse.

The overall requirement for ancillary services is set by security standards for the system. Services are procured by the system operator and charged to the system users.

\section{Transmission Networks}

Transmission constraints and losses in the network introduce distortions in the electricity market which must be resolved. The physical laws governing power flow in the network include Ohm's Law as well as Kirchoff's Current and Voltage Laws. The latter two, respectively, state that the current flow into any point in a circuit is equal to the current flow out of that point;

$$
\text { Scurrent }=0
$$

and the voltage drop around any closed loop circuit must sum to zero:

$$
\sum \text { voltage }=0
$$

\subsection{Losses}

Losses in a transmission line are proportional to the square of the current flowing through it. This is true for direct current (DC) and a good approximation for an alternating current (AC) lines [270]. Transmission lines, therefore, operate at high voltages to deliver power over long distances at low currents to minimise losses. High voltage lines are then stepped down through transformers to deliver power at safe voltage levels for consumers.

Transmission losses cannot be attributed to single generators or individual loads, but depend on the interactions between them. The additional loss associated with transmission of one extra unit of power is called the marginal loss and is twice as high as the average loss [271]. In a perfectly competitive market for transmission, users would be charged the marginal cost of their losses. 


\subsection{Congestion Charging}

The maximum amount of electricity which can be delivered through a power line is limited by its transmission capacity limit. Losses in the line create heat which can cause sagging or, in extreme cases, failure. The thermal limit of a line is dependent on both the real and reactive power flowing through it.

Lines also have stability limits. In AC power lines the voltage at the generator is at its maximum value ahead of the voltage at the load. The difference between these values enables current and power to flow. The larger the voltage difference, the larger the power flow. However, if the generator voltage leads the load by a phase angle of more than $90^{\circ}$, the voltage at the load end rapidly becomes unstable and collapses [272]. To avoid stability limits, maximum phase differences and corresponding maximum power capacities are specified for transmission lines.

There are two classifications of limits on power lines; physical and contingency limits. Contingency limits are stricter than physical limits and ensure that a line's physical limit is not reached if another line or a generator unexpectedly goes offline. Congestion charging is required to account for limits on transmission lines.

When line limits are approached, the transmission resource is considered scarce and the price increases. Transmission rights are traded separately to energy. In order to sell and transmit power from one location to a second, either the buyer or the seller must own transmission rights. The cost of transmission can lead to different costs of energy at different locations.

\section{Generation Investment}

\subsection{System Merit Order}

At any time, a typical generation mix will contain a range of generators with differing fixed and variable costs. Ordering these generators by increasing short run marginal cost provides the system merit order (the order in which generators are economically dispatched). This can be broken down into three general categories as outlined below.

\section{Base load generators}

These have low operating costs, but high capital costs. They are expected to operate during most hours available in order to gain sufficient revenue to recover their fixed costs over their lifetime. Often, base load generators are required to operate with constant power output for technical reasons and are slow to respond to variations in demand. 


\section{Mid-merit generators}

These are generally more flexible with lower capital costs and so are not required to operate continuously to be economic. They usually have higher variable costs and so only come online when prices are higher and sufficient to cover their short run marginal costs.

\section{Peaking generators}

Peaking plant is called upon for a short number of hours per year, typically during times of high demand. They have high variable costs due to low thermal efficiency and expensive fuels.

\subsection{Long Run Costs}

In perfectly competitive energy markets, generators are incentivised to bid their marginal cost for generating electricity. In the short run, this cost covers variable operating costs such as fuel. In the long run, however, they must also cover the initial capital investment associated with building the plant.

Although generators bid their marginal costs, they are paid the market clearing price. The market clearing price is set by the highest merit order generator which is scheduled to run. The difference between the market clearing price and a generator's short run marginal cost is known as a scarcity rent and covers the generator's long run fixed costs [31]. Peaking generators are the highest merit order plant and so bidding at a price equivalent to their short run marginal cost would not allow these generators to ever recover their fixed costs. When electricity is scarce, these generators bid significantly above their short run marginal cost resulting in a price spike. Price spikes allow peaking plant to recover their long run fixed costs.

Price spikes generally occur for a short number of hours a year. Plotting an annual electricity price duration curve gives an indication of the range of electricity prices over a year as shown in Figure 0.8. 


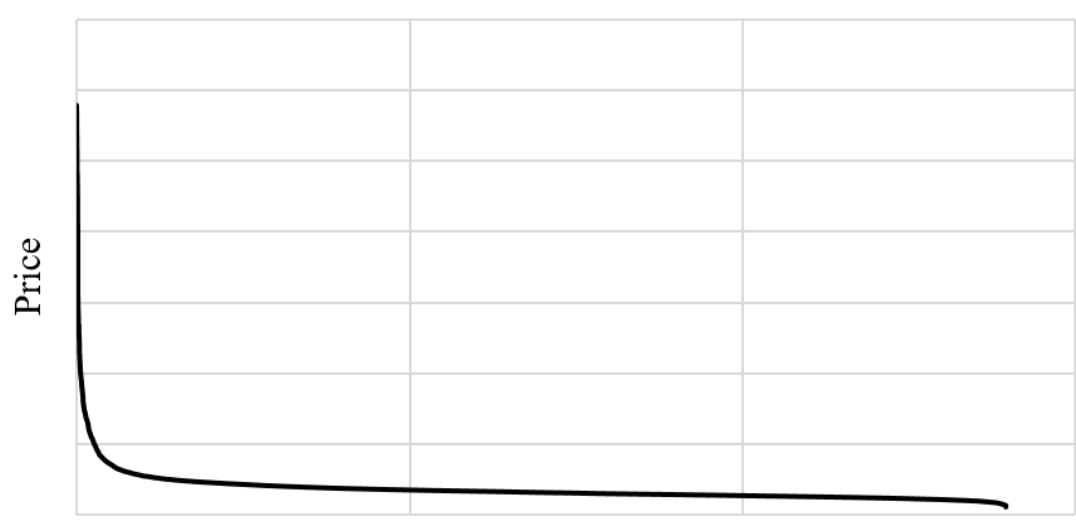

Number of hours

Figure 0.8 Example of electricity price duration curve based on 2003 GB spot market electricity prices [169]

It is price spikes which send long term investment signals to generators [273]. When capacity is scarce, price spikes occur more frequently and are of a larger magnitude. This presents opportunities for generators to make higher profits and so additional plant is built. As more generators come online, there are fewer periods of scarcity and so price spikes reduce, reducing opportunities for profits. This signals investors to retire old plant which is no longer economic. The uncertainty surrounding the height and duration of these price spikes leads to investment risk.

\subsection{Capacity Expansion}

There are issues associated with relying on the market to drive investment in generation adequacy. A lack of demand price elasticity and cyclic demand patterns lead to price increases that are not smooth or gradual. In theory, when capacity is scarce, price spikes should provide adequate signals for investors to build extra generators. Equilibrium should be reached and social welfare maximised. However, the consumers' inability to respond to short term price signals can delay investment. Additionally, generation plant has a lead time which further delays the response of the market. Traditionally generators are large and 'lumpy'. These features can lead to boom and bust cycles with periods of over-investment followed by delayed capacity and energy scarcity [31]

Inadequate provision of capacity could lead to disconnection of consumer loads which is considered largely unacceptable politically. Equally, price spikes are considered by some customers to be unjustified and socially unacceptable [36].

With increasing penetration of intermittent renewable generation making price spikes more difficult to forecast, generation investment becomes higher risk [26]. Increasingly regulators 
are implementing alternative mechanisms to promote generator investment. These are highlighted below.

\section{Capacity payments}

This system is formed on the basis that paying small regular payments to generators providing capacity is preferable to paying extreme price spikes on rare occasions. This provides a revenue stream for peaking generators to cover their fixed costs. Payments are proportional to the capacity provided and the price is set by the regulator. The effect is to moderate the price in the energy market and socialise the cost of peaking plant. In the long term, this reduces incentives for efficient behaviour and could lead to too much capital being invested in generation and too few signals to consumers to control demand in response to price [36].

\section{Capacity market}

A capacity market sets the amount of capacity required relative to a 'generation adequacy' 10 target. Retailers and consumers are required to buy a share of the total requirement from a competitive market. The quantity of capacity required is set by the regulator, but the prices can be volatile. The timescale over which capacity is planned and sold is critical to the market. Retailers prefer shorter time periods (one month or less) as it reduces the capacity they are required to purchase during times of light load. Generators prefer longer periods (seasonal or annual) which encourage investment [36].

\section{Reliability contracts}

Reliability contracts would allow consumers to decide freely and independently how much they value reliability. In current markets this is not possible and would require a central authority to purchase contracts on behalf of consumers [36].

\section{Transmission Investment}

The transmission network is a natural monopoly exhibiting features of high capital costs, bulky investments and significant economies of scale. As such, it must be regulated and so presents a low risk (low return) business for investors [274]. The generation market is deregulated and separated from the transmission network; however, investment in transmission must be optimised in line with generation investment to achieve system level optimisation.

${ }^{10}$ Generation adequacy defines the ability of the system to meet the total power and energy demand of all consumers at virtually all times. It is a statistical measure of the security of supply of the system. 
Investments in transmission are large and irreversible with little or no resale value. Assets have long lifetime of 20-30 years. Changing markets over this lifetime can lead to stranded assets [31].

Cost based transmission expansion allows transmission companies to receive sufficient revenue to attract capital investors looking for a safe return. The transmission company forecasts transmission requirements and submits an expansion plan to the regulator. The regulator reviews the plan and agrees what can be built. The transmission company then makes the upgrades and recovers the costs through charges to the users. Transmission and distribution costs accounted for approximately 20\% of an average UK electricity bill in 2012 [275].

\section{Development of the UK Electricity Industry}

\subsection{Pre-privatisation}

The electricity supply industry in GB was initiated through the Electricity Lighting Act 1882 [276]. In 1933 a 132kV National Grid began interconnected regional grid operation [92]. After World War Two, there were around 560 suppliers in the UK, with approximately one third of these under private ownership [277].

In 1943, the Hydro-electric Development Scotland Act placed responsibility for generation, transmission and distribution in the north of Scotland under a public corporation, the North of Scotland Hydro-Electric Board. A few years later, the Electricity Act 1947 [278] was passed and the industry in England and Wales was also reorganised and nationalised. The British Electricity Authority (BEA) was made responsible for generation and transmission and 14 area boards were created with responsibility for distribution and retail of electricity in their own regions. 12 area boards were based in England and Wales and two in the south of Scotland.

Over a decade later, the two Scottish area boards and BAE's Scottish generating divisions were merged under the Electricity Reorganisation (Scotland) Act 1954. From these, the South of Scotland Electricity Board (SSEB) was established. The SSEB was vertically integrated and was responsible for generation, transmission and distribution of electricity. At this time, the BEA was renamed the Central Electricity Authority (CEA), which, following the Electricity Act 1957, became the Central Electricity Generating Board (CEGB) [277]. The industry structure remained like this until privatisation in 1990. 


\subsection{Privatisation}

Prior to privatisation of the electricity industry, national supply was considered crucial in helping the economic recovery after the Second World War. It enabled expansion of generation capacity and transmission infrastructure [36]. However, by the 1980s, in line with global attitudes towards state-owned sectors, the British regulatory system was regarded as an "inefficient equilibrium that only privatisation appeared capable of upsetting" [279]. In response to criticism of sell offs of previous public monopolies, the industry was restructured prior to privatisation [280] under the Electricity Act 1989 [143].

In March 1990, the CEGB was dissolved and its assets divided between four new companies introducing a new industry structure in England and Wales. Three generating companies were established; National Power, PowerGen and Nuclear Electric. Fossil fuel plant was allocated between National Power and PowerGen and Nuclear Electric was transferred the entire nuclear generating capacity. A single transmission company, National Grid Company (NGC), took responsibility of the transmission system, pumped storage stations and interconnections with Scotland and France. Twelve regional electric companies (RECs) were created to replace the area boards. These were responsible for distribution systems in their respective areas and were required to supply all 'reasonable demand' for electricity [277].

After being vested as public limited companies, National Power and PowerGen sold 60\% of their shares in 1991 and the remainder in 1995. Nuclear reactors, however, were considered too expensive to be privatised and Nuclear Electric remained under state ownership until 1996 [277]. Shares in the RECs were sold in 1990, with the government retaining a golden share in each until 1995 [216]. When NGC was fully privatised in 1996, the pumped storage capacity was sold to an American firm, Edison Mission Energy (acquired by International Power in 2004) as it was deemed anti-competitive for a private company to own both generating and transmission assets [281].

In Scotland, ScottishPower and Scottish Hydro-Electric replaced the SSEB and the HydroElectric Board. Both companies were privatised in 1991. Similarly to England and Wales, nuclear generation was assigned to a separate company, Scottish Nuclear. In 1996, Scottish Nuclear became part of British Energy and, in 1998, Scottish Hydro-Electric merged with Southern Electric to become Scottish and Southern Energy [277].

An electricity pool was established as the mechanism for trading wholesale electricity in England and Wales. An independent regulator, the Office of Electricity Regulation (OFFER), was established to regulate the newly privatised industry [282]. 


\section{The electricity pool}

The purpose of the pool was to enable competitive bidding between generators to establish an electricity price for each half hour period. The principles of the pool were based on the CEGB merit order dispatch. Generators submitted their supply curve a day-ahead. The system operator (NGC) computed the least cost economic dispatch required to meet demand and all generators were paid the system marginal price. NGC was responsible for making real time adjustments to ensure supply and demand were balanced [277].

The pool purchase price was formed from two components; the spot market price for electricity and a capacity payment. The capacity payment was a function of the system loss of load probability and the value of lost load. This payment would increase exponentially as demand approached the total system capacity available. Pool prices were peaky and volatile. To hedge against uncertainty, generators and suppliers would sell the bulk of electricity in OTC forward trades which would usually take the form of contracts for difference [283].

Over a decade of operation, a number of problems were identified with the electricity pool [284]:

- There was a lack of competition in price setting with PowerGen and National Power able to set the spot price over $90 \%$ of the time due to their considerable market share;

- There was a minimal demand side participation in the market;

- Bidding arrangements were considered too complex;

- The capacity payment mechanism was susceptible to producers with a large market share withholding capacity by declaring plant unavailable, leading to poor signals for actual requirements for long term capacity investment;

- Interactions with the gas market were inefficient with trading taking place a day ahead while gas trading occurred closer to real time. The large numbers of gas turbines could influence electricity prices at the day ahead stage, but if prices were not high enough, they could sell their fuel on the gas market much closer to real time. This created further issues for the system operator in matching supply and demand.

Some of these problems were attributed to a lack of competition in the generation market which was addressed during the late 1990s with regulatory responses including enforced divestment of generation capacity [285]. This allowed a number of new players to enter the market and improved competition but many issues with the pool still remained. Subsequently, there was a review of electricity trading arrangements and the New Electricity Trading Arrangements (NETA) were introduced in 2001 [277]. 


\section{NETA}

NETA was intended to maintain a secure and reliable system whilst providing greater competition in the wholesale electricity market. It enabled direct trading between generators, suppliers, traders and consumers through a combination of forward and future markets and short term power exchanges. ELEXON was assigned as the Balancing and Settlement Code Company (BSCCo) and was responsible for the market operation. Key changes from the electricity pool are outlined below [277].

- Each generator was responsible for determining its own output rather than the NGC scheduling supply of all generators;

- Trades were valued at the individual bid price, not the highest overall price bid;

- Trading continued up until gate closure which was closer to real time to allow market participants to tailor their contracted positions more accurately;

- Differences between physical consumption or production and contracted position at gate closure were settled through the balancing mechanism and incurred an associated penalty;

- The demand side was expected to play a more active role in price setting and so there was no capacity payment [286].

In April 2005, the electricity markets in Scotland and England and Wales merged to form a single British wholesale market. NETA was replaced by the British Electricity Trading and Transmission Arrangements (BETTA). The principles and market mechanisms remained largely unchanged but exposed Scottish-based generators to the prevailing transmission charging arrangements. An overview of the market structure is shown in Figure 0.9.

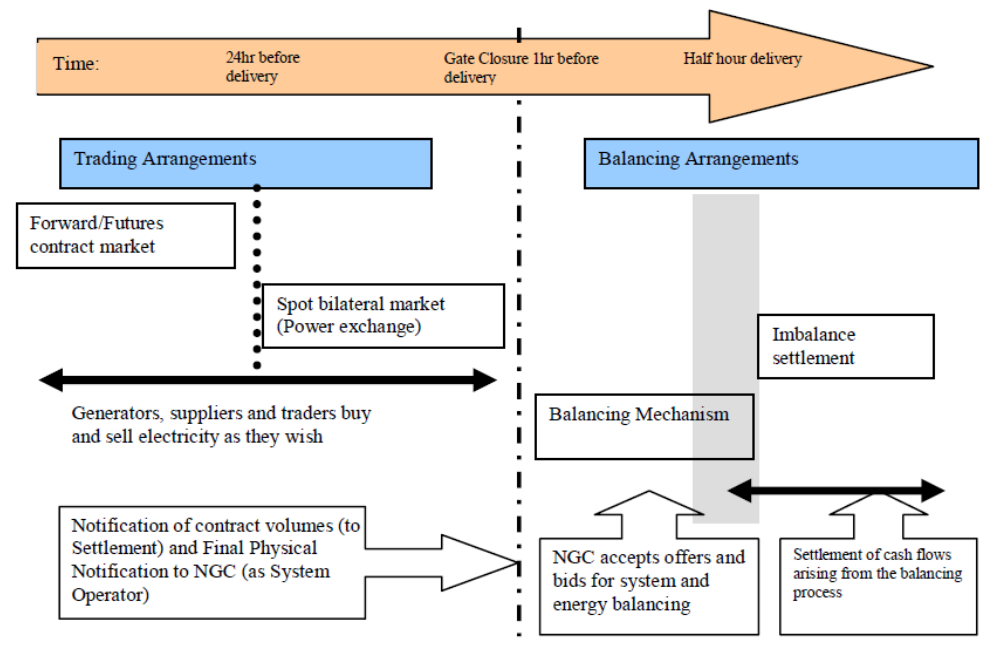

Figure 0.9 BETTA market trading arrangements (source [287]) 


\subsection{Current Market}

\section{Generation}

The 'Big Six' energy companies dominate the generation market in GB. These are Scottish and Southern Energy (SSE), RWE Npower, EDF, E.ON, Centrica and Iberdrola/Scottish Power (SP). The market share of electricity generated by each of the 'Big Six' in 2011 is shown in Figure 0.10. These companies have largely integrated generation and supply businesses. With only six companies controlling almost three quarters of the generation market it is fundamentally an oligopoly. As a result there are significant barriers to new entrants and a high potential for abuse of market power, collusion and price setting [266].

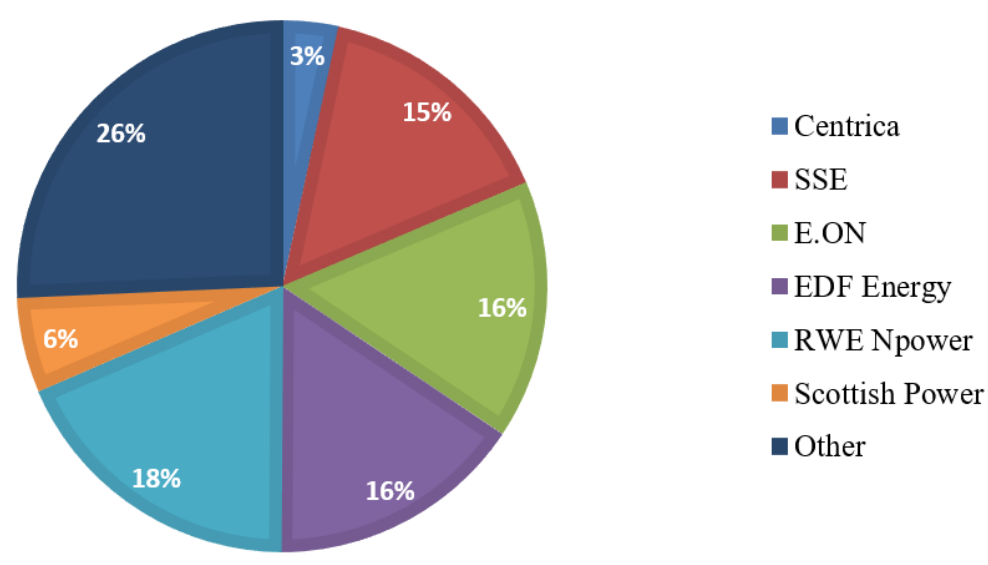

Figure 0.10 Market share of electricity generation in 2011 (source [288])

\section{Transmission}

There are three sets of transmission assets onshore and various offshore assets making up the transmission system in GB. A single system operator, National Grid Electricity Transmission PLC (NGET), owns and operates the system in England and Wales and is responsible for the operation of the entire network. The transmission owners for southern Scotland and northern Scotland are Scottish Power Transmission Ltd (SPTL) and Scottish Hydro-Electric Transmission Ltd (SHETL), respectively [277]. The construction of offshore transmission assets is administered by the Offshore Transmission Owners (OFTOs) regime. OFTOs are selected and licenced by regulator, Ofgem.

\section{Distribution}

Distribution network operators (DNOs) are responsible for the distribution service in their areas. There are 14 licensed DNOs in the UK. These companies are owned by six different organisations in GB and one in Northern Ireland in the regions shown in Figure 0.11. 
Additionally, there are some independent network operators who own and run smaller networks embedded within the DNO networks.

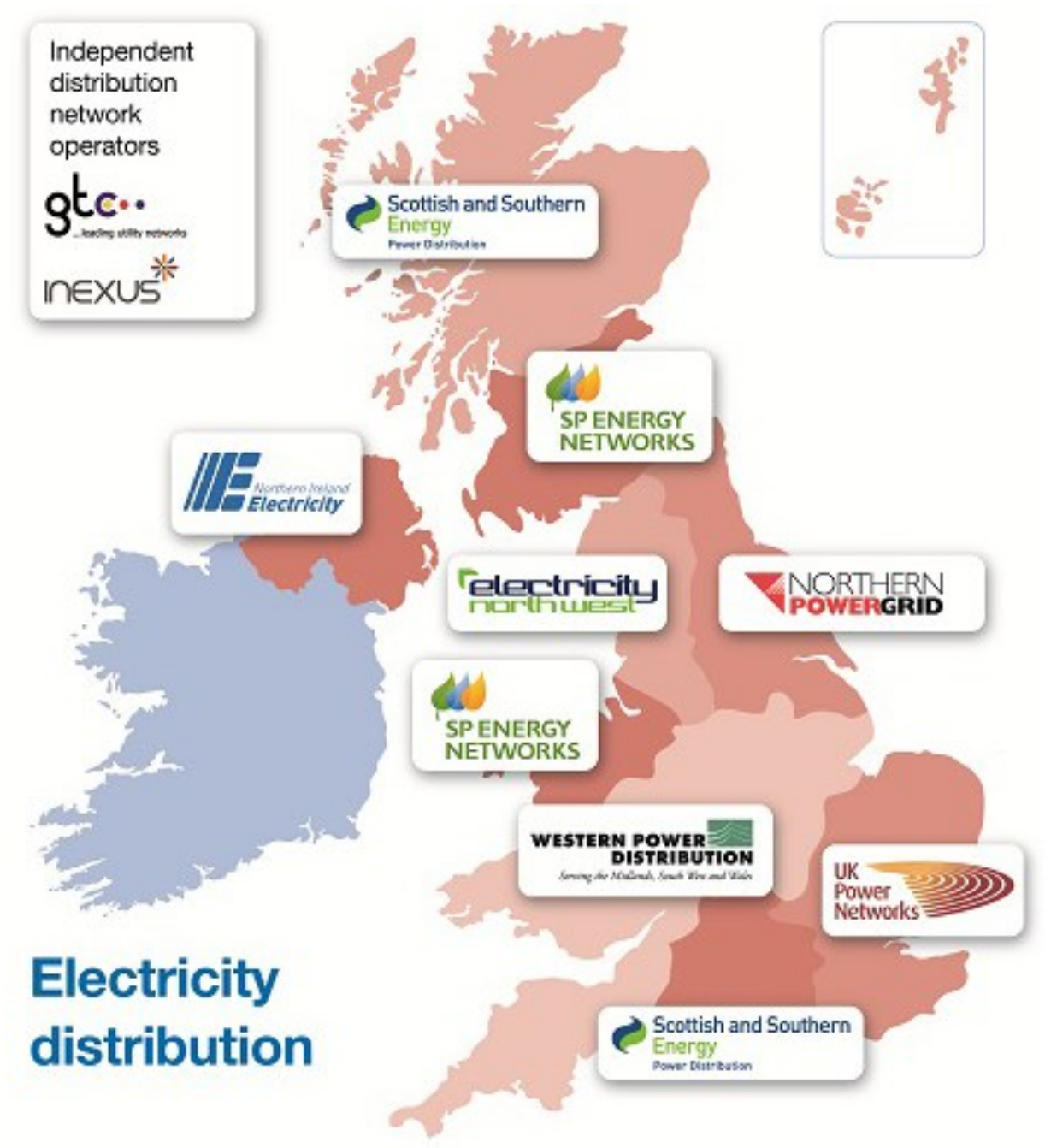

Figure 0.11 Distribution network operator companies (source [289])

\section{Supply}

Electricity suppliers pay DNOs for transporting their customers' electricity in the distribution services area network. Suppliers then pass the costs onto customers. At the end of 2011, the 'Big Six' companies, discussed above, supplied electricity to $99 \%$ of domestic customers. Outside the domestic market, smaller suppliers have gained some share, supplying between $3 \%$ and $9 \%$ of the market in different commercial sectors [290]. 


\section{Regulation}

The regulatory framework in GB was established under the Electricity Act 1989 and has undergone numerous amendments since then [277]. Ofgem was established as the gas and electricity market regulator under the Utilities Act 2000.

Ofgem operates under the direction and governance of the Gas and Electricity Markets Authority (GEMA). Ofgem's principal objective is to protect the interests of customers 'through promotion of value for money, security of supply and sustainability, for present and future generations' [291]. The regulatory regime is based on a licencing system where specific activities cannot be undertaken without a licence. These include generation, transmission, distribution, supply and operation of interconnectors. Ofgem is responsible for approving licences, setting price controls and investigating and penalising companies which breach their licence conditions.

To facilitate competition, the regulatory framework requires separation of monopoly activities from generation and supply. The Electricity Act, 1989, prevents companies which operate under distribution or transmission licences from holding generation or supply licences. In reality, several DNOs are owned by holding companies which also own supply and generation businesses.

Licence conditions require licensees to maintain relevant industry codes. Codes include a procedure for modification of their terms so that they can remain relevant to the changing requirements of the electricity market. Key industry codes are outlined below [292]:

1. the Connection and Use of System Code (CUSC) outlines arrangements for connection to and use of the transmission system, including methods for NGET to determine charges that users pay;

2. the Grid Code contains all technical regulations for the operation and use of the transmission system;

3. the System Operator-Transmission Owner Code (STC) regulates the relationship between system operators and transmission owners;

4. the Balancing and Settlement Code (BSC) governs the arrangements for balancing of electricity and settlement;

5. the Distribution Code contains the technical protocols for connection to and use of each distribution network; 
6. the Distribution Connection and Use of System Agreement (DCUSA) governs licensed distributors, suppliers and generators regarding the use of distribution networks to transport electricity between them;

7. the Master Registration Agreement (MRA) is an agreement between all licences distributors and suppliers which includes the procedures relating to customers switching their supplier.

In addition to Ofgem, DECC is responsible for establishing policies relating to energy and climate change.

Key legislation affecting the electricity industry includes:

1. The Climate Change Act, 2008 placing a legally binding commitment on the UK Government to reduce greenhouse gas emissions by $80 \%$ by 2050 relative to 1990 levels [1];

2. The Renewable Energy Directive requiring the UK to supply $15 \%$ its energy demand from renewable sources by 2020 [293];

3. The Large Combustion Plant Directive (LCPD) requiring combustion plants with a thermal capacity of 50MW or greater to limit their emissions. Under the directive, plant built earlier than 1987 could choose to comply with the limits or to opt out. If the option to opt out was chosen, then the plant was limited to a maximum of 20,000 hours of operation after 2007 and was required to close by 2015 [90].

Other bodies involved in regulation of the electricity industry include:

1. The Health and Safety Executive (HSE), which is responsible for imposing the safety aspects of the Electricity Safety, Quality and Continuity Regulations, 2002 [294];

2. The Office of Nuclear Regulation (ONR), which plays a key role in regulating nuclear power. The ONR previously operated as an agency of the HSE, but was established as an independent statutory body following the Electricity Market Reform [295].

\subsection{Electricity Market Reform}

It is estimated that before 2020 , the electricity industry will require over $£ 110$ billion capital investment [296] to replace and upgrade generating plant and transmission and distribution infrastructure. Historic electricity market arrangements were not expected to deliver the required scale or pace of investment required in lower-carbon generation whilst ensuring adequate security of supply and affordability to consumers. In December 2010, the UK Government announced a proposed Electricity Market Reform (EMR). Following a two year 
consultation period, in November 2012, the Energy Bill was introduced to the UK Parliament. It received Royal Assent at the end of 2013 [295].

There are four key elements to the EMR which are outlined below.

\section{Contracts for Difference (CfD)}

Allocation of the first round of CfD was announced in 2015. They will fully replace the Renewable Obligation, an existing subsidy for renewable generators, from 2017. The mechanism sets a strike price for energy from low carbon generators. If the wholesale price is less than the strike price, generators are paid the difference. If the wholesale price is greater than the strike price, the generators must pay the difference. The cost characteristics of low carbon generators (low operating and high capital cost) mean that they face greater exposure to wholesale price risk than conventional fossil fuel plant, which had a natural hedge as a price setter. The strike price provides a fixed payment for generators to reduce this risk and encourage investment in low carbon generation [297]. Renewable, nuclear and carbon capture and storage plant is eligible for CfD. A government owned company acts as the party responsible for collecting a levy from suppliers to fund the contract payments. The cost of the levy is, in turn, passed onto customers.

\section{Carbon Price Floor}

In addition to incentives for investing in low carbon generation, the government introduced a new carbon price floor on carbon emissions. From 2005, the carbon price was set by the market under the EU Emissions Trading Scheme (EUETS). A fixed number of allowances to emit carbon were created and distributed. Businesses then had to provide allowances to match the emissions they produced. Companies were free to trade allowances enabling supply and demand to create a traded price for carbon [298]. However, it was argued that there was an oversupply of allowances which suppressed the price. DECC noted that "the carbon price resulting from this cap has not been stable, certain or high enough to encourage sufficient investment in low-carbon electricity generation in the UK" [4]. The carbon price floor was introduced in 2013 and set a minimum price for carbon in the UK. The price is collected through a tax imposed on fossil fuels used for power generation on the basis of their carbon content [299].

\section{Emissions Performance Standard (EPS)}

The EPS sets an annual limit on carbon emissions from new fossil fuel power stations. This does not apply to new biomass plant or to energy from waste. The EPS was initially set at a 
level equivalent to $450 \mathrm{~g} \mathrm{CO}_{2} / \mathrm{kWh}$ for all new fossil fuel plant and is reviewed every three years. This level ensures that no new unabated coal-fired power stations can be built [300].

\section{Capacity Market}

There were concerns that with increasingly high levels of intermittent generation and market flaws, such as short term demand side inelasticity, there were insufficient market signals to trigger the required investment in generation capacity. To address this, a capacity mechanism was introduced as outlined below [125]:

- A forecast of future peak demand is made on behalf of Government by Ofgem, the System Operator or other technical experts;

- Ministers agree the total amount of capacity required to ensure security of supply based on this forecast;

- The agreed volume of capacity is contracted through a competitive central auction run by the System Operator 4-5 years ahead of the delivery year;

- The participants successful in the auction enter into capacity agreements and receive an agreed revenue stream to cover the cost of the plant capacity. In return, the plant is committed to provide electricity when called upon for a stress event. If they cannot deliver when required, they face high penalties;

- The cost of the capacity payment is split between the electricity suppliers in the delivery year.

Generators eligible for CfD payments are not allowed to participate in the capacity auction to avoid overcompensation for low carbon plants. The first capacity auction was held by the system operator in 2014 for a delivery year of 2018/19. The duration of contracts offered is between one year for providers of existing capacity and up to ten years for developers of newbuild plant.

\section{Summary}

This appendix has provided an introduction to the electricity market industry and fundamentals of energy system economics which are referred to throughout this thesis. A brief history of the development of the electricity industry in GB was provided and the key rules and regulations governing the sector were presented. Finally, the most recent changes brought about through the EMR were outlined. 


\section{Publications}

The work presented in this thesis has been reported in the following publications and presentations, copies of which are included below:

\section{Journal Papers}

1. A. Dunbar, L. C. Cradden, A. R. Wallace, G. P. Harrison, "The impact of wind power on arbitrage revenue for electricity storage", journal paper published in the IET Generation Transmission and Distribution Special Issue: Optimal Utilization of Storage Systems in Transmission and Distribution Systems, 2015.

2. A. Dunbar, A. R. Wallace, G. P. Harrison, "Energy storage and wind power: Sensitivity of revenue to future market uncertainties", journal paper published in IET Renewable Power Generation Special Issue: Offshore Energy Storage, 2016

\section{Conference Papers}

1. A. Dunbar, F. Tagliaferri, I. M. Viola, G. P. Harrison, "The impact of electricity price forecast accuracy on the optimality of storage revenue", paper presented at the IET Renewable Power Generation Conference, Naples, Italy, 2014.

2. A. Dunbar, A. R. Wallace, G. P. Harrison, "Storage characteristics rewarded by arbitrage and the implications of offshore wind power", paper presented at the Offshore Energy and Storage Symposium, Edinburgh, UK, 2015.

\section{Posters}

3. A. McDowel1"1, G. P. Harrison, "The Value of Electrical Energy Storage: a Comparison between Commercial and System Level Benefits", poster presented at the UKERC Annual Assembly, Warwick, 2014.

4. A. Dunbar, G. P. Harrison, "Could Wind Power be Detrimental to Storage Revenue?”, poster presented at UKES2014 awarded Poster Competition Prize, Warwick, UK, 2014.

5. A. Dunbar, A. R. Wallace, G. P. Harrison, "Catch-22: Could Wind Power Reduce Commercial Opportunities for Electrical Energy Storage", poster presented at the House of Commons at SET for Britain, London, UK, 2016.

\footnotetext{
${ }^{11}$ Work presented under maiden name
} 


\title{
Impact of wind power on arbitrage revenue for electricity storage
}

ISSN 1751-8687

Received on 30th January 2015 Revised on 13th July 2015 Accepted on 24th August 2015 doi: 10.1049/iet-gtd.2015.0139 www.ietdl.org

\author{
Anna Dunbar $\bowtie$, Lucy C. Cradden, Robin Wallace, Gareth P. Harrison \\ School of Engineering, Institute for Energy Systems, University of Edinburgh, Edinburgh, Scotland, UK \\ $\bowtie$ E-mail: a.dunbar@ed.ac.uk
}

\begin{abstract}
Electrical energy storage provides a potential solution to the challenge of integrating large amounts of intermittent renewable energy into the electricity system. To make storage commercially viable, its operators will have to aggregate multiple revenue streams across the electricity industry. Arbitrage is recognised as one potential revenue stream. To date, wind power has provided a small contribution toward electricity generation in Great Britain (GB). Gas generators have delivered a significant proportion of total demand. Historic electricity prices reflect this, being driven principally by variations in gas price and daily demand cycles. The study reported here investigates the potential impact of wind power on electricity prices and arbitrage opportunities for energy storage in GB. Results indicate that increased wind power leads to higher price volatility for low electricity prices, but reduced frequency of higher prices which may be detrimental to storage revenue.
\end{abstract}

\section{Introduction}

Electrical energy storage is a potential solution to the challenge of the Energy Trilemma, facilitating the integration of intermittent renewable energy into the electricity grid. Many potential benefits have been identified throughout the electricity system [1] including:

(i) improved system control, power quality and reliability;

(ii) provision of emergency power and black start services;

(iii) reduced network congestion and deferral of investment in distribution and transmission infrastructure;

(iv) system balancing;

(v) peak shaving and load levelling;

(vi) more efficient use of generation plant;

(vii) avoided curtailment of renewables; and

(viii) firming up intermittent generation and shaping inflexible plant.

Storage has the potential to benefit a range of users in different subsectors of the electricity industry including the system operator, distribution network operators, renewable energy generators, conventional generators, industrial users, and consumers. However, each would traditionally be more likely to invest in established technologies which would be more cost effective to them in the short term, overlooking the system-wide, longer-term benefits of storage [2]. It has been estimated that by 2050 deployment of energy storage could lead to savings of $£ 10 \mathrm{bn} /$ year within the British electricity system [3]. Despite this, accessing commercial rewards remains challenging.

It is increasingly understood that operators will have to aggregate multiple revenue streams for storage to be commercially viable [4]. Arbitrage, purchasing cheap off-peak electricity and selling it on-peak when the price is high, is recognised as one revenue stream which will contribute to a business model. Many studies have investigated revenue available to a storage operator based on historic electricity prices and generally highlight a lack of commercial opportunities for storage despite the wide range of benefits which are provided.

Walawalkar et al. [5] investigated the potential for storage in the New York spot market and found a high sensitivity of arbitrage revenue to the round trip efficiency of the storage technology. This paper highlighted that there may be opportunities for storage in arbitrage and reserve services markets, but that there remained barriers to large-scale integration for wholesale market applications. Connolly et al. [6] compared arbitrage value of a pumped storage plant in 13 different global markets highlighting the range of values which could be achieved. This demonstrated the dependence of value on regulatory frameworks and generation portfolios which other studies also point to. Sioshansi et al. [7] attributed variations in arbitrage value of storage in the Pennsylvania-New Jersey-Maryland (PJM) market to the specific generation mix and cost of fuel. This highlighted the high level of risk associated with an investment in energy storage. Even with perfect foresight of electricity prices, profits vary from year to year with variations of 50 and $75 \%$ identified in $[6,8]$, respectively. Other studies including [9-11] assessed different optimisation methods and the importance of forecast accuracy, but similarly, were based on historic market prices.

Projecting the future scope for arbitrage is more challenging and this is particularly so for markets such as Great Britain (GB) where substantial structural changes will occur. Historically, peak electricity prices in GB tend to have been driven by gas prices as Fig. $1 a$ shows. This occurs as mid-merit and peaking generators include a large proportion of combined and open cycle gas turbines (OCGTs) [12]. Furthermore, as wind power has made only a small contribution and has had little impact on wholesale electricity prices, daily fluctuations in price have been driven principally by variations in demand as shown in Fig. $1 b$. Over the next decade, the contribution from wind generation is expected to increase dramatically. It is often speculated that commercial opportunities for storage will emerge as the penetration of intermittent renewables grows as it has the potential to increase price volatility [13]. More frequent and more acute price differentials would provide additional opportunities for arbitrage which could become a more profitable revenue stream in the future.

A notable analysis of this phenomenon for future GB systems is presented by Grünewald et al. [14] finding that $32 \mathrm{GW}$ of wind capacity would enable the gross value of storage to cover its capital costs and investment could be commercially viable through price arbitrage alone. The framework applied is credible, but there are a number of areas within the model that may have limitations. The simulation of future energy prices using a simple marginal 
generation cost model did not consider the impact of varying fuel and carbon prices, which are both significant sources of uncertainty. Additionally, wind is attributed a marginal value equivalent to the opportunity cost of a Renewable Obligation Certificate (ROC) which has the effect of driving costs negative. An exponential mark-down was also applied to electricity prices when demand was lowest to represent generators' preferences not to curtail output. This subsidy was available for renewable generators although this will not be the case in the future as the UK Government has announced an end to ROCs for all new onshore wind farms as early as 2016 [15]. While adopting a broadly similar framework, this paper specifically addresses these aspects by incorporating changing fuel and carbon prices in future energy scenarios and making the assumption that no subsidy is paid for wind generation meaning that the minimum price of electricity never falls below zero. Subsequently, an alternative price function is applied for periods of low demand. These changes lead to distinct results, observing that increased wind capacity leads to more frequently suppressed electricity prices which may be detrimental to arbitrage revenue. Given the rapidly changing portfolio of generation technologies, this paper makes a valid contribution by enabling understanding of the implications of wind power on energy storage and the business case for developing storage in the future.

\section{Model}

\subsection{Overview}

A model was created to simulate time series of electricity prices under different generating capacity and fuel price assumptions.
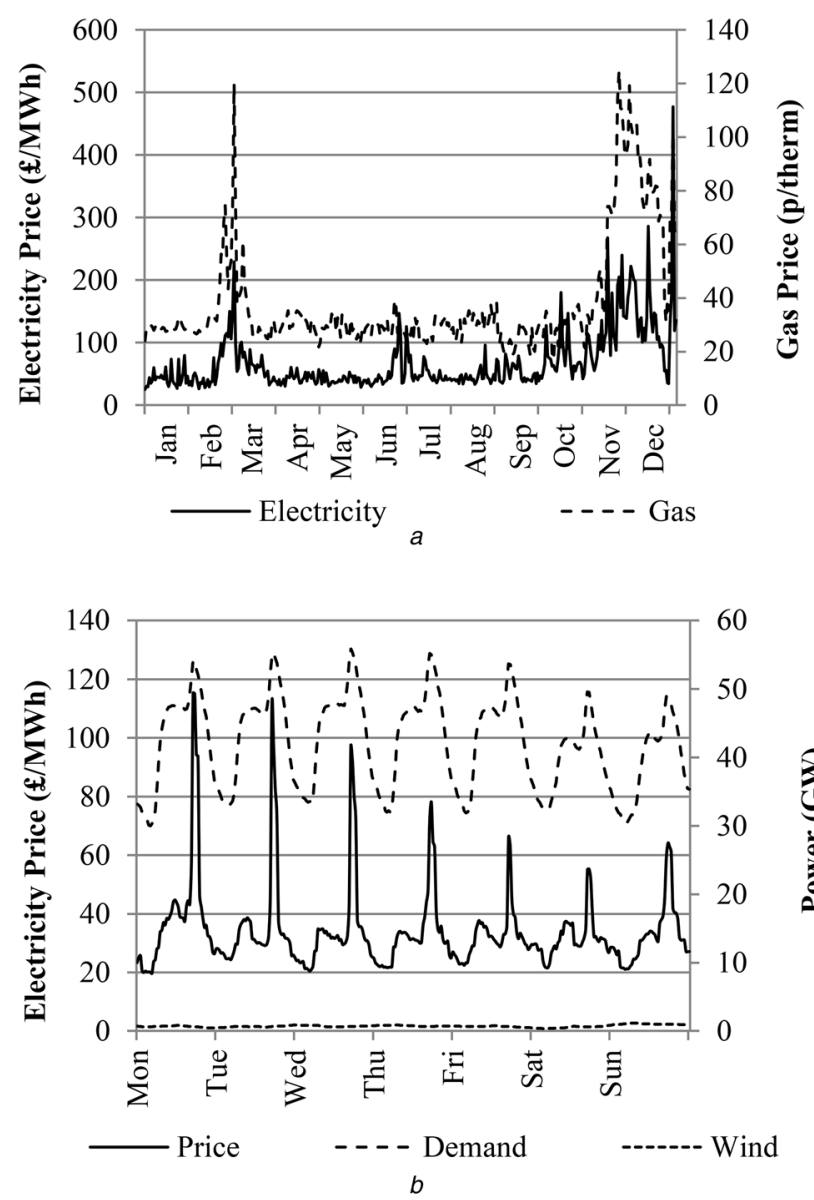

Fig. 1 Historic electricity price variation

$a$ Daily peak electricity price [16] and National Balancing Point gas price [17], 2005 $b$ Half-hourly electricity price [16], demand [18] and wind power output [19], first week of November 2005
The electricity prices were used to define the optimum operating schedule for an energy storage device and determine how much revenue could be expected from arbitrage.

The majority of electricity in GB is traded through private bilateral exchanges in forward markets. It is not possible to model these contracts directly as electricity prices are not published. About 3\% of electricity is traded on the power exchange, a spot market operating up to $1 \mathrm{~h}$ ahead of real time [20]. Although only a small volume of electricity is traded, prices are published and so the market index price is expected to strongly influence pricing in forward markets. Fundamental price-based market models, established on the assumption of perfect competition, have been shown to be representative of the power exchange and are commonly used for modelling scenarios of future energy prices [21].

To model market prices, the dispatchable power which must be delivered by thermal generators in the system was calculated. For each half-hour period, renewable power output was deducted from electricity demand to produce a 'net demand' curve. The generator supply function was formed by stacking thermal plant in merit order of increasing marginal cost. The price of electricity for each time period was determined by the market clearing price.

The electricity price time series and storage system characteristics were used to determine the optimum operating schedule to maximise arbitrage revenue. A summary of the model is shown in Fig. 2, which is based on a fundamental price model coupled with an arbitrage optimisation. This is broadly similar to that proposed by Grünewald et al. [14], but critical aspects differ including:

(i) the approaches used for modelling the meteorological data for renewable energy output;

(ii) the generator supply function;

(iii) the calculation of marginal generation costs; and

(iv) the optimisation of the storage operating schedule.

The details of the model are described below. Section 2.2 describes how the thermal generator supply function was formed. Section 2.3 explains how wind power output was calculated. Section 2.4 outlines the approach used to represent demand data and Section 2.5 describes how the storage operating schedule was determined and the technical characteristics of the device that were used.

\subsection{Generator supply function}

Thermal generators were grouped into four classes of plants: nuclear, coal, combined cycle gas turbine (CCGT) and OCGT. Each class

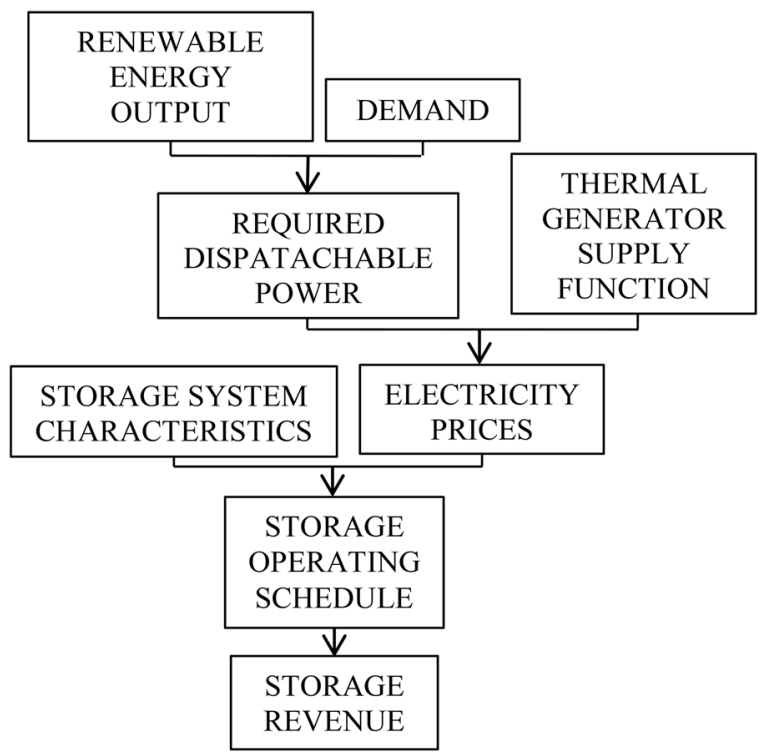

Fig. 2 Model overview 
was bound by a lower limit of its own marginal cost and an upper limit of the marginal cost of the next class in the merit order stack [4]. Between these two values the hyperbolic function shown in (1) was assumed. This smoothed the discontinuities in the step function and better represented the complexities of the supply curve such as differing ages and efficiencies of plant within each class of generation

$$
\mathrm{CE}=\Pi_{x}\left[1+\frac{\Pi_{x+1}-\Pi_{x}}{\Pi_{x}} \frac{\cosh \left(P_{x} / C_{x}\right)-1}{\cosh (1)-1}\right]
$$

where CE is the cost of electricity, $\Pi_{x}$ is the short run marginal generation cost, $x$ denotes the merit order of the marginal class (i.e. for base load $x=1$ and for peaking plant $x=4), P_{x}$ and $C_{x}$ are power output and installed capacity of the marginal class, respectively.

All generators were assumed to be paid the market clearing price which was set by the highest merit order generator that was scheduled to run. The difference between the market clearing price and a generator's short run marginal cost is known as a scarcity rent and covers long run fixed costs [22].

Peaking plant was the last class of generator to be dispatched. Its maximum price was not limited by a more expensive generator and so it bid to produce electricity at a premium price to reflect scarcity of supply. This enabled peaking generators to recover their fixed costs. Following a similar approach to Eager et al. [23], the exponential function in (2) was used:

$$
\mathrm{CE}=\Pi_{x}\left[\beta \mathrm{e}^{\alpha\left(P_{x} / C_{x}\right)}\right]
$$

where $\alpha$ and $\beta$ are constants which were determined empirically and used to define the extent of the uplift applied when capacity was scarce. Fig. 3 shows an example aggregate supply curve.

Historic generator capacity was sourced from the Digest of United Kingdom Energy Statistics [12]. Smaller peaking plant including oil, as well as pumped storage, was grouped with OCGTs. Other small generators, such as run-of-river hydro, were grouped with coal. Plant capacity was assumed to be fixed throughout the year with a constant availability applied to each class of generator reducing its total capacity. The British system was considered an islanded network with no interconnector capacity.

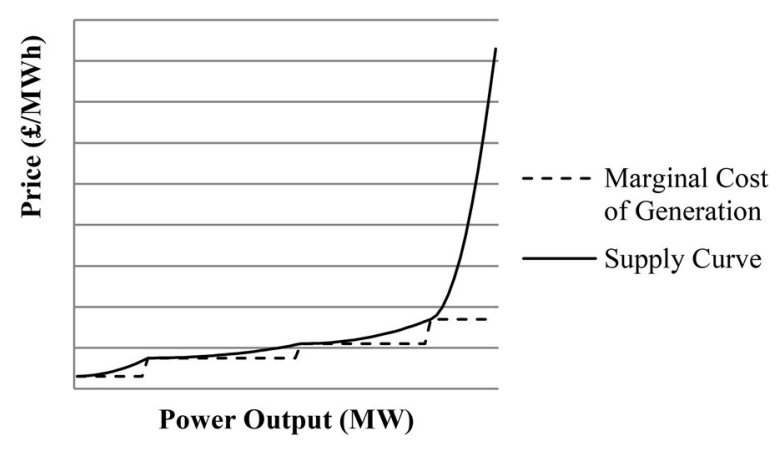

Fig. 3 Electricity supply curve
Marginal generation costs were calculated for each class using (3)

$$
\Pi_{x}=\frac{1}{\eta_{x}}\left(a_{x} F_{x}+v_{x} F_{\text {car }}\right)+V_{x}+e_{x}
$$

where $\eta_{x}$ is the thermal efficiency ( $x$ denoting the generator class), $a_{x} F_{x}$ is the price of fuel, with $a_{x}$ as a conversion coefficient and $F_{x}$ as the fuel price in units relating to the fuel for each class of generator. Specifically, nuclear and coal fuel prices were in $£ / \mathrm{kg}$ while gas prices were in $£ /$ therm. $\nu_{x}$ is the amount of carbon produced from burning fuel at $100 \%$ efficiency, $F_{\text {car }}$ is the price of carbon, $V_{x}$ is the variable operation and maintenance cost and $e_{x}$ is the cost of enriching fuel which only applies to nuclear generators. The data used for each class is listed in Table 1.

Time series of historic fuel, $F_{x}$, and carbon prices, $F_{\text {car }}$, were sourced from the NUEXCO Exchange [27], the Department of Energy and Climate Change [28], the ICE ENDEX [17] and the European Environment Agency [29].

\subsection{Wind power output}

Wind power was the only form of renewable energy considered in the model. It was assumed to have zero marginal cost and was always dispatched when available. GB was conceptually modelled as a single bus transmission system and network constraints were neglected. It was assumed that wind output was not curtailed unless demand was fully satisfied.

Hourly wind speed data for the United Kingdom and surrounding waters were produced by Hawkins [19] using the Weather Research and Forecasting model for the years 2001-2010. Wind speeds were available at a spatial resolution of $3 \mathrm{~km} \times 3 \mathrm{~km}$ and were extracted at $80 \mathrm{~m}$ above ground or sea level.

The DECC RESTATS planning database [30] was used to identify the location and capacity of wind farms in GB. Each farm was considered to be producing power from the start of the year in which the database stated it was fully commissioned and operational. The time series of wind speed data corresponding to each farm location was extracted from the wind model.

Power output from each wind farm was calculated using the equivalent aggregate power curve described by (4) [19], where $P$ is the power output and $U$ is the wind speed. Although this was not representative of all wind farms, it was expected to be typical and accounted for effects due to interactions between turbines that tend to smooth power production, which manufacturers' power curves do not [19]

$$
P=\frac{1}{1+\mathrm{e}^{-(U-9.7 / 1.8)}}
$$

Power output from all wind farms was summed to give the aggregate output for both onshore and offshore winds and reduced by $10 \%$ to account for availability. This was a conservative estimate for onshore, but early offshore availability may be $<90 \%$ [31]. The data was interpolated linearly to obtain a time series of power output at half-hourly intervals.

\subsection{Demand}

\begin{tabular}{|c|c|c|c|c|c|c|}
\hline $\begin{array}{l}\text { Generator } \\
\text { type }\end{array}$ & $\begin{array}{c}\text { Thermal efficiency } \\
\eta, \%\end{array}$ & $\begin{array}{c}\text { Carbon emissions } v, \\
\mathrm{~kg} / \mathrm{MWh}\end{array}$ & $\begin{array}{c}\text { Variable operating costs } V \text {, } \\
\text { f/MWh }\end{array}$ & $\begin{array}{c}\text { Enrichment cost } e, \\
\text { f/MWh }\end{array}$ & $\begin{array}{c}\text { Availability, } \\
\%\end{array}$ & $\begin{array}{l}\text { Conversion } \\
\text { coefficient } a\end{array}$ \\
\hline nuclear & 36 & 0 & 1.8 & 2.5 & 78 & $8.24 \times 10^{-3}$ \\
\hline coal & 36 & 285 & 2 & 0 & 86 & 150 \\
\hline CCGT & 60 & 185 & 2.2 & 0 & 87 & 34.128 \\
\hline OCGT & 46 & 185 & 2.7 & 0 & 95 & 34.128 \\
\hline
\end{tabular}

Historic demand data is available from National Grid [18]. Demand was assumed to have zero price elasticity, a reasonable assumption

Table 1 Thermal generator data [24-26] 
for historic electricity prices. This may change in the future with the introduction of smart grids and demand side response. These effects were not considered in this paper.

\subsection{Storage operating schedule}

The optimum operating schedule for a storage device was established assuming perfect foresight of electricity prices and subject to the technical constraints of the device. Revenue was optimised on a weekly basis with the additional constraint that the state of charge must be zero at the start and end of each week. This had minimal impact on total revenue compared with optimisation on a daily, monthly or annual basis, but reduced the computational time. The following assumptions were applied:

- The storage device had $100 \%$ availability.

- The storage capacity was small compared with total electricity demand. It was a price taker and did not affect the market price of electricity.

- GB was a single bus system and storage was not subject to network constraints.

- The device characteristics were constant over its life time.

- The conversion efficiency was modelled during charging only. The discharge cycle was $100 \%$ efficient.

- The ramp rate was negligible compared with the time period.

- The cost of charging and discharging (in addition to the cost of electricity) was negligible.

- The discount rate was negligible over the time period considered.

The decision variables for the storage operator were how much electricity to buy, $q_{t}^{\mathrm{C}}(\mathrm{MWh})$, and sell, $q_{t}^{\mathrm{D}}(\mathrm{MWh})$, during each time period, $t$. The state of charge of the storage device, $S_{t}$ (MWh), was defined by (5) and subject to the constraints given in (6)-(8)

$$
\begin{gathered}
S_{t}=\eta_{\mathrm{s}} S_{t-1}+\eta_{\mathrm{c}} q_{t}^{\mathrm{C}}-q_{t}^{\mathrm{D}} \\
0 \leq S_{t} \leq S_{\text {max }} \\
0 \leq q_{t}^{\mathrm{C}} \leq q_{\text {max }}^{\mathrm{C}} \\
0 \leq q_{t}^{\mathrm{D}} \leq q_{\text {max }}^{\mathrm{D}}
\end{gathered}
$$

where $\eta_{\mathrm{s}}$ is the storage efficiency (\%), $\eta_{\mathrm{c}}$ is conversion efficiency $(\%), S_{\max }$ is the maximum storage capacity (MWh), $q_{\max }^{\mathrm{C}}$ and $q_{\max }^{\mathrm{D}}$ (MWh) are maximum quantities of energy which can be charged or discharged in a single time period. These are a function of the maximum charging and discharging rates, $Q_{\mathrm{C}}$ and $Q_{\mathrm{D}}(\mathrm{MW})$.

The objective was to maximise annual revenue, $R$, which is the sum of the price, $P_{t}$, multiplied by the net quantity sold during each settlement period. This is defined in (9)

$$
R=\sum P_{t}\left(q_{t}^{\mathrm{D}}-q_{t}^{\mathrm{C}}\right)
$$

The linear optimisation described by Byrne and Silva-Monroy [32] was applied to calculate the optimum operating schedule and determine the maximum revenue available to the storage operator.

There is a range of storage technologies with different technical and cost characteristics. The applications, benefits and disadvantages are discussed by a number of authors [33]. The characteristics presented in Table 2 were used as the storage constraints in this paper. These are representative of a grid scale device which would likely participate in arbitrage markets. This paper does not seek to investigate the impact of varying storage characteristics on arbitrage or to compare the performance of different technologies.
Table 2 Storage characteristics

\begin{tabular}{lcccc}
\hline $\begin{array}{l}\text { Storage } \\
\text { capacity } \\
\begin{array}{l}S_{\text {max }} \\
\text { MWh }\end{array}\end{array}$ & $\begin{array}{c}\text { Maximum } \\
\text { charging } \\
\text { rate } Q^{\mathrm{C}}, \mathrm{MW}\end{array}$ & $\begin{array}{c}\text { Maximum } \\
\text { discharging } \\
\text { rate } Q^{\mathrm{D}}, \mathrm{MW}\end{array}$ & $\begin{array}{c}\text { Conversion } \\
\text { efficiency } \eta_{\mathrm{c}}, \\
\%\end{array}$ & $\begin{array}{c}\text { Storage } \\
\text { efficiency } \\
\eta_{\mathrm{s}}, \%\end{array}$ \\
\hline 200 & 20 & 20 & 75 & 100 \\
\hline
\end{tabular}

\section{Model validation}

The simulation was initially run for three historic years to validate the price model and investigate the revenue available to a storage operator under market conditions with a small penetration of wind power. The years 2005-2007 were selected as robust data sets for coal, gas and nuclear fuel prices were all available, in addition to wind resource data. For other years, this information was not available at as high a temporal resolution.

A number of characteristics of the price time series affected the storage operating schedule including the frequency and magnitude of peaks and troughs as well as the mean price. The uplift coefficients, $\alpha$ and $\beta$, were calibrated using the revenue available from historic and simulated electricity prices. Using revenue, rather than a statistical measure from the time series, allowed multiple characteristics, which may have impacted the storage operating schedule, to be accounted for. Alternative approaches used to calibrate uplift functions include assigning a value of lost load (VOLL) to the price when the capacity margin is zero [24] or pricing to ensure that the investment case for peaking plant remains viable [14]. These methods introduce challenges of accurately defining VOLL and peaking plant costs, respectively. Furthermore, in this model, installed generation capacities were defined exogenously and were not dependent on simulated electricity prices. They were expected to be reflective of future scenarios, but would not necessarily represent the system margin accurately enough to employ these methods.

\subsection{Generation capacity}

The classifications of installed generation capacity for 2005-2007 are shown in Fig. 4. Over these years the installed wind capacity increased from 1.65 to $2.82 \mathrm{GW}$. In 2007, it represented $3.7 \%$ of the total installed capacity.

\subsection{Electricity price}

Historic electricity prices from the UK power exchange [16] were used to validate the price model. For reasons discussed in Section 2.1, the power exchange can be used as a reference for forward and ancillary electricity prices. Additionally, as a market of last

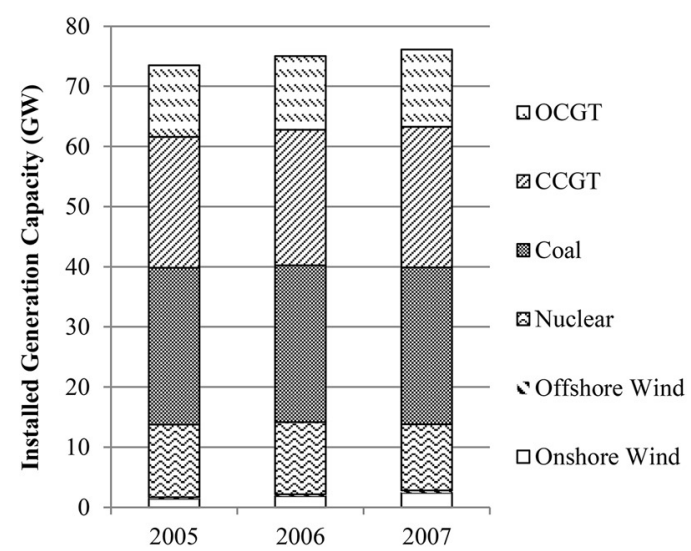

Fig. 4 Installed generation capacity 2005-2007 [12] 
resort, price volatility is high and so it is likely to be the market where energy storage would participate for arbitrage sales.

Fig. 5 shows a comparison between historic spot market index prices and electricity prices simulated using the model.

The model adequately captured the basic characteristics of the market. Critically, the good fit of the half-hourly prices in Fig. 5 shows that the diurnal variations, to which arbitrage is particularly sensitive [14], were well represented.

Fig. 6 shows demand net wind output and corresponding historic electricity price for November and December 2007. This shows that price troughs of a similar magnitude occurred on a daily basis when the net demand was lowest. Price peaks corresponded with the daily peaks in net demand; however, the magnitude varied. The maximum peak, in mid-November, did not coincide with maximum net demand, indicating that other factors were influencing the magnitude of price spikes.

Table 3 presents the annual minimum and maximum electricity prices using historic data from 2005 to 2007. The mean and
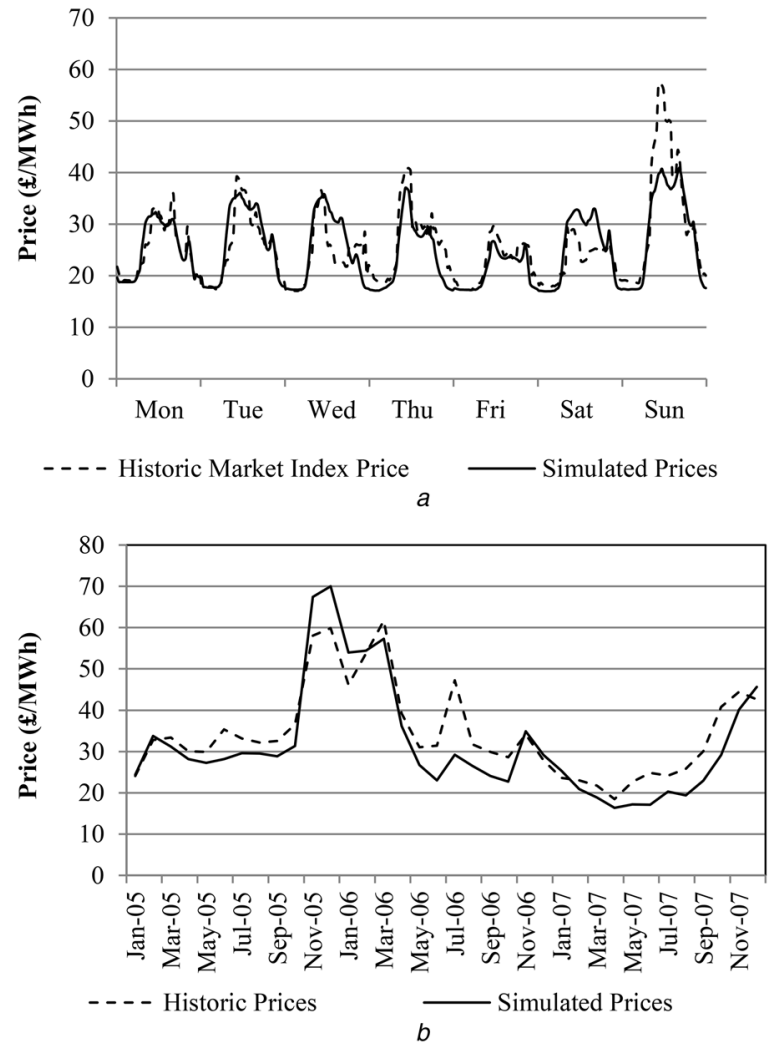

Fig. 5 Historic market index prices and simulated electricity prices

$a$ First week in August 2007

$b$ Average monthly electricity prices

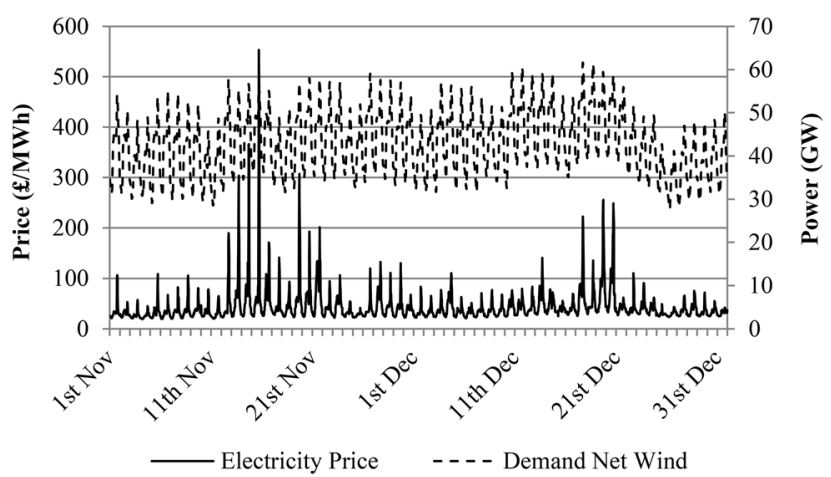

Fig. 6 Demand net wind and electricity price (2007) standard deviation of the daily troughs and daily peaks for each year are also presented. This shows that there was significant variation in maximum daily price, but little variation in the minimum daily price. The same data for the simulated electricity prices is presented in Table 4.

The simulations adequately represented the key characteristics of the historic electricity price time series that were significant to arbitrage. As one-off, extreme measurements the absolute maximum and minimum values were less critical than the mean and standard deviation data which was in better agreement. The peak statistics showed better agreement than the trough statistics. The maximum difference between historic and simulated mean peak values in any year was $7 \%$. The maximum difference between the mean trough values for 2006 was $326 \%$; however, the absolute error for this year was still only $£ 14.29 / \mathrm{MWh}$. The better representation of peak values was possible through calibration of the uplift coefficients $\alpha$ and $\beta$ whose values did not affect the trough characteristics. The simulated results showed slightly higher standard deviation of peak prices compared with historic data which may have provided additional price volatility to compensate for the minor overestimation in minimum prices. The error in the peak standard deviation was negligible in 2007 where the difference between the mean trough values was also smallest.

\subsection{Electricity price forecast error}

The price time series in this model is the out turned price and represents a projection of future prices rather than a forecast. In reality, a storage operator will not have perfect foresight of electricity prices and will have to devise an operating schedule using a price forecast. Errors in the price forecast will lead to sub-optimal decisions being made and storage revenue being reduced. The price forecast error is dependent on the wind forecast error, the demand forecast error and other associated errors. Demand has historically been forecast relatively accurately and wind forecasts are becoming more precise as more capacity is deployed. $\mathrm{Hu}$ and Taylor [34] suggest that electricity price forecasts with errors of $<10 \%$ could be readily achieved in the short-term British electricity market. The implications of price forecast accuracy on the optimality of storage revenue are analysed by Dunbar et al. [11]; relative to perfect foresight, an error of $10 \%$ would typically equate to a few per cent reduction and, in the extreme, a loss of $10 \%$ revenue. These errors would be expected to be consistent across a range of scenarios and are small compared with uncertainties associated with other inputs such as future gas prices and carbon prices and the variation in wind power output from one year to the next. Owing to the high level of uncertainty associated with future energy scenario inputs, the results are used to identify trends in revenue over several years, rather than to compare the absolute value with storage costs and assess the return on investment for a storage device. It is believed that a lack of explicit treatment of forecast error within the modelling will therefore not have a substantial impact on the results and conclusions.

\subsection{Arbitrage revenue}

The storage operation algorithm was applied to both historic market index price and simulated price time series for 2005-2007 to compare the revenue. The results are shown in Table 5.

Using simulated prices, the revenue was not consistently over or under estimated compared with historic prices. The simulated price results were within $10 \%$ of those obtained using historic prices. This gives further confidence that the characteristics critical to arbitrage revenue were captured well in the electricity price model. The revenue varied by $<10 \%$ between years with the same 3 year average of $£ 0.87 \mathrm{~m}$ from both historic and simulated price data.

Fig. 7 shows the optimum operating schedule for November 2007 using simulated prices. This shows that the device was charging and discharging on at least a daily basis in line with the daily cycle of 
Table 3 Annual peak and trough electricity price statistics from historic data

\begin{tabular}{|c|c|c|c|c|c|c|}
\hline Year & $\begin{array}{c}\text { Minimum price, } \\
\text { f/MWh }\end{array}$ & $\begin{array}{c}\text { Daily trough mean, } \\
\text { f/MWh }\end{array}$ & $\begin{array}{l}\text { Daily trough standard } \\
\text { deviation, } \mathrm{f} / \mathrm{MWh}\end{array}$ & $\begin{array}{l}\text { Maximum price, } \\
\text { f/MWh }\end{array}$ & $\begin{array}{c}\text { Daily peak mean, } \\
\text { f/MWh }\end{array}$ & $\begin{array}{l}\text { Daily peak standard } \\
\text { deviation, f/MWh }\end{array}$ \\
\hline 2005 & 11.86 & 23.18 & 4.41 & 476.91 & 67.49 & 47.86 \\
\hline 2006 & 1.09 & 4.38 & 6.7 & 414.71 & 69.93 & 47.92 \\
\hline 2007 & 5.92 & 17.06 & 4.47 & 553.3 & 60.5 & 52.94 \\
\hline
\end{tabular}

Table 4 Annual peak and trough electricity price statistics from simulated data

\begin{tabular}{|c|c|c|c|c|c|c|}
\hline Year & $\begin{array}{l}\text { Minimum price, } \\
\text { f/MWh }\end{array}$ & $\begin{array}{c}\text { Daily trough mean, } \\
\text { f/MWh }\end{array}$ & $\begin{array}{l}\text { Daily trough standard } \\
\text { deviation, } \mathrm{f} / \mathrm{MWh}\end{array}$ & $\begin{array}{l}\text { Maximum price, } \\
\mathrm{f} / \mathrm{MWh}\end{array}$ & $\begin{array}{c}\text { Daily peak mean, } \\
\text { f/MWh }\end{array}$ & $\begin{array}{l}\text { Daily peak standard } \\
\text { deviation, } \mathrm{f} / \mathrm{MWh}\end{array}$ \\
\hline 2005 & 17.38 & 28.41 & 6.44 & 617.18 & 72.26 & 64.26 \\
\hline 2006 & 8.62 & 18.67 & 5.95 & 513.6 & 70.27 & 53.53 \\
\hline 2007 & 9.02 & 13.23 & 4.11 & 451.06 & 63.32 & 53.22 \\
\hline
\end{tabular}

Table 5 Annual arbitrage revenue 2005-2007

\begin{tabular}{ccc}
\hline Year & $\begin{array}{c}\text { Revenue from historic market } \\
\text { index prices, fm }\end{array}$ & $\begin{array}{c}\text { Revenue from simulated } \\
\text { electricity prices, fm }\end{array}$ \\
\hline 2005 & 0.892 & 0.839 \\
2006 & 0.831 & 0.914 \\
2007 & 0.875 & 0.845 \\
\hline
\end{tabular}

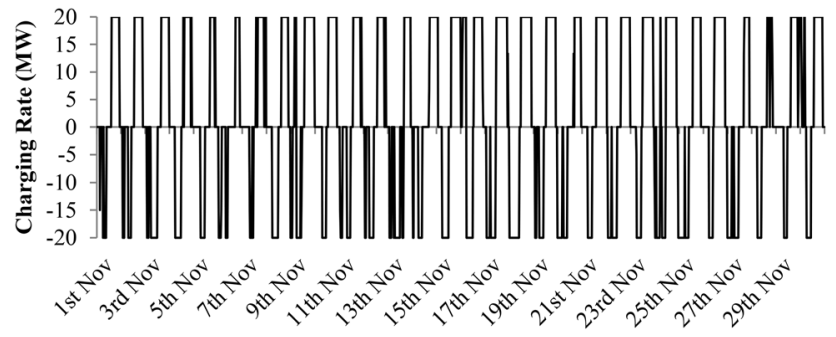

Fig. 7 Storage operating schedule November 2007

electricity prices. This pattern was typical of the operating schedule over the three years investigated.

\section{Future energy scenario case study}

A wide range of scenarios have been developed for future energy systems in GB including scenarios based on system optimisation [35] and capacity investment decisions [23]. Any future scenario or forecast could be applied to the model. In this paper, the National Grid Gone Green Future Energy Scenario 2014 [36] was used as a case study for analysis of future years with a high penetration of wind power. The future energy scenarios are published annually by National Grid and are defined through discussion with stakeholders and operational experience. The input data are clearly defined and publicly available. The scenarios take into account a range of socio- and techno-economic factors to describe plausible future energy systems. For each scenario, there is a range of changing inputs such as fuel costs, generation mix and demand. In the Gone Green scenario, sustainable energy policies are aligned and renewable energy and carbon targets are met. There is strong economic growth and investment in new technologies; particularly wind.

Fig. 8 shows the classification of installed generation capacity from 2020 to 2025 for the Gone Green scenario. The extent to which coal capacity was reduced is not explicit from Fig. 8 as carbon capture and storage and biomass, whose capacity increased, were grouped in the same classification as coal for the purpose of

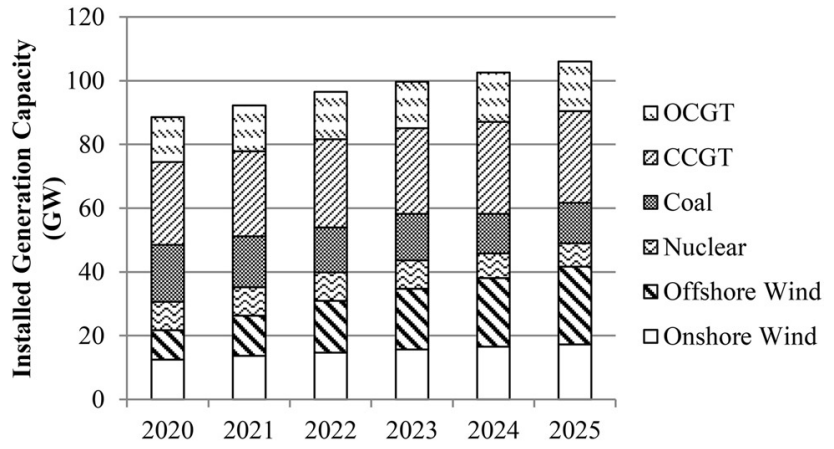

Fig. 8 National Grid Gone Green future installed generation capacity 2020-2025 [36]

the model. From 2020 to 2025 , the total installed wind capacity increased from 22 to $42 \mathrm{GW}$ which represented an increase from 26 to $39 \%$ of the total installed generation capacity.

Several approaches can be taken to determine the future spatial distribution of wind farms. Grunewald et al. [14] used an optimisation algorithm to place wind capacity in geographically diverse areas. This did not consider planning restrictions or network constraints, both of which are critical factors in the development of wind farms. Green and Vasilakos [37] assumed that offshore installations would be focused in areas where National Grid was intending to upgrade transmission lines and placed additional capacity in regions which aligned with these locations. Here, wind capacity was scaled at existing sites. This assumed that the spatial distribution in the future would be the same as it is currently. Onshore and offshore capacities were scaled as separate regions to ensure that variations between onshore and offshore resource were captured. The power output was calculated as described in Section 2.3 using spatially distributed wind speed time series from a historic year - this year is termed the 'wind year'.

Future average annual fuel and carbon prices are detailed in the Gone Green scenario data and summarised for the years 2020 2025 in Table 6. Time series of fuel price data from the wind year

Table 6 National Grid Gone Green future fuel and carbon prices [36]

\begin{tabular}{cccc}
\hline Year & $\begin{array}{c}\text { Wholesale gas } \\
\text { price, pence/therm }\end{array}$ & $\begin{array}{c}\text { Wholesale coal } \\
\text { price, \$/tonne }\end{array}$ & $\begin{array}{c}\text { Wholesale UK } \\
\text { carbon price, f/tonne }\end{array}$ \\
\hline 2020 & 75.9 & 96.5 & 32.7 \\
2021 & 78.5 & 96.5 & 37 \\
2022 & 80.6 & 96.6 & 41.4 \\
2023 & 81.9 & 96.4 & 45.7 \\
2024 & 83.2 & 108.9 & 50.1 \\
2025 & 84.9 & 109.1 & 54.5 \\
\hline
\end{tabular}


was scaled to match the average value to ensure that some intra-annual volatility was maintained. Similarly, a time series of historic demand data from the wind year was transformed to match the average and peak demand for future years. Taking historic time series from the same wind year ensured that the relationship between weather, demand patterns and fuel costs was maintained.

\section{Results and discussion}

\subsection{Electricity price}

Fig. $9 a$ shows the time series of demand and wind power output predicted for November and December 2025 using a 2007 wind year. In 2025, the average demand was similar to that from 2005 shown in Fig. $1 b$; however, the average wind power output was significantly increased. Fig. $9 b$ shows the demand net wind power output and the resulting electricity price for the same period. This can be compared with Fig. 6 which shows the same time series for 2007.

The demand net wind time series represents the output which thermal generators would be required to supply. In 2025, this was less regular than in 2007 due to the increased output from wind power, a similar result to that presented by Cox [13]. As a result, the daily electricity price troughs were more volatile. This is evident from the dips in price seen in Fig. $9 b$ which are absent in Fig. 6. These occurred during periods of low demand and high wind output where base load generation, rather than mid-merit plant, acted as the marginal generator and set the price of electricity. The standard deviation of the daily minimum electricity price in 2025 was $£ 10.76 / \mathrm{MWh}$, double that from 2007. There was also reduced frequency of high electricity prices due to periods of high wind output reducing the peaks in net demand. Peaking generators were no longer needed during these periods and mid-merit plant set the electricity price. These results were largely in agreement with other studies [13, 21], which additionally concluded that these less frequent price spikes were likely to be of a larger magnitude to enable peaking plant to cover its fixed cost over fewer operational hours. Modelling this phenomenon would have required the uplift coefficients $\alpha$ and $\beta$ to be recalibrated each year depending on the capacity margin and peaking plant investment case. This was not carried out here.

The effect of increased wind power was to increase the volatility of the output which thermal generators were required to supply, but to reduce the overall volatility of electricity prices. This was due to the shape of the supply curve, shown in Fig. 3, which was steep during periods of high demand, but shallow during periods of low demand; indicating higher supply-side price elasticity when electricity was scarce. Increased wind power output reduced scarcity of supply and shifted thermal demand down the supply curve to where supply-side price elasticity was at its lowest. This result contradicts previous studies and suggests that the available revenue through price arbitrage is reduced. There would be increased opportunities for storage to charge when prices were low, but reduced opportunities for storage to discharge and sell electricity at an inflated price.

\subsection{Arbitrage revenue}

Fig. 10 shows the storage operating schedule for November 2025 using a 2007 wind year. The device generally cycled on a daily basis, however, unlike Fig. 7, which shows the operating schedule for the same month in 2007, there were some days that the device did not charge or discharge at all. These days coincided with periods of high wind power output where previously inflated prices were diminished.

Table 7 presents the maximum expected annual revenue from 2020 to 2025 using both 2006 and 2007 wind years. The results showed a modest increase in revenue over the years investigated compared with the $90 \%$ increase in installed wind capacity over the same period. Furthermore, the increase in revenue from 2006 to 2020 with a 2006 wind year was $<5 \%$. Over this period, wind capacity increased from just over 2 to $22 \mathrm{GW}$. This suggests that there were other factors influencing the storage revenue and, in accordance with the observation from Section 5.1, the increase in wind capacity was unlikely to be the cause of the rise in revenue.

Increasing gas and carbon prices over the period are likely to have led to the increased revenue. Increases in both gas and carbon prices would increase the marginal cost of generation for peaking plant, but have no impact on the marginal cost of the baseload generator, nuclear, which has zero emissions. As a result, the daily price spread would be larger and the opportunity for revenue from arbitrage increased. In the National Grid Gone Green Scenario, carbon prices increased steadily up to 2025 . The expected increase in revenue from this may have been counteracted by the increase in wind power over the same period. Gas prices, however, decreased from 2015 to 2020 followed by a small, but steady increase from 2020 to 2025 . This trend is consistent with the changes is arbitrage revenue over the period.
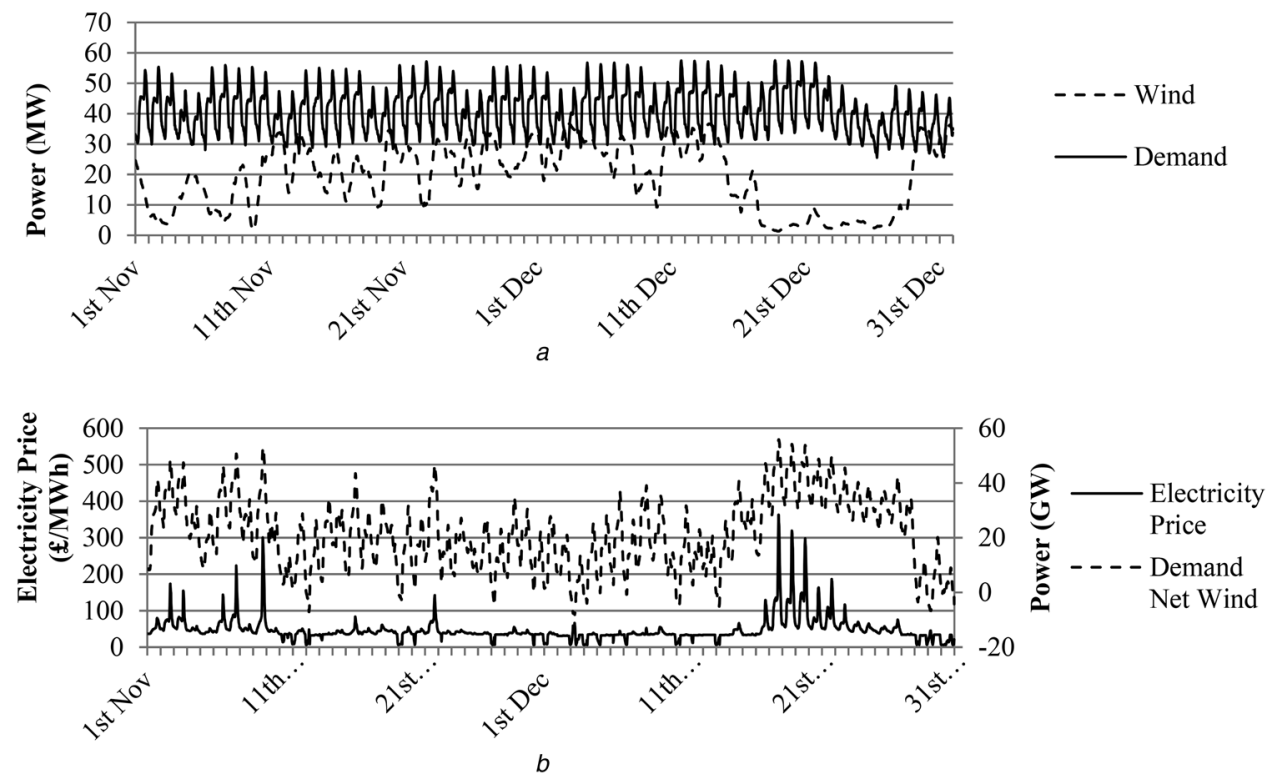

Fig. 9 Outputs for 2025 using 2007 wind year

$a$ Demand and wind power

$b$ Demand net wind and electricity price 


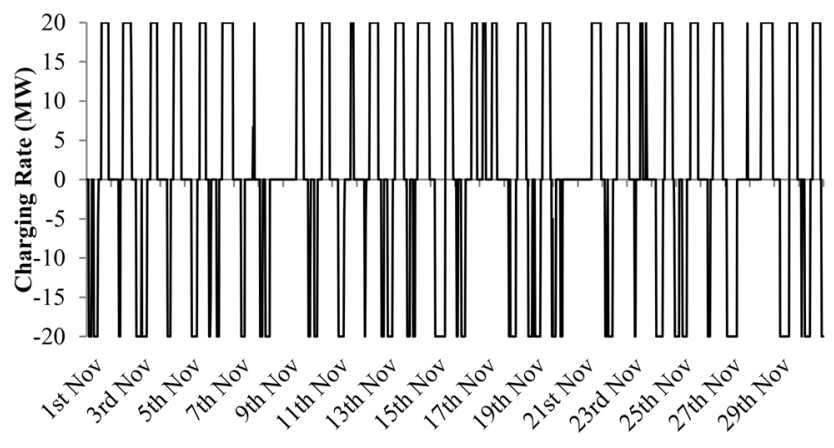

Fig. 10 Storage operating schedule November 2025 using 2007 wind year

Table 7 Maximum annual arbitrage revenue 2020-2025

\begin{tabular}{ccc}
\hline Year & $\begin{array}{c}\text { Revenue based on 2006 wind } \\
\text { year, fm }\end{array}$ & $\begin{array}{c}\text { Revenue based on 2007 wind } \\
\text { year, fm }\end{array}$ \\
\hline 2020 & 0.943 & 0.447 \\
2021 & 0.991 & 0.47 \\
2022 & 0.989 & 0.472 \\
2023 & 1.01 & 0.482 \\
2024 & 1.076 & 0.556 \\
2025 & 1.086 & 0.584 \\
\hline
\end{tabular}

A second observation evident from the results in Table 7 is the reduction in expected revenue when the wind year changed from 2006 to 2007 and a different wind pattern was assumed. This highlights the additional risk to a storage operator when there is a large amount of intermittent generation in the system. Revenue could reduce by up to $50 \%$ from one year to the next.

The results support Grünewald's conclusions that commercialisation of storage, as a facilitating technology, is dependent on a number of uncertainties including the future energy mix, the regulatory environment in the energy sector and the stochastic uncertainty from one year to the next [14]. Arbitrage revenue is dependent on the individual behaviour of each of these characteristics, but also on the complex interactions between them. These uncertainties increase the cost of finance for storage technologies and reduce the chance of successful market uptake.

\subsection{Wind as a price setter}

The analysis above models wind as negative load; however, in the $\mathrm{UK}$, it is only embedded generation below $50 \mathrm{MW}$ capacity which truly behaves in this way. Larger wind farms forecast their output in advance and trade in forward markets. Instead of contributing to negative demand, wind farm output would adjust the supply function for each half-hour period. Thermal generation would be required to respond not only to changes in demand, but also to forecast errors. Moreover, the assumption that thermal plant is dispatched in merit order is a simplification. In reality, the network responds in different ways to variation in wind and demand and, consequently, there are different cost implications. Additionally, if there was a significant market share of wind, wind generators would not bid to produce electricity at their marginal cost of zero. These effects would change the electricity price at the lower end of the supply curve and some cases may drive it negative. In the UK, this would be expected only at high levels of installed capacity and would be infrequent [38]. While this would have some impact on the results, trends in arbitrage revenue would be unlikely to be affected as the magnitude and frequency of price spikes would remain largely unchanged.

\section{Conclusions}

Grid scale electrical energy storage could be a facilitating technology enabling intermittent renewable generation to be connected to the electricity grid. However, the commercial arrangements for storage are complex and it can be challenging to access financial rewards for the benefits which are provided. Electricity price arbitrage is one revenue stream which is available to a storage operator. Electricity prices, currently driven by gas prices and daily demand cycles, are expected to vary in the future as more intermittent wind power is connected to the grid. This will change the potential revenue available from arbitrage.

This paper presents a model developed and used to investigate the revenue available to a storage operator through price arbitrage in future years. The National Grid Gone Green Future Energy Scenario, which has significantly increased wind capacity, was investigated. The results showed a modest increase in arbitrage revenue from 2020 to 2025 which was not expected to be a direct result of the increase in wind capacity, but due to contributions from a number of changing factors including increased fuel and carbon prices. The investigation into electricity prices suggested that, contrary to previous findings, increased wind power may, in fact, be detrimental to storage revenue. With increased wind capacity there were numerous occasions of suppressed electricity prices and a reduced frequency of price spikes. This may create fewer opportunities for storage to discharge and sell electricity at a high price. Furthermore, increased wind capacity is likely to lead to large variations in revenue from one year to the next which will increase the risk of investment in energy storage technology.

\section{References}

1 Akhil, A.A., Huff, G., Currier, A.B., et al.: 'DOE/EPRI 2013 electricity storage handbook in collaboration with NRECA' (Sandia National Laboratories, 2013)

2 Grunewald, P., Cockerill, T., Contestabile, M., et al.: 'The socio-technical transition of distributed electricity storage into future networks - system value and stakeholder views', Energy Policy, 2012, 50, pp. 449-457

3 Strbac, G., Aunedi, M., Pudjianto, D., et al.: 'Strategic assessment of the role and value of energy storage systems in the UK low carbon energy future'. Imperial College report for the Carbon Trust, Energy Futures Lab, London, 2012

4 Grunewald, P.: 'Electricity storage in future GB networks - a market failure?'. Proc. Int. Conf. British Institute of Energy Economics Ninth Conf., Oxford, UK, 2012

5 Walawalkar, R., Apt, J., Mancini, R.: 'Economics of electric energy storage for energy arbitrage and regulation in New York', Energy Policy, 2007, 35, pp. $2558-2568$

6 Connolly, D., Lund, H., Finn, P., et al.: 'Practical operation strategies for pumped hydroelectric energy storage (PHES) utilising electricity price arbitrage', Energy Policy, 2011, 39, pp. 4189-4196

7 Sioshansi, R., Denholm, P., Jenkin, T., et al.: 'Estimating the value of electricity storage in PJM: arbitrage and some welfare effects', Energy Econ., 2009, 31, pp. 269-277

8 Barbour, E., Wilson, I.A.G., Bryden, I.G., et al.: 'Towards an objective method to compare energy storage technologies: development and validation of a model to determine the upper boundary of revenue available from electrical price arbitrage', Energy Environ. Sci., 2012, 5, (1), pp. 5425-5436

9 Lund, H., Salgi, G., Elmegaard, B., et al.: 'Optimal operation strategies of compressed air energy storage (CAES) on electricity spot markets with fluctuating prices', Appl. Therm. Eng., 2009, 29, (5-6), pp. 799-806

10 Khani, H., Zadeh, D.M.R.: 'Online adaptive real-time dispatch of privately owned energy storage systems using public-domain electricity market prices', IEEE Trans. Power Syst., 2015, 30, (2), pp. 930-938

11 Dunbar, A., Tagliaferri, F., Viola, I.M., et al.: 'The impact of electricity price forecast accuracy on the optimality of storage revenue' Proc. Int. Conf. IET Third Renewable Power Generation Conf., Naples, Italy, 2014

12 MacLeay, I., Harris, K., Annut, A.: 'Digest of United Kingdom energy statistics (DUKES)' (Department of Energy and Climate Change, 2010)

13 Cox, J.: 'The impact of intermittency: how wind variability could change the shape of the British and Irish electricity markets' (Pöyry, 2009)

14 Grunewald, P., Cockerill, T., Contestabile, M., et al.: 'The role of large scale storage in a GB low carbon energy future: issues and policy challenges', Energy Policy, 2011, 39, pp. 4807-4815

15 ELEXON, 'ELEXON Portal Website' [online]. Available at https://www. elexonportal.co.uk/news/latest?cachebust=ib0snesorc, accessed 26 June 2013

16 ICE ENDEX, 'ICE Download Centre' [online]. Available at http://www.iceendex. $\mathrm{com} /$, accessed 11th September 2014

17 National Grid: 'Metered half-hourly electricity demands' [online]. Available at http:// www.nationalgrid.com/uk/electricity/data/demand+data/, accessed 28 August 2013

18 Hawkins, S.: "A high resolution reanalysis of wind speeds over the British isles for wind energy integration'. PhD thesis, University of Edinburgh, 2012

19 Department of Energy and Climate Change, UK Government: 'Changes to onshore wind subsidies protect investment and get the best deal for bill payers' [online] Available at https://www.gov.uk/government/news/changes-to-onshore-windAvailable at https://www.gov.uk/government/news/changes-to-onshore-wind-
subsidies-protect-investment-and-get-the-best-deal-for-bill-payers, accessed 24th June 2015 
20 Wilson, G.I.A., McGregor, P.G., Infield, D.G., et al.: 'Grid-connected renewables, storage and the UK electricity market', Renew. Energy, 2011, 36, (8), pp. $2166-2170$

21 Green, R.J., Vasilakos, N.: 'The long-term impact of wind power on electricity prices and generating capacity'. Proc. Int. Conf. IEEE Power and Energy Society General Meeting, San Diego, USA, 2011

22 Kirschen, D., Strbac, G.: 'Fundamentals of power system economics' (Wiley, 2010)

23 Eager, D., Hobbs, B.F., Bailek, J.W.: 'Dynamic modelling of generation capacity investment in markets with high wind penetration', IEEE Trans. Power Syst., 2012, 27, (4), pp. 2127-2137

24 Eager, D.: 'Dynamic modelling of generation capacity investment in electricity markets with high wind penetrations'. PhD thesis, University of Edinburgh, 2012

25 Digest of United Kingdom Energy Statistics: 'Annex A: energy and commodity balances, conversion factors and calorific values' (Department of Energy and Climate Change, 2012)

26 Mott MacDonald: 'UK electricity generation costs update' (Department of Energy and Climate Change, 2010)

27 TradeTech: 'Uranium Prices - NUEXCO Exchange Value' [online]. Available at http://www.uranium.info/nuexco_exchange_value.php, accessed 2 September 2014

28 Department of Energy and Climate Change: 'Quarterly energy prices' (National Statistics, 2009)
29 European Environment Agency: 'EUA Future Prices 2005-2011' [online]. Available at http://www.eea.europa.eu/data-and-maps/figures/eua-future-prices200520132011\#tab-documents, accessed 2nd September 2014

30 Department of Energy and Climate Change: 'DECC Planning Database - Monthly extract' [online]. Available at https://www.restats.decc.gov.uk/app/reporting/decc/ monthlyextract, accessed 13th May 2014

31 Harman, K., Walker, R., Wilkinson, M.: 'Availability trends observed at operational wind farms'. Proc. Int. Conf. European Wind Energy Conf., Brussels, Belgium, 2008

32 Byrne, R.H., Silva-Monroy, C.A.: 'Estimating the maximum potential revenue for grid connected electricity storage: arbitrage and regulation' (Sandia National Laboratories, 2012)

33 Fuchs, G.: 'Technology overview on electricity storage' (Smart Energy for Europe Platform GmbH (SEFEP), 2012)

34 Hu, L., Taylor, G.: 'A novel hybrid technique for short-term electricity price forecasting in UK electricity markets', J. Int. Counc. Electr. Eng., 2014, 4, (2), pp. $114-120$

35 Skea, J., Ekins, P., Winskel, M.: 'Energy 2050: the transition to a secure, low carbon energy system for the UK' (Earthscan, 2011)

36 National Grid: 'UK future energy scenarios' (National Grid Plc., 2014)

37 Green, R., Vasilakos, N.: 'Market behaviour with large amounts of intermittent generation', Energy Policy, 2010, 38, (7), pp. 3211-3220

38 Turner, M., Buckley, D., Sinclair, D.: 'Negative pricing in the GB wholesale electricity market' (Baringa, 2015) 


\title{
Energy storage and wind power: sensitivity of revenue to future market uncertainties

\author{
Anna Dunbar ${ }^{1}$, A. Robin Wallace ${ }^{1}$, Gareth P. Harrison ${ }^{1}$ 凶 \\ ${ }^{1}$ School of Engineering, Institute for Energy Systems, University of Edinburgh, Edinburgh, UK \\ 凶E-mail: gareth.harrison@ed.ac.uk
}

\begin{abstract}
Grid connected electrical energy storage is expected to enable the integration of variable renewable generation in the future. As the electricity sector develops wholesale electricity prices will change, which will change the way in which storage technologies are operated. This study investigates the sensitivity of storage revenue to uncertain market variables. Results indicate that higher gas prices, carbon prices and average demand would increase peak electricity prices, leading to larger daily price spreads and increased storage revenue. Increased wind generation, however, would reduce opportunities for price arbitrage and lessen storage revenue. Wind power also affects the way in which devices are operated and changes the characteristics which are rewarded by the market. With increased wind capacity, storage devices cycle less regularly as operation is driven by substantial changes in wind power output, rather than daily demand patterns. As a result, slower discharge times are more favourable and revenue is more sensitive to rates of self-discharge. Furthermore, there is less variation in wholesale electricity price and consequently conversion efficiency is more critical to performance.
\end{abstract}

\section{Introduction}

Electrical energy storage (EES) is regarded as a potential solution to the challenge of the Energy Trilemma in facilitating the grid integration of renewable energy. Many benefits of storage have been identified including improved system control, reduced network congestion and avoided curtailment of renewable output [1]. In the coming decades deployment of renewable energy capacity is expected to increase significantly leading to a greater requirement for flexibility and a higher value to be placed on these benefits.

There are expected to be bespoke applications, such as in islanded or heavily constrained networks, where business cases for storage would exist [2]. Storing renewable electricity until it could be consumed locally would be a more attractive option than upgrading transmission or distribution network connections in these cases. However, many renewable energy projects will not have the benefit of local consumers. These projects, particularly those located offshore, will be network-connected and will generate into a centralised energy system.

Grid connected EES will be required to aggregate revenue streams from a range of markets if it is to be commercially viable $[3,4]$. One recognised revenue stream is price arbitrage purchasing electricity when it is cheap and selling it back to electricity suppliers during periods of peak demand when the price is high.

Several studies have investigated the revenue available to a storage operator through price arbitrage with [5-9] using historic electricity prices to estimate revenue available under existing market conditions. Barbour et al. [5] compared the performance of pumped hydro, hydrogen and battery storage devices in the Great Britain (GB) market from 2005 to 2010. The results demonstrated increasing revenue with charging rate and technology performance improving with efficiency. Other studies have investigated a single technology in multiple markets. Connolly et al. [10] compared arbitrage value of pumped hydro plant in 13 different regions highlighting the dependence of revenue on local market conditions. Sioshansi et al. [11] concluded that arbitrage value was dependent on the specific generation mix and fuel costs. As larger number of wind farms are deployed, the generation mix will change substantially and wholesale electricity prices will increasingly be driven by wind power output in addition to demand cycles. Gas and carbon prices will also change in the future affecting the daily price spread. These may change the way in which storage devices are operated. Few authors have modelled arbitrage revenue in future electricity markets, however, Grünewald et al. [12] proposed a method to investigate this. Four storage technologies were examined in markets with increasing renewable energy capacity and the sensitivity of net present value was tested against a range of variables. The trading margin, or daily price spread, was highlighted as one of the most sensitive parameters. The model was not, however, capable of reflecting variations in gas and carbon prices in the price spread and consequently the effect of these on storage revenue. Furthermore, wind power was attributed a marginal value equivalent to the opportunity cost of a Renewable Obligation Certificate, which had the effect of driving costs negative. Barbour et al. [13] investigated the impact of negative electricity prices on arbitrage revenue for storage and concluded that whilst creating some opportunities to gain additional revenue during periods of charging, their occurrence would be infrequent and would probably not impact technology choices for storage.

Dunbar et al. [14] used a similar approach to [12] implementing an alternative wind model, price function and including sufficient detail to reflect the impact of changing gas and carbon prices on arbitrage revenue. A single set of storage characteristics was investigated to show the changing annual revenue from 2020 to 2025 in the National Grid 2014 'Gone Green' Future Energy Scenario (FES). This scenario exhibited increasing wind capacity and higher gas and carbon prices, among other changes. The results suggested that increased wind power may lead to reduced arbitrage revenue, while increasing gas and carbon prices may increase revenue. However, these factors were investigated with a single scenario and the impact of their individual effects was not explicitly identified. Furthermore, the impact of these changes on the storage operation strategy was not investigated, nor the implications of this on the device characteristics which would be most favourable in these conditions.

Using the model described in [14], this paper investigates the sensitivity of arbitrage revenue to changes in gas price, carbon price, capacity margin and wind power capacity in the GB market and the impact of these variables on the preferred device characteristics. The potential future value of these variables is highly uncertain and investors will need to understand how a storage investment will perform across a range of outcomes. Scenarios are a useful method for appraising uncertainty, but sensitivity studies allow the influence of individual factors to be 
investigated. It is critical that the relative importance of key storage characteristics is understood in the context of uncertain market variables. This will enable technology choices to be developed which are robust to changing market conditions instead of solutions which are optimal for today's market, but which may become redundant as the sector evolves. Table 1 compares the approach used in this study with other work investigating arbitrage revenue to highlight its contribution.

\section{Storage revenue model}

The model comprises several components which estimate storage revenue in a simulated electricity system: an electricity market price model, a wind generation model and a storage arbitrage revenue model. Each of these components is explained fully in [14] which should be referred to for further details regarding the model assumptions and validation. A summary of the key features is given below.

\subsection{Electricity market model}

The electricity market price model was established on the assumption of perfect competition. This approach has been shown to be representative of the power exchange in GB and is commonly used for modelling scenarios of future electricity prices where variables are significantly different from historic levels [15]. The pricing model operates using estimated aggregate supply and demand functions where the price of electricity for each half hour time period is determined by the market clearing price. Thermal generators were grouped into four classes: nuclear, coal, combined cycle gas turbines (CCGTs) and open cycle gas turbines (OCGTs). The aggregate supply function was formed by stacking the generator classes in merit order of increasing marginal cost, $C$, which was calculated for each technology using:

$$
C=\frac{1}{\eta}\left(a F+v F_{\text {car }}\right)+V+e
$$

where $\eta$ is the thermal efficiency, $F$ is the fuel cost, $v$ is the carbon emitted from combustion, $F_{\text {car }}$ is the carbon price, $V$ is the variable generation cost, $a$ is a conversion coefficient and $e$ is the cost of nuclear fuel enrichment.

Competitive prices were assumed with marginal generators bidding a price between their own marginal generation cost and the cost of the next class of generator in the merit order stack (the fundamental costs and characteristics of each generator type are given in Table 2). Between these two values, a hyperbolic function was used to smooth the discontinuities in the step function and better represent the complexities of the supply curve, such as differing ages and efficiencies of plant within each generation type [14]. An exponential uplift in price which applies to OCGT to represent their ability to set high prices at extreme demand levels (and recover their fixed costs) [14]; similar approaches have been used by other authors $[16,17]$. An example of the supply curve is shown in Fig. 1 where the merit order was nuclear, coal, CCGT then OCGT. The supply curve is notably flat in the regions where base load and mid merit generators fulfil demand. When peaking capacity is required, there is a sharp increase in price.

Historic demand data from National Grid is used to drive the model and this defines the power which conventional generation must serve in each time period. It is common when modelling markets with wind generation to deduct aggregate wind output time series (Section 2.2) from the underlying electricity demand time series; it is this 'net demand' that the remaining generation is dispatched to meet and the resulting intersection with the supply curve defines the market price.

In practice only relatively small, embedded wind generation behaves as negative load with larger wind farms forecasting their output and trading in forward markets. As such, wind farm output would tend to adjust the supply function for each half-hour period. Thermal generation would be required to respond not only to changes in wind and demand, as well as forecast errors, indicating that the assumption that thermal plant is dispatched in merit order is a simplification. With significant market share of wind there is potential for market prices to not only be suppressed, but in certain cases to become negative [21]. First, wind may be the marginal generator and when in receipt of subsidy it may offer negative bids up to the subsidy level to avoid curtailment. Second, an inflexible baseload plant that otherwise would be shut down and re-started may seek to avoid the costs of doing so by offering negative bids to keep generating. The extent and occurrence of negative prices is, however, strongly dependent on a range of factors including the extent of wind generation, levels of demand and the specific subsidy regime in place [21]; they would be expected only at high levels of installed capacity and would tend to be relatively infrequent [21]. As the merit order model applied here does not account for the dynamics of generation dispatch, the assumption that no subsidies are paid for renewable generation means the minimum price of electricity never falls below zero. As such, the shape of the supply curve does not fundamentally change, remaining shallow at low net demand and steep during periods of high net demand.

Historic Market Index Prices from the UK power exchange [22] were used to calibrate and validate the market model. Data from

Table 1 Comparison of scientific literature

\begin{tabular}{|c|c|c|c|c|c|}
\hline Paper & Market & Electricity prices & $\begin{array}{l}\text { Negative } \\
\text { prices }\end{array}$ & $\begin{array}{l}\text { Sensitivities } \\
\text { investigated }\end{array}$ & Implications \\
\hline Figueiredo et al. [8] & various & historic & no & alternative historic markets & $\begin{array}{c}\text { Revenue varies significantly between markets, } \\
\text { dependent on specific generation mix, market } \\
\text { design and participant behaviour }\end{array}$ \\
\hline Connolly et al. [10] & various & historic & no & $\begin{array}{l}\text { market, year, optimisation } \\
\text { strategy }\end{array}$ & $\begin{array}{l}\text { Highlights variation in revenue between historic } \\
\text { years, markets and optimisation strategies }\end{array}$ \\
\hline Sioshansi et al. [11] & PJM & historic & no & $\begin{array}{l}\text { storage characteristics, } \\
\text { forecasting, year }\end{array}$ & $\begin{array}{l}\text { Highlights influence of gas price on arbitrage } \\
\text { revenue from historic prices. Justifies use of perfect } \\
\text { foresight }\end{array}$ \\
\hline Grünewald et al. [12] & GB & $\begin{array}{l}\text { future scenarios - } \\
\text { varies wind/solar }\end{array}$ & yes & $\begin{array}{l}\text { storage characteristics, } \\
\text { generation mix }\end{array}$ & $\begin{array}{c}\text { Concludes arbitrage may be commercially viable for } \\
\text { low cost, long duration storage in future with large } \\
\text { renewable capacity }\end{array}$ \\
\hline Barbour et al. [13] & GB & historic (modified) & yes & $\begin{array}{l}\text { Storage efficiency and } \\
\text { capacity }\end{array}$ & $\begin{array}{l}\text { Negative pricing demonstrated to be beneficial to } \\
\text { storage, but unlikely to have a major impact on } \\
\text { technology choices }\end{array}$ \\
\hline $\begin{array}{l}\text { Imperial College } \\
\text { London [4] }\end{array}$ & GB & future scenarios & no & $\begin{array}{l}\text { Storage size relative to } \\
\text { wind farm size }\end{array}$ & $\begin{array}{c}\text { Focus on 'value of storage'; simple case study of } \\
\text { wind farm }\end{array}$ \\
\hline this paper & GB & $\begin{array}{l}\text { future scenarios - } \\
\text { varies wind, gas } \\
\text { and carbon prices }\end{array}$ & no & $\begin{array}{l}\text { 'Wind year', gas and } \\
\text { carbon prices, wind } \\
\text { capacity, storage } \\
\text { characteristics }\end{array}$ & $\begin{array}{c}\text { Sensitivity to wind power capacity, gas and carbon } \\
\text { prices investigated (independently). Implications of } \\
\text { wind-driven price profile on storage operation } \\
\text { investigated }\end{array}$ \\
\hline
\end{tabular}




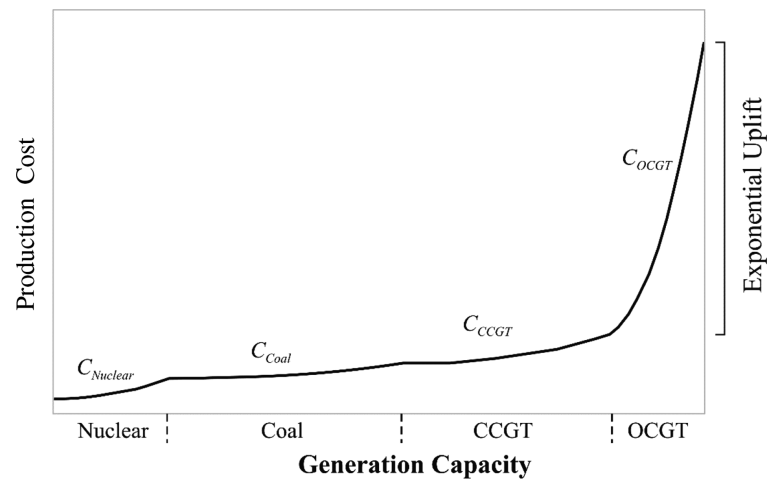

Fig. 1 Example electricity supply curve

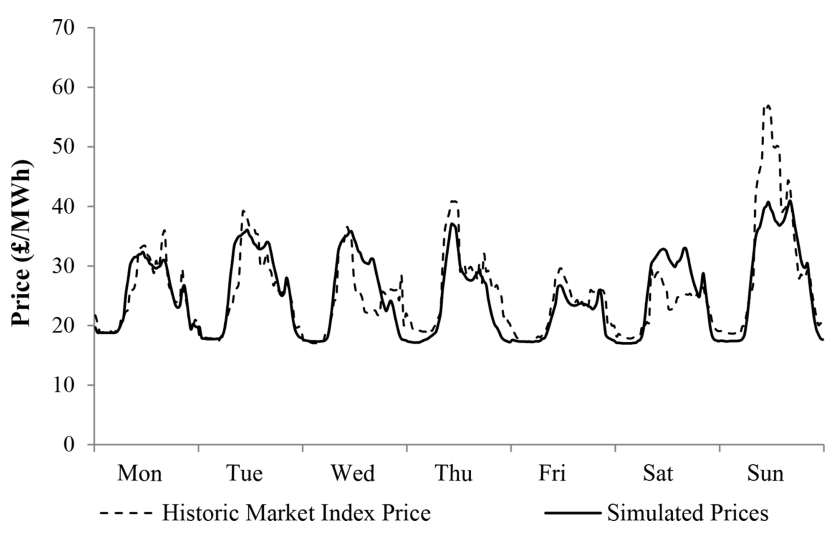

Fig. 2 Historic market prices and simulated electricity prices for first week in August 2007

2005 to 2007 [14] was used as it represents a period prior to the major increase in wind generation in GB. Time series of historic fuel and carbon prices were sourced from [23-26]. Price data at as high a temporal resolution as possible was used; in the case of gas, daily prices were found to substantially better capture underlying electricity market price behaviour. This was deemed credible as although generators will purchase most of their fuel at fixed prices on forward markets, they may also trade on daily gas markets to adjust their position; this leads daily gas prices to better represent the marginal behaviour of gas generation.

The calibration was aimed at ensuring that the intraday spread in electricity prices - of critical importance to arbitrage revenue was represented well by the model. The mean daily peak and trough prices are much more important for arbitrage than the extreme values and these were found to be captured well [14]. Across the 3 years, the absolute error (bias) for mean peak prices was $£ 2.64 / \mathrm{MWh}$ and $£ 5.64 / \mathrm{MWh}$ for mean trough values. When applied to the storage model (Section 2.3), the difference between the modelled and historic electricity prices resulted in revenue differing by at worst $10 \%$ in any one year and virtually zero on average. The quality of the fit is well demonstrated in Fig. 2 which shows the historic and simulated electricity prices for the first week in August 2007.

\subsection{Wind power production}

Aggregate wind production time series were based on high resolution hourly wind simulations for the UK and surrounding waters produced by Hawkins [27]. The DECC RESTATS planning database [28] was used to identify the location and capacity of existing and planned wind farms in GB with the site-specific wind speed series extracted at each location. Power output from each wind farm was calculated using an equivalent aggregate power curve described in [27]. Depending on the assumptions about wind deployment this allows aggregate production for onshore and offshore wind fleets to reflect the diversity of wind speeds across the UK. Aggregate production was reduced by $10 \%$ to account for availability, a conservative assumption onshore but early offshore availability was less than $90 \%$ [29]. The data was interpolated linearly to obtain a time series of wind power output at half-hourly intervals. To represent scenarios with larger amounts of wind generation, the capacities at existing wind farm locations were scaled up; while this does not fully reflect the spatial diversity promoted by larger, more distributed wind fleets it is adequate for the purposes of this paper and not expected to substantially alter the results.

Although the analysis presented here focuses on wind, it could conceivably be extended to other variable renewable generation such as solar PV, wave and tidal using similar atmospheric or oceanographic modelling techniques.

\subsection{Energy arbitrage model}

The time series of electricity prices formed an input for the storage arbitrage revenue model. The revenue was calculated using linear optimisation [30] which determines the quantity of electricity bought and sold during each period, subject to the constraints of the storage capacity, maximum charging/discharging rates as well as efficiencies for conversion (the round-trip ratio of energy delivered to energy consumed) and storage (which measures selfdischarge of the device). The model assumes the storage operator has perfect foresight of electricity prices; previous work showed minimal reduction in revenue using practical operating strategies compared to perfect foresight [31]. The storage device was assumed to be small relative to the total capacity in the market and its operation did not affect the price of electricity. Further details on the optimisation can be found in [14].

\section{Sensitivity study}

The sensitivity study was conducted by individually adjusting key 'external' parameters from initial baseline values to investigate the impact of each factor on arbitrage revenue. These parameters included gas and carbon prices, average capacity margin and installed wind capacity. In a future energy system these would not vary independently of each other and additional variables, such as thermal generation capacity and underlying patterns of demand, would also change; however, these were kept constant to investigate each effect in isolation and gauge its significance.

The baseline case used historic data from 2006, including time series of fuel [25] and carbon [26] prices, generation capacity [32], demand times series [33] and wind speed time series [27]. This ensured a degree of coherence in the underlying data. The installed capacity of each class of generator is: $12 \mathrm{GW}$ nuclear, $26 \mathrm{GW}$ coal, 22.6 GW CCGT, 12 GW OCGT, $1.9 \mathrm{GW}$ onshore wind and 300 MW offshore wind [32]. Installed wind capacity was less than 3\% of the total generation capacity and typically, coal generation was dispatched before CCGT in the merit order.

Initially, the storage characteristics were fixed at the baseline values listed in Table 3 . These depict a moderate scale device with a power-to-storage ratio of $1: 10$, reasonable round trip efficiency and no other losses.

Table 2 Thermal generator data [18-20]

\begin{tabular}{lcccccc}
\hline Generator type & $\begin{array}{c}\text { Thermal } \\
\text { efficiency } \eta, \%\end{array}$ & $\begin{array}{c}\text { Carbon emissions } \\
v, \text { kg/MWh }\end{array}$ & $\begin{array}{c}\text { Variable operating } \\
\text { costs } V, £ / M W h\end{array}$ & $\begin{array}{c}\text { Enrichment cost } e, \text { Availability, \% } \\
£ / \text { MWh }\end{array}$ & $\begin{array}{c}\text { Conversion } \\
\text { coefficient } a\end{array}$ \\
\hline nuclear & 36 & 0 & 1.8 & 2.5 & 0 & 78 \\
coal & 36 & 285 & 2.0 & 0 & 86 & $8.24 \times 10^{-3}$ \\
CCGT & 60 & 185 & 2.2 & 0 & 87 & 150 \\
OCGT & 46 & 185 & 2.7 & 95 & 34.128 \\
\hline
\end{tabular}



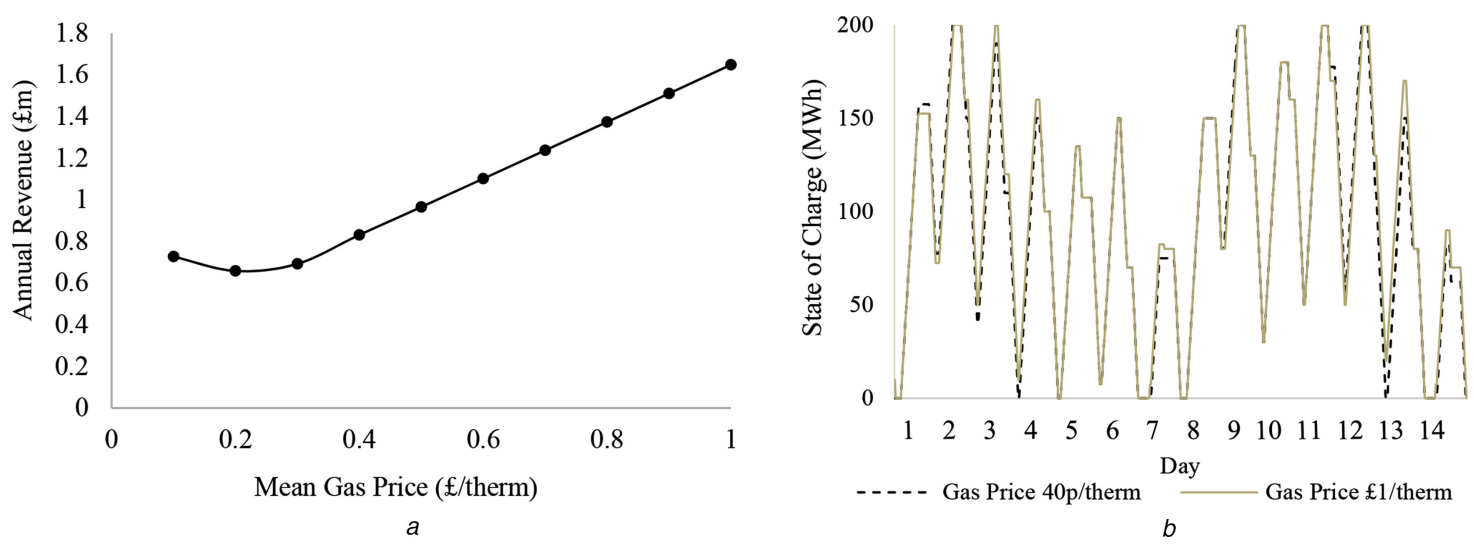

Fig. 3 Impact of gas prices on

(a) Annual storage revenue with range of prices and, (b) Storage state of charge for two winter weeks with gas price of $40 \mathrm{p} /$ therm and $£ 1 /$ therm
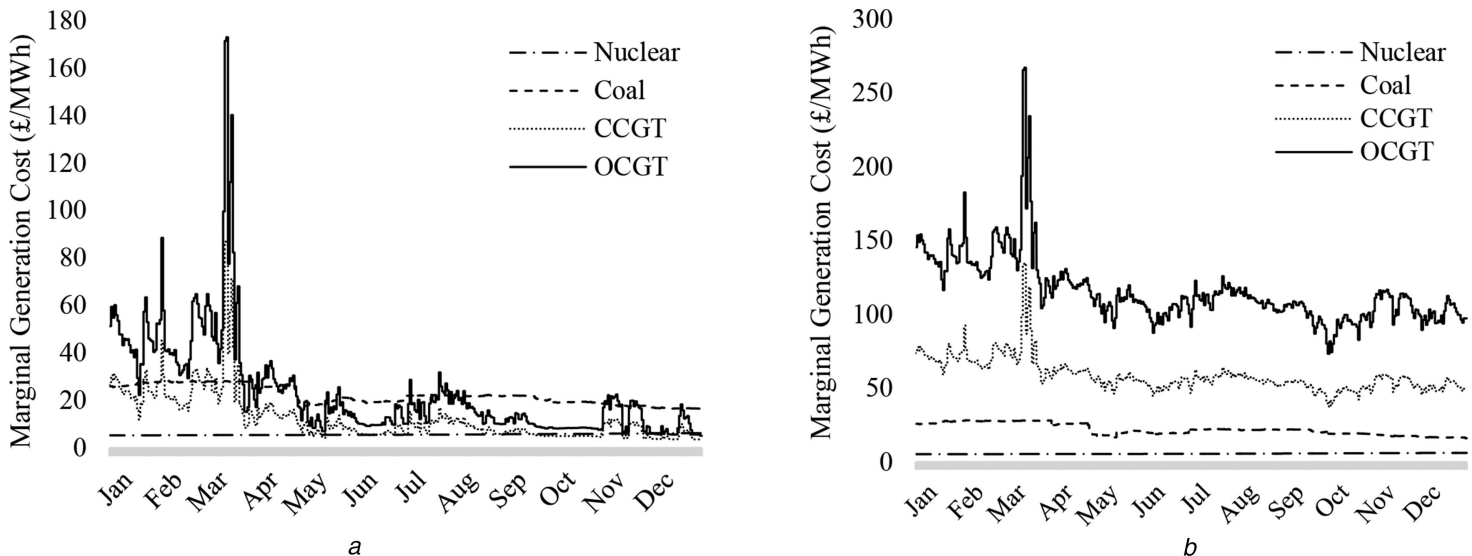

Fig. 4 Marginal generation costs with average gas prices of (a) $10 \mathrm{p} /$ therm and, (b) $£ 1 /$ therm

\section{Market variables}

\subsection{Gas price}

The 2014 National Grid FES [34] estimates that, in a high price scenario, gas prices would be slightly less than $£ 1 /$ therm by 2035 . The arbitrage algorithm was therefore run for simulated electricity prices with average gas prices increasing from $10 \mathrm{p} /$ therm $(£ 3.41 /$ $\mathrm{MWh})$ to $£ 1 /$ therm $(£ 34.13 / \mathrm{MWh})$. Gas prices are volatile and have varied over this range of values in the last ten years. For reference, the average gas price in 2015 was $\sim 50 \mathrm{p} /$ therm $(£ 17.07 / \mathrm{MWh})$ [25]. For each average gas price, the remaining inputs from the baseline year were used and the electricity price simulated at each half hour for 365 days to enable the annual revenue to be determined. The time series of historic gas prices from the 2006 baseline year was scaled in each case to maintain a constant intraannual volatility of gas prices for each run.

Fig. $3 a$ shows that for gas prices greater than $30 \mathrm{p} /$ therm the arbitrage revenue increased approximately linearly with gas price. Gas turbines were the most expensive thermal generators dispatched and their marginal prices set the daily peak electricity prices. Fig. $3 b$ shows the storage device state of charge over a twoweek period with an average gas price of $40 \mathrm{p} /$ therm and $£ 1 /$ therm. This shows that the optimum operating schedule in both cases was almost identical. The device charged and discharged on a daily basis in line with daily demand cycles. Wind power output had little influence on electricity prices compared with variations in

Table 3 Baseline storage characteristics

\begin{tabular}{lcc}
\hline Storage constraint & Unit & Value \\
\hline maximum storage capacity & $\mathrm{MWh}$ & 200 \\
maximum charging/discharging rate & $\mathrm{MW}$ & 20 \\
conversion efficiency (round trip) & $\%$ & 75 \\
storage efficiency & $\% /$ day & 100 \\
\hline
\end{tabular}

demand as the baseline installed capacity was small. Despite the similar storage operational pattern, the higher gas price led to a larger daily price spread enabling more revenue to be made during each cycle.

Interestingly, for the lowest gas prices, revenue increased. This was because the lowest gas prices reduced the marginal generation cost of gas sufficiently that it became cheaper than coal for some periods. During these periods, coal was the marginal generator and CCGT contributed to base load during some off peak hours. Lower gas prices reduced the price of off peak generation which increased the daily price spread, enabling more revenue to be achieved during a storage cycle.

Fig. $4 a$ shows the marginal generation costs across the year with an average gas price of $10 \mathrm{p} /$ therm. This shows periods where coal had the highest marginal generation cost and was dispatched as peaking plant. Fig. $4 b$ shows the marginal generation costs with an average gas price of $£ 1 /$ therm, which shows that OCGTs were the most expensive generator for all periods of the year. The variation in marginal costs for each generator type is a result of the time series of fuel prices used (daily gas, monthly coal and quarterly nuclear prices).

\subsection{Carbon price}

Historically, the carbon price has always been below $£ 20 /$ tonne [26] and the 2014 National Grid FES [34] estimate that by 2035 it could increase to between $£ 30 /$ tonne and $£ 75 /$ tonne. The analysis was repeated for average carbon prices from $£ 10 /$ tonne $(£ 0.01 / \mathrm{kg})$ to $£ 100 /$ tonne $(£ 0.1 / \mathrm{kg})$. The time series of carbon prices within the year was scaled from 2006 to the average value.

Fig. $5 a$ shows that revenue increased with carbon price, albeit at a diminishing rate. Increasing the carbon price increased both gas and coal marginal generation costs, but did not affect nuclear generation costs. For many periods, increasing the carbon price raised the daily peak electricity prices, increasing the price spread 


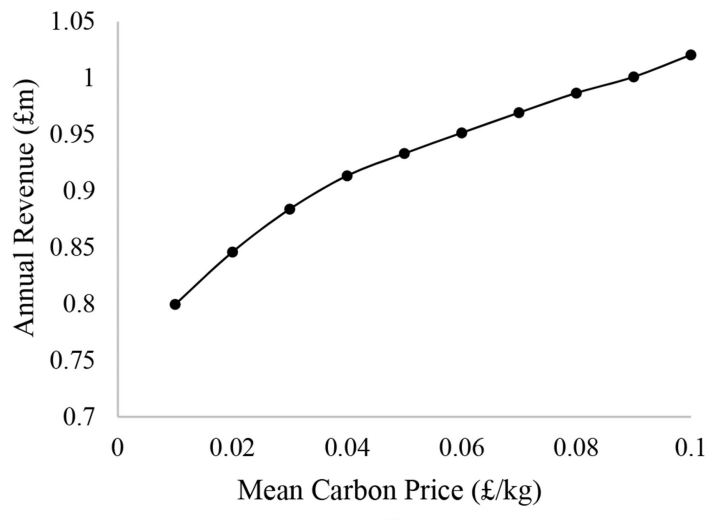

a

Fig. 5 Variation in annual storage revenue with

(a) Carbon price and, (b) Average annual demand as a proxy for capacity margin

and enabling the storage device to gain additional revenue. For other off peak periods the second marginal generator - commonly coal - was required, which set the off peak electricity prices. Increasing the carbon price increased coal generation costs more significantly than gas generation costs, reducing the price spread during periods where coal was required for off peak generation. As the carbon price increased further the price spread - and opportunity for arbitrage - was reduced during these periods, leading to diminishing gains in revenue.

The storage device followed a similar strategy to that shown in Fig. $3 b$ over the range of carbon prices investigated. The storage revenue was significantly less sensitive to carbon price than to gas price. There was an increase in revenue of less than $30 \%$ with an order of magnitude increase in carbon price (from $£ 10$ to $£ 100$ / tonne). This compared with an increase in revenue of over $125 \%$ for an order of magnitude increase in gas price (from $10 p$ to $£ 1 /$ therm). This demonstrates the relatively modest influence of the current range of expected carbon prices on arbitrage revenue compared to the impact of gas prices.

\subsection{Capacity margin}

Retaining the underlying pattern of demand from 2006 and with peak demand kept constant, average demand was varied from 30 to $50 \mathrm{GW}$ to investigate the impact on storage revenue. Generation capacity was fixed at 2006 levels, so increasing demand represented a reduction in the average capacity margin. In a competitive market, this would lead to increased electricity prices incentivising investors to build more generators. This would, in turn, restore a greater average capacity margin and reduce prices restoring market equilibrium. The static market model used does not reflect these changes, but allows variations in demand to be a proxy for the capacity margin.

Fig. $5 b$ indicates annual revenue increasing as capacity margin falls. For low average demand, representing a high average capacity margin, commonly the low merit order generators were able to serve demand throughout the day. This was delivered by the left-hand side of the supply curve shown in Fig. 1. In this region, prices are low and price elasticity of supply is also low, demonstrated by the shallow curve, resulting in a small price spread. As demand grew, reducing the capacity margin, the higher merit order generators including peaking plant were required. This was delivered through generation represented by the right-hand side of the supply curve. Here, prices are higher, but price elasticity of supply is also higher, demonstrated by the steep shape of the curve. As a result, for the same daily variation in demand, the price spread was increasingly larger enabling higher revenue to be achieved.

As the pattern of demand remained unchanged, the optimum operation strategy was similar across the range of average demand investigated, comparable to that shown in Fig. $3 b$.

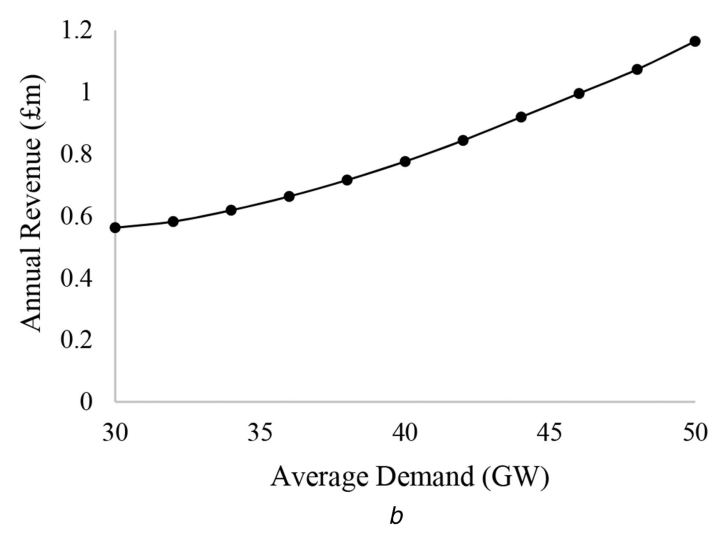

\subsection{Installed wind capacity}

The arbitrage model was run for installed wind capacity increasing from 0 to $40 \mathrm{GW}$. The ratio of offshore to onshore capacity was fixed at 3:2. Again, the remaining inputs, including the wind speed distributions, were taken from the 2006 baseline year. Retaining all other generation capacity as per $2006,40 \mathrm{GW}$ of installed wind represents $35 \%$ of the total generation capacity in GB. Fig. $6 a$ shows that the revenue reduced as the wind capacity increased. This was due to lower variation in electricity price with increased wind power output. To illustrate this Fig. $6 b$ shows the wind power output for two winter weeks with $40 \mathrm{GW}$ of installed wind capacity and Fig. $6 c$ the resulting electricity prices for cases of $40 \mathrm{GW}$ and no installed wind. Prices were similar for both scenarios between days 8 and 9 when the wind power output was nearest to zero. With $40 \mathrm{GW}$ of installed wind capacity, peak prices were significantly reduced during periods of high wind power output, which led generally to lower price variation. This is a result of the shape of the supply curve (Fig. 1) which was steep during periods of low wind production, but shallow during periods of high wind production. $40 \mathrm{GW}$ of wind capacity reduced scarcity of supply, leading to reduced average prices and although there was increased variation in thermal output there was reduced variation in price; these led to decreasing arbitrage revenue. This is shown clearly in the annual price duration curves (Fig. $6 d$ ) with 0 and $40 \mathrm{GW}$ of installed wind capacity. Prices are generally suppressed with 40 GW wind including at extreme low net demand. Higher peak prices have been suggested to be a natural outcome in a system with high penetrations of wind capacity where peaking plant seeks to recover its fixed costs over fewer operational hours [15], although the operation of a capacity market would tend to transfer these costs out of the wholesale energy market. In common with other models that neglect dynamic pricing changes (e.g. [21]), the increases in peak prices are not seen here. However, the consequent impact on revenue estimates is limited as the constraints on operation of a storage device limit their ability to exploit these sporadic and infrequent price spikes.

Fig. 7 shows the state of charge of the storage device for the same two weeks. With no installed wind capacity the device charged and discharged once a day in line with the variation in electricity price driven by demand patterns. The storage device did not reach its maximum storage capacity or fully discharge on every cycle. With $40 \mathrm{GW}$ of installed wind the device charged and discharged less frequently with only four distinct cycles over the two-week period. This is similar to the four distinct cycles of wind power output shown in Fig. $6 b$ and is in line with the typical frequency of synoptic weather patterns that dominate UK climate. In addition with $40 \mathrm{GW}$ of installed wind capacity, the storage device was limited for longer periods of time by its $200 \mathrm{MWh}$ maximum capacity.

\subsection{Base year}

For all the variables discussed above, time series of demand and wind speeds were taken from the same 2006 base year. These 

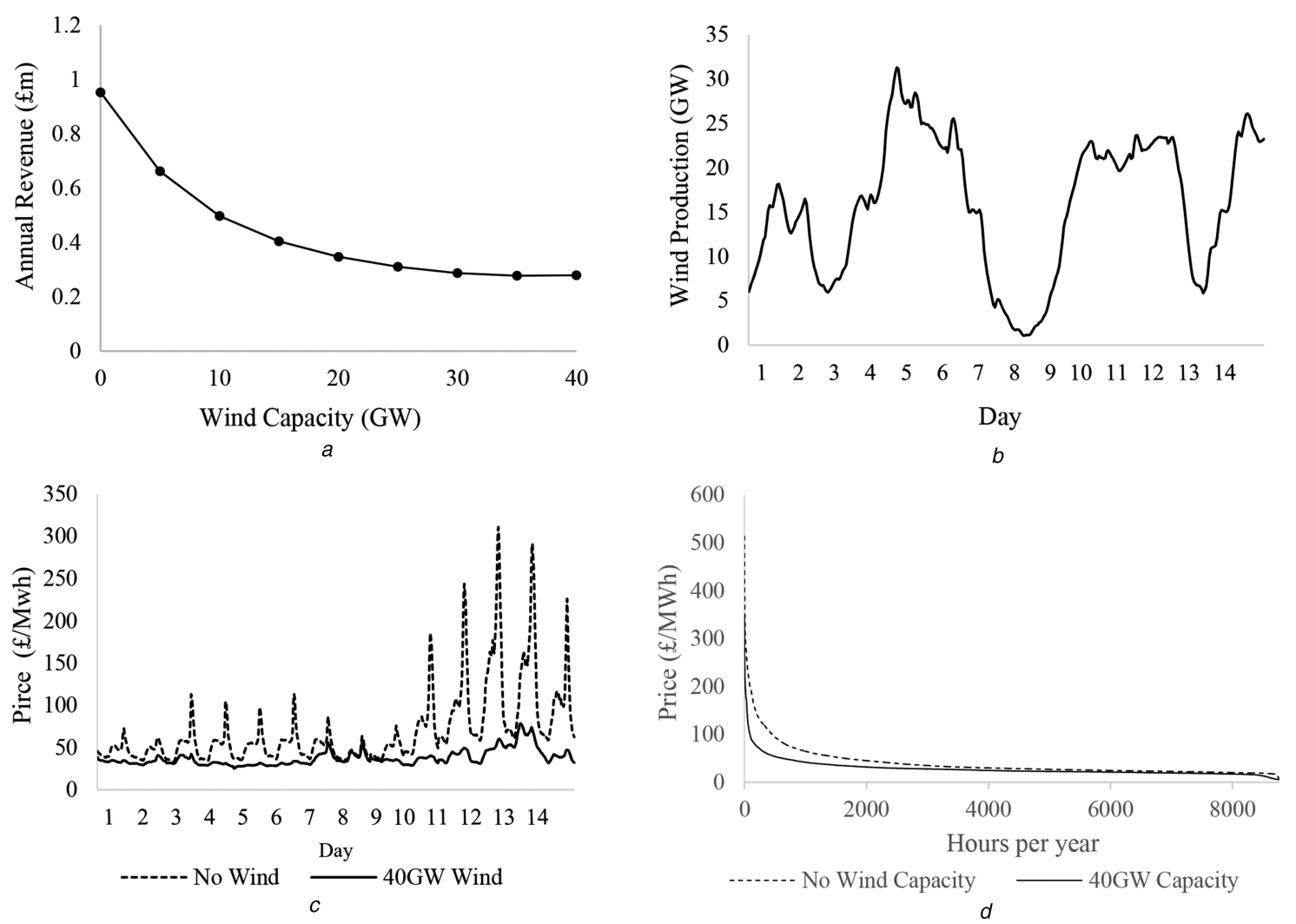

Fig. 6 Revenue reduced as the wind capacity increased

(a) Impact of wind capacity on annual revenue; for case of $40 \mathrm{GW}$ wind, (b) Wind production and, (c) Electricity prices for two winter weeks and, (d) Annual price duration curve

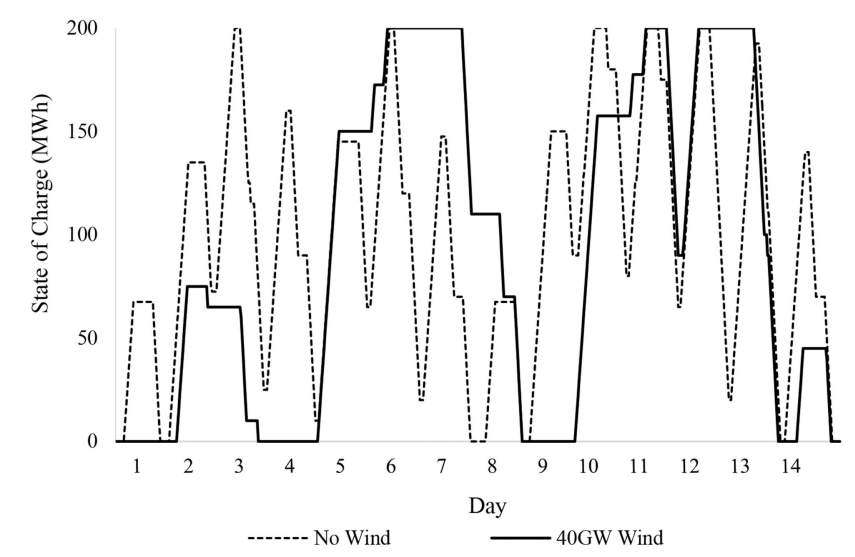

Fig. 7 Storage state of charge for two winter weeks with no wind and 40 GW installed wind capacity

patterns change from one year to the next and will affect electricity storage revenue. To examine the effect of this the underlying time series of demand and wind speed patterns from different base years were employed with fuel and carbon prices remaining fixed at 2006 levels. Table 4 shows the resulting annual revenue for each of the years. It can be seen that there are substantial differences between them with variations of over $100 \%$ in revenue from one year to the next. This demonstrates the inherent risk facing

Table 4 Annual revenue from 2006 using alternative base year time series

\begin{tabular}{lc}
\hline Year & Revenue $(£ 000)$ \\
\hline 2005 & 363 \\
2006 & 821 \\
2007 & 386 \\
2008 & 629 \\
2009 & 401 \\
2010 & 449 \\
\hline
\end{tabular}

electricity storage investors from variations in patterns of demand and wind speeds; factors influenced by circumstances outside even the electricity sector.

\section{Storage system characteristics}

The external factors examined in Section 4 are outside the direct control of electricity storage investors. However, it may be possible to engineer device characteristics to minimise potential negative impacts or enhance the positive impacts of these external factors. Understanding the value of different technology characteristics in the context of changing markets will enable development of storage systems which are robust to uncertain future circumstances.

\subsection{Storage capacity and charging rate}

The majority of the variables investigated in Section 4 did not affect the optimum operating strategy of the storage device. In these cases, the sensitivity of revenue to storage capacity and charging rate did not change significantly as the variables changed. However, Fig. 7 shows that there was a substantial change in the operating schedule with $40 \mathrm{GW}$ of installed wind capacity compared with no installed wind. This suggests that the storage capacity and charging rate may be valued differently in energy systems with different penetrations of wind. This was investigated by comparing the change in annual revenue for devices with increasing storage capacity and charging rate for cases with no installed wind capacity and with $40 \mathrm{GW}$ of wind capacity.

Fig. $8 a$ shows the change in revenue with increasing storage capacity and constant charging rate for cases with 0 and $40 \mathrm{GW}$ of installed wind. The remaining characteristics were fixed at the values listed in Table 3. The results are normalised relative to the revenue from devices with a $600 \mathrm{MWh}$ capacity, the maximum investigated for each case: $£ 0.96 \mathrm{~m}$ and $£ 0.38 \mathrm{~m}$ for the 0 and 40 GW wind cases, respectively. The normalised revenue shows the distinct difference in sensitivity to storage capacity more visibly than the absolute values. Fig. $8 b$ shows the change in revenue with increasing charging rate and constant storage capacity for the same cases, again normalised relative to the revenue from devices with a 

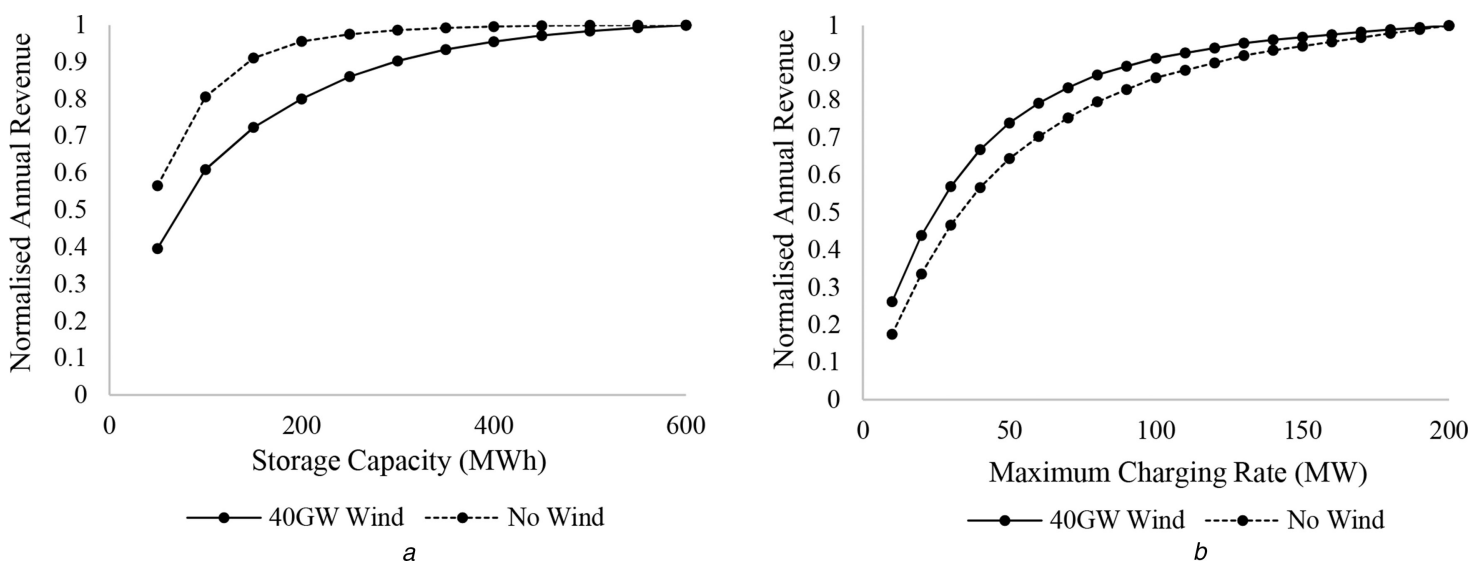

Fig. 8 Annual revenue for 0 and $40 \mathrm{GW}$ installed wind capacity cases with other variables fixed at baseline (a) Varying storage capacity with fixed charging rate, normalised relative to $600 \mathrm{MWh}$ storage capacity and, (b) Varying charging rate with fixed storage capacity, normalised relative to $200 \mathrm{MW}$ charging rate
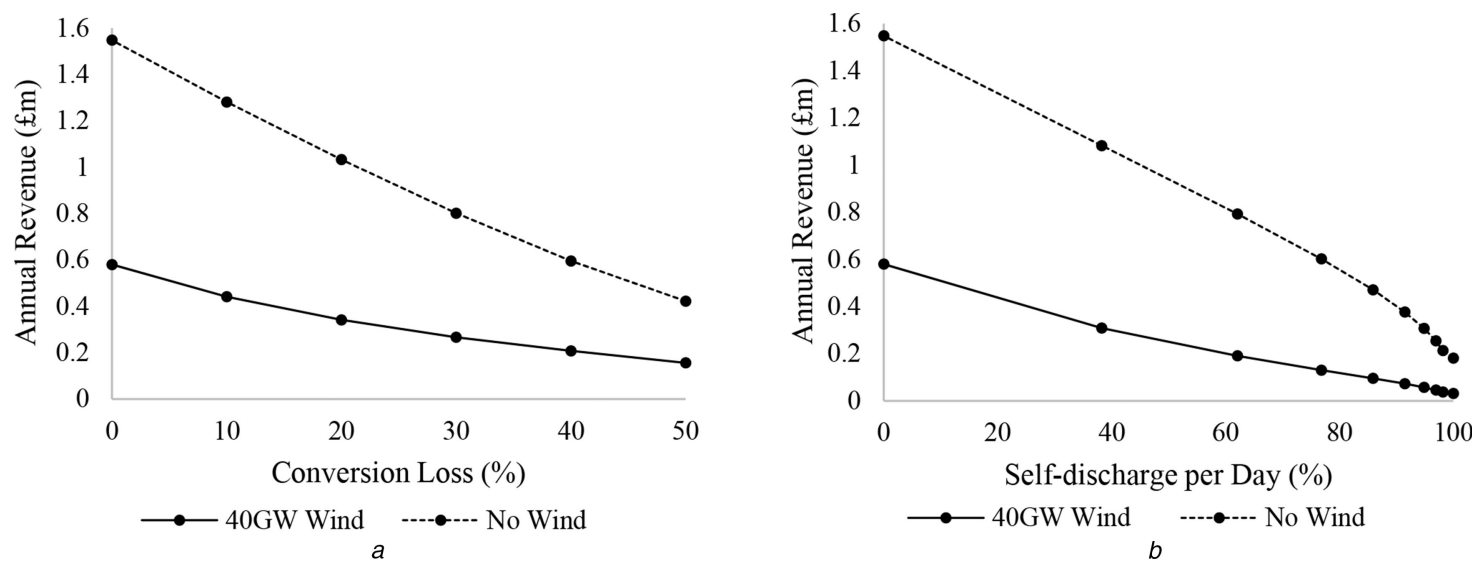

Fig. 9 Annual revenue for high and low installed wind capacities, varying (a) Conversion losses with zero self-discharge and, (b) Self-discharge with zero conversion losses

$200 \mathrm{MW}$ charging rate $(£ 2.72 \mathrm{~m}$ and $£ 0.69 \mathrm{~m}$ for 0 and $40 \mathrm{GW}$ of wind capacity, respectively).

Figs. $8 a$ and $b$ show that the rate of increase in revenue falls as storage capacity and charging rate are independently increased. For larger storage capacities the full range was utilised less frequently and increasing the capacity further yielded fewer opportunities to store more electricity and generate additional revenue. Similarly, the highest charging rates were the least restrictive on revenue so increasing them further, with the storage capacity fixed, yielded fewer benefits. With no wind capacity, the arbitrage revenue was much less sensitive to the storage capacity than with $40 \mathrm{GW}$ of wind, however, it was more sensitive to the charging rate. With no wind, the storage performed best by charging and discharging on a daily basis limited by the maximum charging rate on each cycle, as shown in Fig. 7. With $40 \mathrm{GW}$ of wind capacity, however, the storage performed best by charging, discharging and storing energy over longer periods of time with fewer cycles, leading to the capacity becoming the more restrictive constraint. These results suggest that devices with higher storage capacity to power ratios may perform better in markets with a large penetration of wind power.

\subsection{Efficiency}

In any energy market, arbitrage revenue will be sensitive to the round-trip efficiency and self-discharge of a device. With higher electricity prices, conversion losses are relatively more costly. Increasing fuel and carbon prices led to higher electricity prices and increased sensitivity to round-trip efficiency and selfdischarge. Increased wind capacity, on the other hand, led to frequently reduced electricity prices. However, these led to increased sensitivity to round-trip efficiency and self-discharge, as shown in Figs. $9 a$ and $b$. The higher levels of wind power not only reduced average electricity prices, but also reduced the variation between wholesale price peaks and troughs. As a result, conversion processes need to be more efficient to return the same revenue. Furthermore, with high wind penetrations, electricity prices cycled over longer durations and energy was stored over longer periods making revenue more sensitive to self-discharge.

\section{Conclusions}

As the electricity system incorporates increased renewables there will be changes in electricity price which will affect the operation of and business case for energy storage. This paper demonstrates the impact of a range of uncertain market variables on price arbitrage opportunities and how different storage characteristics are rewarded. Systematic examination of the sensitivity of revenue to key external factors showed that revenue rose with increasing gas price, carbon price and demand, but fell as wind capacity increased. Further, as well as uncertain mid- to long-term market conditions storage revenue was sensitive to inter-annual variations in wind speeds and demand and the economic, climate and behavioural patterns that drive them. Increased wind capacity is found to substantially impact the revenue available to storage, but also the way in which it is operated. With higher wind penetration, wholesale prices became more strongly influenced by wind power output than by diurnal patterns of demand behaviour. This reduces the number of storage cycles and leads to energy being stored for longer periods. As a result, revenue becomes more sensitive to storage capacity and efficiency, but less sensitive to charging rate. Revenue also becomes more dependent on conversion efficiency as the variation between wholesale price peaks and troughs was reduced. As storage characteristics designed for current market conditions appear not be optimal as wind penetration rises, smaller modular storage devices which could be expanded independently as the electricity system evolves, may offer safer investment options than large, monolithic projects. 


\section{References}

[1] Akhil, A., Huff, G., Currier, A.B., et al.: 'DOE/EPRI 2013 electricity storage handbook in collaboration with NRECA' (Sandia National Laboratories, California, 2013)

[2] Mignard, D.: 'Estimating the capital costs of energy storage technologies for levelling the output of renewable energy sources', Int. J. Environ. Studies, 2014, 71, (6), pp. 796-803

[3] Xian, H., Delarue, E., D'haeseleer, W., et al.: 'A novel business model for aggregating the values of electricity storage', Energy Policy, 2011, 39, pp. $1575-1585$

[4] Imperial College London: 'Can storage help reduce the cost of a future UK electricity system?' (Carbon Trust, 2016)

[5] Barbour, E., Wilson, G.I.A., Bryden, I.G., et al.: 'Towards an objective method to compare energy storage technologies: development and validation of a model to determine the upper boundary of revenue available from electrical price arbitrage', Energy Environ. Sci., 2012, 5, pp. 5425-5436

[6] Walawalker, R., Alt, J., Mancini, R.: 'Economics of electric energy storage for energy arbitrage and regulation in New York', Energy Policy, 2007, 35, pp. $2558-2568$

[7] Graves, F., Jenkin, T., Murphy, D.: 'Opportunities for electricity storage in deregulated markets', Electr. J., 1999, 12, pp. 46-56

[8] Figueiredo, F., Flynn, P., Cabral, E.: 'The economics of energy storage in 14 deregulated power markets', Energy Studies Rev., 2006, 14, pp. 131-152

[9] Khani, H., Zadeh, M.R.D.: 'Online adaptive real-time optimal dispatch of privately owned energy storage systems using public-domain electricity market prices', IEEE Trans. Power Syst., 2015, 30, (2), pp. 930-938

[10] Connolly, D., Lund, H., Finn, P., et al.: 'Practical operation strategies for pumped hydroelectric energy storage (PHES) utilising electricity price arbitrage', Energy Policy, 2011, 39, pp. 4189-4196

[11] Sioshansi, R., Denholm, P., Jenkin, T., et al: 'Estimating the value of electricity storage in PJM: arbitrage and some welfare effects', Energy Econ., 2009, 31, pp. 269-277

[12] Grünewald, P., Cockerill, T., Contestabile, M., et al.: 'Role of large scale storage in a GB low carbon energy future: issues and policy challenges', Energy Policy, 2011, 39, (9), pp. 4807-4815

[13] Barbour, E., Wilson, G., Hall, P., et al.: 'Can negative electricity prices encourage inefficient electrical energy storage devices', Int. J. Environ. Studies, 2014, 71, (6), pp. 862-876

[14] Dunbar, A., Cradden, L.C., Wallace, R., et al.: 'Impact of wind power on arbitrage revenue for electricity storage', IET Gener. Transm. Distrib., 2016, 10, (3), pp. 789-806

[15] Green, R., Vasilakos, N.: 'The long-term impact of wind power on electricity prices and generating capacity'. IEEE Power and Energy Society General Meeting, San Diego, 2011
[16] Grunewald, P.: 'Electricity storage in future GB networks - a market failure?'. British Institute of Energy Economics 9th Conf., Oxford, 2012

[17] Eager, D., Hobbs, B.F., Bialek, J.W.: 'Dynamic modelling of generation capacity investment in markets with high wind penetration', IEEE Trans. Power Syst., 2012, 27, (4), pp. 2127-2137

[18] Eager, D.: 'Dynamic modelling of generation capacity investment in electricity markets with high wind penetrations'. PhD Thesis, University of Edinburgh, 2012

[19] Digest of United Kingdom Energy Statistics: 'Annex A: energy and commodity balances, conversion factors and calorific values' (Department of Energy and Climate Change, London, 2012)

[20] Mott MacDonald: 'UK electricity generation costs update' (DECC, Brighton, 2010)

[21] Baringa: 'Negative pricing in the GB while sale electricity market' (DECC, London, 2015)

[22] ELEXON: 'ELEXON Price Portal', Available at: www.elexonportal.co.uk. [Accessed 26 June 2013]

[23] TradeTech: 'Uranium Prices - NUEXCO Exchange Value', Available at http://www.uranium.info/nuexco_exchange_value.php. [Accessed 2 September 2014]

[24] Department of Energy and Climate Change: 'Quarterly energy prices' (National Statistics, London, 2009)

[25] ICE ENDEX: 'ICE Download Centre', Available at: http:/ www.iceendex.com/. [Accessed 11 September 2014]

[26] European Environment Agency: 'EUA Future Prices 2005-2011', Available at: http://www.eea.europa.eu/data-and-maps/figures/eua-futureprices-200520132011\#tab-documents. [Accessed 2 September 2014]

[27] Hawkins, S.: 'A high resolution reanalysis of wind speeds over the British isles for wind energy integration'. PhD Thesis, University of Edinburgh, 2012

[28] DECC: 'DECC Planning Database - Monthly extract', 2014. Available at: https://restats.decc.gov.uk/app/reporting/decc/monthlyextract

[29] Harman, K., Walker, R., Wilkinson, M.: 'Availability trends observed at operational wind farms'. European Wind Energy Conf., Brussels, 2008

[30] Byrne, R.H., Silva-Monroy, C.A.: 'Estimating the maximum potential revenue for grid connected electricity storage: arbitrage and regulation' (Sandia National Laboratories, California, 2012)

[31] Dunbar, A., Tagliaferri, F., Viola, I.M., et al.: 'The impact of electricity price forecast accuracy on the optimality of storage revenue'. Third IET Renewable Power Generation Conf., Naples, 2014

[32] MacLeay, I., Harris, K., Annut, A.: 'Digest of United Kingdom Energy Statistics (DUKES)' (Department of Energy and Climate Change, London, 2010)

[33] National Grid: 'Metered half-hourly electricity demands', Available at: http:// nationalgrid.com/uk/electricity/demand\#data/. [Accessed 28 August 2013]

[34] National Grid: 'UK future energy scenarios' (National Grid Plc., 2014) 


\title{
The impact of electricity price forecast accuracy on the optimality of storage revenue
}

\author{
A. Dunbar*, F. Tagliaferri *, I.M. Viola *, G.P. Harrison *广 \\ ${ }^{\star}$ Institute for Energy Systems, School of Engineering, The University of Edinburgh, Mayfield Road, EH9 3JL, UK \\ ${ }^{+}$Corresponding Author, gareth.harrison@ed.ac.uk
}

Keywords: Electricity Storage; Price Arbitrage; Dynamic programming; Forecast Accuracy

\begin{abstract}
Grid connected electrical energy storage could enable large numbers of intermittent renewable generators to be deployed in the UK. Many studies investigate the revenue which could be achieved through arbitrage assuming perfect foresight of electricity prices. In practice, storage operators will not have perfect foresight and will have to devise operational strategies using price forecasts. This paper investigates the impact of forecast accuracy on the optimality of storage revenue. The optimal revenue available is determined using linear programming and historic electricity prices. The results are compared to those found using dynamic programming and electricity price forecasts with increasing percentage error. A small scale lithium ion battery and a large pumped hydro energy storage (PHES) device are compared. The results show that revenue reduces at an increasing rate with increasing forecast error. The PHES device is more sensitive to forecast accuracy than the lithium ion battery. For both technologies, with a maximum error of $30 \%, 80 \%$ of the optimal revenue can be achieved. With increased capacity and significantly increased power rating, the lithium ion battery becomes more sensitive to price forecast accuracy.
\end{abstract}

\section{Introduction}

Electrical energy storage provides a potential solution to the challenge of integrating large amounts of intermittent generation to the grid. It could reduce the requirement for investment in expensive peaking plant and avoid curtailment of non-dispatchable generators. Additionally, it could reduce capital expenditure in transmission and distribution infrastructure as well as system operating costs. Strbac et al [1] have estimated that by 2050 electrical energy storage could provide savings of up to $£ 10$ bn per year to the British electricity system.

With the potential value of electrical energy storage recognised, understanding the economic and market drivers for widespread storage deployment is a growing area of research. Many studies consider the revenue that could be achieved through arbitrage, i.e. taking advantage of price differentials in the wholesale electricity market. Arbitrage alone is not expected to provide sufficient revenue to storage operators. However, combined with additional services, such as delivery of ancillary services, it is likely to be an essential revenue stream.

It is common practice to assume perfect foresight of electricity prices to assess the revenue that can be achieved through arbitrage [2]. Grunewald [3] investigated the gain in revenue an operator could receive with foresight over an increasing time horizon. He showed that by increasing foresight from 1 hour to 4 hours, revenue improvements of up to $22 \%$ could be achieved. Foresight beyond 12 hours was of no additional value, as storage devices typically follow a daily cycle in line with electricity prices.

In practice, operators will not have perfect foresight and so alternative approximate optimisation methods must be used with price forecasts. Various approaches have been taken to address this. Walawalkar et al [4] assumed a fixed daily storage cycle: the device was charged overnight and discharged during the same pre-defined peak hours each day. Sioshansi et al [5] determined an optimal charging strategy using prices from the preceding two week period and applied this to the current two week period. Using this approach, approximately $85 \%$ of the optimal revenue was achieved.

Lund et al [6] and Connolly et al [7] compared different practical strategies - without foresight of electricity prices to an optimal strategy - with perfect foresight - for compressed air energy storage (CAES) and PHES respectively. With the practical strategies implemented, CAES could achieve $80-90 \%$ of its optimum revenue. For PHES, the operator required "very accurate price predictions" to avoid a significant loss in profit. The accuracy required was not, however, quantified.

In this paper, the impact of price forecast accuracy on the optimality of storage revenue is investigated. Typical characteristics of a lithium ion battery are used as a base case. An upper bound on the revenue available through arbitrage is calculated using linear programming. This optimal solution is compared to results found using dynamic programming with notional price forecasts with increasing percentage error. The results are compared to characteristics of a large scale PHES system. The storage power capacity and energy rating are 
varied to investigate the sensitivity of storage size to forecast accuracy.

Electricity price processes are characterised by high volatility, large spikes, reversion to a daily pattern and seasonality as described by Amjady and Keynia [8]. Price forecasting is a broad and complex field of research in its own right Electricity markets vary between countries depending on geographical and system specific constraints, generator types and demand profiles. Forecast accuracies depend on the electricity market being examined as well as the forecasting method being used. This paper does not attempt to compare forecasting techniques, but investigates the impact of varying forecast accuracy on the optimality of storage revenue in the British electricity market.

\section{Method}

Market index data defines the price, $P_{t}(£ / \mathrm{MWh})$, of electricity for each half hour settlement period, $t$, in the UK. It reflects the value of wholesale electricity in the short-term market.

The storage device is defined by the following characteristics:

$S_{\max } \quad$ Storage capacity (MWh) - the total amount of electricity that can be stored by the device.

$Q^{C} \quad$ Charging rate (MW) - The maximum rate at which the storage device consumes electricity when recharging.

$Q^{D} \quad$ Discharging rate $(\mathrm{MW})$ - The maximum rate at which the storage device can deliver electricity.

$\eta_{c} \quad$ Conversion efficiency (\%) - the ratio of energy delivered to energy consumed excluding any losses due to self-discharge.

$\eta_{s} \quad$ Storage efficiency (\%) - the percentage of electricity retained in storage over each time period.

The following assumptions are applied:

- The storage device has $100 \%$ availability.

- Storage is a price taker and its operation does not affect the market price of electricity.

- The network is a single bus system and storage is not subjected to network constraints.

- The device characteristics are constant.

- The conversion efficiency is modelled during charging only i.e. the discharge cycle is $100 \%$ efficient.
- The ramp rate is negligible compared to the time period.

- The cost of charging and discharging (in addition to the cost of electricity) is negligible.

- The interest rate is negligible over the time period considered.

From the charging and discharging rates, the maximum quantity of electricity (MWh) which can be charged, $q_{\text {max }}^{C}$, or discharged, $q_{\max }^{D}$, in a single half hour time period is defined.

The decision variables for the storage operator are how much electricity to buy, $q_{t}^{C}$, and sell, $q_{t}^{D}$, during each time period. The state of charge of the storage device, $\mathrm{S}_{\mathrm{t}}$, is defined by Equation (1) and subject to the constraints given in Equations (2), (3) and (4).

$$
\begin{aligned}
& S_{t}=\eta_{s} S_{t-1}+\eta_{c} q^{C}{ }_{t}-q^{D}{ }_{t} \\
& 0 \leq S_{t} \leq S_{\text {max }} \\
& 0 \leq q^{C}{ }_{t} \leq q_{\text {max }}^{C} \\
& 0 \leq q^{D}{ }_{t} \leq q_{\text {max }}^{D}
\end{aligned}
$$

The objective is to maximise the annual revenue, $\mathrm{R}$, which is the sum of the price multiplied by the net quantity sold during each settlement period. This is defined in Equation (5).

$$
R=\Sigma P_{t}\left(q_{t}^{D}-q_{t}^{C}\right)
$$

\subsection{Linear Programming}

Linear programming is used to calculate the upper bound on revenue that can be achieved with perfect foresight of electricity prices as demonstrated by Byrne and Silva-Monroy [9]. $R^{*}$ is defined as $-R$ to formulate the problem as a standard minimisation problem with the objective defined by Equation (6), subject to the constraints in Equations (7) and (8).

$$
\begin{array}{ll}
\text { Minimise } & R^{*}=-f^{T} x \\
\text { Subject to } & A x \leq b \\
& l b \leq x \leq u b
\end{array}
$$

where $x$ is a vector of decision variables and $f$ a vector of prices for each half hour period throughout the year. $A$ is a matrix computed from the conversion and storage efficiencies and $b$ a vector based on the maximum storage capacity. $l b$ and $u b$ are lower bounds and upper bounds; zero and the maximum charging/discharging rate respectively. A standard linear programming function implemented in MatLab, "linprog $(f, A, b,[],[], l b, u b)$ ", is used to solve the objective function and define the optimum operation strategy to maximise annual revenue. 


\subsection{Dynamic Programming}

Dynamic programming is a technique used to solve a broad range of optimization problems, and is particularly applicable to multi stage stochastic optimization problems. It was formalised by Berteksas [10] and has since been used for a variety of applications including finance [11] [12].

Dynamic programming divides problems into a number of sub-problems and solves each sub-problem such that the overall solution is optimal to the original problem. In this work dynamic programming is used to solve the stochastic version of the problem defined in Section 2.1. Instead of perfect foresight of future electricity prices, a forecast is assumed with fixed maximum error. The objective of the dynamic programme is to generate a policy $\left\{q_{t}^{* D}-q_{t}^{*{ }^{C}}\right\}$, which is a set of time-dependent optimal decisions, with the objective function defined by Equation (9) and with the same constraints defined in Equations (7) and (8).

Minimise

$$
\mathbf{E}\left(R^{*}\right)=\mathbf{E}\left(\Sigma_{t} P_{t}\left(q^{*}{ }_{t}^{D}-q^{*}{ }_{t}^{C}\right)\right)
$$

E represents the expected value with respect to the probability distribution of the electricity prices. Electricity prices are modelled as a Markov chain, i.e. the probability distribution for the prices at time $t+1$ depend only on the price observed at time $t$.

The price forecasts are artificially generated by adding a random variable with uniform distribution over an interval [$s, s]$ to the actual prices, where $s$ is the maximum error of the forecast. The average error of the forecast is zero, while the absolute average error is $s / 2$.

Further details on the dynamic programming algorithm are detailed in [13].

\subsection{Data}

Market price data for Great Britain is available online from the Elexon Portal [14]. For this study, the annual revenue is calculated using data from 2013. Figure 1 shows the half hourly electricity prices for the first two weeks in January 2013. This demonstrates the typical daily cycle of cheap electricity prices overnight followed by an increase in the morning and a daily peak in the evening. This cyclic pattern presents opportunities for arbitrage on a daily basis.

Characteristics for a lithium ion battery are used for this analysis. These are based on the battery system demonstrated as part of the UK Power Networks Smarter Network Storage Project [15]. This is a small scale storage device connected to the distribution network. For comparison, results are compared to a large scale PHES based on characteristics of Dinorwig [16].

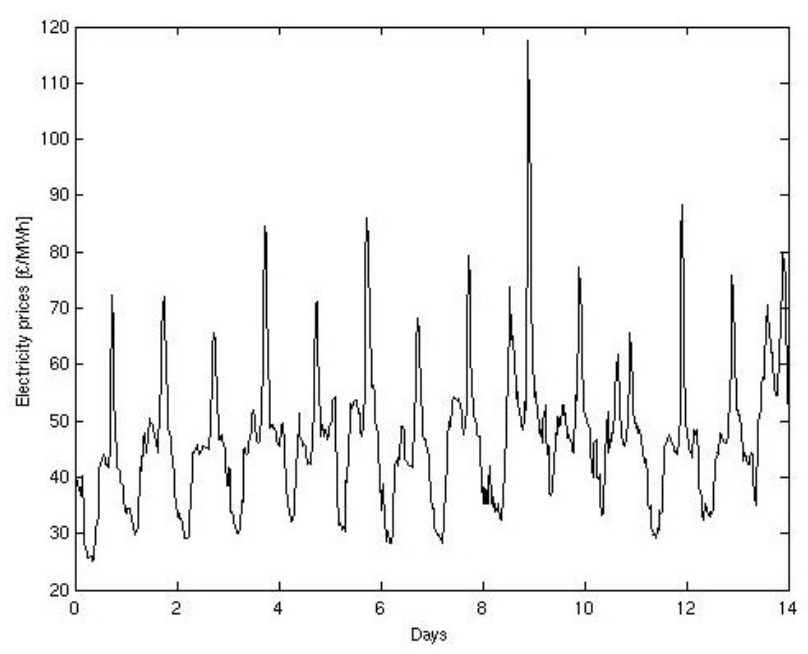

Figure 1: Electricity prices for the first two weeks in 2013

\begin{tabular}{|c|c|c|}
\hline Characteristic & $\begin{array}{c}\text { Lithium Ion } \\
\text { Battery }\end{array}$ & PHES \\
\hline$S_{\max }(\mathrm{MWh})$ & 10 & 10100 \\
$Q^{C}(\mathrm{MW})$ & 6 & 1728 \\
$Q^{D}(\mathrm{MW})$ & 6 & 1728 \\
$\eta_{c}(\%)$ & 65 & 0.75 \\
$\eta_{s}(\%)$ & 99.5 & 100 \\
\hline
\end{tabular}

Table 1: Lithium ion battery and PHES characteristics

The storage efficiency of PHES is approximated to $100 \%$. There will, in fact, be some losses due to evaporation; however, these will be minimal compared to the size of the reservoir.

The dynamic programme is run using randomly generated forecasts with maximum errors of $1 \%, 2 \%, 5 \%, 10 \%, 20 \%$, $30 \%, 40 \%$ and $50 \%$. Ten simulations are run for each level of forecast error. The maximum percentage difference between simulations for the same error is $3 \%$.

\section{Results and Discussion}

\subsection{Increasing Forecast Error}

For the lithium ion battery, with perfect foresight using linear programming, the optimum revenue which could be achieved based on 2013 electricity prices is $£ 47,248$. With dynamic programming, as the maximum error is increased from zero to $50 \%$, the revenue reduces to $63.6 \%$ of the optimum. The results, shown in Figure 2, show that the revenue reduces at an increasing rate with increasing forecast error.

$\mathrm{Hu}$ and Taylor [17] implied that forecast errors of $10 \%$ or less could be readily realised in the short-term British electricity market. If a storage operator could achieve this level of forecast accuracy, $\sim 98 \%$ of the optimum revenue available could be attained. Even if forecast accuracy reduces with a larger number of variable generators in the future, a significant proportion of the optimal revenue would still be available. 


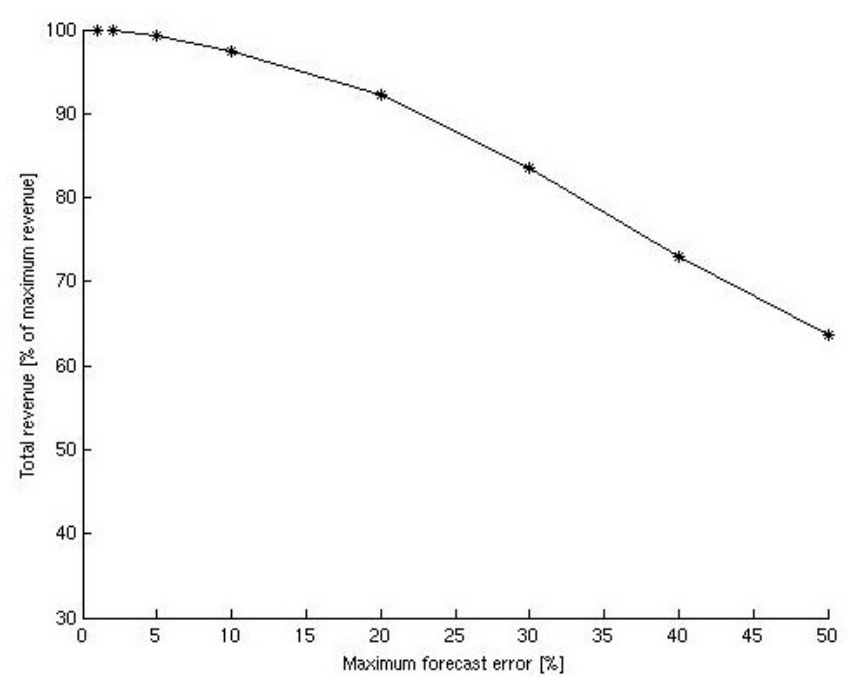

Figure 2: Loss of revenue with increasing forecast error for lithium ion battery

\subsection{Large Scale Storage}

For the large PHES the optimum revenue which could be achieved based on 2013 electricity prices is $£ 50 \mathrm{~m}$. The optimality is more sensitive to forecast accuracy than the smaller scale battery. The results, shown in Figure 3, exhibit a similar pattern to those for the lithium ion battery, however, the revenue reduces at a faster rate as the forecast error increases. As the maximum error is increased from zero to $50 \%$, the revenue reduces to $56 \%$ of the optimum. To maintain $98 \%$ of the optimum revenue, the forecast error must be within $5 \%$. The optimum revenue available to the PHES operator is significantly higher than that available to the lithium ion battery operator, as a result of the differing scales of technology. This may imply that a lower percentage of the optimal revenue is more acceptable to the PHES operator; however, the costs for the PHES will also be significantly higher so this conclusion cannot be made without the cost information being considered which is out with the scope of this study.

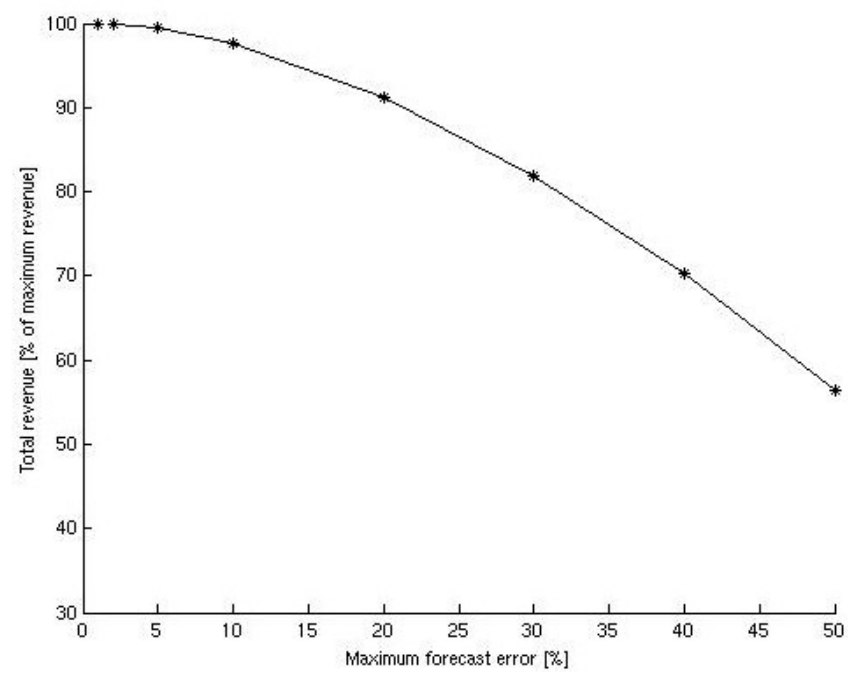

Figure 3: Loss of revenue with increasing forecast error for PHES

\subsection{Variation in Storage Capacity and Power Rating}

The simulations are repeated using the characteristics for the lithium ion battery, with increased storage capacity. The results are shown in Figure 4.

The optimal revenue available for storage devices with $10 \mathrm{MWh}, 15 \mathrm{MWh}$ and 20MWh capacities is $£ 47,248, £ 57,322$ and $£ 61,503$ respectively. The results show that for increased storage capacity, but fixed power rating, the device is more sensitive to price forecast accuracy.

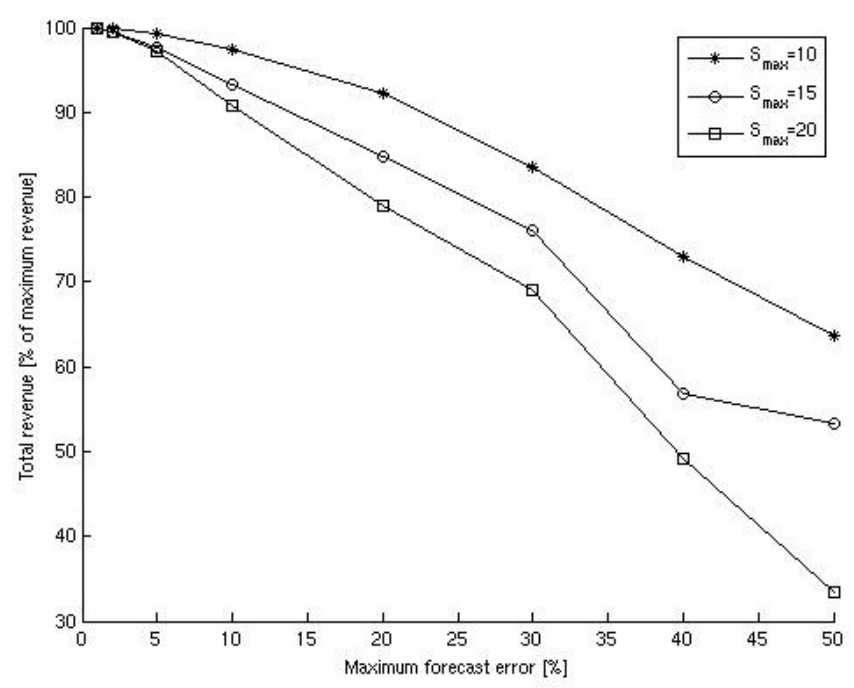

Figure 4: Loss of revenue with increasing forecast error for lithium ion battery with variation in storage capacity

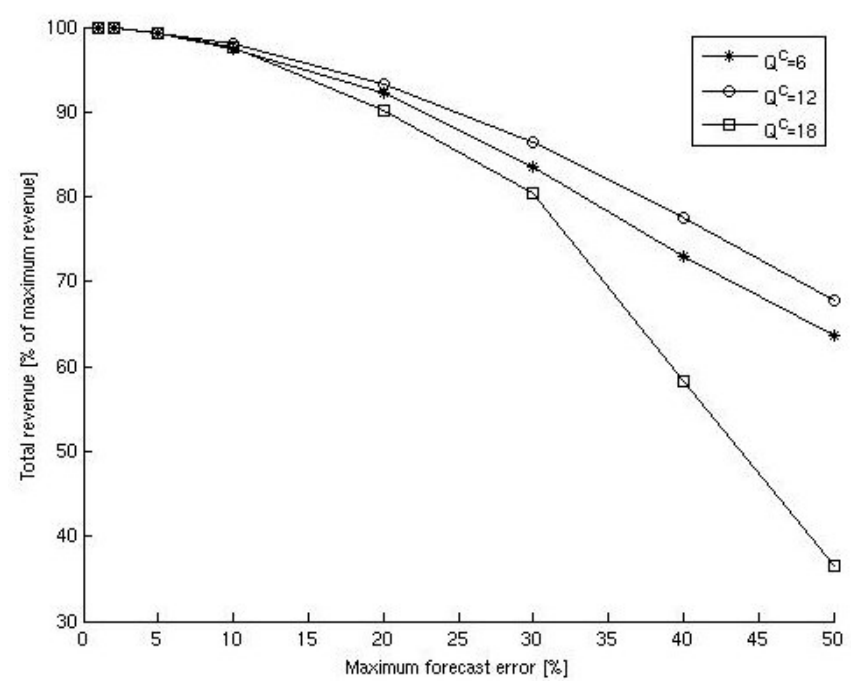

Figure 5: Loss of revenue with increasing forecast error for lithium ion battery with variation in power rating

Figure 5 shows the results using the characteristics for the lithium ion battery with fixed storage capacity but varying power rating. The optimal revenue available for storage devices with $6 \mathrm{MW}, 12 \mathrm{MW}$ and $18 \mathrm{MW}$ power ratings is $£ 47,248, £ 56,772$ and $£ 60,331$ respectively. The results indicate that the different power ratings have differing 
sensitivities to the accuracy of the forecast. Doubling the power rating leads to a small improvement, however, the revenue decreases significantly when tripling the power rating. This suggests the presence of an optimal threshold for the least sensitive power rating.

\section{Conclusions}

Grid connected electrical energy storage could enable a significant number of intermittent renewable generators to be connected to the electricity grid. There is a need to understand the economic case for energy storage to determine how it may be deployed in the future. One way in which storage operators can gain revenue is through price arbitrage, or time-shifting of energy. Many studies have calculated the expected revenue which could be achieved with perfect foresight of electricity prices. In practice, storage operators will not have perfect foresight and must devise operating strategies based on electricity price forecasts. Inevitably, this will lead to a reduction in projected revenue. This paper investigates the impact of price forecast accuracy on the optimality of storage revenue.

The optimal storage revenue is determined using linear programming with historical electricity prices to model a situation where perfect foresight is available. A practical strategy is then implemented, without perfect foresight, using dynamic programming and notional price forecasts with increasing percentage error. Storage characteristics of a lithium ion battery and a large scale PHES device are investigated using price data from the British wholesale electricity market from 2013.

For the technologies investigated, the optimality of storage revenue reduced at an increasing rate as the forecast error increased. The PHES device was more sensitive to forecast error that the smaller scale lithium ion battery. For both technologies, with a maximum error of $30 \%, 80 \%$ of the optimal revenue was achieved. These levels of forecast accuracy can be readily realised for the short term UK market. With increased storage capacity and significantly increased power rating, the lithium ion battery was more sensitive to forecast error.

\section{Acknowledgements}

This project has received funding from the Engineering and Physical Science Research Council as part of a Doctoral Training Grant.

\section{References}

[1] G. Strbac, M. Aunedi, D. Pudjianto, P. Djapic, F. Teng, A. Sturt, D. Jackravut, R. Sansom, V. Yufit, N. Brandon. "Strategic Assessment of the Role and Value of Energy Storage Systems in the UK Low Carbon Energy Future", Energy Futures Lab, Imperial College London, Report for the Carbon Trust, (2012).
[2] E. Barbour, I. A. Wilson, I. G. Bryden, P. G. McGregor, P. A. Mulheran, P. J. Hall. "Towards an objective method to compare energy storage technologies: development and validation of a model to determine the upper boundary of revenue available from electrical price arbitrage", Energy and Environmental Science, 5, pp. 5425-5436, (2012).

[3] P. Grunewald. "The Role of Electricity Storage in Low Carbon Energy Systems: Techno-economic Drivers and Transitional Barriers", PhD Thesis, Imperial College London, Centre for Energy Policy and Technology Faculty of Natural Sciences, (2012).

[4] R. Walawalkar, J. Apt, R. Mancini. "Economics of electric energy storage for energy arbitrage and regulation in New York", Energy Policy, 35, pp. 25582568, (2007).

[5] R. Sioshansi, P. Denholm, T. Jenkin, J. Weiss. "Estimating the value of electricity storage in PJM: Arbitrage and some welfare effects", Energy Economics, 31, pp. 269-277, (2009).

[6] H. Lund, G. Salgi, B. Elmegaard, A. N. Andersen. "Optimal operation strategies of compressed air energy storage (CAES) on electricity spot markets with fluctuating prices", Applied Thermal Engineering, 29, pp. 799-806, (2009).

[7] D. Connolly, H. Lund, P. Finn, B. V. Mathiesen, M. Leahy. "Practical operation strategies for pumped hydroelectric energy storage (PHES) utilising electricity price arbitrage", Energy Policy, 39, pp. 4189-4196, (2011).

[8] N. Amjady, F. Keynia. "Day ahead price forecasting of electricity markets by a mixed data model and hybrid forecast method", International Journal of Electrical Power \& Energy Systems, 30(9), pp. 533-546, (2008).

[9] R. H. Byrne, C. A. Silva-Monroy. "Estimating the Maximum Potential Revenue for Grid Connected Electricity Storage: Arbitrage and Regulation", Sandia National Laboratories, Study for the DOE Energy Storage Systems Programme, (2012).

[10] D. P. Bertsekas. "Dynamic programming and stochastic control." Mathematics in science and engineering, (1976).

[11] H. Ben-Ameur, M. Breton, P. L'Ecuyer, L. Karoui. "A dynamic programming approach for pricing options embedded in bonds", Journal of Economic Dynamics and Control, 31, pp. 2212-2233, (2007).

[12] C. Lui, H. Song, J. Lawarree, R. Dahlgren. "New methods for electric energy contract decision making." Electric Utility Deregulation and Restructuring and Power Technologies, International Conference Proceedings. pp. 125-129, (2000).

[13] D. P. Bertsekas. "Dynamic programming and optimal control Vol. 1. No. 2", Belmont, MA: Athena Scientific, (1995).

[14] ELEXON Portal, "Market Index Price and Volume", https://www.elexonportal.co.uk/article/view/188?cacheb ust=t52ukg8hiq, accessed 22/04/2014.

[15] UK Power Networks. "Smarter Network Storage", http://innovation.ukpowernetworks.co.uk/innovation/en/ 
Projects/tier-2-projects/Smarter-Network-Storage-

(SNS)/Project-Documents/Smarter-Network-StrorageBrochure-Oct+2013.pdf, accessed 22/04/2014.

[16] Electric Mountain, "Dinorwig Power Station", http://www.electricmountain.co.uk/en-GB/Dinorwig, accessed 22/04/2014.

[17] L. Hu, G. Taylor. "A Novel Hybrid Technique for ShortTerm Electricity Price Forecasting in UK Electricity Markets", Journal of International Council on Electrical Engineering, 4(2), pp. 114-120, (2014). 


\title{
STORAGE CHARACTERISTICS REWARDED BY ARBITRAGE AND THE IMPLICATIONS OF OFFSHORE WIND POWER
}

\author{
A. Dunbar, A.R. Wallace, G.P. Harrison \\ Institute for Energy Systems, School of Engineering, University of Edinburgh, Scotland \\ a.dunbar@ed.ac.uk
}

Keywords: Electricity prices; Wind power; Arbitrage; Large scale storage

\begin{abstract}
Grid connected electrical energy storage is expected to enable the integration of many varying output offshore wind farms into the electricity network. As more wind farms are deployed, wholesale electricity prices will change. Peak and off-peak times will be driven by wind power output in addition to demand cycles. This will change the way in which storage technologies will be operated. This paper investigates how different storage characteristics are rewarded and how this may change with a higher penetration of offshore wind generation. Results indicate that increased variable generation reduces opportunities for price arbitrage. Storage devices cycle less regularly as operation tends to be driven by substantial changes in wind power output, rather than daily demand patterns. As a result, slower discharge times are more favourable and revenue is more sensitive to rates of self-discharge. Furthermore, increased wind production leads to less variation in wholesale electricity prices and consequently conversion efficiency is more critical to performance.
\end{abstract}

\section{Introduction}

Electrical energy storage is a potential solution to the challenge of the Energy Trilemma in facilitating the integration of intermittent renewable energy into the electricity network. Many benefits of storage have been identified including improved system control, reduced network congestion and avoided curtailment of renewable output [1]. In the coming decades deployment of offshore wind power is expected to increase significantly leading to a greater requirement for flexibility and a higher value to be placed on these benefits.

There are expected to be bespoke applications in islanded or heavily constrained networks where business cases for commercial scale storage would exist [2]. Storing renewable electricity until it could be consumed locally would be a more attractive option than upgrading transmission or distribution network connections in these cases. However, many offshore renewable energy projects will not have the benefit of local demand consumers. These projects will be connected to the network and will generate electricity into a centralised energy system.

Grid connected electrical energy storage will be required to aggregate revenue streams from a range of markets if it is to be commercially viable. One recognised revenue stream is price arbitrage - purchasing electricity when it is cheap and selling it back to electricity suppliers during periods of peak demand when the price is high. There is a range of storage technologies available with different combinations of characteristics which will be rewarded differently by price arbitrage.

In the future, as large numbers of intermittent offshore wind farms are deployed, wholesale electricity prices will change. Peak and off-peak times will be driven by wind power output in addition to demand cycles. This will change the way in which storage devices will be operated. This paper investigates how different storage characteristics are rewarded by price arbitrage and how this may change in the future. Understanding the changing market and financial drivers for energy storage will help to guide technology developers and provide evidence to de-risk investment. If the characteristics of storage which are promoted through the market are not those which provide the most value to the energy system then alternative support mechanisms may be required.

\section{Method}

\subsection{Storage revenue}

The linear optimisation method described by Byrne and SilvaMonroy [3] was used to determine the maximum revenue available to a storage operator through arbitrage. The decision variables were how much electricity to buy, $q_{t}^{C}(\mathrm{MWh})$, and sell, $q_{t}^{D}(\mathrm{MWh})$, during each time period, $t$. The state of charge of the storage device, $S_{t}(\mathrm{MWh})$, was defined by equation (1) and subject to the technical constraints of the device given in equations (2), (3) and (4):

$$
\begin{aligned}
& S_{t}=\eta_{s} S_{t-1}+\eta_{c} q_{t}^{C}-q_{t}^{D} \\
& 0 \leq S_{t} \leq S_{\max } \\
& 0 \leq q_{t}^{C} \leq q_{\max }^{C}
\end{aligned}
$$




$$
0 \leq q_{t}^{D} \leq q_{\max }^{D}
$$

where $\eta_{s}$ is the storage efficiency $(\%), \eta_{c}$ is conversion efficiency (\%), $S_{\max }$ is maximum storage capacity (MWh), $q_{\max }^{C}$ and $q_{\max }^{D}(\mathrm{MWh})$ are maximum quantities of energy which can be charged or discharged in a single time period. These are a function of the maximum charging and discharging rates, $Q_{C}$ and $Q_{D}(\mathrm{MW})$. The objective function of the optimisation was to maximise annual revenue, $R$, which is the sum of the price, $P_{t}$, multiplied by the net quantity sold during each settlement period. This is defined in equation (5).

$$
R=\sum P_{t}\left(q_{t}^{D}-q_{t}^{C}\right)
$$

The revenue was optimised on a weekly basis with the additional constraint that the state of charge was zero at the start and end of each week. This had minimal impact on total revenue compared to optimisation on a daily, monthly or annual basis, but reduced the computational time. The following assumptions were applied:

- The storage operator had perfect foresight of electricity prices for the week ahead.

- $\quad$ The storage device had $100 \%$ availability.

- The storage capacity was small compared to total electricity demand i.e. it was a price taker and did not affect the market price of electricity.

- Great Britain (GB) was represented as a single bus system and storage was not subject to network constraints.

- The device characteristics were constant over its life time.

- The conversion efficiency was modelled during charging only. The discharge cycle was $100 \%$ efficient.

- The ramp rate was negligible compared to the time period.

- The cost of charging and discharging (in addition to the cost of electricity) was negligible.

- The discount rate was negligible over the time period considered.

\subsection{Electricity prices}

A time series of electricity prices was required as an input for the arbitrage model. For historic years Market Index Prices from the UK power exchange [4] could be used. Although only a small volume of electricity is traded on the power exchange, prices are published and so the Market Index Price is expected to influence electricity prices in forward markets [5]. Furthermore, as a market of last resort, the power exchange exhibits high price volatility and is likely to be the platform where storage operators would trade for arbitrage sales.

A model was created to simulate electricity prices for future energy scenarios with a high penetration of time varying offshore wind power. A fundamental price-based market model, established on the assumption of perfect competition was used. This approach has been shown to be representative of the power exchange in GB and is commonly used for modelling scenarios of future energy prices [6].

A generator supply function was formed by stacking thermal plant in merit order of increasing marginal cost. Wind power output was deducted from electricity demand to produce a 'net demand' curve. The price of electricity for each half hour time period was determined by the market clearing price. An exponential uplift in price was applied for peaking generators to represent scarcity of supply. An example of the aggregate supply curve is shown in Figure 1. Similar approaches have been used by Grünewald [7] and Eager [8].

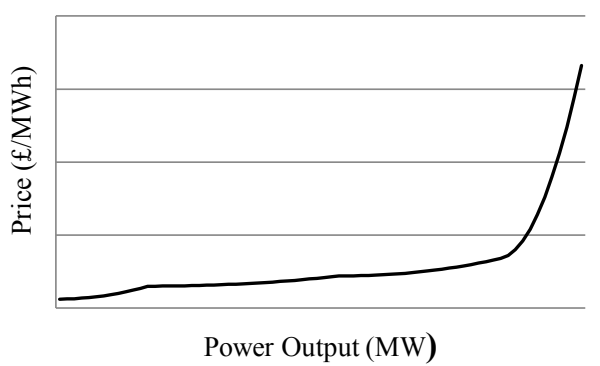

Figure 1: Example electricity supply curve.

\subsubsection{Wind power output}

Wind power was assumed to have zero marginal cost and was always dispatched when available. With GB modelled as a single bus transmission system, network constraints were neglected and it was assumed that wind output was not curtailed unless demand was fully satisfied.

Hourly wind speed data for the United Kingdom and surrounding waters was produced by Hawkins [9] using the Weather Research and Forecasting model for the years 20012010. Wind speeds were available at a spatial resolution of $3 \mathrm{~km}$ by $3 \mathrm{~km}$ and were extracted at $80 \mathrm{~m}$ above ground or sea level. The DECC RESTATS planning database [10] was used to identify the location and capacity of wind farms in GB. The time series of wind speed data corresponding to each farm location was extracted from the wind model. Power output from each wind farm was calculated using the equivalent aggregate power curve described by equation (6) [9].

$$
P=\frac{1}{1+e^{-\frac{U-9.7}{1.8}}}
$$

where $P$ is the power output per unit capacity and $U$ is the wind speed $(\mathrm{m} / \mathrm{s})$. Power output from all wind farms was summed to give the aggregate output for both onshore and offshore wind and reduced by $10 \%$ to account for availability. This was a conservative estimate for onshore but early offshore availability may be less than $90 \%$ [11]. The data was interpolated linearly to obtain a time series of power output at half-hourly intervals. 
Wind capacity was scaled at existing sites to represent scenarios with different penetrations of wind power. This assumed that the spatial distribution in the future would be the same as it is currently. Onshore and offshore capacities were scaled as separate regions to ensure that variations between resource were captured.

For future energy scenarios, other variables would be expected to change including thermal generation capacity, fuel prices and demand patterns. However, these were kept constant in this study to investigate the isolated effect of varying installed wind capacity on arbitrage revenue.

\subsection{Sensitivity study}

Initially, the installed wind capacity was varied and revenue calculated using fixed storage characteristics. Subsequently, to examine the impacts of storage technology and capability in the context of high and low wind scenarios, each of the following factors was then varied:
1. storage capacity
2. charging/discharging rate
3. conversion efficiency
4. storage efficiency.

The revenue was calculated using simulated electricity prices with (a) no installed wind and (b) 40GW installed wind:

\section{Results and discussion}

\subsection{Installed wind capacity}

The arbitrage algorithm was run for simulated electricity prices with increasing installed wind capacity from zero to $40 \mathrm{GW}$. The ratio of offshore to onshore capacity was fixed at $3: 2$. The remaining inputs of fuel prices [12], carbon prices [13], thermal generation capacity [14] and demand profiles [15] were taken from historic data from 2006. 40GW of installed wind represents $35 \%$ of the total generation capacity in GB under these assumptions. The storage characteristics used are listed in Table 1. The results are shown in Figure 2.

\begin{tabular}{|c|c|c|c|}
\hline $\begin{array}{c}\text { Maximum } \\
\text { storage } \\
\text { capacity } \\
S_{\max } \\
(M W h)\end{array}$ & $\begin{array}{c}\text { Maximum } \\
\text { charging/ } \\
\text { discharging } \\
\text { rate } Q_{C} / Q_{D} \\
(M W)\end{array}$ & $\begin{array}{c}\text { Conversion } \\
\text { efficiency } \\
\eta_{c}(\%)\end{array}$ & $\begin{array}{c}\text { Storage } \\
\text { efficiency } \\
\eta_{s}(\%)\end{array}$ \\
\hline 200 & 20 & 75 & 100 \\
\hline
\end{tabular}

Table 1: Storage characteristics.

The results show that the revenue reduced as the wind capacity increased. This was due to the reduced variation in electricity price with increased wind power output. Figure 3 shows the wind power output for the first two weeks in February with $40 \mathrm{GW}$ of installed wind generation capacity. Figure 4 shows the electricity prices for the same period for scenarios with zero installed wind and with $40 \mathrm{GW}$ of installed wind capacity.

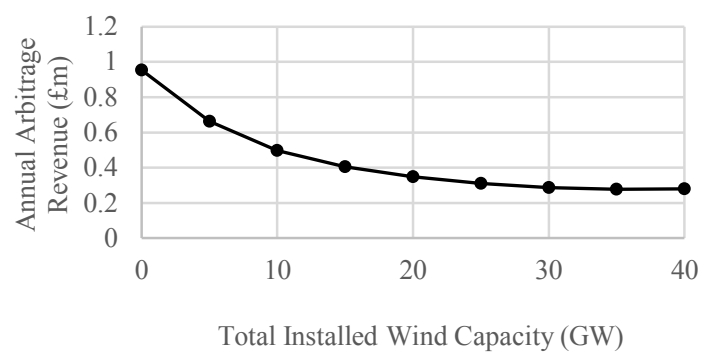

Figure 2: Annual revenue using simulated electricity prices with increasing penetration of installed wind capacity.

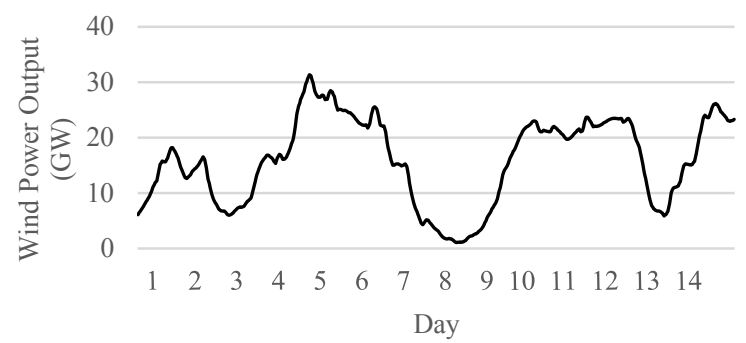

Figure 3: Wind power output with $40 \mathrm{GW}$ installed capacity for first two weeks in February.

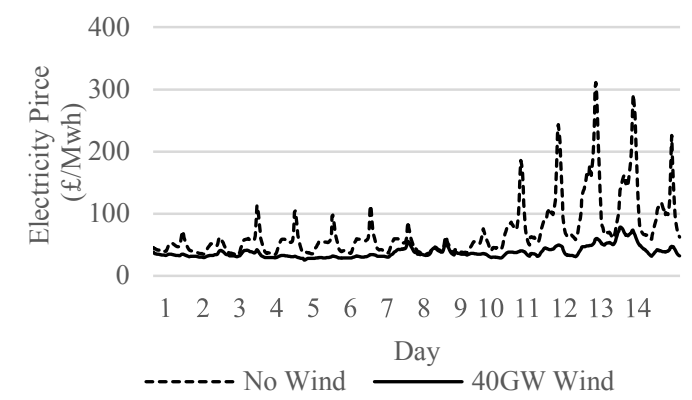

Figure 4: Electricity price with no wind and $40 \mathrm{GW}$ of wind capacity for first two weeks in February.

Prices were similar for both scenarios between days 8 and 9 when the wind power output was nearest to zero. With 40GW of installed wind, during periods of high wind power output peak prices were significantly reduced, which led generally to lower price variation. This is a result of the shape of the supply curve, shown in Figure 1. The curve was steep during periods of high demand, but shallow during periods of low demand; indicating higher price elasticity of supply when electricity was scarce. The increased wind output with $40 \mathrm{GW}$ of capacity reduced scarcity of supply, leading to reduced price elasticity. This led to not only reduced average prices, but also reduced variation in price, despite increased variation in thermal output, decreasing arbitrage revenue.

Figure 5 shows the state of charge of the storage device for the same two weeks. With no installed wind capacity the device charged and discharged once a day in line with the variation in electricity price which was driven by demand patterns. The storage device did not reach its maximum storage capacity or fully discharge on every cycle. With $40 \mathrm{GW}$ of installed wind the device charged and discharged less frequently with only 
four distinct cycles over the two week period. This is similar to the four distinct cycles of wind power output shown in Figure 3 and is in line with the frequency of synoptic weather patterns that dominate UK climate. Additionally with $40 \mathrm{GW}$ of installed wind capacity, the storage device was limited for longer periods of time by its maximum capacity of $200 \mathrm{MWh}$.

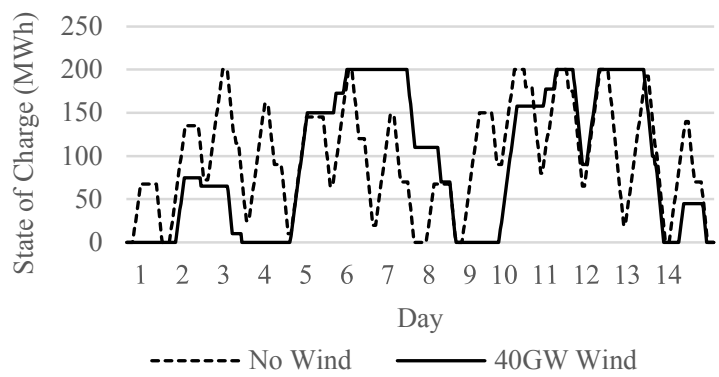

Figure 5: Storage state of charge for first two weeks in

February with no wind and $40 \mathrm{GW}$ installed wind capacity.

The change in the optimum operating schedule for the storage device suggests that storage characteristics may be valued differently in energy systems with a high penetration of intermittent renewables.

\subsection{Storage capacity and charging/discharging rate}

Figure 6(a) shows the change in revenue with increasing storage capacity for scenarios with $40 \mathrm{GW}$ of wind and no wind. The remaining storage characteristics were fixed at the values given in Table 1 . Figure $6(\mathrm{~b})$ shows the same results normalised relative to the revenue from a storage device with $600 \mathrm{MWh}$ storage capacity to show the relative sensitivity in each scenario.

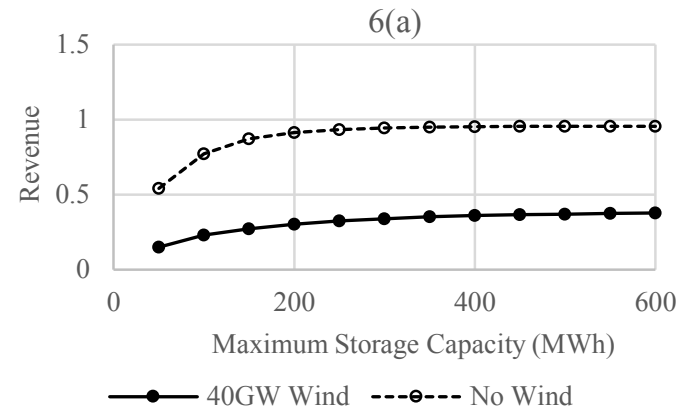

6(b)

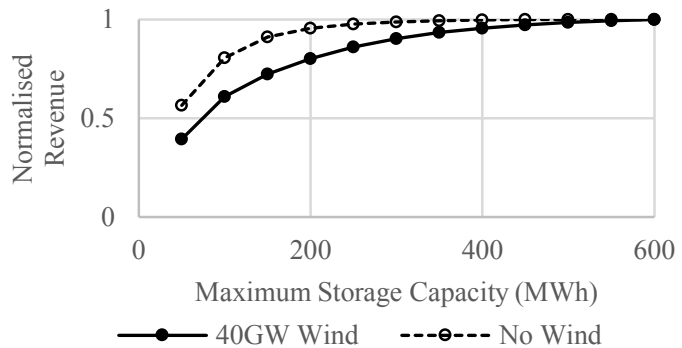

Figure 6(a): Annual revenue with varying storage capacity (b): Normalised relative to 600MWh storage capacity for each scenario.
Figure 7(a) shows the change in revenue with increasing charging/discharging rate for scenarios with $40 \mathrm{GW}$ of wind and no wind. The remaining storage characteristics were fixed at the values given in Table 1. Figure 7(b) shows the same results normalised relative to the revenue from a storage device with $200 \mathrm{MW}$ charging/discharging rate to show the relative sensitivity in each scenario.

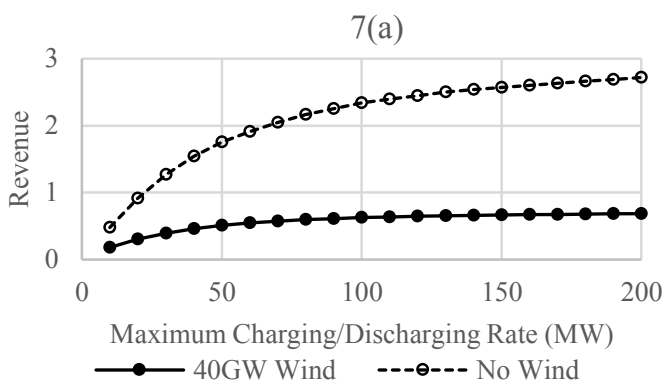

$7(\mathrm{~b})$

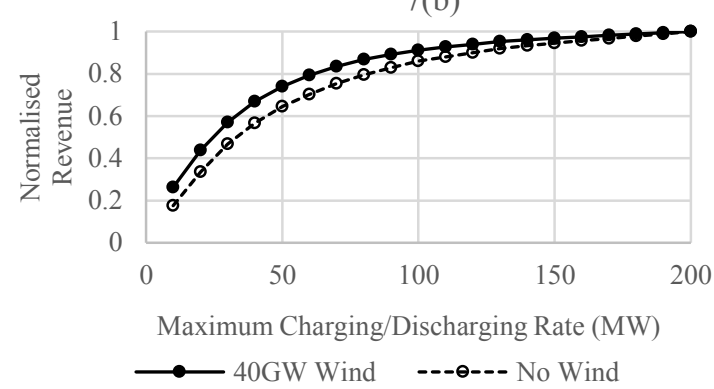

Figure 7(a): Annual revenue with varying charing rate (b): Normalised relative to $200 \mathrm{MW}$ storage capacity for each scenario

Figure 6 and Figure 7 show that, as expected, the increase in revenue from increasing storage capacity and charging/discharging rates decreased as the capacity and charging/discharging rates increased for both scenarios. With no wind, a lower storage capacity returned the maximum normalised revenue, but higher charging/discharging rates were required than with $40 \mathrm{GW}$ of wind.

With no wind devices performed best by charging and discharging on a daily basis and were rarely restricted by storage capacity. In this scenario increasing the charging/discharging rate was more beneficial than increasing capacity. With $40 \mathrm{GW}$ of wind devices performed best by charging, discharging and storing energy over longer periods of time, causing storage capacity to be more restrictive on revenue than charging/discharging rate. In this scenario increasing capacity was more beneficial than increasing the power rating.

\subsection{Efficiency}

Conversion efficiency is a measure of the roundtrip efficiency of a storage device. Examples of conversion losses include those associated with the turbines in a pumped hydro plant. Storage efficiency is a measure of the device self-discharge and is a function the length of time that the energy is stored. Examples of storage losses include the evaporation of fluid 
from a pumped storage reservoir or loss of charge from a battery. For the purpose of this study storage efficiency was defined as the percentage of energy retained in the store at the end of each half hour time period relative to that at the beginning. For example, a storage efficiency of $99 \%$ corresponded to a $1 \%$ loss of energy in a half hour period or a $38 \%$ loss in 24 hours. Conversion efficiency and storage efficiency were varied independently to investigate their impact on revenue.

Figure 8 (a) shows the change in revenue with $100 \%$ storage efficiency and increasing conversion efficiency for scenarios with no wind and $40 \mathrm{GW}$ of wind. The maximum storage capacity and charging/discharging rates were fixed at $200 \mathrm{MWh}$ and 20MW respectively. Figure $8(\mathrm{~b})$ shows the results normalised relative to a device with $100 \%$ conversion efficiency for each scenario. This demonstrates the relative importance of conversion efficiency in each scenario.
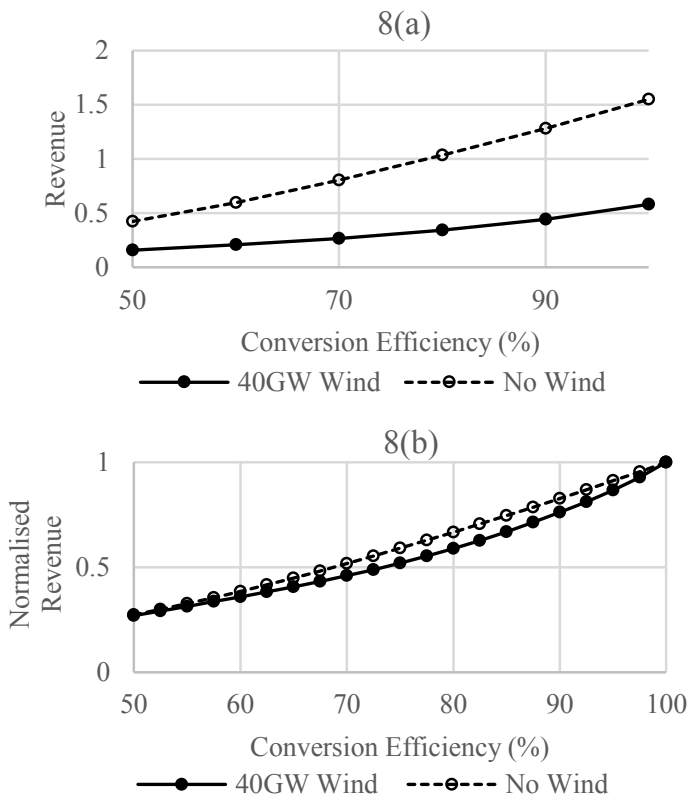

Figure 8(a): Annual revenue with varying conversion efficiency (b) Normalised relative to $100 \%$ conversion efficiency for each scenario.

Figure 8(a) shows that the revenue increased more rapidly with increasing conversion efficiency in the scenario with zero wind than with $40 \mathrm{GW}$ of wind. However, Figure 8(b) shows that for conversion efficiencies greater than around $80 \%$, the increase in normalised revenue was greater with $40 \mathrm{GW}$ of wind than with no wind, indicating that the presence of a large penetration of wind power increased the dependence of revenue on high conversion efficiencies. Previous studies have reported that high penetrations of wind power may lead to lower electricity prices, less value being placed on energy and conversion efficiency becoming less important [16]. However, the higher output of wind power not only reduced average electricity prices but also reduced the variation between wholesale price peaks and troughs. As a result, conversion processes needed to be more efficient to return the same revenue.
Figure 9(a) shows the change in revenue with $100 \%$ conversion efficiency and increasing storage efficiency for scenarios with no wind and $40 \mathrm{GW}$ of wind. The maximum storage capacity and charging/discharging rates were fixed at $200 \mathrm{MWh}$ and 20MW respectively. Figure 9(b) shows the results normalised relative to a device with $100 \%$ storage efficiency for each scenario.
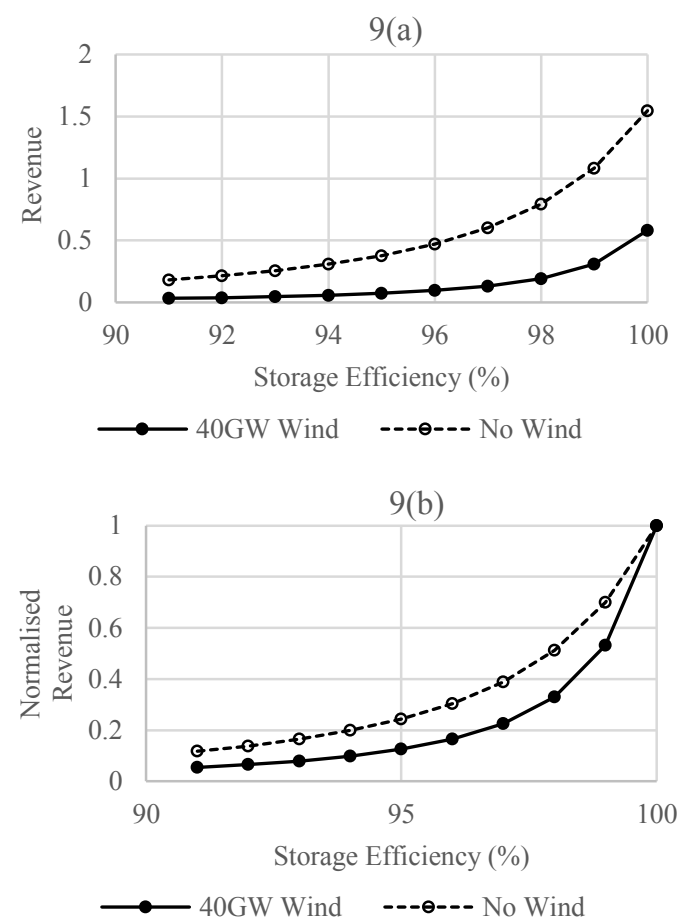

Figure 9(a): Annual revenue with varying storage efficiency (b): Normalised relative to $100 \%$ storage efficiency for each scenario.

As expected, for both scenarios, high storage efficiency was critical to performance. The higher potential revenue from the scenario with zero wind power led to the more apparent increase in revenue with increasing storage efficiency than with 40GW of wind seen in Figure 9(a). However, Figure 9(b) shows that the scenario with $40 \mathrm{GW}$ of wind, has a much more pronounced increase in revenue for the highest storage efficiencies. With lower average electricity prices, revenue should be less dependent on storage efficiency as the losses would be less costly than if average prices were higher [17]. However, with $40 \mathrm{GW}$ of wind, electricity prices cycled over longer durations and energy was stored over longer periods. As a result, revenue was relatively more sensitive to storage efficiency.

\section{Conclusions}

Grid connected electrical energy storage is expected to enable the integration of large amounts of time varying offshore wind power. As more intermittent generation is connected to the network, electricity prices will change which will change the way in which storage is operated. This paper discusses the 
impact of increased offshore wind on price arbitrage opportunities and how different storage characteristics are rewarded.

Simulated electricity prices with increasing installed wind capacity were used in a linear optimisation programme to determine the maximum revenue available to a storage operator. The results show that revenue reduced as wind capacity increased. With $15 \mathrm{GW}$ of installed wind, revenue reduced by $60 \%$ compared to that with no installed wind capacity. This was due to changes in wholesale electricity prices with increased wind power output. The mean price and the price range were reduced lessening opportunities for arbitrage. Energy storage devices will need to rely more heavily on alternative revenue streams with increasing penetration of intermittent renewable power.

With higher wind penetration, wholesale prices became more strongly influenced by wind power output than by diurnal patterns of demand behaviour. This reduced the number of cycles carried out by the storage device and led to energy being stored for longer periods of time. As a result, revenue was more sensitive to storage capacity and storage efficiency, but less sensitive to charging/discharging rate. Revenue was also more dependent on conversion efficiency as the variation between wholesale electricity price peaks and troughs was reduced.

\section{References}

[1] A. Akhil, G. Huff, A. B. Currier, B. C. Kaun, D. M. Tastler, S. B. Chen, A. L. Cotter, D. Bradshaw and W. D. Gauntlett, "DOE/EPRI 2013 Electricity Storage Handbook in Collaboration with NRECA," Sandia National Laboratories, California, 2013.

[2] D. Mignard, "Estimating the capital costs of energy storage technologies for levelling the output of renewbale energy sources," International Journal of Environmental Studies, vol. 71, no. 6, pp. 796-803, 2014.

[3] R. H. Byrne and C. A. Silva-Monroy, "Estimating the Maximum Potential Revenue for Grid Connected Electricity Storage: Arbitrage and Regulation," Sandia National Laboratories, California, 2012.

[4] ELEXON, "ELEXON Price Portal," [Online]. Available: www.elexonportal.co.uk. [Accessed 26th June 2013].

[5] E. Barbour, G. I. A. Wilson, I. G. Bryden, P. G. McGregor, P. A. Mulheran and P. J. Hall, "Towards an objective method to compare energy storage technologies: development and validation of a model to determine the upper boundary of revenue available from electrical price arbitrage," Energy \& Environmental Science, vol. 5, pp. 5425-5436, 2012.
[6] R. Green and N. Vasilakos, "The long-term impact of wind power on electricity prices and generating capacity," in IEEE Power and Energy Society General Meeting, San Diego, 2011.

[7] P. Grunewald, "Electricity storage in future GB networks - a market failure?," in British Institute of Energy Economics 9th Conference, Oxford, 2012.

[8] D. Eager, B. F. Hobbs and J. W. Bialek, "Dynamic modelling of generation capacity investment in markets with high wind penetration," IEEE Transactions on Power Systems, vol. 27, no. 4, pp. 2127-2137, 2012.

[9] S. Hawkins, A High Resolution Reanalysis of Wind Speeds over the British Isles for Wind Energy Integration, University of Edinburgh: PhD Thesis, 2012 .

[10] DECC, "DECC Planning Database - Monthly exctract,"

https://restats.decc.gov.uk/app/reporting/decc/monthl yextract, 2014.

[11] K. Harman, R. Walker and M. Wilkinson, "Availability trends observed at operational wind farms," in European Wind Energy Conference, Brussels, 2008.

[12] ICE ENDEX, "ICE Download Centre," [Online]. Available: http://iceendex.com/. [Accessed 11th September 2014].

[13] European Environment Agency, "EUA Future Prices 2005-2011," [Online]. Available: http://www.eea.europa.eu/data-and-maps/figures/euafuture-prices-200520132011\#tab-documents. [Accessed 2nd September 2014].

[14] I. MacLeay, K. Harris and A. Annut, "Digest of United Kingdom Energy Statistics (DUKES)," Department of Energy and Climate Change, London, 2010.

[15] National Grid, "Metered half-hourly electricity demands," [Online]. Available: http://nationalgrid.com/uk/electricity/demand+data/. [Accessed 28th August 2013].

[16] P. Grünewald, T. Cockerill, M. Contestabile and P. Pearson, "Role of large scale storage in a GB low carbon energy future: Issues and policy challenges," Energy Policy, vol. 39, no. 9, pp. 4807-4815, 2011.

[17] R. Loisel, A. Mercier, C. Gatzen, N. Elms and H. Petric, "Valuation Framework for Large Scale Electricity Storage in a Case with Wind Curtailment," Energy Policy, vol. 38, pp. 7323-7337, 2010. 


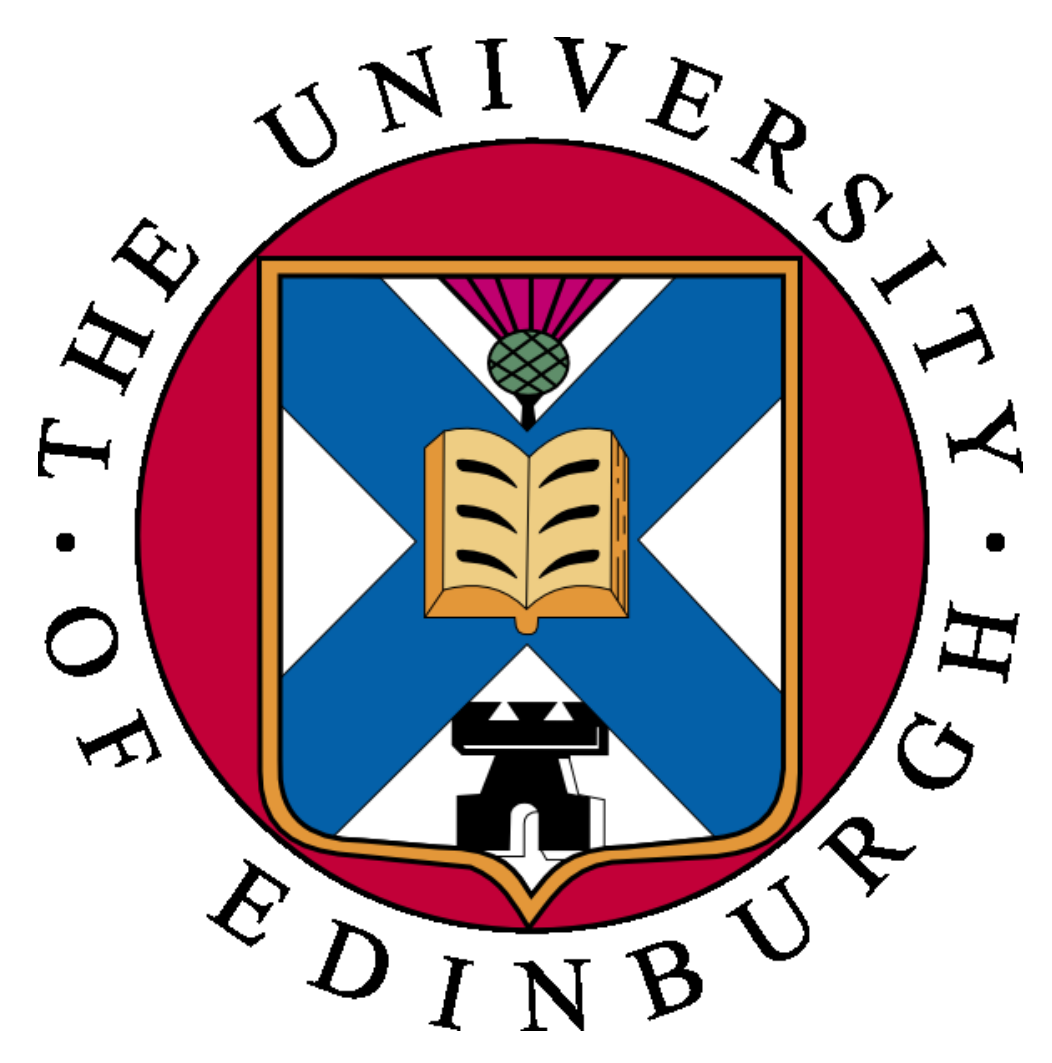

\section{The Value of Electrical Energy Storage}

A comparison of commercial and system level benefits

\section{Anna McDowell a.mcdowell@ed.ac.uk Supervisor: Gareth Harrison}

\section{MOTIVATION}

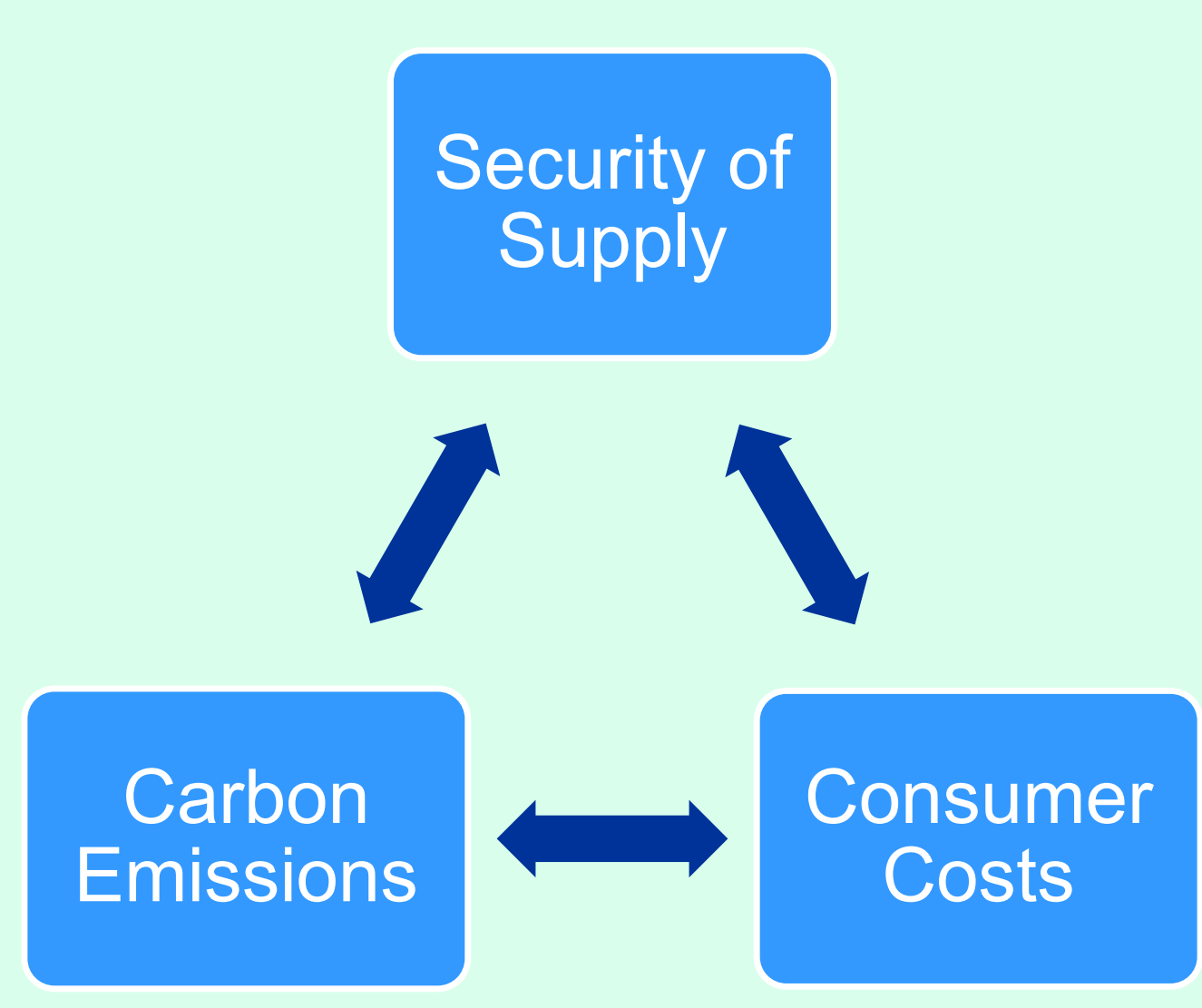

The Climate Change Act, 2008, initiated a drive to transform the electricity industry. Large numbers of intermittent renewable generators and less flexible nuclear power plants are expected to be deployed in the coming decades. The integration of these technologies, whilst maintaining security of supply, is expected to increase costs. This challenge has been dubbed the Energy Trilema.

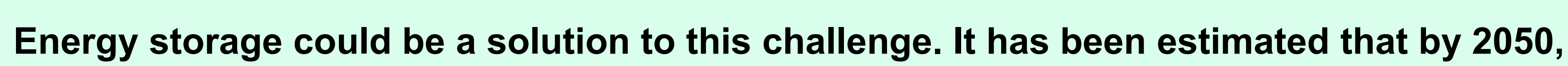
grid scale electrical energy storage could provide savings of over £10bn/year to the British electricity system.

\section{MARKET ISSUES}

Electrical energy storage provides value to a number of stakeholders throughout the electricity industry. Benefits are accrued between regulated and deregulated markets. This leads to complex contractual arrangements and provides little incentive for any individual player to invest in storage technology.

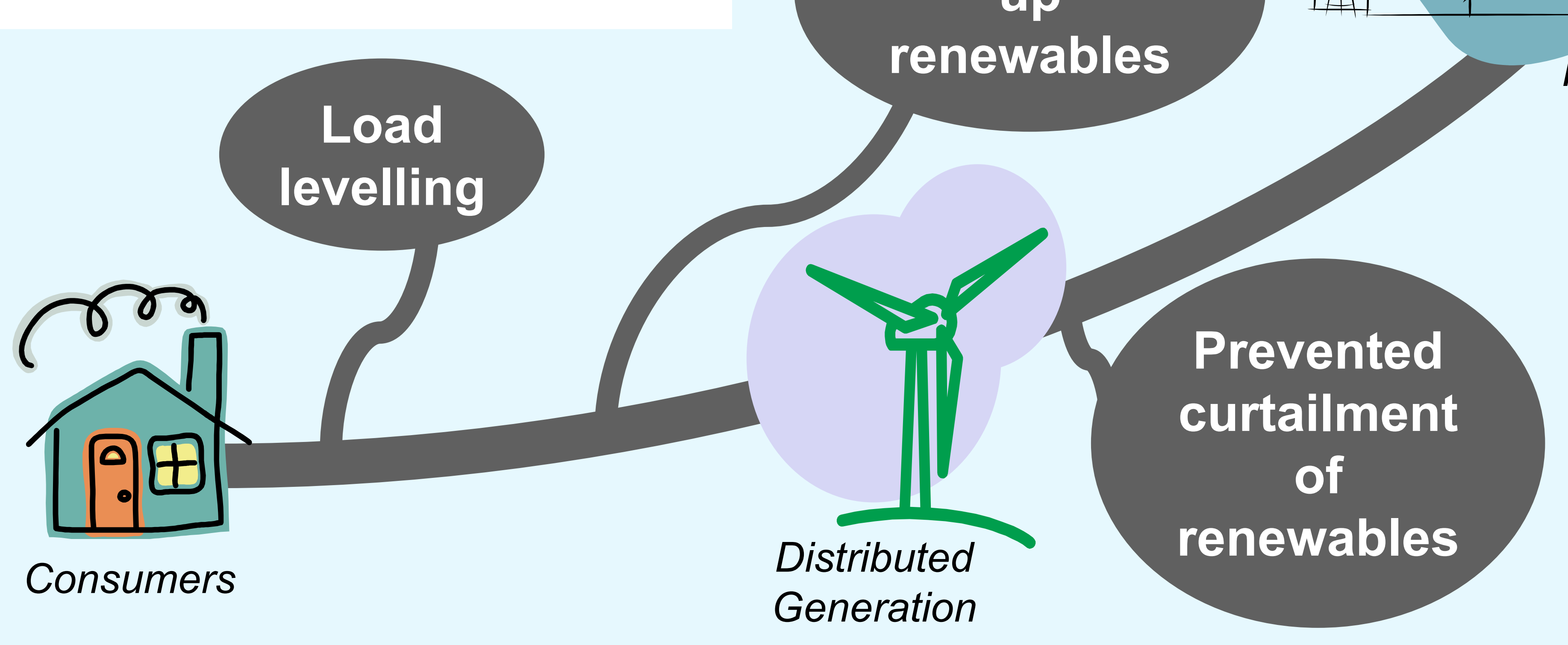

\section{PROGRESS TO DATE}

A linear optimisation programme is used to determine the optimum storage strategy and maximise revenue using historic electricity prices.

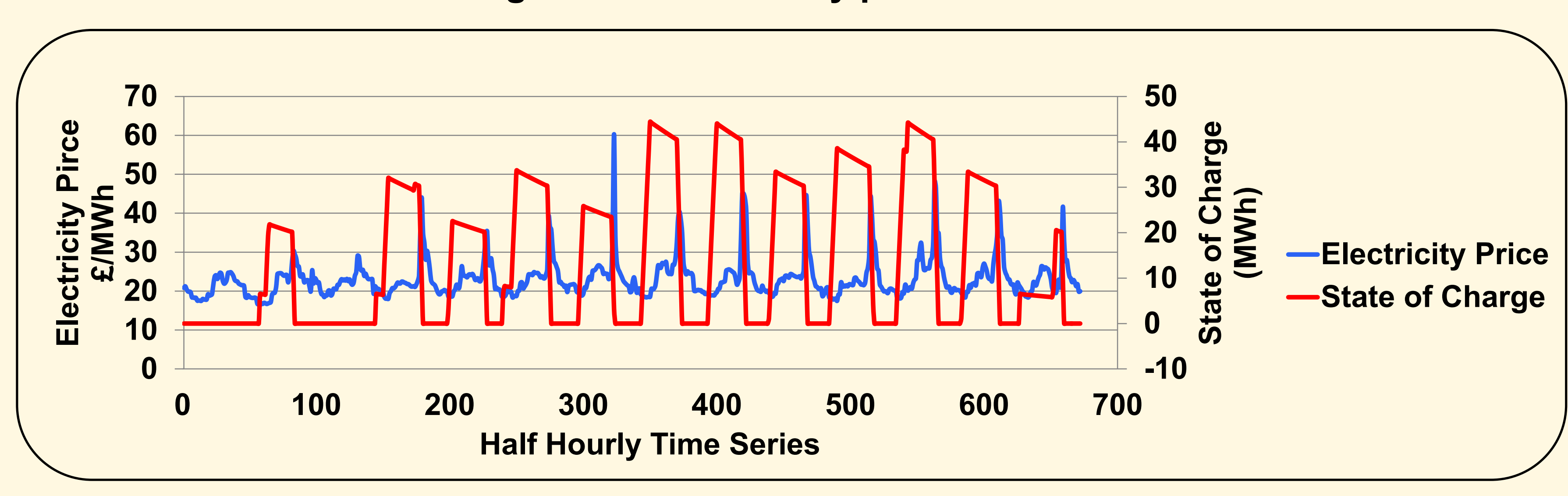

Storage operation strategy for 20MWh, 80MWh lithium ion battery for first two weeks of 2005.

Expected costs are defined as distributed variables and Monte Carlo techniques used to determine expected NPV and investment risk. Preliminary results show:

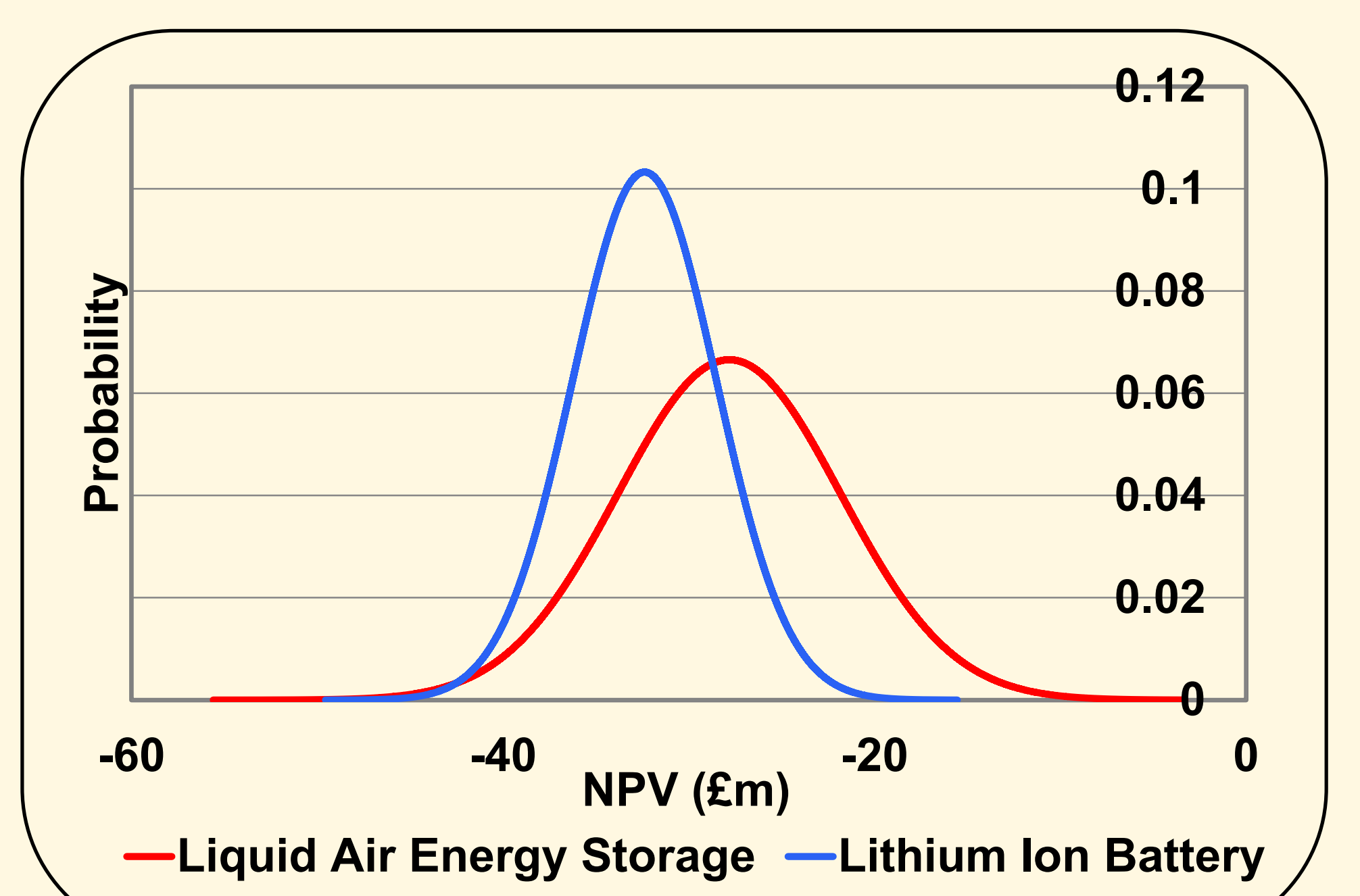

1. Large scale storage is not commercially viable if arbitrage is a sole revenue stream.

2. Technology cost uncertainty causes a wide distribution of expected returns. Uncertainty associated with electricity prices is not yet modelled.

Expected NPV for 20MW, 80MWh storage technologies. Based on 2008 electricity prices.

\section{SYSTEM BENEFITS}

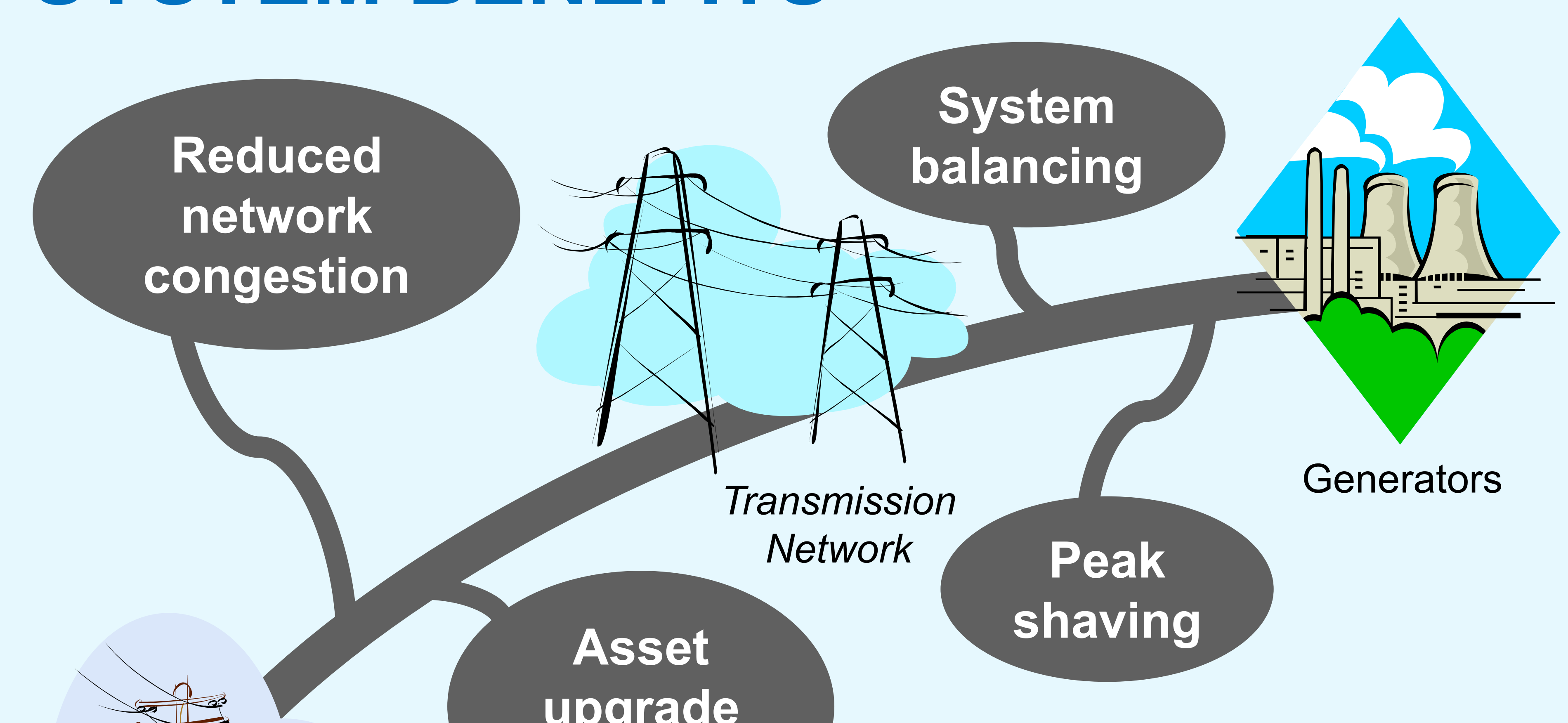

MODELLING

Distribution Network

upgrade

deferral

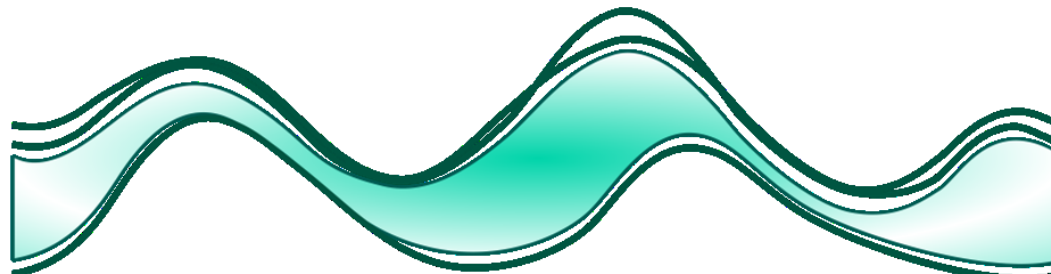


The implications of wind power on price arbitrage opportunities for electrical energy storage

\section{"Could wind power be detrimental to storage?"}

Anna Dunbar; a.dunbar@ed.ac.uk

Supervisor: Gareth Harrison

School of Engineering, The University of Edinburgh, Edinburgh EH9 3JL, UK

\section{MOTIVATION}
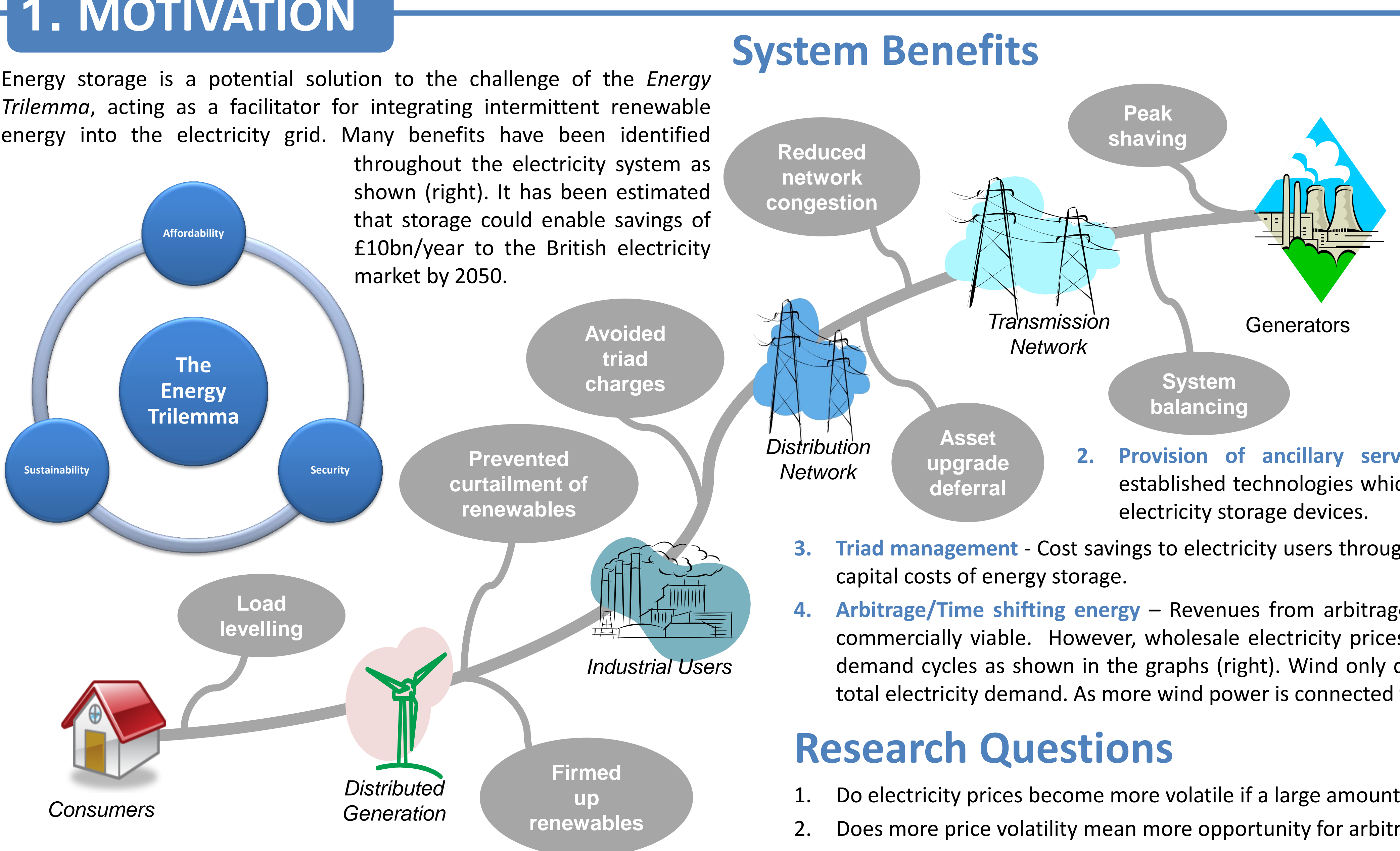

\section{Commercial}

Opportunities

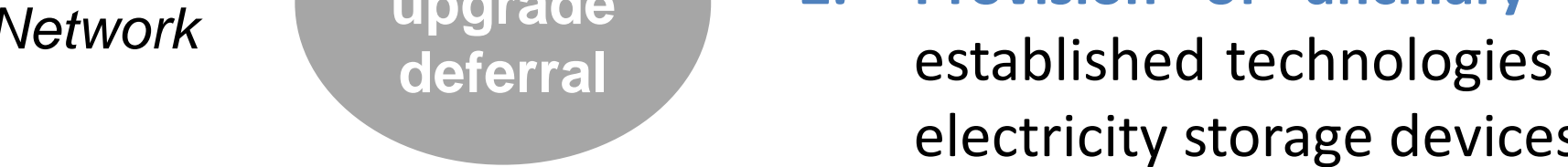

Despite the number of system benefits that

have been identified, commercia

1. Transmission and distribution upgrade

deferral - Ownership models for the

complex. Furthermore, companies face high

penalties for poor reliability and so are risk

averse, favouring established technologies over novel energy storage.

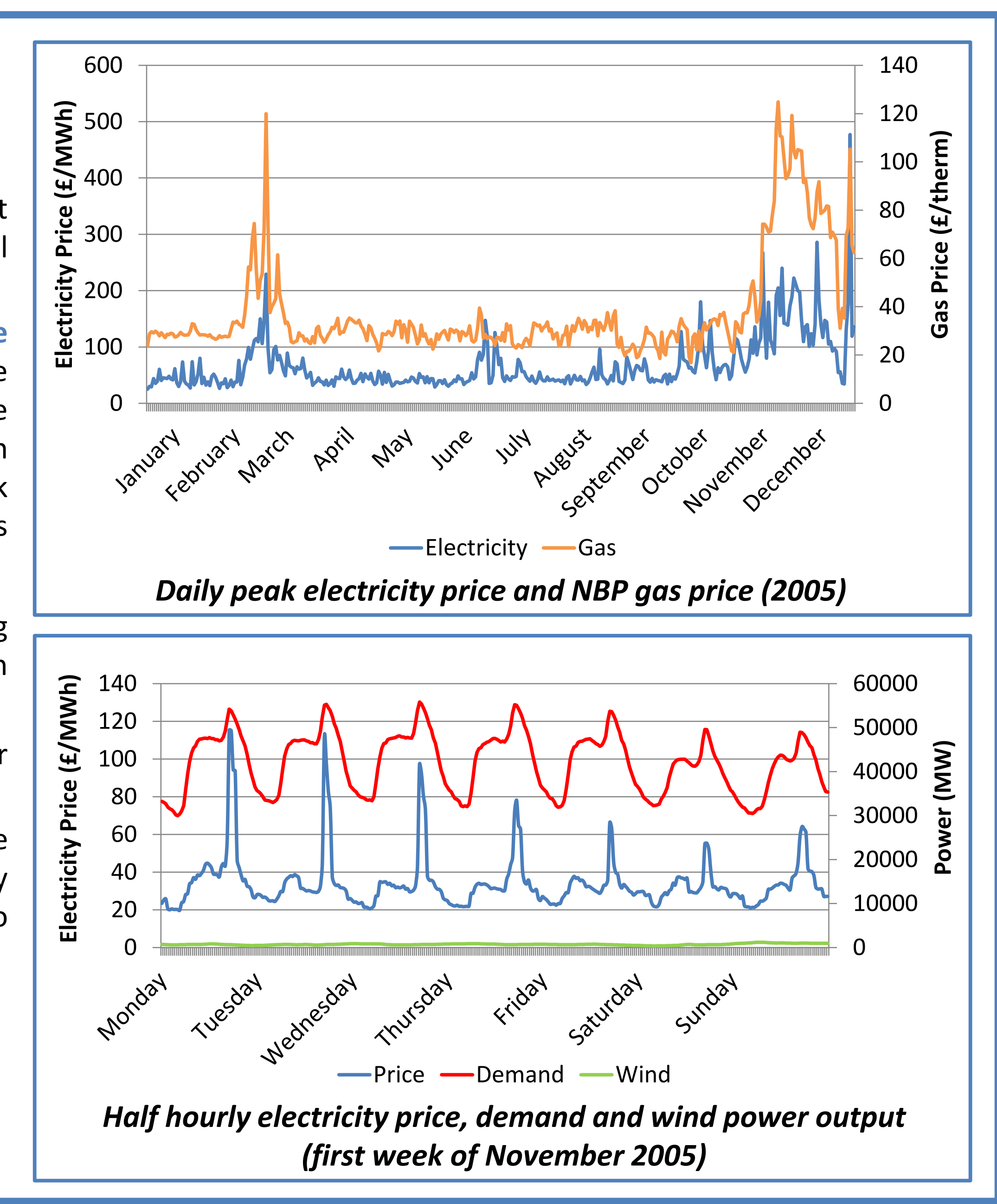
capital costs of energy storage.

Arbitrage/Time shifting energy - Revenues from arbitrage are not currently sufficient to make storage demand cycles as shown in the graphs (right). Wind only delivers a small amount of power comp otal electricity demand. As more wind power is connected to the system, arbitrage may become viable.

\section{Research Questions}

Do electricity prices become more volatile if a large amount of wind power is connected to the grid? . Does more price volatility mean more opportunity for arbitrage for electricity storage? (first week of November 2005)

\section{MODEL}

\section{Overview}

A fundamental price model has been developed and validated Historic demand time series are transformed to using historic electricity prices. Thermal generators are maintain historic patterns of behaviour. model. Base load and mid-merit generators are assumed to bid to produce electricity at a price between their marginal Wind

generation cost and the cost of the next cheapest generator. Installed wind capacity is scaled up at existing sites An exponential uplift function is applied to the prices bid by in the UK to represent the spatial distribution of peaking plant to reflect scarcity of supply. The mode
applied to the National Grid Future Energy Scenarios.

National Grid Future Energy Scenarios
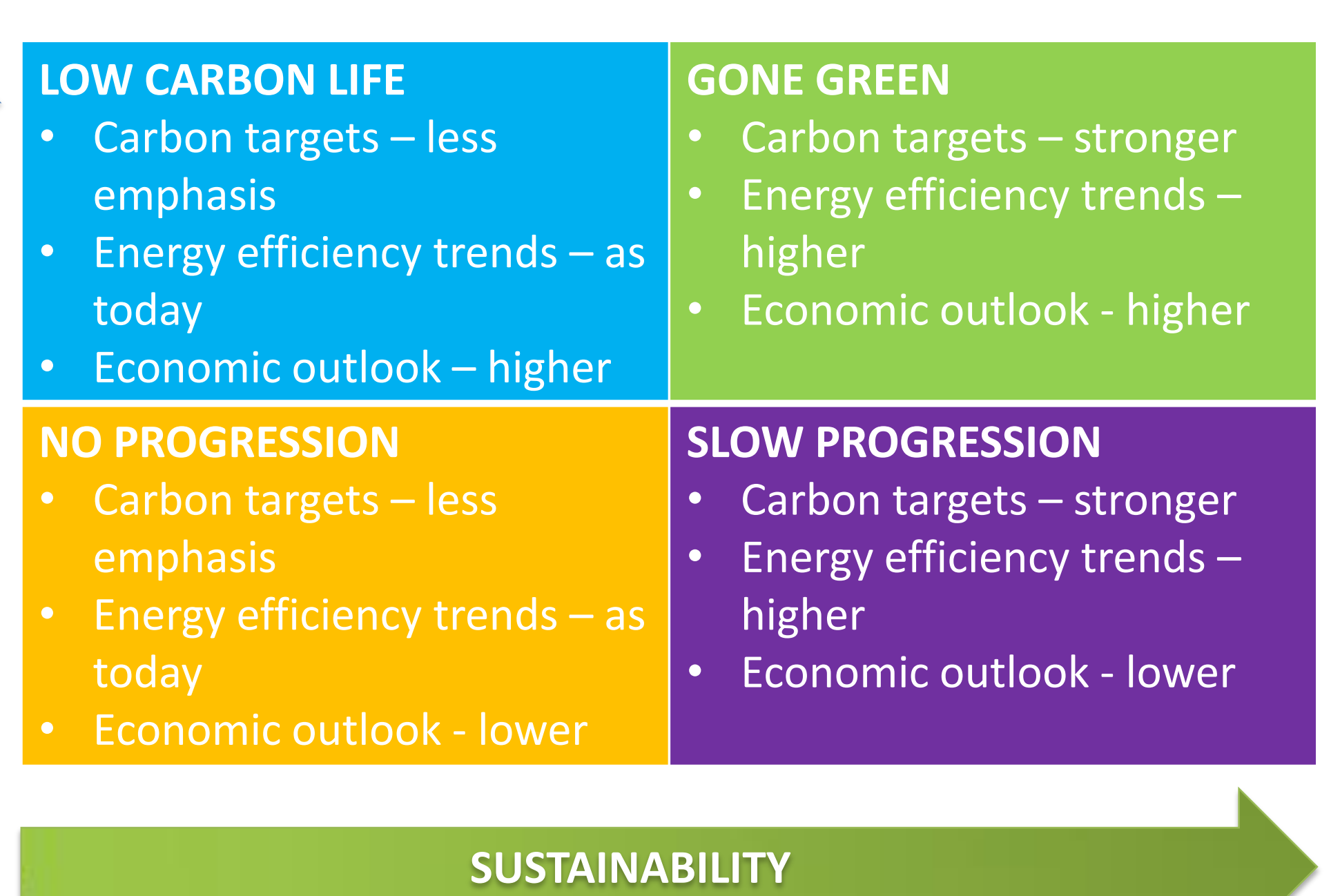

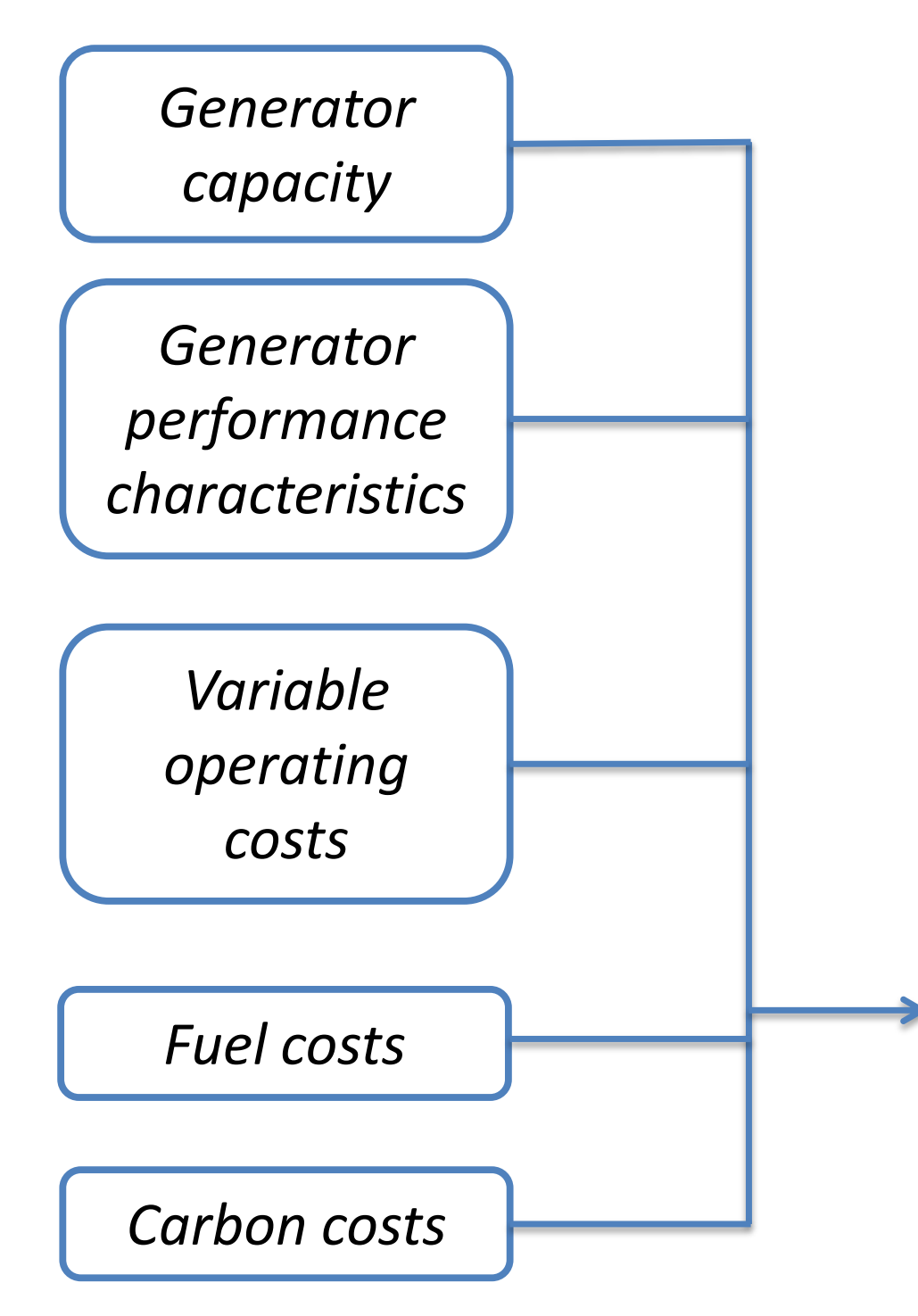

Carbon costs
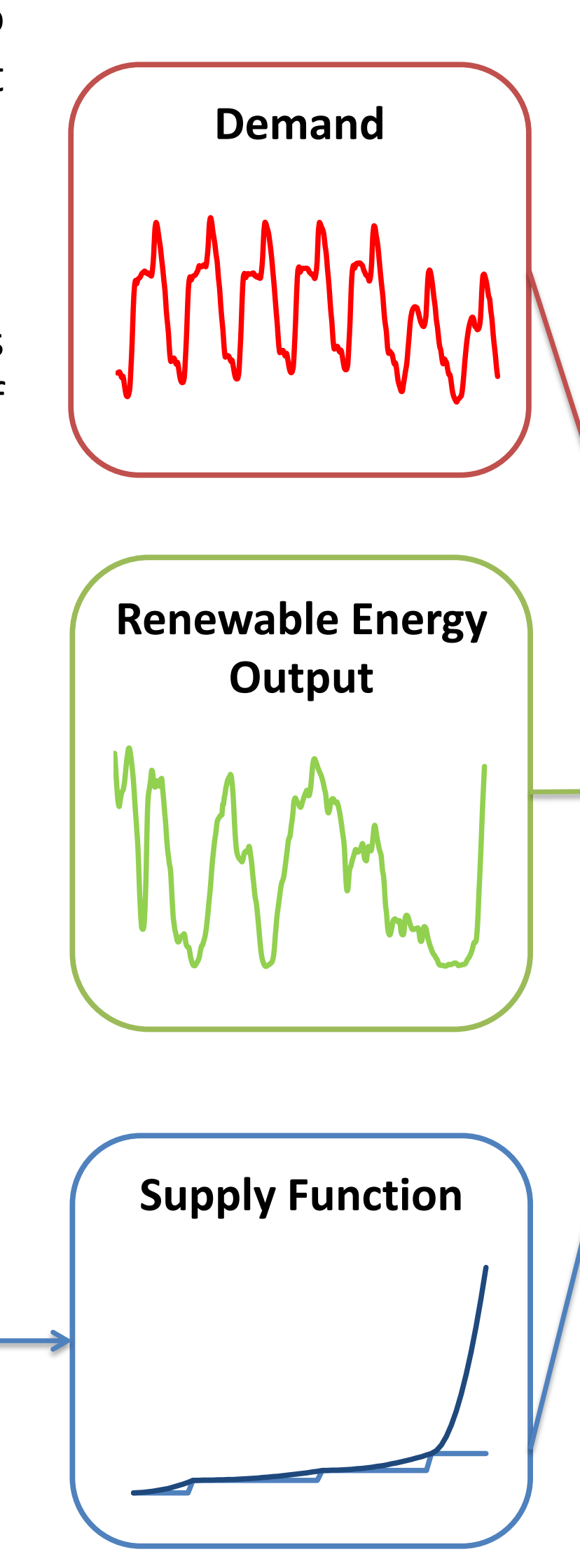

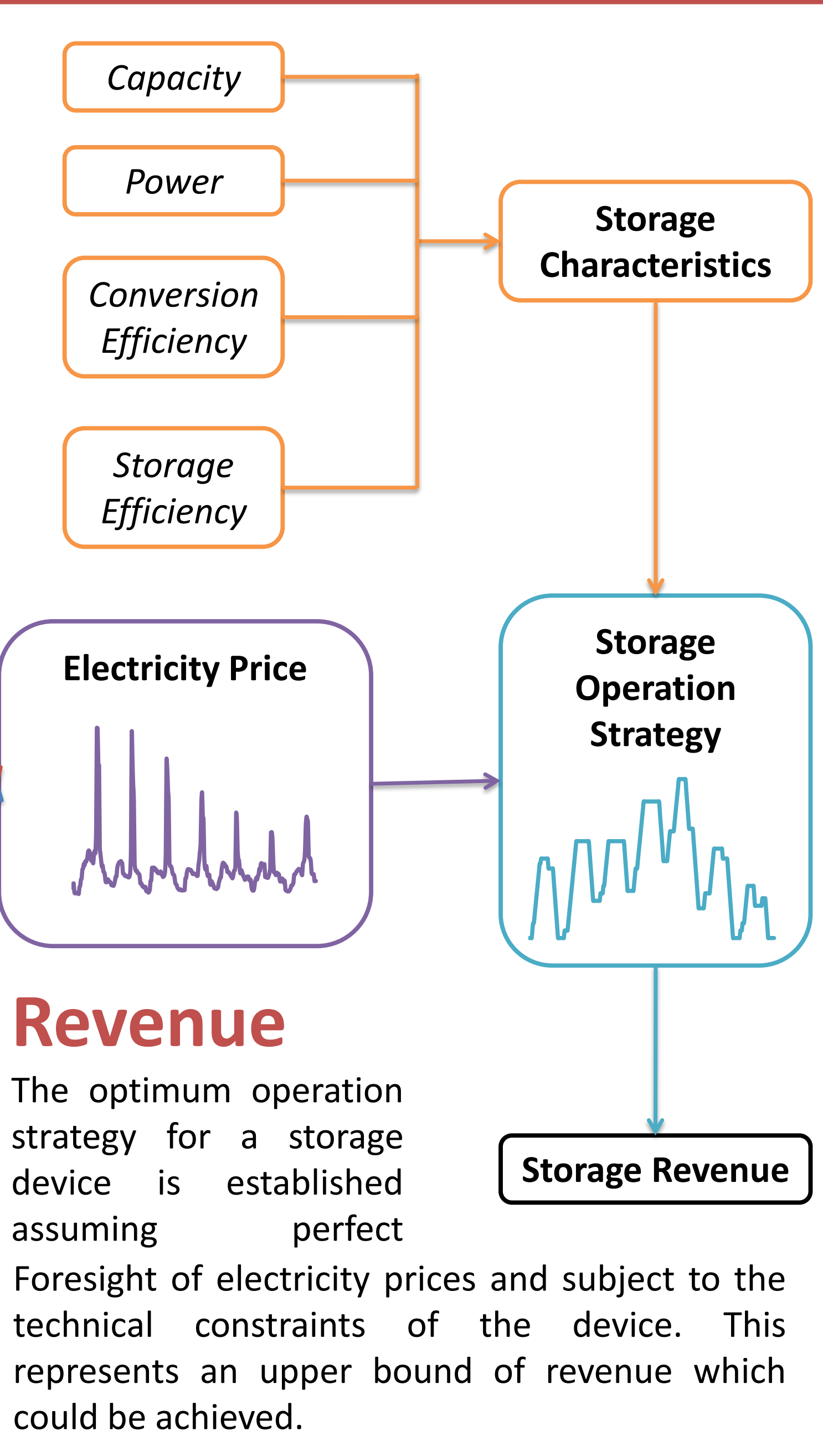

Validation of Electricity Prices

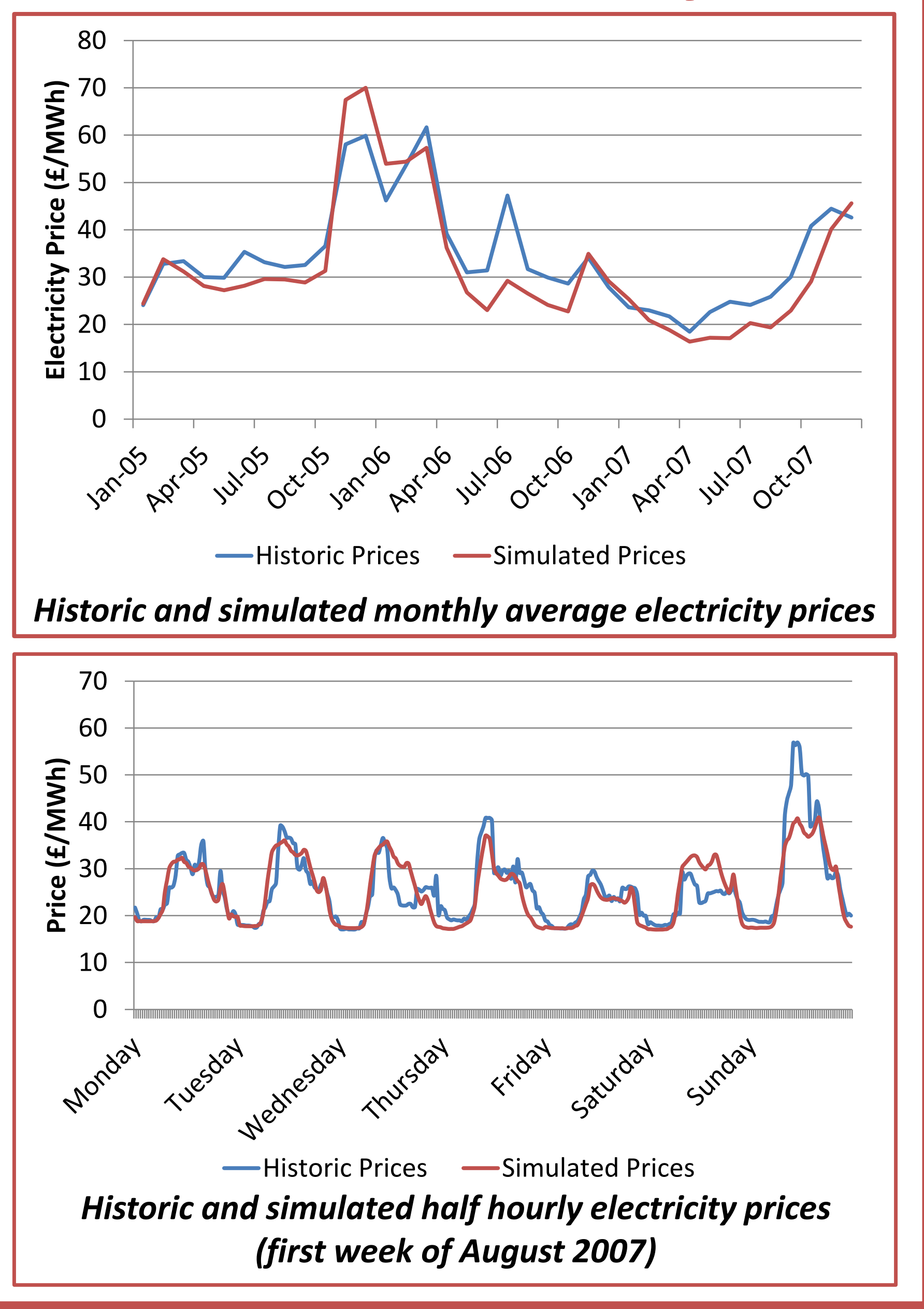

\section{RESULTS}

\section{Input Data}

The National Grid Gone Green Future Energy Scenario results are presented. This is the
scenario with the most power
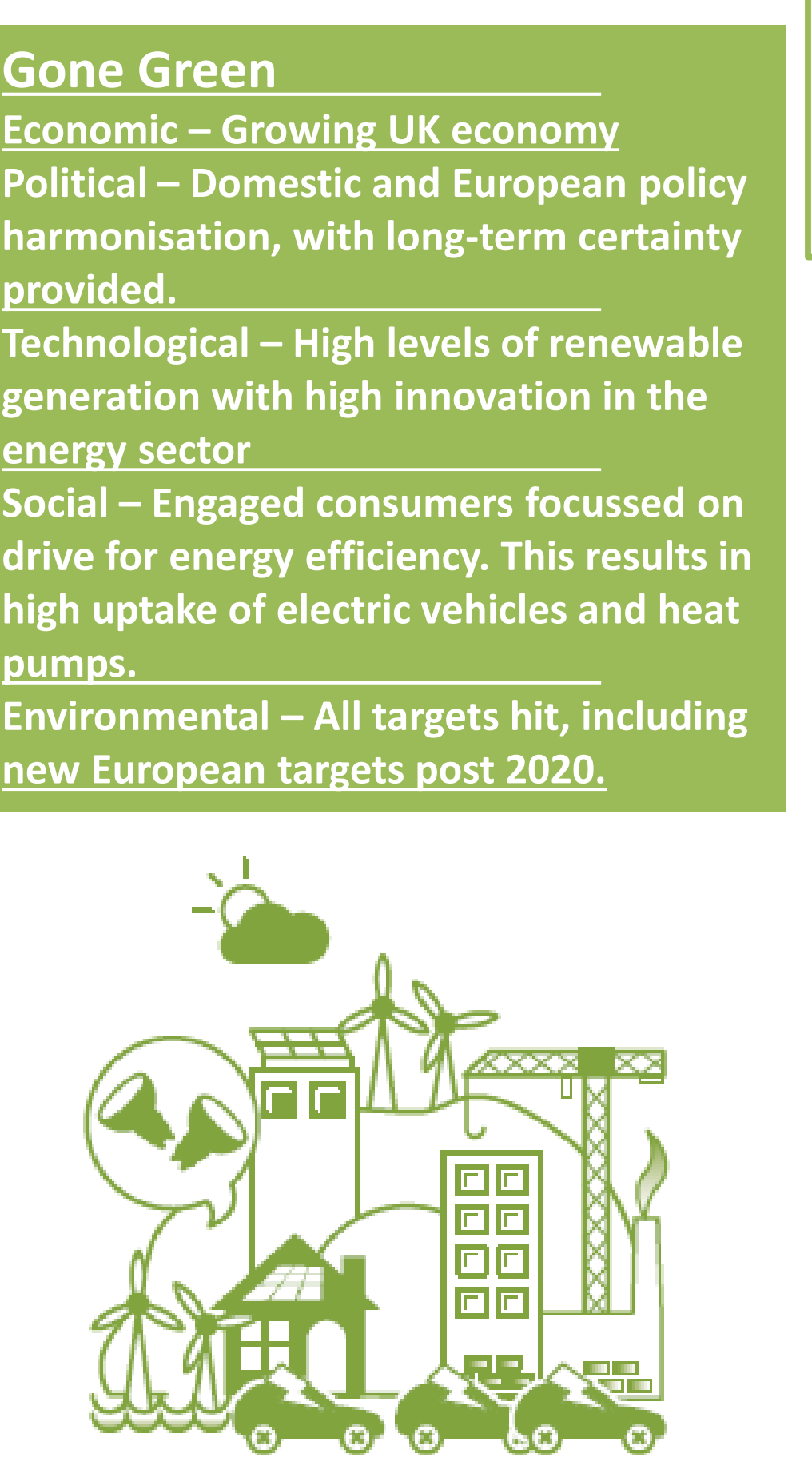
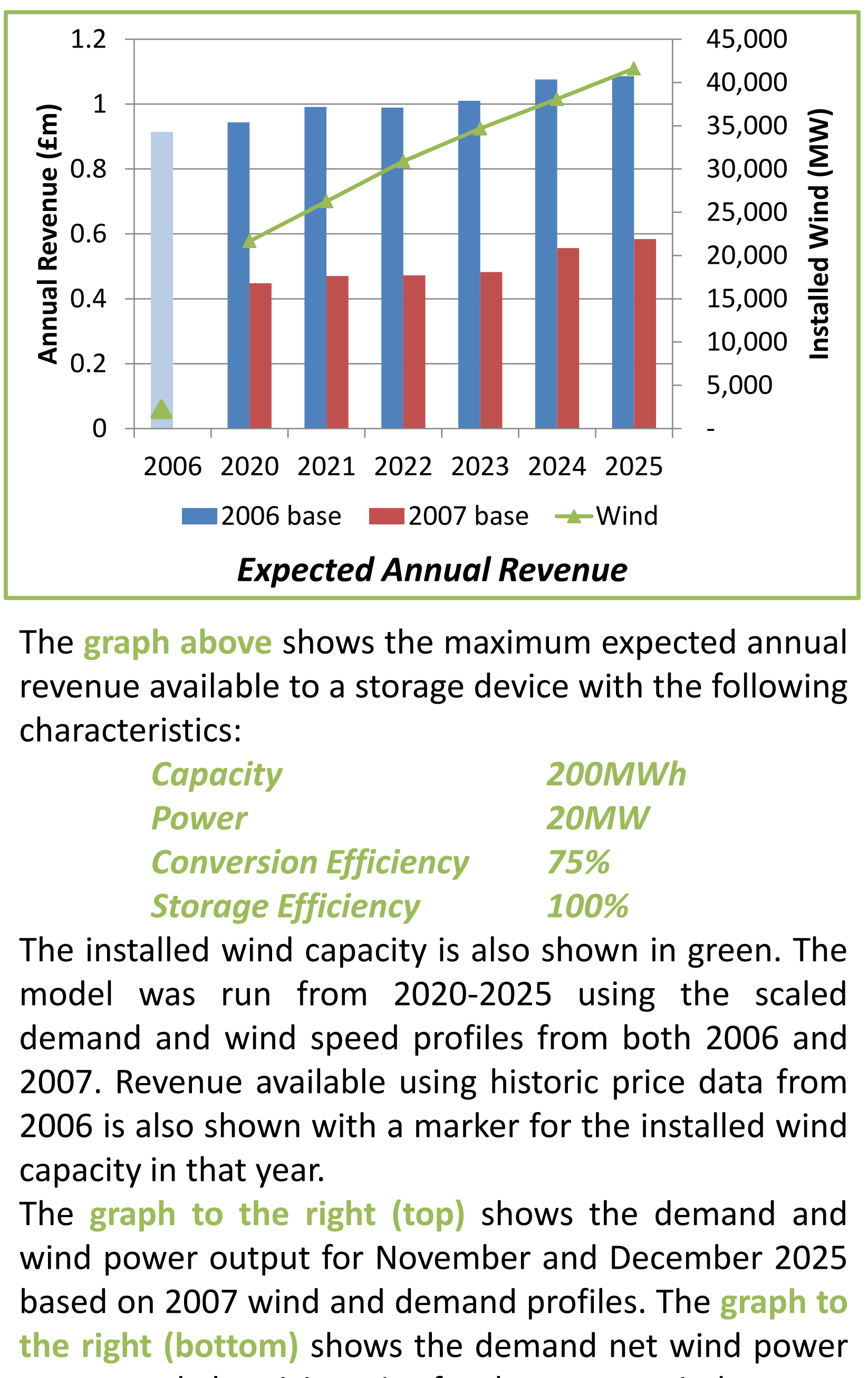
output and electricity price for the same neriod.

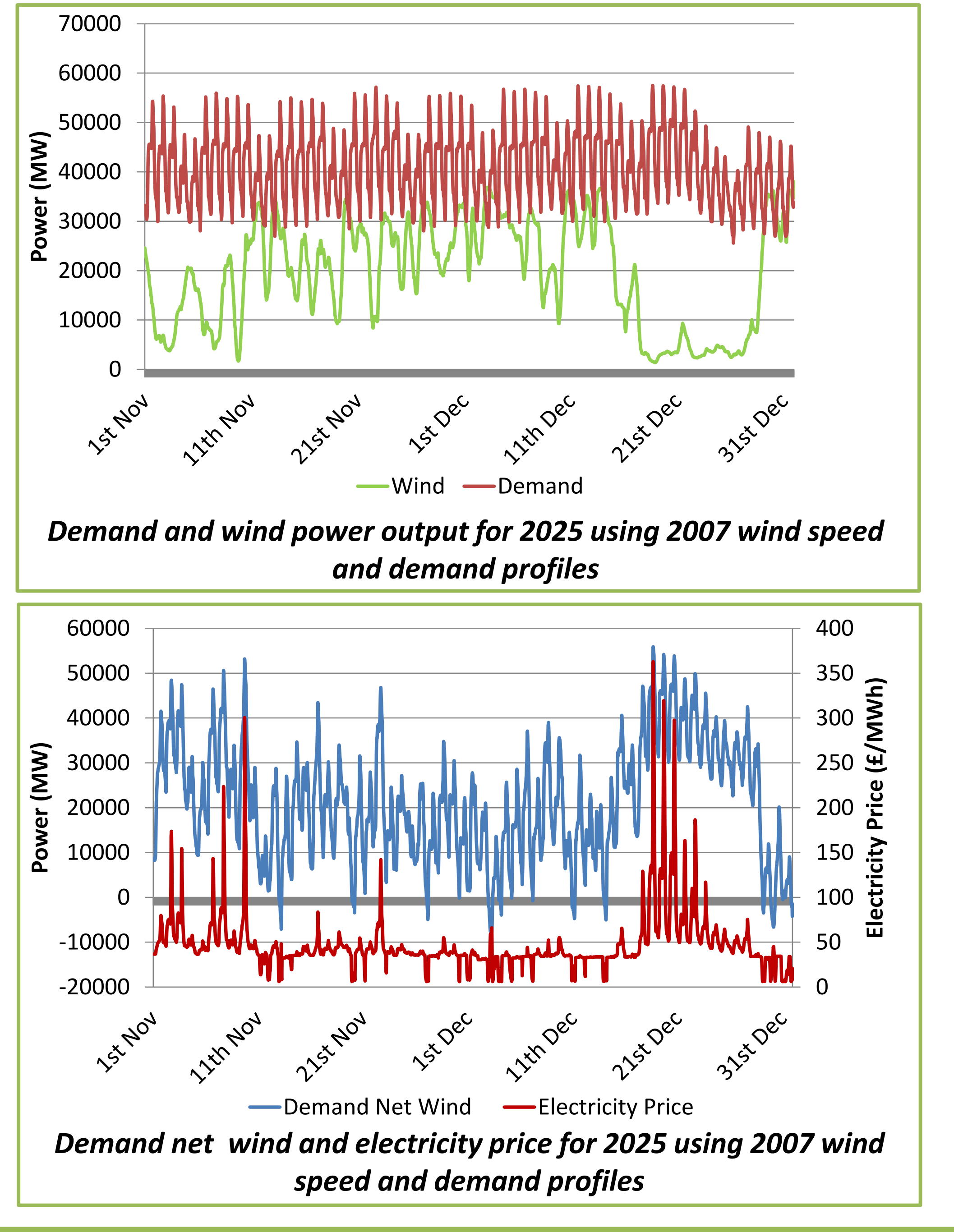
speed and demand profiles

\section{CONCLUSIONS}

Prices

Increased wind power caused higher price vola
_ $\quad$ Multiple instances of supressed prices.

Reduced frequency of inflated prices.

Other factors, including thermal plant capacity and fuel prices, maintain significant influence on electricity prices.

\section{Revenue}

From 2020-2025 there were small increases in annual revenue.

For the same period installed wind capacity increased from $\sim 20 \mathrm{GW}$ to $\sim 40 \mathrm{GW}$. Using an alten
almost $50 \%$.

- More wind led to higher variation in revenue between years.

More opportunity to charge when

Less opportunity to discharge when prices are inflated.

Further Work

Improve inputs

Demand behaviour

Consider alternative future energy scenarios

3. Investigate changes to future electricity price formation

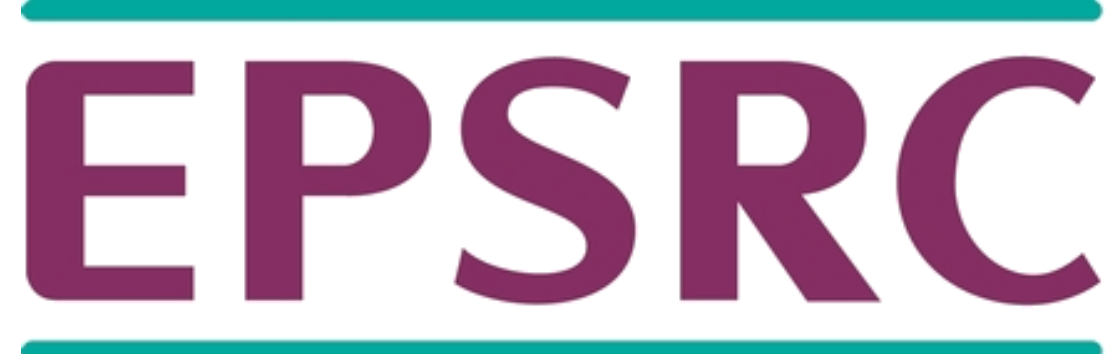

Engineering and Physical Sciences Research Council 


\section{Catch-22: Could Wind Power Reduce Commercial Opportunities for Electrical Energy Storage?}

Anna Dunbar; a.dunbar@ed.ac.uk

Supervisors: Professor Gareth Harrison \& Professor Robin Wallace

School of Engineering, The University of Edinburgh, Edinburgh EH9 3DW, UK

\section{ELECTRICITY STORAGE \\ ELECTRICITY PRICES \\ ARBITRAGE \\ FUTURE ENERGY SCENARIOS}

WIND POWER

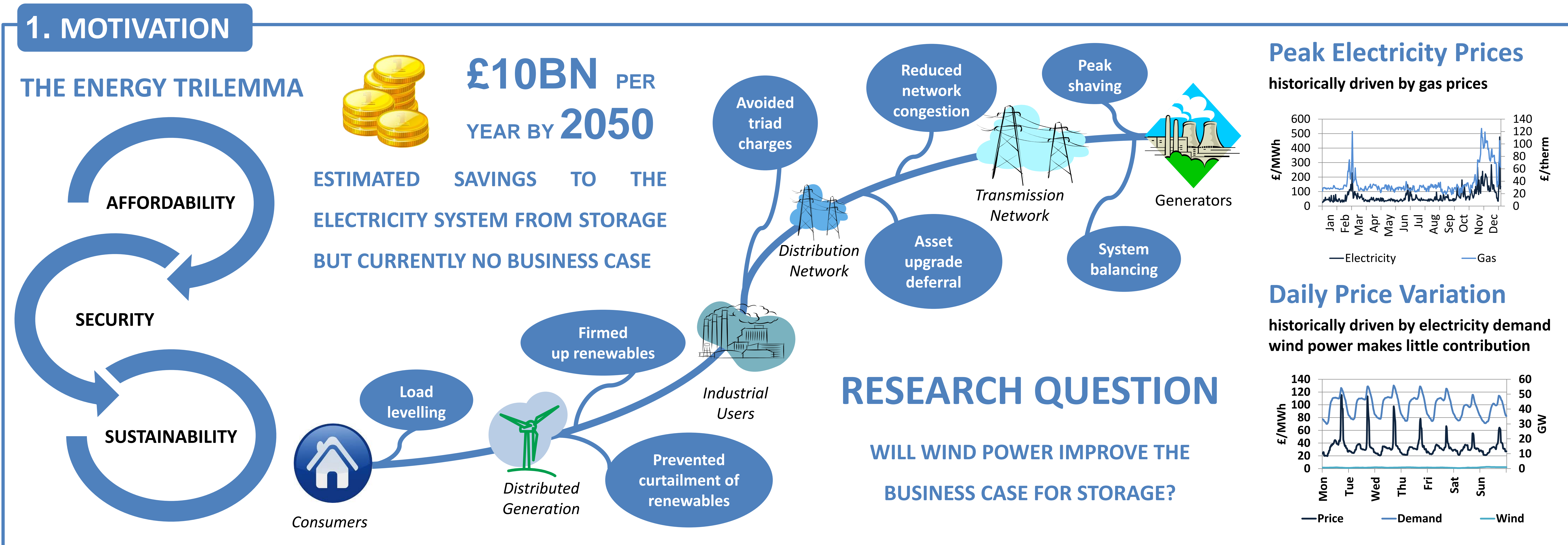

\section{MODEL}

A fundamental price model,
assuming perfect competition
in the electricity market, was
created to simulate electricity
prices with increasing
installed wind capacity.
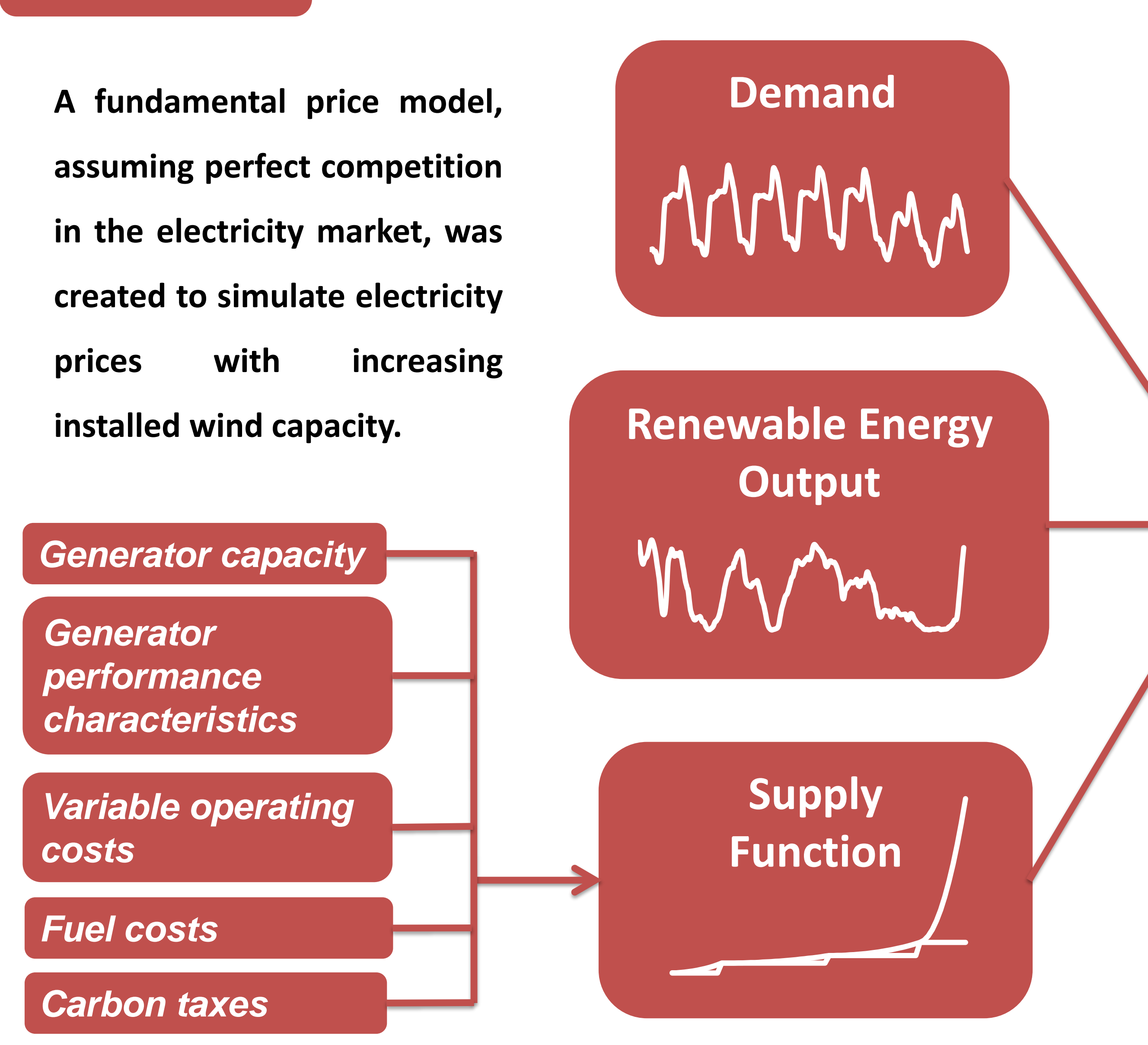

\section{RESULTS}

\section{GW}

coulo REDUCE AвBтRAG
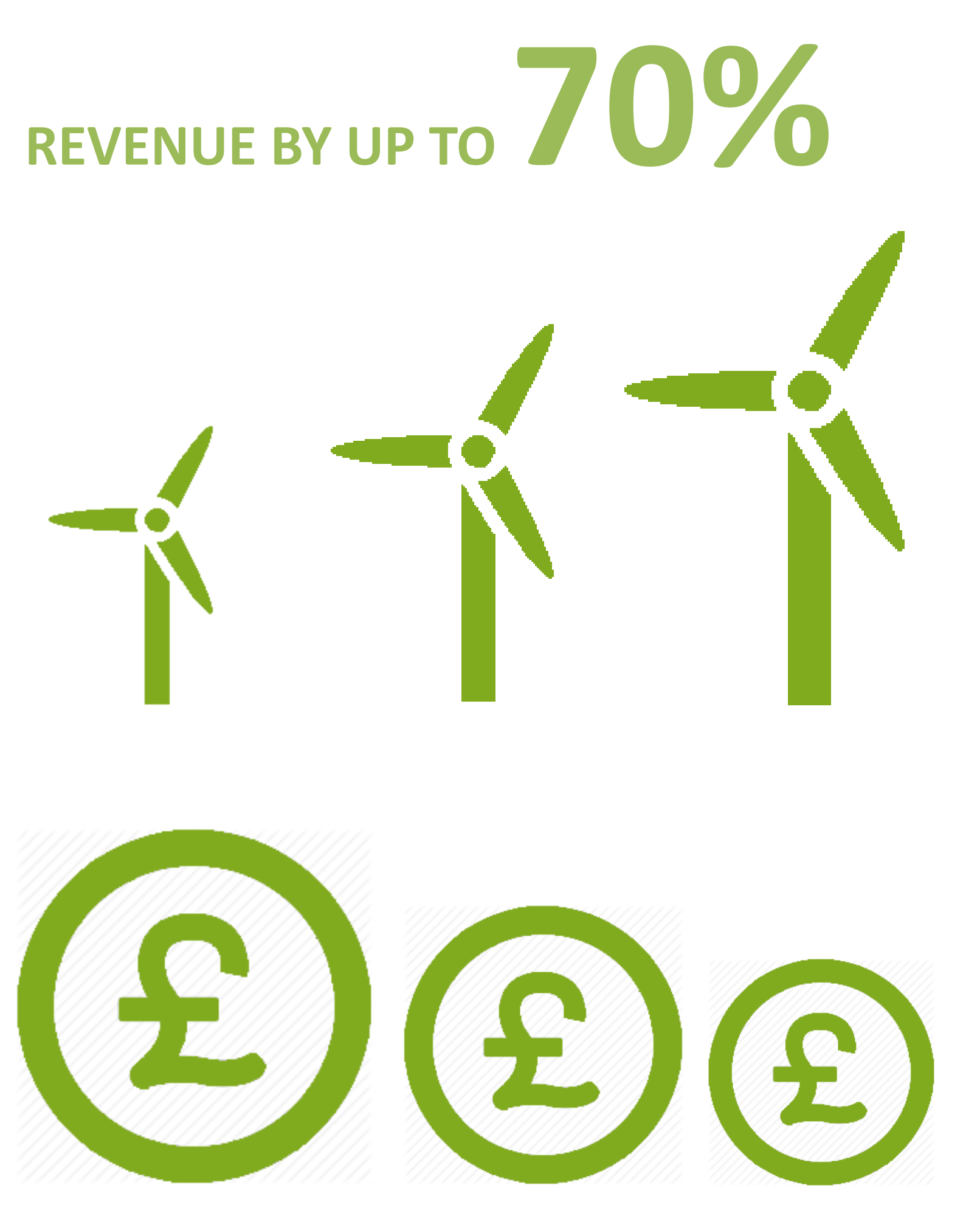

Why does this happen?

Wind power has low marginal generation costs and so increased output reduces daily price peaks reducing the price spread and opportunities for arbitrage.

\section{What does this mean?}

With more installed wind capacity:

there are fewer cycles and operations are more constrained by storage capacity than by charging rate;

there is less variation in electricity price, so revenue is more sensitive to conversion efficiency; and

electricity is stored over longer periods of time, so revenue is more sensitive to self-discharge.

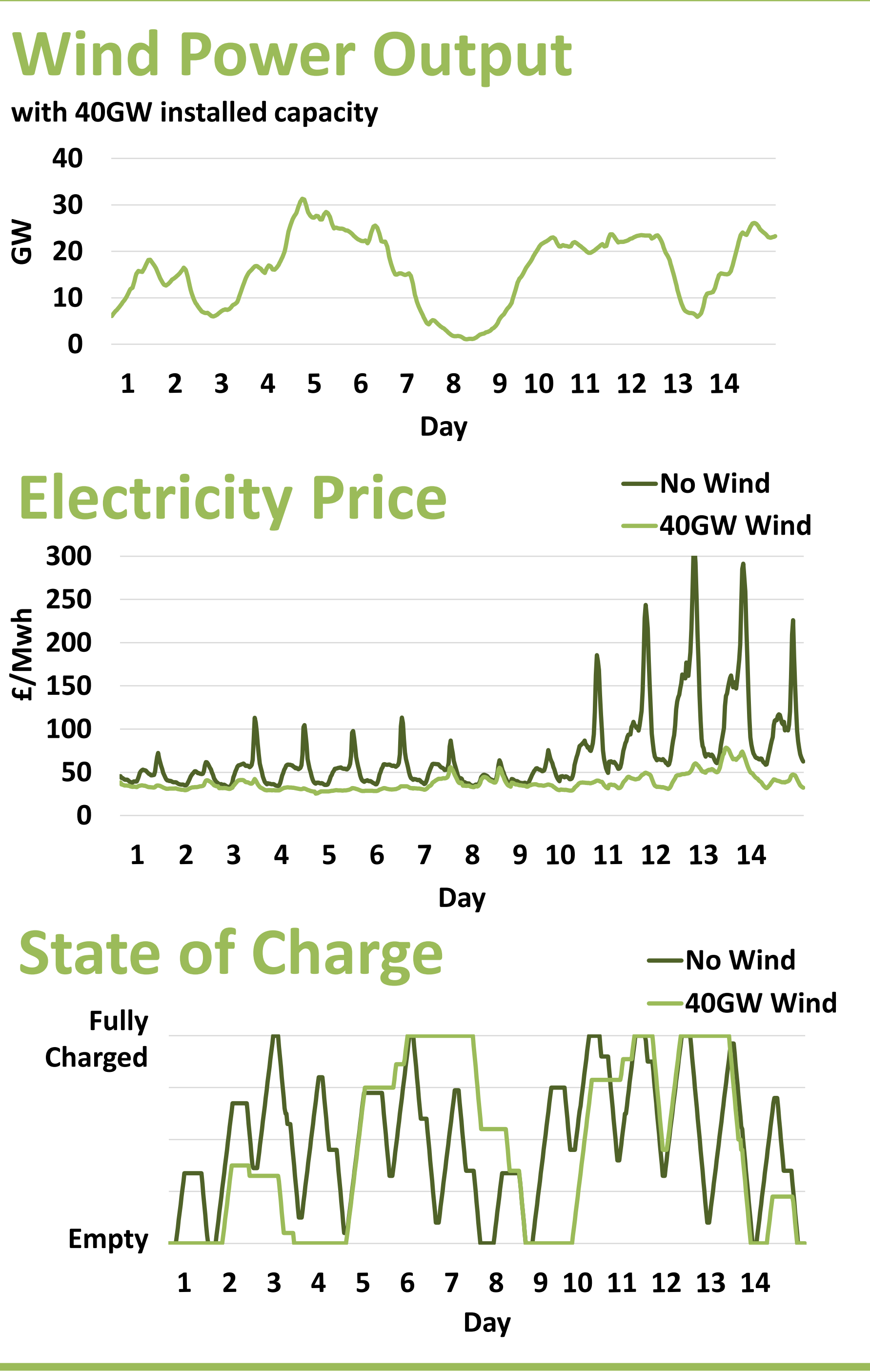

Storage

Revenue
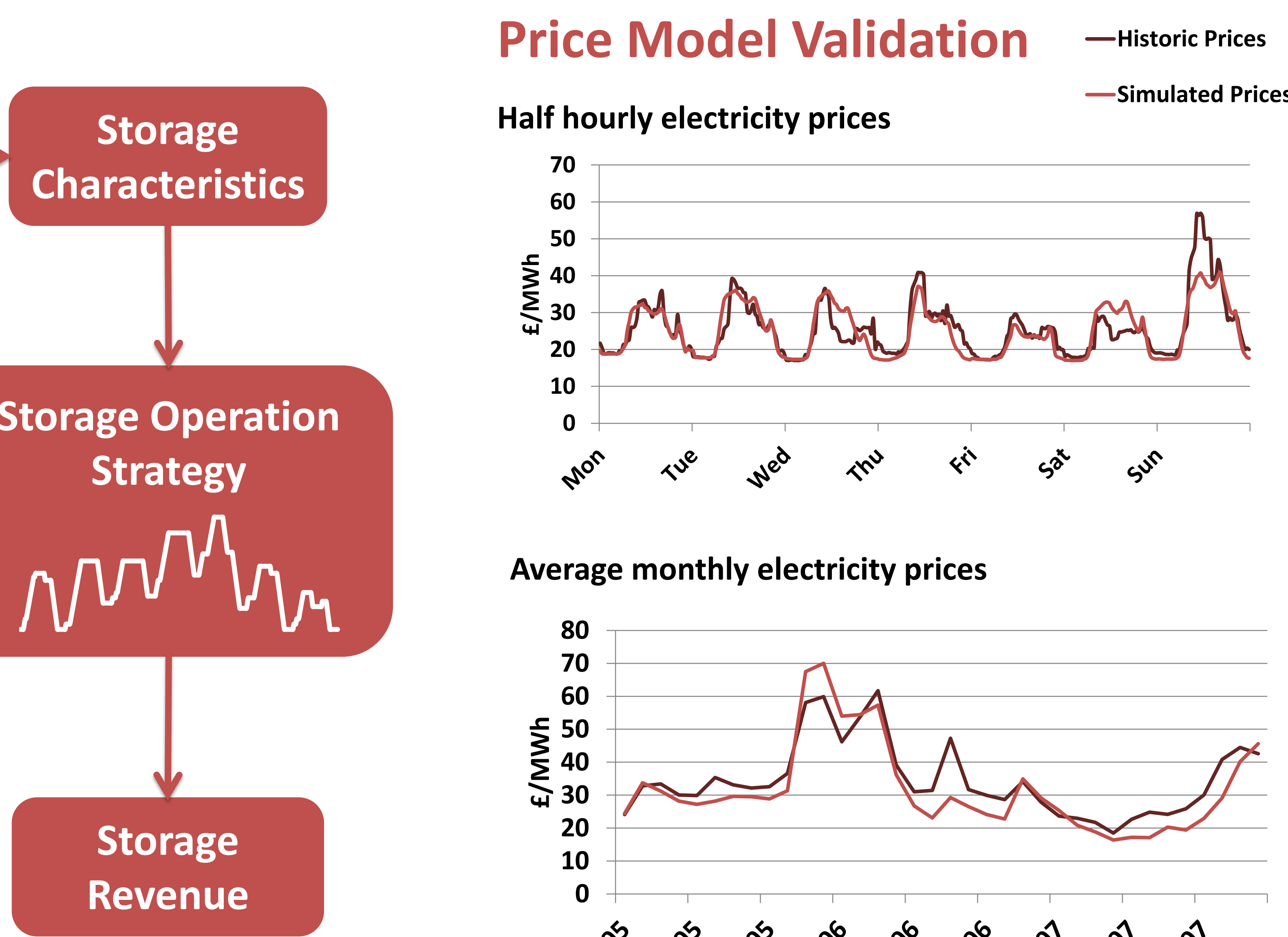

Average monthly electricity prices

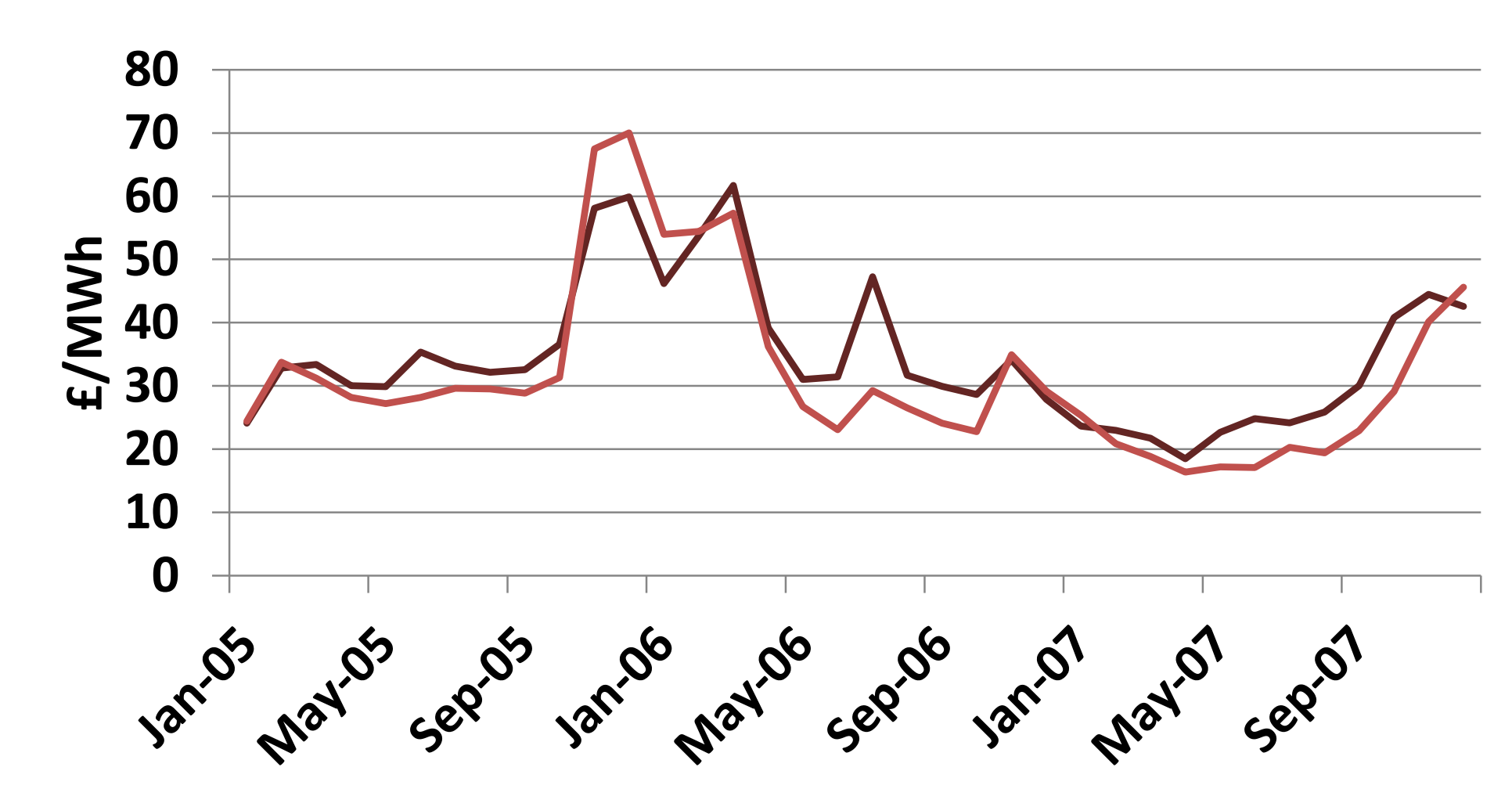

\section{CONCLUSIONS}

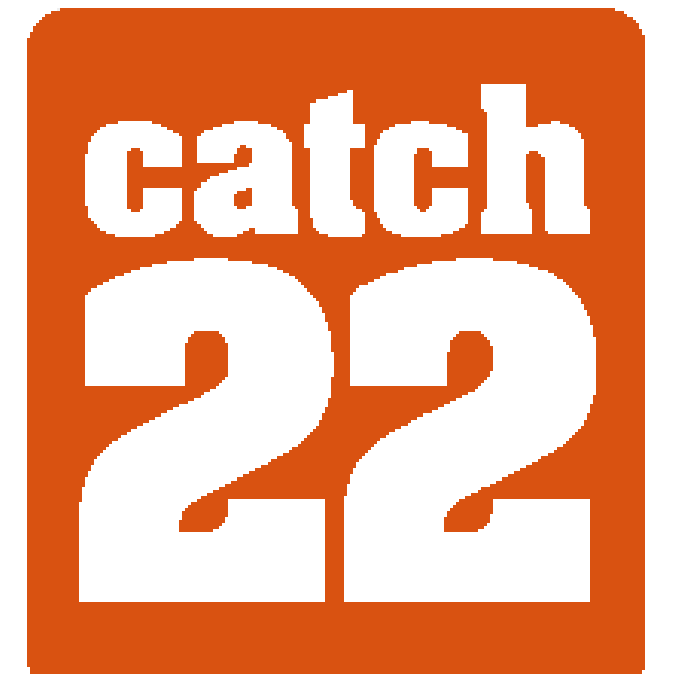

WIND POWER INCREASES THE

SYSTEM VALUE OF ENERGY

STORAGE BUT REDUCES ITS

COMMERCIAL VIABILITY

\section{POLICY IMPLICATIONS}

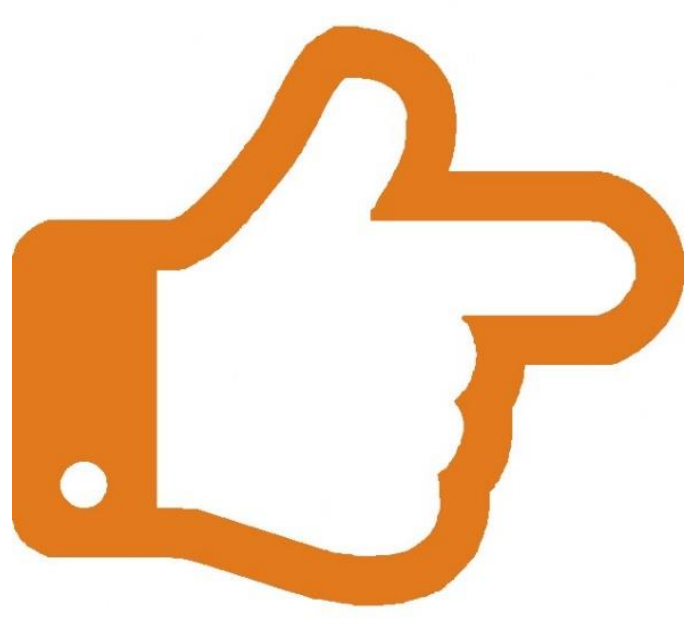

Evidence of a failure in the electricity
market in rewarding the true value of
storage. Without appropriate financial
rewards storage benefits will not be

delivered and, despite

potentially being more

costly in the long term,

conventional fossil fuel

technologies will prevail.

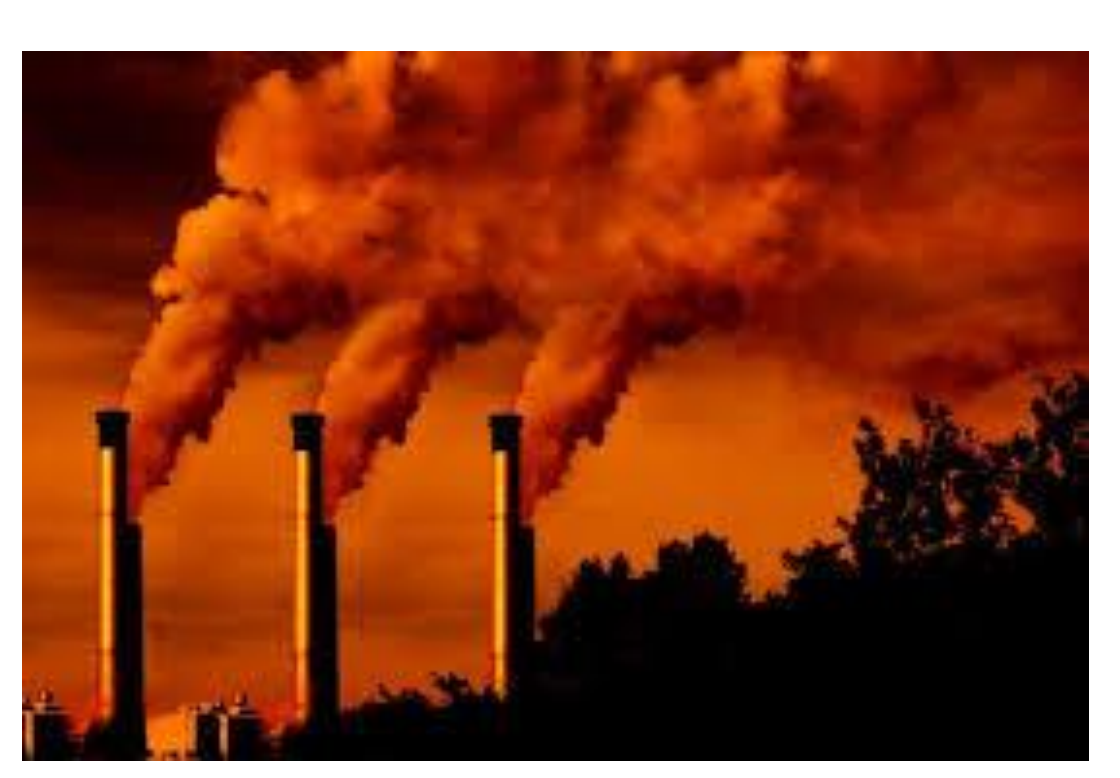

Key References 